\title{
Probing the chemical kinetics of minimalist functional group (MFG) gasoline surrogates
}

Bogdan Dragoș Ilieș ${ }^{1}$, Muralikrishna Khandavilli ${ }^{1}$, Goutham Kukkadapu ${ }^{2}$, Yang Li ${ }^{1}$, S. W. Wagnon $^{2}$, Abdul Gani Abdul Jameel ${ }^{3}$, S. Mani Sarathy ${ }^{1 *}$

${ }^{1}$ Clean Combustion Research Center, King Abdullah University of Science and Technology, Physical Sciences and Engineering Division, Thuwal, Saudi Arabia

${ }^{2}$ Lawrence Livermore National Laboratory, Livermore, CA, USA

${ }^{3}$ Department of Chemical Engineering, King Fahd University of Petroleum \& Minerals, Dhahran 31261, Saudi Arabia

\section{JSR stable products}
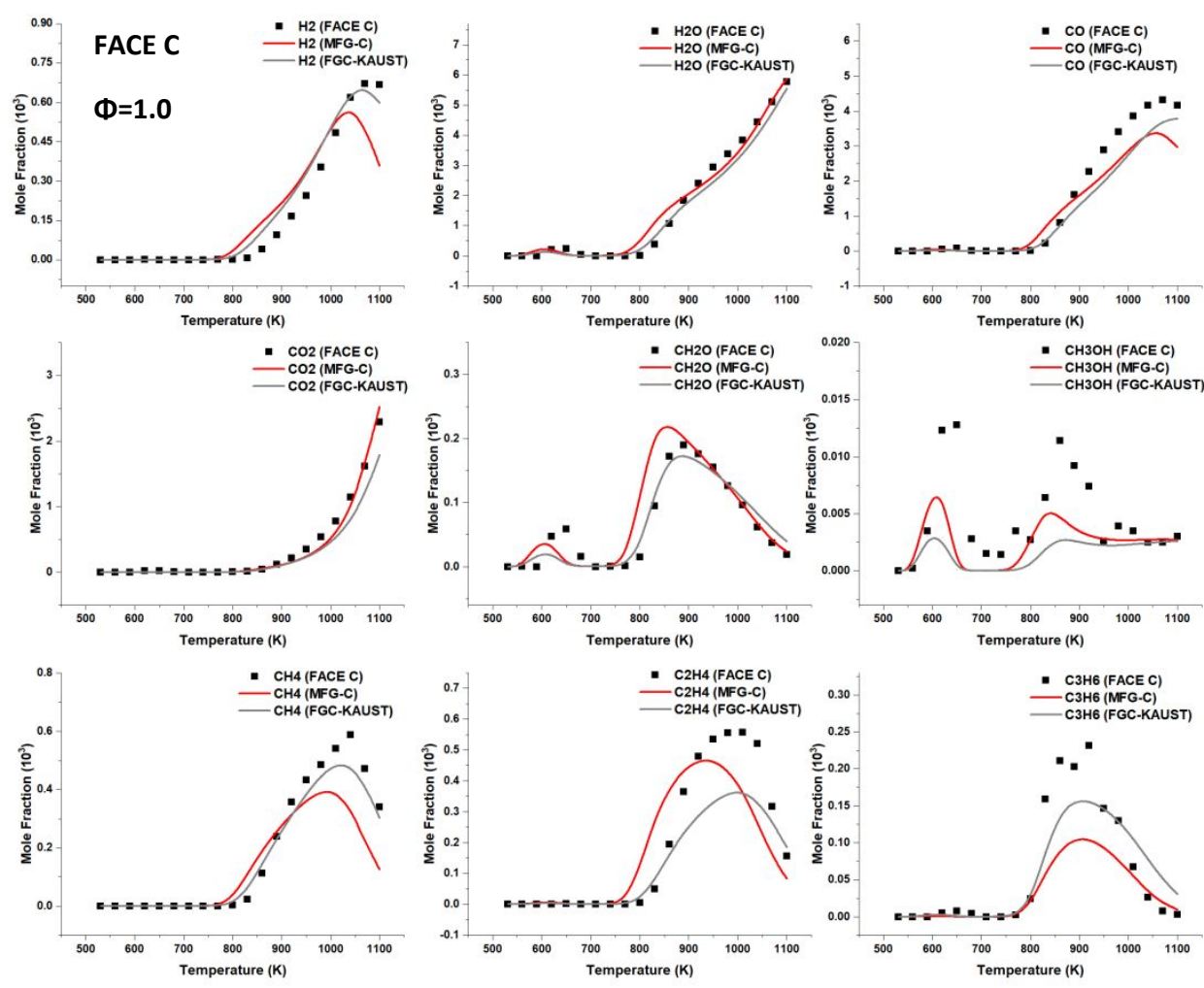

Fig. S1: JSR data for FACE C at 10 atm for stoichiometric $(\phi=1.0)$ conditions 

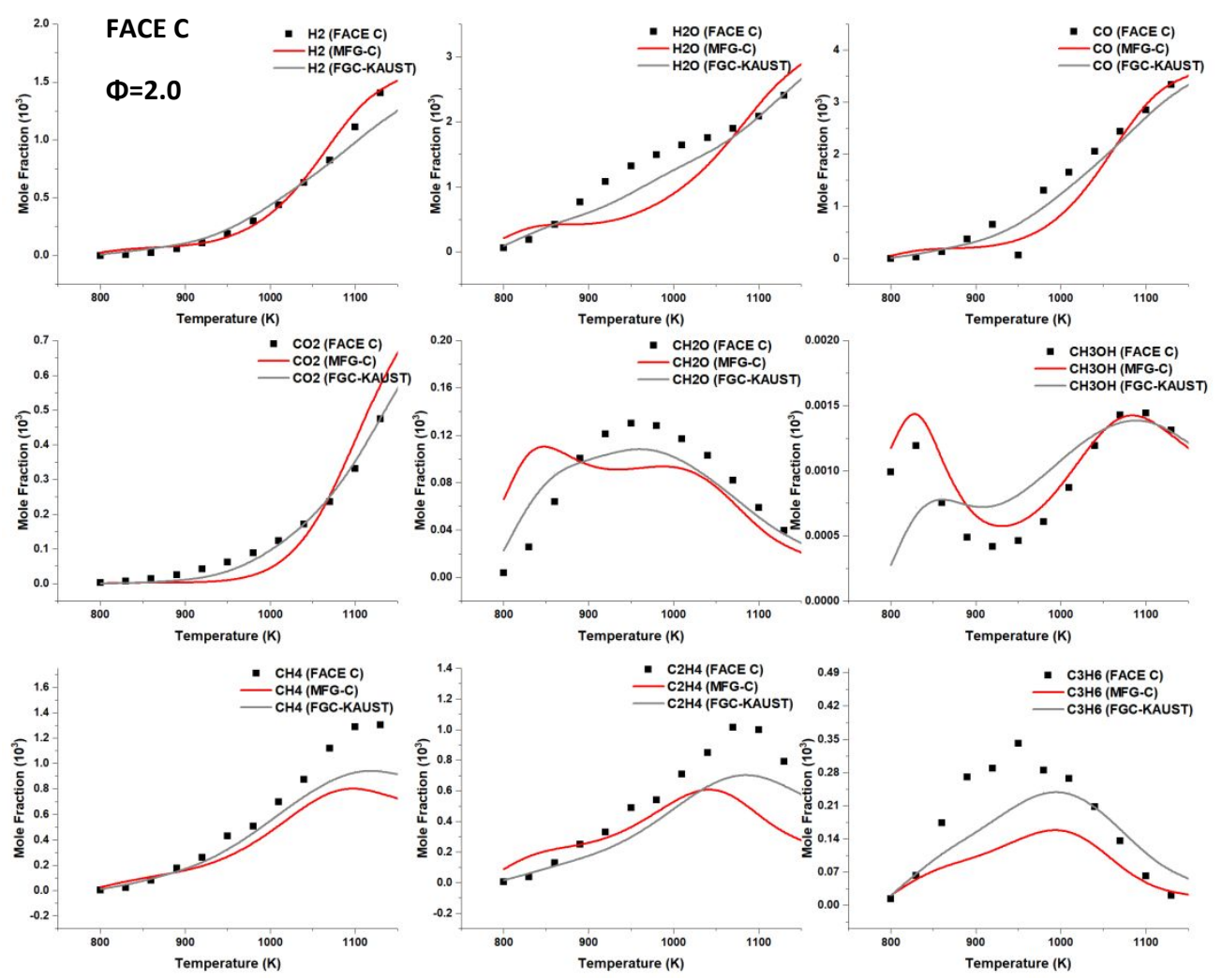

Fig. S2.: JSR data for FACE C at $10 \mathrm{~atm}$ for fuel-rich $(\phi=2.0)$ conditions
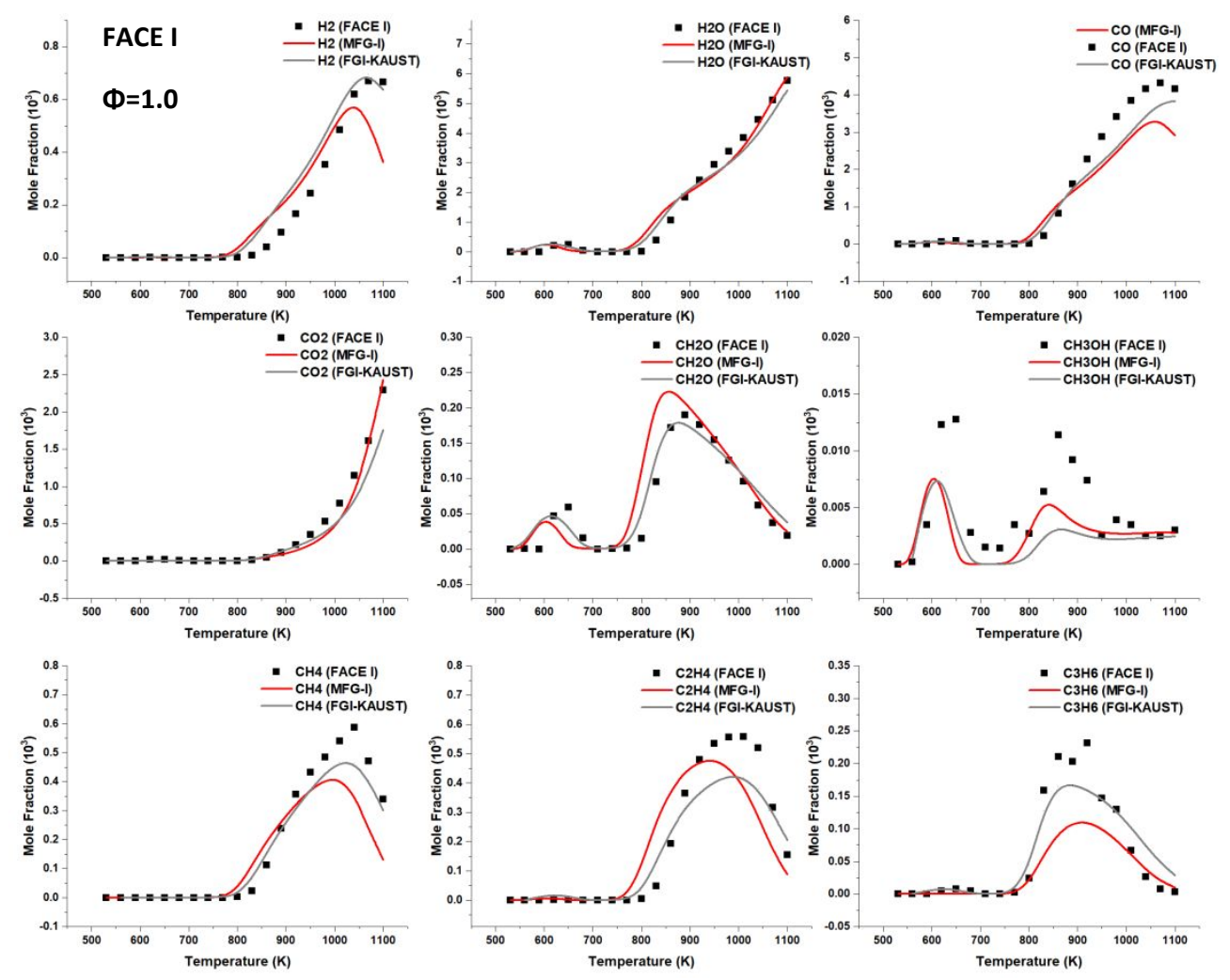

Fig. S3: JSR data for FACE I at $10 \mathrm{~atm}$ for stoichiometric $(\phi=1.0)$ conditions 

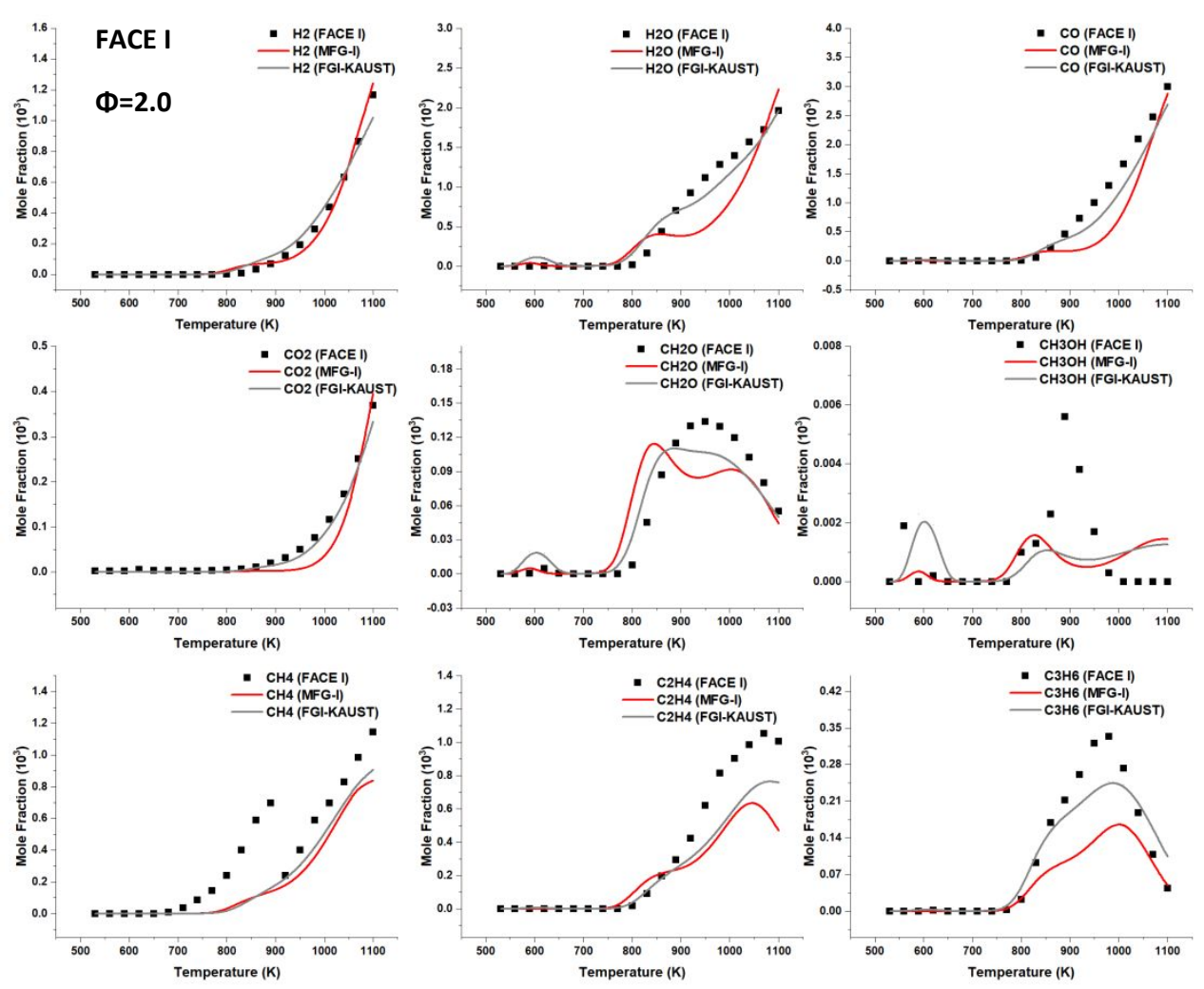

Fig. S4: JSR data for FACE I at 10 atm for fuel-rich $(\phi=2.0)$ conditions
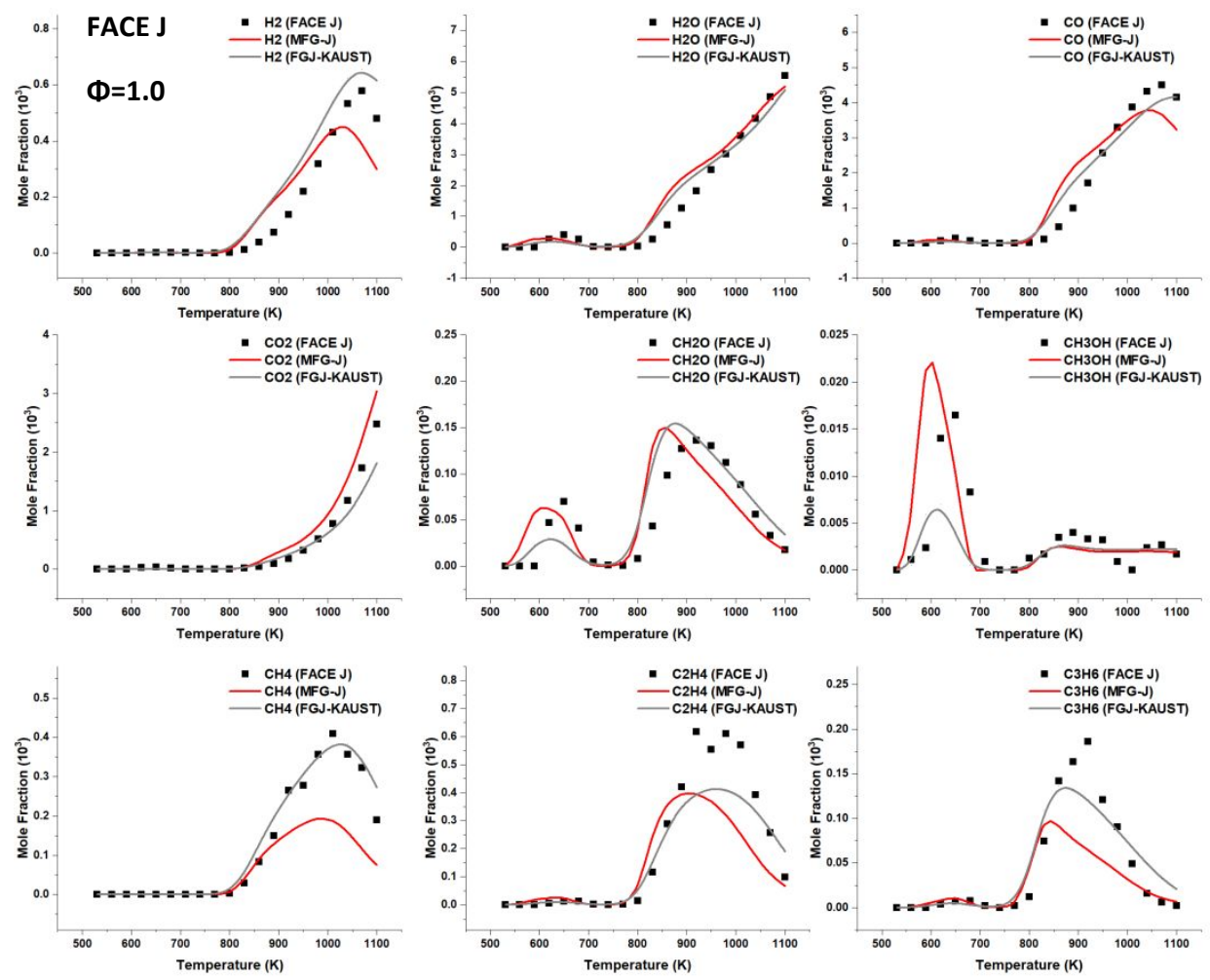

Fig. S5: JSR data for FACE J at $10 \mathrm{~atm}$ for stoichiometric $(\phi=1.0)$ conditions 

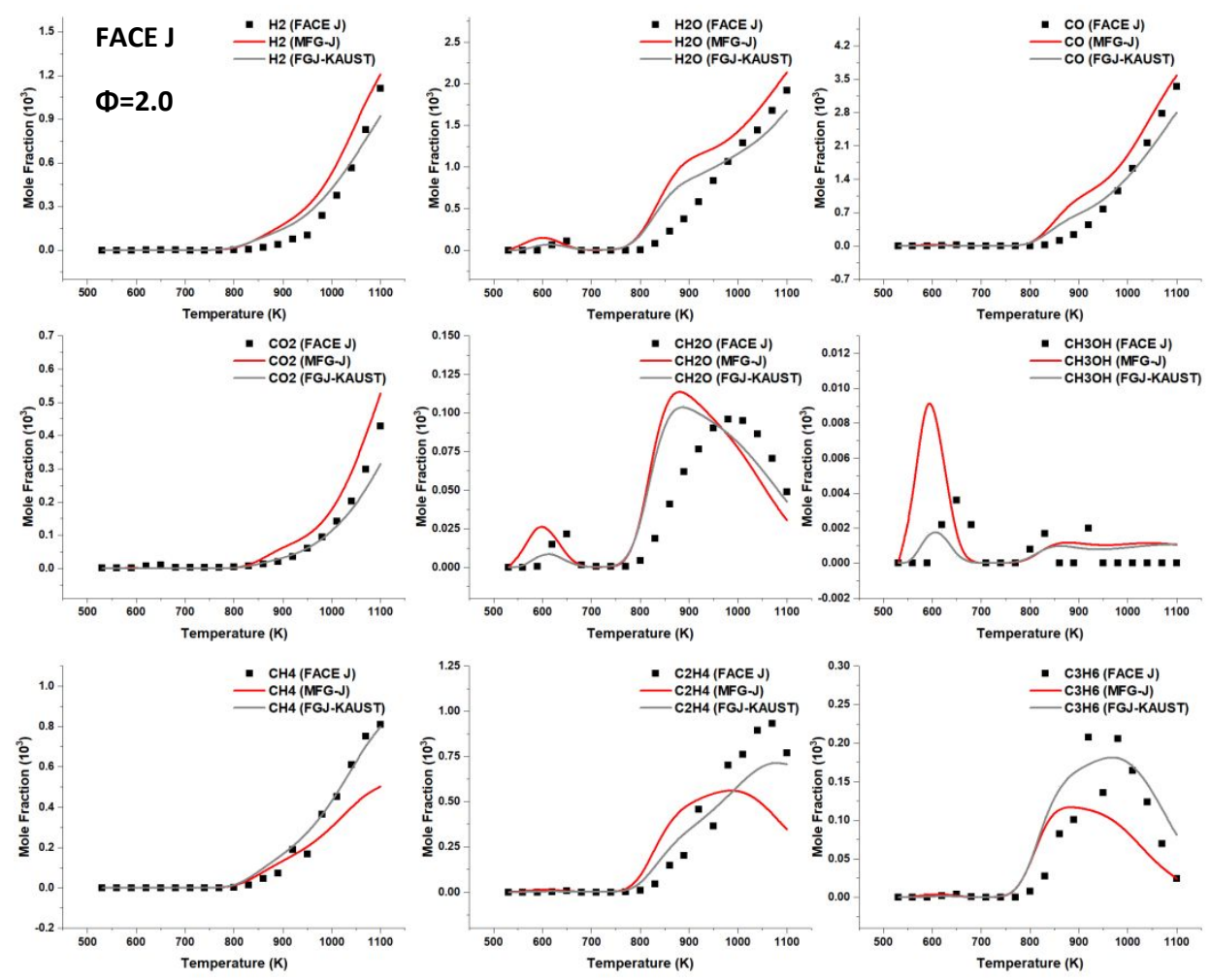

Fig. S6: JSR data for FACE J at $10 \mathrm{~atm}$ for fuel-rich $(\phi=2.0)$ conditions

\section{JSR radical products}
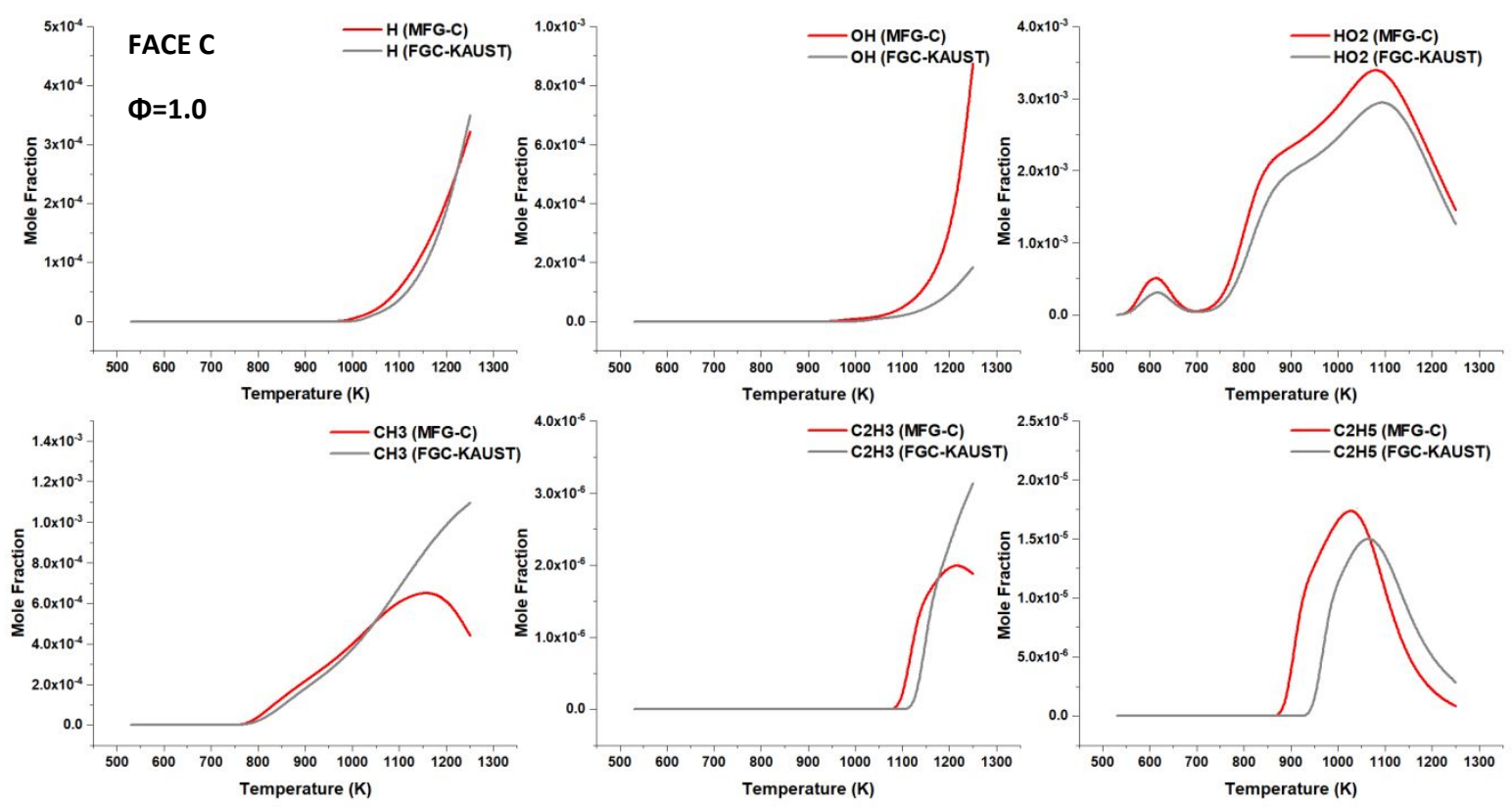

Fig. S7: JSR data for FACE C at 10 atm for stoichiometric $(\phi=1.0)$ conditions 


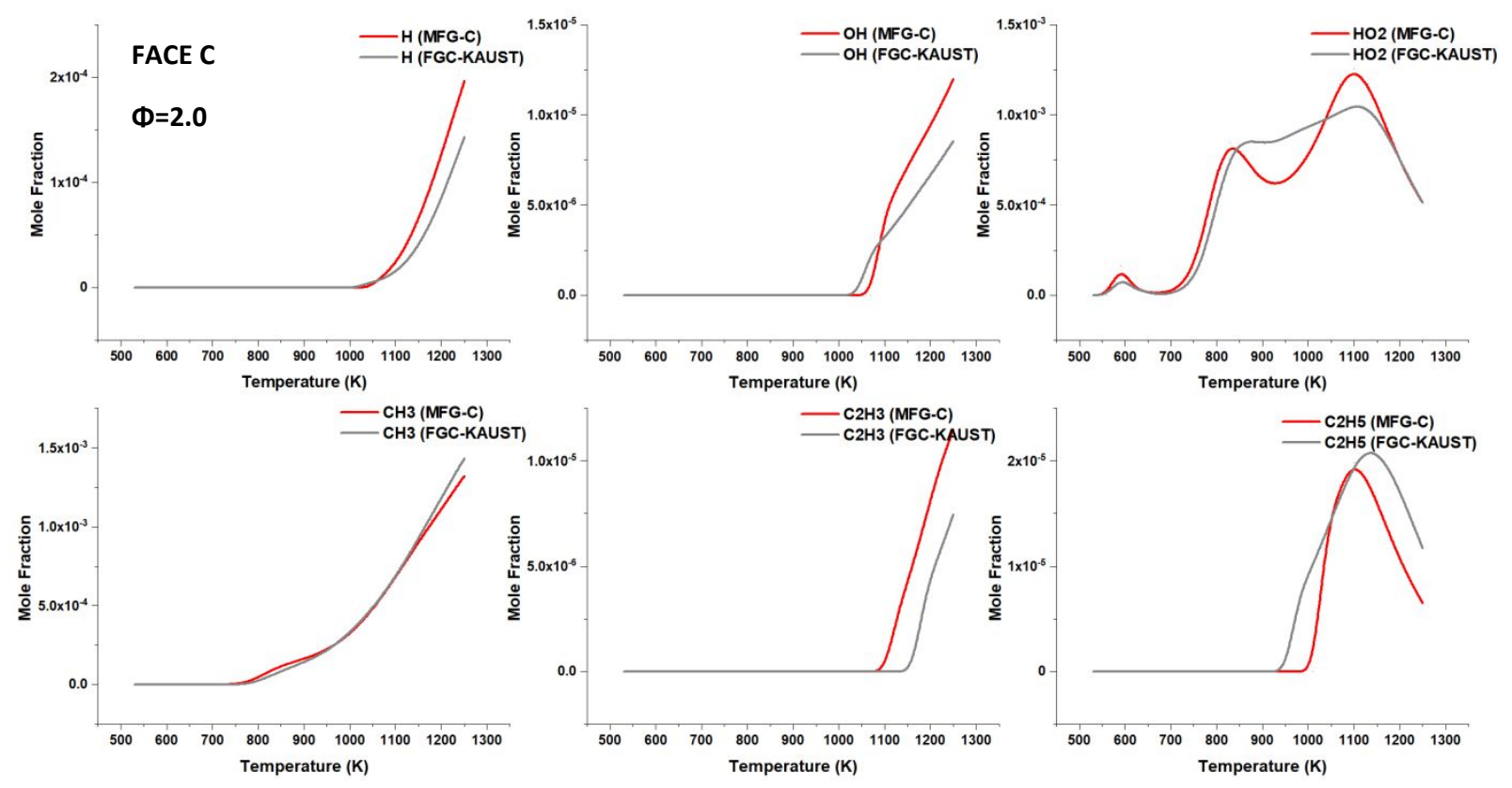

Fig. S8: JSR data for FACE C at $10 \mathrm{~atm}$ for fuel-rich $(\phi=2.0)$ conditions

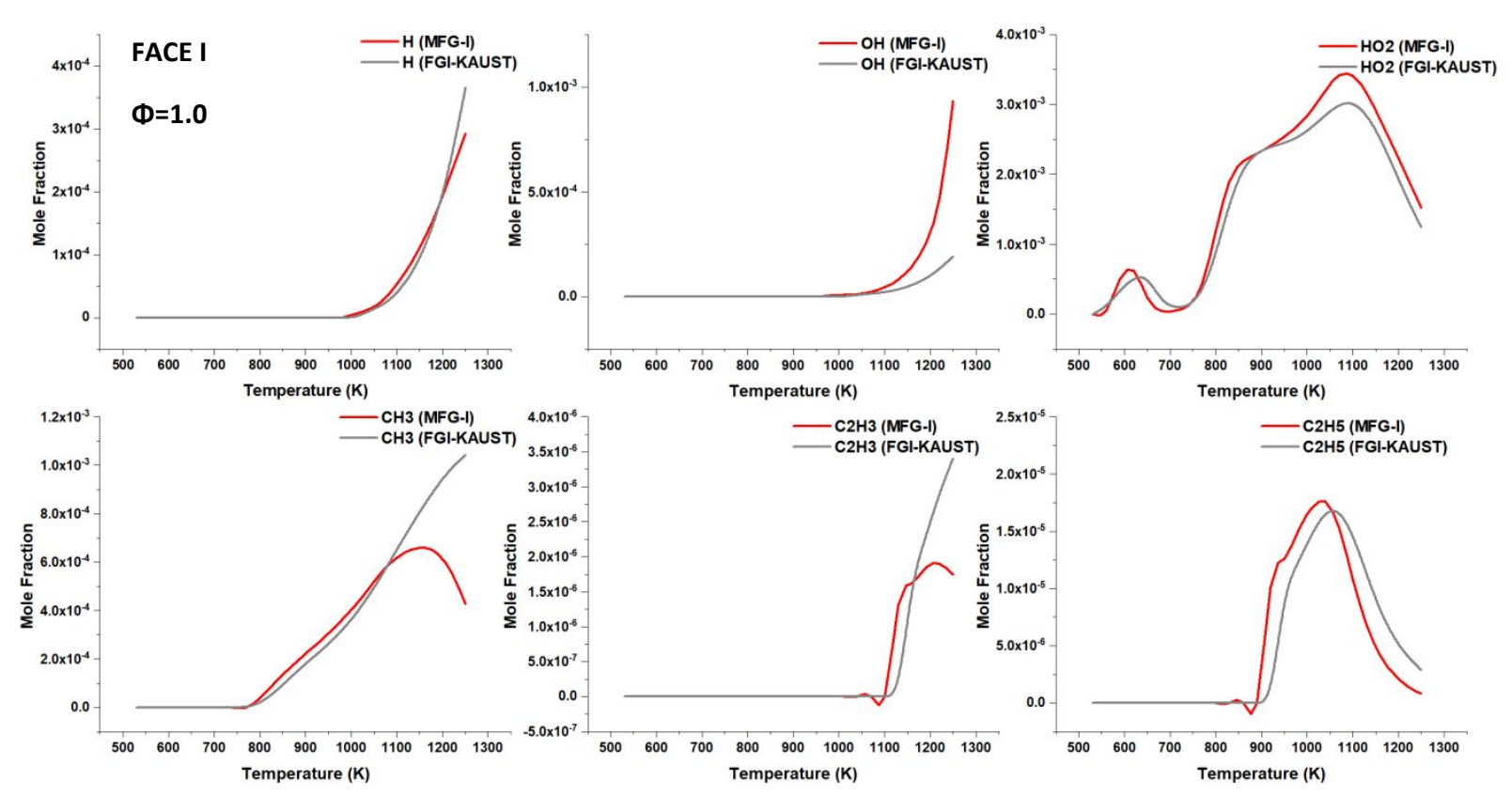

Fig. S9: JSR data for FACE I at $10 \mathrm{~atm}$ for stoichiometric $(\phi=1.0)$ conditions 


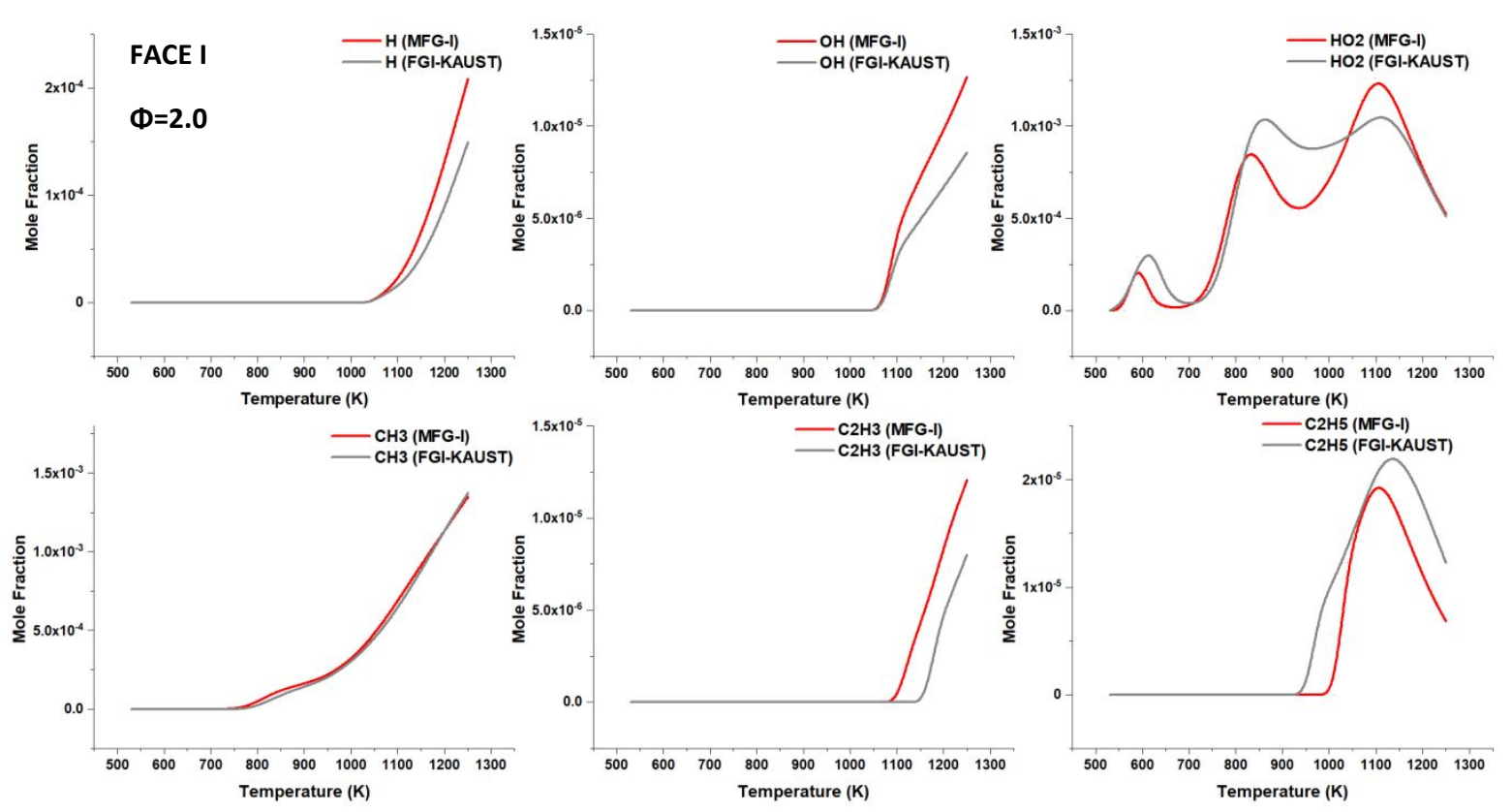

Fig. S10: JSR data for FACE I at 10 atm for fuel-rich $(\phi=2.0)$ conditions
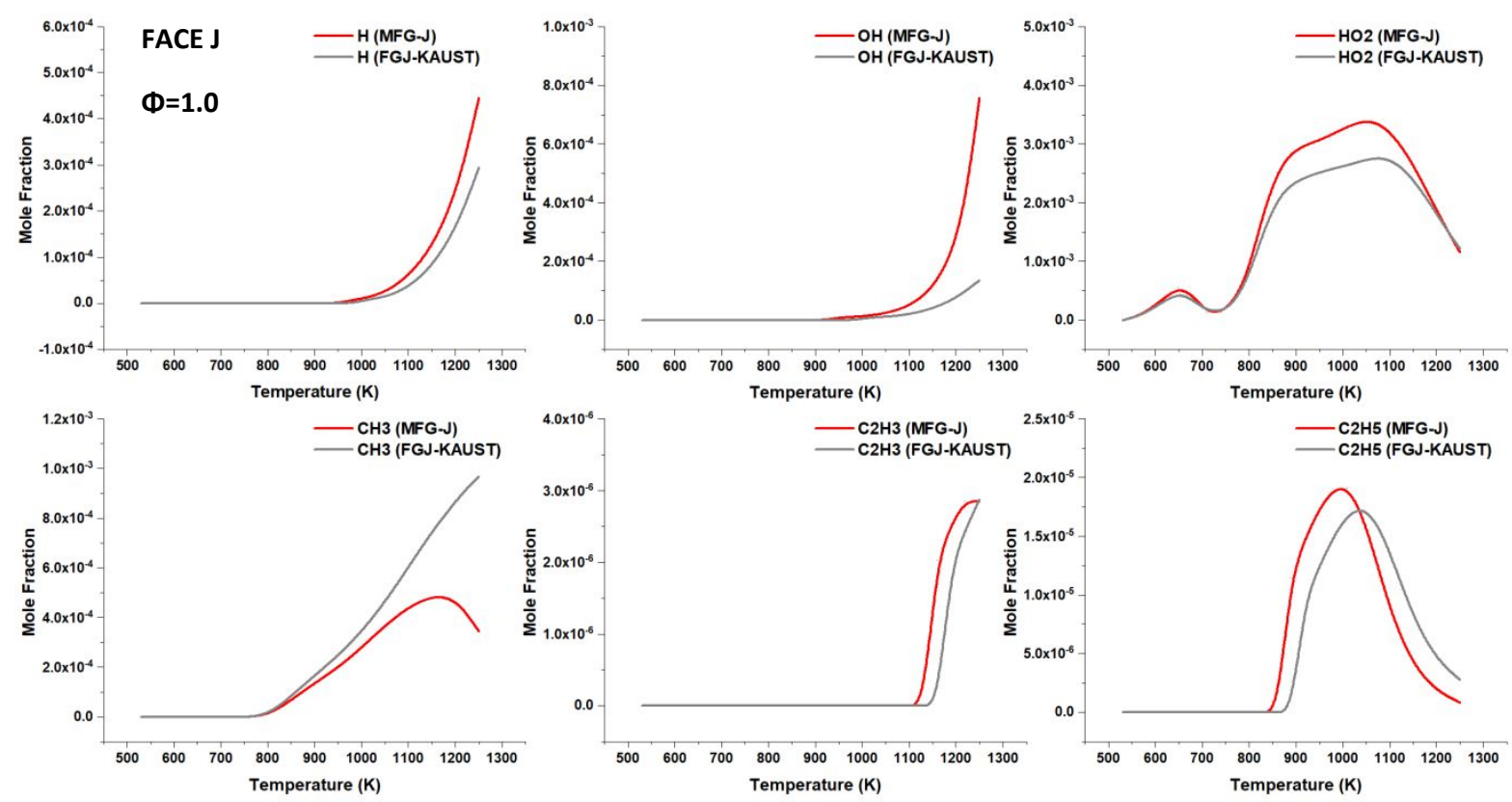

Fig. S11: JSR data for FACE J at $10 \mathrm{~atm}$ for stoichiometric $(\phi=1.0)$ conditions 

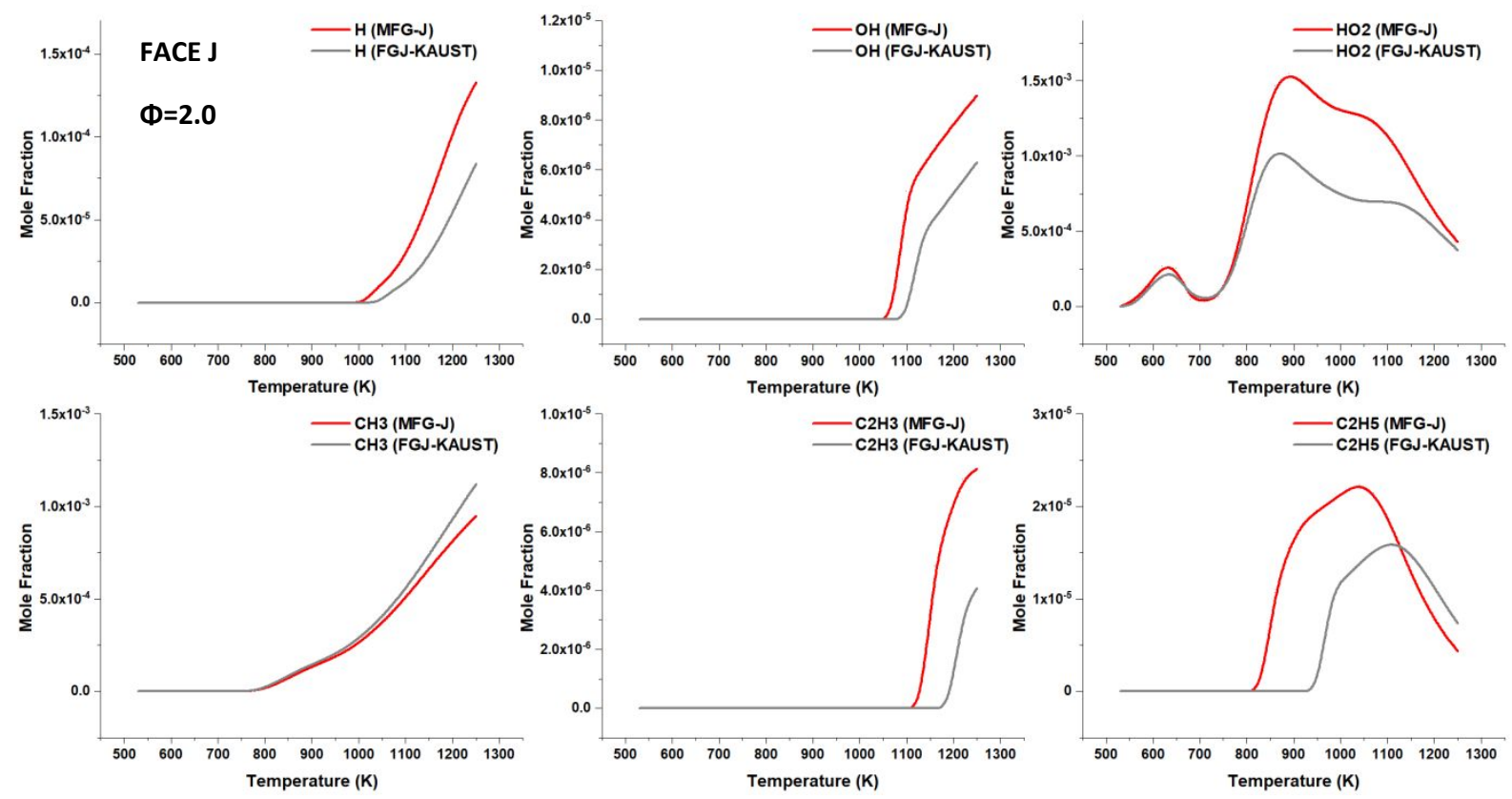

Fig. S12: JSR data for FACE J at 10 atm for fuel-rich $(\phi=2.0)$ conditions 


\section{Glossary of species}

\begin{tabular}{|c|c|c|c|}
\hline No. & MECH_NAME & SMILES & Stdinchl \\
\hline 1 & $\mathrm{H}$ & {$[\mathrm{H}]$} & $\operatorname{lnChl}=1 \mathrm{~S} / \mathrm{H}$ \\
\hline 2 & $\mathrm{~N}$ & {$[\mathrm{~N}]$} & $\operatorname{lnChl}=1 \mathrm{~S} / \mathrm{N}$ \\
\hline 3 & $\mathrm{NH} 2 \mathrm{OH}$ & ON & $\operatorname{lnChl}=1 \mathrm{~S} / \mathrm{H} 3 \mathrm{NO} / \mathrm{c} 1-2 / \mathrm{h} 2 \mathrm{H}, 1 \mathrm{H} 2$ \\
\hline 4 & $\mathrm{~N} 2 \mathrm{H} 2$ & $\mathrm{~N}=\mathrm{N}$ & $\operatorname{lnChl}=1 \mathrm{~S} / \mathrm{H} 2 \mathrm{~N} 2 / \mathrm{c} 1-2 / \mathrm{h} 1-2 \mathrm{H}$ \\
\hline 5 & $\mathrm{H} 2$ & {$[\mathrm{H}][\mathrm{H}]$} & $\operatorname{lnChl}=1 \mathrm{~S} / \mathrm{H} 2 / \mathrm{h} 1 \mathrm{H}$ \\
\hline 6 & $\mathrm{NH}$ & {$[\mathrm{NH}]$} & InChl=1S/HN/h1H \\
\hline 7 & NO2 & {$[\mathrm{N}+](=\mathrm{O})[\mathrm{O}-]$} & InChl=1S/NO2/c2-1-3 \\
\hline 8 & $\mathrm{~N} 2 \mathrm{H} 3$ & $\mathrm{~N}[\mathrm{NH}]$ & $\operatorname{lnChl}=1 \mathrm{~S} / \mathrm{H} 3 \mathrm{~N} 2 / \mathrm{c} 1-2 / \mathrm{h} 1 \mathrm{H}, 2 \mathrm{H} 2$ \\
\hline 9 & 0 & {$[0]$} & $\operatorname{lnChl}=1 \mathrm{~S} / 0$ \\
\hline 10 & $\mathrm{NH} 2$ & [NH2] & $\ln C h \mathrm{l}=1 \mathrm{~S} / \mathrm{H} 2 \mathrm{~N} / \mathrm{h} 1 \mathrm{H} 2$ \\
\hline 11 & $\mathrm{HNO2}$ & 01NO1 & InChl=1S/HNO2/c1-2-3-1/h1H \\
\hline 12 & $\mathrm{~N} 2 \mathrm{H} 4$ & $\mathrm{NN}$ & $\operatorname{lnChl}=1 \mathrm{~S} / \mathrm{H} 4 \mathrm{~N} 2 / \mathrm{c1} 1-2 / \mathrm{h} 1-2 \mathrm{H} 2$ \\
\hline 13 & $\mathrm{OH}$ & {$[\mathrm{OH}]$} & InChl=1S/HO/h1H \\
\hline 14 & $\mathrm{NH3}$ & $\mathrm{N}$ & InChl=1S/H3N/h1H3 \\
\hline 15 & HONO & $\mathrm{ON}=\mathrm{O}$ & InChl=1S/HNO2/c2-1-3/h(H,2,3) \\
\hline 16 & $\mathrm{~N} 2 \mathrm{O}$ & $\mathrm{N} N \mathrm{~N}=\mathrm{O}$ & $\operatorname{lnChl}=1 \mathrm{~S} / \mathrm{N} 2 \mathrm{O} / \mathrm{c1}-2-3$ \\
\hline 17 & $\mathrm{OHV}$ & excited[OH] & EXCITED_InChl=1S/HO/h1H \\
\hline 18 & NO & {$[\mathrm{N}]=\mathrm{O}$} & InChl=15/NO/c1-2 \\
\hline 19 & $\mathrm{NO} 3$ & {$[0] N(=0)=0$} & InChl=1S/NO3/c2-1(3)4 \\
\hline 20 & $\mathrm{~N} 2 \mathrm{O3}$ & $\mathrm{O}=\mathrm{NN}(=\mathrm{O})=\mathrm{O}$ & $\operatorname{lnChl}=15 / \mathrm{N} 2 \mathrm{O} / \mathrm{c} 3-1-2(4) 5$ \\
\hline 21 & $\mathrm{H} 2 \mathrm{O}$ & 0 & $\ln C h \mathrm{~h}=1 \mathrm{~S} / \mathrm{H} 2 \mathrm{O} / \mathrm{h} 1 \mathrm{H} 2$ \\
\hline 22 & HON & $\mathrm{O}[\mathrm{N}]$ & $\operatorname{lnChl}=1 \mathrm{~S} / \mathrm{HNO} / \mathrm{c} 1-2 / \mathrm{h} 2 \mathrm{H}$ \\
\hline 23 & HONO2 & {$[0-][\mathrm{N}+](=0) 0$} & InChl=1S/HNO3/c2-1(3)4/h(H,2,3,4) \\
\hline 24 & $\mathrm{~N} 2 \mathrm{O} 4$ & $\mathrm{O}=\mathrm{N}(\mathrm{N}(=\mathrm{O})=\mathrm{O})=\mathrm{O}$ & $\operatorname{lnChl}=15 / \mathrm{N} 2 \mathrm{O} 4 / \mathrm{c} 3-1(4) 2(5) 6$ \\
\hline 25 & 02 & {$[0][0]$} & InChl=1S/02/c1-2 \\
\hline 26 & HNO & $\mathrm{N}=\mathrm{O}$ & $\operatorname{lnChl}=1 \mathrm{~S} / \mathrm{HNO} / \mathrm{c} 1-2 / \mathrm{h} 1 \mathrm{H}$ \\
\hline 27 & $\mathrm{~N} 2$ & N\#N & InChl=1S/N2/c1-2 \\
\hline 28 & $\mathrm{HE}$ & {$[\mathrm{He}]$} & $\operatorname{lnChl}=1 \mathrm{~S} / \mathrm{He}$ \\
\hline 29 & $\mathrm{HO} 2$ & {$[0] 0$} & InChl=1S/HO2/c1-2/h1H \\
\hline 30 & $\mathrm{HNOH}$ & $\mathrm{O}[\mathrm{NH}]$ & $\operatorname{lnChl}=1 \mathrm{~S} / \mathrm{H} 2 \mathrm{NO} / \mathrm{c} 1-2 / \mathrm{h} 1-2 \mathrm{H}$ \\
\hline 31 & $\mathrm{NNH}$ & {$[\mathrm{N}]=\mathrm{N}$} & $\operatorname{lnChl}=1 \mathrm{~S} / \mathrm{HN} 2 / \mathrm{c} 1-2 / \mathrm{h} 1 \mathrm{H}$ \\
\hline 32 & AR & {$[\mathrm{Ar}]$} & InChl=1S/Ar \\
\hline 33 & $\mathrm{H} 2 \mathrm{O} 2$ & 00 & InChl=1S/H2O2/c1-2/h1-2H \\
\hline 34 & $\mathrm{H} 2 \mathrm{NO}$ & $\mathrm{N}[\mathrm{O}]$ & $\operatorname{lnChl}=1 \mathrm{~S} / \mathrm{H} 2 \mathrm{NO} / \mathrm{c1}-2 / \mathrm{h} 1 \mathrm{H} 2$ \\
\hline 35 & H2NN & $\mathrm{N}[\mathrm{N}]$ & InChl=1S/H2N2/c1-2/h1H2 \\
\hline 36 & $\mathrm{c}$ & [c] & $\operatorname{lnChl}=1 \mathrm{~S} / \mathrm{C}$ \\
\hline 37 & $\mathrm{CO}$ & {$[\mathrm{C}]=0$} & InChl=1S/CO/c1-2 \\
\hline 38 & $\mathrm{CO} 2$ & $\mathrm{O}=\mathrm{C}=\mathrm{O}$ & $\operatorname{lnChl}=1 \mathrm{~S} / \mathrm{CO} / \mathrm{c} 2-1-3$ \\
\hline 39 & $\mathrm{CH} 3 \mathrm{O} 2 \mathrm{H}$ & $\mathrm{COO}$ & InChl=1S/CH4O2/c1-3-2/h2H,1H3 \\
\hline 40 & $\mathrm{CH}$ & {$[\mathrm{CH}]$} & InChl=1S/CH/h1H \\
\hline 41 & $\mathrm{HCO}$ & {$[\mathrm{CH}]=\mathrm{O}$} & InChl=1S/CHO/c1-2/h1H \\
\hline 42 & HOCO & {$[\mathrm{C}](=0) \mathrm{O}$} & $\operatorname{lnChl}=1 \mathrm{~S} / \mathrm{CHO} / \mathrm{c} 2-1-3 / \mathrm{h}(\mathrm{H}, 2,3)$ \\
\hline 43 & $\mathrm{O} 2 \mathrm{CHO}$ & {$[0] \mathrm{OC}=0$} & InChl=1S/CHO3/c2-1-4-3/h1H \\
\hline 44 & $\mathrm{CHV}$ & excited $[\mathrm{CH}]$ & EXCITED_InChl=1S/CH/h1H \\
\hline 45 & $\mathrm{CH} 2 \mathrm{O}$ & $\mathrm{C}=\mathrm{O}$ & InChl=1S/CH2O/c1-2/h1H2 \\
\hline 46 & $\mathrm{OCHO}$ & {$[\mathrm{O}] \mathrm{C}=\mathrm{O}$} & InChl=1S/CHO2/c2-1-3/h1H \\
\hline 47 & $\mathrm{HO} 2 \mathrm{CHO}$ & $\mathrm{O}=\mathrm{COO}$ & $\operatorname{lnChl}=15 / \mathrm{CH} 2 \mathrm{O} 3 / \mathrm{c} 2-1-4-3 / \mathrm{h} 1,3 \mathrm{H}$ \\
\hline 48 & $\mathrm{CH} 2$ & triplet [CH2] & $\operatorname{lnChl}=1 \mathrm{~S} / \mathrm{CH} 2 / \mathrm{h} 1 \mathrm{H} 2$ \\
\hline 49 & $\mathrm{HCOH}$ & singlet $[\mathrm{CH}] \mathrm{O}$ & InChl=1S/CH2O/c1-2/h1-2H \\
\hline 50 & $\mathrm{HOCHO}$ & $\mathrm{O}=\mathrm{CO}$ & InChl=1S/CH2O2/c2-1-3/h1H,(H,2,3) \\
\hline 51 & $\mathrm{HOCH} 2 \mathrm{O} 2$ & {$[\mathrm{O}] \mathrm{OCO}$} & $\operatorname{lnChl}=1 \mathrm{~S} / \mathrm{CH} 3 \mathrm{O} / \mathrm{c} 2-1-4-3 / \mathrm{h} 2 \mathrm{H}, 1 \mathrm{H} 2$ \\
\hline 52 & $\mathrm{CH} 2$ (S) & singlet [CH2] & SINGLET_InChl=1S/CH2/h1H2 \\
\hline 53 & $\mathrm{CH} 3 \mathrm{O}$ & $\mathrm{C}[\mathrm{O}]$ & $\operatorname{lnChl}=1 \mathrm{~S} / \mathrm{CH} 3 \mathrm{O} / \mathrm{c} 1-2 / \mathrm{h} 1 \mathrm{H} 3$ \\
\hline 54 & $\mathrm{CH} 3 \mathrm{O} 2$ & $\mathrm{CO}[\mathrm{O}]$ & $\ln \mathrm{Chl}=1 \mathrm{~S} / \mathrm{CH} 3 \mathrm{O} 2 / \mathrm{c} 1-3-2 / \mathrm{h} 1 \mathrm{H} 3$ \\
\hline 55 & $\mathrm{OCH} 2 \mathrm{O} 2 \mathrm{H}$ & {$[0] \mathrm{COO}$} & $\operatorname{lnChl}=1 \mathrm{~S} / \mathrm{CH} 3 \mathrm{O} / \mathrm{c} 2-1-4-3 / \mathrm{h} 3 \mathrm{H}, 1 \mathrm{H} 2$ \\
\hline 56 & $\mathrm{CH} 3$ & {$[\mathrm{CH} 3]$} & InChl=1S/CH3/h1H3 \\
\hline 57 & $\mathrm{CH} 2 \mathrm{OH}$ & {$[\mathrm{CH} 2] \mathrm{O}$} & $\operatorname{lnChl}=1 \mathrm{~S} / \mathrm{CH} 3 \mathrm{O} / \mathrm{c} 1-2 / \mathrm{h} 2 \mathrm{H}, 1 \mathrm{H} 2$ \\
\hline 58 & $\mathrm{CH} 2 \mathrm{O} 2 \mathrm{H}$ & {$[\mathrm{CH} 2] \mathrm{OO}$} & InChl=1S/CH3O2/c1-3-2/h2H,1H2 \\
\hline 59 & $\mathrm{HOCH} 2 \mathrm{O} 2 \mathrm{H}$ & OCOO & InChl $=1 \mathrm{~S} / \mathrm{CH} 4 \mathrm{O} 3 / \mathrm{c} 2-1-4-3 / \mathrm{h} 2-3 \mathrm{H}, 1 \mathrm{H} 2$ \\
\hline 60 & $\mathrm{CH} 4$ & $\mathrm{c}$ & InChl=1S/CH4/h1H4 \\
\hline 61 & $\mathrm{CH} 3 \mathrm{OH}$ & $\mathrm{CO}$ & $\operatorname{lnChl}=1 \mathrm{~S} / \mathrm{CH} 4 \mathrm{O} / \mathrm{c} 1-2 / \mathrm{h} 2 \mathrm{H}, 1 \mathrm{H} 3$ \\
\hline 62 & $\mathrm{HOCH} 2 \mathrm{O}$ & {$[0] \mathrm{CO}$} & InChl=1S/CH3O2/c2-1-3/h2H,1H2 \\
\hline 63 & $\mathrm{C} 2 \mathrm{H}$ & {$[\mathrm{C}] \# \mathrm{C}$} & $\operatorname{lnChl}=1 \mathrm{~S} / \mathrm{C} 2 \mathrm{H} / \mathrm{c} 1-2 / \mathrm{h} 1 \mathrm{H}$ \\
\hline 64 & $\mathrm{C} 2 \mathrm{H} 4 \mathrm{O} 1-2$ & $\mathrm{C1CO1}$ & $\operatorname{lnChl}=1 \mathrm{~S} / \mathrm{C} 2 \mathrm{H} 4 \mathrm{O} / \mathrm{c} 1-2-3-1 / \mathrm{h} 1-2 \mathrm{H} 2$ \\
\hline 65 & $\mathrm{CH} 2 \mathrm{OCHO}$ & {$[\mathrm{CH} 2] \mathrm{OC}=\mathrm{O}$} & $\ln \mathrm{Chl}=1 \mathrm{~S} / \mathrm{C} 2 \mathrm{H} 3 \mathrm{O} / \mathrm{cc} 1-4-2-3 / \mathrm{h} 2 \mathrm{H}, 1 \mathrm{H} 2$ \\
\hline 66 & $\mathrm{OCH} 2 \mathrm{OCHO}$ & {$[0] \mathrm{COC}=0$} & $\operatorname{lnChl}=1 \mathrm{~S} / \mathrm{C} 2 \mathrm{H} 3 \mathrm{O} 3 / \mathrm{c} 3-1-5-2-4 / \mathrm{h} 1 \mathrm{H}, 2 \mathrm{H} 2$ \\
\hline 67 & $\mathrm{C} 2 \mathrm{H} 2$ & CHC & $\operatorname{lnChl}=1 \mathrm{~S} / \mathrm{C} 2 \mathrm{H} 2 / \mathrm{c} 1-2 / \mathrm{h} 1-2 \mathrm{H}$ \\
\hline 68 & $\mathrm{CH} 3 \mathrm{CHO}$ & $\mathrm{CC}=\mathrm{O}$ & $\operatorname{lnChl}=1 \mathrm{~S} / \mathrm{C} 2 \mathrm{H} 4 \mathrm{O} / \mathrm{c} 1-2-3 / \mathrm{h} 2 \mathrm{H}, 1 \mathrm{H} 3$ \\
\hline 69 & $\mathrm{HOCHCHO}$ & $\mathrm{O}=\mathrm{C}[\mathrm{CH}] \mathrm{O}$ & InChl=1S/C2H3O2/c3-1-2-4/h1-3H \\
\hline 70 & $\mathrm{HOCH} 2 \mathrm{OCO}$ & $\mathrm{O}=[\mathrm{C}] \mathrm{OCO}$ & InChl=1S/C2 $2 \mathrm{H} 3 \mathrm{O} / \mathrm{c} 3-1-5-2-4 / \mathrm{h} 3 \mathrm{H}, 1 \mathrm{H} 2$ \\
\hline 71 & $\mathrm{H} 2 \mathrm{CC}$ & {$[\mathrm{C}]=\mathrm{C}$} & $\operatorname{lnChl}=1 \mathrm{~S} / \mathrm{C} 2 \mathrm{H} 2 / \mathrm{c} 1-2 / \mathrm{h} 1 \mathrm{H} 2$ \\
\hline 72 & $\mathrm{C} 2 \mathrm{H} 3 \mathrm{OH}$ & $\mathrm{C}=\mathrm{CO}$ & $\operatorname{lnChl}=1 \mathrm{~S} / \mathrm{C} 2 \mathrm{H} 4 \mathrm{O} / \mathrm{c1}-2-3 / \mathrm{h} 2-3 \mathrm{H}, 1 \mathrm{H} 2$ \\
\hline 73 & $\mathrm{OCH} 2 \mathrm{CHO}$ & $C(C[0])=0$ & $\operatorname{lnChl}=1 \mathrm{~S} / \mathrm{C} 2 \mathrm{H} 3 \mathrm{O} 2 / \mathrm{c} 3-1-2-4 / \mathrm{h} 1 \mathrm{H}, 2 \mathrm{H} 2$ \\
\hline 74 & CH3CO3H & $\mathrm{CC}(=0) 00$ & InChl=1S/C2H4O3/c1-2(3)5-4/h4H,1H3 \\
\hline 75 & $\mathrm{C} 2 \mathrm{H} 3$ & {$[\mathrm{CH}]=\mathrm{C}$} & $\operatorname{lnChl}=1 \mathrm{~S} / \mathrm{C} 2 \mathrm{H} 3 / \mathrm{c} 1-2 / \mathrm{h} 1 \mathrm{H}, 2 \mathrm{H} 2$ \\
\hline 76 & $\mathrm{C} 2 \mathrm{H} 5 \mathrm{O}$ & $\mathrm{CC}[0]$ & $\operatorname{lnChl}=1 \mathrm{~S} / \mathrm{C} 2 \mathrm{H} 5 \mathrm{O} / \mathrm{c} 1-2-3 / \mathrm{h} 2 \mathrm{H} 2,1 \mathrm{H} 3$ \\
\hline 77 & $\mathrm{CH} 3 \mathrm{COOH}$ & $\mathrm{CC}(=0) \mathrm{O}$ & $\operatorname{lnChl}=1 \mathrm{~S} / \mathrm{C} 2 \mathrm{H} 402 / \mathrm{c} 1-2(3) 4 / \mathrm{h} 1 \mathrm{H} 3,(\mathrm{H}, 3,4)$ \\
\hline 78 & $\mathrm{HO} 2 \mathrm{CH} 2 \mathrm{CHO}$ & $c(\mathrm{COO})=0$ & InChl=1S/C2H403/c3-1-2-5-4/h1,4H,2H2 \\
\hline 79 & $\mathrm{C} 2 \mathrm{H} 4$ & $\mathrm{C}=\mathrm{C}$ & $\operatorname{lnChl}=1 \mathrm{~S} / \mathrm{C} 2 \mathrm{H} 4 / \mathrm{c} 1-2 / \mathrm{h} 1-2 \mathrm{H} 2$ \\
\hline 80 & $\mathrm{PC} 2 \mathrm{H} 4 \mathrm{OH}$ & {$[\mathrm{CH} 2] \mathrm{CO}$} & $\operatorname{lnChl}=1 \mathrm{~S} / \mathrm{C} 2 \mathrm{H} 5 \mathrm{O} / \mathrm{c} 1-2-3 / \mathrm{h} 3 \mathrm{H}, 1-2 \mathrm{H} 2$ \\
\hline 81 & $\mathrm{HOCH} 2 \mathrm{CHO}$ & $\mathrm{OCC}=0$ & InChl=1S/C2H4O2/c3-1-2-4/h1,4H,2H2 \\
\hline 82 & $\mathrm{O} 2 \mathrm{C} 2 \mathrm{H} 4 \mathrm{OH}$ & {$[0] \mathrm{OCCO}$} & InChl=1S/C2H503/c3-1-2-5-4/h3H,1-2H2 \\
\hline 83 & $\mathrm{C} 2 \mathrm{H} 5$ & {$[\mathrm{CH} 2] \mathrm{C}$} & InChl=1S/C2H5/c1-2/h1H2,2H3 \\
\hline 84 & $\mathrm{SC} 2 \mathrm{H} 4 \mathrm{OH}$ & $\mathrm{C}[\mathrm{CH}] \mathrm{O}$ & $\operatorname{lnChl}=1 \mathrm{~S} / \mathrm{C} 2 \mathrm{H} 5 \mathrm{O} / \mathrm{c} 1-2-3 / \mathrm{h} 2-3 \mathrm{H}, 1 \mathrm{H} 3$ \\
\hline 85 & $\mathrm{C} 2 \mathrm{H} 3 \mathrm{OOH}$ & $\mathrm{C}=\mathrm{COO}$ & InChl=1S/C2H4O2/c1-2-4-3/h2-3H,1H2 \\
\hline 86 & $\mathrm{CH} 2 \mathrm{CH} 2 \mathrm{O}-2 \mathrm{OOH}$ & {$[0] \mathrm{CCOO}$} & InChl=1S/C2H5O3/c3-1-2-5-4/h4H,1-2H2 \\
\hline 87 & $\mathrm{C} 2 \mathrm{H} 6$ & $\mathrm{CC}$ & $\operatorname{lnChl}=1 \mathrm{~S} / \mathrm{C} 2 \mathrm{H} 6 / \mathrm{c} 1-2 / \mathrm{h} 1-2 \mathrm{H} 3$ \\
\hline
\end{tabular}




\begin{tabular}{|c|c|c|c|}
\hline 88 & $\mathrm{CH} 3 \mathrm{OCH} 2$ & {$[\mathrm{CH} 2] \mathrm{OC}$} & $\operatorname{lnChl}=1 \mathrm{~S} / \mathrm{C} 2 \mathrm{H} 5 \mathrm{O} / \mathrm{c} 1-3-2 / \mathrm{h} 1 \mathrm{H} 2,2 \mathrm{H} 3$ \\
\hline 89 & $\mathrm{CY}(\mathrm{CCO}) \mathrm{OH}$ & OC1CO1 & InChl=1S/C2H402/c3-2-1-4-2/h2-3H,1H2 \\
\hline 90 & $\mathrm{CH} 2 \mathrm{CHOH}-2 \mathrm{OOH}$ & $\mathrm{O}[\mathrm{CH}] \mathrm{COO}$ & $\operatorname{lnChl}=1 \mathrm{~S} / \mathrm{C} 2 \mathrm{H} 503 / \mathrm{c} 3-1-2-5-4 / \mathrm{h} 1,3-4 \mathrm{H}, 2 \mathrm{H} 2$ \\
\hline 91 & $\mathrm{HCCO}$ & {$[\mathrm{CH}]=\mathrm{C}=\mathrm{O}$} & InChl=1S/C2HO/c1-2-3/h1H \\
\hline 92 & $\mathrm{C} 2 \mathrm{H} 5 \mathrm{OH}$ & CCO & InChl=1S/C2H6O/c1-2-3/h3H,2H2,1H3 \\
\hline 93 & $\mathrm{CH} 3 \mathrm{OCHO}$ & $\mathrm{COC}=0$ & $\operatorname{lnChl}=1 \mathrm{~S} / \mathrm{C} 2 \mathrm{H} 4 \mathrm{O} 2 / \mathrm{c} 1-4-2-3 / \mathrm{h} 2 \mathrm{H}, 1 \mathrm{H} 3$ \\
\hline 94 & CH3CHOH-102 & {$[\mathrm{O}] \mathrm{OC}(\mathrm{O}) \mathrm{C}$} & InChl=1S/C2H503/c1-2(3)5-4/h2-3H,1H3 \\
\hline 95 & $\mathrm{CHCHO}$ & {$[\mathrm{CH}] \mathrm{CO}$} & InChl=1S/C2H2O/c1-2-3/h1-2H \\
\hline 96 & $\mathrm{CH} 3 \mathrm{OCH} 3$ & $\operatorname{coc}$ & $\operatorname{lnChl}=1 \mathrm{~S} / \mathrm{C} 2 \mathrm{H} 6 \mathrm{O} / \mathrm{c} 1-3-2 / \mathrm{h} 1-2 \mathrm{H} 3$ \\
\hline 97 & $\mathrm{C} 2 \mathrm{H} 5 \mathrm{O} 2$ & $\mathrm{CCO}[\mathrm{O}]$ & $\operatorname{lnChl}=1 \mathrm{~S} / \mathrm{C} 2 \mathrm{H} 5 \mathrm{O} 2 / \mathrm{c} 1-2-4-3 / \mathrm{h} 2 \mathrm{H} 2,1 \mathrm{H} 3$ \\
\hline 98 & $\mathrm{CH} 3 \mathrm{COHOOH}$ & $\mathrm{OO}[\mathrm{C}](\mathrm{O}) \mathrm{C}$ & InChl=1S/C2H5O3/c1-2(3)5-4/h3-4H,1H3 \\
\hline 99 & $\mathrm{CH} 2 \mathrm{CO}$ & $\mathrm{C}=\mathrm{C}=\mathrm{O}$ & $\operatorname{lnChl}=1 \mathrm{~S} / \mathrm{C} 2 \mathrm{H} 2 \mathrm{O} / \mathrm{c} 1-2-3 / \mathrm{h} 1 \mathrm{H} 2$ \\
\hline 100 & $\mathrm{COCHO}$ & $\mathrm{O}=[\mathrm{C}] \mathrm{C}=\mathrm{O}$ & InChl=1S/C2HO2/c3-1-2-4/h1H \\
\hline 101 & $\mathrm{C} 2 \mathrm{H} 4 \mathrm{O} 2 \mathrm{H}$ & {$[\mathrm{CH} 2] \mathrm{COO}$} & InChl=1S/C2H5O2/c1-2-4-3/h3H,1-2H2 \\
\hline 102 & $\mathrm{C} 2 \mathrm{H} 4 \mathrm{O}-10 \mathrm{OH}$ & $00 \mathrm{O}([\mathrm{O}]) \mathrm{C}$ & InChl=1S/C2H503/c1-2(3)5-4/h2,4H,1H3 \\
\hline 103 & $\mathrm{HCCOH}$ & $\mathrm{CHCO}$ & $\operatorname{lnChl}=1 \mathrm{~S} / \mathrm{C} 2 \mathrm{H} 2 \mathrm{O} / \mathrm{c} 1-2-3 / \mathrm{h} 1,3 \mathrm{H}$ \\
\hline 104 & $\mathrm{CHOCHO}$ & $\mathrm{O}=\mathrm{CC}=\mathrm{O}$ & InChl=1S/C2H2O2/c3-1-2-4/h1-2H \\
\hline 105 & $\mathrm{CH} 3 \mathrm{OCH} 2 \mathrm{O}$ & $\operatorname{coc}[0]$ & $\operatorname{lnChl}=1 \mathrm{~S} / \mathrm{C} 2 \mathrm{H} 5 \mathrm{O} 2 / \mathrm{c} 1-4-2-3 / \mathrm{h} 2 \mathrm{H} 2,1 \mathrm{H} 3$ \\
\hline 106 & $\mathrm{CH} 2 \mathrm{CHOH}-1 \mathrm{OOH}$ & $\mathrm{OOC}(\mathrm{O})[\mathrm{CH} 2]$ & InChl=1S/C2H5O3/c1-2(3)5-4/h2-4H,1H2 \\
\hline 107 & $\mathrm{C} 2 \mathrm{H} 3 \mathrm{O} 1-2$ & {$[\mathrm{CH}] 1 \mathrm{CO} 1$} & InChl=1S/C2H3O/c1-2-3-1/h1H,2H2 \\
\hline 108 & $\mathrm{CH} 2 \mathrm{COOH}$ & {$[\mathrm{CH} 2] \mathrm{C}(\mathrm{O})=\mathrm{O}$} & InChl=1S/C2H3O2/c1-2(3)4/h1H2,(H,3,4) \\
\hline 109 & $\mathrm{C} 2 \mathrm{H} 5 \mathrm{O} 2 \mathrm{H}$ & $\mathrm{ccoO}$ & $\operatorname{lnChl}=1 \mathrm{~S} / \mathrm{C} 2 \mathrm{H} 6 \mathrm{O} 2 / \mathrm{c} 1-2-4-3 / \mathrm{h} 3 \mathrm{H}, 2 \mathrm{H} 2,1 \mathrm{H} 3$ \\
\hline 110 & $\mathrm{CH} 3 \mathrm{OCH} 2 \mathrm{O} 2$ & $\mathrm{COCO}[\mathrm{O}]$ & $\operatorname{lnChl}=1 \mathrm{~S} / \mathrm{C} 2 \mathrm{H} 503 / \mathrm{cc}-4-2-5-3 / \mathrm{h} 2 \mathrm{H} 2,1 \mathrm{H3}$ \\
\hline 111 & $\mathrm{CH} 3 \mathrm{CO}$ & $\mathrm{C}[\mathrm{C}]=\mathrm{O}$ & InChl $=1 \mathrm{~S} / \mathrm{C} 2 \mathrm{H} 3 \mathrm{O} / \mathrm{c} 1-2-3 / \mathrm{h} 1 \mathrm{H} 3$ \\
\hline 112 & $\mathrm{HOCH} 2 \mathrm{CO}$ & $\mathrm{OC}[\mathrm{C}]=\mathrm{O}$ & $\operatorname{lnChl}=1 \mathrm{~S} / \mathrm{C} 2 \mathrm{H} 3 \mathrm{OO} / \mathrm{c} 3-1-2-4 / \mathrm{h} 3 \mathrm{H}, 1 \mathrm{H} 2$ \\
\hline 113 & $\mathrm{CHOOCO}$ & $\mathrm{C}(=0) \mathrm{O}[\mathrm{C}](=0)$ & InChl=1S/C2HO3/c3-1-5-2-4/h1H \\
\hline 114 & $\mathrm{CH} 2 \mathrm{OCH} 2 \mathrm{O} 2 \mathrm{H}$ & {$[\mathrm{CH} 2] \mathrm{OCOO}$} & InChl=1S/C2H5O3/c1-4-2-5-3/h3H,1-2H2 \\
\hline 115 & $\mathrm{CH} 2 \mathrm{CHO}$ & {$[\mathrm{CH} 2] \mathrm{C}=0$} & InChl=1S/C2H3O/c1-2-3/h2H,1H2 \\
\hline 116 & $\mathrm{C} 2 \mathrm{H} 3 \mathrm{OO}$ & $\mathrm{C}=\mathrm{C}(\mathrm{O}[\mathrm{O}])$ & InChl=1S/C2H3O2/c1-2-4-3/h2H,1H2 \\
\hline 117 & $\mathrm{O} 2 \mathrm{CH} 2 \mathrm{CHO}$ & {$[0] \mathrm{OCC}=\mathrm{O}$} & InChl=1S/C2H3O3/c3-1-2-5-4/h1H,2H2 \\
\hline 118 & $\mathrm{CH} 3 \mathrm{OCH} 2 \mathrm{O} 2 \mathrm{H}$ & COCOO & InChl=1S/C2H6O3/c1-4-2-5-3/h3H,2H2,1H3 \\
\hline 119 & $\mathrm{C} 2 \mathrm{H} 2 \mathrm{OH}$ & {$[\mathrm{CH}]=\mathrm{CO}$} & InChl=1S/C2H3O/c1-2-3/h1-3H \\
\hline 120 & $\mathrm{CH} 3 \mathrm{CO} 2$ & $\mathrm{CC}([0])=0$ & $\operatorname{lnChl}=1 \mathrm{~S} / \mathrm{C} 2 \mathrm{H} 3 \mathrm{O} 2 / \mathrm{c} 1-2(3) 4 / \mathrm{h} 1 \mathrm{H} 3$ \\
\hline 121 & $\mathrm{HO} 2 \mathrm{CH} 2 \mathrm{CO}$ & $\mathrm{O}=[\mathrm{C}] \mathrm{COO}$ & InChl=1S/C2H3O3/c3-1-2-5-4/h4H,2H2 \\
\hline 122 & $\mathrm{HO} 2 \mathrm{CH} 2 \mathrm{OCHO}$ & $\mathrm{O}=\mathrm{COCOO}$ & $\operatorname{lnChl}=1 \mathrm{~S} / \mathrm{C} 2 \mathrm{H} 4 \mathrm{O} 4 / \mathrm{c} 3-1-5-2-6-4 / \mathrm{h} 1,4 \mathrm{H}, 2 \mathrm{H} 2$ \\
\hline 123 & $\mathrm{SC} 2 \mathrm{H} 2 \mathrm{OH}$ & $\mathrm{C}=[\mathrm{C}] \mathrm{O}$ & $\operatorname{lnChl}=1 \mathrm{~S} / \mathrm{C} 2 \mathrm{H} 3 \mathrm{O} / \mathrm{c} 1-2-3 / \mathrm{h} 3 \mathrm{H}, 1 \mathrm{H} 2$ \\
\hline 124 & $\mathrm{CH} 3 \mathrm{OCO}$ & $\mathrm{CO}[\mathrm{C}]=\mathrm{O}$ & $\operatorname{lnChl}=1 \mathrm{~S} / \mathrm{C} 2 \mathrm{H} 3 \mathrm{O} 2 / \mathrm{c} 1-4-2-3 / \mathrm{h} 1 \mathrm{H} 3$ \\
\hline 125 & $\mathrm{CH} 3 \mathrm{CO} 3$ & $\mathrm{CC}(=\mathrm{O}) \mathrm{O}[\mathrm{O}]$ & $\operatorname{lnChl}=1 \mathrm{~S} / \mathrm{C} 2 \mathrm{H} 3 \mathrm{O} / \mathrm{c} 1-2(3) 5-4 / \mathrm{h} 1 \mathrm{H} 3$ \\
\hline 126 & $\mathrm{O} 2 \mathrm{CH} 2 \mathrm{OCH} 2 \mathrm{O} 2 \mathrm{H}$ & {$[0] 0 \mathrm{cocoO}$} & InChl=1S/C2H5O5/c3-6-1-5-2-7-4/h3H,1-2H2 \\
\hline 127 & $\mathrm{C} 3 \mathrm{H} 2$ & {$[\mathrm{CH}]=\mathrm{C}=[\mathrm{CH}]$} & InChl=1S/C3H2/c1-3-2/h1-2H \\
\hline 128 & $\mathrm{C} 2 \mathrm{H} 5 \mathrm{CO}$ & $\mathrm{CC}[\mathrm{C}]=0$ & InChl=1S/C3H5O/c1-2-3-4/h2H2,1H3 \\
\hline 129 & C3H5-TO2 & $\mathrm{CC}(=\mathrm{C}) \mathrm{O}[0]$ & InChl=1S/C3H5O2/c1-3(2)5-4/h1H2,2H3 \\
\hline 130 & C3H501-20OH-3 & $\mathrm{C} 1 \mathrm{C}(\mathrm{O} 1) \mathrm{C}(\mathrm{OO})$ & $\operatorname{lnChl}=15 / \mathrm{C} 3 \mathrm{H} 6 \mathrm{O} / \mathrm{c} / \mathrm{c}-6-2-3-3-1-5-3 / \mathrm{h} 3-4 \mathrm{H}, 1-2 \mathrm{H} 2$ \\
\hline 131 & $\mathrm{H} 2 \mathrm{CCC}(\mathrm{S})$ & $\mathrm{C}=\mathrm{C}=[\mathrm{C}]$ & $\operatorname{lnChl}=1 \mathrm{~S} / \mathrm{C} 3 \mathrm{H} 2 / \mathrm{c} 1-3-2 / \mathrm{h} 1 \mathrm{H} 2$ \\
\hline 132 & $\mathrm{CH} 2 \mathrm{CH} 2 \mathrm{CHO}$ & {$[\mathrm{CH} 2] \mathrm{CC}=\mathrm{O}$} & InChl=1S/C3H5O/c1-2-3-4/h3H,1-2H2 \\
\hline 133 & C3H5-SO2 & $\mathrm{CC}=\mathrm{CO}[\mathrm{O}]$ & InChl=1S/C3H502/c1-2-3-5-4/h2-3H,1H3 \\
\hline 134 & C3H501-30OH-2 & $\mathrm{C} 1 \mathrm{C}(\mathrm{OO}) \mathrm{C}(\mathrm{O} 1)$ & InChl=15/C3H6O3/c4-6-3-1-5-2-3/h3-4H,1-2H2 \\
\hline 135 & $\mathrm{C} 3 \mathrm{H} 2(\mathrm{~S})$ & singlet $[\mathrm{CH}]=\mathrm{C}=[\mathrm{CH}]$ & SINGLET_InChl=1S/C3H2/c1-3-2/h1-2H \\
\hline 136 & $\mathrm{CH} 3 \mathrm{COCH} 2$ & {$[\mathrm{CH} 2] \mathrm{C}(\mathrm{C})=\mathrm{O}$} & $\operatorname{lnChl}=1 \mathrm{~S} / \mathrm{C} 3 \mathrm{H} 5 \mathrm{O} / \mathrm{c} 1-3(2) 4 / \mathrm{h} 1 \mathrm{H} 2,2 \mathrm{H} 3$ \\
\hline 137 & $\mathrm{CH} 2 \mathrm{COOCH} 3$ & $\mathrm{O}=\mathrm{C}([\mathrm{CH} 2]) \mathrm{OC}$ & InChl=1S/C3H5O2/c1-3(4)5-2/h1H2,2H3 \\
\hline 138 & С3КET21 & $\mathrm{CC}(=0) \mathrm{COO}$ & InChl=1S/C3H6O3/c1-3(4)2-6-5/h5H,2H2,1H3 \\
\hline 139 & $\mathrm{C} 3 \mathrm{H} 2 \mathrm{C}$ & $\mathrm{C} 1=\mathrm{C}[\mathrm{C}] 1$ & InChl=1S/C3H2/c1-2-3-1/h1-2H \\
\hline 140 & PC3H4OH-1 & $\mathrm{CC}=[\mathrm{C}] \mathrm{O}$ & InChl=1S/C3H5O/c1-2-3-4/h2,4H,1H3 \\
\hline 141 & $\mathrm{CH} 3 \mathrm{COOCH} 2$ & $\mathrm{O}=\mathrm{C}(\mathrm{C}) \mathrm{O}[\mathrm{CH} 2]$ & InChl=1S/C3H5O2/c1-3(4)5-2/h2H2,1H3 \\
\hline 142 & $\mathrm{CH} 2 \mathrm{COHCH} 2 \mathrm{OOH}$ & $\mathrm{C}=\mathrm{C}(\mathrm{COO}) \mathrm{O}$ & InChl=1S/C3H6O3/c1-3(4)2-6-5/h4-5H,1-2H2 \\
\hline 143 & $\mathrm{C} 3 \mathrm{H} 3$ & $\mathrm{C \# C[CH2]}$ & $\mathrm{InChl}=1 \mathrm{~S} / \mathrm{C} 3 \mathrm{H} 3 / \mathrm{c} 1-3-2 / \mathrm{h} 1 \mathrm{H}, 2 \mathrm{H} 2$ \\
\hline 144 & SC3H5OH & $\mathrm{CC}=\mathrm{CO}$ & InChl=1S/C3H6O/c1-2-3-4/h2-4H,1H3 \\
\hline 145 & $\mathrm{C} 2 \mathrm{H} 5 \mathrm{OCO}$ & $\mathrm{CCO}[\mathrm{C}]=\mathrm{O}$ & InChl=15/C3H5O2/c1-2-5-3-4/h2H2,1H3 \\
\hline 146 & $\mathrm{C} 3 \mathrm{H} 6 \mathrm{OH}-102$ & $\operatorname{ccC}(\mathrm{O}[\mathrm{O}]) \mathrm{O}$ & InChl=15/C3H703/c1-2-3(4)6-5/h3-4H,2H2,1H3 \\
\hline 147 & C3H4-P & $\mathrm{C \# CC}$ & InChl=1S/C3H4/c1-3-2/h1H,2H3 \\
\hline 148 & C3H6O1-2 & CC1CO1 & InChl=1S/C3H6O/c1-3-2-4-3/h3H,2H2,1H3 \\
\hline 149 & $\mathrm{HOC} 2 \mathrm{H} 4 \mathrm{CO}$ & OcC $[\mathrm{C}]=0$ & InChl=1S/C3H502/c4-2-1-3-5/h4H,1-2H2 \\
\hline 150 & C3H4-A & $\mathrm{C}=\mathrm{C}=\mathrm{C}$ & InChl=1S/C3H4/c1-3-2/h1-2H2 \\
\hline 151 & C3H6O1-3 & C1COC1 & InChl=1S/C3H6O/c1-2-4-3-1/h1-3H2 \\
\hline 152 & $\mathrm{CH} 3 \mathrm{COCH} 2 \mathrm{O}$ & $\mathrm{CC}(=\mathrm{O}) \mathrm{C}[0]$ & InChl=1S/C3H5O2/c1-3(5)2-4/h2H2,1H3 \\
\hline 153 & $\mathrm{HOC} 3 \mathrm{H} 6 \mathrm{O} 2$ & $\mathrm{CC}(\mathrm{O}[\mathrm{O}]) \mathrm{CO}$ & $\operatorname{lnChl}=1 \mathrm{~S} / \mathrm{C} 3 \mathrm{H} 703 / \mathrm{c} 1-3(2-4) 6-5 / \mathrm{h} 3-4 \mathrm{H}, 2 \mathrm{H} 2,1 \mathrm{H} 3$ \\
\hline 154 & $\mathrm{CC} 3 \mathrm{H} 4$ & $\mathrm{C} 1=\mathrm{CC} 1$ & InChl=1S/C3H4/c1-2-3-1/h1-2H,3H2 \\
\hline 155 & $\mathrm{IC} 3 \mathrm{H} 5 \mathrm{OH}$ & $\mathrm{C}=\mathrm{C}(\mathrm{C}) \mathrm{O}$ & InChl=1S/C3H6O/c1-3(2)4/h4H,1H2,2H3 \\
\hline 156 & $\mathrm{HOCH} 2 \mathrm{COCH} 2$ & {$[\mathrm{CH} 2] \mathrm{C}(=\mathrm{O}) \mathrm{CO}$} & $\mathrm{InChl}=1 \mathrm{~S} / \mathrm{C} 3 \mathrm{H} 5 \mathrm{O} 2 / \mathrm{cc}-3(5) 2-4 / \mathrm{h} 4 \mathrm{H}, 1-2 \mathrm{H} 2$ \\
\hline 157 & IC3H6OH-102 & {$[\mathrm{O}] \mathrm{OCC}(\mathrm{O}) \mathrm{C}$} & $\operatorname{lnChl}=15 / \mathrm{C} 3 \mathrm{H} 703 / \mathrm{c} 1-3(4) 2-6-5 / \mathrm{h} 3-4 \mathrm{H}, 2 \mathrm{H} 2,1 \mathrm{H3}$ \\
\hline 158 & C3H5-A & {$[\mathrm{CH} 2] \mathrm{C}=\mathrm{C}$} & InChl=1S/C3H5/c1-3-2/h3H,1-2H2 \\
\hline 159 & $\mathrm{C} 3 \mathrm{H} 5 \mathrm{OH}$ & $\mathrm{C}=\mathrm{CCO}$ & InChl=1S/C3H6O/c1-2-3-4/h2,4H,1,3H2 \\
\hline 160 & $\mathrm{CH} 3 \mathrm{COCHOH}$ & $\mathrm{CC}(=\mathrm{O})[\mathrm{CH}] \mathrm{O}$ & InChl=1S/C3H502/c1-3(5)2-4/h2,4H,1H3 \\
\hline 161 & С3H6OH-30OH-1 & $\mathrm{C}(\mathrm{OO}) \mathrm{C}[\mathrm{CH}] \mathrm{O}$ & $\operatorname{lnChl}=15 / \mathrm{C} 3 \mathrm{H} 703 / \mathrm{c} 4-2-1-3-6-5 / \mathrm{h} 2,4-5 \mathrm{H}, 1,3 \mathrm{H} 2$ \\
\hline 162 & C3H5-S & {$[\mathrm{CH}]=\mathrm{CC}$} & InChl=1S/C3H5/c1-3-2/h1,3H,2H3 \\
\hline 163 & $\mathrm{C} 2 \mathrm{H} 5 \mathrm{CHO}$ & $\mathrm{CCC}=0$ & InChl=1S/C3H6O/c1-2-3-4/h3H,2H2,1H3 \\
\hline 164 & $\mathrm{CH} 2 \mathrm{OCH} 2 \mathrm{CHO}$ & {$[\mathrm{CH} 2] \mathrm{OCC}=\mathrm{O}$} & InChl=1S/C3H502/c1-5-3-2-4/h2H,1,3H2 \\
\hline 165 & $\mathrm{C} 3 \mathrm{H} 6 \mathrm{OH}-3 \mathrm{O} 2$ & $\mathrm{C}(\mathrm{O}[\mathrm{O}]) \mathrm{CCO}$ & $\operatorname{lnChl}=1 \mathrm{~S} / \mathrm{C} 3 \mathrm{H} 703 / \mathrm{c} 4-2-1-3-6-5 / \mathrm{h} 4 \mathrm{H}, 1-3 \mathrm{H} 2$ \\
\hline 166 & C3H5-T & $\mathrm{C}=[\mathrm{C}] \mathrm{C}$ & $\operatorname{lnChl}=1 \mathrm{~S} / \mathrm{C} 3 \mathrm{H} 5 / \mathrm{c} 1-3-2 / \mathrm{h} 1 \mathrm{H} 2,2 \mathrm{H} 3$ \\
\hline 167 & $\mathrm{CH} 3 \mathrm{COCH} 3$ & $\mathrm{CC}(\mathrm{C})=0$ & $\operatorname{lnChl}=1 \mathrm{~S} / \mathrm{C} 3 \mathrm{H} 6 \mathrm{O} / \mathrm{c1} 1-3(2) 4 / \mathrm{h} 1-2 \mathrm{H} 3$ \\
\hline 168 & $\mathrm{C} 2 \mathrm{H} 4 \mathrm{OCHO}$ & $\mathrm{CC}(\mathrm{C}=\mathrm{O})[0]$ & $\operatorname{lnChl}=1 \mathrm{~S} / \mathrm{C} 3 \mathrm{H} 502 / \mathrm{cc}-3(5) 2-4 / \mathrm{h} 2-3 \mathrm{H}, 1 \mathrm{H} 3$ \\
\hline 169 & ME2VOMJVO & {$[C](=0) O C(=O) C(=0)$} & $\operatorname{lnChl}=1 \mathrm{~S} / \mathrm{C} 3 \mathrm{HO} 4 / \mathrm{c} 4-1-3(6) 7-2-5 / \mathrm{h} 1 \mathrm{H}$ \\
\hline 170 & $\mathrm{C} 3 \mathrm{H} 6$ & $\mathrm{C}=\mathrm{CC}$ & InChl=1S/C3H6/c1-3-2/h3H,1H2,2H3 \\
\hline 171 & $\mathrm{C} 3 \mathrm{H} 6 \mathrm{OH} 1-2$ & $\mathrm{C}[\mathrm{CH}] \mathrm{CO}$ & $\operatorname{lnChl}=1 \mathrm{~S} / \mathrm{C} 3 \mathrm{H} 7 \mathrm{O} / \mathrm{c1}-2-3-4 / \mathrm{h} 2,4 \mathrm{H}, 3 \mathrm{H} 2,1 \mathrm{H} 3$ \\
\hline 172 & C3Y1-3OR & $C([0]) C C=0$ & InChl=1S/C3H5O2/c4-2-1-3-5/h2H,1,3H2 \\
\hline 173 & ME2JVOMVO & $\mathrm{C}(=0) \mathrm{OC}(=0)[\mathrm{C}](=0)$ & InChl=1S/C3HO4/c4-1-3(6)7-2-5/h2H \\
\hline 174 & $\mathrm{CC} 3 \mathrm{H} 6$ & $\mathrm{C} 1 \mathrm{CC} 1$ & InChl=1S/C3H6/c1-2-3-1/h1-3H2 \\
\hline 175 & C3H6OH1-1 & $\mathrm{CC}[\mathrm{CH}] \mathrm{O}$ & InChl=1S/C3H7O/c1-2-3-4/h3-4H,2H2,1H3 \\
\hline 176 & $\mathrm{CH} 3 \mathrm{COOCH} 3$ & $\mathrm{O}=\mathrm{C}(\mathrm{C}) \mathrm{OC}$ & InChl=1S/C3H6O2/c1-3(4)5-2/h1-2H3 \\
\hline 177 & ME2VOMVO & $C(=0) O C(=0) C(=0)$ & InChl=1S/C3H2O4/c4-1-3(6)7-2-5/h1-2H \\
\hline 178 & $\mathrm{IC} 3 \mathrm{H} 7$ & $\mathrm{C}[\mathrm{CH}] \mathrm{C}$ & InChl=1S/C3H7/c1-3-2/h3H,1-2H3 \\
\hline
\end{tabular}




\begin{tabular}{|c|c|c|c|}
\hline 179 & С3H6OH1-3 & {$[\mathrm{CH} 2] \mathrm{CCO}$} & InChl=1S/C3H7O/c1-2-3-4/h4H,1-3H \\
\hline 180 & $\mathrm{HOC} 2 \mathrm{H} 4 \mathrm{CHO}$ & OCCC $=0$ & InChl=15/C3H6O2/c4-2-1-3-5/h2,5H,1,3H2 \\
\hline 181 & $\mathrm{HO} 2 \mathrm{CH} 2 \mathrm{COCHO}$ & $\mathrm{OOCC}(=0) \mathrm{C}=\mathrm{O}$ & InChl=1S/C3H4O4/c4-1-3(5)2-7-6/h1,6H,2H2 \\
\hline 182 & $\mathrm{NC} 3 \mathrm{H} 7$ & {$[\mathrm{CH} 2] \mathrm{CC}$} & $\operatorname{lnChl}=1 \mathrm{~S} / \mathrm{C} 3 \mathrm{H} 7 / \mathrm{c1}-3-2 / \mathrm{h} 1,3 \mathrm{H} 2,2 \mathrm{H} 3$ \\
\hline 183 & $\mathrm{C} 3 \mathrm{H} 6 \mathrm{OH} 2-1$ & {$[\mathrm{CH} 2] \mathrm{C}(\mathrm{O}) \mathrm{C}$} & InChl=1S/C3H7O/c1-3(2)4/h3-4H,1H2,2H3 \\
\hline 184 & АСЗ $550 \mathrm{OH}$ & $\mathrm{C}=\mathrm{CCOO}$ & InChl=1S/C3H6O2/c1-2-3-5-4/h2,4H,1,3H2 \\
\hline 185 & OOCC(VO)COJ & $\mathrm{OOCC}(=\mathrm{O}) \mathrm{C}[\mathrm{O}]$ & InChl=15/C3H5O4/c4-1-3(5)2-7-6/h6H,1-2H2 \\
\hline 186 & $\mathrm{C} 3 \mathrm{H} 8$ & $\mathrm{CCC}$ & InChl=1S/C3H8/c1-3-2/h3H2,1-2H3 \\
\hline 187 & NC3H7O & $\operatorname{ccc}[0]$ & $\operatorname{lnChl}=1 \mathrm{~S} / \mathrm{C} 3 \mathrm{H} 7 \mathrm{O} / \mathrm{c} 1-2-3-4 / \mathrm{h} 2-3 \mathrm{H} 2,1 \mathrm{H} 3$ \\
\hline 188 & $\mathrm{IC} 3 \mathrm{H} 5 \mathrm{Q}$ & $\mathrm{C}=\mathrm{C}(\mathrm{C}) \mathrm{OO}$ & $\operatorname{lnChl}=1 \mathrm{~S} / \mathrm{C} 3 \mathrm{H} 6 \mathrm{OO} / \mathrm{c1} 1-3(2) 5-4 / \mathrm{h} 4 \mathrm{H}, 1 \mathrm{H} 2,2 \mathrm{H} 3$ \\
\hline 189 & СЗОНКЕТЗ-1 & $\mathrm{C}(=\mathrm{O}) \mathrm{CC}(\mathrm{OO}) \mathrm{O}$ & $\operatorname{lnChl}=1 \mathrm{~S} / \mathrm{C} 3 \mathrm{H} 604 / \mathrm{c} 4-2-1-3(5) 7-6 / \mathrm{h} 2-3,5-6 \mathrm{H}, 1 \mathrm{H} 2$ \\
\hline 190 & $\mathrm{C} 2 \mathrm{HCHO}$ & $\mathrm{CHCC}=\mathrm{O}$ & InChl=1S/C3H2O/c1-2-3-4/h1,3H \\
\hline 191 & IC3H7O & $\mathrm{CC}(\mathrm{C})[0]$ & InChl=1S/C3H7O/c1-3(2)4/h3H,1-2H3 \\
\hline 192 & $\mathrm{C} 2 \mathrm{H} 5 \mathrm{COOH}$ & $\operatorname{CCC}(=0) 0$ & InChl=1S/C3H6O2/c1-2-3(4)5/h2H2,1H3,(H,4,5) \\
\hline 193 & $\mathrm{CHCCJOH}$ & $\mathrm{CHC}[\mathrm{CH}] \mathrm{O}$ & InChl=1S/C3H3O/c1-2-3-4/h1,3-4H \\
\hline 194 & TССH6OH & $\mathrm{C}[\mathrm{C}](\mathrm{C}) \mathrm{O}$ & InChl=1S/C3H7O/c1-3(2)4/h4H,1-2H3 \\
\hline 195 & $\mathrm{NC3H702}$ & $\mathrm{CCCO}[\mathrm{O}]$ & $\operatorname{lnChl}=1 \mathrm{~S} / \mathrm{C} 3 \mathrm{H} 7 \mathrm{O} 2 / \mathrm{c} 1-2-3-5-4 / \mathrm{h} 2-3 \mathrm{H} 2,1 \mathrm{H} 3$ \\
\hline 196 & $\mathrm{C} 3 \mathrm{H} 6 \mathrm{OOH} 1-2 \mathrm{O} 2$ & $\mathrm{CC}(\mathrm{COO}) \mathrm{O}[\mathrm{O}]$ & InChl=1S/C3H7O4/c1-3(7-5)2-6-4/h3-4H,2H2,1H3 \\
\hline 197 & $\mathrm{C} 2 \mathrm{H} 3 \mathrm{CO}$ & $\mathrm{C}=\mathrm{C}[\mathrm{C}]=\mathrm{O}$ & $\operatorname{lnChl}=1 \mathrm{~S} / \mathrm{C} 3 \mathrm{H} 3 \mathrm{O} / \mathrm{c1} 1-2-3-4 / \mathrm{h} 2 \mathrm{H}, 1 \mathrm{H} 2$ \\
\hline 198 & $\mathrm{NC} 3 \mathrm{H} 7 \mathrm{OH}$ & $\mathrm{CCCO}$ & InChl=1S/C3H8O/c1-2-3-4/h4H,2-3H2,1H3 \\
\hline 199 & IC3H7O2 & $\mathrm{CC}(\mathrm{C}) \mathrm{O}[\mathrm{O}]$ & InChl=1S/C3H702/c1-3(2)5-4/h3H,1-2H3 \\
\hline 200 & С3H6OOH1-302 & {$[0] 0 \mathrm{cccoO}$} & InChl=1S/C3H7O4/C4-6-2-1-3-7-5/h4H,1-3H2 \\
\hline 201 & CJVCCVO & {$[\mathrm{CH}]=\mathrm{CC}=\mathrm{O}$} & InChl=1S/C3H3O/c1-2-3-4/h1-3H \\
\hline 202 & $\mathrm{IC} 3 \mathrm{H} 7 \mathrm{OH}$ & $\mathrm{CC}(\mathrm{O}) \mathrm{C}$ & $\operatorname{lnChl}=1 \mathrm{~S} / \mathrm{C} 3 \mathrm{H} 8 \mathrm{O} / \mathrm{c} 1-3(2) 4 / \mathrm{h} 3-4 \mathrm{H}, 1-2 \mathrm{H} 3$ \\
\hline 203 & $\mathrm{C} 3 \mathrm{H} 6 \mathrm{OOH} 1-2$ & $\mathrm{C}[\mathrm{CH}] \mathrm{COO}$ & InChl=1S/C3H7O2/c1-2-3-5-4/h2,4H,3H2,1H3 \\
\hline 204 & $\mathrm{C} 3 \mathrm{H} 6 \mathrm{OOH} 2-1 \mathrm{O} 2$ & $\mathrm{CC}(\mathrm{CO}[\mathrm{O}]) 0 \mathrm{O}$ & InChl=1S/C3H7O4/c1-3(7-5)2-6-4/h3,5H,2H2,1H3 \\
\hline 205 & $\mathrm{C} 3 \mathrm{H} 3 \mathrm{O}$ & $\mathrm{C}=\mathrm{C}=\mathrm{C}([\mathrm{O}])$ & $\operatorname{lnChl}=1 \mathrm{~S} / \mathrm{C} 3 \mathrm{H} 3 \mathrm{O} / \mathrm{c1}-2-3-4 / \mathrm{h} 3 \mathrm{H}, 1 \mathrm{H} 2$ \\
\hline 206 & OVCCVCVO & $\mathrm{O}=\mathrm{CC}=\mathrm{C}=\mathrm{O}$ & InChl=1S/C3H2O2/c4-2-1-3-5/h1-2H \\
\hline 207 & С3H6OOH1-3 & {$[\mathrm{CH} 2] \mathrm{CCOO}$} & InChl=1S/C3H702/c1-2-3-5-4/h4H,1-3H2 \\
\hline 208 & C3H51-2,30OH & {$[\mathrm{CH} 2] \mathrm{C}(\mathrm{COO}) \mathrm{OO}$} & InChl=1S/C3H7O4/c1-3(7-5)2-6-4/h3-5H,1-2H2 \\
\hline 209 & $\mathrm{CHCCH} 2 \mathrm{OH}$ & OCCAC & InChl=1S/C3H4O/c1-2-3-4/h1,4H,3H2 \\
\hline 210 & C3Y12-1R & $\mathrm{CC}(=\mathrm{O})[\mathrm{C}]=0$ & $\operatorname{lnChl}=1 \mathrm{~S} / \mathrm{C} 3 \mathrm{H} 3 \mathrm{O} 2 / \mathrm{c} 1-3(5) 2-4 / \mathrm{h} 1 \mathrm{H} 3$ \\
\hline 211 & $\mathrm{C} 3 \mathrm{H} 6 \mathrm{OOH} 2-1$ & {$[\mathrm{CH} 2] \mathrm{C}(\mathrm{C}) \mathrm{OO}$} & InChl=1S/C3H702/c1-3(2)5-4/h3-4H,1H2,2H3 \\
\hline 212 & C3H52-1,30OH & $\mathrm{OOC}[\mathrm{CH}] \mathrm{COO}$ & InChl=1S/C3H704/c4-6-2-1-3-7-5/h1,4-5H,2-3H2 \\
\hline 213 & $\mathrm{CH} 3 \mathrm{CHCO}$ & $\mathrm{CC}=\mathrm{C}=\mathrm{O}$ & $\operatorname{lnChl}=1 \mathrm{~S} / \mathrm{C} 3 \mathrm{H} 4 \mathrm{O} / \mathrm{c} 1-2-3-4 / \mathrm{h} 2 \mathrm{H}, 1 \mathrm{H} 3$ \\
\hline 214 & OVCCJCVO & {$[\mathrm{O}] \mathrm{C}=\mathrm{CC}=\mathrm{O}$} & InChl=1S/C3H3O2/c4-2-1-3-5/h1-3H \\
\hline 215 & $\mathrm{NC} 3 \mathrm{H} 7 \mathrm{O} 2 \mathrm{H}$ & Cccoo & InChl=1S/C3H8O2/c1-2-3-5-4/h4H,2-3H2,1H3 \\
\hline 216 & ME2VOMOOH & $\mathrm{C}(\mathrm{OO}) \mathrm{OC}(=\mathrm{O}) \mathrm{C}(=\mathrm{O})$ & InChl=1S/C3H4O5/c4-1-3(5)7-2-8-6/h1,6H,2H2 \\
\hline 217 & $\mathrm{C} 2 \mathrm{H} 3 \mathrm{CHO}$ & $\mathrm{C}=\mathrm{CC}=\mathrm{O}$ & InChl=1S/C3H4O/c1-2-3-4/h2-3H,1H2 \\
\hline 218 & OVCccJvo & $\mathrm{O}=[\mathrm{C}] \mathrm{CC}=\mathrm{O}$ & InChl=1S/C3H3O2/c4-2-1-3-5/h2H,1H2 \\
\hline 219 & IC3H7O2H & $\mathrm{CC}(\mathrm{C}) 0 \mathrm{O}$ & InChl=1S/C3H8O2/c1-3(2)5-4/h3-4H,1-2H3 \\
\hline 220 & $\mathrm{O} 2 \mathrm{CH} 2 \mathrm{COCH} 2 \mathrm{O} 2 \mathrm{H}$ & OOCC $(=0) \mathrm{CO}[0]$ & InChl=1S/C3H5O5/c4-3(1-7-5)2-8-6/h5H,1-2H2 \\
\hline 221 & PC3H4OH-3 & $\mathrm{C}=\mathrm{C}[\mathrm{CH}] \mathrm{O}$ & InChl=1S/C3H5O/c1-2-3-4/h2-4H,1H2 \\
\hline 222 & ME2JVO & $\operatorname{coc}(=0)[C](=0)$ & InChl=1S/C3H3O3/c1-6-3(5)2-4/h1H3 \\
\hline 223 & СзҮ3Q1-2J & $\mathrm{OOCC}=\mathrm{C}[\mathrm{O}]$ & InChl=1S/C3H5O3/c4-2-1-3-6-5/h1-2,5H,3H2 \\
\hline 224 & $\mathrm{CYC2OCHO}$ & $\mathrm{C} 1 \mathrm{OC} 1 \mathrm{C}=\mathrm{O}$ & InChl=1S/C3H4O2/c4-1-3-2-5-3/h1,3H,2H2 \\
\hline 225 & $\mathrm{C} 3 \mathrm{H} 5 \mathrm{O}$ & $\mathrm{C}=\mathrm{CC}[\mathrm{O}]$ & $\operatorname{lnChl}=1 \mathrm{~S} / \mathrm{C} 3 \mathrm{H} 50 / \mathrm{c} 1-2-3-4 / \mathrm{h} 2 \mathrm{H}, 1,3 \mathrm{H} 2$ \\
\hline 226 & $\mathrm{CH} 2 \mathrm{COHCO}$ & $\mathrm{C}=\mathrm{C}(\mathrm{O})[\mathrm{C}](=\mathrm{O})$ & InChl=1S/C3H3O2/c1-3(5)2-4/h5H,1H2 \\
\hline 227 & ME2VOMJ & {$[\mathrm{CH} 2] \mathrm{OC}(=\mathrm{O}) \mathrm{C}(=\mathrm{O})$} & InChl=1S/C3H3O3/c1-6-3(5)2-4/h2H,1H2 \\
\hline 228 & $\mathrm{CO}(\mathrm{CH} 2 \mathrm{OOH}) 2$ & $c(c(\mathrm{COO})=0) 00$ & InChl=15/C3H6O5/c4-3(1-7-5)2-8-6/h5-6H,1-2H2 \\
\hline 229 & $\mathrm{CH} 2 \mathrm{CHOCH} 2$ & $\mathrm{C}=\mathrm{CO}[\mathrm{CH} 2]$ & InChl=1S/C3H5O/c1-3-4-2/h3H,1-2H2 \\
\hline 230 & С3Y12 & $\mathrm{CC}(=\mathrm{O}) \mathrm{C}=\mathrm{O}$ & InChl=1S/C3H4O2/c1-3(5)2-4/h2H,1H3 \\
\hline 231 & ME2VO & $\operatorname{coc}(=0) C(=0)$ & InChl=1S/C3H4O3/c1-6-3(5)2-4/h2H,1H3 \\
\hline 232 & CH3CHCHO & $\mathrm{C}[\mathrm{CH}] \mathrm{C}=\mathrm{O}$ & InChl=1S/C3H5O/c1-2-3-4/h2-3H,1H3 \\
\hline 233 & $\mathrm{C} 3 \mathrm{H} 3 \mathrm{O} 2 \mathrm{H}$ & $\mathrm{C}=\mathrm{C}=\mathrm{C}(\mathrm{OO})$ & InChl=1S/C3H4O2/c1-2-3-5-4/h3-4H,1H2 \\
\hline 234 & $\mathrm{CH} 3 \mathrm{COCH} 2 \mathrm{O} 2$ & $\mathrm{CC}(=0) \mathrm{CO}[\mathrm{O}]$ & InChl=1S/C3H5O3/c1-3(4)2-6-5/h2H2,1H3 \\
\hline 235 & C3H6OH-30OH-102 & $\mathrm{C}(\mathrm{OO}) \mathrm{CC}(\mathrm{O}[\mathrm{O}]) \mathrm{O}$ & InChl=1S/C3H705/c4-3(8-6)1-2-7-5/h3-5H,1-2H2 \\
\hline 236 & $\mathrm{CH} 2 \mathrm{CCH} 2 \mathrm{OH}$ & $\mathrm{C}=[\mathrm{C}] \mathrm{CO}$ & $\operatorname{lnChl}=1 \mathrm{~S} / \mathrm{C} 3 \mathrm{H} 50 / \mathrm{c} 1-2-3-4 / \mathrm{h} 4 \mathrm{H}, 1,3 \mathrm{H} 2$ \\
\hline 237 & OVCCCVO & $\mathrm{O}=\mathrm{CCC}=\mathrm{O}$ & InChl=15/C3H4O2/C4-2-1-3-5/h2-3H,1H2 \\
\hline 238 & $\mathrm{CH} 2 \mathrm{COCH} 2 \mathrm{O} 2 \mathrm{H}$ & $\mathrm{OOCC}(=\mathrm{C})[\mathrm{O}]$ & $\operatorname{lnChl}=1 \mathrm{~S} / \mathrm{C} 3 \mathrm{H} 5 \mathrm{O} / \mathrm{c} 1-3(4) 2-6-5 / \mathrm{h} 5 \mathrm{H}, 1-2 \mathrm{H} 2$ \\
\hline 239 & С3Ү3ОН1-2J & $\mathrm{OCC}=\mathrm{C}[\mathrm{O}]$ & InChl=1S/C3H5O2/c4-2-1-3-5/h1-2,5H,3H2 \\
\hline 240 & PC3H4OH-2 & $\mathrm{C}[\mathrm{C}]=\mathrm{CO}$ & InChl=1S/C3H5O/c1-2-3-4/h3-4H,1H3 \\
\hline 241 & $\mathrm{CH} 3 \mathrm{COHCO}$ & $\mathrm{CC}(\mathrm{O})=\mathrm{C}=\mathrm{O}$ & InChl=1S/C3H4O2/c1-3(5)2-4/h5H,1H3 \\
\hline 242 & C3KET12 & $\mathrm{CC}(\mathrm{C}=0) 00$ & InChl=15/C3H6O3/c1-3(2-4)6-5/h2-3,5H,1H3 \\
\hline 243 & С3Y3OH1-2O2 & $\mathrm{OCC}(\mathrm{C}=\mathrm{O}) \mathrm{O}[\mathrm{O}]$ & InChl=1S/C3H5O4/c4-1-3(2-5)7-6/h1,3,5H,2H2 \\
\hline 244 & $\mathrm{SC} 3 \mathrm{H} 4 \mathrm{OH}$ & $\mathrm{C}=\mathrm{C}([\mathrm{CH} 2]) \mathrm{O}$ & InChl=1S/C3H5O/c1-3(2)4/h4H,1-2H2 \\
\hline 245 & $\mathrm{CH} 2 \mathrm{COHCHO}$ & $\mathrm{C}=\mathrm{C}(\mathrm{O}) \mathrm{C}=\mathrm{O}$ & $\operatorname{lnChl}=1 \mathrm{~S} / \mathrm{C} 3 \mathrm{H} 4 \mathrm{OO} / \mathrm{c} 1-3(5) 2-4 / \mathrm{h} 2,5 \mathrm{H}, 1 \mathrm{H} 2$ \\
\hline 246 & C3KET13 & $\mathrm{O}=\mathrm{CCCOO}$ & InChl=1S/C3H6O3/c4-2-1-3-6-5/h2,5H,1,3H2 \\
\hline 247 & СзҮзQ2-1J & {$[\mathrm{CH} 2] \mathrm{C}(\mathrm{C}=\mathrm{O}) \mathrm{OO}$} & InChl=1S/C3H5O3/c1-3(2-4)6-5/h2-3,5H,1H2 \\
\hline 248 & IC3H6OH-10OH-3 & $\mathrm{OOCC}(\mathrm{O})[\mathrm{CH} 2]$ & $\operatorname{lnChl}=15 / \mathrm{C} 3 \mathrm{H} 7 \mathrm{O} / \mathrm{c} 1-3(4) 2-6-5 / \mathrm{h} 3-5 \mathrm{H}, 1-2 \mathrm{H} 2$ \\
\hline 249 & IC3H6OH-10OH-2 & $00 \mathrm{O}[\mathrm{C}](0) \mathrm{C}$ & InChl=1S/C3H703/c1-3(4)2-6-5/h4-5H,2H2,1H3 \\
\hline 250 & $\mathrm{HO} 2 \mathrm{CH} 2 \mathrm{CHOHCHO}$ & $\mathrm{OC}(\mathrm{C}=\mathrm{O}) \mathrm{COO}$ & $\operatorname{lnChl}=1 \mathrm{~S} / \mathrm{C} 3 \mathrm{H} 604 / \mathrm{c} 4-1-3(5) 2-7-6 / \mathrm{h} 1,3,5-6 \mathrm{H}, 2 \mathrm{H} 2$ \\
\hline 251 & IC3H6OH-2O2 & {$[\mathrm{O}] \mathrm{OC}(\mathrm{O})(\mathrm{C}) \mathrm{C}$} & InChl=1S/C3H7O3/c1-3(2,4)6-5/h4H,1-2H3 \\
\hline 252 & IC3H6OH-10OH-302 & OOcc(co[0])O & $\operatorname{lnChl}=15 / C 3 H 705 / c 4-3(1-7-5) 2-8-6 / h 3-5 \mathrm{H}, 1-2 \mathrm{H} 2$ \\
\hline 253 & $\mathrm{C} 2 \mathrm{H} 4 \mathrm{CHO}-2 \mathrm{O} 2$ & $\mathrm{CC}(\mathrm{C}=0) \mathrm{O}[\mathrm{O}]$ & InChl=1S/C3H5O3/c1-3(2-4)6-5/h2-3H,1H3 \\
\hline 254 & $\mathrm{C} 2 \mathrm{H} 4 \mathrm{CHO}-3 \mathrm{O} 2$ & {$[\mathrm{O}] \mathrm{OCCC}=\mathrm{O}$} & InChl=1S/C3H5O3/c4-2-1-3-6-5/h2H,1,3H2 \\
\hline 255 & СH3СНОOHCJO & $\mathrm{CC}([\mathrm{C}]=0) 00$ & InChl=1S/C3H5O3/c1-3(2-4)6-5/h3,5H,1H3 \\
\hline 256 & $\mathrm{HO} 2 \mathrm{CH} 2 \mathrm{CH} 2 \mathrm{CJO}$ & $00 c c[C]=0$ & $\operatorname{lnChl}=1 \mathrm{~S} / \mathrm{C} 3 \mathrm{H} 5 \mathrm{O} / \mathrm{c} 4-2-1-3-6-5 / \mathrm{h} 5 \mathrm{H}, 1,3 \mathrm{H} 2$ \\
\hline 257 & $\mathrm{C} 4 \mathrm{H} 2$ & C\#CC\#C & InChl=1S/C4H2/c1-3-4-2/h1-2H \\
\hline 258 & PC4H8OH-4 & {$[\mathrm{CH} 2] \mathrm{CCCO}$} & InChl=1S/C4H9O/c1-2-3-4-5/h5H,1-4H2 \\
\hline 259 & $\mathrm{PC} 4 \mathrm{H} 9 \mathrm{O} 2$ & CCCCO $[0]$ & InChl=1S/C4H9O2/c1-2-3-4-6-5/h2-4H2,1H3 \\
\hline 260 & SC4H8OH-3O2H & $\mathrm{cC}(\mathrm{OO}) \mathrm{C}(\mathrm{O}) \mathrm{c}$ & InChl=1S/C4H10O3/c1-3(5)4(2)7-6/h3-6H,1-2H3 \\
\hline 261 & $\mathrm{C} 4 \mathrm{H} 3-\mathrm{I}$ & $\mathrm{C \# C[C]=C}$ & InChl=1S/C4H3/c1-3-4-2/h1H,2H2 \\
\hline 262 & SC4H8OH-3 & $\mathrm{C}[\mathrm{CH}] \mathrm{C}(\mathrm{O}) \mathrm{C}$ & InChl=1S/C4H9O/c1-3-4(2)5/h3-5H,1-2H3 \\
\hline 263 & SC4H9O2 & $\operatorname{ccc}(\mathrm{C}) \mathrm{O}[\mathrm{O}]$ & InChl=1S/C4H9O2/c1-3-4(2)6-5/h4H,3H2,1-2H3 \\
\hline 264 & SC4H8OH-2O2H & $\operatorname{ccc}(0)(00) \mathrm{C}$ & InChl=1S/C4H1003/c1-3-4(2,5)7-6/h5-6H,3H2,1-2H3 \\
\hline 265 & C4H3-N & {$[\mathrm{CH}]=\mathrm{CCHC}$} & InChl=1S/C4H3/c1-3-4-2/h1-3H \\
\hline 266 & SC4H8OH-4 & {$[\mathrm{CH} 2] \mathrm{CC}(\mathrm{O}) \mathrm{C}$} & InChl=1S/C4H9O/c1-3-4(2)5/h4-5H,1,3H2,2H3 \\
\hline 267 & $\mathrm{C} 4 \mathrm{H} 8 \mathrm{OOH} 1-2$ & $\mathrm{CC}[\mathrm{CH}] \mathrm{COO}$ & InChl=1S/C4H9O2/c1-2-3-4-6-5/h3,5H,2,4H2,1H3 \\
\hline 268 & IC4H8OH-BOOH & $\mathrm{CC}(\mathrm{C})(\mathrm{OO}) \mathrm{CO}$ & $\operatorname{lnChl}=1 \mathrm{~S} / \mathrm{C} 4 \mathrm{H} 1003 / \mathrm{c} 1-4(2,3-5) 7-6 / \mathrm{h5}-6 \mathrm{H}, 3 \mathrm{H} 2,1-2 \mathrm{H} 3$ \\
\hline 269 & $\mathrm{C} 4 \mathrm{H} 4$ & $\mathrm{CHCC}=\mathrm{C}$ & InChl=1S/C4H4/c1-3-4-2/h1,4H,2H2 \\
\hline
\end{tabular}




\begin{tabular}{|c|c|c|c|}
\hline 270 & $\mathrm{SC} 4 \mathrm{H} 8 \mathrm{OH}-1$ & $\mathrm{CCC}(\mathrm{O})[\mathrm{CH} 2]$ & InChl=15/C4H9O/c1-3-4(2)5/h4-5H,2-3H2,1H3 \\
\hline 271 & C4H8OOH1-3 & $\mathrm{C}[\mathrm{CH}] \mathrm{CCOO}$ & InChl=15/C4H9O2/c1-2-3-4-6-5/h2,5H,3-4H2,1H3 \\
\hline 272 & $\mathrm{IQC4H7OH}$ & $\mathrm{CC}(\mathrm{C})(\mathrm{O}) \mathrm{COO}$ & InChl=1S/C4H1003/c1-4(2,5)3-7-6/h5-6H,3H2,1-2H3 \\
\hline 273 & $\mathrm{CHCCH} 2 \mathrm{CH} 2$ & $\mathrm{C \# CC[CH2]}$ & InChl=1S/C4H5/c1-3-4-2/h1H,2,4H2 \\
\hline 274 & SC4H8OH-2 & $\mathrm{CC}[\mathrm{C}](\mathrm{O}) \mathrm{C}$ & InChl=1S/C4H9O/c1-3-4(2)5/h5H,3H2,1-2H3 \\
\hline 275 & $\mathrm{C} 4 \mathrm{H} 8 \mathrm{OOH} 1-4$ & {$[\mathrm{CH} 2] \mathrm{CCCOO}$} & InChl=1S/C4H9O2/c1-2-3-4-6-5/h5H,1-4H2 \\
\hline 276 & IC4H8OH-AOOH & $\mathrm{C}(\mathrm{OO}) \mathrm{C}(\mathrm{C}) \mathrm{CO}$ & $\mathrm{InChl}=1 \mathrm{~S} / \mathrm{C} 4 \mathrm{H} 1003 / \mathrm{c} 1-4(2-5) 3-7-6 / \mathrm{h} 4-6 \mathrm{H}, 2-3 \mathrm{H} 2,1 \mathrm{H} 3$ \\
\hline 277 & $\mathrm{CHCCHCH} 3$ & $\mathrm{C \# C[CH]C}$ & InChl=1S/C4H5/c1-3-4-2/h1,4H,2H3 \\
\hline 278 & IC4H8OH-C & $\mathrm{CC}(\mathrm{C})[\mathrm{CH}] \mathrm{O}$ & InChl=1S/C4H9O/c1-4(2)3-5/h3-5H,1-2H3 \\
\hline 279 & $\mathrm{C} 4 \mathrm{H} 8 \mathrm{OOH} 2-1$ & {$[\mathrm{CH} 2] \mathrm{C}(\mathrm{CC}) \mathrm{OO}$} & InChl=1S/C4H9O2/c1-3-4(2)6-5/h4-5H,2-3H2,1H3 \\
\hline 280 & $\mathrm{SC} 4 \mathrm{H} 8 \mathrm{OH}-4 \mathrm{O} 2 \mathrm{H}$ & $\mathrm{C}(\mathrm{OO}) \mathrm{cc}(\mathrm{O}) \mathrm{c}$ & $\operatorname{lnChl}=1 \mathrm{~S} / \mathrm{C} 4 \mathrm{H} 1003 / \mathrm{c} 1-4(5) 2-3-7-6 / \mathrm{h} 4-6 \mathrm{H}, 2-3 \mathrm{H} 2,1 \mathrm{H} 3$ \\
\hline 281 & $\mathrm{C} 4 \mathrm{H} 5 \mathrm{-I}$ & $\mathrm{C}=[\mathrm{C}] \mathrm{C}=\mathrm{C}$ & InChl=1S/C4H5/c1-3-4-2/h3H,1-2H2 \\
\hline 282 & $\mathrm{IC} 4 \mathrm{H} 8 \mathrm{OH}$ & {$[\mathrm{CH} 2] \mathrm{C}(\mathrm{C}) \mathrm{CO}$} & InChl=1S/C4H9O/c1-4(2)3-5/h4-5H,1,3H2,2H3 \\
\hline 283 & $\mathrm{C} 4 \mathrm{H} 8 \mathrm{OOH} 2-3$ & $\mathrm{C}[\mathrm{CH}] \mathrm{C}(\mathrm{C}) \mathrm{OO}$ & InChl=1S/C4H9O2/c1-3-4(2)6-5/h3-5H,1-2H3 \\
\hline 284 & PC4H8OH-3O2H & $\mathrm{CC}(\mathrm{OO}) \mathrm{CCO}$ & $\operatorname{lnChl}=1 \mathrm{~S} / \mathrm{C} 4 \mathrm{H} 1003 / \mathrm{c} 1-4(7-6) 2-3-5 / \mathrm{h} 4-6 \mathrm{H}, 2-3 \mathrm{H} 2,1 \mathrm{H} 3$ \\
\hline 285 & C4H5-N & {$[\mathrm{CH}]=\mathrm{CC}=\mathrm{C}$} & $\mathrm{InChl}=1 \mathrm{~S} / \mathrm{C} 4 \mathrm{H} 5 / \mathrm{c} 1-3-4-2 / \mathrm{h} 1,3-4 \mathrm{H}, 2 \mathrm{H} 2$ \\
\hline 286 & IC4H8OH-IT & $\mathrm{C}[\mathrm{C}](\mathrm{C}) \mathrm{CO}$ & $\mathrm{InChl}=1 \mathrm{~S} / \mathrm{C} 4 \mathrm{H} 9 \mathrm{O} / \mathrm{c1}-4(2) 3-5 / \mathrm{h} 5 \mathrm{H}, 3 \mathrm{H} 2,1-2 \mathrm{H} 3$ \\
\hline 287 & $\mathrm{C} 4 \mathrm{H} 8 \mathrm{OOH} 2-4$ & {$[\mathrm{CH} 2] \mathrm{CC}(\mathrm{C}) \mathrm{OO}$} & InChl=15/C4H9O2/c1-3-4(2)6-5/h4-5H,1,3H2,2H3 \\
\hline 288 & РС448OH-402H & $\mathrm{C}(\mathrm{OO}) \mathrm{CCCO}$ & InChl=1S/C4H10O3/c5-3-1-2-4-7-6/h5-6H,1-4H2 \\
\hline 289 & $\mathrm{C} 4 \mathrm{H} 5-2$ & $\mathrm{CC \# C[CH2]}$ & InChl=1S/C4H5/c1-3-4-2/h1H2,2H3 \\
\hline 290 & IC4H8OH-TI & $\mathrm{CC}(\mathrm{C})(\mathrm{O})[\mathrm{CH} 2]$ & $\mathrm{InChl}=1 \mathrm{~S} / \mathrm{C} 4 \mathrm{H} 9 \mathrm{O} / \mathrm{c} 1-4(2,3) 5 / \mathrm{h} 5 \mathrm{H}, 1 \mathrm{H} 2,2-3 \mathrm{H} 3$ \\
\hline 291 & IC4H9O2 & $\mathrm{CC}(\mathrm{C}) \mathrm{CO}[\mathrm{O}]$ & InChl=1S/C4H9O2/c1-4(2)3-6-5/h4H,3H2,1-2H3 \\
\hline 292 & MP3JVO2VO & $\operatorname{coc}(=0) C(=O)[C](=0)$ & InChl=1S/C4H3O4/c1-8-4(7)3(6)2-5/h1H3 \\
\hline 293 & C4H6-1 & C\#CCC & $\operatorname{lnChl}=1 \mathrm{~S} / \mathrm{C} 4 \mathrm{H} 6 / \mathrm{c} 1-3-4-2 / \mathrm{h} 1 \mathrm{H}, 4 \mathrm{H} 2,2 \mathrm{H} 3$ \\
\hline 294 & PC4H9O & $\operatorname{cccC}[0]$ & InChl=1S/C4H9O/c1-2-3-4-5/h2-4H2,1H3 \\
\hline 295 & TC4H9O2 & $\mathrm{CC}(\mathrm{C})(\mathrm{C}) \mathrm{O}[\mathrm{O}]$ & InChl=1S/C4H9O2/c1-4(2,3)6-5/h1-3H3 \\
\hline 296 & MP3VO2VO & $\operatorname{coc}(=0) C(=0) C(=0)$ & InChl=1S/C4H4O4/c1-8-4(7)3(6)2-5/h2H,1H3 \\
\hline 297 & $\mathrm{C} 4 \mathrm{H} 6$ & $\mathrm{C}=\mathrm{CC}=\mathrm{C}$ & $\operatorname{lnChl}=1 \mathrm{~S} / \mathrm{C} 4 \mathrm{H} 6 / \mathrm{c1}-3-4-2 / \mathrm{h} 3-4 \mathrm{H}, 1-2 \mathrm{H} 2$ \\
\hline 298 & SC4H9O & $\operatorname{ccc}(C)[0]$ & $\mathrm{InChl}=1 \mathrm{~S} / \mathrm{C} 4 \mathrm{H} 9 \mathrm{O} / \mathrm{c1}-3-4(2) 5 / \mathrm{h} 4 \mathrm{H}, 3 \mathrm{H} 2,1-2 \mathrm{H} 3$ \\
\hline 299 & $\mathrm{IC} 4 \mathrm{H} 8 \mathrm{O} 2 \mathrm{H}-\mathrm{I}$ & {$[\mathrm{CH} 2] \mathrm{C}(\mathrm{C}) \mathrm{COO}$} & InChl=15/C4H9O2/c1-4(2)3-6-5/h4-5H,1,3H2,2H3 \\
\hline 300 & $\mathrm{CH} 3 \mathrm{OCCOHOOCH} 2$ & $\mathrm{CC}(=0) \mathrm{C}(=0) \mathrm{COO}$ & InChl=1S/C4H6O4/c1-3(5)4(6)2-8-7/h7H,2H2,1H3 \\
\hline 301 & C4H612 & $\mathrm{C}=\mathrm{C}=\mathrm{CC}$ & InChl=1S/C4H6/c1-3-4-2/h4H,1H2,2H3 \\
\hline 302 & TC4H9O & $\mathrm{CC}(\mathrm{C})(\mathrm{C})[\mathrm{O}]$ & InChl=1S/C4H9O/c1-4(2,3)5/h1-3H3 \\
\hline 303 & $\mathrm{IC} 4 \mathrm{H} 8 \mathrm{O} 2 \mathrm{H}-\mathrm{T}$ & $\mathrm{C}[\mathrm{C}](\mathrm{C}) \mathrm{COO}$ & InChl=1S/C4H9O2/c1-4(2)3-6-5/h5H,3H2,1-2H3 \\
\hline 304 & $\mathrm{OCHHOOCHCOCH} 3$ & $\mathrm{C}(=\mathrm{O}) \mathrm{C}(\mathrm{OO}) \mathrm{C}(=\mathrm{O}) \mathrm{C}$ & InChl=1S/C4H6O4/c1-3(6)4(2-5)8-7/h2,4,7H,1H3 \\
\hline 305 & C4H6-2 & CC\#CC & $\operatorname{lnChl}=1 \mathrm{~S} / \mathrm{C} 4 \mathrm{H} 6 / \mathrm{c} 1-3-4-2 / \mathrm{h} 1-2 \mathrm{H} 3$ \\
\hline 306 & $\mathrm{IC4H9O}$ & $\mathrm{CC}(\mathrm{C}) \mathrm{C}[\mathrm{O}]$ & InChl=1S/C4H9O/c1-4(2)3-5/h4H,3H2,1-2H3 \\
\hline 307 & TC4H8O2H-I & {$[\mathrm{CH} 2] \mathrm{C}(\mathrm{C})(\mathrm{C}) \mathrm{OO}$} & InChl=1S/C4H9O2/c1-4(2,3)6-5/h5H,1H2,2-3H3 \\
\hline 308 & CC(OO)CVOCVO & $\operatorname{CC}(\mathrm{C}(=0) \mathrm{C}=0) 00$ & InChl=1S/C4H6O4/c1-3(8-7)4(6)2-5/h2-3,7H,1H3 \\
\hline 309 & IC4H7-I1 & {$[\mathrm{CH}]=\mathrm{C}(\mathrm{C}) \mathrm{C}$} & InChl=1S/C4H7/c1-4(2)3/h1H,2-3H3 \\
\hline 310 & $\mathrm{NC4H9OH}$ & $\mathrm{CCCCO}$ & InChl=1S/C4H100/c1-2-3-4-5/h5H,2-4H2,1H3 \\
\hline 311 & PC4H9O2H & ccccoo & InChl=1S/C4H1002/c1-2-3-4-6-5/h5H,2-4H2,1H3 \\
\hline 312 & $\mathrm{OCHCH} 2 \mathrm{COHOOCH} 2$ & $\mathrm{C}(=0) \mathrm{CC}(=0) \mathrm{COO}$ & InChl=1S/C4H6O4/c5-2-1-4(6)3-8-7/h2,7H,1,3H2 \\
\hline 313 & $\mathrm{IC4H7}$ & {$[\mathrm{CH} 2] \mathrm{C}(=\mathrm{C}) \mathrm{C}$} & InChl=1S/C4H7/c1-4(2)3/h1-2H2,3H3 \\
\hline 314 & $\mathrm{SC} 4 \mathrm{H} 9 \mathrm{OH}$ & $\operatorname{ccc}(0) \mathrm{C}$ & InChl=1S/C4H10O/c1-3-4(2)5/h4-5H,3H2,1-2H3 \\
\hline 315 & $\mathrm{SC} 4 \mathrm{H} 9 \mathrm{OO} \mathrm{H}$ & $\operatorname{ccc}(C) 00$ & InChl=1S/C4H10O2/c1-3-4(2)6-5/h4-5H,3H2,1-2H3 \\
\hline 316 & C4H71-1 & {$[\mathrm{CH}]=\mathrm{CCC}$} & $\operatorname{lnChl}=1 \mathrm{~S} / \mathrm{C} 4 \mathrm{H} 7 / \mathrm{c1} 1-3-4-2 / \mathrm{h} 1,3 \mathrm{H}, 4 \mathrm{H} 2,2 \mathrm{H} 3$ \\
\hline 317 & $\mathrm{IC} 4 \mathrm{H} 9 \mathrm{OH}$ & $\mathrm{CC}(\mathrm{C}) \mathrm{CO}$ & InChl=1S/C4H10O/c1-4(2)3-5/h4-5H,3H2,1-2H3 \\
\hline 318 & $\mathrm{IC} 4 \mathrm{H} 9 \mathrm{O} 2 \mathrm{H}$ & $\mathrm{CC}(\mathrm{C}) \mathrm{COO}$ & InChl=1S/C4H10O2/c1-4(2)3-6-5/h4-5H,3H2,1-2H3 \\
\hline 319 & $\mathrm{C} 4 \mathrm{Y} 2 \mathrm{OH} 4-3 \mathrm{O} 2$ & $\mathrm{OCC}(\mathrm{C}(=\mathrm{O}) \mathrm{C}) \mathrm{O}[\mathrm{O}]$ & InChl=1S/C4H704/c1-3(6)4(2-5)8-7/h4-5H,2H2,1H3 \\
\hline 320 & C4H71-2 & $\mathrm{C}=[\mathrm{C}] \mathrm{CC}$ & InChl=1S/C4H7/c1-3-4-2/h1,4H2,2H3 \\
\hline 321 & TC4H9OH & $\mathrm{CC}(\mathrm{C})(\mathrm{O}) \mathrm{C}$ & $\operatorname{lnChl}=1 \mathrm{~S} / \mathrm{C} 4 \mathrm{H} 10 \mathrm{O} / \mathrm{c} 1-4(2,3) 5 / \mathrm{h} 5 \mathrm{H}, 1-3 \mathrm{H} 3$ \\
\hline 322 & TC4H9O2H & $\mathrm{CC}(\mathrm{C})(\mathrm{C}) 0 \mathrm{O}$ & InChl=1S/C4H10O2/c1-4(2,3)6-5/h5H,1-3H3 \\
\hline 323 & C4H71-3 & $\mathrm{C}=\mathrm{C}[\mathrm{CH}] \mathrm{C}$ & InChl=1S/C4H7/c1-3-4-2/h3-4H,1H2,2H3 \\
\hline 324 & F2OJ & {$[0] \mathrm{C} 1=\mathrm{CC}=\mathrm{CO} 1$} & $\operatorname{lnChl}=1 \mathrm{~S} / \mathrm{C} 4 \mathrm{H} 3 \mathrm{O} 2 / \mathrm{c5}-4-2-1-3-6-4 / \mathrm{h} 1-3 \mathrm{H}$ \\
\hline 325 & $\mathrm{C} 2 \mathrm{H} 5 \mathrm{OCOCHOO}$ & $\cos (\operatorname{co}[0])=0$ & InChl=1S/C4H7O4/c1-2-7-4(5)3-8-6/h2-3H2,1H3 \\
\hline 326 & C4H71-4 & {$[\mathrm{CH} 2] \mathrm{CC}=\mathrm{C}$} & $\mathrm{InChl}=1 \mathrm{~S} / \mathrm{C} 4 \mathrm{H} 7 / \mathrm{c} 1-3-4-2 / \mathrm{h} 3 \mathrm{H}, 1-2,4 \mathrm{H} 2$ \\
\hline 327 & ovcCVCCJVO & $\mathrm{O}=\mathrm{CC}=\mathrm{C}[\mathrm{C}]=\mathrm{O}$ & $\operatorname{lnChl}=15 / \mathrm{C} 4 \mathrm{H} 3 \mathrm{O} 2 / \mathrm{C} 5-3-1-2-4-6 / \mathrm{h} 1-3 \mathrm{H}$ \\
\hline 328 & MP3JVO & $\operatorname{COC}(=0) C[C](=0)$ & $\operatorname{lnChl}=1 \mathrm{~S} / \mathrm{C} 4 \mathrm{H} 5 \mathrm{O} 3 / \mathrm{c} 1-7-4(6) 2-3-5 / \mathrm{h} 2 \mathrm{H} 2,1 \mathrm{H} 3$ \\
\hline 329 & $\mathrm{CVC}(\mathrm{COOJ}) \mathrm{COOH}$ & $\mathrm{OOCC}(=\mathrm{C}) \mathrm{CO}[\mathrm{O}]$ & $\operatorname{lnChl}=1 \mathrm{~S} / \mathrm{C} 4 \mathrm{H} 704 / \mathrm{c} 1-4(2-7-5) 3-8-6 / \mathrm{h} 5 \mathrm{H}, 1-3 \mathrm{H} 2$ \\
\hline 330 & $\mathrm{C} 4 \mathrm{H} 72-2$ & $\mathrm{C}[\mathrm{C}]=\mathrm{CC}$ & InChl=1S/C4H7/c1-3-4-2/h3H,1-2H3 \\
\hline 331 & B23DE104OH & $\mathrm{OC}=\mathrm{C}=\mathrm{CC}=\mathrm{O}$ & InChl=1S/C4H4O2/c5-3-1-2-4-6/h1,3-4,6H \\
\hline 332 & $\mathrm{CC}(\mathrm{OOJ}) \mathrm{CVOCO}$ & {$[\mathrm{O}] \mathrm{OC}(\mathrm{C}(=\mathrm{O}) \mathrm{CO}) \mathrm{C}$} & InChl=1S/C4H704/c1-3(8-7)4(6)2-5/h3,5H,2H2,1H3 \\
\hline 333 & IC4H8 & $\mathrm{C}=\mathrm{C}(\mathrm{C}) \mathrm{C}$ & InChl=1S/C4H8/c1-4(2)3/h1H2,2-3H3 \\
\hline 334 & $\mathrm{~F} 2 \mathrm{OH}$ & $\mathrm{OC} 1=\mathrm{CC}=\mathrm{CO} 1$ & InChl=1S/C4H4O2/c5-4-2-1-3-6-4/h1-3,5H \\
\hline 335 & MP3VOMJ & {$[\mathrm{CH} 2] \mathrm{OC}(=\mathrm{O}) \mathrm{CC}(=\mathrm{O})$} & InChl=1S/C4H5O3/c1-7-4(6)2-3-5/h3H,1-2H2 \\
\hline 336 & $\mathrm{CC}(\mathrm{OO}) \mathrm{CVOCJO}$ & $\mathrm{OOC}(\mathrm{C}(=\mathrm{CO})[\mathrm{O}]) \mathrm{C}$ & InChl=15/C4H7O4/c1-3(8-7)4(6)2-5/h2-3,5,7H,1H3 \\
\hline 337 & C4H8-1 & $\mathrm{C}=\mathrm{CCC}$ & $\operatorname{lnChl}=1 \mathrm{~S} / \mathrm{C} 4 \mathrm{H} 8 / \mathrm{c} 1-3-4-2 / \mathrm{h} 3 \mathrm{H}, 1,4 \mathrm{H} 2,2 \mathrm{H} 3$ \\
\hline 338 & OVCC(VC)CVO & $\mathrm{O}=\mathrm{CC}(=\mathrm{C}) \mathrm{C}=\mathrm{O}$ & $\operatorname{lnChl}=1 \mathrm{~S} / \mathrm{C} 4 \mathrm{H} 4 \mathrm{O} 2 / \mathrm{c} 1-4(2-5) 3-6 / \mathrm{h} 2-3 \mathrm{H}, 1 \mathrm{H} 2$ \\
\hline 339 & MP3VO2J & $\mathrm{COC}(=\mathrm{O})[\mathrm{CH}] \mathrm{C}(=\mathrm{O})$ & $\operatorname{lnChl}=1 \mathrm{~S} / \mathrm{C} 4 \mathrm{H} 5 \mathrm{O} 3 / \mathrm{c} 1-7-4(6) 2-3-5 / \mathrm{h} 2-3 \mathrm{H}, 1 \mathrm{H} 3$ \\
\hline 340 & $\mathrm{C} 4 \mathrm{H} 61-\mathrm{OOH} 34$ & $00 \mathrm{OCC}(\mathrm{C}=\mathrm{C}) 00$ & InChl=1S/C4H8O4/c1-2-4(8-6)3-7-5/h2,4-6H,1,3H2 \\
\hline 341 & C4H8-2 & $\mathrm{CC}=\mathrm{CC}$ & $\operatorname{lnChl}=1 \mathrm{~S} / \mathrm{C} 4 \mathrm{H} 8 / \mathrm{c1} 1-3-4-2 / \mathrm{h} 3-4 \mathrm{H}, 1-2 \mathrm{H} 3$ \\
\hline 342 & ovcCVCCVO & $\mathrm{O}=\mathrm{CC}=\mathrm{CC}=\mathrm{O}$ & $\mathrm{InChl}=1 \mathrm{~S} / \mathrm{C} 4 \mathrm{H} 4 \mathrm{O} 2 / \mathrm{c} 5-3-1-2-4-6 / \mathrm{h} 1-4 \mathrm{H}$ \\
\hline 343 & C4H62-OOH14 & $\mathrm{OOCC}=\mathrm{CCOO}$ & InChl=15/C4H8O4/c5-7-3-1-2-4-8-6/h1-2,5-6H,3-4H2 \\
\hline 344 & PC4H9 & {$[\mathrm{CH} 2] \mathrm{CCC}$} & InChl=1S/C4H9/c1-3-4-2/h1,3-4H2,2H3 \\
\hline 345 & C4H512-004 & {$[\mathrm{O}] \mathrm{OCC}=\mathrm{C}=\mathrm{C}$} & InChl=1S/C4H5O2/c1-2-3-4-6-5/h3H,1,4H2 \\
\hline 346 & OVCCVCCOOH & $\mathrm{OOCC}=\mathrm{CC}=\mathrm{O}$ & InChl=1S/C4H6O3/c5-3-1-2-4-7-6/h1-3,6H,4H2 \\
\hline 347 & NC4KET21OH-1 & $\operatorname{CCC}(=0) \mathrm{C}(00) \mathrm{O}$ & InChl=1S/C4H8O4/c1-2-3(5)4(6)8-7/h4,6-7H,2H2,1H3 \\
\hline 348 & SC4H9 & $\mathrm{C}[\mathrm{CH}] \mathrm{CC}$ & $\operatorname{lnChl}=1 \mathrm{~S} / \mathrm{C} 4 \mathrm{H} 9 / \mathrm{c} 1-3-4-2 / \mathrm{h} 3 \mathrm{H}, 4 \mathrm{H} 2,1-2 \mathrm{H} 3$ \\
\hline 349 & CYCCCOO-3J & $\mathrm{C}=\mathrm{C} 1[\mathrm{CH}] \mathrm{COO} 1$ & $\operatorname{lnChl}=1 \mathrm{~S} / \mathrm{C} 4 \mathrm{H} 5 \mathrm{O} 2 / \mathrm{c} 1-4-2-3-5-6-4 / \mathrm{h} 2 \mathrm{H}, 1,3 \mathrm{H} 2$ \\
\hline 350 & MP3VO & $\operatorname{coc}(=0) \operatorname{CC}(=0)$ & $\operatorname{lnChl}=1 \mathrm{~S} / \mathrm{C} 4 \mathrm{H} 6 \mathrm{O} 3 / \mathrm{c} 1-7-4(6) 2-3-5 / \mathrm{h} 3 \mathrm{H}, 2 \mathrm{H} 2,1 \mathrm{H} 3$ \\
\hline 351 & NC4KET12OH-1 & $\operatorname{ccc}(\mathrm{OOO}) \mathrm{C}(=0) \mathrm{O}$ & InChl=1S/C4H8O4/c1-2-3(8-7)4(5)6/h3,7H,2H2,1H3,(H,5,6) \\
\hline 352 & $\mathrm{IC4H} 9$ & {$[\mathrm{CH} 2] \mathrm{C}(\mathrm{C}) \mathrm{C}$} & InChl=1S/C4H9/c1-4(2)3/h4H,1H2,2-3H3 \\
\hline 353 & OJCCVCCVO & {$[\mathrm{O}] \mathrm{CC}=\mathrm{CC}=\mathrm{O}$} & InChl=1S/C4H5O2/c5-3-1-2-4-6/h1-3H,4H2 \\
\hline 354 & CH3CHOOHCHCO & $\mathrm{CC}(\mathrm{OO}) \mathrm{C}=\mathrm{C}=\mathrm{O}$ & $\operatorname{lnChl}=15 / C 4 H 603 / c 1-4(7-6) 2-3-5 / h 2,4,6 \mathrm{H}, 1 \mathrm{H} 3$ \\
\hline 355 & NC4KET12OH-2 & $\operatorname{ccc}(0)(\mathrm{OO}) \mathrm{C}=\mathrm{O}$ & $\operatorname{lnChl}=1 \mathrm{~S} / \mathrm{C} 4 \mathrm{H} 8 \mathrm{OO} / \mathrm{c1}-2-4(6,3-5) 8-7 / \mathrm{h} 3,6-7 \mathrm{H}, 2 \mathrm{H} 2,1 \mathrm{H} 3$ \\
\hline 356 & TC4H9 & $\mathrm{C}[\mathrm{C}](\mathrm{C}) \mathrm{C}$ & InChl=1S/C4H9/c1-4(2)3/h1-3H3 \\
\hline 357 & $\mathrm{C} 4 \mathrm{H} 513-0 \mathrm{OO}$ & {$[\mathrm{O}] \mathrm{OC}(=\mathrm{C}) \mathrm{C}=\mathrm{C}$} & InChl=1S/C4H5O2/c1-3-4(2)6-5/h3H,1-2H2 \\
\hline 358 & $\mathrm{CH} 2 \mathrm{OOHCH} 2 \mathrm{CHCO}$ & $\mathrm{C}(\mathrm{OO}) \mathrm{CC}=\mathrm{C}=\mathrm{O}$ & InChl=1S/C4H6O3/c5-3-1-2-4-7-6/h1,6H,2,4H2 \\
\hline 359 & IC4KET31OH-1 & $\mathrm{C}(=0) \mathrm{C}(\mathrm{C}) \mathrm{C}(\mathrm{OO}) \mathrm{O}$ & $\mathrm{InChl}=1 \mathrm{~S} / \mathrm{C} 4 \mathrm{H} 8 \mathrm{OO} / \mathrm{c} 1-3(2-5) 4(6) 8-7 / \mathrm{h} 2-4,6-7 \mathrm{H}, 1 \mathrm{H} 3$ \\
\hline 360 & $\mathrm{C} 4 \mathrm{H} 10$ & $\operatorname{cccc}$ & $\operatorname{lnChl}=1 \mathrm{~S} / \mathrm{C} 4 \mathrm{H} 10 / \mathrm{c} 1-3-4-2 / \mathrm{h} 3-4 \mathrm{H} 2,1-2 \mathrm{H} 3$ \\
\hline
\end{tabular}




\begin{tabular}{|c|c|c|c|}
\hline 361 & $\mathrm{CH} 2 \mathrm{CYCOO}-\mathrm{CH} 2$ & $\mathrm{C}=\mathrm{CC} 1([\mathrm{CH} 2]) 001$ & InChl=1S/C4H5O2/c1-3-4(2)5-6-4/h3H,1-2H2 \\
\hline 362 & $\mathrm{OVCC}(\mathrm{VC}) \mathrm{COOH}$ & $\mathrm{OOCC}(=\mathrm{C}) \mathrm{C}=\mathrm{O}$ & InChl=1S/C4H6O3/c1-4(2-5)3-7-6/h2,6H,1,3H2 \\
\hline 363 & IC4KET13OH-1 & $\mathrm{C}(\mathrm{OO}) \mathrm{C}(\mathrm{C}) \mathrm{C}(=0) \mathrm{O}$ & InChl=1S/C4H8O4/c1-3(2-8-7)4(5)6/h3,7H,2H2,1H3,(H,5,6) \\
\hline 364 & $\mathrm{IC} 4 \mathrm{H} 10$ & $\mathrm{CC}(\mathrm{C}) \mathrm{C}$ & InChl=1S/C4H10/c1-4(2)3/h4H,1-3H3 \\
\hline 365 & $\mathrm{CYCOOC}-\mathrm{CH} 2$ & $\mathrm{C}=\mathrm{C} 100 \mathrm{C} 1[\mathrm{CH} 2]$ & InChl=1S/C4H5O2/c1-3-4(2)6-5-3/h3H,1-2H2 \\
\hline 366 & $\mathrm{C} 4 \mathrm{D} 2 \mathrm{OH} 4-2 \mathrm{O} 2$ & $\mathrm{OCC}=\mathrm{C}(\mathrm{O}[\mathrm{O}]) \mathrm{C}$ & $\operatorname{lnChl}=1 \mathrm{~S} / \mathrm{C} 4 \mathrm{H} 703 / \mathrm{c} 1-4(7-6) 2-3-5 / \mathrm{h} 2,5 \mathrm{H}, 3 \mathrm{H} 2,1 \mathrm{H} 3$ \\
\hline 367 & NC4KET31OH-1 & $\mathrm{CC}(=0) \mathrm{CC}(\mathrm{OO}) \mathrm{O}$ & InChl=1S/C4H8O4/c1-3(5)2-4(6)8-7/h4,6-7H,2H2,1H3 \\
\hline 368 & $\mathrm{CHCCHCO}$ & $\mathrm{C \# CC=C=O}$ & InChl=1S/C4H2O/c1-2-3-4-5/h1,3H \\
\hline 369 & $\mathrm{C} 2 \mathrm{H} 3 \mathrm{COOCH} 2$ & {$[\mathrm{CH} 2] \mathrm{OC}(=\mathrm{O}) \mathrm{C}=\mathrm{C}$} & InChl=1S/C4H5O2/c1-3-4(5)6-2/h3H,1-2H \\
\hline 370 & $\mathrm{CH} 2 \mathrm{CHOOHCOCH} 3$ & {$[\mathrm{CH} 2] \mathrm{C}(\mathrm{OO}) \mathrm{C}(\mathrm{C})=\mathrm{O}$} & InChl=1S/C4H7O3/c1-3(5)4(2)7-6/h4,6H,2H2,1H3 \\
\hline 371 & NC4KET32OH-2 & $\mathrm{CC}(=\mathrm{O}) \mathrm{C}(\mathrm{O})(\mathrm{OO}) \mathrm{C}$ & $\operatorname{lnChl}=1 \mathrm{~S} / \mathrm{C} 4 \mathrm{H} 8 \mathrm{O} 4 / \mathrm{c} 1-3(5) 4(2,6) 8-7 / \mathrm{h} 6-7 \mathrm{H}, 1-2 \mathrm{H} 3$ \\
\hline 372 & $\mathrm{H} 2 \mathrm{C} 4 \mathrm{O}$ & $\mathrm{CHCC1}=\mathrm{C}(\mathrm{O} 1)$ & $\operatorname{lnChl}=1 \mathrm{~S} / \mathrm{C} 4 \mathrm{H} 2 \mathrm{O} / \mathrm{c} 1-2-4-3-5-4 / \mathrm{h} 1,3 \mathrm{H}$ \\
\hline 373 & $\mathrm{NC4H5O2}$ & {$[\mathrm{O}] \mathrm{OC}=\mathrm{CC}=\mathrm{C}$} & InChl=1S/C4H5O2/c1-2-3-4-6-5/h2-4H,1H2 \\
\hline 374 & $\mathrm{CH} 3 \mathrm{CHOOCOCH} 3$ & $\mathrm{CC}(=\mathrm{O}) \mathrm{C}(\mathrm{C}) \mathrm{O}[\mathrm{O}]$ & InChl=1S/C4H7O3/c1-3(5)4(2)7-6/h4H,1-2H3 \\
\hline 375 & NC4KET34OH-2 & $\mathrm{C}(\mathrm{OO}) \mathrm{C}(=\mathrm{O}) \mathrm{C}(\mathrm{O}) \mathrm{C}$ & $\operatorname{lnChl}=1 \mathrm{~S} / \mathrm{C} 4 \mathrm{H} 8 \mathrm{OO} 4 \mathrm{c} 1-3(5) 4(6) 2-8-7 / \mathrm{h} 3,5,7 \mathrm{H}, 2 \mathrm{H} 2,1 \mathrm{H} 3$ \\
\hline 376 & СзH3СO & $\mathrm{C}=\mathrm{C}=\mathrm{C}[\mathrm{C}]=\mathrm{O}$ & InChl=1S/C4H3O/c1-2-3-4-5/h3H,1H2 \\
\hline 377 & $\mathrm{C} 2 \mathrm{H} 3 \mathrm{CHOHCH} 2 \mathrm{OO}$ & $\mathrm{OC}(\mathrm{C}=\mathrm{C}) \mathrm{CO}[\mathrm{O}]$ & InChl=1S/C4H703/c1-2-4(5)3-7-6/h2,4-5H,1,3H2 \\
\hline 378 & NC4KET24OH-3 & $\mathrm{CC}(=0) \mathrm{C}(\mathrm{O}) \mathrm{COO}$ & InChl=1S/C4H8O4/c1-3(5)4(6)2-8-7/h4,6-7H,2H2,1H3 \\
\hline 379 & $\mathrm{CH} 2 \mathrm{CHCCO}$ & $\mathrm{C}=\mathrm{C}[\mathrm{C}]=\mathrm{C}=\mathrm{O}$ & InChl=1S/C4H3O/c1-2-3-4-5/h2H,1H2 \\
\hline 380 & $\mathrm{C} 2 \mathrm{H} 5 \mathrm{COCO}$ & {$[\mathrm{C}](=\mathrm{O}) \mathrm{C}(=\mathrm{O}) \mathrm{CC}$} & InChl=1S/C4H5O2/c1-2-4(6)3-5/h2H2,1H3 \\
\hline 381 & MP2OH3J & $\mathrm{COC}(=\mathrm{O}) \mathrm{C}(\mathrm{O})[\mathrm{CH} 2]$ & InChl=1S/C4H7O3/c1-3(5)4(6)7-2/h3,5H,1H2,2H3 \\
\hline 382 & NC4KET43OH-2 & $\mathrm{C}(=0) \mathrm{C}(\mathrm{OO}) \mathrm{C}(\mathrm{O}) \mathrm{C}$ & InChl=1S/C4H8O4/c1-3(6)4(2-5)8-7/h2-4,6-7H,1H3 \\
\hline 383 & $\mathrm{CHCCHCHO}$ & $\mathrm{C \# CC=C[O]}$ & $\operatorname{lnChl}=1 \mathrm{~S} / \mathrm{C} 4 \mathrm{H} 3 \mathrm{O} / \mathrm{c} 1-2-3-4-5 / \mathrm{h} 1,3-4 \mathrm{H}$ \\
\hline 384 & MP2D3J & $\operatorname{COC}(=\mathrm{O}) \mathrm{C}=[\mathrm{CH}]$ & InChl=1S/C4H5O2/c1-3-4(5)6-2/h1,3H,2H3 \\
\hline 385 & $\mathrm{SC} 2 \mathrm{H} 4 \mathrm{COHOOCH} 2$ & $\mathrm{C}[\mathrm{CH}] \mathrm{C}(=0) \mathrm{COO}$ & InChl=15/C4H703/c1-2-4(5)3-7-6/h2,6H,3H2,1H3 \\
\hline 386 & NC4KET32OH-1 & $\mathrm{CC}(=0) \mathrm{C}(\mathrm{OO}) \mathrm{CO}$ & InChl=1S/C4H8O4/c1-3(6)4(2-5)8-7/h4-5,7H,2H2,1H3 \\
\hline 387 & F2J & $\mathrm{O} 1[\mathrm{C}]=\mathrm{CC}=\mathrm{C} 1$ & $\operatorname{lnChl}=1 \mathrm{~S} / \mathrm{C} 4 \mathrm{H} 3 \mathrm{O} / \mathrm{c} 1-2-4-5-3-1 / \mathrm{h} 1-3 \mathrm{H}$ \\
\hline 388 & OVCC(VC)COJ & {$[\mathrm{O}] \mathrm{CC}(=\mathrm{C}) \mathrm{C}=\mathrm{O}$} & InChl=1S/C4H5O2/c1-4(2-5)3-6/h2H,1,3H2 \\
\hline 389 & $\mathrm{C} 2 \mathrm{H} 5 \mathrm{COO} 2 \mathrm{CH} 2$ & $\operatorname{ccC}(=0) \operatorname{CO}[0]$ & InChl=1S/C4H7O3/c1-2-4(5)3-7-6/h2-3H2,1H3 \\
\hline 390 & NC4KET13OH-1 & $\mathrm{CC}(\mathrm{OO}) \mathrm{CC}(=\mathrm{O}) \mathrm{O}$ & $\operatorname{lnChl}=1 \mathrm{~S} / \mathrm{C} 4 \mathrm{H} 8 \mathrm{O} 4 / \mathrm{cc} 1-3(8-7) 2-4(5) 6 / \mathrm{h} 3,7 \mathrm{H}, 2 \mathrm{H} 2,1 \mathrm{H} 3,(\mathrm{H}, 5,6)$ \\
\hline 391 & F3」 & $\mathrm{O} 1 \mathrm{C}=[\mathrm{C}] \mathrm{C}=\mathrm{C} 1$ & InChl=1S/C4H3O/c1-2-4-5-3-1/h1,3-4H \\
\hline 392 & $\mathrm{CC}(\mathrm{VO}) \mathrm{CVCO}$ & $\mathrm{CC}(=\mathrm{O}) \mathrm{C}=\mathrm{CO}$ & InChl=1S/C4H6O2/c1-4(6)2-3-5/h2-3,5H,1H3 \\
\hline 393 & $\mathrm{PC} 2 \mathrm{H} 4 \mathrm{COHOOCH} 2$ & {$[\mathrm{CH} 2] \mathrm{CC}(=\mathrm{O}) \mathrm{COO}$} & InChl=1S/C4H7O3/c1-2-4(5)3-7-6/h6H,1-3H2 \\
\hline 394 & NC4KET13OH-2 & $\mathrm{CC}(\mathrm{OO}) \mathrm{C}(\mathrm{O}) \mathrm{C}=\mathrm{O}$ & InChl=15/C4H8O4/c1-3(8-7)4(6)2-5/h2-4,6-7H,1H3 \\
\hline 395 & CJVCCVCVO & {$[\mathrm{CH}]=\mathrm{CC}=\mathrm{C}=\mathrm{O}$} & InChl=1S/C4H3O/c1-2-3-4-5/h1-3H \\
\hline 396 & C4Y24 & $\mathrm{O}=\mathrm{CCC}(=0) \mathrm{C}$ & InChl=1S/C4H6O2/c1-4(6)2-3-5/h3H,2H2,1H3 \\
\hline 397 & $\mathrm{CH} 3 \mathrm{HOOCHCOCH} 2$ & $\mathrm{CC}(\mathrm{OO}) \mathrm{C}(=\mathrm{O})[\mathrm{CH} 2]$ & InChl=1S/C4H7O3/c1-3(5)4(2)7-6/h4,6H,1H2,2H3 \\
\hline 398 & NC4KET23OH-1 & $\mathrm{CC}(\mathrm{OO}) \mathrm{C}(=0) \mathrm{CO}$ & InChl=1S/C4H8O4/c1-3(8-7)4(6)2-5/h3,5,7H,2H2,1H3 \\
\hline 399 & $\mathrm{C} 2 \mathrm{H} 3 \mathrm{CHCO}$ & $\mathrm{C}=\mathrm{CC}=\mathrm{C}=\mathrm{O}$ & $\operatorname{lnChl}=1 \mathrm{~S} / \mathrm{C} 4 \mathrm{H} 4 \mathrm{O} / \mathrm{c} 1-2-3-4-5 / \mathrm{h} 2-3 \mathrm{H}, 1 \mathrm{H} 2$ \\
\hline 400 & $\mathrm{C} 3 \mathrm{H} 3 \mathrm{CH} 2 \mathrm{OOH}$ & $\mathrm{OOCC}=\mathrm{C}=\mathrm{C}$ & $\operatorname{lnChl}=1 \mathrm{~S} / \mathrm{C} 4 \mathrm{H} 6 \mathrm{O} 2 / \mathrm{c1} 1-2-3-4-6-5 / \mathrm{h} 3,5 \mathrm{H}, 1,4 \mathrm{H} 2$ \\
\hline 401 & $\mathrm{HOOCH} 2 \mathrm{CHCOCH} 3$ & $\mathrm{C}(\mathrm{OO})[\mathrm{CH}] \mathrm{C}(=\mathrm{O}) \mathrm{C}$ & $\mathrm{InChl}=1 \mathrm{~S} / \mathrm{C} 4 \mathrm{H} 7 \mathrm{O} 3 / \mathrm{c} 1-4(5) 2-3-7-6 / \mathrm{h} 2,6 \mathrm{H}, 3 \mathrm{H} 2,1 \mathrm{H} 3$ \\
\hline 402 & HOCOCQ(CH3)2 & $\mathrm{CC}(\mathrm{C})(\mathrm{OO}) \mathrm{C}(=0) 0$ & InChl=1S/C4H8O4/c1-4(2,8-7)3(5)6/h7H,1-2H3,(H,5,6) \\
\hline 403 & $\mathrm{C} 3 \mathrm{H} 3 \mathrm{CHO}$ & $\mathrm{C}=\mathrm{C}=\mathrm{CC}=\mathrm{O}$ & InChl=1S/C4H4O/c1-2-3-4-5/h3-4H,1H2 \\
\hline 404 & MP2D & $\operatorname{coc}(=0) C=C$ & InChl=1S/C4H6O2/c1-3-4(5)6-2/h3H,1H2,2H3 \\
\hline 405 & $\mathrm{O} 2 \mathrm{CH} 2 \mathrm{CH} 2 \mathrm{COCH} 3$ & {$[\mathrm{O}] \mathrm{OCCC}(=\mathrm{O}) \mathrm{C}$} & InChl=1S/C4H7O3/c1-4(5)2-3-7-6/h2-3H2,1H3 \\
\hline 406 & IC4KET32ОH-1 & $\mathrm{Cl}=\mathrm{O}) \mathrm{C}(\mathrm{C})(\mathrm{OO}) \mathrm{CO}$ & InChl=1S/C4H8O4/c1-4(2-5,3-6)8-7/h2,6-7H,3H2,1H3 \\
\hline 407 & $\mathrm{CHCCH} 2 \mathrm{CHO}$ & $\mathrm{C \# CCC}=\mathrm{O}$ & InChl=1S/C4H4O/c1-2-3-4-5/h1,4H,3H2 \\
\hline 408 & VINACET & $\mathrm{C}=\mathrm{COC}(\mathrm{C})=\mathrm{O}$ & InChl=1S/C4H6O2/c1-3-6-4(2)5/h3H,1H2,2H3 \\
\hline 409 & $\mathrm{HOOCH} 2 \mathrm{CH} 2 \mathrm{COCH} 2$ & $\mathrm{C}(\mathrm{OO}) \mathrm{CC}(=\mathrm{O})[\mathrm{CH} 2]$ & InChl=1S/C4H7O3/c1-4(5)2-3-7-6/h6H,1-3H2 \\
\hline 410 & $\mathrm{CHOC}(\mathrm{CH} 3) \mathrm{OHCH} 2 \mathrm{Q}$ & $\mathrm{C}(=\mathrm{O}) \mathrm{C}(\mathrm{C})(\mathrm{O}) \mathrm{COO}$ & InChl=1S/C4H8O4/c1-4(6,2-5)3-8-7/h2,6-7H,3H2,1H3 \\
\hline 411 & FURAN & $\mathrm{C} 1=\mathrm{CC}=\mathrm{CO} 1$ & InChl=1S/C4H4O/c1-2-4-5-3-1/h1-4H \\
\hline 412 & $\mathrm{CH} 3 \mathrm{OCHCOCH} 2$ & $\mathrm{CC}(=0) \mathrm{C} 1 \mathrm{CO} 1$ & InChl=1S/C4H6O2/c1-3(5)4-2-6-4/h4H,2H2,1H3 \\
\hline 413 & TC3H6O2CHO & $\mathrm{CC}(\mathrm{C})(\mathrm{C}=\mathrm{O}) \mathrm{O}[\mathrm{O}]$ & InChl=1S/C4H7O3/c1-4(2,3-5)7-6/h3H,1-2H3 \\
\hline 414 & NC4KET42OH-2 & $\mathrm{C}(=\mathrm{O}) \mathrm{CC}(\mathrm{O})(\mathrm{OO}) \mathrm{C}$ & InChl=1S/C4H8O4/c1-4(6,8-7)2-3-5/h3,6-7H,2H2,1H3 \\
\hline 415 & FUROP1 & $\mathrm{C} 1=\mathrm{C}[\mathrm{C}] \mathrm{CO} 1$ & $\operatorname{lnChl}=1 \mathrm{~S} / \mathrm{C} 4 \mathrm{H} 4 \mathrm{O} / \mathrm{c} 1-2-4-5-3-1 / \mathrm{h} 1,3 \mathrm{H}, 4 \mathrm{H} 2$ \\
\hline 416 & $\mathrm{CH} 3 \mathrm{CHOCH}_{2} \mathrm{CO}$ & $\mathrm{CC} 1 \mathrm{C}(=\mathrm{O}) \mathrm{CO} 1$ & InChl=1S/C4H6O2/c1-3-4(5)2-6-3/h3H,2H2,1H3 \\
\hline 417 & $\mathrm{IC} 3 \mathrm{H} 5 \mathrm{O} 2 \mathrm{HCHO}$ & {$[\mathrm{CH} 2] \mathrm{C}(\mathrm{C})(\mathrm{C}=\mathrm{O}) \mathrm{OO}$} & InChl=1S/C4H7O3/c1-4(2,3-5)7-6/h3,6H,1H2,2H3 \\
\hline 418 & IC4КЕТЗЗОН-1 & $\mathrm{C}(=\mathrm{O}) \mathrm{C}(\mathrm{COO}) \mathrm{CO}$ & InChl=1S/C4H8O4/c5-1-4(2-6)3-8-7/h1,4,6-7H,2-3H2 \\
\hline 419 & FUROP2 & $\mathrm{C} 1=\mathrm{CC}[\mathrm{C}] \mathrm{O} 1$ & $\operatorname{lnChl}=1 \mathrm{~S} / \mathrm{C} 4 \mathrm{H} 4 \mathrm{O} / \mathrm{c} 1-2-4-5-3-1 / \mathrm{h} 1,3 \mathrm{H}, 2 \mathrm{H} 2$ \\
\hline 420 & $\mathrm{OCH} 2 \mathrm{CH} 2 \mathrm{COCH} 2$ & $\mathrm{O}=\mathrm{C} 1 \mathrm{COCC} 1$ & $\operatorname{lnChl}=15 / C 4 H 602 / c 5-4-1-2-6-3-4 / h 1-3 \mathrm{H} 2$ \\
\hline 421 & TC3H6O2HCO & $C C(C)([C]=0) 00$ & InChl=1S/C4H7O3/c1-4(2,3-5)7-6/h6H,1-2H3 \\
\hline 422 & NC4KET42OH-1 & $\mathrm{C}(=\mathrm{O}) \mathrm{CC}(\mathrm{OO}) \mathrm{CO}$ & InChl=1S/C4H8O4/c5-2-1-4(3-6)8-7/h2,4,6-7H,1,3H2 \\
\hline 423 & $\mathrm{C} 4 \mathrm{H} 4 \mathrm{O}$ & $\mathrm{CCHCC}=\mathrm{O}$ & InChl=1S/C4H4O/c1-2-3-4-5/h4H,1H3 \\
\hline 424 & $\mathrm{C} 4 \mathrm{Y} 2 \mathrm{OH} 4-3 \mathrm{~J}$ & $\mathrm{OCC}=\mathrm{C}([\mathrm{O}]) \mathrm{C}$ & $\operatorname{lnChl}=1 \mathrm{~S} / \mathrm{C} 4 \mathrm{H} 702 / \mathrm{cc}-4(6) 2-3-5 / \mathrm{h} 2,5 \mathrm{H}, 3 \mathrm{H} 2,1 \mathrm{H} 3$ \\
\hline 425 & C4H6O1-30OH4 & $\mathrm{C}=\mathrm{CC}([\mathrm{O}]) \mathrm{COO}$ & InChl=1S/C4H703/c1-2-4(5)3-7-6/h2,4,6H,1,3H2 \\
\hline 426 & NC4KET43OH-1 & $\mathrm{C}(=\mathrm{O}) \mathrm{C}(\mathrm{OO}) \mathrm{CCO}$ & InChl=1S/C4H8O4/c5-2-1-4(3-6)8-7/h3-5,7H,1-2H2 \\
\hline 427 & C4H5OH-13-3R & $\mathrm{O}[\mathrm{CH}] \mathrm{C}=\mathrm{C}=\mathrm{C}$ & InChl=1S/C4H5O/c1-2-3-4-5/h3-5H,1H2 \\
\hline 428 & C4Y2OH4-4J & $\mathrm{CC}(=\mathrm{O}) \mathrm{C}[\mathrm{CH}] \mathrm{O}$ & $\operatorname{lnChl}=1 \mathrm{~S} / \mathrm{C} 4 \mathrm{H} 702 / \mathrm{cc}-4(6) 2-3-5 / \mathrm{h} 3,5 \mathrm{H}, 2 \mathrm{H} 2,1 \mathrm{H} 3$ \\
\hline 429 & $\mathrm{C} 4 \mathrm{H} 6 \mathrm{O} 2-10 \mathrm{OH} 4$ & {$[\mathrm{O}] \mathrm{CC}=\mathrm{CCOO}$} & $\operatorname{lnChl}=1 \mathrm{~S} / \mathrm{C} 4 \mathrm{H} 703 / \mathrm{C} 5-3-1-2-4-7-6 / \mathrm{h} 1-2,6 \mathrm{H}, 3-4 \mathrm{H} 2$ \\
\hline 430 & NC4KET41OH-2 & $\mathrm{C}(=0) \mathrm{CC}(0) \mathrm{COO}$ & InChl=1S/C4H8O4/c5-2-1-4(6)3-8-7/h2,4,6-7H,1,3H2 \\
\hline 431 & $\mathrm{CH} 2 \mathrm{CHCHCHO}$ & {$[\mathrm{CH} 2] \mathrm{C}=\mathrm{CC}=\mathrm{O}$} & $\operatorname{lnChl}=1 \mathrm{~S} / \mathrm{C} 4 \mathrm{H} 5 \mathrm{O} / \mathrm{c} 1-2-3-4-5 / \mathrm{h} 2-4 \mathrm{H}, 1 \mathrm{H} 2$ \\
\hline 432 & C4H71-OO3 & $\mathrm{CC}(\mathrm{C}=\mathrm{C}) \mathrm{O}[\mathrm{O}]$ & $\operatorname{lnChl}=1 \mathrm{~S} / \mathrm{C} 4 \mathrm{H} 702 / \mathrm{c1}-3-4(2) 6-5 / \mathrm{h} 3-4 \mathrm{H}, 1 \mathrm{H} 2,2 \mathrm{H} 3$ \\
\hline 433 & $\mathrm{C} 3 \mathrm{H} 4 \mathrm{CH} 2 \mathrm{OH}-1 \mathrm{OOH}$ & $\mathrm{OOCC}=\mathrm{CCO}$ & InChl=15/C4H8O3/c5-3-1-2-4-7-6/h1-2,5-6H,3-4H2 \\
\hline 434 & NC4KET34OH-1 & $\mathrm{C}(\mathrm{OO}) \mathrm{C}(=\mathrm{O}) \mathrm{CCO}$ & InChl=1S/C4H8O4/C5-2-1-4(6)3-8-7/h5,7H,1-3H2 \\
\hline 435 & $\mathrm{C} 4 \mathrm{H} 61-4 \mathrm{OOH} 3$ & {$[\mathrm{CH} 2] \mathrm{C}(\mathrm{C}=\mathrm{C}) \mathrm{OO}$} & InChl=1S/C4H7O2/c1-3-4(2)6-5/h3-5H,1-2H2 \\
\hline 436 & NC4KET21 & $\mathrm{CCC}(=0) \mathrm{COO}$ & InChl=1S/C4H8O3/c1-2-4(5)3-7-6/h6H,2-3H2,1H3 \\
\hline 437 & NC4KET41OH-1 & $\mathrm{C}(=0) \mathrm{CCC}(00) \mathrm{O}$ & InChl=1S/C4H8O4/c5-3-1-2-4(6)8-7/h3-4,6-7H,1-2H2 \\
\hline 438 & AC3H5CO & $\mathrm{C}=\mathrm{CC}[\mathrm{C}]=\mathrm{O}$ & InChl=1S/C4H5O/c1-2-3-4-5/h2H,1,3H2 \\
\hline 439 & $\mathrm{C} 3 \mathrm{H} 4 \mathrm{CH} 2 \mathrm{OH}-3 \mathrm{O}$ & {$[\mathrm{O}] \mathrm{C}(\mathrm{C}=\mathrm{C}) \mathrm{CO}$} & InChl=1S/C4H702/c1-2-4(6)3-5/h2,4-5H,1,3H2 \\
\hline 440 & NC4KET23 & $\mathrm{CC}(\mathrm{OO}) \mathrm{C}(=\mathrm{O}) \mathrm{C}$ & InChl=1S/C4H8O3/c1-3(5)4(2)7-6/h4,6H,1-2H3 \\
\hline 441 & NC4KET14OH-2 & $\mathrm{C}(\mathrm{OO}) \mathrm{CC}(\mathrm{O}) \mathrm{C}=\mathrm{O}$ & InChl=1S/C4H8O4/c5-3-4(6)1-2-8-7/h3-4,6-7H,1-2H2 \\
\hline 442 & $\mathrm{C} 3 \mathrm{H} 3 \mathrm{CH} 2 \mathrm{O}$ & {$[\mathrm{O}] \mathrm{CC}=\mathrm{C}=\mathrm{C}$} & InChl=1S/C4H5O/c1-2-3-4-5/h3H,1,4H2 \\
\hline 443 & $\mathrm{C} 3 \mathrm{H} 4 \mathrm{CH} 2 \mathrm{OH}-1 \mathrm{O}$ & $\mathrm{OCC}=\mathrm{CC}[\mathrm{O}]$ & $\operatorname{lnChl}=1 \mathrm{~S} / \mathrm{C} 4 \mathrm{H} 702 / \mathrm{c} 5-3-1-2-4-6 / \mathrm{h} 1-2,5 \mathrm{H}, 3-4 \mathrm{H} 2$ \\
\hline 444 & NC4KET24 & OOCCC $(=0) \mathrm{C}$ & InChl=1S/C4H8O3/c1-4(5)2-3-7-6/h6H,2-3H2,1H3 \\
\hline 445 & NC4KET24OH-1 & $\mathrm{C}(\mathrm{OO}) \mathrm{CC}(=\mathrm{O}) \mathrm{CO}$ & InChl=1S/C4H8O4/C5-3-4(6)1-2-8-7/h5,7H,1-3H2 \\
\hline 446 & $\mathrm{CJVCC}(\mathrm{C}) \mathrm{VO}$ & $\mathrm{CC}(=\mathrm{O}) \mathrm{C}=[\mathrm{CH}]$ & InChl=1S/C4H5O/c1-3-4(2)5/h1,3H,2H3 \\
\hline 447 & BAOJ & {$[\mathrm{O}] \mathrm{C}(=\mathrm{O}) \mathrm{CCC}$} & $\operatorname{lnChl}=1 \mathrm{~S} / \mathrm{C} 4 \mathrm{H} 7 \mathrm{O} 2 / \mathrm{c} 1-2-3-4(5) 6 / \mathrm{h} 2-3 \mathrm{H} 2,1 \mathrm{H} 3$ \\
\hline 448 & C4H7O1-30OH-4 & $\mathrm{C} 1 \mathrm{CC}(\mathrm{O} 1) \mathrm{C}(\mathrm{OO})$ & InChl=1S/C4H8O3/c5-7-3-4-1-2-6-4/h4-5H,1-3H2 \\
\hline 449 & NC4KET14OH-1 & $\mathrm{C}(\mathrm{OO}) \mathrm{CCC}(=0) \mathrm{O}$ & $\operatorname{lnChl}=1 \mathrm{~S} / \mathrm{C} 4 \mathrm{H} 804 / \mathrm{c5}-4(6) 2-1-3-8-7 / \mathrm{h} 7 \mathrm{H}, 1-3 \mathrm{H} 2,(\mathrm{H}, 5,6)$ \\
\hline 450 & $\mathrm{CH} 2 \mathrm{CCOCH} 3$ & $\mathrm{C}=[\mathrm{C}] \mathrm{C}(=0) \mathrm{C}$ & InChl=1S/C4H5O/c1-3-4(2)5/h1H2,2H3 \\
\hline 451 & MP3J & $\mathrm{COC}(=\mathrm{O}) \mathrm{C}[\mathrm{CH} 2]$ & InChl=1S/C4H702/c1-3-4(5)6-2/h1,3H2,2H3 \\
\hline
\end{tabular}




\begin{tabular}{|c|c|c|c|}
\hline 452 & С4H701-30OH-2 & $\mathrm{C} 1 \mathrm{C}(\mathrm{OO}) \mathrm{C}(\mathrm{O} 1) \mathrm{C}$ & InChl=1S/C4H8O3/c1-3-4(7-5)2-6-3/h3-5H,2H2,1H3 \\
\hline 453 & $\mathrm{C} 2 \mathrm{H} 3 \mathrm{COCH} 2$ & $\mathrm{C}=\mathrm{CC}(=\mathrm{O})[\mathrm{CH} 2]$ & InChl=1S/C4H5O/c1-3-4(2)5/h3H,1-2H2 \\
\hline 454 & $\mathrm{PC} 2 \mathrm{H} 4 \mathrm{OCOC}$ & {$[\mathrm{CH} 2] \operatorname{COC}(\mathrm{C})=\mathrm{O}$} & InChl=1S/C4H702/c1-3-6-4(2)5/h1,3H2,2H3 \\
\hline 455 & C4H701-20OH-4 & $\mathrm{C} 1 \mathrm{C}(\mathrm{O} 1) \mathrm{CC}(\mathrm{OO})$ & InChl=1S/C4H8O3/c5-7-2-1-4-3-6-4/h4-5H,1-3H2 \\
\hline 456 & $\mathrm{C} 4 \mathrm{H} 5 \mathrm{O}-2$ & $\mathrm{C} 1=\mathrm{CC}[\mathrm{CH}] \mathrm{O} 1$ & InChl=1S/C4H5O/c1-2-4-5-3-1/h1,3-4H,2H2 \\
\hline 457 & SC2H4OCOC & $\mathrm{C}[\mathrm{CH}] \mathrm{OC}(\mathrm{C})=\mathrm{O}$ & $\operatorname{lnChl}=1 \mathrm{~S} / \mathrm{C} 4 \mathrm{H} 702 / \mathrm{c} 1-3-6-4(2) 5 / \mathrm{h} 3 \mathrm{H}, 1-2 \mathrm{H} 3$ \\
\hline 458 & $\mathrm{C} 4 \mathrm{H} 701-40 \mathrm{OH}-2$ & $\mathrm{C} 1 \mathrm{C}(\mathrm{OO}) \mathrm{CC}(\mathrm{O} 1)$ & InChl=1S/C4H8O3/c5-7-4-1-2-6-3-4/h4-5H,1-3H2 \\
\hline 459 & IC4H6Q2-II & $\mathrm{C}=\mathrm{C}(\mathrm{COO}) \mathrm{COO}$ & InChl=1S/C4H8O4/c1-4(2-7-5)3-8-6/h5-6H,1-3H2 \\
\hline 460 & $\mathrm{C} 4 \mathrm{H} 5 \mathrm{O}-3$ & $\mathrm{C} 1=\mathrm{C}[\mathrm{CH}] \mathrm{CO} 1$ & InChl=1S/C4H5O/c1-2-4-5-3-1/h1-3H,4H2 \\
\hline 461 & $\mathrm{C} 2 \mathrm{H} 5 \mathrm{OCOCH}$ & $\mathrm{CCOC}([\mathrm{CH} 2])=\mathrm{O}$ & $\mathrm{InChl}=1 \mathrm{~S} / \mathrm{C} 4 \mathrm{H} 7 \mathrm{O} 2 / \mathrm{c} 1-3-6-4(2) 5 / \mathrm{h} 2-3 \mathrm{H} 2,1 \mathrm{H} 3$ \\
\hline 462 & C4H701-20OH-3 & $\mathrm{C} 1 \mathrm{C}(\mathrm{O} 1) \mathrm{C}(\mathrm{OO}) \mathrm{C}$ & $\operatorname{lnChl}=1 \mathrm{~S} / \mathrm{C} 4 \mathrm{H} 8 \mathrm{O} 3 / \mathrm{c} 1-3(7-5) 4-2-6-4 / \mathrm{h} 3-5 \mathrm{H}, 2 \mathrm{H} 2,1 \mathrm{H} 3$ \\
\hline 463 & $\mathrm{C} 4 \mathrm{H} 8 \mathrm{OOH} 1-2 \mathrm{O} 2$ & $\mathrm{CCC}(\mathrm{COO}) \mathrm{O}[\mathrm{O}]$ & InChl=1S/C4H9O4/c1-2-4(8-6)3-7-5/h4-5H,2-3H2,1H3 \\
\hline 464 & SC3H5CO & $\mathrm{CC}=\mathrm{C}[\mathrm{C}]=\mathrm{O}$ & $\operatorname{lnChl}=1 \mathrm{~S} / \mathrm{C} 4 \mathrm{H} 5 \mathrm{O} / \mathrm{c} 1-2-3-4-5 / \mathrm{h} 2-3 \mathrm{H}, 1 \mathrm{H} 3$ \\
\hline 465 & $\mathrm{C} 2 \mathrm{H} 5 \mathrm{COOCH} 2$ & $\mathrm{cCC}(=0) \mathrm{C}[0]$ & InChl=1S/C4H7O2/c1-2-4(6)3-5/h2-3H2,1H3 \\
\hline 466 & C4H702-30OH-1 & $\mathrm{C}(0 \mathrm{O}) \mathrm{C} 1 \mathrm{C}(\mathrm{O} 1) \mathrm{C}$ & InChl=1S/C4H8O3/c1-3-4(7-3)2-6-5/h3-5H,2H2,1H3 \\
\hline 467 & C4H8OOH1-302 & $\mathrm{cc}(\mathrm{CCOO}) \mathrm{O}[0]$ & InChl=1S/C4H9O4/c1-4(8-6)2-3-7-5/h4-5H,2-3H2,1H3 \\
\hline 468 & IC3H5CO & $\mathrm{C}=\mathrm{C}(\mathrm{C})[\mathrm{C}]=\mathrm{O}$ & InChl=1S/C4H5O/c1-4(2)3-5/h1H2,2H3 \\
\hline 469 & CH3OCHCOCH3 & $\operatorname{CC}([0]) C(=0) C$ & InChl=1S/C4H702/c1-3(5)4(2)6/h3H,1-2H3 \\
\hline 470 & NC4KET12 & $\operatorname{ccc}(\mathrm{C}=0) 00$ & InChl=1S/C4H8O3/c1-2-4(3-5)7-6/h3-4,6H,2H2,1H3 \\
\hline 471 & $\mathrm{C} 4 \mathrm{H} 8 \mathrm{OOH} 1-402$ & {$[0]$ occccoo } & InChl=1S/C4H9O4/c5-7-3-1-2-4-8-6/h5H,1-4H2 \\
\hline 472 & $\mathrm{IC} 3 \mathrm{H} 4 \mathrm{CHO}-\mathrm{A}$ & $\mathrm{C}=\mathrm{C}([\mathrm{CH} 2]) \mathrm{C}=\mathrm{O}$ & $\operatorname{lnChl}=1 \mathrm{~S} / \mathrm{C} 4 \mathrm{H} 5 \mathrm{O} / \mathrm{c} 1-4(2) 3-5 / \mathrm{h} 3 \mathrm{H}, 1-2 \mathrm{H} 2$ \\
\hline 473 & $\mathrm{OCH} 2 \mathrm{CH} 2 \mathrm{COCH} 3$ & $C([0]) C C(=0) C$ & $\mathrm{InChl}=1 \mathrm{~S} / \mathrm{C} 4 \mathrm{H} 7 \mathrm{O} 2 / \mathrm{c} 1-4(6) 2-3-5 / \mathrm{h} 2-3 \mathrm{H} 2,1 \mathrm{H} 3$ \\
\hline 474 & NC4KET13 & $\mathrm{CC}(\mathrm{CC}=0) 00$ & InChl=1S/C4H8O3/c1-4(7-6)2-3-5/h3-4,6H,2H2,1H3 \\
\hline 475 & $\mathrm{C} 4 \mathrm{H} 8 \mathrm{OOH} 2-102$ & $\mathrm{CCC}(\mathrm{CO}[\mathrm{O}]) \mathrm{OO}$ & InChl=1S/C4H9O4/c1-2-4(8-6)3-7-5/h4,6H,2-3H2,1H3 \\
\hline 476 & $\mathrm{~B} 1 \mathrm{Y} 4 \mathrm{OH}$ & $\mathrm{C}=\mathrm{C}=\mathrm{CCO}$ & InChl=1S/C4H6O/c1-2-3-4-5/h3,5H,1,4H2 \\
\hline 477 & НОС3 $66 \mathrm{CO}$ & {$[\mathrm{C}](=0) \mathrm{CCCO}$} & InChl=1S/C4H702/c5-3-1-2-4-6/h5H,1-3H2 \\
\hline 478 & NC4KET14 & $\mathrm{O}=\mathrm{CCCCOO}$ & InChl=15/C4H8O3/c5-3-1-2-4-7-6/h3,6H,1-2,4H2 \\
\hline 479 & $\mathrm{C} 4 \mathrm{H} 8 \mathrm{OOH} 2-3 \mathrm{O} 2$ & $\mathrm{CC}(\mathrm{O}[\mathrm{O}]) \mathrm{C}(\mathrm{C}) \mathrm{OO}$ & InChI=1S/C4H9O4/c1-3(7-5)4(2)8-6/h3-5H,1-2H3 \\
\hline 480 & $\mathrm{C} 4 \mathrm{H} 5 \mathrm{OH}-13$ & $\mathrm{C}=\mathrm{CC}=\mathrm{CO}$ & $\operatorname{lnChl}=1 \mathrm{~S} / \mathrm{C} 4 \mathrm{H} 6 \mathrm{O} / \mathrm{c} 1-2-3-4-5 / \mathrm{h} 2-5 \mathrm{H}, 1 \mathrm{H} 2$ \\
\hline 481 & TC3H6OCHO & $\operatorname{CC}(\mathrm{C})([0]) \mathrm{C}=0$ & InChl=1S/C4H702/c1-4(2,6)3-5/h3H,1-2H3 \\
\hline 482 & IC4KETII & $\mathrm{CC}(\mathrm{C}=0) \mathrm{COO}$ & InChl=1S/C4H8O3/c1-4(2-5)3-7-6/h2,4,6H,3H2,1H3 \\
\hline 483 & $\mathrm{C} 4 \mathrm{H} 8 \mathrm{OOH} 2-402$ & $\mathrm{CC}(\mathrm{CCO}[\mathrm{O}]) 0 \mathrm{O}$ & InChl=1S/C4H9O4/c1-4(8-6)2-3-7-5/h4,6H,2-3H2,1H3 \\
\hline 484 & $\mathrm{AC} 3 \mathrm{H} 5 \mathrm{CHO}$ & $\mathrm{C}=\mathrm{CCC}=\mathrm{O}$ & InChl=1S/C4H6O/c1-2-3-4-5/h2,4H,1,3H2 \\
\hline 485 & $\mathrm{IC4H702}$ & $\mathrm{C}=\mathrm{C}(\mathrm{C}) \mathrm{CO}[\mathrm{O}]$ & $\operatorname{lnChl}=1 \mathrm{~S} / \mathrm{C} 4 \mathrm{H} 7 \mathrm{O} 2 / \mathrm{c} 1-4(2) 3-6-5 / \mathrm{h} 1,3 \mathrm{H} 2,2 \mathrm{H} 3$ \\
\hline 486 & IC4KETIT & $\mathrm{CC}(\mathrm{C})(\mathrm{C}=0) 00$ & $\operatorname{lnChl}=1 \mathrm{~S} / \mathrm{C} 4 \mathrm{H} 8 \mathrm{O} 3 / \mathrm{c} 1-4(2,3-5) 7-6 / \mathrm{h} 3,6 \mathrm{H}, 1-2 \mathrm{H} 3$ \\
\hline 487 & $\mathrm{C} 4 \mathrm{H} 71-2,4 \mathrm{OOH}$ & {$[\mathrm{CH} 2] \mathrm{C}(\mathrm{OO}) \mathrm{CC}(\mathrm{OO})$} & InChl=1S/C4H9O4/c1-4(8-6)2-3-7-5/h4-6H,1-3H2 \\
\hline 488 & $\mathrm{C} 2 \mathrm{H} 3 \mathrm{COCH} 3$ & $\mathrm{CC}(=\mathrm{O}) \mathrm{C}=\mathrm{C}$ & InChl=15/C4H6O/c1-3-4(2)5/h3H,1H2,2H3 \\
\hline 489 & $\mathrm{IC} 4 \mathrm{H} 6 \mathrm{OOH}-\mathrm{I}$ & $\mathrm{C}=\mathrm{C}([\mathrm{CH} 2]) \mathrm{COO}$ & InChl=15/C4H702/c1-4(2)3-6-5/h5H,1-3H2 \\
\hline 490 & $\mathrm{IC} 3 \mathrm{H} 5 \mathrm{COHQ}$ & $\mathrm{C}=\mathrm{C}(\mathrm{C}) \mathrm{C}(\mathrm{O}) 0 \mathrm{O}$ & InChl=1S/C4H8O3/c1-3(2)4(5)7-6/h4-6H,1H2,2H3 \\
\hline 491 & $\mathrm{C} 4 \mathrm{H} 72-1,3 \mathrm{OOH}$ & $\mathrm{CC}(\mathrm{OO})[\mathrm{CH}] \mathrm{C}(\mathrm{OO})$ & InChl=1S/C4H9O4/c1-4(8-6)2-3-7-5/h2,4-6H,3H2,1H3 \\
\hline 492 & $\mathrm{C} 4 \mathrm{H} 6 \mathrm{O} 23$ & $01 \mathrm{CCC}=\mathrm{C} 1$ & $\operatorname{lnChl}=1 \mathrm{~S} / \mathrm{C} 4 \mathrm{H} 6 \mathrm{O} / \mathrm{c} 1-2-4-5-3-1 / \mathrm{h} 1,3 \mathrm{H}, 2,4 \mathrm{H} 2$ \\
\hline 493 & CCYCCOOC-T1 & $\mathrm{C}[\mathrm{C}] 1 \mathrm{COOC} 1$ & $\operatorname{lnChl}=1 \mathrm{~S} / \mathrm{C} 4 \mathrm{H} 702 / \mathrm{c} 1-4-2-5-6-3-4 / \mathrm{h} 2-3 \mathrm{H} 2,1 \mathrm{H} 3$ \\
\hline 494 & C4H6OHOOH1-4-3 & $\mathrm{C}=\mathrm{CC}(\mathrm{CO}) \mathrm{OO}$ & InChl=1S/C4H8O3/c1-2-4(3-5)7-6/h2,4-6H,1,3H2 \\
\hline 495 & $\mathrm{C} 4 \mathrm{H} 72-1,4 \mathrm{OOH}$ & $\mathrm{C}(\mathrm{OO}) \mathrm{C}[\mathrm{CH}] \mathrm{C}(\mathrm{OO})$ & InChl=1S/C4H9O4/c5-7-3-1-2-4-8-6/h1,5-6H,2-4H2 \\
\hline 496 & $\mathrm{C} 4 \mathrm{H} 6 \mathrm{O} 25$ & O1CC=CC1 & InChl=1S/C4H6O/c1-2-4-5-3-1/h1-2H,3-4H2 \\
\hline 497 & C2CYCOOC-I1 & {$[\mathrm{CH} 2] \mathrm{C} 1(\mathrm{C}) \mathrm{COO} 1$} & InChl=1S/C4H7O2/c1-4(2)3-5-6-4/h1,3H2,2H3 \\
\hline 498 & $\mathrm{C} 4 \mathrm{H} 6 \mathrm{OHOOH} 2-2-1$ & $\mathrm{CC}=\mathrm{C}(\mathrm{O}) \mathrm{C}(\mathrm{OO})$ & InChl=1S/C4H8O3/c1-2-4(5)3-7-6/h2,5-6H,3H2,1H3 \\
\hline 499 & $\mathrm{C} 4 \mathrm{H} 71-2,30 \mathrm{OH}$ & {$[\mathrm{CH} 2] \mathrm{C}(\mathrm{OO}) \mathrm{C}(\mathrm{OO}) \mathrm{C}$} & InChl=15/C4H9O4/c1-3(7-5)4(2)8-6/h3-6H,1H2,2H3 \\
\hline 500 & $\mathrm{IC} 3 \mathrm{H} 5 \mathrm{CHO}$ & $\mathrm{C}=\mathrm{C}(\mathrm{C}) \mathrm{C}=\mathrm{O}$ & InChl=1S/C4H6O/c1-4(2)3-5/h3H,1H2,2H3 \\
\hline 501 & CCYC2OCO & $\mathrm{CC} 1(\mathrm{C}[\mathrm{O}]) \mathrm{CO} 1$ & InChl=1S/C4H7O2/c1-4(2-5)3-6-4/h2-3H2,1H3 \\
\hline 502 & C4H6OHOOH1-3-4 & $\mathrm{C}=\mathrm{CC}(\mathrm{O}) \mathrm{C}(\mathrm{OO})$ & InChI=1S/C4H8O3/c1-2-4(5)3-7-6/h2,4-6H,1,3H2 \\
\hline 503 & $\mathrm{C} 4 \mathrm{H} 71-3,4 \mathrm{OOH}$ & {$[\mathrm{CH} 2] \mathrm{CC}(\mathrm{OO}) \mathrm{C}(\mathrm{OO})$} & InChl=1S/C4H9O4/c1-2-4(8-6)3-7-5/h4-6H,1-3H2 \\
\hline 504 & IC3H6CO & $\mathrm{CC}(\mathrm{C})=\mathrm{C}=\mathrm{O}$ & InChl=1S/C4H6O/c1-4(2)3-5/h1-2H3 \\
\hline 505 & CCYCCOOC-12 & $\mathrm{CC} 1[\mathrm{CH}] \mathrm{OOC} 1$ & InChl=1S/C4H702/c1-4-2-5-6-3-4/h2,4H,3H2,1H3 \\
\hline 506 & C4H6OHOOH1-2-3 & $\mathrm{C}=\mathrm{C}(\mathrm{O}) \mathrm{C}(\mathrm{C}) \mathrm{OO}$ & InChl=1S/C4H8O3/c1-3(5)4(2)7-6/h4-6H,1H2,2H3 \\
\hline 507 & $\mathrm{C} 4 \mathrm{H} 72-3,4 \mathrm{OOH}$ & $\mathrm{C}[\mathrm{CH}] \mathrm{C}(\mathrm{OO}) \mathrm{C}(\mathrm{OO})$ & InChl=1S/C4H9O4/c1-2-4(8-6)3-7-5/h2,4-6H,3H2,1H3 \\
\hline 508 & $\mathrm{C} 2 \mathrm{H} 3 \mathrm{CHOCH} 2$ & $\mathrm{C}=\mathrm{CC} 1 \mathrm{CO} 1$ & InChl=1S/C4H6O/c1-2-4-3-5-4/h2,4H,1,3H2 \\
\hline 509 & CHOIC3H6O & $\mathrm{CC}(\mathrm{C}=\mathrm{O}) \mathrm{C}[\mathrm{O}]$ & InChl=1S/C4H7O2/c1-4(2-5)3-6/h2,4H,3H2,1H3 \\
\hline 510 & $\mathrm{C} 4 \mathrm{H} 7 \mathrm{OH}-2 \mathrm{OOH}-1$ & $\mathrm{CCC}(\mathrm{OO})[\mathrm{CH}] \mathrm{O}$ & InChl=1S/C4H9O3/c1-2-4(3-5)7-6/h3-6H,2H2,1H3 \\
\hline 511 & $\mathrm{IC4H8OOH-102}$ & $\mathrm{CC}(\mathrm{CO}[\mathrm{O}]) \mathrm{COO}$ & $\operatorname{lnChl}=1 \mathrm{~S} / \mathrm{C} 4 \mathrm{H} 9 \mathrm{O} 4 / \mathrm{c1}-4(2-7-5) 3-8-6 / \mathrm{h} 4-5 \mathrm{H}, 2-3 \mathrm{H} 2,1 \mathrm{H} 3$ \\
\hline 512 & $\mathrm{SC} 3 \mathrm{H} 5 \mathrm{CHO}$ & $\mathrm{CC}=\mathrm{CC}=\mathrm{O}$ & $\operatorname{lnChl}=1 \mathrm{~S} / \mathrm{C} 4 \mathrm{H} 6 \mathrm{O} / \mathrm{c} 1-2-3-4-5 / \mathrm{h} 2-4 \mathrm{H}, 1 \mathrm{H} 3$ \\
\hline 513 & $\mathrm{IC} 3 \mathrm{H} 5 \mathrm{OOCH} 2$ & $\mathrm{C}=\mathrm{C}(\mathrm{C}) \mathrm{OO}[\mathrm{CH} 2]$ & InChl=1S/C4H7O2/c1-4(2)6-5-3/h1,3H2,2H3 \\
\hline 514 & C4H7OH-10OH-3 & $\mathrm{C}[\mathrm{CH}] \mathrm{CC}(\mathrm{OO}) \mathrm{O}$ & InChl=15/C4H9O3/c1-2-3-4(5)7-6/h2,4-6H,3H2,1H3 \\
\hline 515 & IC4H8OOH-TO2 & $\mathrm{CC}(\mathrm{C})(\mathrm{COO}) \mathrm{O}[\mathrm{O}]$ & InChl=1S/C4H9O4/c1-4(2,8-6)3-7-5/h5H,3H2,1-2H3 \\
\hline 516 & $\mathrm{C} 2 \mathrm{H} 5 \mathrm{CHCO}$ & $\mathrm{CCC}=\mathrm{C}=\mathrm{O}$ & InChl=1S/C4H6O/c1-2-3-4-5/h3H,2H2,1H3 \\
\hline 517 & IC4H7-I102 & {$[\mathrm{O}] \mathrm{OC}=\mathrm{C}(\mathrm{C}) \mathrm{C}$} & InChl=1S/C4H702/c1-4(2)3-6-5/h3H,1-2H3 \\
\hline 518 & $\mathrm{C} 4 \mathrm{H} 7 \mathrm{OH}-10 \mathrm{OOH}-2$ & $\mathrm{CC}[\mathrm{CH}] \mathrm{C}(\mathrm{OO}) \mathrm{O}$ & InChl=1S/C4H9O3/c1-2-3-4(5)7-6/h3-6H,2H2,1H3 \\
\hline 519 & TC4H8OOH-IO2 & $\mathrm{CC}(\mathrm{C})(\mathrm{CO}[\mathrm{O}]) \mathrm{OO}$ & InChl=1S/C4H9O4/c1-4(2,8-6)3-7-5/h6H,3H2,1-2H3 \\
\hline 520 & $\mathrm{C} 4 \mathrm{H} 622-4 \mathrm{OH}$ & $\mathrm{C}[\mathrm{C}]=\mathrm{CCO}$ & InChl=1S/C4H7O/c1-2-3-4-5/h3,5H,4H2,1H3 \\
\hline 521 & $\mathrm{C} 2 \mathrm{H} 5 \mathrm{CHOHCO}$ & $\operatorname{ccc}(\mathrm{O})[\mathrm{C}]=\mathrm{O}$ & InChl=1S/C4H702/c1-2-4(6)3-5/h4,6H,2H2,1H3 \\
\hline 522 & PC4H8OH-102 & $\operatorname{cccc}(0[0]) 0$ & InChl=1S/C4H9O3/c1-2-3-4(5)7-6/h4-5H,2-3H2,1H3 \\
\hline 523 & TIC4H7Q2-I & {$[\mathrm{CH} 2] \mathrm{C}(\mathrm{C})(\mathrm{COO}) 0 \mathrm{O}$} & InChl=15/C4H9O4/c1-4(2,8-6)3-7-5/h5-6H,1,3H2,2H3 \\
\hline 524 & SC3H5OCH2-1 & $\mathrm{CC}=\mathrm{CO}[\mathrm{CH} 2]$ & InChl=1S/C4H7O/c1-3-4-5-2/h3-4H,2H2,1H3 \\
\hline 525 & CH3COCOHCH3 & $C[C](0) C(C)=0$ & InChl=1S/C4H702/c1-3(5)4(2)6/h5H,1-2H3 \\
\hline 526 & C4H7OH-10OH-4 & {$[\mathrm{CH} 2] \mathrm{CCC}(\mathrm{OO}) \mathrm{O}$} & InChl=1S/C4H9O3/c1-2-3-4(5)7-6/h4-6H,1-3H2 \\
\hline 527 & IIC4H7Q2-I & {$[\mathrm{CH} 2] \mathrm{C}(\mathrm{COO}) \mathrm{COO}$} & InChl=1S/C4H9O4/c1-4(2-7-5)3-8-6/h4-6H,1-3H2 \\
\hline 528 & $\mathrm{C} 2 \mathrm{H} 3 \mathrm{CHOHCH} 2$ & {$[\mathrm{CH} 2] \mathrm{C}(\mathrm{C}=\mathrm{C}) \mathrm{O}$} & InChl=1S/C4H7O/c1-3-4(2)5/h3-5H,1-2H2 \\
\hline 529 & $\mathrm{C} 2 \mathrm{H} 4 \mathrm{COCH} 2 \mathrm{OH}$ & $\mathrm{C}[\mathrm{CH}] \mathrm{C}(=\mathrm{O}) \mathrm{CO}$ & InChl=1S/C4H702/c1-2-4(6)3-5/h2,5H,3H2,1H3 \\
\hline 530 & C4H7OH-2OOH-3 & $\mathrm{C}[\mathrm{CH}] \mathrm{C}(\mathrm{OO}) \mathrm{CO}$ & InChl=15/C4H9O3/c1-2-4(3-5)7-6/h2,4-6H,3H2,1H3 \\
\hline 531 & IIC4H7Q2-T & $\mathrm{C}[\mathrm{C}](\mathrm{COO}) \mathrm{COO}$ & InChl=1S/C4H9O4/c1-4(2-7-5)3-8-6/h5-6H,2-3H2,1H3 \\
\hline 532 & $\mathrm{CH} 2 \mathrm{CH} 2 \mathrm{COCH} 3$ & {$[\mathrm{CH} 2] \mathrm{CC}(=\mathrm{O}) \mathrm{C}$} & InChl=1S/C4H7O/c1-3-4(2)5/h1,3H2,2H3 \\
\hline 533 & C4H71-402 & $\mathrm{C}=\mathrm{CCCO}[\mathrm{O}]$ & InChl=1S/C4H7O2/c1-2-3-4-6-5/h2H,1,3-4H2 \\
\hline 534 & PC4H8OH-2O2 & $\mathrm{CCC}(\mathrm{O}[\mathrm{O}]) \mathrm{CO}$ & InChl=1S/C4H9O3/c1-2-4(3-5)7-6/h4-5H,2-3H2,1H3 \\
\hline 535 & MP2OH3OO & $\operatorname{COC}(=0) \mathrm{C}(\mathrm{O}) \mathrm{C}(\mathrm{O}[\mathrm{O}])$ & $\operatorname{lnChl}=1 \mathrm{~S} / \mathrm{C} 4 \mathrm{H} 705 / \mathrm{c1}-8-4(6) 3(5) 2-9-7 / \mathrm{h} 3,5 \mathrm{H}, 2 \mathrm{H} 2,1 \mathrm{H} 3$ \\
\hline 536 & $\mathrm{C} 2 \mathrm{H} 5 \mathrm{COCH} 2$ & $\mathrm{CCC}(=\mathrm{O})[\mathrm{CH} 2]$ & InChl=1S/C4H7O/c1-3-4(2)5/h2-3H2,1H3 \\
\hline 537 & $\mathrm{C} 4 \mathrm{H} 61-30 \mathrm{OH} 4$ & $\mathrm{C}=\mathrm{C}[\mathrm{CH}] \mathrm{COO}$ & $\operatorname{lnChl}=1 \mathrm{~S} / \mathrm{C} 4 \mathrm{H} 702 / \mathrm{c} 1-2-3-4-6-5 / \mathrm{h} 2-3,5 \mathrm{H}, 1,4 \mathrm{H} 2$ \\
\hline 538 & SQC4H7OHP-4 & {$[\mathrm{CH} 2] \mathrm{CC}(\mathrm{OO}) \mathrm{CO}$} & InChl=1S/C4H9O3/c1-2-4(3-5)7-6/h4-6H,1-3H2 \\
\hline 539 & $\mathrm{HOOCH} 2 \mathrm{O} 2 \mathrm{CHCOCH} 3$ & $\mathrm{OOCC}(\mathrm{O}[\mathrm{O}]) \mathrm{C}(=\mathrm{O}) \mathrm{C}$ & InChl=1S/C4H705/c1-3(5)4(9-7)2-8-6/h4,6H,2H2,1H3 \\
\hline 540 & $\mathrm{CH} 3 \mathrm{CHCOCH} 3$ & $\mathrm{C}[\mathrm{CH}] \mathrm{C}(=\mathrm{O}) \mathrm{C}$ & InChl=1S/C4H7O/c1-3-4(2)5/h3H,1-2H3 \\
\hline 541 & $\mathrm{C} 4 \mathrm{H} 72-2 \mathrm{O} 2$ & $\mathrm{CC}=\mathrm{C}(\mathrm{C}) \mathrm{O}[\mathrm{O}]$ & InChl=1S/C4H702/c1-3-4(2)6-5/h3H,1-2H3 \\
\hline 542 & PQC4H7OHS-3 & $\mathrm{C}[\mathrm{CH}] \mathrm{C}(\mathrm{O}) \mathrm{COO}$ & InChl=1S/C4H9O3/c1-2-4(5)3-7-6/h2,4-6H,3H2,1H3 \\
\hline
\end{tabular}




\begin{tabular}{|c|c|c|c|}
\hline 543 & О2СН2 $2 \mathrm{HOOCHCOCH} 3$ & {$[0] \mathrm{Occ}(\mathrm{OO}) \mathrm{C}(=\mathrm{O}) \mathrm{C}$} & InChl=1S/C4H705/c1-3(5)4(9-7)2-8-6/h4,7H,2H2,1H3 \\
\hline 544 & $\mathrm{C} 4 \mathrm{H} 64,2-1 \mathrm{OH}$ & $\mathrm{C}=\mathrm{C}[\mathrm{CH}] \mathrm{CO}$ & InChl=1S/C4H7O/c1-2-3-4-5/h2-3,5H,1,4H2 \\
\hline 545 & C4H71-102 & $\mathrm{CCC}=\mathrm{CO}[\mathrm{O}]$ & $\operatorname{lnChl}=1 \mathrm{~S} / \mathrm{C} 4 \mathrm{H} 702 / \mathrm{c1}-2-3-4-6-5 / \mathrm{h} 3-4 \mathrm{H}, 2 \mathrm{H} 2,1 \mathrm{H} 3$ \\
\hline 546 & SC4H8OH-102 & $\operatorname{ccc}(0) \mathrm{CO}[0]$ & InChl=1S/C4H9O3/c1-2-4(5)3-7-6/h4-5H,2-3H2,1H3 \\
\hline 547 & $\mathrm{O} 2 \mathrm{SC} 2 \mathrm{H} 4 \mathrm{COHOOCH} 2$ & $\mathrm{C}(\mathrm{OO}) \mathrm{C}(=\mathrm{O}) \mathrm{C}(\mathrm{O}[\mathrm{O}]) \mathrm{C}$ & $\operatorname{lnChl}=1 \mathrm{~S} / \mathrm{C} 4 \mathrm{H} 705 / \mathrm{c} 1-3(9-7) 4(5) 2-8-6 / \mathrm{h} 3,6 \mathrm{H}, 2 \mathrm{H} 2,1 \mathrm{H} 3$ \\
\hline 548 & $\mathrm{C} 4 \mathrm{H} 63,1-1 \mathrm{OH}$ & $\mathrm{C}[\mathrm{CH}] \mathrm{C}=\mathrm{CO}$ & $\operatorname{lnChl}=1 \mathrm{~S} / \mathrm{C} 4 \mathrm{H} 7 \mathrm{O} / \mathrm{c} 1-2-3-4-5 / \mathrm{h} 2-5 \mathrm{H}, 1 \mathrm{H} 3$ \\
\hline 549 & C4H71-2O2 & $\mathrm{C}=\mathrm{C}(\mathrm{CC}) \mathrm{O}[\mathrm{O}]$ & InChl=1S/C4H7O2/c1-3-4(2)6-5/h2-3H2,1H3 \\
\hline 550 & $\mathrm{SC} 4 \mathrm{H} 7 \mathrm{OH}-10 \mathrm{OOH}-4$ & {$[\mathrm{CH} 2] \mathrm{CC}(\mathrm{O}) \mathrm{COO}$} & InChl=1S/C4H9O3/c1-2-4(5)3-7-6/h4-6H,1-3H2 \\
\hline 551 & $\mathrm{CH} 3 \mathrm{HOOCHCOO} 2 \mathrm{CH} 2$ & $\mathrm{CC}(\mathrm{OO}) \mathrm{C}(=0) \mathrm{CO}[\mathrm{O}]$ & $\operatorname{lnChl}=1 \mathrm{~S} / \mathrm{C} 4 \mathrm{H} 705 / \mathrm{c} 1-3(9-7) 4(5) 2-8-6 / \mathrm{h} 3,7 \mathrm{H}, 2 \mathrm{H} 2,1 \mathrm{H} 3$ \\
\hline 552 & $\mathrm{C} 4 \mathrm{H} 71-\mathrm{O}$ & $\mathrm{CC}([\mathrm{O}]) \mathrm{C}=\mathrm{C}$ & $\operatorname{lnChl}=1 \mathrm{~S} / \mathrm{C} 4 \mathrm{H} 7 \mathrm{O} / \mathrm{c} 1-3-4(2) 5 / \mathrm{h} 3-4 \mathrm{H}, 1 \mathrm{H} 2,2 \mathrm{H} 3$ \\
\hline 553 & C4Y1-3OR & $\mathrm{CC}([\mathrm{O}]) \mathrm{CC}=\mathrm{O}$ & $\operatorname{lnChl}=1 \mathrm{~S} / \mathrm{C} 4 \mathrm{H} 702 / \mathrm{cc}-4(6) 2-3-5 / \mathrm{h} 3-4 \mathrm{H}, 2 \mathrm{H} 2,1 \mathrm{H} 3$ \\
\hline 554 & $\mathrm{SC} 4 \mathrm{H} 7 \mathrm{OH}-10 \mathrm{OH}-2$ & $\mathrm{CC}[\mathrm{C}](\mathrm{O}) \mathrm{COO}$ & InChl=1S/C4H9O3/c1-2-4(5)3-7-6/h5-6H,2-3H2,1H3 \\
\hline 555 & $\mathrm{HOOCH} 2 \mathrm{CH} 2 \mathrm{COO} 2 \mathrm{CH} 2$ & $\mathrm{OOCCC}(=0) \mathrm{CO}[0]$ & InChl=1S/C4H705/c5-4(3-9-7)1-2-8-6/h6H,1-3H2 \\
\hline 556 & THF-2J & $\mathrm{C} 1 \mathrm{CC}[\mathrm{CH}] \mathrm{O} 1$ & InChl=1S/C4H7O/c1-2-4-5-3-1/h3H,1-2,4H2 \\
\hline 557 & $\mathrm{C} 4 \mathrm{Y} 2 \mathrm{OH} 4$ & $\mathrm{CC}(=0) \mathrm{CCO}$ & $\operatorname{lnChl}=1 \mathrm{~S} / \mathrm{C} 4 \mathrm{H} 8 \mathrm{OO} / \mathrm{c1}-4(6) 2-3-5 / \mathrm{h} 5 \mathrm{H}, 2-3 \mathrm{H} 2,1 \mathrm{H} 3$ \\
\hline 558 & $\mathrm{IC} 4 \mathrm{H} 8 \mathrm{OH}-102$ & $\mathrm{CC}(\mathrm{C}) \mathrm{C}(\mathrm{O}[\mathrm{O}]) \mathrm{O}$ & InChl=1S/C4H9O3/c1-3(2)4(5)7-6/h3-5H,1-2H3 \\
\hline 559 & THF-3J & $\mathrm{C} 1 \mathrm{C}[\mathrm{CH}] \mathrm{CO} 1$ & $\operatorname{lnChl}=1 \mathrm{~S} / \mathrm{C} 4 \mathrm{H} 7 \mathrm{O} / \mathrm{c1}-2-4-5-3-1 / \mathrm{h} 1 \mathrm{H}, 2-4 \mathrm{H} 2$ \\
\hline 560 & ETHACET & $\mathrm{CC}(=0) \mathrm{OCC}$ & $\operatorname{lnChl}=1 \mathrm{~S} / \mathrm{C} 4 \mathrm{H} 8 \mathrm{O} 2 / \mathrm{c} 1-3-6-4(2) 5 / \mathrm{h} 3 \mathrm{H} 2,1-2 \mathrm{H} 3$ \\
\hline 561 & IC4H7OH-10OH-3 & {$[\mathrm{CH} 2] \mathrm{C}(\mathrm{C}) \mathrm{C}(\mathrm{OO}) \mathrm{O}$} & InChl=1S/C4H9O3/c1-3(2)4(5)7-6/h3-6H,1H2,2H3 \\
\hline 562 & $\mathrm{C} 4 \mathrm{H} 7 \mathrm{OH}-10 \mathrm{OH}-2 \mathrm{O} 2$ & $\operatorname{CCC}(\mathrm{O}[\mathrm{O}]) \mathrm{C}(\mathrm{OO}) \mathrm{O}$ & $\operatorname{lnChl}=15 / C 4 H 9 O 5 / c 1-2-3(8-6) 4(5) 9-7 / h 3-5,7 \mathrm{H}, 2 \mathrm{H} 2,1 \mathrm{H} 3$ \\
\hline 563 & IC3H7CO & $\mathrm{CC}(\mathrm{C})[\mathrm{C}]=\mathrm{O}$ & $\operatorname{lnChl}=1 \mathrm{~S} / \mathrm{C} 4 \mathrm{H} 7 \mathrm{O} / \mathrm{c} 1-4(2) 3-5 / \mathrm{h} 4 \mathrm{H}, 1-2 \mathrm{H} 3$ \\
\hline 564 & C4H7OHCYO1-2 & $\mathrm{CCC} 1 \mathrm{C}(\mathrm{O} 1) \mathrm{O}$ & InChl=15/C4H8O2/c1-2-3-4(5)6-3/h3-5H,2H2,1H3 \\
\hline 565 & $\mathrm{IC} 4 \mathrm{H} 7 \mathrm{OH}-10 \mathrm{OH}-2$ & $\mathrm{C}[\mathrm{C}](\mathrm{C}) \mathrm{C}(\mathrm{OO}) \mathrm{O}$ & InChl=1S/C4H9O3/c1-3(2)4(5)7-6/h4-6H,1-2H3 \\
\hline 566 & C4H7OH-20OH-102 & $\operatorname{CCC}(\mathrm{OO}) \mathrm{C}(\mathrm{O}[\mathrm{O}]) \mathrm{O}$ & InChl=1S/C4H905/c1-2-3(8-6)4(5)9-7/h3-6H,2H2,1H3 \\
\hline 567 & $\mathrm{IC} 3 \mathrm{H} 6 \mathrm{CHO}$ & {$[\mathrm{CH} 2] \mathrm{C}(\mathrm{C}) \mathrm{C}=0$} & InChl=1S/C4H7O/c1-4(2)3-5/h3-4H,1H2,2H3 \\
\hline 568 & SC4H7OHCYO2-1 & $\mathrm{CCC}(0)(01) \mathrm{C} 1$ & InChl=1S/C4H8O2/c1-2-4(5)3-6-4/h5H,2-3H2,1H3 \\
\hline 569 & $\mathrm{SC} 4 \mathrm{H} 8 \mathrm{OH}-3 \mathrm{O} 2$ & $\mathrm{CC}(\mathrm{O}[\mathrm{O}]) \mathrm{C}(\mathrm{O}) \mathrm{C}$ & InChl=1S/C4H9O3/c1-3(5)4(2)7-6/h3-5H,1-2H3 \\
\hline 570 & TC3H6CHO & $\mathrm{C}[\mathrm{C}](\mathrm{C}) \mathrm{C}=\mathrm{O}$ & $\operatorname{lnChl}=1 \mathrm{~S} / \mathrm{C} 4 \mathrm{H} 7 \mathrm{O} / \mathrm{c} 1-4(2) 3-5 / \mathrm{h} 3 \mathrm{H}, 1-2 \mathrm{H} 3$ \\
\hline 571 & SC4H7OHCYO3-4 & $\mathrm{C} 1 \mathrm{C}(\mathrm{O} 1) \mathrm{C}(\mathrm{O}) \mathrm{C}$ & $\ln C h \mathrm{l}=1 \mathrm{~S} / \mathrm{C} 4 \mathrm{H} 8 \mathrm{O} 2 / \mathrm{c} 1-3(5) 4-2-6-4 / \mathrm{h} 3-5 \mathrm{H}, 2 \mathrm{H} 2,1 \mathrm{H} 3$ \\
\hline 572 & SQC4H7OHS-4 & $\mathrm{CC}(\mathrm{OO}) \mathrm{C}(\mathrm{O})[\mathrm{C}]$ & InChl=1S/C4H9O3/c1-3(5)4(2)7-6/h3-6H,1H2,2H3 \\
\hline 573 & SC4H7OH-10OH-2O2 & $\operatorname{CCC}(0)(O[O]) \operatorname{COO}$ & $\operatorname{lnChl}=15 / C 4 H 9 O 5 / c 1-2-4(5,9-7) 3-8-6 / h 5-6 \mathrm{H}, 2-3 \mathrm{H} 2,1 \mathrm{H} 3$ \\
\hline 574 & IC4H7O & $\mathrm{C}=\mathrm{C}(\mathrm{C}) \mathrm{C}[\mathrm{O}]$ & InChl=1S/C4H7O/c1-4(2)3-5/h1,3H2,2H3 \\
\hline 575 & C4H7OHCYO1-3 & $\mathrm{CC1CC}(\mathrm{O} 1) \mathrm{O}$ & InChl=1S/C4H8O2/c1-3-2-4(5)6-3/h3-5H,2H2,1H3 \\
\hline 576 & SC4H7OH-30OH-4 & {$[\mathrm{CH} 2] \mathrm{C}(\mathrm{OO}) \mathrm{C}(\mathrm{O}) \mathrm{C}$} & InChl=1S/C4H9O3/c1-3(5)4(2)7-6/h3-6H,2H2,1H3 \\
\hline 577 & IC4H7OH-10OH-302 & $\mathrm{C}(\mathrm{O}[\mathrm{O}]) \mathrm{C}(\mathrm{C}) \mathrm{C}(\mathrm{OO}) \mathrm{O}$ & $\operatorname{lnChl}=1 \mathrm{~S} / \mathrm{C} 4 \mathrm{H} 9 \mathrm{O} 5 / \mathrm{c} 1-3(2-8-6) 4(5) 9-7 / \mathrm{h} 3-5,7 \mathrm{H}, 2 \mathrm{H} 2,1 \mathrm{H} 3$ \\
\hline 578 & $\mathrm{IC} 4 \mathrm{H} 6 \mathrm{OH}$ & $\mathrm{C}=\mathrm{C}(\mathrm{C})[\mathrm{CH}] \mathrm{O}$ & $\operatorname{lnChl}=1 \mathrm{~S} / \mathrm{C} 4 \mathrm{H} 7 \mathrm{O} / \mathrm{c} 1-4(2) 3-5 / \mathrm{h} 3,5 \mathrm{H}, 1 \mathrm{H} 2,2 \mathrm{H} 3$ \\
\hline 579 & IC4H7OHCYO1-3 & $\mathrm{C} 1 \mathrm{C}(\mathrm{C}) \mathrm{C}(\mathrm{O} 1) \mathrm{O}$ & InChl=15/C4H8O2/c1-3-2-6-4(3)5/h3-5H,2H2,1H3 \\
\hline 580 & $\mathrm{SC} 4 \mathrm{H} 7 \mathrm{OH}-3 \mathrm{OOH}-2$ & $\mathrm{CC}(\mathrm{OO})[\mathrm{C}](\mathrm{O}) \mathrm{C}$ & InChl=1S/C4H9O3/c1-3(5)4(2)7-6/h4-6H,1-2H3 \\
\hline 581 & IC4H7OH-30OH-102 & $\mathrm{C}(\mathrm{OO}) \mathrm{C}(\mathrm{C}) \mathrm{C}(\mathrm{O}[\mathrm{O}]) \mathrm{O}$ & InChl=1S/C4H9O5/c1-3(2-8-6)4(5)9-7/h3-6H,2H2,1H3 \\
\hline 582 & $\mathrm{IC} 3 \mathrm{H} 5 \mathrm{OCH} 2$ & $\mathrm{C}=\mathrm{C}(\mathrm{C}) \mathrm{O}[\mathrm{CH} 2]$ & $\operatorname{lnChl}=1 \mathrm{~S} / \mathrm{C} 4 \mathrm{H} 7 \mathrm{O} / \mathrm{c} 1-4(2) 5-3 / \mathrm{h} 1,3 \mathrm{H} 2,2 \mathrm{H} 3$ \\
\hline 583 & SC4H7OHCYO2-3 & $\mathrm{CC} 1 \mathrm{C}(\mathrm{O})(\mathrm{O} 1) \mathrm{C}$ & $\operatorname{lnChl}=1 \mathrm{~S} / \mathrm{C} 4 \mathrm{H} 8 \mathrm{O} 2 / \mathrm{c} 1-3-4(2,5) 6-3 / \mathrm{h} 3,5 \mathrm{H}, 1-2 \mathrm{H} 3$ \\
\hline 584 & SC4H7OH-2OOH-3 & $\mathrm{C}[\mathrm{CH}] \mathrm{C}(\mathrm{O})(\mathrm{OO}) \mathrm{C}$ & $\operatorname{lnChl}=1 \mathrm{~S} / \mathrm{C} 4 \mathrm{H} 903 / \mathrm{c} 1-3-4(2,5) 7-6 / \mathrm{h} 3,5-6 \mathrm{H}, 1-2 \mathrm{H} 3$ \\
\hline 585 & SC4H7OH-3OOH-4O2 & $\mathrm{C}(\mathrm{O}[\mathrm{O}]) \mathrm{C}(\mathrm{OO}) \mathrm{C}(\mathrm{O}) \mathrm{C}$ & $\operatorname{lnChl}=15 / C 4 H 905 / c 1-3(5) 4(9-7) 2-8-6 / h 3-5,7 \mathrm{H}, 2 \mathrm{H} 2,1 \mathrm{H} 3$ \\
\hline 586 & $\mathrm{C} 4 \mathrm{H} 702-1$ & $\mathrm{CC}=\mathrm{CC}[\mathrm{O}]$ & InChl=1S/C4H7O/c1-2-3-4-5/h2-3H,4H2,1H3 \\
\hline 587 & C4H7OHCYO2-3 & $\mathrm{CC} 1 \mathrm{C}(\mathrm{O} 1) \mathrm{CO}$ & InChl=1S/C4H8O2/c1-3-4(2-5)6-3/h3-5H,2H2,1H3 \\
\hline 588 & $\mathrm{SC} 4 \mathrm{H} 7 \mathrm{OH}-2 \mathrm{OOH}-4$ & {$[\mathrm{CH} 2] \mathrm{CC}(\mathrm{O})(\mathrm{OO}) \mathrm{C}$} & InChl=1S/C4H9O3/c1-3-4(2,5)7-6/h5-6H,1,3H2,2H3 \\
\hline 589 & SC4H7OH-40OH-3O2 & $\mathrm{C}(\mathrm{OO}) \mathrm{C}(\mathrm{O}[\mathrm{O}]) \mathrm{C}(\mathrm{O}) \mathrm{C}$ & $\operatorname{lnChl}=1 \mathrm{~S} / \mathrm{C} 4 \mathrm{H} 905 / \mathrm{c1}-3(5) 4(9-7) 2-8-6 / \mathrm{h} 3-6 \mathrm{H}, 2 \mathrm{H} 2,1 \mathrm{H} 3$ \\
\hline 590 & $\mathrm{AC} 3 \mathrm{H} 5 \mathrm{OCH} 2$ & $\mathrm{C}=\mathrm{CCO}[\mathrm{CH} 2]$ & $\operatorname{lnChl}=1 \mathrm{~S} / \mathrm{C} 4 \mathrm{H} 7 \mathrm{O} / \mathrm{c} 1-3-4-5-2 / \mathrm{h} 3 \mathrm{H}, 1-2,4 \mathrm{H} 2$ \\
\hline 591 & $\mathrm{CCY}(\mathrm{COCC}) \mathrm{OH}$ & $\mathrm{CC}(\mathrm{O} 1) \mathrm{C}(\mathrm{O}) \mathrm{C} 1$ & InChl=1S/C4H8O2/c1-3-4(5)2-6-3/h3-5H,2H2,1H3 \\
\hline 592 & SC4H7OH-2OOH-1 & $\mathrm{CCC}(\mathrm{O})(\mathrm{OO})[\mathrm{CH} 2]$ & InChl=1S/C4H9O3/c1-3-4(2,5)7-6/h5-6H,2-3H2,1H3 \\
\hline 593 & C4H7OH-10OH-302 & $\mathrm{CC}(\mathrm{O}[\mathrm{O}]) \mathrm{CC}(\mathrm{OO}) \mathrm{O}$ & $\operatorname{lnChl}=15 / C 4 H 9 O 5 / c 1-3(8-6) 2-4(5) 9-7 / h 3-5,7 \mathrm{H}, 2 \mathrm{H} 2,1 \mathrm{H} 3$ \\
\hline 594 & $\mathrm{C} 4 \mathrm{H} 63,1-3 \mathrm{OH}$ & $\mathrm{C}=\mathrm{C}[\mathrm{C}](\mathrm{C}) \mathrm{O}$ & $\operatorname{lnChl}=1 \mathrm{~S} / \mathrm{C} 4 \mathrm{H} 70 / \mathrm{c} 1-3-4(2) 5 / \mathrm{h} 3,5 \mathrm{H}, 1 \mathrm{H} 2,2 \mathrm{H} 3$ \\
\hline 595 & $\mathrm{C} 2 \mathrm{CY}(\mathrm{COC}) \mathrm{OH}$ & $\mathrm{CC} 1(\mathrm{C}) \mathrm{C}(\mathrm{O} 1) \mathrm{O}$ & $\operatorname{lnChl}=1 \mathrm{~S} / \mathrm{C} 4 \mathrm{H} 8 \mathrm{O} 2 / \mathrm{cc} 1-4(2) 3(5) 6-4 / \mathrm{h} 3,5 \mathrm{H}, 1-2 \mathrm{H} 3$ \\
\hline 596 & SC4H8OH-2O2 & $\operatorname{CCC}(\mathrm{O})(\mathrm{O}[\mathrm{O}]) \mathrm{C}$ & $\mathrm{InChl}=1 \mathrm{~S} / \mathrm{C} 4 \mathrm{H} 9 \mathrm{O} / \mathrm{c} 1-3-4(2,5) 7-6 / \mathrm{h} 5 \mathrm{H}, 3 \mathrm{H} 2,1-2 \mathrm{H} 3$ \\
\hline 597 & C4H7OH-30OH-102 & $\mathrm{CC}(\mathrm{OO}) \mathrm{CC}(\mathrm{O}[\mathrm{O}]) \mathrm{O}$ & InChl=1S/C4H905/c1-3(8-6)2-4(5)9-7/h3-6H,2H2,1H3 \\
\hline 598 & $\mathrm{C} 4 \mathrm{H} 63,1-2 \mathrm{OH}$ & $\mathrm{C}=\mathrm{C}(\mathrm{O})[\mathrm{CH}] \mathrm{C}$ & $\operatorname{lnChl}=1 \mathrm{~S} / \mathrm{C} 4 \mathrm{H} 7 \mathrm{O} / \mathrm{c} 1-3-4(2) 5 / \mathrm{h} 3,5 \mathrm{H}, 2 \mathrm{H} 2,1 \mathrm{H} 3$ \\
\hline 599 & $\mathrm{CCY}(\mathrm{CCO}) \mathrm{COH}$ & $\mathrm{C} 1 \mathrm{C}(\mathrm{C})(\mathrm{O} 1) \mathrm{CO}$ & InChl=1S/C4H8O2/c1-4(2-5)3-6-4/h5H,2-3H2,1H3 \\
\hline 600 & TQC4H7OHI & $\mathrm{CC}(\mathrm{C})(\mathrm{OO})[\mathrm{CH}] \mathrm{O}$ & $\operatorname{lnChl}=1 \mathrm{~S} / \mathrm{C} 4 \mathrm{H} 9 \mathrm{O} 3 / \mathrm{c} 1-4(2,3-5) 7-6 / \mathrm{h} 3,5-6 \mathrm{H}, 1-2 \mathrm{H} 3$ \\
\hline 601 & SC4H7OH-2OOH-3O2 & $\mathrm{CC}(\mathrm{O}[\mathrm{O}]) \mathrm{C}(\mathrm{O})(\mathrm{OO}) \mathrm{C}$ & InChl=1S/C4H9O5/c1-3(8-6)4(2,5)9-7/h3,5,7H,1-2H3 \\
\hline 602 & C4H701-4 & $\mathrm{C}=\mathrm{CCC}[\mathrm{O}]$ & $\operatorname{lnChl}=1 \mathrm{~S} / \mathrm{C} 4 \mathrm{H} 70 / \mathrm{c} 1-2-3-4-5 / \mathrm{h} 2 \mathrm{H}, 1,3-4 \mathrm{H} 2$ \\
\hline 603 & SC4H7OHCYO2-4 & $\mathrm{C} 1 \mathrm{CC}(\mathrm{O})(\mathrm{O} 1) \mathrm{C}$ & InChl=1S/C4H8O2/c1-4(5)2-3-6-4/h5H,2-3H2,1H3 \\
\hline 604 & QC4H7OHP & {$[\mathrm{CH} 2] \mathrm{C}(\mathrm{C})(\mathrm{OO}) \mathrm{CO}$} & InChl=1S/C4H9O3/c1-4(2,3-5)7-6/h5-6H,1,3H2,2H3 \\
\hline 605 & SC4H7OH-3OOH-2O2 & $\mathrm{CC}(\mathrm{OO}) \mathrm{C}(\mathrm{O})(\mathrm{O}[\mathrm{O}]) \mathrm{C}$ & InChl=1S/C4H9O5/c1-3(8-6)4(2,5)9-7/h3,5-6H,1-2H3 \\
\hline 606 & NC3H7CO & $\mathrm{CCC}[\mathrm{C}]=0$ & InChl=1S/C4H7O/c1-2-3-4-5/h2-3H2,1H3 \\
\hline 607 & $\mathrm{CCY}(\mathrm{CCOC}) \mathrm{OH}$ & $\mathrm{C} 1 \mathrm{C}(\mathrm{C})(\mathrm{O}) \mathrm{CO} 1$ & $\operatorname{lnChl}=1 \mathrm{~S} / \mathrm{C} 4 \mathrm{H} 8 \mathrm{O} 2 / \mathrm{c} 1-4(5) 2-6-3-4 / \mathrm{h} 5 \mathrm{H}, 2-3 \mathrm{H} 2,1 \mathrm{H} 3$ \\
\hline 608 & TQJC4H8OH & $\mathrm{CC}(\mathrm{C})(\mathrm{O}[\mathrm{O}]) \mathrm{CO}$ & InChl=1S/C4H9O3/c1-4(2,3-5)7-6/h5H,3H2,1-2H3 \\
\hline 609 & C4H7OH-2OOH-302 & $\mathrm{CC}(\mathrm{O}[\mathrm{O}]) \mathrm{C}(\mathrm{OO}) \mathrm{CO}$ & $\operatorname{lnChl}=1 \mathrm{~S} / \mathrm{C} 4 \mathrm{H} 905 / \mathrm{c} 1-3(8-6) 4(2-5) 9-7 / \mathrm{h} 3-5,7 \mathrm{H}, 2 \mathrm{H} 2,1 \mathrm{H} 3$ \\
\hline 610 & $\mathrm{C} 3 \mathrm{H} 6 \mathrm{CHO}-1$ & {$[\mathrm{CH} 2] \mathrm{CCC}=\mathrm{O}$} & InChl=1S/C4H7O/c1-2-3-4-5/h4H,1-3H2 \\
\hline 611 & IC4H7OHCYO3-3 & $\mathrm{C1C}(\mathrm{CO} 1) \mathrm{CO}$ & InChl=1S/C4H8O2/c5-1-4-2-6-3-4/h4-5H,1-3H2 \\
\hline 612 & IQC4H7OHT & {$[\mathrm{CH} 2] \mathrm{C}(\mathrm{C})(\mathrm{O}) \mathrm{COO}$} & InChl=1S/C4H9O3/c1-4(2,5)3-7-6/h5-6H,1,3H2,2H3 \\
\hline 613 & C4H7OH-30OH-2O2 & $\mathrm{CC}(\mathrm{OO}) \mathrm{C}(\mathrm{O}[\mathrm{O}]) \mathrm{CO}$ & InChl=1S/C4H905/c1-3(8-6)4(2-5)9-7/h3-6H,2H2,1H3 \\
\hline 614 & $\mathrm{C} 3 \mathrm{H} 6 \mathrm{CHO}-2$ & $\mathrm{C}[\mathrm{CH}] \mathrm{CC}=\mathrm{O}$ & $\operatorname{lnChl}=1 \mathrm{~S} / \mathrm{C} 4 \mathrm{H} 7 \mathrm{O} / \mathrm{c} 1-2-3-4-5 / \mathrm{h} 2,4 \mathrm{H}, 3 \mathrm{H} 2,1 \mathrm{H} 3$ \\
\hline 615 & C4H7OHCYO3-4 & $\mathrm{C} 1 \mathrm{C}(\mathrm{O} 1) \mathrm{CCO}$ & $\operatorname{lnChl}=1 \mathrm{~S} / \mathrm{C} 4 \mathrm{H} 8 \mathrm{O} 2 / \mathrm{c5}-2-1-4-3-6-4 / \mathrm{h} 4-5 \mathrm{H}, 1-3 \mathrm{H} 2$ \\
\hline 616 & IQJC4H8OH & $\mathrm{CC}(\mathrm{C})(\mathrm{O}) \mathrm{CO}[\mathrm{O}]$ & InChl=1S/C4H9O3/c1-4(2,5)3-7-6/h5H,3H2,1-2H3 \\
\hline 617 & SQC4H7OHS-4O2 & $\mathrm{CC}(\mathrm{OO}) \mathrm{C}(\mathrm{O}) \mathrm{CO}[\mathrm{O}]$ & $\operatorname{lnChl}=15 / \mathrm{C} 4 \mathrm{H} 905 / \mathrm{c} 1-3(9-7) 4(5) 2-8-6 / \mathrm{h} 3-5,7 \mathrm{H}, 2 \mathrm{H} 2,1 \mathrm{H} 3$ \\
\hline 618 & $\mathrm{C} 3 \mathrm{H} 6 \mathrm{CHO}-3$ & $\mathrm{CC}[\mathrm{CH}] \mathrm{C}=\mathrm{O}$ & $\operatorname{lnChl}=1 \mathrm{~S} / \mathrm{C} 4 \mathrm{H} 7 \mathrm{O} / \mathrm{c} 1-2-3-4-5 / \mathrm{h} 3-4 \mathrm{H}, 2 \mathrm{H} 2,1 \mathrm{H} 3$ \\
\hline 619 & HOC3H6CHO & $\mathrm{C}(=0) \mathrm{CCCO}$ & InChl=1S/C4H8O2/c5-3-1-2-4-6/h3,6H,1-2,4H2 \\
\hline 620 & IC4H7OH-30OH-1 & $\mathrm{C}(\mathrm{OO}) \mathrm{C}(\mathrm{C})[\mathrm{CH}] \mathrm{O}$ & InChl=1S/C4H9O3/c1-4(2-5)3-7-6/h2,4-6H,3H2,1H3 \\
\hline 621 & PQC4H7OHS-3O2 & $\mathrm{CC}(\mathrm{O}[\mathrm{O}]) \mathrm{C}(\mathrm{O}) \mathrm{COO}$ & InChl=1S/C4H9O5/c1-3(9-7)4(5)2-8-6/h3-6H,2H2,1H3 \\
\hline 622 & $\mathrm{C} 4 \mathrm{H} 7012-4$ & $\mathrm{C} 1 \mathrm{C}(\mathrm{O} 1) \mathrm{C}[\mathrm{CH} 2]$ & $\ln \mathrm{Chl}=1 \mathrm{~S} / \mathrm{C} 4 \mathrm{H} 7 \mathrm{O} / \mathrm{c1}-2-4-3-5-4 / \mathrm{h} 4 \mathrm{H}, 1-3 \mathrm{H} 2$ \\
\hline 623 & $\mathrm{CY}(\mathrm{CCCO}) \mathrm{COH}$ & $\mathrm{C} 1 \mathrm{CC}(\mathrm{O} 1) \mathrm{CO}$ & InChl=1S/C4H8O2/c5-3-4-1-2-6-4/h4-5H,1-3H2 \\
\hline 624 & IC4H8OH-302 & $\mathrm{C}(\mathrm{O}[\mathrm{O}]) \mathrm{C}(\mathrm{C}) \mathrm{CO}$ & InChl=1S/C4H9O3/c1-4(2-5)3-7-6/h4-5H,2-3H2,1H3 \\
\hline 625 & TQC4H7OHIO2 & $\mathrm{CC}(\mathrm{C})(\mathrm{OO}) \mathrm{C}(\mathrm{O}[\mathrm{O}]) \mathrm{O}$ & InChl=1S/C4H905/c1-4(2,9-7)3(5)8-6/h3,5,7H,1-2H3 \\
\hline 626 & C4H7013-4 & $\mathrm{C} 1 \mathrm{CC}(\mathrm{O} 1)[\mathrm{CH} 2]$ & $\ln \mathrm{Chl}=1 \mathrm{~S} / \mathrm{C} 4 \mathrm{H} 7 \mathrm{O} / \mathrm{c1}-4-2-3-5-4 / \mathrm{h} 4 \mathrm{H}, 1-3 \mathrm{H} 2$ \\
\hline 627 & SC4H7OHCYO4-1 & $\mathrm{C}(\mathrm{O} 1) \mathrm{CC}(\mathrm{O}) \mathrm{C1}$ & InChl=1S/C4H8O2/c5-4-1-2-6-3-4/h4-5H,1-3H2 \\
\hline 628 & $\mathrm{IC} 4 \mathrm{H} 7 \mathrm{OH}-3 \mathrm{OOH}-3$ & $\mathrm{C}(\mathrm{OO}) \mathrm{C}([\mathrm{CH} 2]) \mathrm{CO}$ & InChl=1S/C4H9O3/c1-4(2-5)3-7-6/h4-6H,1-3H2 \\
\hline 629 & TQC4H7OHTO2 & $\mathrm{CC}(\mathrm{C})(\mathrm{O}[\mathrm{O}]) \mathrm{C}(\mathrm{OO}) \mathrm{O}$ & InChl=1S/C4H9O5/c1-4(2,9-7)3(5)8-6/h3,5-6H,1-2H3 \\
\hline 630 & C4H7023-1 & $\mathrm{CC} 1 \mathrm{C}(\mathrm{O} 1)[\mathrm{CH} 2]$ & $\operatorname{lnChl}=1 \mathrm{~S} / \mathrm{C} 4 \mathrm{H} 7 \mathrm{O} / \mathrm{c} 1-3-4(2) 5-3 / \mathrm{h} 3-4 \mathrm{H}, 1 \mathrm{H} 2,2 \mathrm{H} 3$ \\
\hline 631 & C4H7OHCYO1-4 & $\mathrm{C1CCC}(\mathrm{O} 1) \mathrm{O}$ & $\operatorname{lnChl}=1 \mathrm{~S} / \mathrm{C} 4 \mathrm{H} 8 \mathrm{O} 2 / \mathrm{c} 5-4-2-1-3-6-4 / \mathrm{h} 4-5 \mathrm{H}, 1-3 \mathrm{H} 2$ \\
\hline 632 & $\mathrm{IC} 4 \mathrm{H} 7 \mathrm{OH}-30 \mathrm{OH}-2$ & $\mathrm{C}(\mathrm{OO})[\mathrm{C}](\mathrm{C}) \mathrm{CO}$ & $\operatorname{lnChl}=1 \mathrm{~S} / \mathrm{C} 4 \mathrm{H} 9 \mathrm{O} 3 / \mathrm{c} 1-4(2-5) 3-7-6 / \mathrm{h} 5-6 \mathrm{H}, 2-3 \mathrm{H} 2,1 \mathrm{H} 3$ \\
\hline 633 & IC4H7OH-2OOH-3O2 & $\mathrm{C}(\mathrm{O}[\mathrm{O}]) \mathrm{C}(\mathrm{C})(\mathrm{OO}) \mathrm{CO}$ & $\operatorname{lnChl}=15 / C 4 H 905 / c 1-4(2-5,9-7) 3-8-6 / h 5,7 \mathrm{H}, 2-3 \mathrm{H} 2,1 \mathrm{H} 3$ \\
\hline
\end{tabular}




\begin{tabular}{|c|c|c|c|}
\hline 634 & CC4H7O-I נו- & {$\left[\mathrm{CH}_{2}\right] \mathrm{C} 1 \mathrm{COC} 1$} & $\operatorname{lnChl}=1 \mathrm{~S} / \mathrm{C} 4 \mathrm{H} 7 \mathrm{O} / \mathrm{c1}-4-2-5-3-4 / \mathrm{h} 4 \mathrm{H}, 1-3 \mathrm{H} 2$ \\
\hline 635 & AC4H7OOH & $\mathrm{C}=\mathrm{CCOOC}$ & $\operatorname{lnChl}=1 \mathrm{~S} / \mathrm{C} 4 \mathrm{H} 8 \mathrm{OO} / \mathrm{c1} 1-3-4-6-5-2 / \mathrm{h} 3 \mathrm{H}, 1,4 \mathrm{H} 2,2 \mathrm{H} 3$ \\
\hline 636 & SC4H7OH-4OOH-3 & $\mathrm{C}(\mathrm{OO})[\mathrm{CH}] \mathrm{C}(\mathrm{O}) \mathrm{C}$ & InChl=1S/C4H9O3/c1-4(5)2-3-7-6/h2,4-6H,3H2,1H3 \\
\hline 637 & $\mathrm{IC} 4 \mathrm{H} 7 \mathrm{OH}-3 \mathrm{OOH}-2 \mathrm{O} 2$ & $\mathrm{C}(\mathrm{OO}) \mathrm{C}(\mathrm{C})(\mathrm{O}[\mathrm{O}]) \mathrm{CO}$ & $\operatorname{lnChl}=15 / C 4 H 905 / \mathrm{c} 1-4(2-5,9-7) 3-8-6 / h 5-6 \mathrm{H}, 2-3 \mathrm{H} 2,1 \mathrm{H} 3$ \\
\hline 638 & C4H71-4OH & $\mathrm{C}(\mathrm{O}) \mathrm{CC}=\mathrm{C}$ & InChl=1S/C4H8O/c1-2-3-4-5/h2,5H,1,3-4H2 \\
\hline 639 & $\mathrm{C} 4 \mathrm{H} 72-10 \mathrm{OH}$ & $\mathrm{CC}=\mathrm{CCOO}$ & InChl=1S/C4H8O2/c1-2-3-4-6-5/h2-3,5H,4H2,1H3 \\
\hline 640 & SC4H8OH-4O2 & $\mathrm{C}(\mathrm{O}[\mathrm{O}]) \mathrm{CC}(\mathrm{O}) \mathrm{C}$ & InChl=1S/C4H9O3/c1-4(5)2-3-7-6/h4-5H,2-3H2,1H3 \\
\hline 641 & IQC4H7OHTO2 & $\mathrm{C}(\mathrm{O}[\mathrm{O}]) \mathrm{C}(\mathrm{C})(\mathrm{O}) \mathrm{COO}$ & InChl=1S/C4H9O5/c1-4(5,2-8-6)3-9-7/h5-6H,2-3H2,1H3 \\
\hline 642 & $\mathrm{C} 4 \mathrm{H} 72-1 \mathrm{OH}$ & $\mathrm{CC}=\mathrm{CCO}$ & InChl=1S/C4H8O/c1-2-3-4-5/h2-3,5H,4H2,1H3 \\
\hline 643 & $\mathrm{C} 4 \mathrm{H} 71-30 \mathrm{OH}$ & $\mathrm{C}=\mathrm{CC}(\mathrm{C}) \mathrm{OO}$ & InChl=1S/C4H8O2/c1-3-4(2)6-5/h3-5H,1H2,2H3 \\
\hline 644 & SC4H7OH-4OOH-1 & $\mathrm{C}(\mathrm{OO}) \mathrm{CC}(\mathrm{O})[\mathrm{CH} 2]$ & InChl=1S/C4H9O3/c1-4(5)2-3-7-6/h4-6H,1-3H2 \\
\hline 645 & SC4H7OH-2OOH-402 & $\mathrm{C}(\mathrm{O}[\mathrm{O}]) \mathrm{CC}(\mathrm{O})(\mathrm{OO}) \mathrm{C}$ & $\operatorname{lnChl}=1 \mathrm{~S} / \mathrm{C} 4 \mathrm{H} 905 / \mathrm{c} 1-4(5,9-7) 2-3-8-6 / \mathrm{h} 5,7 \mathrm{H}, 2-3 \mathrm{H} 2,1 \mathrm{H} 3$ \\
\hline 646 & $\mathrm{C} 4 \mathrm{H} 71-1 \mathrm{OH}$ & $\mathrm{CCC}=\mathrm{CO}$ & InChl=1S/C4H8O/c1-2-3-4-5/h3-5H,2H2,1H3 \\
\hline 647 & $\mathrm{IC} 4 \mathrm{H} 7 \mathrm{OOH}$ & $\mathrm{C}=\mathrm{C}(\mathrm{C}) \mathrm{COO}$ & InChl=1S/C4H802/c1-4(2)3-6-5/h5H,1,3H2,2H3 \\
\hline 648 & SC4H7OH-4OOH-2 & $\mathrm{C}(\mathrm{OO}) \mathrm{C}[\mathrm{C}](\mathrm{O}) \mathrm{C}$ & InChl=1S/C4H9O3/c1-4(5)2-3-7-6/h5-6H,2-3H2,1H3 \\
\hline 649 & SC4H7OH-4OOH-2O2 & $\mathrm{C}(\mathrm{OO}) \mathrm{CC}(\mathrm{O})(\mathrm{O}[\mathrm{O}]) \mathrm{C}$ & $\operatorname{lnChl}=15 / C 4 H 905 / c 1-4(5,9-7) 2-3-8-6 / h 5-6 \mathrm{H}, 2-3 \mathrm{H} 2,1 \mathrm{H} 3$ \\
\hline 650 & $\mathrm{C} 4 \mathrm{H} 72-2 \mathrm{OH}$ & $\mathrm{CC}=\mathrm{C}(\mathrm{O}) \mathrm{C}$ & InChl=1S/C4H8O/c1-3-4(2)5/h3,5H,1-2H3 \\
\hline 651 & IC3H6OHCHO & $\mathrm{CC}(\mathrm{C})(\mathrm{C}=0) \mathrm{O}$ & 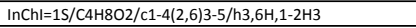 \\
\hline 652 & C4H7OH-30OH-2 & $\mathrm{CC}(\mathrm{OO})[\mathrm{CH}] \mathrm{CO}$ & InChl=1S/C4H9O3/c1-4(7-6)2-3-5/h2,4-6H,3H2,1H3 \\
\hline 653 & IC4H7OH-30OH-302 & $\mathrm{C}(\mathrm{OO}) \mathrm{C}(\mathrm{CO}[\mathrm{O}]) \mathrm{CO}$ & InChl=1S/C4H9O5/c5-1-4(2-8-6)3-9-7/h4-6H,1-3H2 \\
\hline 654 & $\mathrm{C} 4 \mathrm{H} 71-3 \mathrm{OH}$ & $\mathrm{CC}(0) \mathrm{C}=\mathrm{C}$ & InChl=1S/C4H8O/c1-3-4(2)5/h3-5H,1H2,2H3 \\
\hline 655 & NC4KET12OH & $\mathrm{C}(=0) \mathrm{C}(0) \mathrm{CC}$ & InChl=1S/C4H8O2/c1-2-4(6)3-5/h3-4,6H,2H2,1H3 \\
\hline 656 & C4H7OH-30OH-1 & $\mathrm{CC}(\mathrm{OO}) \mathrm{C}[\mathrm{CH}] \mathrm{O}$ & InChl=1S/C4H9O3/c1-4(7-6)2-3-5/h3-6H,2H2,1H3 \\
\hline 657 & C4H7OH-30OH-402 & $\mathrm{C}(\mathrm{O}[\mathrm{O}]) \mathrm{C}(\mathrm{OO}) \mathrm{CCO}$ & InChl=1S/C4H905/c5-2-1-4(9-7)3-8-6/h4-5,7H,1-3H2 \\
\hline 658 & $\mathrm{C} 4 \mathrm{H} 71-2 \mathrm{OH}$ & $\operatorname{ccc}(0)=c$ & InChl=1S/C4H8O/c1-3-4(2)5/h5H,2-3H2,1H3 \\
\hline 659 & NC4KET23OH & $\mathrm{CC}(\mathrm{O}) \mathrm{C}(=\mathrm{O}) \mathrm{C}$ & InChl=1S/C4H8O2/c1-3(5)4(2)6/h3,5H,1-2H3 \\
\hline 660 & PC4H $8 \mathrm{OH}-3 \mathrm{O} 2$ & $\mathrm{CC}(\mathrm{O}[\mathrm{O}]) \mathrm{CCO}$ & InChl=1S/C4H9O3/c1-4(7-6)2-3-5/h4-5H,2-3H2,1H3 \\
\hline 661 & C4H7OH-4OOH-3O2 & $\mathrm{C}(\mathrm{OO}) \mathrm{C}(\mathrm{O}[\mathrm{O}]) \mathrm{CCO}$ & InChl=1S/C4H9O5/c5-2-1-4(9-7)3-8-6/h4-6H,1-3H2 \\
\hline 662 & SC4H7OH-I & $\mathrm{CC}(\mathrm{C})=\mathrm{CO}$ & $\operatorname{lnChl}=1 \mathrm{~S} / \mathrm{C} 4 \mathrm{H} 8 \mathrm{O} / \mathrm{c1} 1-4(2) 3-5 / \mathrm{h} 3,5 \mathrm{H}, 1-2 \mathrm{H} 3$ \\
\hline 663 & NC4KET21OH & $\mathrm{CCC}(=0) \mathrm{CO}$ & $\operatorname{lnChl}=1 \mathrm{~S} / \mathrm{C} 4 \mathrm{H} 8 \mathrm{OO} / \mathrm{c} 1-2-4(6) 3-5 / \mathrm{h} 5 \mathrm{H}, 2-3 \mathrm{H} 2,1 \mathrm{H} 3$ \\
\hline 664 & C4H7OH-30OH-4 & {$[\mathrm{CH} 2] \mathrm{C}(\mathrm{OOO}) \mathrm{CCO}$} & InChl=1S/C4H903/c1-4(7-6)2-3-5/h4-6H,1-3H2 \\
\hline 665 & SQC4H7OHP-4O2 & $\mathrm{C}(\mathrm{O}[\mathrm{O}]) \mathrm{CC}(\mathrm{OO}) \mathrm{CO}$ & InChl=1S/C4H9O5/c5-3-4(9-7)1-2-8-6/h4-5,7H,1-3H2 \\
\hline 666 & $\mathrm{IC} 4 \mathrm{H} 7 \mathrm{OH}$ & $\mathrm{C}=\mathrm{C}(\mathrm{C}) \mathrm{CO}$ & $\operatorname{lnChl}=1 \mathrm{~S} / \mathrm{C} 4 \mathrm{H} 8 \mathrm{O} / \mathrm{c} 1-4(2) 3-5 / \mathrm{h} 5 \mathrm{H}, 1,3 \mathrm{H} 2,2 \mathrm{H} 3$ \\
\hline 667 & $\mathrm{C} 4 \mathrm{H} 71-40 \mathrm{OH}$ & $\mathrm{C}=\mathrm{CCCOO}$ & $\operatorname{lnChl}=1 \mathrm{~S} / \mathrm{C} 4 \mathrm{H} 8 \mathrm{O} 2 / \mathrm{c1} 1-2-3-4-6-5 / \mathrm{h} 2,5 \mathrm{H}, 1,3-4 \mathrm{H} 2$ \\
\hline 668 & $\mathrm{C} 4 \mathrm{H} 7 \mathrm{OH}-4 \mathrm{OOH}-2$ & $\mathrm{C}(\mathrm{OO}) \mathrm{C}[\mathrm{CH}] \mathrm{CO}$ & $\operatorname{lnChl}=1 \mathrm{~S} / \mathrm{C} 4 \mathrm{H} 9 \mathrm{O} / \mathrm{C} 5-3-1-2-4-7-6 / \mathrm{h} 1,5-6 \mathrm{H}, 2-4 \mathrm{H} 2$ \\
\hline 669 & $\mathrm{C} 4 \mathrm{H} 7 \mathrm{OH}-4 \mathrm{OOH}-2 \mathrm{O} 2$ & $\mathrm{C}(\mathrm{OO}) \mathrm{CC}(\mathrm{O}[\mathrm{O}]) \mathrm{CO}$ & InChl=15/C4H9O5/c5-3-4(9-7)1-2-8-6/h4-6H,1-3H2 \\
\hline 670 & C4H8O1-4 & C1CCCO1 & $\operatorname{lnChl}=1 \mathrm{~S} / \mathrm{C} 4 \mathrm{H} 8 \mathrm{O} / \mathrm{c} 1-2-4-5-3-1 / \mathrm{h} 1-4 \mathrm{H} 2$ \\
\hline 671 & $\mathrm{PC} 4 \mathrm{H} 8 \mathrm{OH}-10$ & $\operatorname{cccc}([0]) 0$ & InChl=1S/C4H9O2/c1-2-3-4(5)6/h4-5H,2-3H2,1H3 \\
\hline 672 & C4H7OH-4OOH-3 & $\mathrm{C}(\mathrm{OO})[\mathrm{CH}] \mathrm{CCO}$ & InChl=1S/C4H9O3/c5-3-1-2-4-7-6/h2,5-6H,1,3-4H2 \\
\hline 673 & SC4H7OH-10OH-402 & $\mathrm{C}(\mathrm{O}[\mathrm{O}]) \mathrm{CC}(\mathrm{O}) \mathrm{COO}$ & $\operatorname{lnChl}=1 \mathrm{~S} / \mathrm{C} 4 \mathrm{H} 905 / \mathrm{c5}-4(3-9-7) 1-2-8-6 / \mathrm{h} 4-5,7 \mathrm{H}, 1-3 \mathrm{H} 2$ \\
\hline 674 & C4H8O1-2 & CCC1CO1 & $\operatorname{lnChl}=1 \mathrm{~S} / \mathrm{C} 4 \mathrm{H} 8 \mathrm{O} / \mathrm{c} 1-2-4-3-5-4 / \mathrm{h} 4 \mathrm{H}, 2-3 \mathrm{H} 2,1 \mathrm{H} 3$ \\
\hline 675 & SC4H8OH-1O & $\operatorname{ccc}(0) \mathrm{C}([0])$ & InChl=1S/C4H9O2/c1-2-4(6)3-5/h4,6H,2-3H2,1H3 \\
\hline 676 & C4H7OH-4OOH-1 & $\mathrm{C}(\mathrm{OO}) \mathrm{CC}[\mathrm{CH}] \mathrm{O}$ & $\operatorname{lnChl}=1 \mathrm{~S} / \mathrm{C} 4 \mathrm{H} 9 \mathrm{OO} / \mathrm{c} 5-3-1-2-4-7-6 / \mathrm{h} 3,5-6 \mathrm{H}, 1-2,4 \mathrm{H} 2$ \\
\hline 677 & SC4H7OH-4OOH-102 & $\mathrm{C}(\mathrm{OO}) \mathrm{CC}(\mathrm{O}) \mathrm{C}(\mathrm{O}[\mathrm{O}])$ & InChl=1S/C4H9O5/c5-4(3-9-7)1-2-8-6/h4-6H,1-3H2 \\
\hline 678 & C4H8O1-3 & CC1CCO1 & InChl=1S/C4H8O/c1-4-2-3-5-4/h4H,2-3H2,1H3 \\
\hline 679 & $\mathrm{PC} 4 \mathrm{H} 8 \mathrm{OH}-2 \mathrm{O}$ & $\operatorname{ccc}([0]) \mathrm{CO}$ & InChl=1S/C4H9O2/c1-2-4(6)3-5/h4-5H,2-3H2,1H3 \\
\hline 680 & PC4H8OH-402 & $\mathrm{C}(\mathrm{O}[\mathrm{O}]) \mathrm{CCCO}$ & InChl=1S/C4H9O3/c5-3-1-2-4-7-6/h5H,1-4H2 \\
\hline 681 & C4H7OH-10OH-4O2 & $\mathrm{C}(\mathrm{O}[\mathrm{O}]) \mathrm{CCC}(\mathrm{OO}) \mathrm{O}$ & InChl=1S/C4H9O5/c5-4(9-7)2-1-3-8-6/h4-5,7H,1-3H2 \\
\hline 682 & C4H8O2-3 & CC1OC1C & InChl=1S/C4H8O/c1-3-4(2)5-3/h3-4H,1-2H3 \\
\hline 683 & $\mathrm{IC} 4 \mathrm{H} 8 \mathrm{OH}-10$ & $\mathrm{CC}(\mathrm{C}) \mathrm{C}([0]) \mathrm{O}$ & InChl=1S/C4H9O2/c1-3(2)4(5)6/h3-5H,1-2H3 \\
\hline 684 & TQC4H8OI & $\mathrm{CC}(\mathrm{C})(\mathrm{C}[\mathrm{O}]) 0 \mathrm{O}$ & InChl=1S/C4H9O3/c1-4(2,3-5)7-6/h6H,3H2,1-2H3 \\
\hline 685 & C4H7OH-4OOH-102 & $\mathrm{C}(\mathrm{OO}) \mathrm{CCC}(\mathrm{O}[\mathrm{O}]) \mathrm{O}$ & InChl=1S/C4H9O5/c5-4(9-7)2-1-3-8-6/h4-6H,1-3H2 \\
\hline 686 & IC4H8O & $\mathrm{CC1}(\mathrm{C}) \mathrm{CO} 1$ & InChl=1S/C4H8O/c1-4(2)3-5-4/h3H2,1-2H3 \\
\hline 687 & SC4H8OH-3O & $\mathrm{CC}([\mathrm{O}]) \mathrm{C}(\mathrm{O}) \mathrm{C}$ & InChl=1S/C4H9O2/c1-3(5)4(2)6/h3-5H,1-2H3 \\
\hline 688 & IQC4H8OT & $\mathrm{CC}(\mathrm{C})(\mathrm{COO})[\mathrm{O}]$ & InChl=1S/C4H9O3/c1-4(2,5)3-7-6/h6H,3H2,1-2H3 \\
\hline 689 & TQC4H7OHIQ-I & $\mathrm{CC}(\mathrm{C})(\mathrm{C}([\mathrm{O}]) 00) 00$ & $\operatorname{lnChl}=1 \mathrm{~S} / \mathrm{C} 4 \mathrm{H} 905 / \mathrm{c} 1-4(2,9-7) 3(5) 8-6 / \mathrm{h} 3,6-7 \mathrm{H}, 1-2 \mathrm{H} 3$ \\
\hline 690 & $\mathrm{IC} 3 \mathrm{H} 7 \mathrm{CHO}$ & $C C(C) C=0$ & InChl=1S/C4H8O/c1-4(2)3-5/h3-4H,1-2H3 \\
\hline 691 & $\mathrm{SC} 4 \mathrm{H} 8 \mathrm{OH}-2 \mathrm{O}$ & $\operatorname{cCC}(\mathrm{O})([\mathrm{O}]) \mathrm{C}$ & $\operatorname{lnChl}=1 \mathrm{~S} / \mathrm{C} 4 \mathrm{H} 9 \mathrm{O} 2 / \mathrm{c} 1-3-4(2,5) 6 / \mathrm{h} 5 \mathrm{H}, 3 \mathrm{H} 2,1-2 \mathrm{H} 3$ \\
\hline 692 & SQC4H8OP & $\operatorname{cCC}(00) C([0])$ & $\operatorname{lnChl}=1 \mathrm{~S} / \mathrm{C} 4 \mathrm{H} 9 \mathrm{O} 3 / \mathrm{c1}-2-4(3-5) 7-6 / \mathrm{h} 4,6 \mathrm{H}, 2-3 \mathrm{H} 2,1 \mathrm{H} 3$ \\
\hline 693 & TQC4H7OHIQ-P & {$[\mathrm{CH} 2] \mathrm{C}(\mathrm{C})(\mathrm{C}(\mathrm{O}) \mathrm{OO}) \mathrm{OO}$} & $\operatorname{lnChl}=1 \mathrm{~S} / \mathrm{C} 4 \mathrm{H} 905 / \mathrm{c1}-4(2,9-7) 3(5) 8-6 / \mathrm{h} 3,5-7 \mathrm{H}, 1 \mathrm{H} 2,2 \mathrm{H} 3$ \\
\hline 694 & $\mathrm{CC} 4 \mathrm{H} 8 \mathrm{O}$ & $\mathrm{C} 1 \mathrm{C}(\mathrm{C}) \mathrm{CO} 1$ & InChl=1S/C4H8O/c1-4-2-5-3-4/h4H,2-3H2,1H3 \\
\hline 695 & $\mathrm{IC} 4 \mathrm{H} 8 \mathrm{OH}-2 \mathrm{O}$ & $\mathrm{CC}(\mathrm{C})([\mathrm{O}]) \mathrm{CO}$ & InChl=1S/C4H9O2/c1-4(2,6)3-5/h5H,3H2,1-2H3 \\
\hline 696 & PQC4H8OS & $\operatorname{ccc}([0]) C(O O)$ & InChl=1S/C4H9O3/c1-2-4(5)3-7-6/h4,6H,2-3H2,1H3 \\
\hline 697 & IQC4H8OTQ-I & $\mathrm{CC}(\mathrm{COO})(\mathrm{COO})[\mathrm{O}]$ & InChl=15/C4H9O5/c1-4(5,2-8-6)3-9-7//h6-7H,2-3H2,1H3 \\
\hline 698 & NC3H7CHO & $\mathrm{CCCC}=0$ & InChl=1S/C4H8O/c1-2-3-4-5/h4H,2-3H2,1H3 \\
\hline 699 & TC4H8OH-O & $\mathrm{CC}(\mathrm{C})(\mathrm{O}) \mathrm{C}[\mathrm{O}]$ & $\operatorname{lnChl}=1 \mathrm{~S} / \mathrm{C} 4 \mathrm{H} 9 \mathrm{OO} / \mathrm{C1} 1-4(2,6) 3-5 / \mathrm{h} 6 \mathrm{H}, 3 \mathrm{H} 2,1-2 \mathrm{H} 3$ \\
\hline 700 & SQC4H8OS & $\mathrm{CC}(\mathrm{OO}) \mathrm{C}([\mathrm{O}]) \mathrm{C}$ & $\operatorname{lnChl}=15 / \mathrm{C} 4 \mathrm{H} 9 \mathrm{O} 3 / \mathrm{c} 1-3(5) 4(2) 7-6 / \mathrm{h} 3-4,6 \mathrm{H}, 1-2 \mathrm{H} 3$ \\
\hline 701 & IQC4H7OHTQ-P & {$[\mathrm{CH} 2] \mathrm{C}(\mathrm{COO})(\mathrm{COO}) \mathrm{O}$} & InChl=1S/C4H9O5/c1-4(5,2-8-6)3-9-7/h5-7H,1-3H2 \\
\hline 702 & $\mathrm{C} 2 \mathrm{H} 5 \mathrm{COCH} 3$ & $\operatorname{ccc}(C)=0$ & $\operatorname{lnChl}=1 \mathrm{~S} / \mathrm{C} 4 \mathrm{H} 8 \mathrm{O} / \mathrm{c} 1-3-4(2) 5 / \mathrm{h} 3 \mathrm{H} 2,1-2 \mathrm{H} 3$ \\
\hline 703 & $\mathrm{IC} 4 \mathrm{H} 8 \mathrm{OH}-3 \mathrm{O}$ & $\mathrm{C}([\mathrm{O}]) \mathrm{C}(\mathrm{C}) \mathrm{CO}$ & InChl=1S/C4H9O2/c1-4(2-5)3-6/h4-5H,2-3H2,1H3 \\
\hline 704 & PC4H8OH-102H & $\operatorname{cccc}(00) 0$ & InChl=1S/C4H1003/c1-2-3-4(5)7-6/h4-6H,2-3H2,1H3 \\
\hline 705 & $\mathrm{C} 4 \mathrm{H} 7 \mathrm{O} 2-1,3 \mathrm{OOH}$ & $\mathrm{CC}(\mathrm{OO}) \mathrm{C}([\mathrm{O}]) \mathrm{COO}$ & InChl=1S/C4H9O5/c1-3(9-7)4(5)2-8-6/h3-4,6-7H,2H2,1H3 \\
\hline 706 & PC4H8OH-3 & $\mathrm{C}[\mathrm{CH}] \mathrm{CCO}$ & InChl=1S/C4H9O/c1-2-3-4-5/h2,5H,3-4H2,1H3 \\
\hline 707 & SC4H8OH-4O & $\mathrm{C}([\mathrm{O}]) \mathrm{CC}(\mathrm{O}) \mathrm{C}$ & InChl=1S/C4H9O2/c1-4(6)2-3-5/h4,6H,2-3H2,1H3 \\
\hline 708 & РС448OH-2O2H & $\mathrm{CCC}(\mathrm{OO}) \mathrm{CO}$ & $\operatorname{lnChl}=1 \mathrm{~S} / \mathrm{C} 4 \mathrm{H} 1003 / \mathrm{c1}-2-4(3-5) 7-6 / \mathrm{h} 4-6 \mathrm{H}, 2-3 \mathrm{H} 2,1 \mathrm{H} 3$ \\
\hline 709 & C4D3OH1-20OJ & $\mathrm{OCC}(\mathrm{C}=\mathrm{C}) \mathrm{O}[\mathrm{O}]$ & $\operatorname{lnChl}=1 \mathrm{~S} / \mathrm{C} 4 \mathrm{H} 7 \mathrm{O} / \mathrm{c} 1-2-4(3-5) 7-6 / \mathrm{h} 2,4-5 \mathrm{H}, 1,3 \mathrm{H} 2$ \\
\hline 710 & PC4H8OH-2 & $\mathrm{CC}[\mathrm{CH}] \mathrm{CO}$ & InChl=1S/C4H9O/c1-2-3-4-5/h3,5H,2,4H2,1H3 \\
\hline 711 & PC4H8OH-3O & $\mathrm{CC}([\mathrm{O}]) \mathrm{CCO}$ & InChl=1S/C4H9O2/c1-4(6)2-3-5/h4-5H,2-3H2,1H3 \\
\hline 712 & SC4H8OH-102H & $\mathrm{CCC}(\mathrm{O}) \mathrm{COO}$ & $\operatorname{lnChl}=1 \mathrm{~S} / \mathrm{C} 4 \mathrm{H} 1003 / \mathrm{c1}-2-4(5) 3-7-6 / \mathrm{h} 4-6 \mathrm{H}, 2-3 \mathrm{H} 2,1 \mathrm{H} 3$ \\
\hline 713 & ovccccJvo & $\mathrm{O}=[\mathrm{C}] \mathrm{CCC}=\mathrm{O}$ & $\operatorname{lnChl}=1 \mathrm{~S} / \mathrm{C} 4 \mathrm{H} 5 \mathrm{O} 2 / \mathrm{c} 5-3-1-2-4-6 / \mathrm{h} 3 \mathrm{H}, 1-2 \mathrm{H} 2$ \\
\hline 714 & PC4H8OH-1 & $\mathrm{CCC}[\mathrm{CH}] \mathrm{O}$ & InChl=1S/C4H9O/c1-2-3-4-5/h4-5H,2-3H2,1H3 \\
\hline 715 & PC4H8OH-40 & $\mathrm{C}([0]) \mathrm{CCCO}$ & $\operatorname{lnChl}=1 \mathrm{~S} / \mathrm{C} 4 \mathrm{H} 9 \mathrm{OO} / \mathrm{c} 5-3-1-2-4-6 / \mathrm{h} 5 \mathrm{H}, 1-4 \mathrm{H} 2$ \\
\hline 716 & $\mathrm{IC4H8OH-COOH}$ & $\mathrm{CC}(\mathrm{C}) \mathrm{C}(\mathrm{OO}) \mathrm{O}$ & $\operatorname{lnChl}=1 \mathrm{~S} / \mathrm{C} 4 \mathrm{H} 10 \mathrm{OO} / \mathrm{c} 1-3(2) 4(5) 7-6 / \mathrm{h} 3-6 \mathrm{H}, 1-2 \mathrm{H} 3$ \\
\hline 717 & ovccccvo & $\mathrm{O}=\mathrm{CCCC}=\mathrm{O}$ & InChl=1S/C4H6O2/c5-3-1-2-4-6/h3-4H,1-2H2 \\
\hline 718 & ovccescVo & $\mathrm{O}=\mathrm{CCC}=\mathrm{C}[\mathrm{O}]$ & InChl=1S/C4H5O2/c5-3-1-2-4-6/h1,3-4H,2H2 \\
\hline 719 & C4Y14-2QJ & $\mathrm{O}=\mathrm{CCC}(\mathrm{C}=0) \mathrm{O}[0]$ & $\operatorname{lnChl}=1 \mathrm{~S} / \mathrm{C} 4 \mathrm{H} 504 / \mathrm{C5}-2-1-4(3-6) 8-7 / \mathrm{h} 2-4 \mathrm{H}, 1 \mathrm{H} 2$ \\
\hline 720 & C5H5-1 & $\mathrm{C} 1[\mathrm{C}]=\mathrm{CC}=\mathrm{C} 1$ & InChl=1S/C5H5/c1-2-4-5-3-1/h1-3H,4H2 \\
\hline 721 & A-CC5H10O & CC1COC1C & InChl=1S/C5H100/c1-4-3-6-5(4)2/h4-5H,3H2,1-2H3 \\
\hline 722 & $\mathrm{C} 5 \mathrm{H} 11 \mathrm{O} 2 \mathrm{H}-3$ & $\mathrm{CCC}(\mathrm{OO}) \mathrm{CC}$ & $\operatorname{lnChl}=1 \mathrm{~S} / \mathrm{C} 5 \mathrm{H} 12 \mathrm{O} 2 / \mathrm{c1}-3-5(4-2) 7-6 / \mathrm{h} 5-6 \mathrm{H}, 3-4 \mathrm{H} 2,1-2 \mathrm{H} 3$ \\
\hline 723 & MB203VO & $\operatorname{coc}(=0) C([0]) C(=0) C$ & $\operatorname{lnChl}=15 / \mathrm{C5} 5704 / \mathrm{c} 1-3(6) 4(7) 5(8) 9-2 / \mathrm{h} 4 \mathrm{H}, 1-2 \mathrm{H} 3$ \\
\hline 724 & $\mathrm{C} 5 \mathrm{H} 5-2$ & $\mathrm{C} 1 \mathrm{C}=[\mathrm{C}] \mathrm{C}=\mathrm{C} 1$ & InChl=1S/C5H5/c1-2-4-5-3-1/h1-2,5H,3H2 \\
\hline
\end{tabular}




\begin{tabular}{|c|c|c|c|}
\hline 725 & A-DC5H10O & CC1CCOC1 & InChl=1S/C5H10O/c1-5-2-3-6-4-5/h5H,2-4H2,1H3 \\
\hline 726 & AC5H11O2H & $\operatorname{ccc}(\mathrm{C}) \mathrm{COO}$ & InChl=15/C5H12O2/c1-3-5(2)4-7-6/h5-6H,3-4H2,1-2H3 \\
\hline 727 & MB204VO & $\operatorname{coC}(=0) C([0]) C C(=0)$ & InChl=1S/C5H7O4/c1-9-5(8)4(7)2-3-6/h3-4H,2H2,1H3 \\
\hline 728 & C\#CCVCCS & $\mathrm{C}=\mathrm{C}[\mathrm{CH}] \mathrm{CHC}$ & InChl=1S/C5H5/c1-3-5-4-2/h1,4-5H,2H2 \\
\hline 729 & B-CC5H10O & $\mathrm{CC1C(C)(C)01}$ & InChl=1S/C5H10O/c1-4-5(2,3)6-4/h4H,1-3H3 \\
\hline 730 & BC5H11O2H & $\operatorname{ccc}(C)(C) 00$ & InChl=1S/C5H12O2/c1-4-5(2,3)7-6/h6H,4H2,1-3H3 \\
\hline 731 & MBзомVO & $\mathrm{C}(=0) O C(=0) C C([0]) C$ & InChl=1S/C5H7O4/c1-4(7)2-5(8)9-3-6/h3-4H,2H2,1H3 \\
\hline 732 & $\mathrm{C} 5 \mathrm{H} 5$ & {$[\mathrm{CH}] 1 \mathrm{C}=\mathrm{CC}=\mathrm{C} 1$} & InChl=1S/C5H5/c1-2-4-5-3-1/h1-5H \\
\hline 733 & B-DC5H10O & $\mathrm{CC} 1(\mathrm{C}) \mathrm{CCO} 1$ & InChl=1S/C5H10O/c1-5(2)3-4-6-5/h3-4H2,1-2H3 \\
\hline 734 & $\mathrm{CC} 5 \mathrm{H} 11 \mathrm{O} 2 \mathrm{H}$ & $\mathrm{CC}(\mathrm{C}) \mathrm{C}(\mathrm{C}) \mathrm{OO}$ & InChl=15/C5H12O2/c1-4(2)5(3)7-6/h4-6H,1-3H3 \\
\hline 735 & MB3O2VO & $\operatorname{coc}(=0) C(=0) C([0]) C$ & InChl=1S/C5H7O4/c1-3(6)4(7)5(8)9-2/h3H,1-2H3 \\
\hline 736 & NC5D124-5R & $\mathrm{C}=\mathrm{C}=\mathrm{CC}=[\mathrm{CH}]$ & $\operatorname{lnChl}=1 \mathrm{~S} / \mathrm{C} 5 \mathrm{H} 5 / \mathrm{c} 1-3-5-4-2 / \mathrm{h} 1,3,5 \mathrm{H}, 2 \mathrm{H} 2$ \\
\hline 737 & C-DC5H10O & $\mathrm{CC}(\mathrm{C}) \mathrm{C1CO1}$ & InChl=1S/C5H100/c1-4(2)5-3-6-5/h4-5H,3H2,1-2H3 \\
\hline 738 & DC5H11O2H & $\mathrm{CC}(\mathrm{C}) \mathrm{CCOO}$ & InChl=15/C5H12O2/c1-5(2)3-4-7-6/h5-6H,3-4H2,1-2H3 \\
\hline 739 & MB304VO & $\operatorname{coc}(=0) \operatorname{cc}([0]) C(=0)$ & InChl=15/C5H7O4/c1-9-5(8)2-4(7)3-6/h3-4H,2H2,1H3 \\
\hline 740 & P1Y4E & $\mathrm{CHCCC}=\mathrm{C}$ & InChl=1S/C5H6/c1-3-5-4-2/h1,4H,2,5H2 \\
\hline 741 & NEO-C5H10O & CC1(C)COC1 & $\operatorname{lnChl}=15 / C 5 H 100 / c 1-5(2) 3-6-4-5 / h 3-4 H 2,1-2 \mathrm{H} 3$ \\
\hline 742 & NEOC5H11O2H & $\mathrm{CC}(\mathrm{C})(\mathrm{C}) \mathrm{COO}$ & InChl=1S/C5H12O2/c1-5(2,3)4-7-6/h6H,4H2,1-3H3 \\
\hline 743 & MB4O2VO & $\operatorname{coc}(=0) C(=0) \operatorname{cc}([0])$ & InChl=1S/C5H7O4/c1-9-5(8)4(7)2-3-6/h2-3H2,1H3 \\
\hline 744 & $\mathrm{C} 5 \mathrm{H} 6$ & $\mathrm{C} 1 \mathrm{C}=\mathrm{CC}=\mathrm{C} 1$ & InChl=15/C5H6/c1-2-4-5-3-1/h1-4H,5H2 \\
\hline 745 & $\mathrm{NC} 3 \mathrm{H} 7 \mathrm{COCH} 3$ & $\mathrm{CC}(=0) \mathrm{CCC}$ & InChl=1S/C5H100/c1-3-4-5(2)6/h3-4H2,1-2H3 \\
\hline 746 & C5Y135-1R & $\mathrm{C}(=0) \mathrm{CC}(=\mathrm{O}) \mathrm{C}[\mathrm{C}](=\mathrm{O})$ & InChl=1S/C5H503/c6-3-1-5(8)2-4-7/h3H,1-2H2 \\
\hline 747 & MB4O3VO & $\operatorname{coc}(=0) \operatorname{CC}(=0) C([0])$ & InChl=1S/C5H704/c1-9-5(8)2-4(7)3-6/h2-3H2,1H3 \\
\hline 748 & NC5D124 & $\mathrm{C}=\mathrm{C}=\mathrm{CC}=\mathrm{C}$ & $\operatorname{lnChl}=1 \mathrm{~S} / \mathrm{C} 5 \mathrm{H} 6 / \mathrm{c} 1-3-5-4-2 / \mathrm{h} 3,5 \mathrm{H}, 1-2 \mathrm{H} 2$ \\
\hline 749 & $\mathrm{C} 2 \mathrm{H} 5 \mathrm{COC} 2 \mathrm{H} 5$ & $\operatorname{CCC}(=0) C C$ & InChl=1S/C5H100/c1-3-5(6)4-2/h3-4H2,1-2H3 \\
\hline 750 & C5Y125-1R & $\mathrm{C}(=\mathrm{O}) \mathrm{CCC}(=\mathrm{O})[\mathrm{C}](=\mathrm{O})$ & InChl=1S/C5H5O3/c6-3-1-2-5(8)4-7/h3H,1-2H2 \\
\hline 751 & $\mathrm{OOCC}(\mathrm{VC}) \mathrm{C}(\mathrm{VO}) \mathrm{CO}$ & $\mathrm{OOCC}(=\mathrm{C}) \mathrm{C}(=\mathrm{O}) \mathrm{CO}$ & InChl=1S/C5H8O4/c1-4(3-9-8)5(7)2-6/h6,8H,1-3H2 \\
\hline 752 & B13DE2M-1J & $\mathrm{C}=\mathrm{CC}(=[\mathrm{CH}]) \mathrm{C}$ & $\operatorname{lnChl}=15 / C 5 H 7 / c 1-4-5(2) 3 / \mathrm{h} 2,4 \mathrm{H}, 1 \mathrm{H} 2,3 \mathrm{H} 3$ \\
\hline 753 & IC5Y3 & $\mathrm{CC}(=\mathrm{O}) \mathrm{C}(\mathrm{C}) \mathrm{C}$ & InChl=1S/C5H100/c1-4(2)5(3)6/h4H,1-3H3 \\
\hline 754 & C5Y125-5R & $C(=0) C(=0) C C[C](=0)$ & $\operatorname{lnChl}=1 \mathrm{~S} / \mathrm{C} 5 \mathrm{H} 5 \mathrm{O} / \mathrm{C} / \mathrm{c6}-3-1-2-5(8) 4-7 / \mathrm{h} 4 \mathrm{H}, 1-2 \mathrm{H} 2$ \\
\hline 755 & OVCC(COO)VCCO & OOCC $(=\mathrm{CCO}) \mathrm{C}=\mathrm{O}$ & InChl=1S/C5H8O4/c6-2-1-5(3-7)4-9-8/h1,3,6,8H,2,4H2 \\
\hline 756 & B13DE2M-4J & {$[\mathrm{CH}]=\mathrm{CC}(=\mathrm{C}) \mathrm{C}$} & $\operatorname{lnChl}=1 \mathrm{~S} / \mathrm{C5H} 7 / \mathrm{C1}-4-5(2) 3 / \mathrm{h} 1,4 \mathrm{H}, 2 \mathrm{H} 2,3 \mathrm{H} 3$ \\
\hline 757 & $\mathrm{TC} 4 \mathrm{H} 9 \mathrm{CHO}$ & $\mathrm{CC}(\mathrm{C})(\mathrm{C}) \mathrm{C}=\mathrm{O}$ & $\operatorname{lnChl}=1 \mathrm{~S} / \mathrm{C} 5 \mathrm{H} 100 / \mathrm{c} 1-5(2,3) 4-6 / \mathrm{h} 4 \mathrm{H}, 1-3 \mathrm{H} 3$ \\
\hline 758 & CPNKET2-3OR & $\mathrm{C} 1 \mathrm{CC}(=0) \mathrm{C}(=0) \mathrm{C} 1([0])$ & InChl=1S/C5H5O3/c6-3-1-2-4(7)5(3)8/h3H,1-2H2 \\
\hline 759 & BC5Y1OH4-30OH & $\mathrm{OCC}(\mathrm{C}(=\mathrm{C}) \mathrm{C}=0) 00$ & InChl=1S/C5H8O4/c1-4(2-6)5(3-7)9-8/h2,5,7-8H,1,3H2 \\
\hline 760 & C5H714-1 & {$[\mathrm{CH}]=\mathrm{CCC}=\mathrm{C}$} & $\operatorname{lnChl}=1 \mathrm{~S} / \mathrm{C} 5 \mathrm{H} 7 / \mathrm{c} 1-3-5-4-2 / \mathrm{h} 1,3-4 \mathrm{H}, 2,5 \mathrm{H} 2$ \\
\hline 761 & $\mathrm{C} 5 \mathrm{H} 10 \mathrm{OH} 14$ & $\mathrm{C}[\mathrm{CH}] \mathrm{CCCO}$ & InChl=1S/C5H110/c1-2-3-4-5-6/h2,6H,3-5H2,1H3 \\
\hline 762 & CPNKET2-4OR & $\mathrm{C} 1([0]) \mathrm{CC}(=\mathrm{O}) \mathrm{C}(=\mathrm{O}) \mathrm{C} 1$ & $\operatorname{lnChl}=1 \mathrm{~S} / \mathrm{C} 5 \mathrm{H} 5 \mathrm{O} / \mathrm{c} 6-3-1-4(7) 5(8) 2-3 / \mathrm{h} 3 \mathrm{H}, 1-2 \mathrm{H} 2$ \\
\hline 763 & MB2-MOOH & $\mathrm{C}(\mathrm{OO}) \mathrm{OC}(=\mathrm{O}) \mathrm{C}=\mathrm{CC}$ & $\operatorname{lnChl}=15 / C 5 H 804 / c 1-2-3-5(6) 8-4-9-7 / h 2-3,7 \mathrm{H}, 4 \mathrm{H} 2,1 \mathrm{H} 3$ \\
\hline 764 & B13DE2MJ & {$[\mathrm{CH} 2] \mathrm{C}(=\mathrm{C}) \mathrm{C}=\mathrm{C}$} & InChl=1S/C5H7/c1-4-5(2)3/h4H,1-3H2 \\
\hline 765 & C5H11O-1 & $\mathrm{ccccc}[0]$ & InChl=1S/C5H110/c1-2-3-4-5-6/h2-5H2,1H3 \\
\hline 766 & CPNKET3-2OR & $\mathrm{C} 1 \mathrm{CC}(=0) \mathrm{C}([0]) \mathrm{C} 1(=0)$ & $\operatorname{lnChl}=1 \mathrm{~S} / \mathrm{C} 5 \mathrm{H} 5 \mathrm{O} / \mathrm{c} 6-3-1-2-4(7) 5(3) 8 / \mathrm{h} 5 \mathrm{H}, 1-2 \mathrm{H} 2$ \\
\hline 767 & MB2-40OH & $\operatorname{coc}(=0) \mathrm{C}=\mathrm{CC}(\mathrm{OO})$ & $\operatorname{lnChl}=15 / C 5 H 804 / c 1-8-5(6) 3-2-4-9-7 / h 2-3,7 \mathrm{H}, 4 \mathrm{H} 2,1 \mathrm{H} 3$ \\
\hline 768 & C\#CCJCC & $\mathrm{CHC}[\mathrm{CH}] \mathrm{CC}$ & InChl=1S/C5H7/c1-3-5-4-2/h1,5H,4H2,2H3 \\
\hline 769 & $\mathrm{C} 5 \mathrm{H} 10 \mathrm{OH} 13$ & $\mathrm{CC}[\mathrm{CH}] \mathrm{CCO}$ & InChl=1S/C5H11O/c1-2-3-4-5-6/h3,6H,2,4-5H2,1H3 \\
\hline 770 & CPNKET3-4OR & $\mathrm{C} 1([0]) \mathrm{CC}(=0) \mathrm{CC} 1(=0)$ & InChl=15/C5H5O3/c6-3-1-4(7)5(8)2-3/h4H,1-2H2 \\
\hline 771 & MB3-MOOH & $\mathrm{C}(\mathrm{OO}) \mathrm{OC}(=\mathrm{O}) \mathrm{CC}=\mathrm{C}$ & InChl=1S/C5H8O4/c1-2-3-5(6)8-4-9-7/h2,7H,1,3-4H2 \\
\hline 772 & P13DE1J & {$[\mathrm{CH}]=\mathrm{CC}=\mathrm{CC}$} & $\operatorname{lnChl}=1 \mathrm{~S} / \mathrm{C} 5 \mathrm{H} 7 / \mathrm{c} 1-3-5-4-2 / \mathrm{h} 1,3-5 \mathrm{H}, 2 \mathrm{H} 3$ \\
\hline 773 & $\mathrm{C} 5 \mathrm{H} 10 \mathrm{OH} 12$ & $\mathrm{CCC}[\mathrm{CH}] \mathrm{CO}$ & InChl=1S/C5H110/c1-2-3-4-5-6/h4,6H,2-3,5H2,1H3 \\
\hline 774 & CPND2-402R & $\mathrm{C} 1=\mathrm{CC}(=0) \mathrm{CC} 1(\mathrm{O}[\mathrm{O}])$ & InChl=1S/C5H5O3/c6-4-1-2-5(3-4)8-7/h1-2,5H,3H2 \\
\hline 775 & MB3-2OOH & $\operatorname{coc}(=0) C(O O) C=C$ & $\operatorname{lnChl}=15 / C 5 H 804 / c 1-3-4(9-7) 5(6) 8-2 / h 3-4,7 \mathrm{H}, 1 \mathrm{H} 2,2 \mathrm{H} 3$ \\
\hline 776 & $\mathrm{C} 5 \mathrm{H} 10 \mathrm{OH} 11$ & $\mathrm{CCCC}[\mathrm{CH}] \mathrm{O}$ & InChl=15/C5H110/c1-2-3-4-5-6/h5-6H,2-4H2,1H3 \\
\hline 777 & CPND2-502R & $\mathrm{C} 1=\mathrm{CC}(=0) \mathrm{C}(\mathrm{O}[\mathrm{O}]) \mathrm{C} 1$ & $\mathrm{InChl}=1 \mathrm{~S} / \mathrm{C} 5 \mathrm{H} 5 \mathrm{O} / \mathrm{c} 6-4-2-1-3-5(4) 8-7 / \mathrm{h} 1-2,5 \mathrm{H}, 3 \mathrm{H} 2$ \\
\hline 778 & IC5Y4OH2-302 & {$[\mathrm{O}] \mathrm{OC}(\mathrm{C}(\mathrm{O})(\mathrm{C}) \mathrm{C}) \mathrm{C}=\mathrm{O}$} & InChl=1S/C5H9O4/c1-5(2,7)4(3-6)9-8/h3-4,7H,1-2H3 \\
\hline 779 & CYC5H71-3 & $\mathrm{C} 1=\mathrm{C}[\mathrm{CH}] \mathrm{CC} 1$ & $\operatorname{lnChl}=15 / C 5 H 7 / c 1-2-4-5-3-1 / h 1-3 H, 4-5 H 2$ \\
\hline 780 & $\mathrm{C} 5 \mathrm{H} 10 \mathrm{OH} 15$ & {$[\mathrm{CH} 2] \mathrm{CCCCO}$} & $\operatorname{lnChl}=1 \mathrm{~S} / \mathrm{C} 5 \mathrm{H} 110 / \mathrm{c} 1-2-3-4-5-6 / \mathrm{h} 6 \mathrm{H}, 1-5 \mathrm{H} 2$ \\
\hline 781 & CPND2OOH4-5R & $\mathrm{C} 1=\mathrm{CC}(=\mathrm{O})[\mathrm{CH}] \mathrm{C} 1(\mathrm{OO})$ & InChl=1S/C5H5O3/c6-4-1-2-5(3-4)8-7/h1-3,5,7H \\
\hline 782 & IC5Y4OH3-2O2 & {$[0] \mathrm{OC}(\mathrm{C}(\mathrm{C}=\mathrm{O}) \mathrm{O})(\mathrm{C}) \mathrm{C}$} & InChl=1S/C5H9O4/c1-5(2,9-8)4(7)3-6/h3-4,7H,1-2H3 \\
\hline 783 & CYC5H71-4 & $\mathrm{C} 1=\mathrm{CC}[\mathrm{CH}] \mathrm{C} 1$ & $\operatorname{lnChl}=1 \mathrm{~S} / \mathrm{C} 5 \mathrm{H} 7 / \mathrm{cc} 1-2-4-5-3-1 / \mathrm{h} 1-2,5 \mathrm{H}, 3-4 \mathrm{H} 2$ \\
\hline 784 & $\mathrm{C} 5 \mathrm{H} 11 \mathrm{O}-2$ & $\operatorname{cccc}([0]) \mathrm{C}$ & InChl=1S/C5H110/c1-3-4-5(2)6/h5H,3-4H2,1-2H3 \\
\hline 785 & CPND2OOH5-4R & $\mathrm{C} 1=\mathrm{CC}(=\mathrm{O}) \mathrm{C}(\mathrm{OO})[\mathrm{CH}] 1$ & InChl=1S/C5H503/c6-4-2-1-3-5(4)8-7/h1-3,5,7H \\
\hline 786 & MBMOO & $\mathrm{C}(\mathrm{O}[\mathrm{O}]) \mathrm{OC}(=\mathrm{O}) \mathrm{CCC}$ & InChl=1S/C5H9O4/c1-2-3-5(6)8-4-9-7/h2-4H2,1H3 \\
\hline 787 & CVCCVCCJ & $\mathrm{C}=\mathrm{CC}=\mathrm{C}[\mathrm{CH} 2]$ & InChl=1S/C5H7/c1-3-5-4-2/h3-5H,1-2H2 \\
\hline 788 & C5H11O-3 & $\operatorname{CCC}([0]) \mathrm{CC}$ & InChl=15/C5H110/c1-3-5(6)4-2/h5H,3-4H2,1-2H3 \\
\hline 789 & C5Y125 & $\mathrm{C}(=0) \mathrm{C}(=0) \mathrm{CCC}(=0)$ & InChl=15/C5H6O3/c6-3-1-2-5(8)4-7/h3-4H,1-2H2 \\
\hline 790 & MB200 & $\operatorname{coc}(=0) C(O[O]) C C$ & InChl=1S/C5H9O4/c1-3-4(9-7)5(6)8-2/h4H,3H2,1-2H3 \\
\hline 791 & $\mathrm{CC} 3 \mathrm{H} 5-\mathrm{C} 2 \mathrm{H} 3$ & $\mathrm{C}=\mathrm{CC} 1 \mathrm{CC} 1$ & $\operatorname{lnChl}=1 \mathrm{~S} / \mathrm{C} 5 \mathrm{H} 8 / \mathrm{c} 1-2-5-3-4-5 / \mathrm{h} 2,5 \mathrm{H}, 1,3-4 \mathrm{H} 2$ \\
\hline 792 & DC5H10OH-C & $\mathrm{CC}(\mathrm{C})[\mathrm{CH}] \mathrm{CO}$ & InChl=1S/C5H11O/c1-5(2)3-4-6/h3,5-6H,4H2,1-2H3 \\
\hline 793 & C5Y135 & $\mathrm{C}(=0) \mathrm{CC}(=0) \mathrm{CC}(=0)$ & $\operatorname{lnChl}=15 / C 5 H 6 O 3 / c 6-3-1-5(8) 2-4-7 / h 3-4 \mathrm{H}, 1-2 \mathrm{H} 2$ \\
\hline 794 & MBзоO & $\operatorname{coc}(=0) \operatorname{cc}(0[0]) C$ & $\operatorname{lnChl}=1 \mathrm{~S} / \mathrm{C} 5 \mathrm{H} 904 / \mathrm{c} 1-4(9-7) 3-5(6) 8-2 / \mathrm{h} 4 \mathrm{H}, 3 \mathrm{H} 2,1-2 \mathrm{H} 3$ \\
\hline 795 & C5H81-3 & $\mathrm{C}=\mathrm{CC}=\mathrm{CC}$ & InChl=1S/C5H8/c1-3-5-4-2/h3-5H,1H2,2H3 \\
\hline 796 & DC5H10OH-D & $\mathrm{CC}(\mathrm{C}) \mathrm{C}[\mathrm{CH}] \mathrm{O}$ & InChl=1S/C5H110/c1-5(2)3-4-6/h4-6H,3H2,1-2H3 \\
\hline 797 & CPND3-2OOH & $\mathrm{C} 1 \mathrm{C}(=\mathrm{O}) \mathrm{C}(\mathrm{OO}) \mathrm{C}=\mathrm{C} 1$ & InChl=1S/C5H6O3/c6-4-2-1-3-5(4)8-7/h1,3,5,7H,2H2 \\
\hline 798 & MB400 & $\operatorname{coc}(=0) \operatorname{ccc}(0[0])$ & InChl=15/C5H9O4/c1-8-5(6)3-2-4-9-7/h2-4H2,1H3 \\
\hline 799 & B13DE2M & $\mathrm{CC}(=\mathrm{C}) \mathrm{C}=\mathrm{C}$ & $\operatorname{lnChl}=1 \mathrm{~S} / \mathrm{C} 5 \mathrm{H} 8 / \mathrm{c1}-4-5(2) 3 / \mathrm{h} 4 \mathrm{H}, 1-2 \mathrm{H} 2,3 \mathrm{H} 3$ \\
\hline 800 & DC5H10OH-A & {$[\mathrm{CH} 2] \mathrm{C}(\mathrm{C}) \mathrm{CCO}$} & InChl=1S/C5H110/c1-5(2)3-4-6/h5-6H,1,3-4H2,2H3 \\
\hline 801 & CPND2-50OH & $\mathrm{C} 1(\mathrm{OO}) \mathrm{C}(=0) \mathrm{C}=\mathrm{CC} 1$ & InChl=1S/C5H6O3/c6-4-2-1-3-5(4)8-7/h1-2,5,7H,3H2 \\
\hline 802 & MBMOOH2J & $\mathrm{C}(\mathrm{OO}) \mathrm{OC}(=\mathrm{O})[\mathrm{CH}] \mathrm{CC}$ & InChl=15/C5H9O4/c1-2-3-5(6)8-4-9-7/h3,7H,2,4H2,1H3 \\
\hline 803 & DC5H110 & $\mathrm{CC}(\mathrm{C}) \mathrm{CC}[\mathrm{O}]$ & InChl=15/C5H110/c1-5(2)3-4-6/h5H,3-4H2,1-2H3 \\
\hline 804 & CPND2-4OOH & $\mathrm{C} 1 \mathrm{C}(=\mathrm{O}) \mathrm{C}=\mathrm{CC} 1(\mathrm{OO})$ & $\operatorname{lnChl}=1 \mathrm{~S} / \mathrm{C} 5 \mathrm{H} 603 / \mathrm{c} 6-4-1-2-5(3-4) 8-7 / \mathrm{h} 1-2,5,7 \mathrm{H}, 3 \mathrm{H} 2$ \\
\hline 805 & MBMOOHЗЈ & $\mathrm{C}(\mathrm{OO}) \mathrm{OC}(=\mathrm{O}) \mathrm{C}[\mathrm{CH}] \mathrm{C}$ & $\operatorname{lnChl}=15 / C 5 H 9 O 4 / c 1-2-3-5(6) 8-4-9-7 / h 2,7 \mathrm{H}, 3-4 \mathrm{H} 2,1 \mathrm{H} 3$ \\
\hline 806 & P1Y & $\mathrm{CHCCCC}$ & $\operatorname{lnChl}=1 \mathrm{~S} / \mathrm{C} 5 \mathrm{H} 8 / \mathrm{c} 1-3-5-4-2 / \mathrm{h} 1 \mathrm{H}, 4-5 \mathrm{H} 2,2 \mathrm{H} 3$ \\
\hline 807 & DC5H10OH-B & $\mathrm{C}[\mathrm{C}](\mathrm{C}) \mathrm{CCO}$ & InChl=1S/C5H110/c1-5(2)3-4-6/h6H,3-4H2,1-2H3 \\
\hline 808 & BC5Y1OH4-3OJ & {$[\mathrm{O}] \mathrm{C}(\mathrm{C}(=\mathrm{C}) \mathrm{C}=\mathrm{O}) \mathrm{CO}$} & InChl=1S/C5H7O3/c1-4(2-6)5(8)3-7/h2,5,7H,1,3H2 \\
\hline 809 & MB2OOHMJ & {$[\mathrm{CH} 2] \mathrm{OC}(=\mathrm{O}) \mathrm{C}(\mathrm{OO}) \mathrm{CC}$} & $\operatorname{lnChl}=15 / C 5 H 9 O 4 / c 1-3-4(9-7) 5(6) 8-2 / h 4,7 \mathrm{H}, 2-3 \mathrm{H} 2,1 \mathrm{H} 3$ \\
\hline 810 & $\mathrm{C} 5 \mathrm{H} 10 \mathrm{OH}-2 \mathrm{~A}$ & $\mathrm{CCC}(\mathrm{C})[\mathrm{CH}] \mathrm{O}$ & InChl=1S/C5H110/c1-3-5(2)4-6/h4-6H,3H2,1-2H3 \\
\hline 811 & $\mathrm{C} 4 \mathrm{H} 6 \mathrm{CHO} 2-5 \mathrm{OOH} 4 \mathrm{~J}$ & $\mathrm{OOC}[\mathrm{CH}] \mathrm{C}=\mathrm{CC}=\mathrm{O}$ & $\operatorname{lnChl}=15 / C 5 H 703 / C 6-4-2-1-3-5-8-7 / h 1-4,7 \mathrm{H}, 5 \mathrm{H} 2$ \\
\hline 812 & MB2OOH3J & $\mathrm{COC}(=\mathrm{O}) \mathrm{C}(\mathrm{OO})[\mathrm{CH}] \mathrm{C}$ & InChl=1S/C5H9O4/c1-3-4(9-7)5(6)8-2/h3-4,7H,1-2H3 \\
\hline 813 & P2E4CAR & $\mathrm{C} / \mathrm{C}=\mathrm{C} /[\mathrm{C}] \mathrm{C}$ & InChl=1S/C5H8/c1-3-5-4-2/h3,5H,1-2H3 \\
\hline 814 & AC5H10OH & $\mathrm{CC}[\mathrm{C}](\mathrm{C}) \mathrm{CO}$ & InChl=1S/C5H110/c1-3-5(2)4-6/h6H,3-4H2,1-2H3 \\
\hline 815 & $\mathrm{C} 4 \mathrm{H} 6 \mathrm{CHO}-5 \mathrm{O} 2$ & {$[\mathrm{O}] \mathrm{OCCC}=\mathrm{CC}=\mathrm{O}$} & $\operatorname{lnChl}=1 \mathrm{~S} / \mathrm{C} 5 \mathrm{H} 703 / \mathrm{c6} 6-4-2-1-3-5-8-7 / \mathrm{h} 1-2,4 \mathrm{H}, 3,5 \mathrm{H} 2$ \\
\hline
\end{tabular}




\begin{tabular}{|c|c|c|c|}
\hline 816 & $\mathrm{MB2OOH} 4 \mathrm{~J}$ & $\operatorname{COC}(=\mathrm{O}) \mathrm{C}(\mathrm{OO}) \mathrm{C}[\mathrm{CH} 2]$ & InChl=15/C5H9O4/c1-3-4(9-7)5(6)8-2/h4,7H,1,3H2,2H3 \\
\hline 817 & $\mathrm{C} 5 \mathrm{H} 10 \mathrm{OH}-2 \mathrm{C}$ & $\mathrm{C}[\mathrm{CH}] \mathrm{C}(\mathrm{C}) \mathrm{CO}$ & InChl=1S/C5H110/c1-3-5(2)4-6/h3,5-6H,4H2,1-2H3 \\
\hline 818 & CPN-2O2R & $\mathrm{C} 1 \mathrm{CC}(=\mathrm{O}) \mathrm{C}(\mathrm{O}[\mathrm{O}]) \mathrm{C} 1$ & InChl=1S/C5H703/c6-4-2-1-3-5(4)8-7/h5H,1-3H2 \\
\hline 819 & МВзОонМJ & {$[\mathrm{CH} 2] \mathrm{OC}(=0) \mathrm{CC}(\mathrm{OO}) \mathrm{C}$} & $\mathrm{InChl}=1 \mathrm{~S} / \mathrm{C} 5 \mathrm{H} 9 \mathrm{O} 4 / \mathrm{c1}-4(9-7) 3-5(6) 8-2 / \mathrm{h} 4,7 \mathrm{H}, 2-3 \mathrm{H} 2,1 \mathrm{H} 3$ \\
\hline 820 & CYC5H8 & $\mathrm{C} 1=\mathrm{CCCC} 1$ & $\operatorname{lnChl}=15 / \mathrm{C5} H 8 / \mathrm{c} 1-2-4-5-3-1 / \mathrm{h} 1-2 \mathrm{H}, 3-5 \mathrm{H} 2$ \\
\hline 821 & $\mathrm{C} 5 \mathrm{H} 10 \mathrm{OH}-2 \mathrm{D}$ & {$[\mathrm{CH} 2] \mathrm{CC}(\mathrm{C}) \mathrm{CO}$} & $\operatorname{lnChl}=1 \mathrm{~S} / \mathrm{C} 5 \mathrm{H} 11 \mathrm{O} / \mathrm{c} 1-3-5(2) 4-6 / \mathrm{h} 5-6 \mathrm{H}, 1,3-4 \mathrm{H} 2,2 \mathrm{H} 3$ \\
\hline 822 & CPN-302R & $\mathrm{C} 1 \mathrm{CC}(=0) \mathrm{CC} 1(\mathrm{O}[\mathrm{O}])$ & InChl=1S/C5H7O3/c6-4-1-2-5(3-4)8-7/h5H,1-3H2 \\
\hline 823 & $\mathrm{MB} 3 \mathrm{OOH} 2 \mathrm{~J}$ & $\mathrm{COC}(=\mathrm{O})[\mathrm{CH}] \mathrm{C}(\mathrm{OO}) \mathrm{C}$ & $\mathrm{InChl}=1 \mathrm{~S} / \mathrm{C} 5 \mathrm{H} 904 / \mathrm{c} 1-4(9-7) 3-5(6) 8-2 / \mathrm{h} 3-4,7 \mathrm{H}, 1-2 \mathrm{H} 3$ \\
\hline 824 & C5H8D14 & $\mathrm{C}=\mathrm{CCC}=\mathrm{C}$ & InChl=1S/C5H8/c1-3-5-4-2/h3-4H,1-2,5H2 \\
\hline 825 & $\mathrm{C} 5 \mathrm{H} 10 \mathrm{OH}-2 \mathrm{E}$ & $\mathrm{CCC}([\mathrm{CH} 2]) \mathrm{CO}$ & $\operatorname{lnChl}=1 \mathrm{~S} / \mathrm{C} 5 \mathrm{H} 11 \mathrm{O} / \mathrm{c} 1-3-5(2) 4-6 / \mathrm{h} 5-6 \mathrm{H}, 2-4 \mathrm{H} 2,1 \mathrm{H} 3$ \\
\hline 826 & CPNOOH2-3R & $\mathrm{C} 1 \mathrm{CC}(=0) \mathrm{C}(\mathrm{OO})[\mathrm{CH}] 1$ & InChl=1S/C5H703/c6-4-2-1-3-5(4)8-7/h3,5,7H,1-2H2 \\
\hline 827 & $\mathrm{MB} 3 \mathrm{OOH} 4 \mathrm{~J}$ & $\mathrm{COC}(=\mathrm{O}) \mathrm{CC}(\mathrm{OO})[\mathrm{CH} 2]$ & InChl=15/C5H9O4/c1-4(9-7)3-5(6)8-2/h4,7H,1,3H2,2H3 \\
\hline 828 & C5H91-3 & $\mathrm{CC}[\mathrm{CH}] \mathrm{C}=\mathrm{C}$ & InChl=1S/C5H9/c1-3-5-4-2/h3,5H,1,4H2,2H3 \\
\hline 829 & $\mathrm{C} 5 \mathrm{H} 10 \mathrm{OH} 23$ & $\mathrm{CC}[\mathrm{CH}] \mathrm{C}(\mathrm{C}) \mathrm{O}$ & InChl=1S/C5H110/c1-3-4-5(2)6/h4-6H,3H2,1-2H3 \\
\hline 830 & CPNOOH2-4R & {$[\mathrm{CH}] 1 \mathrm{CC}(=\mathrm{O}) \mathrm{C}(\mathrm{OO}) \mathrm{C} 1$} & $\mathrm{InChl}=1 \mathrm{~S} / \mathrm{C} 5 \mathrm{H} 703 / \mathrm{c} 6-4-2-1-3-5(4) 8-7 / \mathrm{h} 1,5,7 \mathrm{H}, 2-3 \mathrm{H} 2$ \\
\hline 831 & MB4OOH2J & $\mathrm{COC}(=\mathrm{O})[\mathrm{CH}] \mathrm{CC}(\mathrm{OO})$ & InChl=15/C5H9O4/c1-8-5(6)3-2-4-9-7/h3,7H,2,4H2,11H3 \\
\hline 832 & C5H91-4 & $\mathrm{C}[\mathrm{CH}] \mathrm{CC}=\mathrm{C}$ & $\operatorname{lnChl}=15 / C 5 H 9 / c 1-3-5-4-2 / h 3-4 \mathrm{H}, 1,5 \mathrm{H} 2,2 \mathrm{H} 3$ \\
\hline 833 & AC5H11O & $\mathrm{CCC}(\mathrm{C}) \mathrm{C}[\mathrm{O}]$ & $\operatorname{lnChl}=1 \mathrm{~S} / \mathrm{C} 5 \mathrm{H} 110 / \mathrm{c} 1-3-5(2) 4-6 / \mathrm{h} 5 \mathrm{H}, 3-4 \mathrm{H} 2,1-2 \mathrm{H} 3$ \\
\hline 834 & CPNOOH2-5R & $\mathrm{C} 1[\mathrm{CH}] \mathrm{C}(=0) \mathrm{C}(\mathrm{OO}) \mathrm{C} 1$ & $\mathrm{InChl}=1 \mathrm{~S} / \mathrm{C} 5 \mathrm{H} 703 / \mathrm{c} 6-4-2-1-3-5(4) 8-7 / \mathrm{h} 2,5,7 \mathrm{H}, 1,3 \mathrm{H} 2$ \\
\hline 835 & MB4OOH3J & $\mathrm{COC}(=\mathrm{O}) \mathrm{C}[\mathrm{CH}] \mathrm{C}(\mathrm{OO})$ & InChl=15/C5H9O4/c1-8-5(6)3-2-4-9-7/h2,7H,3-4H2,1H3 \\
\hline 836 & $\mathrm{C} 5 \mathrm{H} 92-4$ & $\mathrm{C}[\mathrm{CH}] \mathrm{C}=\mathrm{CC}$ & $\operatorname{lnChl}=15 / C 5 H 9 / c 1-3-5-4-2 / h 3-5 \mathrm{H}, 1-2 \mathrm{H} 3$ \\
\hline 837 & BC5H11O & $\operatorname{ccc}(C)(C)[0]$ & $\mathrm{InChl}=1 \mathrm{~S} / \mathrm{C} 5 \mathrm{H} 11 \mathrm{O} / \mathrm{c1} 1-4-5(2,3) 6 / \mathrm{h} 4 \mathrm{H} 2,1-3 \mathrm{H} 3$ \\
\hline 838 & CPNOOH3-2R & $\mathrm{C} 1 \mathrm{CC}(=0)[\mathrm{CH}] \mathrm{C} 1(\mathrm{OO})$ & $\operatorname{lnChl}=1 \mathrm{~S} / \mathrm{C} 5 \mathrm{H} 703 / \mathrm{c6}-4-1-2-5(3-4) 8-7 / \mathrm{h} 3,5,7 \mathrm{H}, 1-2 \mathrm{H} 2$ \\
\hline 839 & CPTQ2QJ & $\mathrm{C} 1 \mathrm{C}(\mathrm{OO}) \mathrm{C}(\mathrm{O}[\mathrm{O}]) \mathrm{CC} 1$ & $\mathrm{InChl}=1 \mathrm{~S} / \mathrm{C} 5 \mathrm{H} 904 / \mathrm{c6}-8-4-2-1-3-5(4) 9-7 / \mathrm{h} 4-6 \mathrm{H}, 1-3 \mathrm{H} 2$ \\
\hline 840 & C5H91-5 & {$[\mathrm{CH} 2] \mathrm{CCC}=\mathrm{C}$} & InChl=15/C5H9/c1-3-5-4-2/h3H,1-2,4-5H2 \\
\hline 841 & CC5H11O & $\mathrm{CC}(\mathrm{C}) \mathrm{C}(\mathrm{C})[\mathrm{O}]$ & InChl=1S/C5H11O/c1-4(2)5(3)6/h4-5H,1-3H3 \\
\hline 842 & CPNOOH3-4R & {$[\mathrm{CH}] 1 \mathrm{CC}(=0) \mathrm{CC} 1(\mathrm{OO})$} & $\mathrm{InChl}=1 \mathrm{~S} / \mathrm{C} 5 \mathrm{H} 7 \mathrm{O} / \mathrm{c} 6-4-1-2-5(3-4) 8-7 / \mathrm{h} 2,5,7 \mathrm{H}, 1,3 \mathrm{H} 2$ \\
\hline 843 & CPTQ3QJ & $\mathrm{C} 1 \mathrm{C}(\mathrm{OO}) \mathrm{CC}(\mathrm{O}[\mathrm{O}]) \mathrm{C} 1$ & $\mathrm{InChl}=1 \mathrm{~S} / \mathrm{C5H} 9 \mathrm{OO} / \mathrm{c} 6-8-4-1-2-5(3-4) 9-7 / \mathrm{h} 4-6 \mathrm{H}, 1-3 \mathrm{H} 2$ \\
\hline 844 & C5H92-5 & {$[\mathrm{CH} 2] \mathrm{CC}=\mathrm{CC}$} & $\operatorname{lnChl}=1 \mathrm{~S} / \mathrm{C} 5 \mathrm{H} 9 / \mathrm{c1}-3-5-4-2 / \mathrm{h} 4-5 \mathrm{H}, 1,3 \mathrm{H} 2,2 \mathrm{H} 3$ \\
\hline 845 & BC5H10OH & $\mathrm{C}[\mathrm{CH}] \mathrm{C}(\mathrm{C})(\mathrm{C}) \mathrm{O}$ & InChl=1S/C5H11O/c1-4-5(2,3)6/h4,6H,1-3H3 \\
\hline 846 & CPNOOH3-5R & $\mathrm{C} 1[\mathrm{CH}] \mathrm{C}(=0) \mathrm{CC} 1(\mathrm{OO})$ & $\mathrm{InChl}=1 \mathrm{~S} / \mathrm{C} 5 \mathrm{H} 703 / \mathrm{c} 6-4-1-2-5(3-4) 8-7 / \mathrm{h} 1,5,7 \mathrm{H}, 2-3 \mathrm{H} 2$ \\
\hline 847 & CPT2Q4Q1J & $\mathrm{OOC} 1[\mathrm{CH}] \mathrm{CC}(\mathrm{C} 1) \mathrm{OO}$ & InChl=1S/C5H9O4/c6-8-4-1-2-5(3-4)9-7/h1,4-7H,2-3H2 \\
\hline 848 & AC5H9-C & $\mathrm{CC}(=\mathrm{C})[\mathrm{CH}] \mathrm{C}$ & $\operatorname{lnChl}=1 \mathrm{~S} / \mathrm{CSH} / \mathrm{c} 1-4-5(2) 3 / \mathrm{h} 4 \mathrm{H}, 2 \mathrm{H} 2,1,3 \mathrm{H} 3$ \\
\hline 849 & $\mathrm{CC} 5 \mathrm{H} 10 \mathrm{OH}$ & $\mathrm{CC}(\mathrm{C}) \mathrm{C}([\mathrm{CH} 2]) \mathrm{O}$ & InChl=1S/C5H110/c1-4(2)5(3)6/h4-6H,3H2,1-2H3 \\
\hline 850 & MBMJVO & {$[\mathrm{C}](=0) \mathrm{OC}(=0) \mathrm{CCC}$} & InChl=1S/C5H7O3/c1-2-3-5(7)8-4-6/h2-3H2,1H3 \\
\hline 851 & $\mathrm{MBMOOH}$ & $\mathrm{C}(\mathrm{OO}) \mathrm{OC}(=\mathrm{O}) \mathrm{CCC}$ & $\operatorname{lnChl}=1 \mathrm{~S} / \mathrm{C5} \mathrm{H} 1004 / \mathrm{c1}-2-3-5(6) 8-4-9-7 / \mathrm{h} 7 \mathrm{H}, 2-4 \mathrm{H} 2,1 \mathrm{H} 3$ \\
\hline 852 & AC5H9-D & $\mathrm{CC}(=\mathrm{C}) \mathrm{C}[\mathrm{CH} 2]$ & InChl=1S/C5H9/c1-4-5(2)3/h1-2,4H2,3H3 \\
\hline 853 & NEOC5H11O & $\mathrm{CC}(\mathrm{C})(\mathrm{C}) \mathrm{C}[\mathrm{O}]$ & InChl=1S/C5H11O/c1-5(2,3)4-6/h4H2,1-3H3 \\
\hline 854 & MB4JVO & $\operatorname{coc}(=0) \operatorname{cc}[C](=0)$ & InChl=15/C5H7O3/c1-8-5(7)3-2-4-6/h2-3H2,1H3 \\
\hline 855 & $\mathrm{MB} 2 \mathrm{OOH}$ & $\mathrm{COC}(=\mathrm{O}) \mathrm{C}(\mathrm{OO}) \mathrm{CC}$ & $\operatorname{lnChl}=15 / \mathrm{C} 5 \mathrm{H} 10 \mathrm{OO} / \mathrm{c} 1-3-4(9-7) 5(6) 8-2 / \mathrm{h} 4,7 \mathrm{H}, 3 \mathrm{H} 2,1-2 \mathrm{H} 3$ \\
\hline 856 & CC5H9-B & $\mathrm{C}=\mathrm{C}[\mathrm{C}](\mathrm{C}) \mathrm{C}$ & InChl=1S/C5H9/c1-4-5(2)3/h4H,1H2,2-3H3 \\
\hline 857 & NC5H11OH & CCccCO & $\operatorname{lnChl}=1 \mathrm{~S} / \mathrm{C5} \mathrm{H} 12 \mathrm{O} / \mathrm{c1}-2-3-4-5-6 / \mathrm{h} 6 \mathrm{H}, 2-5 \mathrm{H} 2,1 \mathrm{H} 3$ \\
\hline 858 & IC5D2Y4-10OH & $\mathrm{CC}(=\mathrm{CC}=0) \mathrm{COO}$ & InChl=1S/C5H8O3/c1-5(2-3-6)4-8-7/h2-3,7H,4H2,1H3 \\
\hline 859 & $\mathrm{MB} 3 \mathrm{OOH}$ & $\operatorname{COC}(=0) \mathrm{CC}(\mathrm{OO}) \mathrm{C}$ & $\mathrm{InChl}=1 \mathrm{~S} / \mathrm{C} 5 \mathrm{H} 10 \mathrm{OO} / \mathrm{c} 1-4(9-7) 3-5(6) 8-2 / \mathrm{h} 4,7 \mathrm{H}, 3 \mathrm{H} 2,1-2 \mathrm{H} 3$ \\
\hline 860 & CC5H9-A & $\mathrm{C}=\mathrm{CC}(\mathrm{C})[\mathrm{CH} 2]$ & InChl=1S/C5H9/c1-4-5(2)3/h4-5H,1-2H2,3H3 \\
\hline 861 & $\mathrm{IC} 5 \mathrm{H} 11 \mathrm{OH}$ & $\mathrm{CC}(\mathrm{C}) \mathrm{CCO}$ & InChl=1S/C5H12O/c1-5(2)3-4-6/h5-6H,3-4H2,1-2H3 \\
\hline 862 & ACC5H7-DOH-AROOH & $\mathrm{OOCC}(=\mathrm{C}) \mathrm{C}=\mathrm{CO}$ & $\operatorname{lnChl}=1 \mathrm{~S} / \mathrm{C} 5 \mathrm{H} 8 \mathrm{OO} / \mathrm{c1} 1-5(2-3-6) 4-8-7 / \mathrm{h} 2-3,6-7 \mathrm{H}, 1,4 \mathrm{H} 2$ \\
\hline 863 & $\mathrm{MB} 4 \mathrm{OOH}$ & $\operatorname{coc}(=0) \operatorname{ccc}(00)$ & $\operatorname{lnChl}=15 / \mathrm{C5} H 1004 / \mathrm{c} 1-8-5(6) 3-2-4-9-7 / \mathrm{h} 7 \mathrm{H}, 2-4 \mathrm{H} 2,1 \mathrm{H} 3$ \\
\hline 864 & IC5H9 & $\mathrm{CC}(\mathrm{C}) \mathrm{C}=[\mathrm{CH}]$ & InChl=1S/C5H9/c1-4-5(2)3/h1,4-5H,2-3H3 \\
\hline 865 & $\mathrm{C} 5 \mathrm{H} 11 \mathrm{OH}-2$ & $\mathrm{CCC}(\mathrm{C}) \mathrm{CO}$ & InChl=1S/C5H12O/c1-3-5(2)4-6/h5-6H,3-4H2,1-2H3 \\
\hline 866 & IC5D1Y4-3OOH & $\mathrm{CC}(=\mathrm{C}) \mathrm{C}(\mathrm{C}=0) 00$ & $\mathrm{InChl}=1 \mathrm{~S} / \mathrm{C} 5 \mathrm{H} 8 \mathrm{OO} / \mathrm{c1}-4(2) 5(3-6) 8-7 / \mathrm{h} 3,5,7 \mathrm{H}, 1 \mathrm{H} 2,2 \mathrm{H} 3$ \\
\hline 867 & C5OHKET2-1 & $\operatorname{cccc}(=0) c(00) 0$ & InChl=1S/C5H1004/c1-2-3-4(6)5(7)9-8/h5,7-8H,2-3H2,1H3 \\
\hline 868 & CYC5H9 & $\mathrm{C} 1[\mathrm{CH}] \mathrm{CCC} 1$ & $\mathrm{InChl}=1 \mathrm{~S} / \mathrm{C} 5 \mathrm{H} 9 / \mathrm{c} 1-2-4-5-3-1 / \mathrm{h} 1 \mathrm{H}, 2-5 \mathrm{H} 2$ \\
\hline 869 & $\mathrm{C} 5 \mathrm{H} 3 \mathrm{O}-2 \mathrm{O}$ & $\mathrm{O}=\mathrm{C} 1 \mathrm{C}=\mathrm{CC}=\mathrm{C} 1[\mathrm{O}]$ & $\mathrm{InChl}=1 \mathrm{~S} / \mathrm{C5} 3 \mathrm{OO} / \mathrm{c} 6-4-2-1-3-5(4) 7 / \mathrm{h} 1-3 \mathrm{H}$ \\
\hline 870 & CPN-2OOH & $\mathrm{C} 1 \mathrm{CC}(=\mathrm{O}) \mathrm{C}(\mathrm{OO}) \mathrm{C} 1$ & InChl=1S/C5H8O3/c6-4-2-1-3-5(4)8-7/h5,7H,1-3H2 \\
\hline 871 & C5OHKET1-2 & $\operatorname{cccc}(00) C(=0) 0$ & InChl=1S/C5H1004/c1-2-3-4(9-8)5(6)7/h4,8H,2-3H2,1H3,(H,6,7) \\
\hline 872 & AC5H9-A2 & $\mathrm{CCC}(=\mathrm{C})[\mathrm{CH} 2]$ & $\operatorname{lnChl}=1 \mathrm{~S} / \mathrm{C} 5 \mathrm{H} 9 / \mathrm{c} 1-4-5(2) 3 / \mathrm{h} 2-4 \mathrm{H} 2,1 \mathrm{H} 3$ \\
\hline 873 & $\mathrm{C} 5 \mathrm{H} 3 \mathrm{O}-3 \mathrm{O}$ & {$[0] \mathrm{C} 1=\mathrm{CC}(=\mathrm{O}) \mathrm{C}=\mathrm{C} 1$} & InChl=1S/C5H3O2/c6-4-1-2-5(7)3-4/h1-3H \\
\hline 874 & $\mathrm{CPN}-3 \mathrm{OOH}$ & $\mathrm{C} 1 \mathrm{CC}(=0) \mathrm{CC} 1(\mathrm{OO})$ & InChl=1S/C5H8O3/c6-4-1-2-5(3-4)8-7/h5,7H,1-3H2 \\
\hline 875 & C5OHKET3-1 & $\mathrm{CCC}(=0) \mathrm{CC}(\mathrm{OO}) \mathrm{O}$ & $\mathrm{InChl}=1 \mathrm{~S} / \mathrm{C} 5 \mathrm{H} 1004 / \mathrm{c1}-2-4(6) 3-5(7) 9-8 / \mathrm{h} 5,7-8 \mathrm{H}, 2-3 \mathrm{H} 2,1 \mathrm{H} 3$ \\
\hline 876 & C5H91-1 & $\mathrm{CCCC}=[\mathrm{CH}]$ & $\mathrm{InChl}=1 \mathrm{~S} / \mathrm{C} 5 \mathrm{Hg} / \mathrm{c1}-3-5-4-2 / \mathrm{h} 1,3 \mathrm{H}, 4-5 \mathrm{H} 2,2 \mathrm{H} 3$ \\
\hline 877 & F2CHO5J & $\mathrm{O}=\mathrm{CC} 1=\mathrm{CC}=[\mathrm{C}] \mathrm{O} 1$ & $\mathrm{InChl}=1 \mathrm{~S} / \mathrm{C5} 3 \mathrm{HO} / \mathrm{c} 6-4-5-2-1-3-7-5 / \mathrm{h} 1-2,4 \mathrm{H}$ \\
\hline 878 & MBMVO & $\mathrm{C}(=0) O C(=0) C C C$ & $\operatorname{lnChl}=1 \mathrm{~S} / \mathrm{C} 5 \mathrm{H} 8 \mathrm{O} 3 / \mathrm{c} 1-2-3-5(7) 8-4-6 / \mathrm{h} 4 \mathrm{H}, 2-3 \mathrm{H} 2,1 \mathrm{H} 3$ \\
\hline 879 & C5OHKET3-2 & $\mathrm{CCC}(=0) \mathrm{C}(\mathrm{OO}) \mathrm{CO}$ & $\mathrm{InChl}=1 \mathrm{~S} / \mathrm{C} 5 \mathrm{H} 1004 / \mathrm{c1}-2-4(7) 5(3-6) 9-8 / \mathrm{h} 5-6,8 \mathrm{H}, 2-3 \mathrm{H} 2,1 \mathrm{H} 3$ \\
\hline 880 & C5H10-2 & $\mathrm{CCC}=\mathrm{CC}$ & InChl=1S/C5H10/c1-3-5-4-2/h3,5H,4H2,1-2H3 \\
\hline 881 & F2CJO & $\mathrm{O}=[\mathrm{C}] \mathrm{C} 1=\mathrm{CC}=\mathrm{CO} 1$ & InChl=1S/C5H3O2/c6-4-5-2-1-3-7-5/h1-3H \\
\hline 882 & MB4VO & $\operatorname{coC}(=0) \operatorname{ccC}(=0)$ & $\operatorname{lnChl}=1 \mathrm{~S} / \mathrm{C} 5 \mathrm{H} 8 \mathrm{O} 3 / \mathrm{c} 1-8-5(7) 3-2-4-6 / \mathrm{h} 4 \mathrm{H}, 2-3 \mathrm{H} 2,1 \mathrm{H} 3$ \\
\hline 883 & C5OHKET1-3 & $\operatorname{ccc}(00) \operatorname{cc}(=0) 0$ & InChl=1S/C5H1004/c1-2-4(9-8)3-5(6)7/h4,8H,2-3H2,1H3,(H,6,7) \\
\hline 884 & C5H10-1 & $\mathrm{CCCC}=\mathrm{C}$ & InChl=1S/C5H10/c1-3-5-4-2/h3H,1,4-5H2,2H3 \\
\hline 885 & $\mathrm{~F} 2 \mathrm{CHO}$ & $\mathrm{O}=\mathrm{CC} 1=\mathrm{CC}=\mathrm{CO} 1$ & InChl=1S/C5H4O2/c6-4-5-2-1-3-7-5/h1-4H \\
\hline 886 & MBCY2OM & $\mathrm{C}(01) \mathrm{OC}(=0) \mathrm{C} 1 \mathrm{CC}$ & InChl=1S/C5H8O3/c1-2-4-5(6)8-3-7-4/h4H,2-3H2,1H3 \\
\hline 887 & C5OHKET2-3 & $\mathrm{CCC}(\mathrm{OO}) \mathrm{C}(=0) \mathrm{CO}$ & $\mathrm{InChl}=1 \mathrm{~S} / \mathrm{C} 5 \mathrm{H} 1004 / \mathrm{c1}-2-5(9-8) 4(7) 3-6 / \mathrm{h} 5-6,8 \mathrm{H}, 2-3 \mathrm{H} 2,1 \mathrm{H} 3$ \\
\hline 888 & $\mathrm{CC} 5 \mathrm{H} 10$ & $\mathrm{CC}(\mathrm{C}) \mathrm{C}=\mathrm{C}$ & $\operatorname{lnChl}=1 \mathrm{~S} / \mathrm{C} 5 \mathrm{H} 10 / \mathrm{cc} 1-4-5(2) 3 / \mathrm{h} 4-5 \mathrm{H}, 1 \mathrm{H} 2,2-3 \mathrm{H} 3$ \\
\hline 889 & P23DE150 & $\mathrm{O}=\mathrm{CC}=\mathrm{C}=\mathrm{CC}=\mathrm{O}$ & InChl=1S/C5H4O2/c6-4-2-1-3-5-7/h2-5H \\
\hline 890 & $\mathrm{MBCY} 3 \mathrm{O} 2$ & $\mathrm{COC}(=0) \mathrm{C} 1 \mathrm{C}(\mathrm{O} 1) \mathrm{C}$ & InChl=1S/C5H8O3/c1-3-4(8-3)5(6)7-2/h3-4H,1-2H3 \\
\hline 891 & DC5OHKETC-D & $\mathrm{CC}(\mathrm{C}) \mathrm{C}(=0) \mathrm{C}(\mathrm{OO}) \mathrm{O}$ & InChl=1S/C5H10O4/c1-3(2)4(6)5(7)9-8/h3,5,7-8H,1-2H3 \\
\hline 892 & CPT & C1CCCC1 & $\mathrm{InChl}=1 \mathrm{~S} / \mathrm{C5} \mathrm{H} 10 / \mathrm{c} 1-2-4-5-3-1 / \mathrm{h} 1-5 \mathrm{H} 2$ \\
\hline 893 & OVCCJCVCCVO & {$[\mathrm{O}] \mathrm{C}=\mathrm{CC}=\mathrm{CC}=\mathrm{O}$} & $\mathrm{InChl}=1 \mathrm{~S} / \mathrm{C} 5 \mathrm{H} 5 \mathrm{OO} / \mathrm{c} 6-4-2-1-3-5-7 / \mathrm{h} 1-5 \mathrm{H}$ \\
\hline 894 & МВСРзОМ & $\mathrm{C} 1 \mathrm{OC}(=0) \mathrm{CC}(\mathrm{O} 1) \mathrm{C}$ & $\operatorname{lnChl}=1 \mathrm{~S} / \mathrm{C} 5 \mathrm{H} 803 / \mathrm{c} 1-4-2-5(6) 8-3-7-4 / \mathrm{h} 4 \mathrm{H}, 2-3 \mathrm{H} 2,1 \mathrm{H} 3$ \\
\hline 895 & DC5OHKETD-C & $\mathrm{CC}(\mathrm{C}) \mathrm{C}(\mathrm{OOO}) \mathrm{C}(=0) \mathrm{O}$ & InChl=1S/C5H1004/c1-3(2)4(9-8)5(6)7/h3-4,8H,1-2H3,(H,6,7) \\
\hline 896 & BC5H10 & $\mathrm{CC}=\mathrm{C}(\mathrm{C}) \mathrm{C}$ & InChl=1S/C5H10/c1-4-5(2)3/h4H,1-3H3 \\
\hline 897 & HMFURAN-S & $\mathrm{O}[\mathrm{CH}] \mathrm{C} 1=\mathrm{CC}=\mathrm{CO} 1$ & $\operatorname{lnChl}=1 \mathrm{~S} / \mathrm{C} 5 \mathrm{H} 5 \mathrm{O} 2 / \mathrm{c6}-4-5-2-1-3-7-5 / \mathrm{h} 1-4,6 \mathrm{H}$ \\
\hline 898 & MBCY402 & $\operatorname{COC}(=0) \mathrm{C} 1 \mathrm{CC}(\mathrm{O} 1)$ & $\operatorname{lnChl}=1 \mathrm{~S} / \mathrm{C} 5 \mathrm{H} 8 \mathrm{O} / \mathrm{C} / \mathrm{c1}-7-5(6) 4-2-3-8-4 / \mathrm{h} 4 \mathrm{H}, 2-3 \mathrm{H} 2,1 \mathrm{H} 3$ \\
\hline 899 & DC5OHKETA-C & $\mathrm{C}(=\mathrm{O}) \mathrm{C}(\mathrm{C}) \mathrm{C}(\mathrm{OO}) \mathrm{CO}$ & $\operatorname{lnChl}=1 \mathrm{~S} / \mathrm{C5} \mathrm{H} 1004 / \mathrm{c1}-4(2-6) 5(3-7) 9-8 / \mathrm{h} 2,4-5,7-8 \mathrm{H}, 3 \mathrm{H} 2,1 \mathrm{H} 3$ \\
\hline 900 & AC5H10 & $\operatorname{cCC}(=\mathrm{C}) \mathrm{C}$ & InChl=15/C5H10/c1-4-5(2)3/h2,4H2,1,3H3 \\
\hline 901 & MF22OJ & {$[0] \mathrm{CC} 1=\mathrm{CC}=\mathrm{CO} 1$} & $\operatorname{lnChl}=1 \mathrm{~S} / \mathrm{C} 5 \mathrm{H} 5 \mathrm{O} 2 / \mathrm{c6} 6-4-5-2-1-3-7-5 / \mathrm{h} 1-3 \mathrm{H}, 4 \mathrm{H} 2$ \\
\hline 902 & MBCY4O3 & $\operatorname{COC}(=0) \operatorname{CC} 1 \mathrm{C}(\mathrm{O} 1)$ & $\mathrm{InChl}=1 \mathrm{~S} / \mathrm{C5} \mathrm{H} 8 \mathrm{O} / \mathrm{c} 1-7-5(6) 2-4-3-8-4 / \mathrm{h} 4 \mathrm{H}, 2-3 \mathrm{H} 2,1 \mathrm{H} 3$ \\
\hline 903 & DC5OHKETA-D & $\mathrm{C}(=0) \mathrm{C}(\mathrm{C}) \mathrm{CC}(\mathrm{OO}) \mathrm{O}$ & InChl=1S/C5H1004/c1-4(3-6)2-5(7)9-8/h3-5,7-8H,2H2,1H3 \\
\hline 904 & C5H11-1 & $\mathrm{CCCC}[\mathrm{CH} 2]$ & $\operatorname{lnChl}=1 \mathrm{~S} / \mathrm{CSH} 11 / \mathrm{c1}-3-5-4-2 / \mathrm{h} 1,3-5 \mathrm{H} 2,2 \mathrm{H} 3$ \\
\hline 905 & MF250J & $\mathrm{CC} 1=\mathrm{CC}=\mathrm{C}([\mathrm{O}]) 01$ & $\mathrm{InChl}=1 \mathrm{~S} / \mathrm{C} 5 \mathrm{H} 5 \mathrm{O} 2 / \mathrm{c1}-4-2-3-5(6) 7-4 / \mathrm{h} 2-3 \mathrm{H}, 1 \mathrm{H} 3$ \\
\hline 906 & $\mathrm{C} 2 \mathrm{H} 3 \mathrm{C}(\mathrm{OO}) \mathrm{CCVO}$ & $\mathrm{O}=\mathrm{CCC}(\mathrm{C}=\mathrm{C}) 0 \mathrm{O}$ & $\mathrm{InChl}=1 \mathrm{~S} / \mathrm{C} 5 \mathrm{H} 8 \mathrm{O} 3 / \mathrm{c} 1-2-5(8-7) 3-4-6 / \mathrm{h} 2,4-5,7 \mathrm{H}, 1,3 \mathrm{H} 2$ \\
\hline
\end{tabular}




\begin{tabular}{|c|c|c|c|}
\hline 907 & DC5OHKETD-A & $\mathrm{C}(\mathrm{OO}) \mathrm{C}(\mathrm{C}) \mathrm{CC}(=\mathrm{O}) \mathrm{O}$ & InChl=1S/C5H1004/c1-4(3-9-8)2-5(6)7/h4,8H,2-3H2,1H3,(H,6,7) \\
\hline 908 & DC5H11 & $\mathrm{CC}(\mathrm{C}) \mathrm{C}[\mathrm{CH} 2]$ & InChl=1S/C5H11/c1-4-5(2)3/h5H,1,4H2,2-3H3 \\
\hline 909 & P3E1501J & $\mathrm{O}=[\mathrm{C}] \mathrm{CC}=\mathrm{CC}=\mathrm{O}$ & $\operatorname{lnChl}=1 \mathrm{~S} / \mathrm{C} 5 \mathrm{H} 5 \mathrm{O} 2 / \mathrm{c6}-4-2-1-3-5-7 / \mathrm{h} 1-2,4 \mathrm{H}, 3 \mathrm{H} 2$ \\
\hline 910 & BC5H8-DOHAOO & {$[\mathrm{O}] \mathrm{OCC}(=\mathrm{CCO}) \mathrm{C}$} & InChl=1S/C5H9O3/c1-5(2-3-6)4-8-7/h2,6H,3-4H2,1H3 \\
\hline 911 & DC5OHKETC-A & $\mathrm{C}(\mathrm{OO}) \mathrm{C}(\mathrm{C}) \mathrm{C}(=\mathrm{O}) \mathrm{CO}$ & InChl=1S/C5H1004/c1-4(3-9-8)5(7)2-6/h4,6,8H,2-3H2,1H3 \\
\hline 912 & C5H11-2 & $\mathrm{CCC}[\mathrm{CH}] \mathrm{C}$ & $\operatorname{lnChl}=1 \mathrm{~S} / \mathrm{C} 5 \mathrm{H} 11 / \mathrm{c} 1-3-5-4-2 / \mathrm{h} 3 \mathrm{H}, 4-5 \mathrm{H} 2,1-2 \mathrm{H} 3$ \\
\hline 913 & P3E2501J & {$[\mathrm{CH} 2] \mathrm{C}(=\mathrm{O}) \mathrm{C}=\mathrm{CC}=\mathrm{O}$} & InChl=1S/C5H5O2/c1-5(7)3-2-4-6/h2-4H,1H2 \\
\hline 914 & AC5H8-DOHCOO & $\mathrm{OCC}(\mathrm{C}(=\mathrm{C}) \mathrm{C}) \mathrm{O}[\mathrm{O}]$ & InChl=1S/C5H9O3/c1-4(2)5(3-6)8-7/h5-6H,1,3H2,2H3 \\
\hline 915 & C5OHKET4-1 & $\mathrm{CC}(=0) \mathrm{CCC}(\mathrm{OO}) \mathrm{O}$ & InChl=1S/C5H1004/c1-4(6)2-3-5(7)9-8/h5,7-8H,2-3H2,1H3 \\
\hline 916 & C5H11-3 & $\mathrm{CC}[\mathrm{CH}] \mathrm{CC}$ & InChl=1S/C5H11/c1-3-5-4-2/h5H,3-4H2,1-2H3 \\
\hline 917 & P3E2505J & $\mathrm{CC}(=0) \mathrm{C}=\mathrm{C}[\mathrm{C}]=\mathrm{O}$ & InChl=1S/C5H5O2/c1-5(7)3-2-4-6/h2-3H,1H3 \\
\hline 918 & CC5H8-DOHBOO & $\mathrm{OC}=\mathrm{CC}(\mathrm{O}[\mathrm{O}])(\mathrm{C}) \mathrm{C}$ & InChl=1S/C5H9O3/c1-5(2,8-7)3-4-6/h3-4,6H,1-2H3 \\
\hline 919 & C5OHKET4-2 & $\mathrm{CC}(=0) \mathrm{CC}(\mathrm{OO}) \mathrm{CO}$ & InChl=1S/C5H1004/c1-4(7)2-5(3-6)9-8/h5-6,8H,2-3H2,1H3 \\
\hline 920 & AC5H11 & $\mathrm{CCC}([\mathrm{CH} 2]) \mathrm{C}$ & InChl=1S/C5H11/c1-4-5(2)3/h5H,2,4H2,1,3H3 \\
\hline 921 & $\mathrm{C} 5 \mathrm{H} 5 \mathrm{OOO}$ & $\mathrm{C} 1=\mathrm{CC}(\mathrm{O}[\mathrm{O}]) \mathrm{C}=\mathrm{C} 1$ & $\operatorname{lnChl}=15 / C 5 H 502 / C 6-7-5-3-1-2-4-5 / h 1-5 \mathrm{H}$ \\
\hline 922 & BC5H8-DOHDOO & {$[0] O C(C=C(C) C) O$} & InChl=1S/C5H9O3/c1-4(2)3-5(6)8-7/h3,5-6H,1-2H3 \\
\hline 923 & C5OHKET4-3 & $\mathrm{CC}(=\mathrm{O}) \mathrm{C}(\mathrm{OO}) \mathrm{CCO}$ & InChl=1S/C5H1004/c1-4(7)5(9-8)2-3-6/h5-6,8H,2-3H2,1H3 \\
\hline 924 & BC5H11 & $\mathrm{CC}[\mathrm{C}](\mathrm{C}) \mathrm{C}$ & InChl=1S/C5H11/c1-4-5(2)3/h4H2,1-3H3 \\
\hline 925 & IC5D2Y14 & $\mathrm{O}=\mathrm{CC}=\mathrm{C}(\mathrm{C}=\mathrm{O}) \mathrm{C}$ & InChl=1S/C5H6O2/c1-5(4-7)2-3-6/h2-4H,1H3 \\
\hline 926 & BC5H8-DOHAQAJ & $\mathrm{OC}[\mathrm{CH}] \mathrm{C}(=\mathrm{C}) \mathrm{COO}$ & InChl=1S/C5H9O3/c1-5(2-3-6)4-8-7/h2,6-7H,1,3-4H2 \\
\hline 927 & C5OHKET1-4 & $\mathrm{CC}(\mathrm{OO}) \mathrm{CCC}(=0) 0$ & InChl=1S/C5H10O4/c1-4(9-8)2-3-5(6)7/h4,8H,2-3H2,1H3,(H,6,7) \\
\hline 928 & CC5H11 & $\mathrm{C}[\mathrm{CH}] \mathrm{C}(\mathrm{C}) \mathrm{C}$ & $\operatorname{lnChl}=15 / \mathrm{C} 5 \mathrm{H} 11 / \mathrm{c} 1-4-5(2) 3 / \mathrm{h} 4-5 \mathrm{H}, 1-3 \mathrm{H} 3$ \\
\hline 929 & ACC5H7-DOH-ARCHO & $\mathrm{OC}=\mathrm{CC}(=\mathrm{C}) \mathrm{C}=\mathrm{O}$ & InChl=1S/C5H6O2/c1-5(4-7)2-3-6/h2-4,6H,1H2 \\
\hline 930 & AC5H8-DOHCQAJ & $\mathrm{OCC}(\mathrm{C}(=\mathrm{C})[\mathrm{CH} 2]) \mathrm{OO}$ & $\operatorname{lnChl}=1 \mathrm{~S} / \mathrm{C} 5 \mathrm{H} 9 \mathrm{O} / \mathrm{c} 1-4(2) 5(3-6) 8-7 / \mathrm{h} 5-7 \mathrm{H}, 1-3 \mathrm{H} 2$ \\
\hline 931 & C5OHKET2-4 & $\mathrm{CC}(\mathrm{OO}) \mathrm{CC}(=0) \mathrm{CO}$ & InChl=1S/C5H1004/c1-4(9-8)2-5(7)3-6/h4,6,8H,2-3H2,1H3 \\
\hline 932 & NEOC5H11 & {$[\mathrm{CH} 2] \mathrm{C}(\mathrm{C})(\mathrm{C}) \mathrm{C}$} & InChl=1S/C5H11/c1-5(2,3)4/h1H2,2-4H3 \\
\hline 933 & CPNO2-3 & $\mathrm{C} 1 \mathrm{CC}(=\mathrm{O}) \mathrm{C}(\mathrm{O} 2) \mathrm{C} 12$ & InChl=1S/C5H6O2/c6-3-1-2-4-5(3)7-4/h4-5H,1-2H2 \\
\hline 934 & C5OHKET3-4 & $\mathrm{CC}(\mathrm{OO}) \mathrm{C}(=\mathrm{O}) \mathrm{CCO}$ & InChl=1S/C5H1004/c1-4(9-8)5(7)2-3-6/h4,6,8H,2-3H2,1H3 \\
\hline 935 & NC5H12 & $\operatorname{cCCCC}$ & $\operatorname{lnChl}=1 \mathrm{~S} / \mathrm{C} 5 \mathrm{H} 12 / \mathrm{c1}-3-5-4-2 / \mathrm{h} 3-5 \mathrm{H} 2,1-2 \mathrm{H} 3$ \\
\hline 936 & CPNO2-4 & $\mathrm{C} 12 \mathrm{CC}(=\mathrm{O}) \mathrm{C}(\mathrm{O} 2) \mathrm{C} 1$ & InChl=1S/C5H6O2/c6-4-1-3-2-5(4)7-3/h3,5H,1-2H2 \\
\hline 937 & AC5H8-DOCQ & {$[\mathrm{O}] \mathrm{CC}(\mathrm{C}(=\mathrm{C}) \mathrm{C}) 0 \mathrm{O}$} & $\operatorname{lnChl}=1 \mathrm{~S} / \mathrm{C} 5 \mathrm{H} 9 \mathrm{O} 3 / \mathrm{c} 1-4(2) 5(3-6) 8-7 / \mathrm{h} 5,7 \mathrm{H}, 1,3 \mathrm{H} 2,2 \mathrm{H3}$ \\
\hline 938 & DC5OHKETD-B & $\mathrm{CC}(\mathrm{C})(\mathrm{OO}) \mathrm{CC}(=\mathrm{O}) \mathrm{O}$ & InChl=1S/C5H10O4/c1-5(2,9-8)3-4(6)7/h8H,3H2,1-2H3,(H,6,7) \\
\hline 939 & $\mathrm{ICSH} 12$ & $\operatorname{cCC}(\mathrm{C}) \mathrm{C}$ & $\operatorname{lnChl}=1 \mathrm{~S} / \mathrm{C} 5 \mathrm{H} 12 / \mathrm{c1}-4-5(2) 3 / \mathrm{h} 5 \mathrm{H}, 4 \mathrm{H} 2,1-3 \mathrm{H} 3$ \\
\hline 940 & CPNO3-4 & $\mathrm{C} 12 \mathrm{CC}(=0) \mathrm{CC} 1(\mathrm{O} 2)$ & $\operatorname{lnChl}=1 \mathrm{~S} / \mathrm{C} 5 \mathrm{H} 6 \mathrm{O} 2 / \mathrm{c6}-3-1-4-5(2-3) 7-4 / \mathrm{h} 4-5 \mathrm{H}, 1-2 \mathrm{H} 2$ \\
\hline 941 & NEOC5H12 & $\mathrm{CC}(\mathrm{C})(\mathrm{C}) \mathrm{C}$ & $\operatorname{lnChl}=15 / C 5 H 12 / c 1-5(2,3) 4 / h 1-4 H 3$ \\
\hline 942 & CPNO2-5 & $\mathrm{C} 1 \mathrm{C} 2 \mathrm{C}(=\mathrm{O}) \mathrm{C}(\mathrm{O} 2) \mathrm{C} 1$ & $\operatorname{lnChl}=15 / C 5 H 6 O 2 / c 6-5-3-1-2-4(5) 7-3 / h 3-4 H, 1-2 \mathrm{H} 2$ \\
\hline 943 & DC5OHKETA-B & $\mathrm{C}(=\mathrm{O}) \mathrm{C}(\mathrm{C})(\mathrm{OO}) \mathrm{CCO}$ & $\operatorname{lnChl}=1 \mathrm{~S} / \mathrm{C} 5 \mathrm{H} 1004 / \mathrm{c} 1-5(4-7,9-8) 2-3-6 / \mathrm{h} 4,6,8 \mathrm{H}, 2-3 \mathrm{H} 2,1 \mathrm{H} 3$ \\
\hline 944 & $\mathrm{C} 5 \mathrm{H} 3 \mathrm{O}-2$ & {$[\mathrm{O}] \mathrm{C} 1=\mathrm{C}=\mathrm{CC}=\mathrm{C} 1$} & InChl=1S/C5H3O/c6-5-3-1-2-4-5/h1-3H \\
\hline 945 & HMFURAN & $\mathrm{OCC} 1=\mathrm{CC}=\mathrm{CO} 1$ & $\operatorname{lnChl}=1 \mathrm{~S} / \mathrm{C} 5 \mathrm{H} 6 \mathrm{O} 2 / \mathrm{C6} 6-4-5-2-1-3-7-5 / \mathrm{h} 1-3,6 \mathrm{H}, 4 \mathrm{H} 2$ \\
\hline 946 & AC5H8DOH-DOOJ & $\mathrm{C}=\mathrm{C}(\mathrm{C}) \mathrm{CC}(\mathrm{O}[\mathrm{O}]) \mathrm{O}$ & $\operatorname{lnChl}=1 \mathrm{~S} / \mathrm{C} 5 \mathrm{H} 903 / \mathrm{c} 1-4(2) 3-5(6) 8-7 / \mathrm{h} 5-6 \mathrm{H}, 1,3 \mathrm{H} 2,2 \mathrm{H} 3$ \\
\hline 947 & DC5OHKETA-A & $\mathrm{C}(=\mathrm{O}) \mathrm{C}(\mathrm{COO}) \mathrm{CCO}$ & $\operatorname{lnChl}=1 \mathrm{~S} / \mathrm{C} 5 \mathrm{H} 1004 / \mathrm{c} 6-2-1-5(3-7) 4-9-8 / \mathrm{h} 3,5-6,8 \mathrm{H}, 1-2,4 \mathrm{H} 2$ \\
\hline 948 & C5H3O-3 & $\mathrm{C} 1=\mathrm{C}[\mathrm{C}]=\mathrm{CC} 1=\mathrm{O}$ & InChl=1S/C5H3O/c6-5-3-1-2-4-5/h1,3-4H \\
\hline 949 & $\mathrm{MF} 25 \mathrm{OH}$ & $\mathrm{CC} 1=\mathrm{CC}=\mathrm{C}(0) 01$ & $\operatorname{lnChl}=1 \mathrm{~S} / \mathrm{C} 5 \mathrm{H} 6 \mathrm{O} 2 / \mathrm{c1}-4-2-3-5(6) 7-4 / \mathrm{h} 2-3,6 \mathrm{H}, 1 \mathrm{H} 3$ \\
\hline 950 & BC5H8-DOHAQCJ & $\mathrm{OOC}[\mathrm{C}](\mathrm{C}=\mathrm{CO}) \mathrm{C}$ & $\mathrm{InChl}=1 \mathrm{~S} / \mathrm{C} 5 \mathrm{H} 903 / \mathrm{c} 1-5(2-3-6) 4-8-7 / \mathrm{h} 2-3,6-7 \mathrm{H}, 4 \mathrm{H} 2,1 \mathrm{H} 3$ \\
\hline 951 & C5OHKET5-2 & $\mathrm{C}(=0) \mathrm{CCC}(\mathrm{OO}) \mathrm{CO}$ & InChl=1S/C5H1004/c6-3-1-2-5(4-7)9-8/h3,5,7-8H,1-2,4H2 \\
\hline 952 & P134TE10 & $\mathrm{O}=\mathrm{C}=\mathrm{CC}=\mathrm{C}=\mathrm{C}$ & $\ln \mathrm{Chl}=1 \mathrm{~S} / \mathrm{C} 5 \mathrm{H} 4 \mathrm{O} / \mathrm{c1}-2-3-4-5-6 / \mathrm{h} 3-4 \mathrm{H}, 1 \mathrm{H} 2$ \\
\hline 953 & P23DE101OH & $\mathrm{CC}=\mathrm{C}=\mathrm{CC}(=\mathrm{O}) \mathrm{O}$ & $\operatorname{lnChl}=1 \mathrm{~S} / \mathrm{C} 5 \mathrm{H} 6 \mathrm{O} / \mathrm{c} 1-2-3-4-5(6) 7 / \mathrm{h} 2,4 \mathrm{H}, 1 \mathrm{H} 3,(\mathrm{H}, 6,7)$ \\
\hline 954 & IC5Y4OOH2-3J & {$[\mathrm{O}] \mathrm{C}=\mathrm{CC}(\mathrm{OO})(\mathrm{C}) \mathrm{C}$} & InChl=1S/C5H9O3/c1-5(2,8-7)3-4-6/h3-4,7H,1-2H3 \\
\hline 955 & C5OHKET5-4 & $\mathrm{C}(=0) \mathrm{C}(\mathrm{OO}) \mathrm{CCCO}$ & $\operatorname{lnChl}=1 \mathrm{~S} / \mathrm{C} 5 \mathrm{H} 1004 / \mathrm{c} 6-3-1-2-5(4-7) 9-8 / \mathrm{h4}-6,8 \mathrm{H}, 1-3 \mathrm{H} 2$ \\
\hline 956 & P1E3Y10 & $\mathrm{O}=\mathrm{C}=\mathrm{CCHCC}$ & $\operatorname{lnChl}=1 \mathrm{~S} / \mathrm{C} 5 \mathrm{H} 4 \mathrm{O} / \mathrm{c} 1-2-3-4-5-6 / \mathrm{h} 4 \mathrm{H}, 1 \mathrm{H} 3$ \\
\hline 957 & P23DE105OH & $\mathrm{O}=\mathrm{CC}=\mathrm{C}=\mathrm{CCO}$ & $\mathrm{InChl}=1 \mathrm{~S} / \mathrm{C} 5 \mathrm{H} 6 \mathrm{O} 2 / \mathrm{c} 6-4-2-1-3-5-7 / \mathrm{h} 2-4,7 \mathrm{H}, 5 \mathrm{H} 2$ \\
\hline 958 & IC5Y4OOH3-2J & $00 C([C](C) C) C=0$ & InChl=15/C5H9O3/c1-4(2)5(3-6)8-7/h3,5,7H,1-2H3 \\
\hline 959 & C5OHKET4-5 & $\mathrm{C}(\mathrm{OO}) \mathrm{C}(=\mathrm{O}) \mathrm{CCCO}$ & $\operatorname{lnChl}=1 \mathrm{~S} / \mathrm{C} 5 \mathrm{H} 1004 / \mathrm{c} 6-3-1-2-5(7) 4-9-8 / \mathrm{h} 6,8 \mathrm{H}, 1-4 \mathrm{H} 2$ \\
\hline 960 & $\mathrm{C} 5 \mathrm{H} 4 \mathrm{O}$ & $\mathrm{C} 1=0) \mathrm{C}=\mathrm{CC}=\mathrm{C} 1$ & InChl=1S/C5H4O/c6-5-3-1-2-4-5/h1-4H \\
\hline 961 & P34DE2O1OH & $\mathrm{OCC}(=\mathrm{O}) \mathrm{C}=\mathrm{C}=\mathrm{C}$ & $\operatorname{lnChl}=1 \mathrm{~S} / \mathrm{C} 5 \mathrm{H} 6 \mathrm{O} 2 / \mathrm{c1} 1-2-3-5(7) 4-6 / \mathrm{h} 3,6 \mathrm{H}, 1,4 \mathrm{H} 2$ \\
\hline 962 & IC5D3OH2-102 & $\mathrm{CC}(\mathrm{C}=\mathrm{C})(\mathrm{CO}[\mathrm{O}]) \mathrm{O}$ & $\mathrm{InChl}=1 \mathrm{~S} / \mathrm{C} 5 \mathrm{H} 903 / \mathrm{c} 1-3-5(2,6) 4-8-7 / \mathrm{h} 3,6 \mathrm{H}, 1,4 \mathrm{H} 2,2 \mathrm{H} 3$ \\
\hline 963 & C5OHKET3-5 & $\mathrm{C}(\mathrm{OO}) \mathrm{CC}(=\mathrm{O}) \mathrm{CCO}$ & InChl=1S/C5H10O4/c6-3-1-5(7)2-4-9-8/h6,8H,1-4H2 \\
\hline 964 & C5Y1D24-1R & $[C]=O) C=C C=C$ & $\operatorname{lnChl}=15 / C 5 H 5 O / c 1-2-3-4-5-6 / h 2-4 \mathrm{H}, 1 \mathrm{H} 2$ \\
\hline 965 & P34DE2O5OH & $\mathrm{CC}(=\mathrm{O}) \mathrm{C}=\mathrm{C}=\mathrm{CO}$ & InChl=1S/C5H6O2/c1-5(7)3-2-4-6/h3-4,6H,1H3 \\
\hline 966 & IC5D1OH3-402 & $\mathrm{CC}(=\mathrm{C}) \mathrm{C}(\mathrm{CO}[\mathrm{O}]) \mathrm{O}$ & InChl=1S/C5H9O3/c1-4(2)5(6)3-8-7/h5-6H,1,3H2,2H3 \\
\hline 967 & C5ОНKET5-3 & $\mathrm{C}(=\mathrm{O}) \mathrm{CC}(\mathrm{OO}) \mathrm{CCO}$ & $\operatorname{lnChl}=1 \mathrm{~S} / \mathrm{C} 5 \mathrm{H} 1004 / \mathrm{c} 6-3-1-5(9-8) 2-4-7 / \mathrm{h} 3,5,7-8 \mathrm{H}, 1-2,4 \mathrm{H} 2$ \\
\hline 968 & CYC5ODEJ & $01[\mathrm{CH}] \mathrm{C}=\mathrm{CC}=\mathrm{C} 1$ & InChl=1S/C5H5O/c1-2-4-6-5-3-1/h1-5H \\
\hline 969 & P3E250 & $\mathrm{CC}(=\mathrm{O}) \mathrm{C}=\mathrm{CC}=\mathrm{O}$ & InChl=1S/C5H6O2/c1-5(7)3-2-4-6/h2-4H,1H3 \\
\hline 970 & CPTOH-2O2 & [0]OC1CCCC10 & InChl=1S/C5H9O3/c6-4-2-1-3-5(4)8-7/h4-6H,1-3H2 \\
\hline 971 & C5OHKET2-5 & $\mathrm{C}(\mathrm{OO}) \mathrm{CCC}(=\mathrm{O}) \mathrm{CO}$ & InChl=1S/C5H10O4/c6-4-5(7)2-1-3-9-8/h6,8H,1-4H2 \\
\hline 972 & C4H5-ICO & $\mathrm{C}=\mathrm{CC}(=\mathrm{C})[\mathrm{C}]=\mathrm{O}$ & $\operatorname{lnChl}=1 \mathrm{~S} / \mathrm{C} 5 \mathrm{H} 5 \mathrm{O} / \mathrm{c1}-3-5(2) 4-6 / \mathrm{h} 3 \mathrm{H}, 1-2 \mathrm{H} 2$ \\
\hline 973 & $\mathrm{C} 5 \mathrm{H} 5 \mathrm{OOH}$ & $\mathrm{C} 1=\mathrm{CC}(\mathrm{OO}) \mathrm{C}=\mathrm{C} 1$ & $\ln \mathrm{Chl}=1 \mathrm{~S} / \mathrm{C} 5 \mathrm{H} 6 \mathrm{O} 2 / \mathrm{c} 6-7-5-3-1-2-4-5 / \mathrm{h} 1-6 \mathrm{H}$ \\
\hline 974 & CPTO2H-2O & OOC1CCCC1 $[0]$ & InChl=1S/C5H9O3/c6-4-2-1-3-5(4)8-7/h4-5,7H,1-3H2 \\
\hline 975 & С5OH2KETA-B & $\operatorname{ccc}(\mathrm{C})(\mathrm{OO}) \mathrm{C}(=0) \mathrm{O}$ & InChl=1S/C5H10O4/c1-3-5(2,9-8)4(6)7/h8H,3H2,1-2H3,(H,6,7) \\
\hline 976 & CPND2-4R & {$[\mathrm{CH}] 1 \mathrm{CC}(=\mathrm{O}) \mathrm{C}=\mathrm{C} 1$} & InChl=1S/C5H5O/c6-5-3-1-2-4-5/h1-3H,4H2 \\
\hline 977 & BC5Y1OH4-1AJ & $\mathrm{OC}[\mathrm{CH}] \mathrm{C}(=\mathrm{C}) \mathrm{C}=\mathrm{O}$ & $\operatorname{lnChl}=15 / C 5 H 702 / c 1-5(4-7) 2-3-6 / h 2,4,6 \mathrm{H}, 1,3 \mathrm{H} 2$ \\
\hline 978 & MBMO & $\mathrm{C}([\mathrm{O}]) \mathrm{OC}(=0) \mathrm{CCC}$ & $\operatorname{lnChl}=15 / C 5 H 9 O 3 / c 1-2-3-5(7) 8-4-6 / h 2-4 \mathrm{H} 2,1 \mathrm{H} 3$ \\
\hline 979 & C5OH2KETA-C & $\mathrm{CC}(\mathrm{OO}) \mathrm{C}(\mathrm{C}) \mathrm{C}(=\mathrm{O}) \mathrm{O}$ & $\operatorname{lnChl}=15 / C 5 H 1004 / c 1-3(5(6) 7) 4(2) 9-8 / h 3-4,8 \mathrm{H}, 1-2 \mathrm{H} 3,(\mathrm{H}, 6,7)$ \\
\hline 980 & CPND2-5R & $\mathrm{C} 1[\mathrm{CH}] \mathrm{C}(=0) \mathrm{C}=\mathrm{C} 1$ & $\operatorname{lnChl}=1 \mathrm{~S} / \mathrm{C} 5 \mathrm{H} 5 \mathrm{O} / \mathrm{C6} 6-5-3-1-2-4-5 / \mathrm{h} 1,3-4 \mathrm{H}, 2 \mathrm{H} 2$ \\
\hline 981 & IC5D1Y4-30J & $\mathrm{CC}(=\mathrm{C}) \mathrm{C}(\mathrm{C}=0)[\mathrm{O}]$ & InChl=1S/C5H7O2/c1-4(2)5(7)3-6/h3,5H,1H2,2H3 \\
\hline 982 & MB2O & $\operatorname{COC}(=0) c([0]) C C$ & $\operatorname{lnChl}=1 \mathrm{~S} / \mathrm{C} 5 \mathrm{H} 9 \mathrm{O} 3 / \mathrm{c} 1-3-4(6) 5(7) 8-2 / \mathrm{h} 4 \mathrm{H}, 3 \mathrm{H} 2,1-2 \mathrm{H} 3$ \\
\hline 983 & C5OH2KETA-D & $00 \operatorname{ccc}(\mathrm{C}) \mathrm{C}(=0) 0$ & $\operatorname{lnChl}=1 \mathrm{~S} / \mathrm{C} 5 \mathrm{H} 1004 / \mathrm{c} 1-4(5(6) 7) 2-3-9-8 / \mathrm{h} 4,8 \mathrm{H}, 2-3 \mathrm{H} 2,1 \mathrm{H} 3,(\mathrm{H}, 6,7)$ \\
\hline 984 & MF22J & {$[\mathrm{CH} 2] \mathrm{C} 1=\mathrm{CC}=\mathrm{CO} 1$} & $\operatorname{lnChl}=1 \mathrm{~S} / \mathrm{C} 5 \mathrm{H} 5 \mathrm{O} / \mathrm{c} 1-5-3-2-4-6-5 / \mathrm{h} 2-4 \mathrm{H}, 1 \mathrm{H} 2$ \\
\hline 985 & IC5D2Y4-10J & $\mathrm{CC}(=\mathrm{CC}=\mathrm{O}) \mathrm{C}[\mathrm{O}]$ & $\operatorname{lnChl}=1 \mathrm{~S} / \mathrm{C} 5 \mathrm{H} 702 / \mathrm{c} 1-5(4-7) 2-3-6 / \mathrm{h} 2-3 \mathrm{H}, 4 \mathrm{H} 2,1 \mathrm{H} 3$ \\
\hline 986 & MB30 & $\operatorname{coc}(=0) \operatorname{cc}([0]) \mathrm{C}$ & InChl=1S/C5H9O3/c1-4(6)3-5(7)8-2/h4H,3H2,1-2H3 \\
\hline 987 & C5OH2KETA-E & $\operatorname{ccc}(\operatorname{coO}) \mathrm{C}(=0) 0$ & InChl=1S/C5H10O4/c1-2-4(3-9-8)5(6)7/h4,8H,2-3H2,1H3,(H,6,7) \\
\hline 988 & MF23J & $\mathrm{CC} 1=[\mathrm{C}] \mathrm{C}=\mathrm{CO} 1$ & $\operatorname{lnChl}=1 \mathrm{~S} / \mathrm{C} 5 \mathrm{H} 5 \mathrm{O} / \mathrm{c} 1-5-3-2-4-6-5 / \mathrm{h} 2,4 \mathrm{H}, 1 \mathrm{H} 3$ \\
\hline 989 & CYC5E1-402 & {$[0] \mathrm{OC} 1 \mathrm{CC}=\mathrm{CC} 1$} & InChl=1S/C5H7O2/c6-7-5-3-1-2-4-5/h1-2,5H,3-4H2 \\
\hline 990 & MB4O & $\operatorname{coc}(=0) \operatorname{ccc}([0])$ & $\operatorname{lnChl}=15 / C 5 H 9 O 3 / c 1-8-5(7) 3-2-4-6 / h 2-4 \mathrm{H} 2,1 \mathrm{H} 3$ \\
\hline 991 & C5OH2KETC-A & $\mathrm{CC}(=0) \mathrm{C}(\mathrm{C}) \mathrm{C}(\mathrm{OO}) \mathrm{O}$ & InChl=1S/C5H10O4/c1-3(4(2)6)5(7)9-8/h3,5,7-8H,1-2H3 \\
\hline 992 & MF24J & $\mathrm{CC} 1=\mathrm{C}[\mathrm{C}]=\mathrm{CO} 1$ & InChl=1S/C5H5O/c1-5-3-2-4-6-5/h3-4H,1H3 \\
\hline 993 & COCCCCHO & $\mathrm{O}=[\mathrm{C}] \mathrm{CCCC}=0$ & InChl=1S/C5H7O2/c6-4-2-1-3-5-7/h4H,1-3H2 \\
\hline 994 & МВ2ОН3Ј & $\operatorname{COC}(=0) \mathrm{C}(\mathrm{O})[\mathrm{CH}] \mathrm{C}$ & InChl=1S/C5H9O3/c1-3-4(6)5(7)8-2/h3-4,6H,1-2H3 \\
\hline 995 & C5OH2KETC-B & $\mathrm{CC}(=0) \mathrm{C}(\mathrm{C})(\mathrm{OO}) \mathrm{CO}$ & $\operatorname{lnChl}=1 \mathrm{~S} / \mathrm{C5} \mathrm{H} 10 \mathrm{OO} 4 / \mathrm{c} 1-4(7) 5(2,3-6) 9-8 / \mathrm{h} 6,8 \mathrm{H}, 3 \mathrm{H} 2,1-2 \mathrm{H} 3$ \\
\hline 996 & MF25J & $\mathrm{CC} 1=\mathrm{CC}=[\mathrm{C}] \mathrm{O} 1$ & InChl=1S/C5H5O/c1-5-3-2-4-6-5/h2-3H,1H3 \\
\hline 997 & CHOCHCCCHO & $\mathrm{O}=\mathrm{CCCC}=\mathrm{C}[\mathrm{O}]$ & $\operatorname{lnChl}=15 / C 5 H 702 / c 6-4-2-1-3-5-7 / h 2,4-5 \mathrm{H}, 1,3 \mathrm{H} 2$ \\
\hline
\end{tabular}




\begin{tabular}{|c|c|c|c|}
\hline 998 & Мвзон4J & $\mathrm{COC}(=\mathrm{O}) \mathrm{CC}(\mathrm{O})[\mathrm{CH} 2]$ & InChl=1S/C5H903/c1-4(6)3-5(7)8-2/h4,6H,1,3H2,2H3 \\
\hline 999 & C5OH2KETC-D & $\mathrm{OOCC}(=\mathrm{O}) \mathrm{C}(\mathrm{C}) \mathrm{CO}$ & InChl=1S/C5H1004/c1-4(2-6)5(7)3-9-8/h4,6,8H,2-3H2,1H3 \\
\hline 1000 & P13DE104J & $\mathrm{O}=\mathrm{C}=\mathrm{CC}=[\mathrm{C}] \mathrm{C}$ & $\operatorname{lnChl}=1 \mathrm{~S} / \mathrm{C} 5 \mathrm{H} 5 \mathrm{O} / \mathrm{c1}-2-3-4-5-6 / \mathrm{h} 3-4 \mathrm{H}, 1 \mathrm{H} 3$ \\
\hline 1001 & CHOCCHCCHO & $\mathrm{O}=\mathrm{CC}[\mathrm{CH}] \mathrm{CC}=\mathrm{O}$ & $\operatorname{lnChl}=1 \mathrm{~S} / \mathrm{C} 5 \mathrm{H} 702 / \mathrm{C} 6-4-2-1-3-5-7 / \mathrm{h} 1,4-5 \mathrm{H}, 2-3 \mathrm{H} 2$ \\
\hline 1002 & DC5H9O-BOO & {$[\mathrm{O}] \mathrm{OC}(\mathrm{CC}=\mathrm{O})(\mathrm{C}) \mathrm{C}$} & InChl=1S/C5H9O3/c1-5(2,8-7)3-4-6/h4H,3H2,1-2H3 \\
\hline 1003 & C5OH2KETC-E & $\mathrm{CC}(=\mathrm{O}) \mathrm{C}(\mathrm{COO}) \mathrm{CO}$ & $\operatorname{lnChl}=1 \mathrm{~S} / \mathrm{C} 5 \mathrm{H} 1004 / \mathrm{c} 1-4(7) 5(2-6) 3-9-8 / \mathrm{h} 5-6,8 \mathrm{H}, 2-3 \mathrm{H} 2,1 \mathrm{H} 3$ \\
\hline 1004 & P23DE104J & $\mathrm{O}=\mathrm{CC}=\mathrm{C}=[\mathrm{C}] \mathrm{C}$ & $\operatorname{lnChl}=1 \mathrm{~S} / \mathrm{C} 5 \mathrm{H} 5 \mathrm{O} / \mathrm{c1} 1-2-3-4-5-6 / \mathrm{h} 4-5 \mathrm{H}, 1 \mathrm{H} 3$ \\
\hline 1005 & CPN-2OR & $\mathrm{C} 1 \mathrm{CC}(=0) \mathrm{C}([0]) \mathrm{C} 1$ & InChl=1S/C5H7O2/c6-4-2-1-3-5(4)7/h4H,1-3H2 \\
\hline 1006 & DC5H9O-COO & {$[\mathrm{O}] \mathrm{OC}(\mathrm{C}(\mathrm{C}) \mathrm{C}) \mathrm{C}=\mathrm{O}$} & $\operatorname{lnChl}=1 \mathrm{~S} / \mathrm{C5} 59 \mathrm{OO} / \mathrm{c1} 1-4(2) 5(3-6) 8-7 / \mathrm{h} 3-5 \mathrm{H}, 1-2 \mathrm{H} 3$ \\
\hline 1007 & C5OH2KETD-A & $\mathrm{O}=\mathrm{CCC}(\mathrm{C}) \mathrm{C}(\mathrm{OO}) \mathrm{O}$ & InChl=1S/C5H1004/c1-4(2-3-6)5(7)9-8/h3-5,7-8H,2H2,1H3 \\
\hline 1008 & P34DE1O2J-C2 & $\mathrm{C}=\mathrm{C}=\mathrm{C}[\mathrm{CH}] \mathrm{C}=\mathrm{O}$ & $\operatorname{lnChl}=1 \mathrm{~S} / \mathrm{C} 5 \mathrm{H} 5 \mathrm{O} / \mathrm{c} 1-2-3-4-5-6 / \mathrm{h} 3-5 \mathrm{H}, 1 \mathrm{H} 2$ \\
\hline 1009 & CPN-3OR & $\mathrm{C} 1 \mathrm{CC}(=0) \mathrm{CC} 1([\mathrm{O}])$ & InChl=1S/C5H7O2/c6-4-1-2-5(7)3-4/h4H,1-3H2 \\
\hline 1010 & PC4H8CHO-102 & $\operatorname{cccc}(\mathrm{O}[\mathrm{O}]) \mathrm{C}(=\mathrm{O})$ & InChl=1S/C5H9O3/c1-2-3-5(4-6)8-7/h4-5H,2-3H2,1H3 \\
\hline 1011 & C5OH2KETD-B & $\mathrm{O}=\mathrm{CCC}(\mathrm{C})(\mathrm{OO}) \mathrm{CO}$ & InChl=1S/C5H1004/c1-5(4-7,9-8)2-3-6/h3,7-8H,2,4H2,1H3 \\
\hline 1012 & P34DE205J & {$[\mathrm{CH}]=\mathrm{C}=\mathrm{CC}(=\mathrm{O}) \mathrm{C}$} & $\operatorname{lnChl}=1 \mathrm{~S} / \mathrm{C} 5 \mathrm{H} 5 \mathrm{O} / \mathrm{c1} 1-3-4-5(2) 6 / \mathrm{h} 1,4 \mathrm{H}, 2 \mathrm{H} 3$ \\
\hline 1013 & CPNOH3-2R & $\mathrm{C} 1 \mathrm{CC}(=\mathrm{O})[\mathrm{CH}] \mathrm{C} 1(\mathrm{O})$ & InChl=1S/C5H7O2/c6-4-1-2-5(7)3-4/h3-4,6H,1-2H2 \\
\hline 1014 & PC4H8CHO-2O2 & $\mathrm{CCC}(\mathrm{O}[\mathrm{O}]) \mathrm{CC}(=\mathrm{O})$ & $\mathrm{InChl}=1 \mathrm{~S} / \mathrm{C} 5 \mathrm{H} 9 \mathrm{OO} / \mathrm{c1}-2-5(8-7) 3-4-6 / \mathrm{h} 4-5 \mathrm{H}, 2-3 \mathrm{H} 2,1 \mathrm{H3}$ \\
\hline 1015 & C5OH2KETD-C & $\mathrm{O}=\mathrm{CC}(\mathrm{OO}) \mathrm{C}(\mathrm{C}) \mathrm{CO}$ & $\operatorname{lnChl}=1 \mathrm{~S} / \mathrm{C} 5 \mathrm{H} 1004 / \mathrm{c} 1-4(2-6) 5(3-7) 9-8 / \mathrm{h} 3-6,8 \mathrm{H}, 2 \mathrm{H} 2,1 \mathrm{H} 3$ \\
\hline 1016 & $\mathrm{C} 5 \mathrm{H} 5 \mathrm{O}$ & $\mathrm{C} 1([0]) \mathrm{C}=\mathrm{CC}=\mathrm{C} 1$ & InChl=1S/C5H5O/c6-5-3-1-2-4-5/h1-5H \\
\hline 1017 & CPNOH3-4R & {$[\mathrm{CH}] 1 \mathrm{CC}(=\mathrm{O}) \mathrm{CC} 1(\mathrm{O})$} & $\operatorname{lnChl}=15 / C 5 H 7 O 2 / c 6-4-1-2-5(7) 3-4 / h 1,4,6 \mathrm{H}, 2-3 \mathrm{H} 2$ \\
\hline 1018 & PC4H8CHO-3O2 & $\mathrm{CC}(\mathrm{O}[\mathrm{O}]) \mathrm{CCC}(=0)$ & $\operatorname{lnChl}=1 \mathrm{~S} / \mathrm{C} 5 \mathrm{H} 9 \mathrm{OO} / \mathrm{c1}-5(8-7) 3-2-4-6 / \mathrm{h} 4-5 \mathrm{H}, 2-3 \mathrm{H} 2,1 \mathrm{H} 3$ \\
\hline 1019 & C5OH2KETD-E & $\mathrm{O}=\mathrm{CCC}(\mathrm{COO}) \mathrm{CO}$ & InChl=1S/C5H10O4/c6-2-1-5(3-7)4-9-8/h2,5,7-8H,1,3-4H2 \\
\hline 1020 & $\mathrm{C} 5 \mathrm{H} 4 \mathrm{OH}$ & {$[\mathrm{C}](0) 1 \mathrm{C}=\mathrm{CC}=\mathrm{C} 1$} & InChl=1S/C5H5O/c6-5-3-1-2-4-5/h1-4,6H \\
\hline 1021 & CPNOH2-3R & $\mathrm{C} 1 \mathrm{CC}(=\mathrm{O}) \mathrm{C}(\mathrm{O})[\mathrm{CH}] 1$ & InChl=1S/C5H7O2/c6-4-2-1-3-5(4)7/h2,4,6H,1,3H2 \\
\hline 1022 & PC4H8CHO-4O2 & $\mathrm{C}(\mathrm{O}[\mathrm{O}]) \operatorname{CCCC}(=0)$ & $\operatorname{lnChl}=15 / C 5 H 9 O 3 / C 6-4-2-1-3-5-8-7 / h 4 H, 1-3,5 \mathrm{H} 2$ \\
\hline 1023 & C5OH2KETE-A & $\operatorname{ccc}(\mathrm{C}=0) \mathrm{C}(\mathrm{OO}) \mathrm{O}$ & InChl=1S/C5H1004/c1-2-4(3-6)5(7)9-8/h3-5,7-8H,2H2,1H3 \\
\hline 1024 & CJVCCVCCVO & {$[\mathrm{CH}]=\mathrm{CC}=\mathrm{CC}=\mathrm{O}$} & InChl=1S/C5H5O/c1-2-3-4-5-6/h1-5H \\
\hline 1025 & $\mathrm{C} 5 \mathrm{H} 702$ & $\mathrm{COC}(=\mathrm{O}) \mathrm{C}=\mathrm{C}[\mathrm{CH} 2]$ & InChl=1S/C5H702/c1-3-4-5(6)7-2/h3-4H,1H2,2H3 \\
\hline 1026 & $\mathrm{CVCC}(\mathrm{OOJ})(\mathrm{C}) \mathrm{CO}$ & $\mathrm{OCC}(\mathrm{C}=\mathrm{C})(\mathrm{O}[\mathrm{O}]) \mathrm{C}$ & $\mathrm{InChl}=1 \mathrm{~S} / \mathrm{C} 5 \mathrm{H} 9 \mathrm{O} 3 / \mathrm{c} 1-3-5(2,4-6) 8-7 / \mathrm{h} 3,6 \mathrm{H}, 1,4 \mathrm{H} 2,2 \mathrm{H} 3$ \\
\hline 1027 & C5OH2KETE-B & $\operatorname{ccc}(\mathrm{C}=0)(\mathrm{OO}) \mathrm{CO}$ & $\operatorname{lnChl}=1 \mathrm{~S} / \mathrm{C} 5 \mathrm{H} 1004 / \mathrm{c} 1-2-5(3-6,4-7) 9-8 / \mathrm{h} 3,7-8 \mathrm{H}, 2,4 \mathrm{H} 2,1 \mathrm{H} 3$ \\
\hline 1028 & NC5D12Y4-5R & $\mathrm{C}=\mathrm{C}=\mathrm{CC}(=\mathrm{O})[\mathrm{CH} 2]$ & $\operatorname{lnChl}=15 / C 5 H 5 O / c 1-3-4-5(2) 6 / h 4 H, 1-2 \mathrm{H} 2$ \\
\hline 1029 & MF22OH3J & $\mathrm{O} 1 \mathrm{C}(\mathrm{C})(\mathrm{O})[\mathrm{CH}] \mathrm{C}=\mathrm{C} 1$ & InChl=1S/C5H7O2/c1-5(6)3-2-4-7-5/h2-4,6H,1H3 \\
\hline 1030 & $\mathrm{O} 2 \mathrm{C} 4 \mathrm{H} 8 \mathrm{CHO}$ & $\mathrm{CC}(\mathrm{C})(\mathrm{C}=\mathrm{O}) \mathrm{CO}[\mathrm{O}]$ & InChl=1S/C5H9O3/c1-5(2,3-6)4-8-7/h3H,4H2,1-2H3 \\
\hline 1031 & C5OH2KETE-C & $\mathrm{CC}(\mathrm{OO}) \mathrm{C}(\mathrm{C}=\mathrm{O}) \mathrm{CO}$ & $\operatorname{lnChl}=1 \mathrm{~S} / \mathrm{C} 5 \mathrm{H} 1004 / \mathrm{c1}-4(9-8) 5(2-6) 3-7 / \mathrm{h} 2,4-5,7-8 \mathrm{H}, 3 \mathrm{H} 2,1 \mathrm{H} 3$ \\
\hline 1032 & $\mathrm{C} 5 \mathrm{H} 5 \mathrm{OH}-1$ & $\mathrm{OC} 1=\mathrm{CC}=\mathrm{CC} 1$ & InChl=1S/C5H6O/c6-5-3-1-2-4-5/h1-3,6H,4H2 \\
\hline 1033 & MF25OH4J & $\mathrm{CC} 1=\mathrm{C}[\mathrm{CH}] \mathrm{C}(\mathrm{O}) \mathrm{O} 1$ & InChl=15/C5H7O2/c1-4-2-3-5(6)7-4/h2-3,5-6H,1H3 \\
\hline 1034 & $\mathrm{O} 2 \mathrm{HC} 4 \mathrm{H} 8 \mathrm{CO}$ & $\mathrm{CC}(\mathrm{C})([\mathrm{C}]=0) \mathrm{COO}$ & InChl=1S/C5H9O3/c1-5(2,3-6)4-8-7/h7H,4H2,1-2H3 \\
\hline 1035 & C5OH2KETE-D & OOCCC(C=0)CO & $\operatorname{lnChl}=1 \mathrm{~S} / \mathrm{C} 5 \mathrm{H} 1004 / \mathrm{c} 6-3-5(4-7) 1-2-9-8 / \mathrm{h} 3,5,7-8 \mathrm{H}, 1-2,4 \mathrm{H} 2$ \\
\hline 1036 & $\mathrm{C} 5 \mathrm{H} 5 \mathrm{OH}-2$ & $\mathrm{OC} 1=\mathrm{CCC}=\mathrm{C} 1$ & $\operatorname{lnChl}=1 \mathrm{~S} / \mathrm{C} 5 \mathrm{H} 6 \mathrm{O} / \mathrm{c} 6-5-3-1-2-4-5 / \mathrm{h} 1,3-4,6 \mathrm{H}, 2 \mathrm{H} 2$ \\
\hline 1037 & P1403J & $\mathrm{CC}(=\mathrm{O})[\mathrm{CH}] \mathrm{CC}=\mathrm{O}$ & InChl=1S/C5H702/c1-5(7)3-2-4-6/h3-4H,2H2,1H3 \\
\hline 1038 & NC52ONEO2-3 & $\mathrm{CC}(=\mathrm{O}) \mathrm{C}(\mathrm{O}[\mathrm{O}]) \mathrm{CC}$ & InChl=1S/C5H9O3/c1-3-5(8-7)4(2)6/h5H,3H2,1-2H3 \\
\hline 1039 & C5H9-BDOHCO2 & $\mathrm{OCC}(\mathrm{C}(\mathrm{O})(\mathrm{C}) \mathrm{C}) \mathrm{O}[\mathrm{O}]$ & InChl=1S/C5H1104/c1-5(2,7)4(3-6)9-8/h4,6-7H,3H2,1-2H3 \\
\hline 1040 & $\mathrm{C} 4 \mathrm{H} 5-\mathrm{ICHO}$ & $\mathrm{C}=\mathrm{CC}(=\mathrm{C}) \mathrm{C}=\mathrm{O}$ & $\operatorname{lnChl}=1 \mathrm{~S} / \mathrm{C} 5 \mathrm{H} 6 \mathrm{O} / \mathrm{c1}-3-5(2) 4-6 / \mathrm{h} 3-4 \mathrm{H}, 1-2 \mathrm{H} 2$ \\
\hline 1041 & P2504J & $\mathrm{CC}(=\mathrm{O}) \mathrm{C}[\mathrm{CH}] \mathrm{C}=\mathrm{O}$ & InChl=1S/C5H7O2/c1-5(7)3-2-4-6/h2,4H,3H2,1H3 \\
\hline 1042 & NC52ONEO2-4 & $\mathrm{CC}(=\mathrm{O}) \mathrm{CC}(\mathrm{O}[\mathrm{O}]) \mathrm{C}$ & InChl=1S/C5H9O3/c1-4(6)3-5(2)8-7/h5H,3H2,1-2H3 \\
\hline 1043 & C5H9-CDOHBO2 & $\mathrm{OCC}(\mathrm{C}(\mathrm{O}[\mathrm{O}])(\mathrm{C}) \mathrm{C}) \mathrm{O}$ & InChl=1S/C5H1104/c1-5(2,9-8)4(7)3-6/h4,6-7H,3H2,1-2H3 \\
\hline 1044 & CPND2 & $\mathrm{C} 1 \mathrm{CC}(=0) \mathrm{C}=\mathrm{C} 1$ & InChl=1S/C5H6O/c6-5-3-1-2-4-5/h1,3H,2,4H2 \\
\hline 1045 & P3E1O4OH2J & $\mathrm{CC}(\mathrm{O})=\mathrm{C}[\mathrm{CH}] \mathrm{C}=\mathrm{O}$ & InChl=1S/C5H702/c1-5(7)3-2-4-6/h2-4,7H,1H3 \\
\hline 1046 & NC52ONEO2-5 & $\mathrm{CC}(=0) \mathrm{CCC}(\mathrm{O}[\mathrm{O}])$ & $\operatorname{lnChl}=15 / C 5 H 903 / c 1-5(6) 3-2-4-8-7 / h 2-4 H 2,1 \mathrm{H3}$ \\
\hline 1047 & C5H9-ADOHBO2 & $\mathrm{OCCC}(\mathrm{O}[\mathrm{O}])(\mathrm{CO}) \mathrm{C}$ & InChl=1S/C5H11O4/c1-5(4-7,9-8)2-3-6/h6-7H,2-4H2,1H3 \\
\hline 1048 & CPND3 & $\mathrm{C} 1 \mathrm{C}(=\mathrm{O}) \mathrm{CC}=\mathrm{C} 1$ & $\operatorname{lnChl}=1 \mathrm{~S} / \mathrm{C} 5 \mathrm{H} 6 \mathrm{O} / \mathrm{c} 6-5-3-1-2-4-5 / \mathrm{h} 1-2 \mathrm{H}, 3-4 \mathrm{H} 2$ \\
\hline 1049 & P4E205OH3J & $\mathrm{CC}(=\mathrm{O})[\mathrm{CH}] \mathrm{C}=\mathrm{CO}$ & InChl=1S/C5H7O2/c1-5(7)3-2-4-6/h2-4,6H,1H3 \\
\hline 1050 & NC53ONEO2-1 & $\mathrm{C}(\mathrm{O}[\mathrm{O}]) \mathrm{CC}(=\mathrm{O}) \mathrm{CC}$ & $\operatorname{lnChl}=15 / C 5 H 9 O 3 / \mathrm{c} 1-2-5(6) 3-4-8-7 / \mathrm{h} 2-4 \mathrm{H} 2,1 \mathrm{H} 3$ \\
\hline 1051 & C5H9-BDOHAO2 & $\mathrm{OCCC}(\mathrm{CO}[\mathrm{O}])(\mathrm{O}) \mathrm{C}$ & $\operatorname{lnChl}=15 / C 5 H 11 O 4 / c 1-5(7,2-3-6) 4-9-8 / h 6-7 \mathrm{H}, 2-4 \mathrm{H} 2,1 \mathrm{H} 3$ \\
\hline 1052 & AC3H5CHCO & $\mathrm{C}=\mathrm{CCC}=\mathrm{C}=\mathrm{O}$ & $\operatorname{lnChl}=1 \mathrm{~S} / \mathrm{C} 5 \mathrm{H} 6 \mathrm{O} / \mathrm{c} 1-2-3-4-5-6 / \mathrm{h} 2,4 \mathrm{H}, 1,3 \mathrm{H} 2$ \\
\hline 1053 & BC5H7-DOHACHO & $\mathrm{CC}(=\mathrm{CCO}) \mathrm{C}=\mathrm{O}$ & InChl=1S/C5H8O2/c1-5(4-7)2-3-6/h2,4,6H,3H2,1H3 \\
\hline 1054 & NC53ONEO2-2 & $\mathrm{CC}(\mathrm{O}[\mathrm{O}]) \mathrm{C}(=\mathrm{O}) \mathrm{CC}$ & InChl=1S/C5H9O3/c1-3-5(6)4(2)8-7/h4H,3H2,1-2H3 \\
\hline 1055 & $\mathrm{C} 5 \mathrm{H} 10 \mathrm{OOH} 2-1 \mathrm{O} 2$ & $\operatorname{cccc}(\mathrm{OO}) \mathrm{C}(\mathrm{O}[\mathrm{O}])$ & InChl=1S/C5H11O4/c1-2-3-5(9-7)4-8-6/h5,7H,2-4H2,1H3 \\
\hline 1056 & C5Y3D14 & $\mathrm{C}=\mathrm{CC}(=\mathrm{O}) \mathrm{C}=\mathrm{C}$ & InChl=1S/C5H6O/c1-3-5(6)4-2/h3-4H,1-2H2 \\
\hline 1057 & BCSH8-DOHAOOH & $\mathrm{OOCC}(=\mathrm{CCO}) \mathrm{C}$ & InChl=15/C5H10O3/c1-5(2-3-6)4-8-7/h2,6-7H,3-4H2,1H3 \\
\hline 1058 & C5H10OOH1-2O2 & $\operatorname{cccc}(\mathrm{O}[\mathrm{O}]) \mathrm{COO}$ & InChl=1S/C5H11O4/c1-2-3-5(9-7)4-8-6/h5-6H,2-4H2,1H3 \\
\hline 1059 & $\mathrm{C} 5 \mathrm{H} 6 \mathrm{O}$ & $\mathrm{C \# CC}(\mathrm{C}) \mathrm{C}=\mathrm{O}$ & InChl=1S/C5H6O/c1-3-5(2)4-6/h1,4-5H,2H3 \\
\hline 1060 & AC5H8-DOHCOOH & $\mathrm{OCC}(\mathrm{C}(=\mathrm{C}) \mathrm{C}) \mathrm{OO}$ & $\operatorname{lnChl}=15 / \mathrm{C} 5 \mathrm{H} 1003 / \mathrm{c} 1-4(2) 5(3-6) 8-7 / \mathrm{h} 5-7 \mathrm{H}, 1,3 \mathrm{H} 2,2 \mathrm{H3}$ \\
\hline 1061 & $\mathrm{C} 5 \mathrm{H} 10 \mathrm{OOH} 3-1 \mathrm{O} 2$ & $\mathrm{CCC}(\mathrm{OO}) \mathrm{CC}(\mathrm{O}[\mathrm{O}])$ & InChl=1S/C5H11O4/c1-2-5(9-7)3-4-8-6/h5,7H,2-4H2,1H3 \\
\hline 1062 & MF2 & $\mathrm{CC} 1=\mathrm{CC}=\mathrm{CO} 1$ & InChl=1S/C5H6O/c1-5-3-2-4-6-5/h2-4H,1H3 \\
\hline 1063 & CC5H8-DOHBOOH & $\mathrm{OC}=\mathrm{CC}(\mathrm{OO})(\mathrm{C}) \mathrm{C}$ & $\operatorname{lnChl}=1 \mathrm{~S} / \mathrm{C} 5 \mathrm{H} 1003 / \mathrm{c} 1-5(2,8-7) 3-4-6 / \mathrm{h} 3-4,6-7 \mathrm{H}, 1-2 \mathrm{H} 3$ \\
\hline 1064 & C5H10OOH1-302 & $\mathrm{CCC}(\mathrm{O}[\mathrm{O}]) \mathrm{CCOO}$ & $\operatorname{lnChl}=15 / C 5 H 1104 / c 1-2-5(9-7) 3-4-8-6 / h 5-6 \mathrm{H}, 2-4 \mathrm{H} 2,1 \mathrm{H} 3$ \\
\hline 1065 & MF2ACAR5 & $\mathrm{CC} 1=\mathrm{CC}[\mathrm{C}] \mathrm{O} 1$ & InChl=1S/C5H6O/c1-5-3-2-4-6-5/h3H,2H2,1H3 \\
\hline 1066 & BC5H8-DOHDOOH & $\mathrm{OOCC}(\mathrm{C}=\mathrm{C}(\mathrm{C}) \mathrm{C}) \mathrm{O}$ & InChl=1S/C5H1003/c1-4(2)3-5(6)8-7/h3,5-7H,1-2H3 \\
\hline 1067 & $\mathrm{C} 5 \mathrm{H} 10 \mathrm{OOH} 3-2 \mathrm{O} 2$ & $\operatorname{CCC}(0 O) C(O[O]) C$ & InChl=1S/C5H1104/c1-3-5(9-7)4(2)8-6/h4-5,7H,3H2,1-2H3 \\
\hline 1068 & P23DE1O-T & $\mathrm{CC}=\mathrm{C}=\mathrm{CC}=\mathrm{O}$ & InChl=1S/C5H6O/c1-2-3-4-5-6/h2,4-5H,1H3 \\
\hline 1069 & AC5H8DOH-A2OOH & $\mathrm{C}=\mathrm{C}(\mathrm{COO}) \mathrm{CCO}$ & InChl=1S/C5H10O3/c1-5(2-3-6)4-8-7/h6-7H,1-4H2 \\
\hline 1070 & $\mathrm{C} 5 \mathrm{H} 10 \mathrm{OOH} 2-3 \mathrm{O} 2$ & $\operatorname{CCC}(\mathrm{O}[\mathrm{O}]) \mathrm{C}(\mathrm{OO}) \mathrm{C}$ & InChl=1S/C5H11O4/c1-3-5(9-7)4(2)8-6/h4-6H,3H2,1-2H3 \\
\hline 1071 & P4Y2O & $\mathrm{CC}(=\mathrm{O}) \mathrm{CCHC}$ & InChl=15/C5H6O/c1-3-4-5(2)6/h1H,4H2,2H3 \\
\hline 1072 & AC5H8DOH-A2VO & $\mathrm{C}=\mathrm{C}(\mathrm{C}=\mathrm{O}) \mathrm{CCO}$ & InChl=1S/C5H8O2/c1-5(4-7)2-3-6/h4,6H,1-3H2 \\
\hline 1073 & NC5KET12 & $\operatorname{cccc}(00) \mathrm{C}=0$ & InChl=1S/C5H10O3/c1-2-3-5(4-6)8-7/h4-5,7H,2-3H2,1H3 \\
\hline 1074 & $\mathrm{C} 5 \mathrm{H} 10 \mathrm{OOH} 2-4 \mathrm{O} 2$ & $\mathrm{CC}(\mathrm{O}[\mathrm{O}]) \mathrm{CC}(\mathrm{OO}) \mathrm{C}$ & InChl=15/C5H11O4/c1-4(8-6)3-5(2)9-7/h4-6H,3H2,1-2H3 \\
\hline 1075 & $\mathrm{C} 5 \mathrm{H} 5 \mathrm{OH}$ & $\mathrm{C} 1(\mathrm{O}) \mathrm{C}=\mathrm{CC}=\mathrm{C} 1$ & InChl=1S/C5H6O/c6-5-3-1-2-4-5/h1-6H \\
\hline 1076 & $\mathrm{IC5CHO1-3O}$ & $\mathrm{O}=\mathrm{CC} 10 \mathrm{C} 1(\mathrm{C}) \mathrm{C}$ & $\operatorname{lnChl}=1 \mathrm{~S} / \mathrm{C} 5 \mathrm{H} 8 \mathrm{O} 2 / \mathrm{c} 1-5(2) 4(3-6) 7-5 / \mathrm{h} 3-4 \mathrm{H}, 1-2 \mathrm{H} 3$ \\
\hline 1077 & NC5KET21 & $\operatorname{cccC}(=0) \operatorname{coO}$ & InChl=1S/C5H1003/c1-2-3-5(6)4-8-7/h7H,2-4H2,1H3 \\
\hline 1078 & $\mathrm{C} 5 \mathrm{H} 100 \mathrm{OH} 2-5 \mathrm{O} 2$ & $\mathrm{C}(\mathrm{O}[\mathrm{O}]) \mathrm{CCC}(\mathrm{OO}) \mathrm{C}$ & InChl=1S/C5H11O4/c1-5(9-7)3-2-4-8-6/h5,7H,2-4H2,1H3 \\
\hline 1079 & CVCCVCCVO & $\mathrm{C}=\mathrm{CC}=\mathrm{CC}=\mathrm{O}$ & $\operatorname{lnChl}=1 \mathrm{~S} / \mathrm{C} 5 \mathrm{H} 6 \mathrm{O} / \mathrm{c1} 1-2-3-4-5-6 / \mathrm{h} 2-5 \mathrm{H}, 1 \mathrm{H} 2$ \\
\hline 1080 & B13DE2MOOH & $\mathrm{OOCC}(=\mathrm{C}) \mathrm{C}=\mathrm{C}$ & $\operatorname{lnChl}=1 \mathrm{~S} / \mathrm{C} 5 \mathrm{H} 8 \mathrm{O} 2 / \mathrm{c1}-3-5(2) 4-7-6 / \mathrm{h} 3,6 \mathrm{H}, 1-2,4 \mathrm{H} 2$ \\
\hline 1081 & NC5KET31 & $\mathrm{CCC}(=0) \mathrm{CCOO}$ & $\operatorname{lnChl}=1 \mathrm{~S} / \mathrm{C} 5 \mathrm{H} 1003 / \mathrm{c1}-2-5(6) 3-4-8-7 / \mathrm{h} 7 \mathrm{H}, 2-4 \mathrm{H} 2,1 \mathrm{H} 3$ \\
\hline 1082 & C5H10OOH1-4O2 & $\mathrm{CC}(\mathrm{O}[0]) \mathrm{CCCOO}$ & InChl=1S/C5H11O4/c1-5(9-7)3-2-4-8-6/h5-6H,2-4H2,1H3 \\
\hline 1083 & NC5D12Y4 & $\mathrm{C}=\mathrm{C}=\mathrm{CC}(=\mathrm{O}) \mathrm{C}$ & $\operatorname{lnChl}=1 \mathrm{~S} / \mathrm{C5} \mathrm{H} 6 \mathrm{O} / \mathrm{c} 1-3-4-5(2) 6 / \mathrm{h} 4 \mathrm{H}, 1 \mathrm{H} 2,2 \mathrm{H} 3$ \\
\hline 1084 & CVCCVCCOO & $\mathrm{OOCC}=\mathrm{CC}=\mathrm{C}$ & $\operatorname{lnChl}=15 / C 5 H 802 / c 1-2-3-4-5-7-6 / h 2-4,6 \mathrm{H}, 1,5 \mathrm{H} 2$ \\
\hline 1085 & NC5KET13 & $\mathrm{CCC}(\mathrm{OO}) \mathrm{CC}=0$ & InChl=1S/C5H10O3/c1-2-5(8-7)3-4-6/h4-5,7H,2-3H2,1H3 \\
\hline 1086 & C5H10OOH1-5O2 & $\mathrm{C}(\mathrm{O}[\mathrm{O}]) \mathrm{CCCCOO}$ & $\operatorname{lnChl}=1 \mathrm{~S} / \mathrm{C} 5 \mathrm{H} 11 \mathrm{O} 4 / \mathrm{c} 6-8-4-2-1-3-5-9-7 / \mathrm{h} 6 \mathrm{H}, 1-5 \mathrm{H} 2$ \\
\hline 1087 & IC5D2Y4-4J & $C[C](C=C=O) C$ & $\operatorname{lnChl}=1 \mathrm{~S} / \mathrm{C} 5 \mathrm{H} 7 \mathrm{O} / \mathrm{c1}-5(2) 3-4-6 / \mathrm{h} 3 \mathrm{H}, 1-2 \mathrm{H} 3$ \\
\hline 1088 & $\mathrm{CVCC}(\mathrm{OO}) \mathrm{CVC}$ & $\mathrm{OOC}(\mathrm{C}=\mathrm{C}) \mathrm{C}=\mathrm{C}$ & $\operatorname{lnChl}=1 \mathrm{~S} / \mathrm{C} 5 \mathrm{H} 8 \mathrm{OO} / \mathrm{c} 1-3-5(4-2) 7-6 / \mathrm{h} 3-6 \mathrm{H}, 1-2 \mathrm{H} 2$ \\
\hline
\end{tabular}




\begin{tabular}{|c|c|c|c|}
\hline 1089 & NC5KET32 & $\operatorname{ccc}(=0) c(00) c$ & InChl=1S/C5H1003/c1-3-5(6)4(2)8-7/h4,7H,3H2,1-2H3 \\
\hline 1090 & C5H91-2,30OH & $\operatorname{CCC}(\mathrm{C}([\mathrm{CH} 2]) 00) 00$ & $\operatorname{lnChl}=15 / \mathrm{C5} 11 \mathrm{OO} 4 / \mathrm{c} 1-3-5(9-7) 4(2) 8-6 / \mathrm{h} 4-7 \mathrm{H}, 2-3 \mathrm{H} 2,1 \mathrm{H} 3$ \\
\hline 1091 & IC5D2Y4-1J & $\mathrm{CC}(=\mathrm{C}) \mathrm{C}=\mathrm{C}[\mathrm{O}]$ & InChl=1S/C5H7O/c1-5(2)3-4-6/h3-4H,1H2,2H3 \\
\hline 1092 & CYC5E1-4O2H & $\mathrm{OOC1CC}=\mathrm{CC} 1$ & InChl=1S/C5H8O2/c6-7-5-3-1-2-4-5/h1-2,5-6H,3-4H2 \\
\hline 1093 & NC5KET23 & $\operatorname{ccc}(\mathrm{OOO}) \mathrm{C}(=0) \mathrm{C}$ & InChl=1S/C5H10O3/c1-3-5(8-7)4(2)6/h5,7H,3H2,1-2H3 \\
\hline 1094 & C5H91-2,40OH & {$[\mathrm{CH} 2] \mathrm{C}(\mathrm{CC}(\mathrm{C}) \mathrm{OO}) \mathrm{OO}$} & $\operatorname{lnChl}=1 \mathrm{~S} / \mathrm{C5} 11104 / \mathrm{c} 1-4(8-6) 3-5(2) 9-7 / \mathrm{h} 4-7 \mathrm{H}, 1,3 \mathrm{H} 2,2 \mathrm{H} 3$ \\
\hline 1095 & ACC5H7-DOH-1AR & {$[\mathrm{CH} 2] \mathrm{C}(=\mathrm{C}) \mathrm{C}=\mathrm{CO}$} & InChl=1S/C5H7O/c1-5(2)3-4-6/h3-4,6H,1-2H2 \\
\hline 1096 & CYC5E1-302H & OOC1CCC=C1 & InChl=1S/C5H8O2/c6-7-5-3-1-2-4-5/h1,3,5-6H,2,4H2 \\
\hline 1097 & ICSKETDC & $\mathrm{CC}(\mathrm{C}) \mathrm{C}(\mathrm{OO}) \mathrm{C}=\mathrm{O}$ & InChl=1S/C5H1003/c1-4(2)5(3-6)8-7/h3-5,7H,1-2H3 \\
\hline 1098 & C5H91-2,50OH & {$[\mathrm{CH} 2] \mathrm{C}(\mathrm{CCCOO}) \mathrm{OO}$} & InChl=1S/C5H1104/c1-5(9-7)3-2-4-8-6/h5-7H,1-4H2 \\
\hline 1099 & C4H6CHO2-4 & $\mathrm{CC}=\mathrm{CC}=\mathrm{C}[\mathrm{O}]$ & $\operatorname{lnChl}=15 / C 5 H 70 / c 1-2-3-4-5-6 / \mathrm{h} 2-5 \mathrm{H}, 1 \mathrm{H} 3$ \\
\hline 1100 & CHOCCCCHO & $\mathrm{O}=\mathrm{CCCCC}=\mathrm{O}$ & InChl=1S/C5H8O2/c6-4-2-1-3-5-7/h4-5H,1-3H2 \\
\hline 1101 & NC5KET24 & $\mathrm{CC}(\mathrm{OO}) \mathrm{CC}(=\mathrm{O}) \mathrm{C}$ & $\operatorname{lnChl}=15 / C 5 H 1003 / c 1-4(6) 3-5(2) 8-7 / \mathrm{h} 5,7 \mathrm{H}, 3 \mathrm{H} 2,1-2 \mathrm{H} 3$ \\
\hline 1102 & C5H91-3,40OH & {$[\mathrm{CH} 2] \mathrm{CC}(\mathrm{C}(\mathrm{C}) \mathrm{OO}) \mathrm{OO}$} & InChl=15/C5H1104/c1-3-5(9-7)4(2)8-6/h4-7H,1,3H2,2H3 \\
\hline 1103 & CVCCVCCOJ & {$[\mathrm{O}] \mathrm{CC}=\mathrm{CC}=\mathrm{C}$} & InChl=1S/C5H7O/c1-2-3-4-5-6/h2-4H,1,5H2 \\
\hline 1104 & MB2D & $\operatorname{COC}(=0) \mathrm{C}=\mathrm{CC}$ & InChl=1S/C5H8O2/c1-3-4-5(6)7-2/h3-4H,1-2H3 \\
\hline 1105 & IC5KETDB & $\mathrm{CC}(\mathrm{OO})(\mathrm{C}) \mathrm{CC}=\mathrm{O}$ & $\operatorname{lnChl}=15 / C 5 H 1003 / c 1-5(2,8-7) 3-4-6 / h 4,7 \mathrm{H}, 3 \mathrm{H} 2,1-2 \mathrm{H} 3$ \\
\hline 1106 & C5H91-3,50OH & {$[\mathrm{CH} 2] \mathrm{CC}(\mathrm{CCOO}) \mathrm{OO}$} & InChl=1S/C5H1104/c1-2-5(9-7)3-4-8-6/h5-7H,1-4H2 \\
\hline 1107 & $\mathrm{C} 4 \mathrm{H} 6 \mathrm{CHO}-43$ & $\mathrm{C}=\mathrm{C}[\mathrm{CH}] \mathrm{CC}=\mathrm{O}$ & InChl=1S/C5H7O/c1-2-3-4-5-6/h2-3,5H,1,4H2 \\
\hline 1108 & MB3D & $\mathrm{COC}(=\mathrm{O}) \mathrm{CC}=\mathrm{C}$ & InChl=15/C5H8O2/c1-3-4-5(6)7-2/h3H,1,4H2,2H3 \\
\hline 1109 & IC5KETDA & $\mathrm{C}(\mathrm{OO}) \mathrm{C}(\mathrm{C}) \mathrm{CC}=\mathrm{O}$ & InChl=1S/C5H1003/c1-5(2-3-6)4-8-7/h3,5,7H,2,4H2,1H3 \\
\hline 1110 & C5H91-4,50OH & {$[\mathrm{CH} 2] \mathrm{CCC}(\mathrm{COO}) 0 \mathrm{O}$} & InChl=1S/C5H1104/c1-2-3-5(9-7)4-8-6/h5-7H,1-4H2 \\
\hline 1111 & C5D1CHO-4R & $\mathrm{C}=\mathrm{CCC}=\mathrm{C}[\mathrm{O}]$ & $\operatorname{lnChl}=1 \mathrm{~S} / \mathrm{C} 5 \mathrm{H} 7 \mathrm{O} / \mathrm{c} 1-2-3-4-5-6 / \mathrm{h} 2,4-5 \mathrm{H}, 1,3 \mathrm{H} 2$ \\
\hline 1112 & NC5DIONE13 & $C(=0) C C(=0) C C$ & InChl=1S/C5H8O2/c1-2-5(7)3-4-6/h4H,2-3H2,1H3 \\
\hline 1113 & NC5KET25 & $\mathrm{C}(\mathrm{OOO}) \mathrm{CCC}(=0) \mathrm{C}$ & InChl=15/C5H1003/c1-5(6)3-2-4-8-7/h7H,2-4H2,1H3 \\
\hline 1114 & $\mathrm{C} 5 \mathrm{H} 92-1,30 \mathrm{OH}$ & $\mathrm{CCC}([\mathrm{CH}] \mathrm{COO}) \mathrm{OO}$ & InChl=1S/C5H1104/c1-2-5(9-7)3-4-8-6/h3,5-7H,2,4H2,1H3 \\
\hline 1115 & C5Y2D4-1R & {$[\mathrm{CH} 2] \mathrm{C}(=\mathrm{O}) \mathrm{CC}=\mathrm{C}$} & $\operatorname{lnChl}=1 \mathrm{~S} / \mathrm{C} 5 \mathrm{H} 7 \mathrm{O} / \mathrm{c1} 1-3-4-5(2) 6 / \mathrm{h} 3 \mathrm{H}, 1-2,4 \mathrm{H} 2$ \\
\hline 1116 & NC5DIONE24 & $\mathrm{CC}(=0) \mathrm{CC}(=0) \mathrm{C}$ & $\mathrm{InChl}=1 \mathrm{~S} / \mathrm{C} 5 \mathrm{H} 8 \mathrm{O} 2 / \mathrm{c} 1-4(6) 3-5(2) 7 / \mathrm{h} 3 \mathrm{H} 2,1-2 \mathrm{H} 3$ \\
\hline 1117 & NC5KET14 & $\mathrm{CC}(\mathrm{OO}) \mathrm{CCC}=\mathrm{O}$ & InChl=1S/C5H10O3/c1-5(8-7)3-2-4-6/h4-5,7H,2-3H2,1H3 \\
\hline 1118 & C5H92-1,40OH & $\mathrm{CC}(\mathrm{C}[\mathrm{CH}] \mathrm{COO}) \mathrm{OO}$ & InChl=1S/C5H1104/c1-5(9-7)3-2-4-8-6/h2,5-7H,3-4H2,1H3 \\
\hline 1119 & B13DE2MOJ & {$[\mathrm{O}] \mathrm{CC}(=\mathrm{C}) \mathrm{C}=\mathrm{C}$} & InChl=1S/C5H7O/c1-3-5(2)4-6/h3H,1-2,4H2 \\
\hline 1120 & BCSH8-DOHAOJ & $\mathrm{OCC}=\mathrm{C}(\mathrm{C}[\mathrm{O}]) \mathrm{C}$ & InChl=1S/C5H9O2/c1-5(4-7)2-3-6/h2,6H,3-4H2,1H3 \\
\hline 1121 & NC5KET15 & $\mathrm{C}(\mathrm{OO}) \mathrm{CCCC}=\mathrm{O}$ & InChl=1S/C5H1003/c6-4-2-1-3-5-8-7/h4,7H,1-3,5H2 \\
\hline 1122 & C5H92-1,50OH & $\mathrm{C}([\mathrm{CH}] \mathrm{COO}) \mathrm{CCOO}$ & InChl=1S/C5H1104/c6-8-4-2-1-3-5-9-7/h2,6-7H,1,3-5H2 \\
\hline 1123 & $\mathrm{CVCC}(\mathrm{OJ}) \mathrm{CVC}$ & $\mathrm{C}=\mathrm{CC}(\mathrm{C}=\mathrm{C})[\mathrm{O}]$ & InChl $=1 \mathrm{~S} / \mathrm{C} 5 \mathrm{H} 7 \mathrm{O} / \mathrm{c1} 1-3-5(6) 4-2 / \mathrm{h} 3-5 \mathrm{H}, 1-2 \mathrm{H} 2$ \\
\hline 1124 & AC5H8-DOHCOJ & $\mathrm{CC}(=\mathrm{C}) \mathrm{C}(\mathrm{CO})[\mathrm{O}]$ & InChl=1S/C5H9O2/c1-4(2)5(7)3-6/h5-6H,1,3H2,2H3 \\
\hline 1125 & IC5KETAD & $\mathrm{CC}(\mathrm{CCOO}) \mathrm{C}=\mathrm{O}$ & InChl=15/C5H10O3/c1-5(4-6)2-3-8-7/h4-5,7H,2-3H2,1H3 \\
\hline 1126 & $\mathrm{C} 5 \mathrm{H} 92-3,4 \mathrm{OOH}$ & $\mathrm{C}[\mathrm{CH}] \mathrm{C}(\mathrm{C}(\mathrm{C}) \mathrm{OO}) \mathrm{OO}$ & InChl=1S/C5H1104/c1-3-5(9-7)4(2)8-6/h3-7H,1-2H3 \\
\hline 1127 & CYC5E1-40 & {$[\mathrm{O}] \mathrm{C} 1 \mathrm{CC}=\mathrm{CC} 1$} & $\operatorname{lnChl}=15 / C 5 H 70 / c 6-5-3-1-2-4-5 / h 1-2,5 \mathrm{H}, 3-4 \mathrm{H} 2$ \\
\hline 1128 & BC5H8-DOHDOJ & $\mathrm{OC}(\mathrm{C}=\mathrm{C}(\mathrm{C}) \mathrm{C})[\mathrm{O}]$ & InChl=1S/C5H9O2/c1-4(2)3-5(6)7/h3,5-6H,1-2H3 \\
\hline 1129 & ICSKETAC & $\mathrm{CC}(\mathrm{C}=\mathrm{O}) \mathrm{C}(\mathrm{C}) \mathrm{OO}$ & InChl=1S/C5H1003/c1-4(3-6)5(2)8-7/h3-5,7H,1-2H3 \\
\hline 1130 & C5H92-3,50OH & $\mathrm{C}[\mathrm{CH}] \mathrm{C}(\mathrm{CCOO}) \mathrm{OO}$ & InChl=1S/C5H1104/c1-2-5(9-7)3-4-8-6/h2,5-7H,3-4H2,1H3 \\
\hline 1131 & CPN-2R & $\mathrm{C} 1[\mathrm{CH}] \mathrm{C}(=\mathrm{O}) \mathrm{CC} 1$ & InChl=1S/C5H7O/c6-5-3-1-2-4-5/h3H,1-2,4H2 \\
\hline 1132 & CC5H8-DOHBOJ & $\mathrm{OC}=\mathrm{CC}([\mathrm{O}])(\mathrm{C}) \mathrm{C}$ & InChl=1S/C5H9O2/c1-5(2,7)3-4-6/h3-4,6H,1-2H3 \\
\hline 1133 & IC5KETAB & $\operatorname{ccC}(C)(C=0) 00$ & InChl=15/C5H10O3/c1-3-5(2,4-6)8-7/h4,7H,3H2,1-2H3 \\
\hline 1134 & C5H92-4,50OH & $\mathrm{C}[\mathrm{CH}] \mathrm{CC}(\mathrm{COO}) \mathrm{OO}$ & InChl=1S/C5H1104/c1-2-3-5(9-7)4-8-6/h2,5-7H,3-4H2,1H3 \\
\hline 1135 & CPN-3R & $\mathrm{C} 1 \mathrm{CC}(=\mathrm{O}) \mathrm{C}[\mathrm{CH}] 1$ & $\operatorname{lnChl}=1 \mathrm{~S} / \mathrm{C} 5 \mathrm{H} 7 \mathrm{O} / \mathrm{c} 6-5-3-1-2-4-5 / \mathrm{h} 1 \mathrm{H}, 2-4 \mathrm{H} 2$ \\
\hline 1136 & AC5H8DOH-A2OJ & $\mathrm{C}=\mathrm{C}(\mathrm{C}[\mathrm{O}]) \mathrm{CCO}$ & InChl=1S/C5H9O2/c1-5(4-7)2-3-6/h6H,1-4H2 \\
\hline 1137 & IC5KETAA & $\operatorname{ccc}(\mathrm{C}=0) \operatorname{coO}$ & InChl=1S/C5H1003/c1-2-5(3-6)4-8-7/h3,5,7H,2,4H2,1H3 \\
\hline 1138 & C5H93-1,20OH & $\mathrm{CC}[\mathrm{CH}] \mathrm{C}(\mathrm{COO}) \mathrm{OO}$ & InChl=1S/C5H1104/c1-2-3-5(9-7)4-8-6/h3,5-7H,2,4H2,1H3 \\
\hline 1139 & C5Y3D1-5R & {$[\mathrm{CH} 2] \mathrm{CC}(=\mathrm{O}) \mathrm{C}=\mathrm{C}$} & InChl=1S/C5H7O/c1-3-5(6)4-2/h3H,1-2,4H2 \\
\hline 1140 & IC5Y4OH2-3J & {$[\mathrm{O}] \mathrm{C}=\mathrm{CC}(\mathrm{O})(\mathrm{C}) \mathrm{C}$} & InChl=1S/C5H9O2/c1-5(2,7)3-4-6/h3-4,7H,1-2H3 \\
\hline 1141 & C5H9O1-2OOH-3 & $\mathrm{CCC}(\mathrm{C} 1 \mathrm{CO} 1) 0 \mathrm{O}$ & InChl=1S/C5H10O3/c1-2-4(8-6)5-3-7-5/h4-6H,2-3H2,1H3 \\
\hline 1142 & C5H93-1,40OH & $\mathrm{CC}([\mathrm{CH}] \mathrm{CCOO}) \mathrm{OO}$ & InChl=1S/C5H1104/c1-5(9-7)3-2-4-8-6/h3,5-7H,2,4H2,1H3 \\
\hline 1143 & PC3H6CHCO & {$[\mathrm{CH} 2] \mathrm{CCC}=\mathrm{C}=\mathrm{O}$} & $\operatorname{lnChl}=1 \mathrm{~S} / \mathrm{C} 5 \mathrm{H} 7 \mathrm{O} / \mathrm{c1}-2-3-4-5-6 / \mathrm{h} 4 \mathrm{H}, 1-3 \mathrm{H} 2$ \\
\hline 1144 & IC5Y4OH3-2J & $\mathrm{O}=\mathrm{CC}([\mathrm{C}](\mathrm{C}) \mathrm{C}) \mathrm{O}$ & InChl=1S/C5H9O2/c1-4(2)5(7)3-6/h3,5,7H,1-2H3 \\
\hline 1145 & C5H9O1-20OH-4 & $\mathrm{CC}(\mathrm{CC} 1 \mathrm{CO}) \mathrm{O}) \mathrm{OO}$ & InChl=1S/C5H10O3/c1-4(8-6)2-5-3-7-5/h4-6H,2-3H2,1H3 \\
\hline 1146 & C5H93-1,50OH & {$[\mathrm{CH}](\mathrm{CCOO}) \mathrm{CCOO}$} & $\operatorname{lnChl}=15 / C 5 H 1104 / c 6-8-4-2-1-3-5-9-7 / \mathrm{h} 1,6-7 \mathrm{H}, 2-5 \mathrm{H} 2$ \\
\hline 1147 & C5Y1D2-1R & $\mathrm{CCC}=\mathrm{C}[\mathrm{C}](=\mathrm{O})$ & $\operatorname{lnChl}=1 \mathrm{~S} / \mathrm{C} 5 \mathrm{H} 7 \mathrm{O} / \mathrm{c1}-2-3-4-5-6 / \mathrm{h} 3-4 \mathrm{H}, 2 \mathrm{H} 2,1 \mathrm{H} 3$ \\
\hline 1148 & C5H81OOH5-3 & $\mathrm{OOCC}[\mathrm{CH}] \mathrm{C}=\mathrm{C}$ & InChl=15/C5H9O2/c1-2-3-4-5-7-6/h2-3,6H,1,4-5H2 \\
\hline 1149 & C5H9O1-2OOH-5 & $\mathrm{C}(\mathrm{CC} 1 \mathrm{CO} 1) \mathrm{COO}$ & $\operatorname{lnChl}=1 \mathrm{~S} / \mathrm{C} 5 \mathrm{H} 1003 / \mathrm{c} 6-8-3-1-2-5-4-7-5 / \mathrm{h} 5-6 \mathrm{H}, 1-4 \mathrm{H} 2$ \\
\hline 1150 & C5H93-2,40OH & $\mathrm{CC}([\mathrm{CH}] \mathrm{C}(\mathrm{C}) \mathrm{OO}) \mathrm{OO}$ & InChl=15/C5H1104/c1-4(8-6)3-5(2)9-7/h3-7H,1-2H3 \\
\hline 1151 & $\mathrm{C} 5 \mathrm{H} 73 \mathrm{JO} 12$ & {$[\mathrm{CH}](\mathrm{O} 1) \mathrm{C} 1 \mathrm{C}=\mathrm{CC}$} & InChl $=1 \mathrm{~S} / \mathrm{C} 5 \mathrm{H} 7 \mathrm{O} / \mathrm{c1} 1-2-3-5-4-6-5 / \mathrm{h} 2-5 \mathrm{H}, 1 \mathrm{H} 3$ \\
\hline 1152 & C5H91-5O2 & {$[\mathrm{O}] \mathrm{OCCCC}=\mathrm{C}$} & InChl=1S/C5H9O2/c1-2-3-4-5-7-6/h2H,1,3-5H2 \\
\hline 1153 & C5H9O1-30OH-2 & $\mathrm{CCC1C( \textrm {CO } ) 0 0}$ & InChl=1S/C5H10O3/c1-2-4-5(8-6)3-7-4/h4-6H,2-3H2,1H3 \\
\hline 1154 & AC5H10OOH-AO2 & $\mathrm{CCC}(\mathrm{COO}) \mathrm{CO}[\mathrm{O}]$ & InChl=1S/C5H1104/c1-2-5(3-8-6)4-9-7/h5-6H,2-4H2,1H3 \\
\hline 1155 & CYC-C5H8O2J & {$[\mathrm{CH}](\mathrm{O} 1) \mathrm{C} 1 \mathrm{CC}=\mathrm{C}$} & $\operatorname{lnChl}=1 \mathrm{~S} / \mathrm{C} 5 \mathrm{H} 7 \mathrm{O} / \mathrm{c1} 1-2-3-5-4-6-5 / \mathrm{h} 2,4-5 \mathrm{H}, 1,3 \mathrm{H} 2$ \\
\hline 1156 & MBMJ & {$[\mathrm{CH} 2] \mathrm{OC}(=\mathrm{O}) \mathrm{CCC}$} & InChl=1S/C5H9O2/c1-3-4-5(6)7-2/h2-4H2,1H3 \\
\hline 1157 & C5H9O1-30OH-4 & $\mathrm{CC}(\mathrm{C} 1 \mathrm{CCO}) \mathrm{OO}$ & InChl=1S/C5H10O3/c1-4(8-6)5-2-3-7-5/h4-6H,2-3H2,1H3 \\
\hline 1158 & AC5H10OOH-BO2 & $\mathrm{CCC}(\mathrm{C})(\mathrm{COO}) \mathrm{O}[\mathrm{O}]$ & InChl=1S/C5H11O4/c1-3-5(2,9-7)4-8-6/h6H,3-4H2,1-2H3 \\
\hline 1159 & EJVK-SA & $\mathrm{C}[\mathrm{CH}] \mathrm{C}(=\mathrm{O}) \mathrm{C}=\mathrm{C}$ & $\operatorname{lnChl}=1 \mathrm{~S} / \mathrm{C} 5 \mathrm{H} 7 \mathrm{O} / \mathrm{c} 1-3-5(6) 4-2 / \mathrm{h} 3-4 \mathrm{H}, 1 \mathrm{H} 2,2 \mathrm{H} 3$ \\
\hline 1160 & MB2J & $\mathrm{COC}(=\mathrm{O})[\mathrm{CH}] \mathrm{CC}$ & InChl=1S/C5H9O2/c1-3-4-5(6)7-2/h4H,3H2,1-2H3 \\
\hline 1161 & C5H9O1-30OH-5 & $\mathrm{C} 1 \mathrm{COC} 1 \mathrm{CCOO}$ & $\operatorname{lnChl}=1 \mathrm{~S} / \mathrm{C} 5 \mathrm{H} 1003 / \mathrm{c6}-8-4-2-5-1-3-7-5 / \mathrm{h} 5-6 \mathrm{H}, 1-4 \mathrm{H} 2$ \\
\hline 1162 & AC5H10OOH-CO2 & $\mathrm{CC}(\mathrm{COO}) \mathrm{C}(\mathrm{C}) \mathrm{O}[\mathrm{O}]$ & InChl=15/C5H11O4/c1-4(3-8-6)5(2)9-7/h4-6H,3H2,1-2H3 \\
\hline 1163 & EVJK-P & $\mathrm{CCC}(=\mathrm{O}) \mathrm{C}=[\mathrm{CH}]$ & InChl=1S/C5H7O/c1-3-5(6)4-2/h1,3H,4H2,2H3 \\
\hline 1164 & MB3」 & $\mathrm{COC}(=\mathrm{O}) \mathrm{C}[\mathrm{CH}] \mathrm{C}$ & InChl=1S/C5H9O2/c1-3-4-5(6)7-2/h3H,4H2,1-2H3 \\
\hline 1165 & C5H9O1-4OOH-2 & CC1CC(CO1)OO & InChl=1S/C5H10O3/c1-4-2-5(8-6)3-7-4/h4-6H,2-3H2,1H3 \\
\hline 1166 & AC5H10OOH-DO2 & $\mathrm{CC}(\mathrm{CCO}[\mathrm{O}]) \mathrm{COO}$ & InChl=1S/C5H11O4/c1-5(4-9-7)2-3-8-6/h5,7H,2-4H2,1H3 \\
\hline 1167 & EVJK-S & $\operatorname{cCC}(=O)[C]=C$ & InChl=1S/C5H7O/c1-3-5(6)4-2/h1,4H2,2H3 \\
\hline 1168 & MB4J & $\mathrm{COC}(=0) \mathrm{CC}[\mathrm{CH} 2]$ & $\operatorname{lnChl}=1 \mathrm{~S} / \mathrm{C} 5 \mathrm{H} 9 \mathrm{O} 2 / \mathrm{c} 1-3-4-5(6) 7-2 / \mathrm{h} 1,3-4 \mathrm{H} 2,2 \mathrm{H} 3$ \\
\hline 1169 & C5H9O1-4OOH-3 & $\mathrm{CC} 1 \mathrm{C}(\mathrm{CCO}) \mathrm{OO}$ & InChl=1S/C5H10O3/c1-4-5(8-6)2-3-7-4/h4-6H,2-3H2,1H3 \\
\hline 1170 & BC5H10OOH-AO2 & $\mathrm{CCC}(\mathrm{C})(\mathrm{CO}[\mathrm{O}]) 0 \mathrm{O}$ & InChl=1S/C5H11O4/c1-3-5(2,9-7)4-8-6/h7H,3-4H2,1-2H3 \\
\hline 1171 & $\mathrm{MF} 22 \mathrm{H}$ & $\mathrm{CC} 1[\mathrm{CH}] \mathrm{C}=\mathrm{CO} 1$ & InChl $=1 \mathrm{~S} / \mathrm{C} 5 \mathrm{H} 7 \mathrm{O} / \mathrm{c1} 1-5-3-2-4-6-5 / \mathrm{h} 2-5 \mathrm{H}, 1 \mathrm{H} 3$ \\
\hline 1172 & CPTO2J & $\mathrm{C} 1 \mathrm{CCCC} 1 \mathrm{O}[0]$ & $\operatorname{lnChl}=1 \mathrm{~S} / \mathrm{C} 5 \mathrm{H} 902 / \mathrm{c} 6-7-5-3-1-2-4-5 / \mathrm{h} 5 \mathrm{H}, 1-4 \mathrm{H} 2$ \\
\hline 1173 & C5H9O1-4OOH-5 & C1CC(COO)OC1 & InChl=1S/C5H1003/c6-8-4-5-2-1-3-7-5/h5-6H,1-4H2 \\
\hline 1174 & $\mathrm{BC} 5 \mathrm{H} 10 \mathrm{OOH}-\mathrm{CO} 2$ & $\mathrm{CC}(\mathrm{C}(\mathrm{C})(\mathrm{C}) \mathrm{OO}) \mathrm{O}[\mathrm{O}]$ & InChl=1S/C5H1104/c1-4(8-6)5(2,3)9-7/h4,7H,1-3H3 \\
\hline 1175 & $\mathrm{MF} 23 \mathrm{H}$ & $\mathrm{O} 1[\mathrm{C}](\mathrm{C}) \mathrm{CC}=\mathrm{C} 1$ & $\operatorname{lnChl}=15 / C 5 H 70 / c 1-5-3-2-4-6-5 / h 2,4 \mathrm{H}, 3 \mathrm{H} 2,1 \mathrm{H} 3$ \\
\hline 1176 & CPT1Q2J & $\mathrm{C} 1 \mathrm{C}(\mathrm{OO})[\mathrm{CH}] \mathrm{CC} 1$ & InChl=1S/C5H9O2/c6-7-5-3-1-2-4-5/h3,5-6H,1-2,4H2 \\
\hline 1177 & C5H9O1-50OH-2 & $\mathrm{C} 1 \mathrm{CC}(\mathrm{COC} 1) \mathrm{OO}$ & InChl=1S/C5H1003/c6-8-5-2-1-3-7-4-5/h5-6H,1-4H2 \\
\hline 1178 & BC5H10OOH-DO2 & $\mathrm{CC}(\mathrm{C})(\mathrm{CCO}[\mathrm{O}]) 0 \mathrm{O}$ & InChl=1S/C5H11O4/c1-5(2,9-7)3-4-8-6/h7H,3-4H2,1-2H3 \\
\hline 1179 & MF24H & $\mathrm{CC} 1=\mathrm{CC}[\mathrm{CH}] \mathrm{O} 1$ & $\operatorname{lnChl}=15 / C 5 H 70 / c 1-5-3-2-4-6-5 / h 3-4 H, 2 \mathrm{H} 2,1 \mathrm{H} 3$ \\
\hline
\end{tabular}




\begin{tabular}{|c|c|c|c|}
\hline 1180 & CPT1Q3Ј & $\mathrm{C} 1 \mathrm{C}(\mathrm{OO}) \mathrm{C}[\mathrm{CH}] \mathrm{C} 1$ & InChl=1S/C5H9O2/c6-7-5-3-1-2-4-5/h1,5-6H,2-4H2 \\
\hline 1181 & $\mathrm{C} 5 \mathrm{H} 9 \mathrm{O} 1-5 \mathrm{OOH}-3$ & $\mathrm{C} 1 \mathrm{Coccc} 100$ & InChl=1S/C5H1003/c6-8-5-1-3-7-4-2-5/h5-6H,1-4H2 \\
\hline 1182 & CC5H10OOH-AO2 & $\mathrm{CC}(\mathrm{CO}[\mathrm{O}]) \mathrm{C}(\mathrm{C}) \mathrm{OO}$ & InChl=1S/C5H1104/c1-4(3-8-6)5(2)9-7/h4-5,7H,3H2,1-2H3 \\
\hline 1183 & MF25H & $\mathrm{CC} 1=\mathrm{C}[\mathrm{CH}] \mathrm{CO} 1$ & $\operatorname{lnChl}=15 / C 5 H 7 O / c 1-5-3-2-4-6-5 / h 2-3 \mathrm{H}, 4 \mathrm{H} 2,1 \mathrm{H} 3$ \\
\hline 1184 & PT1N1Q5J & $\mathrm{OOC}=\mathrm{CCC}[\mathrm{CH} 2]$ & $\mathrm{InChl}=1 \mathrm{~S} / \mathrm{C} 5 \mathrm{H} 902 / \mathrm{c} 1-2-3-4-5-7-6 / \mathrm{h} 4-6 \mathrm{H}, 1-3 \mathrm{H} 2$ \\
\hline 1185 & $\mathrm{C} 5 \mathrm{H} 9 \mathrm{O} 2-3 \mathrm{OOH}-1$ & $\mathrm{CCC1C(COO)O1}$ & $\operatorname{lnChl}=15 / \mathrm{C} 5 \mathrm{H} 10 \mathrm{OO} / \mathrm{c} 1-2-4-5(8-4) 3-7-6 / \mathrm{h} 4-6 \mathrm{H}, 2-3 \mathrm{H} 2,1 \mathrm{H} 3$ \\
\hline 1186 & CC5H10OOH-BO2 & $\mathrm{CC}(\mathrm{C}(\mathrm{C})(\mathrm{C}) \mathrm{O}[\mathrm{O}]) 0 \mathrm{O}$ & InChl=1S/C5H11O4/c1-4(8-6)5(2,3)9-7/h4,6H,1-3H3 \\
\hline 1187 & P3E101J-C1 & $\mathrm{CC}=\mathrm{CC}[\mathrm{C}]=\mathrm{O}$ & InChl=1S/C5H7O/c1-2-3-4-5-6/h2-3H,4H2,1H3 \\
\hline 1188 & PT1N3Q5J & $\mathrm{C}=\mathrm{CC}(\mathrm{OO}) \mathrm{C}[\mathrm{CH} 2]$ & $\operatorname{lnChl}=1 \mathrm{~S} / \mathrm{C5} \mathrm{H} 9 \mathrm{O} 2 / \mathrm{c1}-3-5(4-2) 7-6 / \mathrm{h} 3,5-6 \mathrm{H}, 1-2,4 \mathrm{H} 2$ \\
\hline 1189 & $\mathrm{C} 5 \mathrm{H} 9 \mathrm{O} 2-3 \mathrm{OOH}-4$ & $\mathrm{CC} 1 \mathrm{C}(\mathrm{C}(\mathrm{C}) 0 \mathrm{O}) 01$ & InChl=1S/C5H1003/c1-3-5(7-3)4(2)8-6/h3-6H,1-2H3 \\
\hline 1190 & CC5H10OOH-DO2 & $\mathrm{CC}(\mathrm{C}) \mathrm{C}(\mathrm{CO}[\mathrm{O}]) \mathrm{OO}$ & InChl=1S/C5H1104/c1-4(2)5(9-7)3-8-6/h4-5,7H,3H2,1-2H3 \\
\hline 1191 & P3E104J-C3 & $C[C]=C C C=O$ & $\operatorname{lnChl}=1 \mathrm{~S} / \mathrm{C} 5 \mathrm{H} 7 \mathrm{O} / \mathrm{c} 1-2-3-4-5-6 / \mathrm{h} 3,5 \mathrm{H}, 4 \mathrm{H} 2,1 \mathrm{H} 3$ \\
\hline 1192 & PT1N4Q5J & $\mathrm{C}=\mathrm{CCC}(\mathrm{OO})[\mathrm{CH} 2]$ & InChl=15/C5H9O2/c1-3-4-5(2)7-6/h3,5-6H,1-2,4H2 \\
\hline 1193 & $\mathrm{C} 5 \mathrm{H} 9 \mathrm{O} 2-3 \mathrm{OOH}-5$ & $\mathrm{CC1C}(\mathrm{CCOO}) 01$ & InChl=1S/C5H10O3/c1-4-5(8-4)2-3-7-6/h4-6H,2-3H2,1H3 \\
\hline 1194 & $\mathrm{DC5H} 100 \mathrm{OH}-\mathrm{AO} 2$ & $\mathrm{CC}(\mathrm{CCOO}) \mathrm{CO}[\mathrm{O}]$ & $\operatorname{lnChl}=15 / C 5 H 1104 / c 1-5(4-9-7) 2-3-8-6 / h 5-6 \mathrm{H}, 2-4 \mathrm{H} 2,1 \mathrm{H} 3$ \\
\hline 1195 & P3E2O1J-C2 & {$[\mathrm{CH} 2] \mathrm{C}(=\mathrm{O}) \mathrm{C}=\mathrm{CC}$} & $\operatorname{lnChl}=1 \mathrm{~S} / \mathrm{C} 5 \mathrm{H} 7 \mathrm{O} / \mathrm{c1}-3-4-5(2) 6 / \mathrm{h} 3-4 \mathrm{H}, 2 \mathrm{H} 2,1 \mathrm{H} 3$ \\
\hline 1196 & C5H9O1-2O-5 & $\mathrm{C}(\mathrm{CC} 1 \mathrm{CO} 1) \mathrm{C}[\mathrm{O}]$ & $\mathrm{InChl}=1 \mathrm{~S} / \mathrm{C} 5 \mathrm{H} 9 \mathrm{O} 2 / \mathrm{c6}-3-1-2-5-4-7-5 / \mathrm{h} 5 \mathrm{H}, 1-4 \mathrm{H} 2$ \\
\hline 1197 & $\mathrm{C} 5 \mathrm{H} 9 \mathrm{O} 2-4 \mathrm{OOH}-1$ & $\mathrm{CC1CC}(\mathrm{COO}) 01$ & $\operatorname{lnChl}=15 / \mathrm{C}^{2} \mathrm{H} 10 \mathrm{OO} / \mathrm{c} 1-4-2-5(8-4) 3-7-6 / \mathrm{h} 4-6 \mathrm{H}, 2-3 \mathrm{H} 2,1 \mathrm{H} 3$ \\
\hline 1198 & DC5H10OOH-BO2 & $\mathrm{CC}(\mathrm{C})(\mathrm{CCOO}) \mathrm{O}[\mathrm{O}]$ & $\mathrm{InChl}=1 \mathrm{~S} / \mathrm{C} 5 \mathrm{H} 11 \mathrm{O} 4 / \mathrm{c} 1-5(2,9-7) 3-4-8-6 / \mathrm{h} 6 \mathrm{H}, 3-4 \mathrm{H} 2,1-2 \mathrm{H} 3$ \\
\hline 1199 & P4E2O3J-C1 & $\mathrm{CC}(=\mathrm{O})[\mathrm{CH}] \mathrm{C}=\mathrm{C}$ & InChl=1S/C5H7O/c1-3-4-5(2)6/h3-4H,1H2,2H3 \\
\hline 1200 & $\mathrm{CH} 2 \mathrm{CH} 2 \mathrm{OCH} 2 \mathrm{CH} 2 \mathrm{CHO}$ & {$[\mathrm{CH} 2] \mathrm{COCCC}=\mathrm{O}$} & InChl=1S/C5H9O2/c1-2-7-5-3-4-6/h4H,1-3,5H2 \\
\hline 1201 & $\mathrm{C} 5 \mathrm{H} 9 \mathrm{O} 2-4 \mathrm{OOH}-3$ & $\mathrm{CC1C(C(C)01)00}$ & InChl=1S/C5H1003/c1-3-5(8-6)4(2)7-3/h3-6H,1-2H3 \\
\hline 1202 & $\mathrm{DC} 5 \mathrm{H} 10 \mathrm{OOH}-\mathrm{CO} 2$ & $\mathrm{CC}(\mathrm{C}) \mathrm{C}(\mathrm{COO}) \mathrm{O}[\mathrm{O}]$ & $\operatorname{lnChl}=15 / C 5 H 1104 / c 1-4(2) 5(9-7) 3-8-6 / h 4-6 \mathrm{H}, 3 \mathrm{H} 2,1-2 \mathrm{H} 3$ \\
\hline 1203 & P4E2O5J-C2 & {$[\mathrm{CH}]=\mathrm{CCC}(=\mathrm{O}) \mathrm{C}$} & InChl=1S/C5H7O/c1-3-4-5(2)6/h1,3H,4H2,2H3 \\
\hline 1204 & NC5KET12O & $\operatorname{cccc}(\mathrm{C}=0)[0]$ & InChl=1S/C5H9O2/c1-2-3-5(7)4-6/h4-5H,2-3H2,1H3 \\
\hline 1205 & NC5CYCPER13 & $\mathrm{C} 1(0) \mathrm{CC}(001) \mathrm{CC}$ & InChl=15/C5H10O3/c1-2-4-3-5(6)8-7-4/h4-6H,2-3H2,1H3 \\
\hline 1206 & С5H9A-A,BOOH & $\mathrm{CCC}([\mathrm{CH} 2])(\mathrm{COO}) \mathrm{OO}$ & InChl=1S/C5H11O4/c1-3-5(2,9-7)4-8-6/h6-7H,2-4H2,1H3 \\
\hline 1207 & CYC51EN3OJ & $\mathrm{C} 1=\mathrm{CC}([0]) \mathrm{CC} 1$ & $\operatorname{lnChl}=1 \mathrm{~S} / \mathrm{C} 5 \mathrm{H} 7 \mathrm{O} / \mathrm{c} 6-5-3-1-2-4-5 / \mathrm{h} 1,3,5 \mathrm{H}, 2,4 \mathrm{H} 2$ \\
\hline 1208 & NC5KET130 & $\operatorname{CCC}(\mathrm{CC}=0)[0]$ & $\operatorname{lnChl}=1 \mathrm{~S} / \mathrm{C} 5 \mathrm{H} 902 / \mathrm{c} 1-2-5(7) 3-4-6 / \mathrm{h} 4-5 \mathrm{H}, 2-3 \mathrm{H} 2,1 \mathrm{H} 3$ \\
\hline 1209 & NC5CYCPER24 & $\mathrm{CC} 1(0) \mathrm{CC}(0 \mathrm{O} 1) \mathrm{C}$ & $\operatorname{lnChl}=1 \mathrm{~S} / \mathrm{C5} \mathrm{H} 10 \mathrm{OO} / \mathrm{c} 1-4-3-5(2,6) 8-7-4 / \mathrm{h} 4,6 \mathrm{H}, 3 \mathrm{H} 2,1-2 \mathrm{H} 3$ \\
\hline 1210 & C5H9A-A,COOH & {$[\mathrm{CH} 2] \mathrm{C}(\mathrm{COO}) \mathrm{C}(\mathrm{C}) \mathrm{OO}$} & InChl=1S/C5H11O4/c1-4(3-8-6)5(2)9-7/h4-7H,1,3H2,2H3 \\
\hline 1211 & $\mathrm{C} 4 \mathrm{H} 6 \mathrm{CHO}-5$ & {$[\mathrm{CH} 2] \mathrm{CC}=\mathrm{CC}=\mathrm{O}$} & InChl=1S/C5H7O/c1-2-3-4-5-6/h3-5H,1-2H2 \\
\hline 1212 & NC5KET140 & $\mathrm{CC}(\mathrm{CCC}=0)[0]$ & InChl=1S/C5H9O2/c1-5(7)3-2-4-6/h4-5H,2-3H2,1H3 \\
\hline 1213 & NC5CYCPER31 & $\mathrm{C}(\mathrm{OO} 1) \mathrm{CC} 1(\mathrm{O}) \mathrm{CC}$ & $\ln \mathrm{Chl}=1 \mathrm{~S} / \mathrm{C} 5 \mathrm{H} 10 \mathrm{OO} / \mathrm{c1}-2-5(6) 3-4-7-8-5 / \mathrm{h} 6 \mathrm{H}, 2-4 \mathrm{H} 2,1 \mathrm{H} 3$ \\
\hline 1214 & C5H9A-A,DOOH & {$[\mathrm{CH} 2] \mathrm{C}(\mathrm{CCOO}) \mathrm{COO}$} & InChl=1S/C5H1104/c1-5(4-9-7)2-3-8-6/h5-7H,1-4H2 \\
\hline 1215 & $\mathrm{C} 4 \mathrm{H} 6 \mathrm{CHO} 4-5$ & {$[\mathrm{CH}]=\mathrm{CCCC}=\mathrm{O}$} & InChl=1S/C5H7O/c1-2-3-4-5-6/h1-2,5H,3-4H2 \\
\hline 1216 & NC5KET150 & $\mathrm{C}(\mathrm{CC}=0) \mathrm{CC}[\mathrm{O}]$ & InChl=1S/C5H9O2/c6-4-2-1-3-5-7/h4H,1-3,5H2 \\
\hline 1217 & С5Н9ОА-АООН-В & $\mathrm{CCC} 1(\mathrm{COC} 1) 0 \mathrm{O}$ & $\operatorname{lnChl}=15 / C 5 H 1003 / c 1-2-5(8-6) 3-7-4-5 / h 6 \mathrm{H}, 2-4 \mathrm{H} 2,1 \mathrm{H} 3$ \\
\hline 1218 & C5H9A-B,COOH & $\mathrm{CC}(\mathrm{C}([\mathrm{CH} 2])(\mathrm{C}) \mathrm{OO}) \mathrm{OO}$ & InChl=1S/C5H1104/c1-4(8-6)5(2,3)9-7/h4,6-7H,2H2,1,3H3 \\
\hline 1219 & CPTYO123J & $\mathrm{C} 1 \mathrm{C}(\mathrm{O} 2) \mathrm{C} 2[\mathrm{CH}] \mathrm{C} 1$ & $\operatorname{lnChl}=15 / C 5 H 7 O / c 1-2-4-5(3-1) 6-4 / \mathrm{h} 2,4-5 \mathrm{H}, 1,3 \mathrm{H} 2$ \\
\hline 1220 & NC5KET210 & $\operatorname{cccc}(\operatorname{C}[0])=0$ & $\operatorname{lnChl}=1 \mathrm{~S} / \mathrm{C} 5 \mathrm{H} 9 \mathrm{O} 2 / \mathrm{c} 1-2-3-5(7) 4-6 / \mathrm{h} 2-4 \mathrm{H} 2,1 \mathrm{H} 3$ \\
\hline 1221 & С5H9OA-АООН-С & $\mathrm{CC}(\mathrm{C} 1 \mathrm{COC} 1) 0 \mathrm{O}$ & $\operatorname{lnChl}=1 \mathrm{~S} / \mathrm{C} 5 \mathrm{H} 1003 / \mathrm{c} 1-4(8-6) 5-2-7-3-5 / \mathrm{h} 4-6 \mathrm{H}, 2-3 \mathrm{H} 2,1 \mathrm{H} 3$ \\
\hline 1222 & C5H9A-B,DOOH & {$[\mathrm{CH} 2] \mathrm{C}(\mathrm{C})(\mathrm{CCOO}) \mathrm{OO}$} & $\operatorname{lnChl}=1 \mathrm{~S} / \mathrm{C} 5 \mathrm{H} 1104 / \mathrm{c} 1-5(2,9-7) 3-4-8-6 / \mathrm{h} 6-7 \mathrm{H}, 1,3-4 \mathrm{H} 2,2 \mathrm{H} 3$ \\
\hline 1223 & CYC5O1EN5J & {$[\mathrm{CH}] 1 \mathrm{CCC}=\mathrm{CO} 1$} & $\operatorname{lnChl}=1 \mathrm{~S} / \mathrm{C} 5 \mathrm{H} 7 \mathrm{O} / \mathrm{c1} 1-2-4-6-5-3-1 / \mathrm{h} 2,4-5 \mathrm{H}, 1,3 \mathrm{H} 2$ \\
\hline 1224 & NC5KET230 & $\operatorname{ccc}(\mathrm{C}(\mathrm{C})=0)[0]$ & InChl=1S/C5H9O2/c1-3-5(7)4(2)6/h5H,3H2,1-2H3 \\
\hline 1225 & C5H9OA-AOOH-D & $\mathrm{C}(\mathrm{COO}) \mathrm{C} 1 \mathrm{COC} 1$ & InChl=1S/C5H1003/c6-8-2-1-5-3-7-4-5/h5-6H,1-4H2 \\
\hline 1226 & C5H9A-C,DOOH & {$[\mathrm{CH} 2] \mathrm{C}(\mathrm{C}) \mathrm{C}(\mathrm{COO}) \mathrm{OO}$} & $\operatorname{lnChl}=15 / C 5 H 1104 / c 1-4(2) 5(9-7) 3-8-6 / h 4-7 \mathrm{H}, 1,3 \mathrm{H} 2,2 \mathrm{H} 3$ \\
\hline 1227 & $\mathrm{C} 2 \mathrm{H} 3 \mathrm{OC} 3 \mathrm{H} 4 \mathrm{~A}$ & $\mathrm{C}=\mathrm{COC}=\mathrm{C}[\mathrm{CH} 2]$ & InChl=1S/C5H7O/c1-3-5-6-4-2/h3-5H,1-2H2 \\
\hline 1228 & NC5KET24O & $\mathrm{CC}(\mathrm{CC}(\mathrm{C})=0)[0]$ & InChl=1S/C5H9O2/c1-4(6)3-5(2)7/h4H,3H2,1-2H3 \\
\hline 1229 & С5Н9ОА-ВООН-A & $\mathrm{CCC} 1(\mathrm{CO} 1) \mathrm{COO}$ & InChl=1S/C5H10O3/c1-2-5(3-7-5)4-8-6/h6H,2-4H2,1H3 \\
\hline 1230 & С5H9B-A,AOOH & $\mathrm{CC}[\mathrm{C}](\mathrm{COO}) \mathrm{COO}$ & InChl=15/C5H11O4/c1-2-5(3-8-6)4-9-7/h6-7H,2-4H2,1H3 \\
\hline 1231 & $\mathrm{C} 4 \mathrm{H} 7 \mathrm{CO}-4$ & $\mathrm{C}=\mathrm{CCC}[\mathrm{C}]=\mathrm{O}$ & $\operatorname{lnChl}=1 \mathrm{~S} / \mathrm{C} 5 \mathrm{H} 7 \mathrm{O} / \mathrm{c} 1-2-3-4-5-6 / \mathrm{h} 2 \mathrm{H}, 1,3-4 \mathrm{H} 2$ \\
\hline 1232 & NC5KET250 & $\mathrm{CC}(\mathrm{CCC}[\mathrm{O}])=0$ & $\operatorname{lnChl}=1 \mathrm{~S} / \mathrm{C} 5 \mathrm{H} 9 \mathrm{O} 2 / \mathrm{c} 1-5(7) 3-2-4-6 / \mathrm{h} 2-4 \mathrm{H} 2,1 \mathrm{H} 3$ \\
\hline 1233 & C5H9OA-BOOH-C & $\mathrm{CC}(\mathrm{C} 1(\mathrm{C}) \mathrm{CO} 1) 0 \mathrm{O}$ & InChl=15/C5H10O3/c1-4(8-6)5(2)3-7-5/h4,6H,3H2,1-2H3 \\
\hline 1234 & C5H9B-A,COOH & $\mathrm{C}[\mathrm{C}](\mathrm{COO}) \mathrm{C}(\mathrm{C}) \mathrm{OO}$ & $\operatorname{lnChl}=15 / C 5 H 11 O 4 / c 1-4(3-8-6) 5(2) 9-7 / h 5-7 \mathrm{H}, 3 \mathrm{H} 2,1-2 \mathrm{H} 3$ \\
\hline 1235 & $\mathrm{IC} 3 \mathrm{H} 5 \mathrm{COCH} 2$ & $\mathrm{C}=\mathrm{C}(\mathrm{C}) \mathrm{C}(=\mathrm{C})[\mathrm{O}]$ & InChl=1S/C5H7O/c1-4(2)5(3)6/h1,3H2,2H3 \\
\hline 1236 & NC5KET310 & $\operatorname{CCC}(\operatorname{CC}[0])=0$ & InChl=15/C5H9O2/c1-2-5(7)3-4-6/h2-4H2,1H3 \\
\hline 1237 & C5H9OA-BOOH-D & $\mathrm{CC} 1(\mathrm{CCOO}) \mathrm{CO} 1$ & InChl=1S/C5H10O3/c1-5(4-7-5)2-3-8-6/h6H,2-4H2,1H3 \\
\hline 1238 & C5H9B-A,DOOH & $\mathrm{C}[\mathrm{C}](\mathrm{COOO}) \mathrm{COO}$ & $\operatorname{lnChl}=15 / C 5 H 1104 / c 1-5(4-9-7) 2-3-8-6 / h 6-7 \mathrm{H}, 2-4 \mathrm{H} 2,1 \mathrm{H} 3$ \\
\hline 1239 & $\mathrm{AC} 3 \mathrm{H} 4 \mathrm{COCH} 3$ & $\mathrm{C}=\mathrm{C}([\mathrm{CH} 2]) \mathrm{C}(\mathrm{C})=\mathrm{O}$ & InChl=1S/C5H7O/c1-4(2)5(3)6/h1-2H2,3H3 \\
\hline 1240 & NC5KET32O & $\operatorname{ccc}(C(C)[0])=0$ & InChl=15/C5H9O2/c1-3-5(7)4(2)6/h4H,3H2,1-2H3 \\
\hline 1241 & С5H9OA-СООН-A & $\mathrm{CC1C}(\mathrm{CO} 1) \mathrm{COO}$ & InChl=1S/C5H10O3/c1-4-5(2-7-4)3-8-6/h4-6H,2-3H2,1H3 \\
\hline 1242 & C5H9B-C,DOOH & $\mathrm{C}[\mathrm{C}](\mathrm{C}) \mathrm{C}(\mathrm{COO}) \mathrm{OO}$ & $\operatorname{lnChl}=15 / C 5 H 11 O 4 / c 1-4(2) 5(9-7) 3-8-6 / h 5-7 \mathrm{H}, 3 \mathrm{H} 2,1-2 \mathrm{H} 3$ \\
\hline 1243 & $\mathrm{IC4H7CO}$ & $\mathrm{CC}(=\mathrm{C}) \mathrm{C}[\mathrm{C}]=\mathrm{O}$ & $\operatorname{lnChl}=1 \mathrm{~S} / \mathrm{C} 5 \mathrm{H} 70 / \mathrm{c} 1-5(2) 3-4-6 / \mathrm{h} 1,3 \mathrm{H} 2,2 \mathrm{H} 3$ \\
\hline 1244 & $\mathrm{CH} 3 \mathrm{COCH} 2 \mathrm{OCH} 2 \mathrm{CH} 2$ & {$[\mathrm{CH} 2] \mathrm{COCC}(\mathrm{C})=\mathrm{O}$} & $\operatorname{lnChl}=15 / C 5 H 9 O 2 / c 1-3-7-4-5(2) 6 / h 1,3-4 H 2,2 \mathrm{H} 3$ \\
\hline 1245 & C5H9OA-COOH-B & $\mathrm{CC1C(C)(CO1)00}$ & $\operatorname{lnChl}=1 \mathrm{~S} / \mathrm{C5} \mathrm{H} 10 \mathrm{OO} / \mathrm{c} 1-4-5(2,8-6) 3-7-4 / \mathrm{h} 4,6 \mathrm{H}, 3 \mathrm{H} 2,1-2 \mathrm{H} 3$ \\
\hline 1246 & C5H9C-A,AOOH & $\mathrm{C}[\mathrm{CH}] \mathrm{C}(\mathrm{COO}) \mathrm{COO}$ & $\operatorname{lnChl}=1 \mathrm{~S} / \mathrm{C} 5 \mathrm{H} 1104 / \mathrm{c} 1-2-5(3-8-6) 4-9-7 / \mathrm{h} 2,5-7 \mathrm{H}, 3-4 \mathrm{H} 2,1 \mathrm{H} 3$ \\
\hline 1247 & ACC5H7-DOH & $\mathrm{CC}(=\mathrm{C}) \mathrm{C}=\mathrm{CO}$ & $\operatorname{lnChl}=15 / C 5 H 80 / c 1-5(2) 3-4-6 / h 3-4,6 \mathrm{H}, 1 \mathrm{H} 2,2 \mathrm{H} 3$ \\
\hline 1248 & IC5KETAAO & $\operatorname{ccc}(\mathrm{C}=0) \mathrm{C}[0]$ & InChl=1S/C5H9O2/c1-2-5(3-6)4-7/h3,5H,2,4H2,1H3 \\
\hline 1249 & C5H9OA-COOH-D & $\mathrm{CC} 1 \mathrm{COC} 1 \mathrm{COO}$ & $\mathrm{InChl}=1 \mathrm{~S} / \mathrm{C} 5 \mathrm{H} 10 \mathrm{OO} / \mathrm{c} 1-4-2-7-5(4) 3-8-6 / \mathrm{h} 4-6 \mathrm{H}, 2-3 \mathrm{H} 2,1 \mathrm{H} 3$ \\
\hline 1250 & С5H9C-A,BOOH & $\mathrm{C}[\mathrm{CH}] \mathrm{C}(\mathrm{C})(\mathrm{COO}) \mathrm{OO}$ & InChl=1S/C5H1104/c1-3-5(2,9-7)4-8-6/h3,6-7H,4H2,1-2H3 \\
\hline 1251 & IC5D3O1-2 & $\mathrm{CC} 1(\mathrm{CO} 1) \mathrm{C}=\mathrm{C}$ & $\operatorname{lnChl}=15 / C 5 H 8 O / c 1-3-5(2) 4-6-5 / h 3 H, 1,4 \mathrm{H} 2,2 \mathrm{H} 3$ \\
\hline 1252 & IC5KETABO & $\operatorname{ccc}(C)(C=0)[0]$ & InChl=1S/C5H9O2/c1-3-5(2,7)4-6/h4H,3H2,1-2H3 \\
\hline 1253 & C5H9OA-DOOH-A & C1COCC1COO & InChl=1S/C5H1003/c6-8-4-5-1-2-7-3-5/h5-6H,1-4H2 \\
\hline 1254 & C5H9C-A,DOOH & $\mathrm{CC}([\mathrm{CH}] \mathrm{COO}) \mathrm{COO}$ & InChl=1S/C5H1104/c1-5(4-9-7)2-3-8-6/h2,5-7H,3-4H2,1H3 \\
\hline 1255 & IC5D103-4 & $\mathrm{CC}(=\mathrm{C}) \mathrm{C} 1 \mathrm{CO} 1$ & $\operatorname{lnChl}=15 / C 5 H 80 / c 1-4(2) 5-3-6-5 / h 5 H, 1,3 \mathrm{H} 2,2 \mathrm{H} 3$ \\
\hline 1256 & IC5KETACO & $C C(C=0) C(C)[O]$ & InChl=1S/C5H9O2/c1-4(3-6)5(2)7/h3-5H,1-2H3 \\
\hline 1257 & C5H9OA-DOOH-B & $\mathrm{CC} 1(\mathrm{CCOC} 1) \mathrm{OO}$ & $\operatorname{lnChl}=15 / C 5 H 1003 / c 1-5(8-6) 2-3-7-4-5 / h 6 \mathrm{H}, 2-4 \mathrm{H} 2,1 \mathrm{H} 3$ \\
\hline 1258 & C5H9C-B,DOOH & $\mathrm{CC}(\mathrm{C})([\mathrm{CH}] \mathrm{COO}) 0 \mathrm{O}$ & $\operatorname{lnChl}=1 \mathrm{~S} / \mathrm{C} 5 \mathrm{H} 1104 / \mathrm{c} 1-5(2,9-7) 3-4-8-6 / \mathrm{h} 3,6-7 \mathrm{H}, 4 \mathrm{H} 2,1-2 \mathrm{H} 3$ \\
\hline 1259 & $\mathrm{C} 4 \mathrm{H} 7 \mathrm{CHO}-2$ & $\mathrm{CCC}=\mathrm{CC}(=0)$ & $\operatorname{lnChl}=1 \mathrm{~S} / \mathrm{C} 5 \mathrm{H} 8 \mathrm{O} / \mathrm{c1}-2-3-4-5-6 / \mathrm{h} 3-5 \mathrm{H}, 2 \mathrm{H} 2,1 \mathrm{H} 3$ \\
\hline 1260 & ICSKETADO & $\mathrm{CC}(\mathrm{CC}[\mathrm{O}]) \mathrm{C}=\mathrm{O}$ & InChl=1S/C5H9O2/c1-5(4-7)2-3-6/h4-5H,2-3H2,1H3 \\
\hline 1261 & C5H9OA-DOOH-C & CC1COCC100 & InChl=1S/C5H10O3/c1-4-2-7-3-5(4)8-6/h4-6H,2-3H2,1H3 \\
\hline 1262 & C5H9D-A,AOOH & {$[\mathrm{CH} 2] \mathrm{CC}(\mathrm{COO}) \mathrm{COO}$} & InChl=1S/C5H1104/c1-2-5(3-8-6)4-9-7/h5-7H,1-4H2 \\
\hline 1263 & CPN & $\mathrm{C} 1 \mathrm{CC}(=0) \mathrm{CC} 1$ & $\operatorname{lnChl}=1 \mathrm{~S} / \mathrm{C} 5 \mathrm{H} 8 \mathrm{O} / \mathrm{c} 6-5-3-1-2-4-5 / \mathrm{h} 1-4 \mathrm{H} 2$ \\
\hline 1264 & IC5KETCAO & $\operatorname{CC}(\mathrm{C}[\mathrm{O}]) \mathrm{C}(\mathrm{C})=\mathrm{O}$ & InChl=15/C5H9O2/c1-4(3-6)5(2)7/h4H,3H2,1-2H3 \\
\hline 1265 & C5H9OB-COOH-A & $\mathrm{CC1C(C)(COO)O1}$ & $\operatorname{lnChl}=1 \mathrm{~S} / \mathrm{C} 5 \mathrm{H} 1003 / \mathrm{c} 1-4-5(2,8-4) 3-7-6 / \mathrm{h} 4,6 \mathrm{H}, 3 \mathrm{H} 2,1-2 \mathrm{H} 3$ \\
\hline 1266 & C5H9D-A,BOOH & {$[\mathrm{CH} 2] \mathrm{CC}(\mathrm{C})(\mathrm{COO}) \mathrm{OO}$} & $\operatorname{lnChl}=1 \mathrm{~S} / \mathrm{CH} 1104 / \mathrm{c} 1-3-5(2,9-7) 4-8-6 / \mathrm{h} 6-7 \mathrm{H}, 1,3-4 \mathrm{H} 2,2 \mathrm{H} 3$ \\
\hline 1267 & $\mathrm{CVCCVCCOH}$ & $\mathrm{C}=\mathrm{CC}=\mathrm{CCO}$ & $\operatorname{lnChl}=1 \mathrm{~S} / \mathrm{C} 5 \mathrm{H} 8 \mathrm{O} / \mathrm{c} 1-2-3-4-5-6 / \mathrm{h} 2-4,6 \mathrm{H}, 1,5 \mathrm{H} 2$ \\
\hline 1268 & IC5KETCBO & $\mathrm{CC}(\mathrm{C}(\mathrm{C})(\mathrm{C})[\mathrm{O}])=\mathrm{O}$ & InChl=15/C5H9O2/c1-4(6)5(2,3)7/h1-3H3 \\
\hline 1269 & C5H9OB-COOH-D & $\mathrm{CC} 1(\mathrm{C}) \mathrm{C}(\mathrm{COO}) 01$ & $\operatorname{lnChl}=1 \mathrm{~S} / \mathrm{C5} \mathrm{H} 10 \mathrm{OO} / \mathrm{c} 1-5(2) 4(8-5) 3-7-6 / \mathrm{h} 4,6 \mathrm{H}, 3 \mathrm{H} 2,1-2 \mathrm{H} 3$ \\
\hline 1270 & C5H9D-A,COOH & $\mathrm{CC}(\mathrm{COO}) \mathrm{C}([\mathrm{CH} 2]) \mathrm{OO}$ & InChl=1S/C5H11O4/c1-4(3-8-6)5(2)9-7/h4-7H,2-3H2,1H3 \\
\hline
\end{tabular}




\begin{tabular}{|c|c|c|c|}
\hline 1271 & $\mathrm{CCVCCVCOH}$ & $\mathrm{CC}=\mathrm{CC}=\mathrm{CO}$ & $\operatorname{lnChl}=1 \mathrm{~S} / \mathrm{C} 5 \mathrm{H} 8 \mathrm{O} / \mathrm{c} 1-2-3-4-5-6 / \mathrm{h} 2-6 \mathrm{H}, 1 \mathrm{H} 3$ \\
\hline 1272 & IC5KETCDO & $\operatorname{cC}(C) C(C[0])=0$ & InChl=15/C5H9O2/c1-4(2)5(7)3-6/h4H,3H2,1-2H3 \\
\hline 1273 & C5H9OB-DOOH-A & $\mathrm{CC1}(\mathrm{CCO} 1) \mathrm{COO}$ & $\operatorname{lnChl}=15 / C 5 H 1003 / \mathrm{c} 1-5(4-8-6) 2-3-7-5 / h 6 \mathrm{H}, 2-4 \mathrm{H} 2,1 \mathrm{H} 3$ \\
\hline 1274 & C5H9D-B,COOH & {$[\mathrm{CH} 2] \mathrm{C}(\mathrm{C}(\mathrm{C})(\mathrm{C}) \mathrm{OO}) \mathrm{OO}$} & InChl=1S/C5H1104/c1-4(8-6)5(2,3)9-7/h4,6-7H,1H2,2-3H3 \\
\hline 1275 & $\mathrm{C} 5 \mathrm{H} 8 \mathrm{O}$ & $\mathrm{C}=\mathrm{CC}(\mathrm{C}) \mathrm{C}=\mathrm{O}$ & $\mathrm{InChl}=1 \mathrm{~S} / \mathrm{C} 5 \mathrm{H} 8 \mathrm{O} / \mathrm{c1}-3-5(2) 4-6 / \mathrm{h} 3-5 \mathrm{H}, 1 \mathrm{H} 2,2 \mathrm{H} 3$ \\
\hline 1276 & IC5KETDAO & $\mathrm{CC}(\mathrm{CC}=\mathrm{O}) \mathrm{C}[\mathrm{O}]$ & $\operatorname{lnChl}=1 \mathrm{~S} / \mathrm{C} 5 \mathrm{H} 9 \mathrm{O} 2 / \mathrm{c} 1-5(4-7) 2-3-6 / \mathrm{h} 3,5 \mathrm{H}, 2,4 \mathrm{H} 2,1 \mathrm{H} 3$ \\
\hline 1277 & C5H9OB-DOOH-C & $\mathrm{CC} 1(\mathrm{C}) \mathrm{C}(\mathrm{CO} 1) 0 \mathrm{O}$ & $\operatorname{lnChl}=1 \mathrm{~S} / \mathrm{C5} \mathrm{H} 10 \mathrm{OO} / \mathrm{c} 1-5(2) 4(8-6) 3-7-5 / \mathrm{h} 4,6 \mathrm{H}, 3 \mathrm{H} 2,1-2 \mathrm{H} 3$ \\
\hline 1278 & NEOC5H10OOH-O2 & $\mathrm{CC}(\mathrm{C})(\mathrm{COO}) \mathrm{CO}[\mathrm{O}]$ & InChl=1S/C5H11O4/c1-5(2,3-8-6)4-9-7/h6H,3-4H2,1-2H3 \\
\hline 1279 & NC53ONE-1 & $\operatorname{CCC}(=0) \mathrm{C}=\mathrm{C}$ & $\operatorname{lnChl}=1 \mathrm{~S} / \mathrm{C} 5 \mathrm{H} 8 \mathrm{O} / \mathrm{c} 1-3-5(6) 4-2 / \mathrm{h} 3 \mathrm{H}, 1,4 \mathrm{H} 2,2 \mathrm{H} 3$ \\
\hline 1280 & IC5KETDBO & $\mathrm{CC}(\mathrm{C})(\mathrm{CC}=\mathrm{O})[\mathrm{O}]$ & InChl=1S/C5H9O2/c1-5(2,7)3-4-6/h4H,3H2,1-2H3 \\
\hline 1281 & C5H9OC-DOOH-A & $\mathrm{CC}(\mathrm{COO}) \mathrm{C} 1 \mathrm{CO} 1$ & $\mathrm{InChl}=1 \mathrm{~S} / \mathrm{C} 5 \mathrm{H} 10 \mathrm{OO} / \mathrm{c} 1-4(2-8-6) 5-3-7-5 / \mathrm{h} 4-6 \mathrm{H}, 2-3 \mathrm{H} 2,1 \mathrm{H} 3$ \\
\hline 1282 & NEOC5H9Q2 & {$[\mathrm{CH} 2] \mathrm{C}(\mathrm{C})(\mathrm{COO}) \mathrm{COO}$} & InChl=1S/C5H1104/c1-5(2,3-8-6)4-9-7/h6-7H,1,3-4H2,2H3 \\
\hline 1283 & NC52ONE-3 & $\mathrm{CC}(=\mathrm{O}) \mathrm{C}=\mathrm{CC}$ & InChl=1S/C5H8O/c1-3-4-5(2)6/h3-4H,1-2H3 \\
\hline 1284 & ICSKETDCO & $\mathrm{CC}(\mathrm{C}) \mathrm{C}(\mathrm{C}=\mathrm{O})[0]$ & InChl=1S/C5H9O2/c1-4(2)5(7)3-6/h3-5H,1-2H3 \\
\hline 1285 & C5H9OC-DOOH-B & $\mathrm{CC}(\mathrm{C})(\mathrm{C} 1 \mathrm{CO} 1) 0 \mathrm{O}$ & $\operatorname{lnChl}=15 / \mathrm{C} 5 \mathrm{H} 1003 / \mathrm{c} 1-5(2,8-6) 4-3-7-4 / \mathrm{h} 4,6 \mathrm{H}, 3 \mathrm{H} 2,1-2 \mathrm{H} 3$ \\
\hline 1286 & CPNOOH2-3O2R & $\mathrm{C} 1 \mathrm{CC}(=0) \mathrm{C}(00) \mathrm{C} 1(\mathrm{O}[\mathrm{O}])$ & $\operatorname{lnChl}=1 \mathrm{~S} / \mathrm{C} 5 \mathrm{H} 705 / \mathrm{c} 6-3-1-2-4(9-7) 5(3) 10-8 / \mathrm{h} 4-5,8 \mathrm{H}, 1-2 \mathrm{H} 2$ \\
\hline 1287 & $\mathrm{C} 5 \mathrm{H} 7 \mathrm{OH}-13$ & $\mathrm{C}=\mathrm{CC}(\mathrm{C})=\mathrm{CO}$ & $\operatorname{lnChl}=15 / C 5 H 80 / c 1-3-5(2) 4-6 / h 3-4,6 \mathrm{H}, 1 \mathrm{H} 2,2 \mathrm{H} 3$ \\
\hline 1288 & NEOC5KETOX & $\mathrm{CC}(\mathrm{C})(\mathrm{C}=\mathrm{O}) \mathrm{C}[\mathrm{O}]$ & $\operatorname{lnChl}=1 \mathrm{~S} / \mathrm{C} 5 \mathrm{H} 9 \mathrm{OO} / \mathrm{c} 1-5(2,3-6) 4-7 / \mathrm{h} 3 \mathrm{H}, 4 \mathrm{H} 2,1-2 \mathrm{H} 3$ \\
\hline 1289 & IC5KETCA & $\mathrm{CC}(\mathrm{COO}) \mathrm{C}(\mathrm{C})=0$ & $\operatorname{lnChl}=15 / C 5 H 1003 / c 1-4(3-8-7) 5(2) 6 / h 4,7 \mathrm{H}, 3 \mathrm{H} 2,1-2 \mathrm{H} 3$ \\
\hline 1290 & CPNOOH2-402R & $\mathrm{C} 1(\mathrm{O}[\mathrm{O}]) \mathrm{CC}(=0) \mathrm{C}(\mathrm{OO}) \mathrm{C1}$ & InChl=1S/C5H705/c6-4-1-3(9-7)2-5(4)10-8/h3,5,8H,1-2H2 \\
\hline 1291 & CPTYO12 & $\mathrm{C} 1 \mathrm{C}(\mathrm{O} 2) \mathrm{C} 2 \mathrm{CC} 1$ & $\operatorname{lnChl}=1 \mathrm{~S} / \mathrm{C} 5 \mathrm{H} 8 \mathrm{O} / \mathrm{c1}-2-4-5(3-1) 6-4 / \mathrm{h} 4-5 \mathrm{H}, 1-3 \mathrm{H} 2$ \\
\hline 1292 & NEOC5KEJOL & $\mathrm{CC}(\mathrm{C})(\mathrm{CO})[\mathrm{C}]=\mathrm{O}$ & InChl=15/C5H9O2/c1-5(2,3-6)4-7/h6H,3H2,1-2H3 \\
\hline 1293 & IC5KETCB & $\mathrm{CC}(\mathrm{C}(\mathrm{C})(\mathrm{C}) \mathrm{OO})=0$ & $\ln \mathrm{Chl}=1 \mathrm{~S} / \mathrm{C} 5 \mathrm{H} 1003 / \mathrm{c} 1-4(6) 5(2,3) 8-7 / \mathrm{h} 7 \mathrm{H}, 1-3 \mathrm{H} 3$ \\
\hline 1294 & CPNOOH2-502R & $\mathrm{C} 1 \mathrm{C}(\mathrm{O}[\mathrm{O}]) \mathrm{C}(=0) \mathrm{C}(\mathrm{OO}) \mathrm{C} 1$ & InChl=1S/C5H705/c6-5-3(9-7)1-2-4(5)10-8/h3-4,7H,1-2H2 \\
\hline 1295 & CPTYO13 & $\mathrm{C} 1 \mathrm{C}(\mathrm{O} 2) \mathrm{CC} 2 \mathrm{C} 1$ & $\operatorname{lnChl}=15 / C 5 H 80 / c 1-2-5-3-4(1) 6-5 / h 4-5 \mathrm{H}, 1-3 \mathrm{H} 2$ \\
\hline 1296 & C5H91-102 & $\mathrm{CCCC}=\mathrm{CO}[\mathrm{O}]$ & InChl=1S/C5H9O2/c1-2-3-4-5-7-6/h4-5H,2-3H2,1H3 \\
\hline 1297 & IC5KETCD & $\mathrm{CC}(\mathrm{C}) \mathrm{C}(\mathrm{COO})=\mathrm{O}$ & $\operatorname{lnChl}=15 / \mathrm{C} 5 \mathrm{H} 1003 / \mathrm{c} 1-4(2) 5(6) 3-8-7 / \mathrm{h} 4,7 \mathrm{H}, 3 \mathrm{H} 2,1-2 \mathrm{H} 3$ \\
\hline 1298 & CPNOOH3-2O2R & $\mathrm{C} 1 \mathrm{CC}(=0) \mathrm{C}(\mathrm{O}[0]) \mathrm{C} 1(00)$ & InChl=15/C5H705/c6-3-1-2-4(9-7)5(3)10-8/h4-5,7H,1-2H2 \\
\hline 1299 & $\mathrm{C} 4 \mathrm{H} 7 \mathrm{CHO}-4$ & $\mathrm{C}=\mathrm{CCCC}(=0)$ & InChl=1S/C5H8O/c1-2-3-4-5-6/h2,5H,1,3-4H2 \\
\hline 1300 & AC5H9-DO2 & {$[\mathrm{O}] \mathrm{OCCC}(=\mathrm{C}) \mathrm{C}$} & $\operatorname{lnChl}=15 / C 5 H 902 / c 1-5(2) 3-4-7-6 / h 1,3-4 \mathrm{H} 2,2 \mathrm{H} 3$ \\
\hline 1301 & NEOC5KET & $\mathrm{CC}(\mathrm{C})(\mathrm{C}=0) \mathrm{COO}$ & $\operatorname{lnChl}=15 / C 5 H 1003 / \mathrm{c} 1-5(2,3-6) 4-8-7 / h 3,7 \mathrm{H}, 4 \mathrm{H} 2,1-2 \mathrm{H} 3$ \\
\hline 1302 & CPNOOH3-402R & $\mathrm{C} 1(\mathrm{O}[\mathrm{O}]) \mathrm{CC}=0) \mathrm{CC} 1(\mathrm{OO})$ & InChl=1S/C5H705/c6-3-1-4(9-7)5(2-3)10-8/h4-5,7H,1-2H2 \\
\hline 1303 & C4H7CHO-3 & $\mathrm{CC}=\mathrm{CCC}(=0)$ & $\operatorname{lnChl}=15 / C 5 H 8 O / c 1-2-3-4-5-6 / h 2-3,5 \mathrm{H}, 4 \mathrm{H} 2,1 \mathrm{H} 3$ \\
\hline 1304 & AC5H9-CO2 & $\mathrm{CC}(\mathrm{C}(=\mathrm{C}) \mathrm{C}) \mathrm{O}[\mathrm{O}]$ & InChl=1S/C5H9O2/c1-4(2)5(3)7-6/h5H,1H2,2-3H3 \\
\hline 1305 & NEOC5H9O-OOH & $\mathrm{CC} 1(\mathrm{COC} 1) \mathrm{COO}$ & $\operatorname{lnChl}=15 / C 5 \mathrm{H} 1003 / \mathrm{c} 1-5(4-8-6) 2-7-3-5 / h 6 \mathrm{H}, 2-4 \mathrm{H} 2,1 \mathrm{H} 3$ \\
\hline 1306 & CPNOOH3-502R & $\mathrm{C} 1 \mathrm{C}(\mathrm{O}[\mathrm{O}]) \mathrm{C}(=0) \mathrm{CC} 1(\mathrm{OO})$ & InChl=1S/C5H705/c6-4-1-3(9-7)2-5(4)10-8/h3,5,7H,1-2H2 \\
\hline 1307 & IC3H7CHCO & $\mathrm{CC}(\mathrm{C}) \mathrm{C}=\mathrm{C}=\mathrm{O}$ & $\operatorname{lnChl}=1 \mathrm{~S} / \mathrm{C} 5 \mathrm{H} 8 \mathrm{O} / \mathrm{c} 1-5(2) 3-4-6 / \mathrm{h} 3,5 \mathrm{H}, 1-2 \mathrm{H} 3$ \\
\hline 1308 & CC5H9-BO2 & {$[\mathrm{O}] \mathrm{OC}(\mathrm{C}=\mathrm{C})(\mathrm{C}) \mathrm{C}$} & InChl=1S/C5H9O2/c1-4-5(2,3)7-6/h4H,1H2,2-3H3 \\
\hline 1309 & DC5H10OJ-AOOH & {$[0] \operatorname{ccc}(\mathrm{COO}) \mathrm{C}$} & $\operatorname{lnChl}=15 / C 5 H 1103 / \mathrm{c} 1-5(2-3-6) 4-8-7 / \mathrm{h} 5,7 \mathrm{H}, 2-4 \mathrm{H} 2,1 \mathrm{H} 3$ \\
\hline 1310 & $\mathrm{MBCY} 2 \mathrm{OM}-3 \mathrm{OOH}$ & $\mathrm{C}(\mathrm{O} 1) \mathrm{OC}(=0) \mathrm{C} 1 \mathrm{C}(\mathrm{OOO}) \mathrm{C}$ & $\operatorname{lnChl}=15 / C 5 H 805 / c 1-3(10-7) 4-5(6) 9-2-8-4 / h 3-4,7 \mathrm{H}, 2 \mathrm{H} 2,1 \mathrm{H3} 3$ \\
\hline 1311 & $\mathrm{IC} 3 \mathrm{H} 5 \mathrm{COCH} 3$ & $\mathrm{C}=\mathrm{C}(\mathrm{C}) \mathrm{C}(\mathrm{C})=\mathrm{O}$ & $\operatorname{lnChl}=1 \mathrm{~S} / \mathrm{C} 5 \mathrm{H} 8 \mathrm{O} / \mathrm{c} 1-4(2) 5(3) 6 / \mathrm{h} 1 \mathrm{H} 2,2-3 \mathrm{H} 3$ \\
\hline 1312 & CC5H9-AO2 & $\mathrm{CC}(\mathrm{C}=\mathrm{C}) \mathrm{CO}[\mathrm{O}]$ & InChl=1S/C5H9O2/c1-3-5(2)4-7-6/h3,5H,1,4H2,2H3 \\
\hline 1313 & DC5H10OJ-BOOH & {$[0] \mathrm{ccc}(\mathrm{OOO})(\mathrm{C}) \mathrm{C}$} & $\operatorname{lnChl}=15 / C 5 H 1103 / c 1-5(2,8-7) 3-4-6 / h 7 H, 3-4 H 2,1-2 \mathrm{H} 3$ \\
\hline 1314 & MBCY2OM-4OOH & $\mathrm{C}(\mathrm{O} 1) \mathrm{OC}(=\mathrm{O}) \mathrm{C} 1 \mathrm{CC}(\mathrm{OO})$ & InChl=1S/C5H8O5/c6-5-4(1-2-10-7)8-3-9-5/h4,7H,1-3H2 \\
\hline 1315 & NC52ONE-4 & $\mathrm{CC}(=\mathrm{O}) \mathrm{CC}=\mathrm{C}$ & InChl=1S/C5H8O/c1-3-4-5(2)6/h3H,1,4H2,2H3 \\
\hline 1316 & $\mathrm{MB}$ & $\operatorname{coc}(=0) \operatorname{ccc}$ & InChl=1S/C5H10O2/c1-3-4-5(6)7-2/h3-4H2,1-2H3 \\
\hline 1317 & DC5H10OJ-COOH & {$[0] \mathrm{CC}(\mathrm{C}(\mathrm{C}) \mathrm{C}) \mathrm{OO}$} & InChl=1S/C5H11O3/c1-4(2)5(3-6)8-7/h4-5,7H,3H2,1-2H3 \\
\hline 1318 & МВСҮзОМ-20OH & $\mathrm{C}(\mathrm{O} 1) \mathrm{OC}(=0) \mathrm{C}(\mathrm{OO}) \mathrm{C} 1 \mathrm{C}$ & $\operatorname{lnChl}=15 / C 5 H 805 / c 1-3-4(10-7) 5(6) 9-2-8-3 / h 3-4,7 \mathrm{H}, 2 \mathrm{H2}, 1 \mathrm{H3}$ \\
\hline 1319 & $\mathrm{C} 4 \mathrm{H} 72-2 \mathrm{CHO}$ & $\mathrm{CC}=\mathrm{C}(\mathrm{C}=\mathrm{O}) \mathrm{C}$ & InChl=1S/C5H8O/c1-3-5(2)4-6/h3-4H,1-2H3 \\
\hline 1320 & C5H9O1-2OH-1 & $\operatorname{cccc}(01) \operatorname{c10}$ & InChl=1S/C5H10O2/c1-2-3-4-5(6)7-4/h4-6H,2-3H2,1H3 \\
\hline 1321 & C5H9OH-10OH-4 & $\mathrm{C}[\mathrm{CH}] \mathrm{CCC}(\mathrm{OO}) \mathrm{O}$ & InChl=15/C5H11O3/c1-2-3-4-5(6)8-7/h2,5-7H,3-4H2,1H3 \\
\hline 1322 & МВСҮзОМ-4OOH & $\mathrm{C}(\mathrm{O} 1) \mathrm{OC}(=\mathrm{O}) \mathrm{CC} 1 \mathrm{C}(\mathrm{OO})$ & $\operatorname{lnChl}=1 \mathrm{~S} / \mathrm{C} 5 \mathrm{H} 8 \mathrm{O} 5 / \mathrm{c} 6-5-1-4(2-10-7) 8-3-9-5 / \mathrm{h} 4,7 \mathrm{H}, 1-3 \mathrm{H} 2$ \\
\hline 1323 & $\mathrm{IC} 4 \mathrm{H} 7 \mathrm{CHO}$ & $\mathrm{C}=\mathrm{C}(\mathrm{C}) \mathrm{CC}=\mathrm{O}$ & $\operatorname{lnChl}=1 \mathrm{~S} / \mathrm{C} 5 \mathrm{H} 8 \mathrm{O} / \mathrm{c} 1-5(2) 3-4-6 / \mathrm{h} 4 \mathrm{H}, 1,3 \mathrm{H} 2,2 \mathrm{H} 3$ \\
\hline 1324 & С5H9O1-3OH-1 & $\operatorname{ccc} 1 c c(01) 0$ & InChl=15/C5H10O2/c1-2-4-3-5(6)7-4/h4-6H,2-3H2,1H3 \\
\hline 1325 & $\mathrm{C} 5 \mathrm{H} 9 \mathrm{OH}-10 \mathrm{OOH}-3$ & $\mathrm{CC}[\mathrm{CH}] \mathrm{CC}(\mathrm{OO}) \mathrm{O}$ & $\operatorname{lnChl}=1 \mathrm{~S} / \mathrm{C} 5 \mathrm{H} 11 \mathrm{O} 3 / \mathrm{c} 1-2-3-4-5(6) 8-7 / \mathrm{h} 3,5-7 \mathrm{H}, 2,4 \mathrm{H} 2,1 \mathrm{H} 3$ \\
\hline 1326 & МВСҮзО2-МОOH & $\mathrm{C}(\mathrm{OO}) \mathrm{OC}(=\mathrm{O}) \mathrm{C}(\mathrm{O} 1) \mathrm{C} 1 \mathrm{C}$ & $\operatorname{lnChl}=15 / C 5 H 805 / c 1-3-4(10-3) 5(6) 8-2-9-7 / h 3-4,7 \mathrm{H}, 2 \mathrm{H} 2,1 \mathrm{H} 3$ \\
\hline 1327 & IC5D2Y4 & $\mathrm{CC}(\mathrm{C})=\mathrm{CC}=\mathrm{O}$ & InChl=1S/C5H8O/c1-5(2)3-4-6/h3-4H,1-2H3 \\
\hline 1328 & C5H9O2-3OH-1 & $\operatorname{cCC} 1 \mathrm{C}(01) \mathrm{CO}$ & InChl=1S/C5H10O2/c1-2-4-5(3-6)7-4/h4-6H,2-3H2,1H3 \\
\hline 1329 & $\mathrm{C} 5 \mathrm{H} 9 \mathrm{OH}-10 \mathrm{OH}-2$ & $\mathrm{CCC}[\mathrm{CH}] \mathrm{C}(\mathrm{OO}) \mathrm{O}$ & $\operatorname{lnChl}=15 / C 5 H 1103 / c 1-2-3-4-5(6) 8-7 / h 4-7 \mathrm{H}, 2-3 \mathrm{H} 2,1 \mathrm{H} 3$ \\
\hline 1330 & $\mathrm{MBCY} 3 \mathrm{O} 2-4 \mathrm{OOH}$ & $\mathrm{COC}(=0) \mathrm{C}(01) \mathrm{C} 1 \mathrm{C}(\mathrm{OO})$ & $\operatorname{lnChl}=15 / C 5 H 805 / c 1-8-5(6) 4-3(10-4) 2-9-7 / h 3-4,7 \mathrm{H}, 2 \mathrm{H} 2,1 \mathrm{H3}$ \\
\hline 1331 & AC5H8-DOH-C & $\mathrm{C}=\mathrm{C}(\mathrm{C})[\mathrm{CH}] \mathrm{CO}$ & $\operatorname{lnChl}=15 / C 5 H 9 O / c 1-5(2) 3-4-6 / h 3,6 \mathrm{H}, 1,4 \mathrm{H} 2,2 \mathrm{H} 3$ \\
\hline 1332 & DC5OHOD-C & $\mathrm{CC}(\mathrm{C}) \mathrm{C} 1 \mathrm{C}(\mathrm{O} 1) \mathrm{O}$ & InChl=1S/C5H10O2/c1-3(2)4-5(6)7-4/h3-6H,1-2H3 \\
\hline 1333 & $\mathrm{C} 5 \mathrm{H} 10 \mathrm{OH}-102$ & $\operatorname{CCCCC}(\mathrm{O}[\mathrm{O}]) \mathrm{O}$ & $\operatorname{lnChl}=15 / C 5 H 1103 / c 1-2-3-4-5(6) 8-7 / h 5-6 \mathrm{H}, 2-4 \mathrm{H} 2,1 \mathrm{H} 3$ \\
\hline 1334 & MBCY4O2-MOOH & $\mathrm{C}(\mathrm{OO}) \mathrm{OC}(=\mathrm{O}) \mathrm{C}(\mathrm{O} 1) \mathrm{CC} 1$ & InChl=1S/C5H8O5/c6-5(9-3-10-7)4-1-2-8-4/h4,7H,1-3H2 \\
\hline 1335 & BC5H8DJ-DOH & $\mathrm{OC}=\mathrm{C}[\mathrm{C}](\mathrm{C}) \mathrm{C}$ & InChl=15/C5H9O/c1-5(2)3-4-6/h3-4,6H,1-2H3 \\
\hline 1336 & C5H9O1-4OH-1 & $\mathrm{CC1CCC}(\mathrm{O} 1) \mathrm{O}$ & $\operatorname{lnChl}=1 \mathrm{~S} / \mathrm{C} 5 \mathrm{H} 1002 / \mathrm{c1}-4-2-3-5(6) 7-4 / \mathrm{h} 4-6 \mathrm{H}, 2-3 \mathrm{H} 2,1 \mathrm{H} 3$ \\
\hline 1337 & C5H9OH-2OOH-4 & $\mathrm{C}[\mathrm{CH}] \mathrm{CC}(\mathrm{OO}) \mathrm{CO}$ & $\operatorname{lnChl}=1 \mathrm{~S} / \mathrm{C} 5 \mathrm{H} 11 \mathrm{O} / \mathrm{c} 1-2-3-5(4-6) 8-7 / \mathrm{h} 2,5-7 \mathrm{H}, 3-4 \mathrm{H} 2,1 \mathrm{H} 3$ \\
\hline 1338 & MBCY4O2-3OOH & $\operatorname{COC}(=0) C(01) C(00) C 1$ & InChl=1S/C5H8O5/c1-8-5(6)4-3(10-7)2-9-4/h3-4,7H,2H2,1H3 \\
\hline 1339 & AC5H8DJ-DOH & $\mathrm{C}=\mathrm{C}(\mathrm{C}) \mathrm{C}[\mathrm{CH}] \mathrm{O}$ & InChl=15/C5H9O/c1-5(2)3-4-6/h4,6H,1,3H2,2H3 \\
\hline 1340 & C5H9O2-4OH-1 & $\mathrm{CC} 1 \mathrm{CC}(\mathrm{O} 1) \mathrm{CO}$ & InChl=1S/C5H10O2/c1-4-2-5(3-6)7-4/h4-6H,2-3H2,1H3 \\
\hline 1341 & C5H9OH-2OOH-3 & $\mathrm{CC}[\mathrm{CH}] \mathrm{C}(\mathrm{OO}) \mathrm{CO}$ & InChl=1S/C5H11O3/c1-2-3-5(4-6)8-7/h3,5-7H,2,4H2,1H3 \\
\hline 1342 & MBCY4O3-MOOH & $\mathrm{C}(\mathrm{OO}) \mathrm{OC}(=\mathrm{O}) \mathrm{CC}(\mathrm{O} 1) \mathrm{C} 1$ & InChl=1S/C5H8O5/c6-5(9-3-10-7)1-4-2-8-4/h4,7H,1-3H2 \\
\hline 1343 & AC5H8A2J-DOH & $\mathrm{C}=\mathrm{C}(\mathrm{CCO})[\mathrm{CH} 2]$ & $\operatorname{lnChl}=15 / C 5 H 90 / c 1-5(2) 3-4-6 / h 6 H, 1-4 H 2$ \\
\hline 1344 & DC5OHOD-A & $\mathrm{C} 1 \mathrm{C}(\mathrm{C}) \mathrm{CC}(01) \mathrm{O}$ & $\operatorname{lnChl}=15 / C 5 H 1002 / c 1-4-2-5(6) 7-3-4 / h 4-6 \mathrm{H}, 2-3 \mathrm{H} 2,1 \mathrm{H} 3$ \\
\hline 1345 & $\mathrm{C} 5 \mathrm{H} 9 \mathrm{OH}-2 \mathrm{OOH}-1$ & $\mathrm{CCCC}(\mathrm{OO})[\mathrm{CH}] \mathrm{O}$ & $\operatorname{lnChl}=15 / C 5 H 1103 / c 1-2-3-5(4-6) 8-7 / h 4-7 \mathrm{H}, 2-3 \mathrm{H} 2,1 \mathrm{H} 3$ \\
\hline 1346 & $\mathrm{MBCY} 403-2 \mathrm{OOH}$ & $\operatorname{coc}(=0) c(00) c(01) C 1$ & $\operatorname{lnChl}=15 / \mathrm{C} 5 \mathrm{H} 805 / \mathrm{c} 1-8-5(6) 4(10-7) 3-2-9-3 / \mathrm{h} 3-4,7 \mathrm{H}, 2 \mathrm{H} 2,1 \mathrm{H} 3$ \\
\hline 1347 & IC5D3OH2-1J & $\mathrm{C}=\mathrm{CC}(\mathrm{O})([\mathrm{CH} 2]) \mathrm{C}$ & $\operatorname{lnChl}=15 / C 5 H 9 O / c 1-4-5(2,3) 6 / h 4,6 \mathrm{H}, 1-2 \mathrm{H} 2,3 \mathrm{H} 3$ \\
\hline 1348 & DC5OHOC-A & $\mathrm{C} 1 \mathrm{C}(\mathrm{C}) \mathrm{C}(\mathrm{O} 1) \mathrm{CO}$ & $\operatorname{lnChl}=1 \mathrm{~S} / \mathrm{C} 5 \mathrm{H} 1002 / \mathrm{c1}-4-3-7-5(4) 2-6 / \mathrm{h} 4-6 \mathrm{H}, 2-3 \mathrm{H} 2,1 \mathrm{H} 3$ \\
\hline 1349 & $\mathrm{C} 5 \mathrm{H} 10 \mathrm{OH}-2 \mathrm{O} 2$ & $\operatorname{CCCC}(\mathrm{O}[\mathrm{O}]) \mathrm{CO}$ & $\operatorname{lnChl}=15 / C 5 H 1103 / c 1-2-3-5(4-6) 8-7 / h 5-6 \mathrm{H}, 2-4 \mathrm{H} 2,1 \mathrm{H} 3$ \\
\hline 1350 & MBMOOH2VO & $\mathrm{C}(\mathrm{OO}) \mathrm{OC}(=\mathrm{O}) \mathrm{C}(=\mathrm{O}) \mathrm{CC}$ & $\operatorname{lnChl}=1 \mathrm{~S} / \mathrm{C} 5 \mathrm{H} 8 \mathrm{O} 5 / \mathrm{c} 1-2-4(6) 5(7) 9-3-10-8 / \mathrm{h} 8 \mathrm{H}, 2-3 \mathrm{H} 2,1 \mathrm{H} 3$ \\
\hline 1351 & IC5D1OH3-4] & $\mathrm{CC}(=\mathrm{C}) \mathrm{C}(\mathrm{O})[\mathrm{CH} 2]$ & InChl=15/C5H9O/c1-4(2)5(3)6/h5-6H,1,3H2,2H3 \\
\hline 1352 & C5H9O3-4OH-1 & $\mathrm{CC} 1 \mathrm{C}(\mathrm{O} 1) \mathrm{CCO}$ & InChl=1S/C5H10O2/c1-4-5(7-4)2-3-6/h4-6H,2-3H2,1H3 \\
\hline 1353 & C5H9OH-2OOH-5 & {$[\mathrm{CH} 2] \mathrm{CCC}(\mathrm{OO}) \mathrm{CO}$} & InChl=1S/C5H11O3/c1-2-3-5(4-6)8-7/h5-7H,1-4H2 \\
\hline 1354 & MBMOOH3VO & $\mathrm{C}(\mathrm{OO}) \mathrm{OC}(=\mathrm{O}) \mathrm{CC}(=\mathrm{O}) \mathrm{C}$ & $\operatorname{lnChl}=15 / C 5 H 8 O 5 / c 1-4(6) 2-5(7) 9-3-10-8 / h 8 H, 2-3 \mathrm{H} 2,1 \mathrm{H} 3$ \\
\hline 1355 & IC4H9CO & $\mathrm{CC}(\mathrm{C}) \mathrm{C}[\mathrm{C}]=0$ & InChl=1S/C5H9o/c1-5(2)3-4-6/h5H,3H2,1-2H3 \\
\hline 1356 & DC5OHOD-B & $\mathrm{CC1}(\mathrm{C}) \mathrm{CC}(01) \mathrm{O}$ & $\operatorname{lnChl}=15 / C 5 H 1002 / c 1-5(2) 3-4(6) 7-5 / h 4,6 \mathrm{H}, 3 \mathrm{H} 2,1-2 \mathrm{H} 3$ \\
\hline 1357 & $\mathrm{C} 5 \mathrm{H} 9 \mathrm{OH}-3 \mathrm{OOH}-4$ & $\mathrm{C}[\mathrm{CH}] \mathrm{C}(\mathrm{OO}) \mathrm{CCO}$ & $\operatorname{lnChl}=1 \mathrm{~S} / \mathrm{C} 5 \mathrm{H} 1103 / \mathrm{c} 1-2-5(8-7) 3-4-6 / \mathrm{h} 2,5-7 \mathrm{H}, 3-4 \mathrm{H} 2,1 \mathrm{H} 3$ \\
\hline 1358 & MB2OOHMVO & $\mathrm{C}(=0) \mathrm{OC}(=0) \mathrm{C}(\mathrm{OO}) \mathrm{CC}$ & InChl=1S/C5H8O5/c1-2-4(10-8)5(7)9-3-6/h3-4,8H,2H2,1H3 \\
\hline 1359 & C5H9O2-1 & $\mathrm{CCC}=\mathrm{CC}[\mathrm{O}]$ & $\operatorname{lnChl}=15 / C 5 H 9 O / c 1-2-3-4-5-6 / h 3-4 \mathrm{H}, 2,5 \mathrm{H} 2,1 \mathrm{H} 3$ \\
\hline 1360 & DC5OHOC-B & $\mathrm{CC} 1(\mathrm{C}) \mathrm{C}(\mathrm{O} 1) \mathrm{CO}$ & $\operatorname{lnChl}=15 / C 5 H 1002 / c 1-5(2) 4(3-6) 7-5 / h 4,6 \mathrm{H}, 3 \mathrm{H} 2,1-2 \mathrm{H} 3$ \\
\hline 1361 & С5H9OH-3OOH-2 & $\mathrm{CCC}(\mathrm{OO})[\mathrm{CH}] \mathrm{CO}$ & InChl=1S/C5H11O3/c1-2-5(8-7)3-4-6/h3,5-7H,2,4H2,1H3 \\
\hline
\end{tabular}




\begin{tabular}{|c|c|c|c|}
\hline 1362 & MB2ОOHзVO & $\operatorname{coc}(=0) c(00) c(=0) c$ & InChl=1S/C5H8O5/c1-3(6)4(10-8)5(7)9-2/h4,8H,1-2H3 \\
\hline 1363 & СРTOH-2 & $\mathrm{OC} 1[\mathrm{CH}] \mathrm{CCC} 1$ & InChl=15/C5H9O/c6-5-3-1-2-4-5/h3,5-6H,1-2,4H2 \\
\hline 1364 & DC5OHOB-A & $\mathrm{C} 1 \mathrm{C}(\mathrm{C})(\mathrm{O} 1) \mathrm{CCO}$ & InChl=1S/C5H1002/c1-5(2-3-6)4-7-5/h6H,2-4H2,1H3 \\
\hline 1365 & $\mathrm{C} 5 \mathrm{H} 9 \mathrm{OH}-3 \mathrm{OOH}-1$ & $\mathrm{CCC}(\mathrm{OO}) \mathrm{C}[\mathrm{CH}] \mathrm{O}$ & $\operatorname{lnChl}=15 / C 5 H 1103 / c 1-2-5(8-7) 3-4-6 / h 4-7 \mathrm{H}, 2-3 \mathrm{H} 2,1 \mathrm{H} 3$ \\
\hline 1366 & $\mathrm{MB} 2 \mathrm{OOH} 4 \mathrm{VO}$ & $\operatorname{coc}(=0) C(00) \operatorname{CC}(=0)$ & $\mathrm{InChl}=1 \mathrm{~S} / \mathrm{C5H} 8 \mathrm{O} 5 / \mathrm{c1} 1-9-5(7) 4(10-8) 2-3-6 / \mathrm{h} 3-4,8 \mathrm{H}, 2 \mathrm{H} 2,1 \mathrm{H} 3$ \\
\hline 1367 & $\mathrm{C} 5 \mathrm{H} 911 \mathrm{OH}-5$ & {$[\mathrm{CH} 2] \mathrm{CCC}=\mathrm{CO}$} & $\operatorname{lnChl}=1 \mathrm{~S} / \mathrm{C} 5 \mathrm{H} 9 \mathrm{O} / \mathrm{c1} 1-2-3-4-5-6 / \mathrm{h} 4-6 \mathrm{H}, 1-3 \mathrm{H} 2$ \\
\hline 1368 & DC5OHOA-A & $\mathrm{C1C}(\mathrm{CO} 1) \mathrm{CCO}$ & $\mathrm{InChl}=1 \mathrm{~S} / \mathrm{C} 5 \mathrm{H} 10 \mathrm{O} 2 / \mathrm{c6}-2-1-5-3-7-4-5 / \mathrm{h} 5-6 \mathrm{H}, 1-4 \mathrm{H} 2$ \\
\hline 1369 & $\mathrm{C} 5 \mathrm{H} 10 \mathrm{OH}-3 \mathrm{O} 2$ & $\mathrm{CCC}(\mathrm{O}[\mathrm{O}]) \mathrm{CCO}$ & $\operatorname{lnChl}=15 / C 5 H 1103 / c 1-2-5(8-7) 3-4-6 / h 5-6 \mathrm{H}, 2-4 \mathrm{H} 2,1 \mathrm{H} 3$ \\
\hline 1370 & МвзоОНMVO & $\mathrm{C}(=0) \mathrm{OC}(=0) \mathrm{CC}(\mathrm{OO}) \mathrm{C}$ & InChl=1S/C5H8O5/c1-4(10-8)2-5(7)9-3-6/h3-4,8H,2H2,1H3 \\
\hline 1371 & $\mathrm{C} 5 \mathrm{H} 8 \mathrm{OH} 1-23$ & $\mathrm{CC}=\mathrm{C}[\mathrm{CH}] \mathrm{CO}$ & $\operatorname{lnChl}=15 / C 5 H 9 O / c 1-2-3-4-5-6 / h 2-4,6 \mathrm{H}, 5 \mathrm{H} 2,1 \mathrm{H} 3$ \\
\hline 1372 & C5H9O4-5OH-1 & $\mathrm{C} 1 \mathrm{C}(01) \mathrm{CCCO}$ & $\operatorname{lnChl}=1 \mathrm{~S} / \mathrm{C} 5 \mathrm{H} 10 \mathrm{O} 2 / \mathrm{c} 6-3-1-2-5-4-7-5 / \mathrm{h} 5-6 \mathrm{H}, 1-4 \mathrm{H} 2$ \\
\hline 1373 & $\mathrm{C} 5 \mathrm{H} 9 \mathrm{OH}-3 \mathrm{OOH}-5$ & {$[\mathrm{CH} 2] \mathrm{CC}(\mathrm{OO}) \mathrm{CCO}$} & InChl=1S/C5H11O3/c1-2-5(8-7)3-4-6/h5-7H,1-4H2 \\
\hline 1374 & $\mathrm{MB} 3 \mathrm{OOH} 2 \mathrm{VO}$ & $\operatorname{coc}(=0) c(=0) c(00) c$ & InChl=15/C5H8O5/c1-3(10-8)4(6)5(7)9-2/h3,8H,1-2H3 \\
\hline 1375 & $\mathrm{C} 5 \mathrm{H} 8 \mathrm{OH} 1-12$ & $\mathrm{CCC}=\mathrm{C}[\mathrm{CH}] \mathrm{O}$ & InChl=1S/C5H9o/c1-2-3-4-5-6/h3-6H,2H2,1H3 \\
\hline 1376 & C5H9O3-5OH-1 & $\mathrm{C} 1 \mathrm{CC}(\mathrm{O} 1) \mathrm{CCO}$ & $\operatorname{lnChl}=15 / C 5 H 10 O 2 / \mathrm{c} 6-3-1-5-2-4-7-5 / \mathrm{h} 5-6 \mathrm{H}, 1-4 \mathrm{H} 2$ \\
\hline 1377 & DC5H9OOH-DOH-C & $\mathrm{CC}(\mathrm{C})[\mathrm{CH}] \mathrm{C}(\mathrm{OO}) \mathrm{O}$ & InChl=1S/C5H11O3/c1-4(2)3-5(6)8-7/h3-7H,1-2H3 \\
\hline 1378 & MB3OOH4VO & $\operatorname{coc}(=0) \operatorname{CC}(00) \mathrm{C}(=0)$ & $\mathrm{InChl}=1 \mathrm{~S} / \mathrm{C} 5 \mathrm{H} 8 \mathrm{O} 5 / \mathrm{c1} 1-9-5(7) 2-4(3-6) 10-8 / \mathrm{h3}-4,8 \mathrm{H}, 2 \mathrm{H} 2,1 \mathrm{H} 3$ \\
\hline 1379 & $\mathrm{C} 5 \mathrm{H} 8 \mathrm{OH} 1-34$ & $\mathrm{C}=\mathrm{C}[\mathrm{CH}] \mathrm{CCO}$ & $\operatorname{lnChl}=1 \mathrm{~S} / \mathrm{C} 5 \mathrm{H} 90 / \mathrm{c} 1-2-3-4-5-6 / \mathrm{h} 2-3,6 \mathrm{H}, 1,4-5 \mathrm{H} 2$ \\
\hline 1380 & C5H9O2-50H-1 & $\mathrm{C} 1 \mathrm{CCC}(01) \mathrm{CO}$ & InChl=1S/C5H10O2/c6-4-5-2-1-3-7-5/h5-6H,1-4H2 \\
\hline 1381 & DC5H10OH-DO2 & $\mathrm{CC}(\mathrm{C}) \mathrm{CC}(\mathrm{O}[\mathrm{O}]) \mathrm{O}$ & $\operatorname{lnChl}=15 / C 5 H 1103 / c 1-4(2) 3-5(6) 8-7 / h 4-6 \mathrm{H}, 3 \mathrm{H} 2,1-2 \mathrm{H} 3$ \\
\hline 1382 & $\mathrm{MB} 4 \mathrm{OOH} 2 \mathrm{VO}$ & $\operatorname{coc}(=0) \mathrm{C}(=0) \operatorname{cc}(00)$ & InChl=1S/C5H8O5/c1-9-5(7)4(6)2-3-10-8/h8H,2-3H2,1H3 \\
\hline 1383 & $\mathrm{PC} 4 \mathrm{H} 8 \mathrm{CHO}-3$ & $\mathrm{C}[\mathrm{CH}] \mathrm{CCC}=\mathrm{O}$ & $\operatorname{lnChl}=15 / C 5 H 9 O / c 1-2-3-4-5-6 / h 2,5 \mathrm{H}, 3-4 \mathrm{H} 2,1 \mathrm{H} 3$ \\
\hline 1384 & С5Н9OHO2А-B & $\operatorname{ccc} 1(\mathrm{C}) \mathrm{C}(\mathrm{O} 1) \mathrm{O}$ & InChl=15/C5H10O2/c1-3-5(2)4(6)7-5/h4,6H,3H2,1-2H3 \\
\hline 1385 & DC5H9OOH-DOH-A & {$[\mathrm{CH} 2] \mathrm{C}(\mathrm{C}) \mathrm{CC}(\mathrm{OO}) \mathrm{O}$} & $\operatorname{lnChl}=15 / C 5 H 1103 / \mathrm{c} 1-4(2) 3-5(6) 8-7 / \mathrm{h} 4-7 \mathrm{H}, 1,3 \mathrm{H} 2,2 \mathrm{H} 3$ \\
\hline 1386 & MB4OOH3VO & $\operatorname{COC}(=0) \mathrm{CC}(=0) \mathrm{C}(\mathrm{OO})$ & InChl=15/C5H8O5/c1-9-5(7)2-4(6)3-10-8/h8H,2-3H2,1H3 \\
\hline 1387 & PC4H9CO & $\operatorname{cccc}[\mathrm{C}]=0$ & InChl=15/C5H9O/c1-2-3-4-5-6/h2-4H2,1H3 \\
\hline 1388 & C5H9OHO2A-C & $\mathrm{CC1C}(\mathrm{C}) \mathrm{C}(\mathrm{O} 1) \mathrm{O}$ & InChl=1S/C5H10O2/c1-3-4(2)7-5(3)6/h3-6H,1-2H3 \\
\hline 1389 & DC5H9OOH-DOH-B & $\mathrm{C}[\mathrm{C}](\mathrm{C}) \mathrm{CC}(\mathrm{OO}) \mathrm{O}$ & InChl=15/C5H11O3/c1-4(2)3-5(6)8-7/h5-7H,3H2,1-2H3 \\
\hline 1390 & AC5H8-DOHCQAOO & {$[\mathrm{O}] \mathrm{OCC}(=\mathrm{C}) \mathrm{C}(\mathrm{OO}) \mathrm{CO}$} & InChl=1S/C5H9O5/c1-4(3-9-7)5(2-6)10-8/h5-6,8H,1-3H2 \\
\hline 1391 & PC4H8CHO-2 & $\mathrm{CC}[\mathrm{CH}] \mathrm{CC}=\mathrm{O}$ & $\operatorname{lnChl}=15 / C 5 H 9 O / c 1-2-3-4-5-6 / h 3,5 \mathrm{H}, 2,4 \mathrm{H} 2,1 \mathrm{H} 3$ \\
\hline 1392 & C5H9OHO2A-D & $\mathrm{C} 1 \mathrm{CC}(\mathrm{C}) \mathrm{C}(01) \mathrm{O}$ & $\operatorname{lnChl}=1 \mathrm{~S} / \mathrm{C} 5 \mathrm{H} 10 \mathrm{OO} / \mathrm{c1}-4-2-3-7-5(4) 6 / \mathrm{h} 4-6 \mathrm{H}, 2-3 \mathrm{H} 2,1 \mathrm{H} 3$ \\
\hline 1393 & CC5H9OOH-DOH-D & $\mathrm{CC}(\mathrm{C}) \mathrm{C}(\mathrm{OO})[\mathrm{CH}] \mathrm{O}$ & $\operatorname{lnChl}=1 \mathrm{~S} / \mathrm{C5} H 1103 / \mathrm{c} 1-4(2) 5(3-6) 8-7 / \mathrm{h} 3-7 \mathrm{H}, 1-2 \mathrm{H} 3$ \\
\hline 1394 & BC5H8-DOHAQAOO & $\mathrm{OOCC}(=\mathrm{CCO}) \mathrm{CO}[\mathrm{O}]$ & InChl=1S/C5H9O5/c6-2-1-5(3-9-7)4-10-8/h1,6-7H,2-4H2 \\
\hline 1395 & $\mathrm{PC} 4 \mathrm{H} 8 \mathrm{CHO}-1$ & $\mathrm{CCC}[\mathrm{CH}] \mathrm{C}=\mathrm{O}$ & $\ln \mathrm{Chl}=1 \mathrm{~S} / \mathrm{C} 5 \mathrm{H} 9 \mathrm{O} / \mathrm{c1}-2-3-4-5-6 / \mathrm{h} 4-5 \mathrm{H}, 2-3 \mathrm{H} 2,1 \mathrm{H} 3$ \\
\hline 1396 & С5H9OHO2A-E & $\operatorname{ccc}(\mathrm{C} 1) \mathrm{C}(01) \mathrm{O}$ & $\operatorname{lnChl}=15 / C 5 \mathrm{H} 1002 / \mathrm{c} 1-2-4-3-7-5(4) 6 / \mathrm{h} 4-6 \mathrm{H}, 2-3 \mathrm{H} 2,1 \mathrm{H} 3$ \\
\hline 1397 & $\mathrm{DC5} \mathrm{H} 10 \mathrm{OH}-\mathrm{CO} 2$ & $\mathrm{CC}(\mathrm{C}) \mathrm{C}(\mathrm{O}[\mathrm{O}]) \mathrm{CO}$ & $\operatorname{lnChl}=15 / C 5 H 1103 / c 1-4(2) 5(3-6) 8-7 / h 4-6 \mathrm{H}, 3 \mathrm{H} 2,1-2 \mathrm{H} 3$ \\
\hline 1398 & $\mathrm{MB} 2 \mathrm{OH} 3 \mathrm{OO}$ & $\operatorname{coc}(=0) C(0) C(O[O]) C$ & InChl=1S/C5H9O5/c1-3(10-8)4(6)5(7)9-2/h3-4,6H,1-2H3 \\
\hline 1399 & $\mathrm{PC} 4 \mathrm{H} 8 \mathrm{CHO}-4$ & {$[\mathrm{CH} 2] \mathrm{CCCC}=\mathrm{O}$} & $\operatorname{lnChl}=15 / \mathrm{C} 5 \mathrm{H} 9 \mathrm{O} / \mathrm{c1} 1-2-3-4-5-6 / \mathrm{h} 5 \mathrm{H}, 1-4 \mathrm{H} 2$ \\
\hline 1400 & С5H9OHO2B-C & $\mathrm{CC1C}(\mathrm{C})(\mathrm{O} 1) \mathrm{CO}$ & InChl=15/C5H10O2/c1-4-5(2,3-6)7-4/h4,6H,3H2,1-2H3 \\
\hline 1401 & CC5H9OOH-DOH-A & {$[\mathrm{CH} 2] \mathrm{C}(\mathrm{C}) \mathrm{C}(\mathrm{OO}) \mathrm{CO}$} & $\operatorname{lnChl}=15 / C 5 H 1103 / c 1-4(2) 5(3-6) 8-7 / h 4-7 \mathrm{H}, 1,3 \mathrm{H} 2,2 \mathrm{H} 3$ \\
\hline 1402 & $\mathrm{MB} 3 \mathrm{OH} 4 \mathrm{OO}$ & $\mathrm{COC}(=0) \mathrm{CC}(0) \mathrm{C}(\mathrm{O}[\mathrm{O}])$ & InChl=1S/C5H9O5/c1-9-5(7)2-4(6)3-10-8/h4,6H,2-3H2,1H3 \\
\hline 1403 & C5H9O2-4 & $\mathrm{CC}([\mathrm{O}]) \mathrm{C}=\mathrm{CC}$ & InChl=1S/C5H9O/c1-3-4-5(2)6/h3-5H,1-2H3 \\
\hline 1404 & C5H9OHO2B-D & $\mathrm{C} 1 \mathrm{CC}(\mathrm{C})(\mathrm{O} 1) \mathrm{CO}$ & $\operatorname{lnChl}=1 \mathrm{~S} / \mathrm{C} 5 \mathrm{H} 1002 / \mathrm{c1}-5(4-6) 2-3-7-5 / \mathrm{h} 6 \mathrm{H}, 2-4 \mathrm{H} 2,1 \mathrm{H3}$ \\
\hline 1405 & CC5H9OOH-DOH-B & $\mathrm{C}[\mathrm{C}](\mathrm{C}) \mathrm{C}(\mathrm{OO}) \mathrm{CO}$ & $\operatorname{lnChl}=15 / C 5 H 1103 / c 1-4(2) 5(3-6) 8-7 / h 5-7 \mathrm{H}, 3 \mathrm{H} 2,1-2 \mathrm{H} 3$ \\
\hline 1406 & C5H9OH-10OH-2O2 & $\operatorname{cccc}(0[0]) C(00) 0$ & InChl=1S/C5H1105/c1-2-3-4(9-7)5(6)10-8/h4-6,8H,2-3H2,1H3 \\
\hline 1407 & C5H9O1-3 & $\operatorname{ccc}([0]) \mathrm{C}=\mathrm{C}$ & $\operatorname{lnChl}=15 / C 5 H 9 O / c 1-3-5(6) 4-2 / h 3,5 \mathrm{H}, 1,4 \mathrm{H} 2,2 \mathrm{H} 3$ \\
\hline 1408 & С5H9OHO2B-E & $\mathrm{CCC}(\mathrm{C} 1)(\mathrm{O} 1) \mathrm{CO}$ & InChl=1S/C5H1002/c1-2-5(3-6)4-7-5/h6H,2-4H2,1H3 \\
\hline 1409 & BC5H9OOH-DOH-C & $\mathrm{CC}(\mathrm{C})(\mathrm{OO})[\mathrm{CH}] \mathrm{CO}$ & $\operatorname{lnChl}=1 \mathrm{~S} / \mathrm{C5} \mathrm{H} 11 \mathrm{O} 3 / \mathrm{c} 1-5(2,8-7) 3-4-6 / \mathrm{h} 3,6-7 \mathrm{H}, 4 \mathrm{H} 2,1-2 \mathrm{H} 3$ \\
\hline 1410 & $\mathrm{C} 5 \mathrm{H} 9 \mathrm{OH}-2 \mathrm{OOH}-102$ & $\operatorname{cccc}(00) c(0[0]) 0$ & InChl=1S/C5H1105/c1-2-3-4(9-7)5(6)10-8/h4-7H,2-3H2,1H3 \\
\hline 1411 & DC5H9O-C & $\mathrm{CC}(\mathrm{C})[\mathrm{CH}] \mathrm{C}=\mathrm{O}$ & InChl=1S/C5H9O/c1-5(2)3-4-6/h3-5H,1-2H3 \\
\hline 1412 & C5H9OHO2C-D & $\mathrm{C} 1 \mathrm{C}(\mathrm{O} 1) \mathrm{C}(\mathrm{C}) \mathrm{CO}$ & $\operatorname{lnChl}=15 / C 5 H 10 O 2 / c 1-4(2-6) 5-3-7-5 / h 4-6 \mathrm{H}, 2-3 \mathrm{H} 2,1 \mathrm{H} 3$ \\
\hline 1413 & BC5H9OOH-DOH-D & $\mathrm{CC}(\mathrm{C})(\mathrm{OO}) \mathrm{C}[\mathrm{CH}] \mathrm{O}$ & $\operatorname{lnChl}=1 \mathrm{~S} / \mathrm{C5} \mathrm{H} 11 \mathrm{O} 3 / \mathrm{c} 1-5(2,8-7) 3-4-6 / \mathrm{h} 4,6-7 \mathrm{H}, 3 \mathrm{H} 2,1-2 \mathrm{H} 3$ \\
\hline 1414 & C5H9OH-10OH-302 & $\mathrm{CCC}(\mathrm{O}[\mathrm{O}]) \mathrm{CC}(\mathrm{OO}) \mathrm{O}$ & InChl=1S/C5H1105/c1-2-4(9-7)3-5(6)10-8/h4-6,8H,2-3H2,1H3 \\
\hline 1415 & DC5H9O-A & {$[\mathrm{CH} 2] \mathrm{C}(\mathrm{C}) \mathrm{CC}=\mathrm{O}$} & $\operatorname{lnChl}=1 \mathrm{~S} / \mathrm{C} 5 \mathrm{H} 9 \mathrm{O} / \mathrm{c1}-5(2) 3-4-6 / \mathrm{h} 4-5 \mathrm{H}, 1,3 \mathrm{H} 2,2 \mathrm{H} 3$ \\
\hline 1416 & C5H9OHO2C-E & $\mathrm{CC}(\mathrm{O} 1) \mathrm{C}(\mathrm{C} 1) \mathrm{CO}$ & $\operatorname{lnChl}=15 / C 5 H 10 O 2 / c 1-4-5(2-6) 3-7-4 / h 4-6 \mathrm{H}, 2-3 \mathrm{H} 2,1 \mathrm{H} 3$ \\
\hline 1417 & BC5H9OOH-DOH-A & {$[\mathrm{CH} 2] \mathrm{C}(\mathrm{C})(\mathrm{OO}) \mathrm{CCO}$} & $\operatorname{lnChl}=1 \mathrm{~S} / \mathrm{C5} \mathrm{H} 1103 / \mathrm{c} 1-5(2,8-7) 3-4-6 / \mathrm{h6}-7 \mathrm{H}, 1,3-4 \mathrm{H} 2,2 \mathrm{H} 3$ \\
\hline 1418 & $\mathrm{C} 5 \mathrm{H} 9 \mathrm{OH}-3 \mathrm{OOH}-1 \mathrm{O} 2$ & $\operatorname{ccC}(00) \operatorname{cc}(0[0]) 0$ & InChl=1S/C5H1105/c1-2-4(9-7)3-5(6)10-8/h4-7H,2-3H2,1H3 \\
\hline 1419 & DC5H9O-B & $C[C](C) C C=O$ & InChl=1S/C5H9O/c1-5(2)3-4-6/h4H,3H2,1-2H3 \\
\hline 1420 & C5H9OHO2D-E & $\mathrm{C}(\mathrm{O} 1) \mathrm{CC}(\mathrm{C} 1) \mathrm{CO}$ & $\operatorname{lnChl}=1 \mathrm{~S} / \mathrm{C} 5 \mathrm{H} 10 \mathrm{O} 2 / \mathrm{c} 6-3-5-1-2-7-4-5 / \mathrm{h} 5-6 \mathrm{H}, 1-4 \mathrm{H} 2$ \\
\hline 1421 & DC5H10OH-BO2 & $\mathrm{CC}(\mathrm{C})(\mathrm{O}[\mathrm{O}]) \mathrm{CCO}$ & $\operatorname{lnChl}=15 / C 5 H 1103 / \mathrm{c} 1-5(2,8-7) 3-4-6 / h 6 H, 3-4 \mathrm{H} 2,1-2 \mathrm{H} 3$ \\
\hline 1422 & $\mathrm{C} 5 \mathrm{H} 9 \mathrm{OH}-2 \mathrm{OOH}-3 \mathrm{O} 2$ & $\operatorname{ccc}(\mathrm{O}[\mathrm{O}]) \mathrm{C}(\mathrm{OO}) \mathrm{CO}$ & InChl=1S/C5H11O5/c1-2-4(9-7)5(3-6)10-8/h4-6,8H,2-3H2,1H3 \\
\hline 1423 & AC5H9O-C & $\mathrm{CC}(=\mathrm{C}) \mathrm{C}([\mathrm{O}]) \mathrm{C}$ & InChl=1S/C5H9O/c1-4(2)5(3)6/h5H,1H2,2-3H3 \\
\hline 1424 & CPTO2H & $\mathrm{C1C}(\mathrm{OO}) \mathrm{CCC1}$ & $\operatorname{lnChl}=1 \mathrm{~S} / \mathrm{C} 5 \mathrm{H} 10 \mathrm{OO} / \mathrm{c6}-7-5-3-1-2-4-5 / \mathrm{h} 5-6 \mathrm{H}, 1-4 \mathrm{H} 2$ \\
\hline 1425 & AC5H9OOH-DOH-C & $\mathrm{CC}(\mathrm{COO})[\mathrm{CH}] \mathrm{CO}$ & $\operatorname{lnChl}=15 / C 5 H 1103 / c 1-5(2-3-6) 4-8-7 / h 2,5-7 \mathrm{H}, 3-4 \mathrm{H} 2,1 \mathrm{H} 3$ \\
\hline 1426 & $\mathrm{C} 5 \mathrm{H} 9 \mathrm{OH}-3 \mathrm{OOH}-2 \mathrm{O} 2$ & $\operatorname{ccc}(\mathrm{OO}) \mathrm{C}(\mathrm{O}[\mathrm{O}]) \mathrm{CO}$ & InChl=15/C5H1105/c1-2-4(9-7)5(3-6)10-8/h4-7H,2-3H2,1H3 \\
\hline 1427 & CC5H9O-B & $\mathrm{C}=\mathrm{CC}(\mathrm{C})([\mathrm{O}]) \mathrm{C}$ & $\operatorname{lnChl}=1 \mathrm{~S} / \mathrm{C} 5 \mathrm{H} 9 \mathrm{O} / \mathrm{c} 1-4-5(2,3) 6 / \mathrm{h} 4 \mathrm{H}, 1 \mathrm{H} 2,2-3 \mathrm{H} 3$ \\
\hline 1428 & $\mathrm{IC4H} 7 \mathrm{OOCH} 3$ & $\mathrm{C}=\mathrm{C}(\mathrm{C}) \mathrm{COOC}$ & $\operatorname{lnChl}=1 \mathrm{~S} / \mathrm{C} 5 \mathrm{H} 1002 / \mathrm{c} 1-5(2) 4-7-6-3 / \mathrm{h} 1,4 \mathrm{H} 2,2-3 \mathrm{H} 3$ \\
\hline 1429 & AC5H9OOH-DOH-D & $\mathrm{CC}(\mathrm{COO}) \mathrm{C}[\mathrm{CH}] \mathrm{O}$ & $\operatorname{lnChl}=1 \mathrm{~S} / \mathrm{C} 5 \mathrm{H} 11 \mathrm{O} / \mathrm{c} 1-5(2-3-6) 4-8-7 / \mathrm{h3}, 5-7 \mathrm{H}, 2,4 \mathrm{H} 2,1 \mathrm{H} 3$ \\
\hline 1430 & DC5H9OOH-DOH-CO2 & $\mathrm{CC}(\mathrm{C}) \mathrm{C}(\mathrm{O}[\mathrm{O}]) \mathrm{C}(\mathrm{OO}) \mathrm{O}$ & InChl=1S/C5H11O5/c1-3(2)4(9-7)5(6)10-8/h3-6,8H,1-2H3 \\
\hline 1431 & B2E2M1OJ & {$[0] \mathrm{CC}(=\mathrm{CC}) \mathrm{C}$} & InChl=1S/C5H9O/c1-3-5(2)4-6/h3H,4H2,1-2H3 \\
\hline 1432 & C4H71-30OCH3 & $\mathrm{C}=\mathrm{CC}(\mathrm{C}) \mathrm{OOC}$ & InChl=1S/C5H1002/c1-4-5(2)7-6-3/h4-5H,1H2,2-3H3 \\
\hline 1433 & $\mathrm{DC5H} 10 \mathrm{OH}-\mathrm{AO} 2$ & $\mathrm{C}(\mathrm{O}[\mathrm{O}]) \mathrm{C}(\mathrm{C}) \mathrm{CCO}$ & $\operatorname{lnChl}=15 / C 5 H 1103 / c 1-5(2-3-6) 4-8-7 / h 5-6 \mathrm{H}, 2-4 \mathrm{H} 2,1 \mathrm{H} 3$ \\
\hline 1434 & CC5H9OOH-DOH-DO2 & $\mathrm{CC}(\mathrm{C}) \mathrm{C}(\mathrm{OO}) \mathrm{C}(\mathrm{O}[\mathrm{O}]) \mathrm{O}$ & InChl=1S/C5H1105/c1-3(2)4(9-7)5(6)10-8/h3-7H,1-2H3 \\
\hline 1435 & B2E3M1OJ & {$[\mathrm{O}] \mathrm{CC}=\mathrm{C}(\mathrm{C}) \mathrm{C}$} & InChl=1S/C5H9o/c1-5(2)3-4-6/h3H,4H2,1-2H3 \\
\hline 1436 & $\mathrm{C} 4 \mathrm{H} 72-10 \mathrm{OCH} 3$ & $\mathrm{CC}=\mathrm{CCOOC}$ & $\mathrm{InChl}=1 \mathrm{~S} / \mathrm{C} 5 \mathrm{H} 1002 / \mathrm{c1}-3-4-5-7-6-2 / \mathrm{h} 3-4 \mathrm{H}, 5 \mathrm{H} 2,1-2 \mathrm{H3}$ \\
\hline 1437 & AC5H9OOH-DOH-A & {$[\mathrm{CH} 2] \mathrm{C}(\mathrm{COO}) \mathrm{CCO}$} & $\operatorname{lnChl}=15 / C 5 H 1103 / c 1-5(2-3-6) 4-8-7 / h 5-7 \mathrm{H}, 1-4 \mathrm{H} 2$ \\
\hline 1438 & DC5H9OOH-DOH-AO2 & $\mathrm{C}(\mathrm{O}[\mathrm{O}]) \mathrm{C}(\mathrm{C}) \mathrm{CC}(\mathrm{OO}) \mathrm{O}$ & InChl=1S/C5H1105/c1-4(3-9-7)2-5(6)10-8/h4-6,8H,2-3H2,1H3 \\
\hline 1439 & $\mathrm{C} 5 \mathrm{H} 8 \mathrm{OH} 2-13$ & $\mathrm{C}[\mathrm{CH}] \mathrm{C}(\mathrm{C})=\mathrm{CO}$ & $\operatorname{lnChl}=1 \mathrm{~S} / \mathrm{C} 5 \mathrm{H} 9 \mathrm{O} / \mathrm{c} 1-3-5(2) 4-6 / \mathrm{h} 3-4,6 \mathrm{H}, 1-2 \mathrm{H} 3$ \\
\hline 1440 & $\mathrm{C} 5 \mathrm{H} 91-30 \mathrm{OH}$ & $\mathrm{C}=\mathrm{CC}(\mathrm{CC}) 0 \mathrm{O}$ & $\operatorname{lnChl}=15 / C 5 H 1002 / c 1-3-5(4-2) 77-6 / h 3,5-6 \mathrm{H}, 1,4 \mathrm{H} 2,2 \mathrm{H} 3$ \\
\hline 1441 & AC5H9OOH-DOH-B & $\mathrm{C}[\mathrm{C}](\mathrm{COO}) \mathrm{CCO}$ & $\operatorname{lnChl}=15 / C 5 H 1103 / \mathrm{c} 1-5(2-3-6) 4-8-7 / \mathrm{h} 6-7 \mathrm{H}, 2-4 \mathrm{H} 2,1 \mathrm{H} 3$ \\
\hline 1442 & AC5H9OOH-DOH-DO2 & $\mathrm{CC}(\mathrm{COO}) \mathrm{CC}(\mathrm{O}[\mathrm{O}]) \mathrm{O}$ & InChl=1S/C5H1105/c1-4(3-9-7)2-5(6)10-8/h4-7H,2-3H2,1H3 \\
\hline 1443 & NC4H9CO-2 & $\mathrm{CC}([\mathrm{C}]=0) \mathrm{CC}$ & InChl=1S/C5H9O/c1-3-5(2)4-6/h5H,3H2,1-2H3 \\
\hline 1444 & $\mathrm{C} 5 \mathrm{H} 91-40 \mathrm{OH}$ & $\mathrm{C}=\mathrm{CCC}(\mathrm{C}) 0 \mathrm{O}$ & $\operatorname{lnChl}=15 / C 5 H 1002 / c 1-3-4-5(2) 7-6 / h 3,5-6 \mathrm{H}, 1,4 \mathrm{H} 2,2 \mathrm{H} 3$ \\
\hline 1445 & $\mathrm{C} 5 \mathrm{H} 9 \mathrm{OH}-4 \mathrm{OOH}-2$ & $\mathrm{CC}(\mathrm{OO}) \mathrm{C}[\mathrm{CH}] \mathrm{CO}$ & $\operatorname{lnChl}=15 / \mathrm{C}^{2} \mathrm{H} 11 \mathrm{O} 3 / \mathrm{c} 1-5(8-7) 3-2-4-6 / \mathrm{h} 2,5-7 \mathrm{H}, 3-4 \mathrm{H} 2,1 \mathrm{H} 3$ \\
\hline 1446 & CC5H9OOH-DOH-AO2 & $\mathrm{C}(\mathrm{O}[\mathrm{O}]) \mathrm{C}(\mathrm{C}) \mathrm{C}(\mathrm{OO}) \mathrm{CO}$ & InChl=1S/C5H1105/c1-4(3-9-7)5(2-6)10-8/h4-6,8H,2-3H2,1H3 \\
\hline 1447 & $\mathrm{C} 4 \mathrm{H} 8 \mathrm{CHO1-2}$ & {$[\mathrm{CH} 2] \mathrm{C}(\mathrm{C}=\mathrm{O}) \mathrm{CC}$} & $\operatorname{lnChl}=1 \mathrm{~S} / \mathrm{C} 5 \mathrm{H} 9 \mathrm{O} / \mathrm{c1}-3-5(2) 4-6 / \mathrm{h} 4-5 \mathrm{H}, 2-3 \mathrm{H} 2,1 \mathrm{H} 3$ \\
\hline 1448 & $\mathrm{C} 5 \mathrm{H} 91-5 \mathrm{OOH}$ & $\mathrm{C}=\mathrm{CCCCOO}$ & InChl=1S/C5H1002/c1-2-3-4-5-7-6/h2,6H,1,3-5H2 \\
\hline 1449 & C5H9OH-4OOH-3 & $\mathrm{CC}(\mathrm{OO})[\mathrm{CH}] \mathrm{CCO}$ & InChl=1S/C5H11O3/c1-5(8-7)3-2-4-6/h3,5-7H,2,4H2,1H3 \\
\hline 1450 & AC5H9OOH-DOH-CO2 & $\mathrm{CC}(\mathrm{COO}) \mathrm{C}(\mathrm{O}[\mathrm{O}]) \mathrm{CO}$ & $\operatorname{lnChl}=1 \mathrm{~S} / \mathrm{C} 5 \mathrm{H} 1105 / \mathrm{c} 1-4(3-9-7) 5(2-6) 10-8 / \mathrm{h} 4-7 \mathrm{H}, 2-3 \mathrm{H} 2,1 \mathrm{H} 3$ \\
\hline 1451 & $\mathrm{C} 4 \mathrm{H} 8 \mathrm{CHO}-2$ & $\mathrm{CC}(=\mathrm{C}[\mathrm{O}]) \mathrm{CC}$ & $\mathrm{InChl}=1 \mathrm{~S} / \mathrm{C} 5 \mathrm{H} 9 \mathrm{O} / \mathrm{c1}-3-5(2) 4-6 / \mathrm{h} 4 \mathrm{H}, 3 \mathrm{H} 2,1-2 \mathrm{H} 3$ \\
\hline 1452 & $\mathrm{C} 5 \mathrm{H} 92-10 \mathrm{OH}$ & $\mathrm{CCC}=\mathrm{CCOO}$ & $\operatorname{lnChl}=1 \mathrm{~S} / \mathrm{C} 5 \mathrm{H} 1002 / \mathrm{c} 1-2-3-4-5-7-6 / \mathrm{h} 3-4,6 \mathrm{H}, 2,5 \mathrm{H} 2,1 \mathrm{H} 3$ \\
\hline
\end{tabular}




\begin{tabular}{|c|c|c|c|}
\hline 1453 & С5H9OH-4OOH-1 & $\mathrm{CC}(\mathrm{OO}) \mathrm{CC}[\mathrm{CH}] \mathrm{O}$ & InChl=1S/C5H11O3/c1-5(8-7)3-2-4-6/h4-7H,2-3H2,1H3 \\
\hline 1454 & C5H9OH-10OH-4O2 & $\mathrm{CC}(\mathrm{O}[\mathrm{O}]) \mathrm{CCC}(\mathrm{OO}) \mathrm{O}$ & InChl=1S/C5H1105/c1-4(9-7)2-3-5(6)10-8/h4-6,8H,2-3H2,1H3 \\
\hline 1455 & $\mathrm{C} 4 \mathrm{H} 8 \mathrm{CHO}-2$ & $\mathrm{CC}(\mathrm{C}=\mathrm{O})[\mathrm{CH}] \mathrm{C}$ & InChl=1S/C5H9O/c1-3-5(2)4-6/h3-5H,1-2H3 \\
\hline 1456 & $\mathrm{C} 5 \mathrm{H} 92-4 \mathrm{OOH}$ & $\mathrm{CC}=\mathrm{CC}(\mathrm{C}) \mathrm{OO}$ & $\operatorname{lnChl}=15 / C 5 H 1002 / c 1-3-4-5(2) 7-6 / h 3-6 \mathrm{H}, 1-2 \mathrm{H} 3$ \\
\hline 1457 & $\mathrm{C} 5 \mathrm{H} 10 \mathrm{OH}-4 \mathrm{O} 2$ & $\mathrm{CC}(\mathrm{O}[\mathrm{O}]) \mathrm{CCCO}$ & InChl=1S/C5H1103/c1-5(8-7)3-2-4-6/h5-6H,2-4H2,1H3 \\
\hline 1458 & С5H9OH-4OOH-102 & $\mathrm{CC}(\mathrm{OOO}) \mathrm{CCC}(\mathrm{O}[0]) \mathrm{O}$ & InChl=1S/C5H1105/c1-4(9-7)2-3-5(6)10-8/h4-7H,2-3H2,1H3 \\
\hline 1459 & C53OH1EN5J & $\mathrm{C}=\mathrm{CC}(\mathrm{O}) \mathrm{C}[\mathrm{CH} 2]$ & InChl=1S/C5H9O/c1-3-5(6)4-2/h3,5-6H,1-2,4H2 \\
\hline 1460 & $\mathrm{C} 5 \mathrm{H} 92-50 \mathrm{OH}$ & $\mathrm{CC}=\mathrm{CCCOO}$ & InChl=1S/C5H10O2/c1-2-3-4-5-7-6/h2-3,6H,4-5H2,1H3 \\
\hline 1461 & C5H9OH-4OOH-5 & {$[\mathrm{CH} 2] \mathrm{C}(\mathrm{OO}) \mathrm{CCCO}$} & InChl=1S/C5H11O3/c1-5(8-7)3-2-4-6/h5-7H,1-4H2 \\
\hline 1462 & C5H9OH-2OOH-4O2 & $\mathrm{CC}(\mathrm{O}[\mathrm{O}]) \mathrm{CC}(\mathrm{OO}) \mathrm{CO}$ & InChl=1S/C5H1105/c1-4(9-7)2-5(3-6)10-8/h4-6,8H,2-3H2,1H3 \\
\hline 1463 & CPTOJ & $\mathrm{C} 1 \mathrm{CCCC} 1[0]$ & InChl=1S/C5H9O/c6-5-3-1-2-4-5/h5H,1-4H2 \\
\hline 1464 & C5H9A-AOOH & $\mathrm{CCC}(=\mathrm{C}) \mathrm{COO}$ & InChl=1S/C5H10O2/c1-3-5(2)4-7-6/h6H,2-4H2,1H3 \\
\hline 1465 & C5H9OH-5OOH-3 & $\mathrm{C}(\mathrm{OO}) \mathrm{C}[\mathrm{CH}] \mathrm{CCO}$ & InChl=1S/C5H1103/c6-4-2-1-3-5-8-7/h1,6-7H,2-5H2 \\
\hline 1466 & $\mathrm{C} 5 \mathrm{H} 9 \mathrm{OH}-4 \mathrm{OOH}-2 \mathrm{O} 2$ & $\mathrm{CC}(\mathrm{OO}) \mathrm{CC}(\mathrm{O}[\mathrm{O}]) \mathrm{CO}$ & InChl=1S/C5H1105/c1-4(9-7)2-5(3-6)10-8/h4-7H,2-3H2,1H3 \\
\hline 1467 & $\mathrm{C} 4 \mathrm{H} 8 \mathrm{CHO} 4-2$ & $\mathrm{O}=\mathrm{CC}(\mathrm{C}) \mathrm{C}[\mathrm{CH} 2]$ & $\operatorname{lnChl}=15 / C 5 H 90 / c 1-3-5(2) 4-6 / h 4-5 \mathrm{H}, 1,3 \mathrm{H} 2,2 \mathrm{H} 3$ \\
\hline 1468 & $\mathrm{C} 5 \mathrm{H} 9 \mathrm{~A}-\mathrm{COOH}$ & $\mathrm{C}=\mathrm{C}(\mathrm{C}) \mathrm{C}(\mathrm{C}) \mathrm{OO}$ & InChl=1S/C5H10O2/c1-4(2)5(3)7-6/h5-6H,1H2,2-3H3 \\
\hline 1469 & С5H9OH-5OOH-2 & $\mathrm{C}(\mathrm{OO}) \mathrm{CC}[\mathrm{CH}] \mathrm{CO}$ & InChl=1S/C5H11O3/c6-4-2-1-3-5-8-7/h2,6-7H,1,3-5H2 \\
\hline 1470 & С5H9OH-30OH-4O2 & $\mathrm{CC}(\mathrm{O}[\mathrm{O}]) \mathrm{C}(\mathrm{OO}) \mathrm{CCO}$ & InChl=1S/C5H1105/c1-4(9-7)5(10-8)2-3-6/h4-6,8H,2-3H2,1H3 \\
\hline 1471 & $\mathrm{IC} 3 \mathrm{H} 7 \mathrm{COCH} 2$ & $\mathrm{CC}(\mathrm{C}) \mathrm{C}(=0)[\mathrm{CH} 2]$ & InChl=1S/C5H9O/c1-4(2)5(3)6/h4H,3H2,1-2H3 \\
\hline 1472 & C5H9A-DOOH & $\mathrm{C}=\mathrm{C}(\mathrm{C}) \mathrm{CCOO}$ & InChl=1S/C5H1002/c1-5(2)3-4-7-6/h6H,1,3-4H2,2H3 \\
\hline 1473 & C5H9OH-5OOH-4 & $\mathrm{C}(\mathrm{OO})[\mathrm{CH}] \mathrm{CCCO}$ & InChl=1S/C5H11O3/c6-4-2-1-3-5-8-7/h3,6-7H,1-2,4-5H2 \\
\hline 1474 & $\mathrm{C} 5 \mathrm{H} 9 \mathrm{OH}-4 \mathrm{OOH}-3 \mathrm{O} 2$ & $\mathrm{CC}(\mathrm{OO}) \mathrm{C}(\mathrm{O}[\mathrm{O}]) \mathrm{CCO}$ & $\operatorname{lnChl}=15 / C 5 H 1105 / c 1-4(9-7) 5(10-8) 2-3-6 / h 4-7 \mathrm{H}, 2-3 \mathrm{H} 2,1 \mathrm{H} 3$ \\
\hline 1475 & AC5H9O-A2 & $\operatorname{ccC}(=\mathrm{C}) \mathrm{C}[\mathrm{O}]$ & $\operatorname{lnChl}=1 \mathrm{~S} / \mathrm{C} 5 \mathrm{H} 9 \mathrm{O} / \mathrm{c} 1-3-5(2) 4-6 / \mathrm{h} 2-4 \mathrm{H} 2,1 \mathrm{H} 3$ \\
\hline 1476 & С5H9B-AOOH & $\mathrm{CC}=\mathrm{C}(\mathrm{C}) \mathrm{COO}$ & InChl=1S/C5H1002/c1-3-5(2)4-7-6/h3,6H,4H2,1-2H3 \\
\hline 1477 & $\mathrm{C} 5 \mathrm{H} 10 \mathrm{OH}-5 \mathrm{O} 2$ & $\mathrm{C}(\mathrm{O}[\mathrm{O}]) \mathrm{CCCCO}$ & InChl=1S/C5H11O3/c6-4-2-1-3-5-8-7/h6H,1-5H2 \\
\hline 1478 & ВС5H9OOH-DOH-AO2 & $\mathrm{C}(\mathrm{O}[\mathrm{O}]) \mathrm{C}(\mathrm{C})(\mathrm{OO}) \mathrm{CCO}$ & InChl=1S/C5H1105/c1-5(10-8,2-3-6)4-9-7/h6,8H,2-4H2,1H3 \\
\hline 1479 & $\operatorname{CVCCJ}(\mathrm{C}) \mathrm{CO}$ & $\mathrm{C}[\mathrm{C}](\mathrm{C}=\mathrm{C}) \mathrm{CO}$ & InChl=1S/C5H9O/c1-3-5(2)4-6/h3,6H,1,4H2,2H3 \\
\hline 1480 & C5H9B-DOOH & $\mathrm{CC}(\mathrm{C})=\mathrm{CCOO}$ & InChl=15/C5H1002/c1-5(2)3-4-7-6/h3,6H,4H2,1-2H3 \\
\hline 1481 & $\mathrm{C} 5 \mathrm{H} 10 \mathrm{OH} 2-\mathrm{AO} 2$ & $\operatorname{ccc}(\mathrm{C}) \mathrm{C}(\mathrm{O}[\mathrm{O}]) \mathrm{O}$ & InChl=1S/C5H1103/c1-3-4(2)5(6)8-7/h4-6H,3H2,1-2H3 \\
\hline 1482 & AC5H9OOH-DOH-BO2 & $\mathrm{CC}(\mathrm{COO})(\mathrm{O}[\mathrm{O}]) \mathrm{CCO}$ & InChl=1S/C5H1105/c1-5(10-8,2-3-6)4-9-7/h6-7H,2-4H2,1H3 \\
\hline 1483 & $\mathrm{TC} 4 \mathrm{H} 8 \mathrm{CHO}$ & {$[\mathrm{CH} 2] \mathrm{C}(\mathrm{C})(\mathrm{C}) \mathrm{C}=\mathrm{O}$} & InChl=1S/C5H9O/c1-5(2,3)4-6/h4H,1H2,2-3H3 \\
\hline 1484 & С5H9C-AOOH & $\mathrm{C}=\mathrm{CC}(\mathrm{C}) \mathrm{COO}$ & InChl=1S/C5H10O2/c1-3-5(2)4-7-6/h3,5-6H,1,4H2,2H3 \\
\hline 1485 & $\mathrm{AO} 2 \mathrm{C} 5 \mathrm{H} 10 \mathrm{OH}$ & $\mathrm{CCC}(\mathrm{C})(\mathrm{CO}) \mathrm{O}[\mathrm{O}]$ & $\operatorname{lnChl}=1 \mathrm{~S} / \mathrm{C} 5 \mathrm{H} 1103 / \mathrm{c} 1-3-5(2,4-6) 8-7 / \mathrm{h} 6 \mathrm{H}, 3-4 \mathrm{H} 2,1-2 \mathrm{H} 3$ \\
\hline 1486 & BC5H9OOH-DOH-DO2 & $\mathrm{CC}(\mathrm{C})(\mathrm{OO}) \mathrm{CC}(\mathrm{O}[\mathrm{O}]) \mathrm{O}$ & InChl=1S/C5H1105/c1-5(2,10-8)3-4(6)9-7/h4,6,8H,3H2,1-2H3 \\
\hline 1487 & $\mathrm{NC} 3 \mathrm{H} 7 \mathrm{COCH} 2$ & {$[\mathrm{CH} 2] \mathrm{C}(=\mathrm{O}) \mathrm{CCC}$} & $\operatorname{lnChl}=1 \mathrm{~S} / \mathrm{C} 5 \mathrm{H} 9 \mathrm{O} / \mathrm{c} 1-3-4-5(2) 6 / \mathrm{h} 2-4 \mathrm{H} 2,1 \mathrm{H} 3$ \\
\hline 1488 & С5H9C-BOOH & $\mathrm{C}=\mathrm{CC}(\mathrm{C})(\mathrm{C}) \mathrm{OO}$ & InChl=1S/C5H1002/c1-4-5(2,3)7-6/h4,6H,1H2,2-3H3 \\
\hline 1489 & $\mathrm{C} 5 \mathrm{H} 10 \mathrm{OH} 2-\mathrm{CO} 2$ & $\mathrm{CC}(\mathrm{O}[\mathrm{O}]) \mathrm{C}(\mathrm{C}) \mathrm{CO}$ & InChl=1S/C5H11O3/c1-4(3-6)5(2)8-7/h4-6H,3H2,1-2H3 \\
\hline 1490 & DC5H9OOH-DOH-BO2 & $\mathrm{CC}(\mathrm{C})(\mathrm{O}[\mathrm{O}]) \mathrm{CC}(\mathrm{OO}) \mathrm{O}$ & InChl=1S/C5H1105/c1-5(2,10-8)3-4(6)9-7/h4,6-7H,3H2,1-2H3 \\
\hline 1491 & $\mathrm{CH} 3 \mathrm{CH} 2 \mathrm{CHCOCH} 3$ & $\mathrm{CC}(=\mathrm{O})[\mathrm{CH}] \mathrm{CC}$ & InChl=1S/C5H9O/c1-3-4-5(2)6/h4H,3H2,1-2H3 \\
\hline 1492 & C5H9-BDOHCJ & $\mathrm{OC}[\mathrm{CH}] \mathrm{C}(\mathrm{O})(\mathrm{C}) \mathrm{C}$ & InChl=15/C5H1102/c1-5(2,7)3-4-6/h3,6-7H,4H2,1-2H3 \\
\hline 1493 & C5H10OH2-DO2 & {$[0] \mathrm{OCCC}(\mathrm{C}) \mathrm{CO}$} & InChl=1S/C5H1103/c1-5(4-6)2-3-8-7/h5-6H,2-4H2,1H3 \\
\hline 1494 & BC5H9OOH-DOH-CO2 & $\mathrm{CC}(\mathrm{C})(\mathrm{OO}) \mathrm{C}(\mathrm{O}[\mathrm{O}]) \mathrm{CO}$ & InChl=1S/C5H1105/c1-5(2,10-8)4(3-6)9-7/h4,6,8H,3H2,1-2H3 \\
\hline 1495 & $\mathrm{CH} 3 \mathrm{CHCH} 2 \mathrm{COCH} 3$ & $\mathrm{CC}(=\mathrm{O}) \mathrm{C}[\mathrm{CH}] \mathrm{C}$ & InChl=1S/C5H9O/c1-3-4-5(2)6/h3H,4H2,1-2H3 \\
\hline 1496 & C5H9-CDOHBJ & $\operatorname{OCC}([C](C) C) O$ & InChl=1S/C5H1102/c1-4(2)5(7)3-6/h5-7H,3H2,1-2H3 \\
\hline 1497 & $\mathrm{C} 5 \mathrm{H} 10 \mathrm{OH} 2-\mathrm{EO} 2$ & $\operatorname{ccc}(\mathrm{CO}[0]) \mathrm{CO}$ & InChl=1S/C5H11O3/c1-2-5(3-6)4-8-7/h5-6H,2-4H2,1H3 \\
\hline 1498 & CC5H9OOH-DOH-BO2 & $\mathrm{CC}(\mathrm{C})(\mathrm{O}[\mathrm{O}]) \mathrm{C}(\mathrm{OO}) \mathrm{CO}$ & InChl=1S/C5H1105/c1-5(2,10-8)4(3-6)9-7/h4,6-7H,3H2,1-2H3 \\
\hline 1499 & $\mathrm{CH} 2 \mathrm{CH} 2 \mathrm{CH} 2 \mathrm{COCH} 3$ & $\mathrm{CC}(=\mathrm{O}) \mathrm{CC}[\mathrm{CH} 2]$ & InChl=1S/C5H9O/c1-3-4-5(2)6/h1,3-4H2,2H3 \\
\hline 1500 & C5H9-ADOHBJ & $\mathrm{OCC}[\mathrm{C}](\mathrm{CO}) \mathrm{C}$ & InChl=1S/C5H1102/c1-5(4-7)2-3-6/h6-7H,2-4H2,1H3 \\
\hline 1501 & С5H9OH2-AOOH-B & $\mathrm{CC}[\mathrm{C}](\mathrm{C}) \mathrm{C}(\mathrm{OO}) \mathrm{O}$ & $\operatorname{lnChl}=15 / C 5 H 1103 / c 1-3-4(2) 5(6) 8-7 / h 5-7 \mathrm{H}, 3 \mathrm{H} 2,1-2 \mathrm{H} 3$ \\
\hline 1502 & AC5H9OOH-DOH-AO2 & $\mathrm{C}(\mathrm{O}[\mathrm{O}]) \mathrm{C}(\mathrm{COO}) \mathrm{CCO}$ & InChl=1S/C5H1105/c6-2-1-5(3-9-7)4-10-8/h5-7H,1-4H2 \\
\hline 1503 & $\mathrm{CH} 2 \mathrm{CH} 2 \mathrm{COC} 2 \mathrm{H} 5$ & {$[\mathrm{CH} 2] \mathrm{CC}(=0) \mathrm{CC}$} & InChl=1S/C5H9O/c1-3-5(6)4-2/h1,3-4H2,2H3 \\
\hline 1504 & C5H9-BDOHAJ & $\mathrm{OCCC}(\mathrm{O})([\mathrm{CH} 2]) \mathrm{C}$ & InChl=15/C5H1102/c1-5(2,7)3-4-6/h6-7H,1,3-4H2,2H3 \\
\hline 1505 & $\mathrm{C} 5 \mathrm{H} 9 \mathrm{OH} 2-\mathrm{AOOH}-\mathrm{C}$ & $\mathrm{C}[\mathrm{CH}] \mathrm{C}(\mathrm{C}) \mathrm{C}(\mathrm{OO}) \mathrm{O}$ & InChl=1S/C5H1103/c1-3-4(2)5(6)8-7/h3-7H,1-2H3 \\
\hline 1506 & C5H9OH-4OOH-5O2 & $\mathrm{C}(\mathrm{O}[\mathrm{O}]) \mathrm{C}(\mathrm{OO}) \mathrm{CCCO}$ & InChl=1S/C5H1105/c6-3-1-2-5(10-8)4-9-7/h5-6,8H,1-4H2 \\
\hline 1507 & $\mathrm{CH} 3 \mathrm{CHCOC} 2 \mathrm{H} 5$ & $\mathrm{C}[\mathrm{CH}] \mathrm{C}(=\mathrm{O}) \mathrm{CC}$ & InChl=1S/C5H9O/c1-3-5(6)4-2/h3H,4H2,1-2H3 \\
\hline 1508 & $\mathrm{C} 5 \mathrm{H} 10 \mathrm{OH}-1 \mathrm{O}$ & $\operatorname{ccccc}([0]) 0$ & InChl=1S/C5H1102/c1-2-3-4-5(6)7/h5-6H,2-4H2,1H3 \\
\hline 1509 & C5H9OH2-AOOH-D & {$[\mathrm{CH} 2] \mathrm{CC}(\mathrm{C}) \mathrm{C}(\mathrm{OO}) \mathrm{O}$} & InChl=1S/C5H11O3/c1-3-4(2)5(6)8-7/h4-7H,1,3H2,2H3 \\
\hline 1510 & C5H9OH-5OOH-4O2 & $\mathrm{C}(\mathrm{OO}) \mathrm{C}(\mathrm{O}[\mathrm{O}]) \mathrm{CCCO}$ & InChl=1S/C5H1105/c6-3-1-2-5(10-8)4-9-7/h5-7H,1-4H2 \\
\hline 1511 & C5H9O12-5 & $\mathrm{C} 1 \mathrm{C}(\mathrm{O} 1) \mathrm{CC}[\mathrm{CH} 2]$ & InChl=1S/C5H9O/c1-2-3-5-4-6-5/h5H,1-4H2 \\
\hline 1512 & C5H10OOH1-4 & $\mathrm{C}[\mathrm{CH}] \mathrm{CCCOO}$ & $\operatorname{lnChl}=1 \mathrm{~S} / \mathrm{C} 5 \mathrm{H} 1102 / \mathrm{c} 1-2-3-4-5-7-6 / \mathrm{h} 2,6 \mathrm{H}, 3-5 \mathrm{H} 2,1 \mathrm{H} 3$ \\
\hline 1513 & C5H9OH2-AOOH-E & $\mathrm{CCC}([\mathrm{CH} 2]) \mathrm{C}(\mathrm{OO}) \mathrm{O}$ & $\operatorname{lnChl}=1 \mathrm{~S} / \mathrm{C} 5 \mathrm{H} 11 \mathrm{O} 3 / \mathrm{c1}-3-4(2) 5(6) 8-7 / \mathrm{h} 4-7 \mathrm{H}, 2-3 \mathrm{H} 2,1 \mathrm{H} 3$ \\
\hline 1514 & C5H9OH-30OH-5O2 & $\mathrm{C}(\mathrm{O}[\mathrm{O}]) \mathrm{CC}(\mathrm{OO}) \mathrm{CCO}$ & InChl=1S/C5H1105/c6-3-1-5(10-8)2-4-9-7/h5-6,8H,1-4H2 \\
\hline 1515 & C5H9O13-5 & $\mathrm{C} 1 \mathrm{CC}(\mathrm{O} 1) \mathrm{C}[\mathrm{CH} 2]$ & InChl=1S/C5H9O/c1-2-5-3-4-6-5/h5H,1-4H2 \\
\hline 1516 & C5H1102-1 & CCCCCO $[0]$ & $\operatorname{lnChl}=1 \mathrm{~S} / \mathrm{C} 5 \mathrm{H} 1102 / \mathrm{c} 1-2-3-4-5-7-6 / \mathrm{h} 2-5 \mathrm{H} 2,1 \mathrm{H} 3$ \\
\hline 1517 & С5H9OH2-BOOH-A & $\mathrm{CCC}(\mathrm{C})(\mathrm{OO})[\mathrm{CH}] \mathrm{O}$ & $\operatorname{lnChl=1S/C5H11O3/c1-3-5(2,4-6)8-7/h4,6-7H,3H2,1-2H3~}$ \\
\hline 1518 & C5H9OH-5OOH-3O2 & $\mathrm{C}(\mathrm{OO}) \mathrm{CC}(\mathrm{O}[\mathrm{O}]) \mathrm{CCO}$ & InChl=1S/C5H1105/c6-3-1-5(10-8)2-4-9-7/h5-7H,1-4H2 \\
\hline 1519 & C5H9O14-5 & $\mathrm{C} 1 \mathrm{CCC}(\mathrm{O} 1)[\mathrm{CH} 2]$ & InChl=1S/C5H9O/c1-5-3-2-4-6-5/h5H,1-4H2 \\
\hline 1520 & $\mathrm{C} 5 \mathrm{H} 100 \mathrm{OH} 1-3$ & $\mathrm{CC}[\mathrm{CH}] \mathrm{CCOO}$ & InChl=1S/C5H11O2/c1-2-3-4-5-7-6/h3,6H,2,4-5H2,1H3 \\
\hline 1521 & $\mathrm{C} 5 \mathrm{H} 9 \mathrm{OH} 2-\mathrm{BOOH}-\mathrm{C}$ & $\mathrm{C}[\mathrm{CH}] \mathrm{C}(\mathrm{C})(\mathrm{OO}) \mathrm{CO}$ & InChl=1S/C5H11O3/c1-3-5(2,4-6)8-7/h3,6-7H,4H2,1-2H3 \\
\hline 1522 & C5H9OH-2OOH-5O2 & $\mathrm{C}(\mathrm{O}[\mathrm{O}]) \mathrm{CCC}(\mathrm{OO}) \mathrm{CO}$ & InChl=1S/C5H1105/c6-4-5(10-8)2-1-3-9-7/h5-6,8H,1-4H2 \\
\hline 1523 & C5H9O23-1 & $\mathrm{CCC1C}(\mathrm{O} 1)[\mathrm{CH} 2]$ & InChl=1S/C5H9O/c1-3-5-4(2)6-5/h4-5H,2-3H2,1H3 \\
\hline 1524 & $\mathrm{C5H} 10 \mathrm{OOH} 1-2$ & $\mathrm{CCC}[\mathrm{CH}] \mathrm{COO}$ & $\operatorname{lnChl}=15 / C 5 H 1102 / c 1-2-3-4-5-7-6 / h 4,6 \mathrm{H}, 2-3,5 \mathrm{H} 2,1 \mathrm{H} 3$ \\
\hline 1525 & C5H9OH2-BOOH-D & {$[\mathrm{CH} 2] \mathrm{CC}(\mathrm{C})(\mathrm{OO}) \mathrm{CO}$} & $\operatorname{lnChl=1S/C5H11O3/c1-3-5(2,4-6)8-7/h6-7H,1,3-4H2,2H3~}$ \\
\hline 1526 & C5H9OH-5OOH-2O2 & $\mathrm{C}(\mathrm{OO}) \mathrm{CCC}(\mathrm{O}[\mathrm{O}]) \mathrm{CO}$ & InChl=15/C5H1105/c6-4-5(10-8)2-1-3-9-7/h5-7H,1-4H2 \\
\hline 1527 & C5H9O23-5 & {$[\mathrm{CH} 2] \mathrm{CC} 1 \mathrm{C}(\mathrm{O} 1) \mathrm{C}$} & InChl=1S/C5H9O/c1-3-5-4(2)6-5/h4-5H,1,3H2,2H3 \\
\hline 1528 & $\mathrm{C} 5 \mathrm{H} 10 \mathrm{OOH} 1-5$ & {$[\mathrm{CH} 2] \mathrm{CCCCOO}$} & $\operatorname{lnChl}=1 \mathrm{~S} / \mathrm{C} 5 \mathrm{H} 1102 / \mathrm{c1} 1-2-3-4-5-7-6 / \mathrm{h} 6 \mathrm{H}, 1-5 \mathrm{H} 2$ \\
\hline 1529 & $\mathrm{C} 5 \mathrm{H} 9 \mathrm{OH} 2-\mathrm{BOOH}-\mathrm{E}$ & $\mathrm{CCC}([\mathrm{CH} 2])(\mathrm{OO}) \mathrm{CO}$ & InChl=1S/C5H11O3/c1-3-5(2,4-6)8-7/h6-7H,2-4H2,1H3 \\
\hline 1530 & С5H9OH2-AOOH-BO2 & $\operatorname{CCC}(\mathrm{C})(\mathrm{O}[\mathrm{O}]) \mathrm{C}(\mathrm{OO}) \mathrm{O}$ & InChl=1S/C5H1105/c1-3-5(2,10-8)4(6)9-7/h4,6-7H,3H2,1-2H3 \\
\hline 1531 & C5H9O24-1 & {$[\mathrm{CH} 2] \mathrm{C} 1 \mathrm{CC}(\mathrm{O} 1) \mathrm{C}$} & InChl=1S/C5H9O/c1-4-3-5(2)6-4/h4-5H,1,3H2,2H3 \\
\hline 1532 & $\mathrm{C} 5 \mathrm{H} 10 \mathrm{OH}-2 \mathrm{O}$ & $\operatorname{cccc}([0]) \operatorname{co}$ & InChl=1S/C5H1102/c1-2-3-5(7)4-6/h5-6H,2-4H2,1H3 \\
\hline 1533 & $\mathrm{C} 5 \mathrm{H} 9 \mathrm{OH} 2-\mathrm{COOH}-\mathrm{A}$ & $\mathrm{CC}(\mathrm{OO}) \mathrm{C}(\mathrm{C})[\mathrm{CH}] \mathrm{O}$ & InChl=1S/C5H11O3/c1-4(3-6)5(2)8-7/h3-7H,1-2H3 \\
\hline 1534 & С5H9OH2-АOOH-CO2 & $\mathrm{CC}(\mathrm{O}[\mathrm{O}]) \mathrm{C}(\mathrm{C}) \mathrm{C}(\mathrm{OO}) \mathrm{O}$ & InChl=1S/C5H1105/c1-3(4(2)9-7)5(6)10-8/h3-6,8H,1-2H3 \\
\hline 1535 & $\mathrm{C} 5 \mathrm{H} 10 \mathrm{OH}-3 \mathrm{O}$ & $\operatorname{CCC}([0]) \mathrm{CCO}$ & InChl=1S/C5H1102/c1-2-5(7)3-4-6/h5-6H,2-4H2,1H3 \\
\hline 1536 & $\mathrm{C} 5 \mathrm{H} 9 \mathrm{OH} 2-\mathrm{COOH}-\mathrm{B}$ & $\mathrm{CC}(\mathrm{OO})[\mathrm{C}](\mathrm{C}) \mathrm{CO}$ & InChl=15/C5H11O3/c1-4(3-6)5(2)8-7/h5-7H,3H2,1-2H3 \\
\hline 1537 & C5H9OH2-AOOH-DO2 & {$[0] \mathrm{OCCC}(\mathrm{C}) \mathrm{C}(\mathrm{OO}) \mathrm{O}$} & InChl=1S/C5H1105/c1-4(2-3-9-7)5(6)10-8/h4-6,8H,2-3H2,1H3 \\
\hline 1538 & A-BC5H10O-DJ & {$[\mathrm{CH} 2] \mathrm{CC} 1(\mathrm{C}) \mathrm{CO} 1$} & InChl=15/C5H9O/c1-3-5(2)4-6-5/h1,3-4H2,2H3 \\
\hline 1539 & $\mathrm{C} 5 \mathrm{H} 10 \mathrm{OOH} 2-4$ & $\mathrm{C}[\mathrm{CH}] \mathrm{CC}(\mathrm{OO}) \mathrm{C}$ & $\operatorname{lnChl}=15 / C 5 H 1102 / c 1-3-4-5(2) 7-6 / h 3,5-6 \mathrm{H}, 4 \mathrm{H} 2,1-2 \mathrm{H} 3$ \\
\hline 1540 & $\mathrm{C} 5 \mathrm{H} 9 \mathrm{OH} 2-\mathrm{COOH}-\mathrm{D}$ & {$[\mathrm{CH} 2] \mathrm{C}(\mathrm{OO}) \mathrm{C}(\mathrm{C}) \mathrm{CO}$} & InChl=1S/C5H11O3/c1-4(3-6)5(2)8-7/h4-7H,2-3H2,1H3 \\
\hline 1541 & С5H9OH2-AOOH-EO2 & $\mathrm{CCC}(\mathrm{CO}[\mathrm{O}]) \mathrm{C}(\mathrm{OO}) \mathrm{O}$ & InChl=1S/C5H1105/c1-2-4(3-9-7)5(6)10-8/h4-6,8H,2-3H2,1H3 \\
\hline 1542 & AC5H9O-D & $\mathrm{CC} 1([\mathrm{CH} 2]) \mathrm{CCO} 1$ & InChl=1S/C5H9O/c1-5(2)3-4-6-5/h1,3-4H2,2H3 \\
\hline 1543 & $\mathrm{C} 5 \mathrm{H} 100 \mathrm{OH} 2-3$ & $\mathrm{CC}[\mathrm{CH}] \mathrm{C}(\mathrm{OO}) \mathrm{C}$ & InChl=15/C5H1102/c1-3-4-5(2)7-6/h4-6H,3H2,1-2H3 \\
\hline
\end{tabular}




\begin{tabular}{|c|c|c|c|}
\hline 1544 & С5H9OH2-СOOH-E & $\mathrm{CC}(\mathrm{OO}) \mathrm{C}([\mathrm{CH} 2]) \mathrm{CO}$ & InChl=1S/C5H11O3/c1-4(3-6)5(2)8-7/h4-7H,1,3H2,2H3 \\
\hline 1545 & $\mathrm{C} 5 \mathrm{H} 9 \mathrm{OH} 2-\mathrm{BOOH}-\mathrm{AO} 2$ & $\operatorname{ccc}(\mathrm{C})(\mathrm{OO}) \mathrm{C}(\mathrm{O}[\mathrm{O}]) \mathrm{O}$ & InChl=1S/C5H1105/c1-3-5(2,10-8)4(6)9-7/h4,6,8H,3H2,1-2H3 \\
\hline 1546 & $\mathrm{C} 5 \mathrm{H} 10 \mathrm{OOH} 2-5$ & {$[\mathrm{CH} 2] \mathrm{CCC}(\mathrm{OO}) \mathrm{C}$} & $\operatorname{lnChl}=15 / C 5 H 1102 / c 1-3-4-5(2) 7-6 / h 5-6 \mathrm{H}, 1,3-4 \mathrm{H} 2,2 \mathrm{H} 3$ \\
\hline 1547 & C5H9OH2-DOOH-A & $\mathrm{OOCCC}(\mathrm{C})[\mathrm{CH}] \mathrm{O}$ & $\operatorname{lnChl}=1 \mathrm{~S} / \mathrm{C} 5 \mathrm{H} 11 \mathrm{O} 3 / \mathrm{c} 1-5(4-6) 2-3-8-7 / \mathrm{h} 4-7 \mathrm{H}, 2-3 \mathrm{H} 2,1 \mathrm{H} 3$ \\
\hline 1548 & C5H9OH2-BOOH-CO2 & $\mathrm{CC}(\mathrm{O}[\mathrm{O}]) \mathrm{C}(\mathrm{C})(\mathrm{OO}) \mathrm{CO}$ & InChl=1S/C5H1105/c1-4(9-7)5(2,3-6)10-8/h4,6,8H,3H2,1-2H3 \\
\hline 1549 & C5H9O12-4 & $\mathrm{C}[\mathrm{CH}] \mathrm{CC} 1 \mathrm{CO} 1$ & InChl=1S/C5H9O/c1-2-3-5-4-6-5/h2,5H,3-4H2,1H3 \\
\hline 1550 & $\mathrm{C} 5 \mathrm{H} 100 \mathrm{OH} 2-1$ & $\mathrm{CCCC}(\mathrm{OO})[\mathrm{CH} 2]$ & InChl=1S/C5H1102/c1-3-4-5(2)7-6/h5-6H,2-4H2,1H3 \\
\hline 1551 & $\mathrm{C} 5 \mathrm{H} 9 \mathrm{OH} 2-\mathrm{DOOH}-\mathrm{B}$ & $\mathrm{OOCC}[\mathrm{C}](\mathrm{C}) \mathrm{CO}$ & $\operatorname{lnChl}=1 \mathrm{~S} / \mathrm{C} 5 \mathrm{H} 1103 / \mathrm{c} 1-5(4-6) 2-3-8-7 / \mathrm{h} 6-7 \mathrm{H}, 2-4 \mathrm{H} 2,1 \mathrm{H} 3$ \\
\hline 1552 & C5H9OH2-BOOH-DO2 & {$[\mathrm{O}] \mathrm{OCCC}(\mathrm{C})(\mathrm{OO}) \mathrm{CO}$} & InChl=1S/C5H1105/c1-5(4-6,10-8)2-3-9-7/h6,8H,2-4H2,1H3 \\
\hline 1553 & C5H9O15-3 & $\mathrm{C} 1[\mathrm{CH}] \mathrm{CCOC} 1$ & InChl=1S/C5H9O/c1-2-4-6-5-3-1/h1H,2-5H2 \\
\hline 1554 & $\mathrm{C} 5 \mathrm{H} 1102-2$ & $\operatorname{cccc}(\mathrm{O}[\mathrm{O}]) \mathrm{C}$ & InChl=1S/C5H1102/c1-3-4-5(2)7-6/h5H,3-4H2,1-2H3 \\
\hline 1555 & C5H9OH2-DOOH-C & $\mathrm{OOC}[\mathrm{CH}] \mathrm{C}(\mathrm{C}) \mathrm{CO}$ & InChl=1S/C5H11O3/c1-5(4-6)2-3-8-7/h2,5-7H,3-4H2,1H3 \\
\hline 1556 & С5H9OH2-BOOH-EO2 & $\operatorname{CCC}(\mathrm{CO}[\mathrm{O}])(\mathrm{OO}) \mathrm{CO}$ & InChl=1S/C5H1105/c1-2-5(3-6,10-8)4-9-7/h6,8H,2-4H2,1H3 \\
\hline 1557 & C5H9O23-4 & $\mathrm{C}[\mathrm{CH}] \mathrm{C} 1 \mathrm{OC} 1 \mathrm{C}$ & InChl=1S/C5H9O/c1-3-5-4(2)6-5/h3-5H,1-2H3 \\
\hline 1558 & С5H10OOH3-2 & $\mathrm{CCC}(\mathrm{OO})[\mathrm{CH}] \mathrm{C}$ & $\operatorname{lnChl}=15 / C 5 H 1102 / c 1-3-5(4-2) 77-6 / h 3,5-6 \mathrm{H}, 4 \mathrm{H} 2,1-2 \mathrm{H} 3$ \\
\hline 1559 & C5H9OH2-DOOH-E & $\mathrm{OOCCC}([\mathrm{CH} 2]) \mathrm{CO}$ & InChl=1S/C5H11O3/c1-5(4-6)2-3-8-7/h5-7H,1-4H2 \\
\hline 1560 & $\mathrm{C} 5 \mathrm{H} 9 \mathrm{OH} 2-\mathrm{COOH}-\mathrm{AO} 2$ & $\mathrm{CC}(\mathrm{OO}) \mathrm{C}(\mathrm{C}) \mathrm{C}(\mathrm{O}[\mathrm{O}]) \mathrm{O}$ & InChl=1S/C5H1105/c1-3(4(2)9-7)5(6)10-8/h3-7H,1-2H3 \\
\hline 1561 & $\mathrm{C} 5 \mathrm{H} 100 \mathrm{OH} 3-1$ & $\mathrm{CCC}(\mathrm{OO}) \mathrm{C}[\mathrm{CH} 2]$ & InChl=1S/C5H1102/c1-3-5(4-2)7-6/h5-6H,1,3-4H2,2H3 \\
\hline 1562 & $\mathrm{C} 5 \mathrm{H} 9 \mathrm{OH} 2-\mathrm{EOOH}-\mathrm{A}$ & $\mathrm{CCC}(\mathrm{COO})[\mathrm{CH}] \mathrm{O}$ & InChl=1S/C5H1103/c1-2-5(3-6)4-8-7/h3,5-7H,2,4H2,1H3 \\
\hline 1563 & $\mathrm{C} 5 \mathrm{H} 9 \mathrm{OH} 2-\mathrm{COOH}-\mathrm{BO} 2$ & $\mathrm{CC}(\mathrm{OO}) \mathrm{C}(\mathrm{C})(\mathrm{O}[\mathrm{O}]) \mathrm{CO}$ & InChl=1S/C5H1105/c1-4(9-7)5(2,3-6)10-8/h4,6-7H,3H2,1-2H3 \\
\hline 1564 & C5H1102-3 & $\operatorname{CCC}(\mathrm{O}[0]) \mathrm{CC}$ & InChl=1S/C5H1102/c1-3-5(4-2)7-6/h5H,3-4H2,1-2H3 \\
\hline 1565 & С5H9OH2-EOOH-B & $\mathrm{CC}[\mathrm{C}](\mathrm{COO}) \mathrm{CO}$ & $\operatorname{lnChl}=15 / \mathrm{C} 5 \mathrm{H} 1103 / \mathrm{c} 1-2-5(3-6) 4-8-7 / \mathrm{h} 6-7 \mathrm{H}, 2-4 \mathrm{H} 2,1 \mathrm{H} 3$ \\
\hline 1566 & C5H9OH2-COOH-DO2 & {$[\mathrm{O}] \mathrm{OCC}(\mathrm{OO}) \mathrm{C}(\mathrm{C}) \mathrm{CO}$} & InChl=1S/C5H1105/c1-4(2-6)5(10-8)3-9-7/h4-6,8H,2-3H2,1H3 \\
\hline 1567 & DC5H10OH-DO & $\mathrm{CC}(\mathrm{C}) \mathrm{CC}([\mathrm{O}]) \mathrm{O}$ & InChl=1S/C5H1102/c1-4(2)3-5(6)7/h4-6H,3H2,1-2H3 \\
\hline 1568 & $\mathrm{C} 5 \mathrm{H} 9 \mathrm{OH} 2-\mathrm{EOOH}-\mathrm{C}$ & $\mathrm{C}[\mathrm{CH}] \mathrm{C}(\mathrm{COO}) \mathrm{CO}$ & InChl=1S/C5H11O3/c1-2-5(3-6)4-8-7/h2,5-7H,3-4H2,1H3 \\
\hline 1569 & C5H9OH2-COOH-EO2 & $\mathrm{CC}(\mathrm{OO}) \mathrm{C}(\mathrm{CO}[\mathrm{O}]) \mathrm{CO}$ & InChl=1S/C5H1105/c1-4(10-8)5(2-6)3-9-7/h4-6,8H,2-3H2,1H3 \\
\hline 1570 & DC5H10OH-CO & $\mathrm{CC}(\mathrm{C}) \mathrm{C}([\mathrm{O}]) \mathrm{CO}$ & InChl=1S/C5H1102/c1-4(2)5(7)3-6/h4-6H,3H2,1-2H3 \\
\hline 1571 & $\mathrm{C} 5 \mathrm{H} 9 \mathrm{OH} 2-\mathrm{EOOH}-\mathrm{D}$ & {$[\mathrm{CH} 2] \mathrm{CC}(\mathrm{COO}) \mathrm{CO}$} & InChl=1S/C5H1103/c1-2-5(3-6)4-8-7/h5-7H,1-4H2 \\
\hline 1572 & C5H9OH2-DOOH-AO2 & $00 \operatorname{Occ}(\mathrm{C}) \mathrm{C}(\mathrm{O}[\mathrm{O}]) \mathrm{O}$ & InChl=1S/C5H1105/c1-4(2-3-9-7)5(6)10-8/h4-7H,2-3H2,1H3 \\
\hline 1573 & IC5Y3-1] & $\mathrm{CC}(=\mathrm{O}) \mathrm{C}([\mathrm{CH} 2]) \mathrm{C}$ & $\operatorname{lnChl}=1 \mathrm{~S} / \mathrm{C} 5 \mathrm{H} 9 \mathrm{O} / \mathrm{c} 1-4(2) 5(3) 6 / \mathrm{h} 4 \mathrm{H}, 1 \mathrm{H} 2,2-3 \mathrm{H} 3$ \\
\hline 1574 & DC5H10OH-BO & $\mathrm{CC}(\mathrm{C})([\mathrm{O}]) \mathrm{CCO}$ & InChl=1S/C5H1102/c1-5(2,7)3-4-6/h6H,3-4H2,1-2H3 \\
\hline 1575 & $\mathrm{O} 2 \mathrm{C} 5 \mathrm{H} 10 \mathrm{OH} 23$ & $\operatorname{ccC}(\mathrm{C}(\mathrm{C}) \mathrm{O}) \mathrm{O}[\mathrm{O}]$ & InChl=1S/C5H11O3/c1-3-5(8-7)4(2)6/h4-6H,3H2,1-2H3 \\
\hline 1576 & C5H9OH2-DOOH-BO2 & $\mathrm{OOCCC}(\mathrm{C})(\mathrm{O}[\mathrm{O}]) \mathrm{CO}$ & InChl=1S/C5H1105/c1-5(4-6,10-8)2-3-9-7/h6-7H,2-4H2,1H3 \\
\hline 1577 & $\mid \mathrm{IC5Y3-2J}$ & $\mathrm{CC}(=\mathrm{C}(\mathrm{C}) \mathrm{C})[0]$ & InChl=1S/C5H9O/c1-4(2)5(3)6/h1-3H3 \\
\hline 1578 & DC5H10OH-AO & $\mathrm{C}([\mathrm{O}]) \mathrm{C}(\mathrm{C}) \mathrm{CCO}$ & InChl=1S/C5H1102/c1-5(4-7)2-3-6/h5-6H,2-4H2,1H3 \\
\hline 1579 & $\mathrm{BO} 2 \mathrm{C} 5 \mathrm{H} 10 \mathrm{OH}$ & $\mathrm{CC}(\mathrm{C}(\mathrm{C})(\mathrm{C}) \mathrm{O}) \mathrm{O}[\mathrm{O}]$ & InChl=1S/C5H11O3/c1-4(8-7)5(2,3)6/h4,6H,1-3H3 \\
\hline 1580 & C5H9OH2-DOOH-CO2 & $\mathrm{OOCC}(\mathrm{O}[\mathrm{O}]) \mathrm{C}(\mathrm{C}) \mathrm{CO}$ & InChl=1S/C5H1105/c1-4(2-6)5(10-8)3-9-7/h4-7H,2-3H2,1H3 \\
\hline 1581 & C5H91-40 & $\mathrm{CC}(\mathrm{CC}=\mathrm{C})[\mathrm{O}]$ & $\operatorname{lnChl}=15 / \mathrm{C} 5 \mathrm{H} 9 \mathrm{O} / \mathrm{c1} 1-3-4-5(2) 6 / \mathrm{h} 3,5 \mathrm{H}, 1,4 \mathrm{H} 2,2 \mathrm{H} 3$ \\
\hline 1582 & $\mathrm{C} 5 \mathrm{H} 10 \mathrm{OH}-4 \mathrm{O}$ & $\mathrm{CC}([0]) \mathrm{CCCO}$ & InChl=15/C5H1102/c1-5(7)3-2-4-6/h5-6H,2-4H2,1H3 \\
\hline 1583 & $\mathrm{CO} 2 \mathrm{C} 5 \mathrm{H} 10 \mathrm{OH}$ & $\mathrm{CC}(\mathrm{C}) \mathrm{C}(\mathrm{CO}[\mathrm{O}]) \mathrm{O}$ & InChl=15/C5H1103/c1-4(2)5(6)3-8-7/h4-6H,3H2,1-2H3 \\
\hline 1584 & C5H9OH2-DOOH-EO2 & $\mathrm{OOCCC}(\mathrm{CO}[\mathrm{O}]) \mathrm{CO}$ & $\operatorname{lnChl}=1 \mathrm{~S} / \mathrm{C} 5 \mathrm{H} 11 \mathrm{O} 5 / \mathrm{c} 6-3-55(4-10-8) 1-2-9-7 / \mathrm{h} 5-7 \mathrm{H}, 1-4 \mathrm{H} 2$ \\
\hline 1585 & $\mathrm{C} 5 \mathrm{H} 10 \mathrm{OH}-5 \mathrm{O}$ & $\mathrm{C}([\mathrm{O}]) \mathrm{CCCCO}$ & $\operatorname{lnChl}=1 \mathrm{~S} / \mathrm{C} 5 \mathrm{H} 1102 / \mathrm{c} 6-4-2-1-3-5-7 / \mathrm{h} 6 \mathrm{H}, 1-5 \mathrm{H} 2$ \\
\hline 1586 & $\mathrm{C} 5 \mathrm{H} 100 \mathrm{OH} 1-1 \mathrm{OH}$ & $\mathrm{OC}(\mathrm{OO}) \mathrm{CCCC}$ & InChl=1S/C5H12O3/c1-2-3-4-5(6)8-7/h5-7H,2-4H2,1H3 \\
\hline 1587 & $\mathrm{C} 5 \mathrm{H} 9 \mathrm{OH} 2-\mathrm{EOOH}-\mathrm{AO} 2$ & $\mathrm{CCC}(\mathrm{COO}) \mathrm{C}(\mathrm{O}[\mathrm{O}]) \mathrm{O}$ & InChl=1S/C5H1105/c1-2-4(3-9-7)5(6)10-8/h4-7H,2-3H2,1H3 \\
\hline 1588 & C5H92-50 & $\mathrm{CC}=\mathrm{CCC}[\mathrm{O}]$ & InChl=1S/C5H9O/c1-2-3-4-5-6/h2-3H,4-5H2,1H3 \\
\hline 1589 & $\mathrm{C} 5 \mathrm{H} 10 \mathrm{OH} 2-\mathrm{AO}$ & $\operatorname{ccc}(\mathrm{C}) \mathrm{C}([\mathrm{O}]) \mathrm{O}$ & InChl=1S/C5H1102/c1-3-4(2)5(6)7/h4-6H,3H2,1-2H3 \\
\hline 1590 & $\mathrm{C} 5 \mathrm{H} 10 \mathrm{OOH} 2-1 \mathrm{OH}$ & $\mathrm{CCCC}(\mathrm{OO}) \mathrm{CO}$ & $\operatorname{lnChl}=1 \mathrm{~S} / \mathrm{C} 5 \mathrm{H} 12 \mathrm{O} / \mathrm{c1} 1-2-3-5(4-6) 8-7 / \mathrm{h} 5-7 \mathrm{H}, 2-4 \mathrm{H} 2,1 \mathrm{H} 3$ \\
\hline 1591 & С5H9OH2-EOOH-BO2 & $\mathrm{CCC}(\mathrm{COO})(\mathrm{O}[\mathrm{O}]) \mathrm{CO}$ & InChl=1S/C5H1105/c1-2-5(3-6,10-8)4-9-7/h6-7H,2-4H2,1H3 \\
\hline 1592 & CC5H9-AO & $\mathrm{CC}(\mathrm{C}=\mathrm{C}) \mathrm{C}[\mathrm{O}]$ & $\operatorname{lnChl}=15 / C 5 H 90 / c 1-3-5(2) 4-6 / h 3,5 \mathrm{H}, 1,4 \mathrm{H} 2,2 \mathrm{H} 3$ \\
\hline 1593 & $\mathrm{C} 5 \mathrm{H} 10 \mathrm{OH} 2-\mathrm{BO}$ & $\operatorname{CCC}(\mathrm{C})([\mathrm{O}]) \mathrm{CO}$ & InChl=1S/C5H1102/c1-3-5(2,7)4-6/h6H,3-4H2,1-2H3 \\
\hline 1594 & $\mathrm{C} 5 \mathrm{H} 100 \mathrm{OH} 3-1 \mathrm{OH}$ & $\operatorname{ccc}(00) \operatorname{cco}$ & InChl=1S/C5H12O3/c1-2-5(8-7)3-4-6/h5-7H,2-4H2,1H3 \\
\hline 1595 & C5H9OH2-EOOH-CO2 & $\mathrm{CC}(\mathrm{O}[\mathrm{O}]) \mathrm{C}(\mathrm{COO}) \mathrm{CO}$ & InChl=1S/C5H1105/c1-4(10-8)5(2-6)3-9-7/h4-7H,2-3H2,1H3 \\
\hline 1596 & NEO-PC5H9O & $\mathrm{C}(\mathrm{O} 1) \mathrm{C}(\mathrm{C} 1)(\mathrm{C})[\mathrm{CH} 2]$ & InChl=1S/C5H9O/c1-5(2)3-6-4-5/h1,3-4H2,2H3 \\
\hline 1597 & $\mathrm{C} 5 \mathrm{H} 10 \mathrm{OH} 2-\mathrm{CO}$ & $\mathrm{CC}([\mathrm{O}]) \mathrm{C}(\mathrm{C}) \mathrm{CO}$ & InChl=1S/C5H1102/c1-4(3-6)5(2)7/h4-6H,3H2,1-2H3 \\
\hline 1598 & DC5H10OOH-DOH & $\mathrm{CC}(\mathrm{C}) \mathrm{CC}(\mathrm{OO}) \mathrm{O}$ & InChl=1S/C5H12O3/c1-4(2)3-5(6)8-7/h4-7H,3H2,1-2H3 \\
\hline 1599 & C5H9OH2-EOOH-DO2 & {$[\mathrm{O}] \mathrm{OCCC}(\mathrm{COO}) \mathrm{CO}$} & InChl=1S/C5H1105/c6-3-5(4-10-8)1-2-9-7/h5-6,8H,1-4H2 \\
\hline 1600 & TC4H9CO & $\mathrm{CC}(\mathrm{C})(\mathrm{C})[\mathrm{C}]=\mathrm{O}$ & InChl=1S/C5H9O/c1-5(2,3)4-6/h1-3H3 \\
\hline 1601 & $\mathrm{C} 5 \mathrm{H} 10 \mathrm{OH} 2-\mathrm{DO}$ & {$[\mathrm{O}] \mathrm{CCC}(\mathrm{C}) \mathrm{CO}$} & InChl=1S/C5H1102/c1-5(4-7)2-3-6/h5,7H,2-4H2,1H3 \\
\hline 1602 & CC5H10OOH-DOH & $\mathrm{CC}(\mathrm{C}) \mathrm{C}(\mathrm{OO}) \mathrm{CO}$ & $\operatorname{lnChl}=1 \mathrm{~S} / \mathrm{C} 5 \mathrm{H} 12 \mathrm{O} 3 / \mathrm{c1}-4(2) 5(3-6) 8-7 / \mathrm{h} 4-7 \mathrm{H}, 3 \mathrm{H} 2,1-2 \mathrm{H} 3$ \\
\hline 1603 & MBMOOH2OO & $\mathrm{C}(\mathrm{OO}) \mathrm{OC}(=\mathrm{O}) \mathrm{C}(\mathrm{O}[\mathrm{O}]) \mathrm{CC}$ & InChl=1S/C5H9O6/c1-2-4(11-8)5(6)9-3-10-7/h4,7H,2-3H2,1H3 \\
\hline 1604 & BCSH9-DOH & $\mathrm{CC}(\mathrm{C})=\mathrm{CCO}$ & $\operatorname{lnChl}=1 \mathrm{~S} / \mathrm{C} 5 \mathrm{H} 100 / \mathrm{c} 1-5(2) 3-4-6 / \mathrm{h} 3,6 \mathrm{H}, 4 \mathrm{H} 2,1-2 \mathrm{H} 3$ \\
\hline 1605 & C5H10OH2-EO & $\operatorname{CCC}(\mathrm{C}[\mathrm{O}]) \mathrm{CO}$ & InChl=1S/C5H1102/c1-2-5(3-6)4-7/h5-6H,2-4H2,1H3 \\
\hline 1606 & BC5H10OOH-DOH & $\mathrm{CC}(\mathrm{C})(\mathrm{OO}) \mathrm{CCO}$ & InChl=15/C5H12O3/c1-5(2,8-7)3-4-6/h6-7H,3-4H2,1-2H3 \\
\hline 1607 & МВМООНЗОО & $\mathrm{C}(\mathrm{OO}) \mathrm{OC}(=\mathrm{O}) \mathrm{CC}(\mathrm{O}[\mathrm{O}]) \mathrm{C}$ & InChl=1S/C5H9O6/c1-4(11-8)2-5(6)9-3-10-7/h4,7H,2-3H2,1H3 \\
\hline 1608 & C5H9OH1-4 & $\mathrm{C}=\mathrm{CCCCO}$ & InChl=1S/C5H100/c1-2-3-4-5-6/h2,6H,1,3-5H2 \\
\hline 1609 & AC5H1102 & $\operatorname{CCC}(\mathrm{C}) \mathrm{CO}[\mathrm{O}]$ & InChl=1S/C5H1102/c1-3-5(2)4-7-6/h5H,3-4H2,1-2H3 \\
\hline 1610 & AC5H10OOH-DOH & $\mathrm{CC}(\mathrm{COO}) \mathrm{CCO}$ & $\operatorname{lnChl}=1 \mathrm{~S} / \mathrm{C} 5 \mathrm{H} 12 \mathrm{O} / \mathrm{c} 1-5(2-3-6) 4-8-7 / \mathrm{h} 5-7 \mathrm{H}, 2-4 \mathrm{H} 2,1 \mathrm{H} 3$ \\
\hline 1611 & MB2OOHMOO & $\mathrm{C}(\mathrm{O}[\mathrm{O}]) \mathrm{OC}(=0) \mathrm{C}(\mathrm{OO}) \mathrm{CC}$ & InChl=1S/C5H9O6/c1-2-4(11-8)5(6)9-3-10-7/h4,8H,2-3H2,1H3 \\
\hline 1612 & C5H9OH1-3 & $\mathrm{CC}=\mathrm{CCCO}$ & InChl=1S/C5H10O/c1-2-3-4-5-6/h2-3,6H,4-5H2,1H3 \\
\hline 1613 & BC5H11O2 & $\operatorname{ccC}(\mathrm{C})(\mathrm{C}) \mathrm{O}[\mathrm{O}]$ & InChl=1S/C5H1102/c1-4-5(2,3)7-6/h4H2,1-3H3 \\
\hline 1614 & $\mathrm{C} 5 \mathrm{H} 10 \mathrm{OOH} 4-1 \mathrm{OH}$ & $\mathrm{CC}(\mathrm{OO}) \mathrm{CCCO}$ & $\operatorname{lnChl}=1 \mathrm{~S} / \mathrm{C} 5 \mathrm{H} 12 \mathrm{O} / \mathrm{c1}-5(8-7) 3-2-4-6 / \mathrm{h} 5-7 \mathrm{H}, 2-4 \mathrm{H} 2,1 \mathrm{H} 3$ \\
\hline 1615 & MB2OOH3OO & $\operatorname{coc}(=0) \mathrm{C}(\mathrm{OO}) \mathrm{C}(\mathrm{O}[\mathrm{O}]) \mathrm{C}$ & InChl=1S/C5H9O6/c1-3(10-7)4(11-8)5(6)9-2/h3-4,8H,1-2H3 \\
\hline 1616 & C5H9OH1-2 & $\mathrm{CCC}=\mathrm{CCO}$ & InChl=1S/C5H100/c1-2-3-4-5-6/h3-4,6H,2,5H2,1H3 \\
\hline 1617 & CC5H1102 & $\mathrm{CC}(\mathrm{C}) \mathrm{C}(\mathrm{C}) \mathrm{O}[\mathrm{O}]$ & InChl=1S/C5H11O2/c1-4(2)5(3)7-6/h4-5H,1-3H3 \\
\hline 1618 & $\mathrm{C} 5 \mathrm{H} 100 \mathrm{OH} 5-1 \mathrm{OH}$ & $\mathrm{C}(\mathrm{OO}) \mathrm{cccco}$ & InChl=1S/C5H12O3/c6-4-2-1-3-5-8-7/h6-7H,1-5H2 \\
\hline 1619 & $\mathrm{MB} 2 \mathrm{OOH} 4 \mathrm{OO}$ & $\operatorname{coc}(=0) \mathrm{C}(\mathrm{OO}) \mathrm{CC}(\mathrm{O}[\mathrm{O}])$ & InChl=15/C5H9O6/c1-9-5(6)4(11-8)2-3-10-7/h4,8H,2-3H2,1H3 \\
\hline 1620 & C5H9OH1-1 & $\mathrm{CCCC}=\mathrm{CO}$ & InChl=1S/C5H10O/c1-2-3-4-5-6/h4-6H,2-3H2,1H3 \\
\hline 1621 & DC5H1102 & $\mathrm{CC}(\mathrm{C}) \mathrm{CCO}[\mathrm{O}]$ & InChl=1S/C5H1102/c1-5(2)3-4-7-6/h5H,3-4H2,1-2H3 \\
\hline 1622 & $\mathrm{C} 5 \mathrm{H} 10 \mathrm{OH} 2-\mathrm{AOOH}$ & $\operatorname{ccc}(\mathrm{C}) \mathrm{C}(\mathrm{OOO}) \mathrm{O}$ & InChl=1S/C5H12O3/c1-3-4(2)5(6)8-7/h4-7H,3H2,1-2H3 \\
\hline 1623 & MBзООНМОO & $\mathrm{C}(\mathrm{O}[\mathrm{O}]) \mathrm{OC}(=\mathrm{O}) \mathrm{CC}(\mathrm{OO}) \mathrm{C}$ & InChl=1S/C5H9O6/c1-4(11-8)2-5(6)9-3-10-7/h4,8H,2-3H2,1H3 \\
\hline 1624 & PC4H9CHO & $\operatorname{CCCCC}=0$ & InChl=1S/C5H10O/c1-2-3-4-5-6/h5H,2-4H2,1H3 \\
\hline 1625 & AC5H10OOH-A & $\mathrm{CCC}([\mathrm{CH} 2]) \mathrm{COO}$ & InChl=1S/C5H1102/c1-3-5(2)4-7-6/h5-6H,2-4H2,1H3 \\
\hline 1626 & $\mathrm{C} 5 \mathrm{H} 10 \mathrm{OH} 2-\mathrm{BOOH}$ & $\operatorname{ccc}(\mathrm{C})(\mathrm{OO}) \mathrm{CO}$ & InChl=1S/C5H12O3/c1-3-5(2,4-6)8-7/h6-7H,3-4H2,1-2H3 \\
\hline 1627 & $\mathrm{MB} 3 \mathrm{OOH} 2 \mathrm{OO}$ & $\operatorname{coc}(=0) \mathrm{C}(\mathrm{O}[\mathrm{O}]) \mathrm{C}(\mathrm{OO}) \mathrm{C}$ & InChl=1S/C5H9O6/c1-3(10-7)4(11-8)5(6)9-2/h3-4,7H,1-2H3 \\
\hline 1628 & $\mathrm{C} 5 \mathrm{H} 1001-2$ & $\operatorname{cCCC1C(01)}$ & InChl=1S/C5H10O/c1-2-3-5-4-6-5/h5H,2-4H2,1H3 \\
\hline 1629 & AC5H10OOH-B & $\mathrm{CC}[\mathrm{C}](\mathrm{C}) \mathrm{COO}$ & $\operatorname{lnChl}=15 / C 5 H 1102 / c 1-3-5(2) 4-7-6 / h 6 \mathrm{H}, 3-4 \mathrm{H} 2,1-2 \mathrm{H3}$ \\
\hline 1630 & $\mathrm{C} 5 \mathrm{H} 10 \mathrm{OH} 2-\mathrm{COOH}$ & $\mathrm{CC}(\mathrm{OO}) \mathrm{C}(\mathrm{C}) \mathrm{CO}$ & $\operatorname{lnChl}=15 / C 5 H 12 \mathrm{O} / \mathrm{c} 1-4(3-6) 5(2) 8-7 / \mathrm{h} 4-7 \mathrm{H}, 3 \mathrm{H} 2,1-2 \mathrm{H} 3$ \\
\hline 1631 & $\mathrm{MB} 30 \mathrm{OH} 4 \mathrm{OO}$ & $\operatorname{coc}(=0) \operatorname{cc}(00) \mathrm{C}(\mathrm{O}[\mathrm{O}])$ & InChl=1S/C5H9O6/c1-9-5(6)2-4(11-8)3-10-7/h4,8H,2-3H2,1H3 \\
\hline 1632 & C5H10O1-5 & $\operatorname{c1cccc}(01)$ & $\operatorname{lnChl}=15 / C 5 H 100 / c 1-2-4-6-5-3-1 / h 1-5 \mathrm{H} 2$ \\
\hline 1633 & AC5H10OOH-C & $\mathrm{C}[\mathrm{CH}] \mathrm{C}(\mathrm{C}) \mathrm{COO}$ & $\operatorname{lnChl}=1 \mathrm{~S} / \mathrm{C} 5 \mathrm{H} 11 \mathrm{O} 2 / \mathrm{c} 1-3-5(2) 4-7-6 / \mathrm{h} 3,5-6 \mathrm{H}, 4 \mathrm{H} 2,1-2 \mathrm{H} 3$ \\
\hline 1634 & $\mathrm{C} 5 \mathrm{H} 10 \mathrm{OH} 2-\mathrm{DOOH}$ & $\mathrm{OOCCC}(\mathrm{C}) \mathrm{CO}$ & InChl=1S/C5H12O3/c1-5(4-6)2-3-8-7/h5-7H,2-4H2,1H3 \\
\hline
\end{tabular}




\begin{tabular}{|c|c|c|c|}
\hline 1635 & МВ40OH2OO & $\mathrm{COC}(=\mathrm{O}) \mathrm{C}(\mathrm{O}[\mathrm{O}]) \mathrm{CC}(\mathrm{OO})$ & InChl=1S/C5H9O6/c1-9-5(6)4(11-8)2-3-10-7/h4,7H,2-3H2,1H3 \\
\hline 1636 & $\mathrm{C} 5 \mathrm{H} 1001-3$ & $\operatorname{ccc} 1 \mathrm{CC}(01)$ & InChl=1S/C5H10O/c1-2-5-3-4-6-5/h5H,2-4H2,1H3 \\
\hline 1637 & AC5H10OOH-D & {$[\mathrm{CH} 2] \mathrm{CC}(\mathrm{C}) \mathrm{COO}$} & InChl=1S/C5H1102/c1-3-5(2)4-7-6/h5-6H,1,3-4H2,2H3 \\
\hline 1638 & $\mathrm{C} 5 \mathrm{H} 10 \mathrm{OH} 2-\mathrm{EOOH}$ & $\mathrm{CCC}(\mathrm{COO}) \mathrm{CO}$ & $\operatorname{lnChl}=1 \mathrm{~S} / \mathrm{C} 5 \mathrm{H} 12 \mathrm{O} 3 / \mathrm{c1}-2-5(3-6) 4-8-7 / \mathrm{h} 5-7 \mathrm{H}, 2-4 \mathrm{H} 2,1 \mathrm{H} 3$ \\
\hline 1639 & MB4OOH3OO & $\operatorname{coc}(=0) \operatorname{cC}(O[0]) C(00)$ & InChl=1S/C5H9O6/c1-9-5(6)2-4(11-8)3-10-7/h4,7H,2-3H2,1H3 \\
\hline 1640 & $\mathrm{C} 5 \mathrm{H} 10 \mathrm{O} 2-3$ & $\operatorname{ccC1C}(\mathrm{O} 1) \mathrm{C}$ & $\operatorname{lnChl}=1 \mathrm{~S} / \mathrm{C} 5 \mathrm{H} 10 \mathrm{O} / \mathrm{c} 1-3-5-4(2) 6-5 / \mathrm{h} 4-5 \mathrm{H}, 3 \mathrm{H} 2,1-2 \mathrm{H} 3$ \\
\hline 1641 & BC5H10OOH-A & $\mathrm{CCC}([\mathrm{CH} 2])(\mathrm{C}) \mathrm{OO}$ & InChl=1S/C5H1102/c1-4-5(2,3)7-6/h6H,2,4H2,1,3H3 \\
\hline 1642 & $\mathrm{C} 5 \mathrm{H} 10 \mathrm{OOH} 1-3 \mathrm{OH}$ & $\mathrm{C}(\mathrm{OOH}) \mathrm{CC}(\mathrm{OH}) \mathrm{CC}$ & InChl=1S/C5H12O3/c1-2-5(6)3-4-8-7/h5-7H,2-4H2,1H3 \\
\hline 1643 & $\mathrm{MBM}-2,30 \mathrm{OH}$ & {$[\mathrm{CH} 2] \mathrm{OC}(=\mathrm{O}) \mathrm{C}(\mathrm{OO}) \mathrm{C}(\mathrm{OO}) \mathrm{C}$} & InChl=1S/C5H9O6/c1-3(10-7)4(11-8)5(6)9-2/h3-4,7-8H,2H2,1H3 \\
\hline 1644 & $\mathrm{C} 5 \mathrm{H} 10 \mathrm{OO}-4$ & $\mathrm{CC1CC}(\mathrm{O} 1) \mathrm{C}$ & InChl=1S/C5H100/c1-4-3-5(2)6-4/h4-5H,3H2,1-2H3 \\
\hline 1645 & BC5H10OOH-C & $\mathrm{C}[\mathrm{CH}] \mathrm{C}(\mathrm{C})(\mathrm{C}) \mathrm{OO}$ & $\operatorname{lnChl}=1 \mathrm{~S} / \mathrm{C} 5 \mathrm{H} 11 \mathrm{O} 2 / \mathrm{c} 1-4-5(2,3) 7-6 / \mathrm{h} 4,6 \mathrm{H}, 1-3 \mathrm{H} 3$ \\
\hline 1646 & $\mathrm{C} 5 \mathrm{H} 10 \mathrm{OOH} 2-4 \mathrm{OH}$ & $\mathrm{CC}(\mathrm{OOH}) \mathrm{CC}(\mathrm{OH}) \mathrm{C}$ & $\operatorname{lnChl}=1 \mathrm{~S} / \mathrm{C} 5 \mathrm{H} 12 \mathrm{O} 3 / \mathrm{c1}-4(6) 3-5(2) 8-7 / \mathrm{h} 4-7 \mathrm{H}, 3 \mathrm{H} 2,1-2 \mathrm{H} 3$ \\
\hline 1647 & MBM-2,40OH & {$[\mathrm{CH} 2] \mathrm{OC}(=\mathrm{O}) \mathrm{C}(\mathrm{OO}) \mathrm{CC}(\mathrm{OO})$} & InChl=1S/C5H9O6/c1-9-5(6)4(11-8)2-3-10-7/h4,7-8H,1-3H2 \\
\hline 1648 & $\mathrm{CCSH9-DOH}$ & $\mathrm{CC}(\mathrm{C}) \mathrm{C}=\mathrm{CO}$ & InChl=1S/C5H10O/c1-5(2)3-4-6/h3-6H,1-2H3 \\
\hline 1649 & BC5H10OOH-D & {$[\mathrm{CH} 2] \mathrm{CC}(\mathrm{C})(\mathrm{C}) \mathrm{OO}$} & $\operatorname{lnChl}=1 \mathrm{~S} / \mathrm{C} 5 \mathrm{H} 1102 / \mathrm{c} 1-4-5(2,3) 7-6 / \mathrm{h} 6 \mathrm{H}, 1,4 \mathrm{H} 2,2-3 \mathrm{H} 3$ \\
\hline 1650 & CPNKET2-3 & $\mathrm{C} 1 \mathrm{CC}(=0) \mathrm{C}(=0) \mathrm{C} 1(00)$ & InChl=1S/C5H6O4/c6-3-1-2-4(9-8)5(3)7//h4,8H,1-2H2 \\
\hline 1651 & MBM-3,40OH & {$[\mathrm{CH} 2] \mathrm{OC}(=\mathrm{O}) \mathrm{CC}(\mathrm{OO}) \mathrm{C}(\mathrm{OO})$} & InChl=1S/C5H9O6/c1-9-5(6)2-4(11-8)3-10-7/h4,7-8H,1-3H2 \\
\hline 1652 & DC5H10O & $\mathrm{CC}(\mathrm{C}) \mathrm{CC}=\mathrm{O}$ & InChl=1S/C5H10O/c1-5(2)3-4-6/h4-5H,3H2,1-2H3 \\
\hline 1653 & CC5H10OOH-A & {$[\mathrm{CH} 2] \mathrm{C}(\mathrm{C}) \mathrm{C}(\mathrm{C}) \mathrm{OO}$} & InChl=1S/C5H1102/c1-4(2)5(3)7-6/h4-6H,1H2,2-3H3 \\
\hline 1654 & CPNKET2-4 & $\mathrm{C} 1(\mathrm{OO}) \mathrm{CC}(=\mathrm{O}) \mathrm{C}(=\mathrm{O}) \mathrm{C} 1$ & InChl=1S/C5H6O4/c6-4-1-3(9-8)2-5(4)7/h3,8H,1-2H2 \\
\hline 1655 & $\mathrm{MB2}-\mathrm{M}, 3 \mathrm{OOH}$ & $\mathrm{C}(\mathrm{OO}) \mathrm{OC}(=\mathrm{O})[\mathrm{CH}] \mathrm{C}(\mathrm{OO}) \mathrm{C}$ & InChl=1S/C5H9O6/c1-4(11-8)2-5(6)9-3-10-7/h2,4,7-8H,3H2,1H3 \\
\hline 1656 & AC5H9-DOH & $\mathrm{C}=\mathrm{C}(\mathrm{C}) \mathrm{CCO}$ & InChl $=1 \mathrm{~S} / \mathrm{C} 5 \mathrm{H} 10 \mathrm{O} / \mathrm{c} 1-5(2) 3-4-6 / \mathrm{h} 6 \mathrm{H}, 1,3-4 \mathrm{H} 2,2 \mathrm{H} 3$ \\
\hline 1657 & CC5H10OOH-B & $\mathrm{C}[\mathrm{C}](\mathrm{C}) \mathrm{C}(\mathrm{C}) 0 \mathrm{OO}$ & $\operatorname{lnChl}=1 \mathrm{~S} / \mathrm{C} 5 \mathrm{H} 11 \mathrm{O} 2 / \mathrm{c} 1-4(2) 5(3) 7-6 / \mathrm{h} 5-6 \mathrm{H}, 1-3 \mathrm{H} 3$ \\
\hline 1658 & CPNKET3-2 & $\mathrm{C1CC}(=0) \mathrm{C}(\mathrm{OO}) \mathrm{C} 1(=0)$ & InChl=1S/C5H6O4/c6-3-1-2-4(7)5(3)9-8/h5,8H,1-2H2 \\
\hline 1659 & $\mathrm{MB2}-3,4 \mathrm{OOH}$ & $\mathrm{COC}(=\mathrm{O})[\mathrm{CH}] \mathrm{C}(\mathrm{OO}) \mathrm{C}(\mathrm{OO})$ & InChl=1S/C5H9O6/c1-9-5(6)2-4(11-8)3-10-7/h2,4,7-8H,3H2,1H3 \\
\hline 1660 & C5H10O1-4 & $\mathrm{CC1CCC}(01)$ & InChl=1S/C5H100/c1-5-3-2-4-6-5/h5H,2-4H2,1H3 \\
\hline 1661 & CC5H10OOH-D & $\mathrm{CC}(\mathrm{C}) \mathrm{C}([\mathrm{CH} 2]) 0 \mathrm{O}$ & $\operatorname{lnChl}=1 \mathrm{~S} / \mathrm{C} 5 \mathrm{H} 1102 / \mathrm{c} 1-4(2) 5(3) 7-6 / \mathrm{h4} 4-6 \mathrm{H}, 3 \mathrm{H} 2,1-2 \mathrm{H} 3$ \\
\hline 1662 & CPNKET3-4 & $\mathrm{C} 1(\mathrm{OOO}) \mathrm{CC}(=0) \mathrm{CC} 1(=0)$ & InChl=15/C5H6O4/c6-3-1-4(7)5(2-3)9-8/h5,8H,1-2H2 \\
\hline 1663 & MB3-M,2OOH & $\mathrm{C}(\mathrm{OO}) \mathrm{OC}(=\mathrm{O}) \mathrm{C}(\mathrm{OO})[\mathrm{CH}] \mathrm{C}$ & InChl=1S/C5H9O6/c1-2-4(11-8)5(6)9-3-10-7/h2,4,7-8H,3H2,1H3 \\
\hline 1664 & $\mathrm{C5H} 9 \mathrm{OH} 21-1$ & $\mathrm{CCC}(\mathrm{C})=\mathrm{CO}$ & InChl=1S/C5H100/c1-3-5(2)4-6/h4,6H,3H2,1-2H3 \\
\hline 1665 & DC5H10OOH-A & {$[\mathrm{CH} 2] \mathrm{C}(\mathrm{C}) \mathrm{CCOO}$} & $\operatorname{lnChl}=1 \mathrm{~S} / \mathrm{C} 5 \mathrm{H} 11 \mathrm{O} 2 / \mathrm{c} 1-5(2) 3-4-7-6 / \mathrm{h5}-6 \mathrm{H}, 1,3-4 \mathrm{H} 2,2 \mathrm{H} 3$ \\
\hline 1666 & BC5Y1OH4-3O2 & $\mathrm{OCC}(\mathrm{C}(=\mathrm{C}) \mathrm{C}=0) \mathrm{O}[\mathrm{O}]$ & InChl=1S/C5H7O4/c1-4(2-6)5(3-7)9-8/h2,5,7H,1,3H2 \\
\hline 1667 & $\mathrm{MB} 3-2,4 \mathrm{OOH}$ & $\mathrm{COC}(=\mathrm{O}) \mathrm{C}(\mathrm{OO})[\mathrm{CH}] \mathrm{C}(\mathrm{OO})$ & InChl=1S/C5H9O6/c1-9-5(6)4(11-8)2-3-10-7/h2,4,7-8H,3H2,1H3 \\
\hline 1668 & $\mathrm{C} 5 \mathrm{H} 9 \mathrm{OH} 21-2$ & $\mathrm{CC}=\mathrm{C}(\mathrm{C}) \mathrm{CO}$ & InChl=1S/C5H100/c1-3-5(2)4-6/h3,6H,4H2,1-2H3 \\
\hline 1669 & DC5H10OOH-B & $\mathrm{C}[\mathrm{C}](\mathrm{C}) \mathrm{CCOO}$ & InChl=1S/C5H1102/c1-5(2)3-4-7-6/h6H,3-4H2,1-2H3 \\
\hline 1670 & CPNOH3-2O2R & $\mathrm{C1CC}(=0) \mathrm{C}(\mathrm{O}[0]) \mathrm{C} 1(\mathrm{O})$ & $\operatorname{lnChl}=15 / C 5 H 704 / c 6-3-1-2-4(7) 5(3) 9-8 / h 3,5-6 \mathrm{H}, 1-2 \mathrm{H} 2$ \\
\hline 1671 & MB4-M,2OOH & $\mathrm{C}(\mathrm{OO}) \mathrm{OC}(=\mathrm{O}) \mathrm{C}(\mathrm{OO}) \mathrm{C}[\mathrm{CH} 2]$ & InChl=1S/C5H9O6/c1-2-4(11-8)5(6)9-3-10-7/h4,7-8H,1-3H2 \\
\hline 1672 & $\mathrm{C5H} 9 \mathrm{OH} 21-3$ & $\mathrm{C}=\mathrm{CC}(\mathrm{C}) \mathrm{CO}$ & InChl=1S/C5H10O/c1-3-5(2)4-6/h3,5-6H,1,4H2,2H3 \\
\hline 1673 & DC5H10OOH-C & $\mathrm{CC}(\mathrm{C})[\mathrm{CH}] \mathrm{COO}$ & InChl=15/C5H11O2/c1-5(2)3-4-7-6/h3,5-6H,4H2,1-2H3 \\
\hline 1674 & CPNOH2-3O2R & $\mathrm{C} 1 \mathrm{CC}(=0) \mathrm{C}(\mathrm{O}) \mathrm{C} 1(\mathrm{O}[0])$ & InChl=15/C5H7O4/c6-3-1-2-4(9-8)5(3)7//h4-5,7H,1-2H2 \\
\hline 1675 & MB4-M,3OOH & $\mathrm{C}(\mathrm{OO}) \mathrm{OC}(=\mathrm{O}) \mathrm{CC}(\mathrm{OO})[\mathrm{CH} 2]$ & InChl=1S/C5H9O6/c1-4(11-8)2-5(6)9-3-10-7/h4,7-8H,1-3H2 \\
\hline 1676 & $\mathrm{C} 5 \mathrm{H} 9 \mathrm{OH} 21-4$ & $\mathrm{CcC}=\mathrm{C}) \mathrm{CO}$ & $\operatorname{lnChl}=1 \mathrm{~S} / \mathrm{C} 5 \mathrm{H} 10 \mathrm{O} / \mathrm{c1} 1-3-5(2) 4-6 / \mathrm{h} 6 \mathrm{H}, 2-4 \mathrm{H} 2,1 \mathrm{H} 3$ \\
\hline 1677 & NEOC5H11O2 & $\mathrm{CC}(\mathrm{C})(\mathrm{C}) \mathrm{CO}[\mathrm{O}]$ & InChl=1S/C5H11O2/c1-5(2,3)4-7-6/h4H2,1-3H3 \\
\hline 1678 & CPNOH3-4O2R & $\mathrm{C} 1(\mathrm{O}[\mathrm{O}]) \mathrm{CC}(=\mathrm{O}) \mathrm{CC} 1(\mathrm{O})$ & InChl=1S/C5H7O4/c6-3-1-4(7)5(2-3)9-8/h4-5,7H,1-2H2 \\
\hline 1679 & MB4-2,30OH & $\mathrm{COC}(=\mathrm{O}) \mathrm{C}(\mathrm{OO}) \mathrm{C}(\mathrm{OO})[\mathrm{CH} 2]$ & InChl=1S/C5H9O6/c1-3(10-7)4(11-8)5(6)9-2/h3-4,7-8H,1H2,2H3 \\
\hline 1680 & $\mathrm{NC4H9CHO}-2$ & $\mathrm{CC}(\mathrm{C}=\mathrm{O}) \mathrm{CC}$ & InChl=1S/C5H100/c1-3-5(2)4-6/h4-5H,3H2,1-2H3 \\
\hline 1681 & NEOC5H10OOH & {$[\mathrm{CH} 2] \mathrm{C}(\mathrm{C})(\mathrm{C}) \mathrm{COO}$} & InChl=1S/C5H1102/c1-5(2,3)4-7-6/h6H,1,4H2,2-3H3 \\
\hline 1682 & MBMO2VO & $C([0]) O C(=0) C(=0) C C$ & InChl=1S/C5H7O4/c1-2-4(7)5(8)9-3-6/h2-3H2,1H3 \\
\hline 1683 & BC5CDOHBOOHDJ & $\mathrm{O}[\mathrm{CH}] \mathrm{C}(\mathrm{C}(\mathrm{OO})(\mathrm{C}) \mathrm{C}) \mathrm{O}$ & InChl=1S/C5H1104/c1-5(2,9-8)4(7)3-6/h3-4,6-8H,1-2H3 \\
\hline 1684 & A-AC5H10O & CCC1COC1 & InChl=15/C5H100/c1-2-5-3-6-4-5/h5H,2-4H2,1H3 \\
\hline 1685 & $\mathrm{C} 5 \mathrm{H} 1102 \mathrm{H}-1$ & cccccoo & InChl=15/C5H12O2/c1-2-3-4-5-7-6/h6H,2-5H2,1H3 \\
\hline 1686 & MBMO3VO & $c([0]) O C(=0) C C(=0) C$ & InChl=1S/C5H7O4/c1-4(7)2-5(8)9-3-6/h2-3H2,1H3 \\
\hline 1687 & BC5DYCOHBOOH & $\operatorname{OOC}(\mathrm{C}(\mathrm{C}=0) 0)(\mathrm{C}) \mathrm{C}$ & InChl=1S/C5H10O4/c1-5(2,9-8)4(7)3-6/h3-4,7-8H,1-2H3 \\
\hline 1688 & A-BC5H10O & $\operatorname{cCC} 1(\mathrm{C}) \mathrm{CO} 1$ & InChl=1S/C5H100/c1-3-5(2)4-6-5/h3-4H2,1-2H3 \\
\hline 1689 & $\mathrm{C} 5 \mathrm{H} 11 \mathrm{O} 2 \mathrm{H}-2$ & $\operatorname{cccc}(\mathrm{OO}) \mathrm{C}$ & InChl=1S/C5H12O2/c1-3-4-5(2)7-6/h5-6H,3-4H2,1-2H3 \\
\hline 1690 & MB2OMVO & $C(=0) O C(=0) C([0]) C C$ & InChl=1S/C5H704/c1-2-4(7)5(8)9-3-6/h3-4H,2H2,1H3 \\
\hline 1691 & BCSBDOHCOOHAJ & $\mathrm{OCC}(\mathrm{C}(\mathrm{O})([\mathrm{CH} 2]) \mathrm{C}) \mathrm{OO}$ & InChl=1S/C5H1104/c1-5(2,7)4(3-6)9-8/h4,6-8H,1,3H2,2H3 \\
\hline 1692 & BC5ВDOHCOOHAO2J & {$[\mathrm{O}] \mathrm{OCC}(\mathrm{C}(\mathrm{OO}) \mathrm{CO})(\mathrm{O}) \mathrm{C}$} & InChl=1S/C5H1106/c1-5(7,3-10-8)4(2-6)11-9/h4,6-7,9H,2-3H2,1H3 \\
\hline 1693 & BC5CYBDOHAOOH & $\mathrm{OOCC}(\mathrm{C}(=0) \mathrm{CO})(\mathrm{O}) \mathrm{C}$ & InChl=1S/C5H1005/c1-5(8,3-10-9)4(7)2-6/h6,8-9H,2-3H2,1H3 \\
\hline 1694 & C4Y2OH13-3J & $\mathrm{OCC}(=\mathrm{C}(\mathrm{O}) \mathrm{C})[\mathrm{O}]$ & InChl=1S/C4H7O3/c1-3(6)4(7)2-5/h5-6H,2H2,1H3 \\
\hline 1695 & $\mathrm{C} 4 \mathrm{Y} 23 \mathrm{OH} 1$ & $\mathrm{CC}(=0) \mathrm{C}(=0) \mathrm{CO}$ & $\operatorname{lnChl}=1 \mathrm{~S} / \mathrm{C} 4 \mathrm{H} 6 \mathrm{OO} / \mathrm{c1}-3(6) 4(7) 2-5 / \mathrm{h} 5 \mathrm{H}, 2 \mathrm{H} 2,1 \mathrm{H} 3$ \\
\hline 1696 & $\mathrm{C} 6 \mathrm{H} 2$ & С\#CC\#СC\#C & InChl=1S/C6H2/c1-3-5-6-4-2/h1-2H \\
\hline 1697 & IC603-4 & $\mathrm{CC}(\mathrm{C} 10 \mathrm{C} 1 \mathrm{C}) \mathrm{C}$ & InChl=15/C6H12O/c1-4(2)6-5(3)7-6/h4-6H,1-3H3 \\
\hline 1698 & NEC6OOH1-1A & $\mathrm{CCC}(\mathrm{COO})([\mathrm{CH} 2]) \mathrm{C}$ & InChl=1S/C6H13O2/c1-4-6(2,3)5-8-7/h7H,2,4-5H2,1,3H3 \\
\hline 1699 & $\mathrm{XC6023-10OH}$ & $00 \mathrm{OCC} 1(\mathrm{C}) 0 \mathrm{OC} 1(\mathrm{C}) \mathrm{C}$ & InChl=1S/C6H12O3/c1-5(2)6(3,9-5)4-8-7/h7H,4H2,1-3H3 \\
\hline 1700 & $\mathrm{C} 6 \mathrm{H} 3$ & 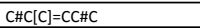 & $\operatorname{lnChl}=1 \mathrm{~S} / \mathrm{C} 6 \mathrm{H} 3 / \mathrm{c} 1-3-5-6-4-2 / \mathrm{h} 1-2,5 \mathrm{H}$ \\
\hline 1701 & IC6O3-5 & $\mathrm{CC}(\mathrm{C} 1 \mathrm{CCO}) \mathrm{C}$ & $\operatorname{lnChl}=1 \mathrm{~S} / \mathrm{C} 6 \mathrm{H} 12 \mathrm{O} / \mathrm{c1} 1-5(2) 6-3-4-7-6 / \mathrm{h} 5-6 \mathrm{H}, 3-4 \mathrm{H} 2,1-2 \mathrm{H} 3$ \\
\hline 1702 & $\mathrm{IC6OOH2-1}$ & $\operatorname{CCCC}(\mathrm{OO})([\mathrm{CH} 2]) \mathrm{C}$ & InChl=1S/C6H13O2/c1-4-5-6(2,3)8-7/h7H,2,4-5H2,1,3H3 \\
\hline 1703 & $13 \mathrm{C} 6 \mathrm{OO} 15-2 \mathrm{OOH}$ & OOC1COC(C1C)C & InChl=1S/C6H12O3/c1-4-5(2)8-3-6(4)9-7/h4-7H,3H2,1-2H3 \\
\hline 1704 & $\mathrm{~L}-\mathrm{C} 6 \mathrm{H} 4$ & 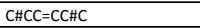 & InChl=1S/C6H4/c1-3-5-6-4-2/h1-2,5-6H \\
\hline 1705 & IC6O4-5 & $\mathrm{CC}(\mathrm{CC} 1 \mathrm{OC} 1) \mathrm{C}$ & InChl=15/C6H12O/c1-5(2)3-6-4-7-6/h5-6H,3-4H2,1-2H3 \\
\hline 1706 & NEC6OOH1-3 & $\mathrm{C}[\mathrm{CH}] \mathrm{C}(\mathrm{COO})(\mathrm{C}) \mathrm{C}$ & $\operatorname{lnChl}=15 / \mathrm{C} 6 \mathrm{H} 13 \mathrm{O} 2 / \mathrm{c1}-4-6(2,3) 5-8-7 / \mathrm{h} 4,7 \mathrm{H}, 5 \mathrm{H} 2,1-3 \mathrm{H} 3$ \\
\hline 1707 & 13C6O15-30OH & $\mathrm{CC} 1(\mathrm{CCOC} 1 \mathrm{C}) \mathrm{OO}$ & $\operatorname{lnChl}=1 \mathrm{~S} / \mathrm{C} 6 \mathrm{H} 12 \mathrm{O} / \mathrm{c} 1-5-6(2,9-7) 3-4-8-5 / \mathrm{h} 5,7 \mathrm{H}, 3-4 \mathrm{H} 2,1-2 \mathrm{H} 3$ \\
\hline 1708 & $\mathrm{C}-\mathrm{C} 6 \mathrm{H} 4$ & $\mathrm{C} 1 \# \mathrm{CC}=\mathrm{CC}=\mathrm{C} 1$ & $\operatorname{lnChl}=15 / \mathrm{C} 6 \mathrm{H} 4 / \mathrm{c} 1-2-4-6-5-3-1 / \mathrm{h} 1-4 \mathrm{H}$ \\
\hline 1709 & NEC601-1A & $\operatorname{ccc} 1(\mathrm{C}) \operatorname{coc} 1$ & InChl=1S/C6H12O/c1-3-6(2)4-7-5-6/h3-5H2,1-2H3 \\
\hline 1710 & $\mathrm{IC6OOH2-3}$ & $\mathrm{CC}[\mathrm{CH}] \mathrm{C}(\mathrm{OO})(\mathrm{C}) \mathrm{C}$ & InChl=15/C6H13O2/c1-4-5-6(2,3)8-7/h5,7H,4H2,1-3H3 \\
\hline 1711 & 13C6O15-40OH & CC10CCC1COO & InChl=1S/C6H12O3/c1-5-6(4-9-7)2-3-8-5/h5-7H,2-4H2,1H3 \\
\hline 1712 & C6H5 & $\mathrm{C} 1=[\mathrm{C}] \mathrm{C}=\mathrm{CC}=\mathrm{C} 1$ & InChl=1S/C6H5/c1-2-4-6-5-3-1/h1-5H \\
\hline 1713 & NEC601-3 & $\mathrm{CC} 10 \mathrm{OCC} 1 \mathrm{C}) \mathrm{C}$ & InChl=1S/C6H12O/c1-5-6(2,3)4-7-5/h5H,4H2,1-3H3 \\
\hline 1714 & NEC6OOH1-4 & {$[\mathrm{CH} 2] \mathrm{CC}(\mathrm{COO})(\mathrm{C}) \mathrm{C}$} & InChl=1S/C6H13O2/c1-4-6(2,3)5-8-7/h7H,1,4-5H2,2-3H3 \\
\hline 1715 & $13 \mathrm{C} 6 \mathrm{O} 15-6 \mathrm{OOH}$ & oocc10ccC1C & InChl=1S/C6H12O3/c1-5-2-3-8-6(5)4-9-7/h5-7H,2-4H2,1H3 \\
\hline 1716 & NEC601-4 & $\mathrm{CC} 1(\mathrm{C}) \operatorname{COCC} 1$ & InChl=1S/C6H12O/c1-6(2)3-4-7-5-6/h3-5H2,1-2H3 \\
\hline 1717 & $\mathrm{IC} 60 \mathrm{OH} 2-4$ & $\mathrm{C}[\mathrm{CH}] \mathrm{CC}(\mathrm{OO})(\mathrm{C}) \mathrm{C}$ & $\operatorname{lnChl}=1 \mathrm{~S} / \mathrm{C} 6 \mathrm{H} 13 \mathrm{O} 2 / \mathrm{c1}-4-5-6(2,3) 8-7 / \mathrm{h} 4,7 \mathrm{H}, 5 \mathrm{H} 2,1-3 \mathrm{H} 3$ \\
\hline 1718 & $13 \mathrm{C} 6 \mathrm{O} 16-2 \mathrm{OOH}$ & OOC1COCCC1C & InChl=1S/C6H12O3/c1-5-2-3-8-4-6(5)9-7/h5-7H,2-4H2,1H3 \\
\hline 1719 & $\mathrm{C6H6}$ & $\mathrm{C} 1=\mathrm{CC}=\mathrm{CC}=\mathrm{C} 1$ & InChl=1S/C6H6/c1-2-4-6-5-3-1/h1-6H \\
\hline 1720 & NEC603-4 & $\mathrm{CC}(\mathrm{C} 10 \mathrm{OC} 1)(\mathrm{C}) \mathrm{C}$ & InChl=1S/C6H12O/c1-6(2,3)5-4-7-5/h5H,4H2,1-3H3 \\
\hline 1721 & NEC6OOH3-1 & $\mathrm{OOC}(\mathrm{C}([\mathrm{CH} 2])(\mathrm{C}) \mathrm{C}) \mathrm{C}$ & InChl=1S/C6H13O2/c1-5(8-7)6(2,3)4/h5,7H,2H2,1,3-4H3 \\
\hline 1722 & FULVENE & $\mathrm{C}=\mathrm{C} 1 \mathrm{C}=\mathrm{CC}=\mathrm{C} 1$ & InChl=1S/C6H6/c1-6-4-2-3-5-6/h2-5H,1H2 \\
\hline 1723 & $\mathrm{XC601-1 \textrm {A }}$ & $\mathrm{CC}(\mathrm{C} 1 \mathrm{COC} 1) \mathrm{C}$ & InChl=1S/C6H12O/c1-5(2)6-3-7-4-6/h5-6H,3-4H2,1-2H3 \\
\hline 1724 & $\mathrm{IC} 6 \mathrm{OOH} 2-5$ & {$[\mathrm{CH} 2] \mathrm{CCC}(\mathrm{OO})(\mathrm{C}) \mathrm{C}$} & InChl=1S/C6H13O2/c1-4-5-6(2,3)8-7/h7H,1,4-5H2,2-3H3 \\
\hline 1725 & 13C6O16-40OH & OOCC1CCOCC1 & InChl=1S/C6H12O3/c7-9-5-6-1-3-8-4-2-6/h6-7H,1-5H2 \\
\hline
\end{tabular}




\begin{tabular}{|c|c|c|c|}
\hline 1726 & $\mathrm{C} 5 \mathrm{H} 4 \mathrm{CH} 3$ & $\mathrm{C} 1=\mathrm{C}[\mathrm{C}](\mathrm{C}) \mathrm{C}=\mathrm{C} 1$ & InChl=1S/C6H7/c1-6-4-2-3-5-6/h2-5H,1H3 \\
\hline 1727 & XC601-2 & $\mathrm{CC}(\mathrm{C} 1(\mathrm{C}) \mathrm{CO} 1) \mathrm{C}$ & InChl=1S/C6H12O/c1-5(2)6(3)4-7-6/h5H,4H2,1-3H3 \\
\hline 1728 & NEC6OOH3-4 & $\mathrm{OOC}(\mathrm{C}(\mathrm{C})(\mathrm{C}) \mathrm{C})[\mathrm{CH} 2]$ & InChl=1S/C6H13O2/c1-5(8-7)6(2,3)4/h5,7H,1H2,2-4H3 \\
\hline 1729 & $13 \mathrm{C} 6023-10 \mathrm{OH}$ & $\mathrm{CC} 1(\mathrm{CC}) \mathrm{OC} 1 \mathrm{COO}$ & InChl=1S/C6H12O3/c1-3-6(2)5(9-6)4-8-7/h5,7H,3-4H2,1-2H3 \\
\hline 1730 & $\mathrm{C} 5 \mathrm{H} 5 \mathrm{CH} 2$ & $\mathrm{C} 1=\mathrm{CC}([\mathrm{CH} 2]) \mathrm{C}=\mathrm{C} 1$ & $\operatorname{lnChl}=15 / \mathrm{C} 6 \mathrm{H} 7 / \mathrm{c1}-6-4-2-3-5-6 / \mathrm{h} 2-6 \mathrm{H}, 1 \mathrm{H} 2$ \\
\hline 1731 & XC601-3 & $\operatorname{cC} 1 \operatorname{coc} 1(\mathrm{C}) \mathrm{C}$ & InChl=1S/C6H12O/c1-5-4-7-6(5,2)3/h5H,4H2,1-3H3 \\
\hline 1732 & IC6OOH3-1 & $\mathrm{CCC}(\mathrm{C}([\mathrm{CH} 2]) \mathrm{C}) \mathrm{OO}$ & InChl=1S/C6H13O2/c1-4-6(8-7)5(2)3/h5-7H,2,4H2,1,3H3 \\
\hline 1733 & $13 \mathrm{C} 6023-40 \mathrm{OH}$ & OOCC1(CC)OC1C & InChl=15/C6H12O3/c1-3-6(4-8-7)5(2)9-6/h5,7H,3-4H2,1-2H3 \\
\hline 1734 & $\mathrm{C} 5 \mathrm{H} 5 \mathrm{CH} 2-1$ & $\mathrm{C} 1=\mathrm{CC}=\mathrm{C}([\mathrm{CH} 2]) \mathrm{C} 1$ & InChl=1S/C6H7/c1-6-4-2-3-5-6/h2-4H,1,5H2 \\
\hline 1735 & $\mathrm{xC601-4}$ & CC1COCC1C & InChl=1S/C6H12O/c1-5-3-7-4-6(5)2/h5-6H,3-4H2,1-2H3 \\
\hline 1736 & $\mathrm{NEC6OOH4-1}$ & $\mathrm{OOCCC}([\mathrm{CH} 2])(\mathrm{C}) \mathrm{C}$ & InChl=1S/C6H13O2/c1-6(2,3)4-5-8-7/h7H,1,4-5H2,2-3H3 \\
\hline 1737 & $13 \mathrm{C} 6023-5 \mathrm{OOH}$ & $\mathrm{OOC}(\mathrm{C} 1(\mathrm{C}) \mathrm{OC} 1 \mathrm{C}) \mathrm{C}$ & InChl=15/C6H12O3/c1-4-6(3,8-4)5(2)9-7/h4-5,7H,1-3H3 \\
\hline 1738 & $\mathrm{C} 5 \mathrm{H} 5 \mathrm{CH} 2-2$ & $\mathrm{C} 1=\mathrm{CC}([\mathrm{CH} 2])=\mathrm{CC} 1$ & InChl=15/C6H7/c1-6-4-2-3-5-6/h2,4-5H,1,3H2 \\
\hline 1739 & $\mathrm{xC602-3}$ & $\mathrm{CC} 1(\mathrm{C}) \mathrm{OC} 1(\mathrm{C}) \mathrm{C}$ & InChl=1S/C6H12O/c1-5(2)6(3,4)7-5/h1-4H3 \\
\hline 1740 & IC6OOH3-2 & $\operatorname{ccc}([\mathrm{C}](\mathrm{C}) \mathrm{C}) \mathrm{OO}$ & InChl=1S/C6H13O2/c1-4-6(8-7)5(2)3/h6-7H,4H2,1-3H3 \\
\hline 1741 & C6H12OOH1-2O & $\mathrm{C}(\mathrm{OO}) \mathrm{C}([0]) \mathrm{CCCC}$ & InChl=1S/C6H13O3/c1-2-3-4-6(7)5-9-8/h6,8H,2-5H2,1H3 \\
\hline 1742 & $\mathrm{CYC6H7}$ & $\mathrm{C} 1=\mathrm{CC}[\mathrm{CH}] \mathrm{C}=\mathrm{C} 1$ & $\operatorname{lnChl}=15 / C 6 H 7 / c 1-2-4-6-5-3-1 / h 1-5 H, 6 \mathrm{H} 2$ \\
\hline 1743 & $13 \mathrm{C} 601-2$ & $\mathrm{CC}(\mathrm{C} 1 \mathrm{CO} 1) \mathrm{CC}$ & $\operatorname{lnChl}=1 \mathrm{~S} / \mathrm{C} 6 \mathrm{H} 12 \mathrm{O} / \mathrm{c1}-3-5(2) 6-4-7-6 / \mathrm{h} 5-6 \mathrm{H}, 3-4 \mathrm{H} 2,1-2 \mathrm{H} 3$ \\
\hline 1744 & $\mathrm{NEC6OOH4-3}$ & $\mathrm{OOC}[\mathrm{CH}] \mathrm{C}(\mathrm{C})(\mathrm{C}) \mathrm{C}$ & $\operatorname{lnChl}=1 \mathrm{~S} / \mathrm{C} 6 \mathrm{H} 13 \mathrm{O} 2 / \mathrm{c1} 1-6(2,3) 4-5-8-7 / \mathrm{h} 4,7 \mathrm{H}, 5 \mathrm{H} 2,1-3 \mathrm{H} 3$ \\
\hline 1745 & C6H12OOH1-3O & $\mathrm{C}(\mathrm{OO}) \mathrm{CC}([\mathrm{O}]) \mathrm{CCC}$ & InChl=1S/C6H13O3/c1-2-3-6(7)4-5-9-8/h6,8H,2-5H2,1H3 \\
\hline 1746 & IC6D134-1AR & $\mathrm{C}=\mathrm{C}([\mathrm{CH} 2]) \mathrm{C}=\mathrm{C}=\mathrm{C}$ & InChl=15/C6H7/c1-4-5-6(2)3/h5H,1-3H2 \\
\hline 1747 & $13 \mathrm{C601-3}$ & $\mathrm{CC} 1(\mathrm{CC}) \mathrm{CCO} 1$ & InChl=1S/C6H12O/c1-3-6(2)4-5-7-6/h3-5H2,1-2H3 \\
\hline 1748 & $\mathrm{IC6OOH} 3-4$ & $\mathrm{C}[\mathrm{CH}] \mathrm{C}(\mathrm{C}(\mathrm{C}) \mathrm{C}) \mathrm{OO}$ & InChl=1S/C6H13O2/c1-4-6(8-7)5(2)3/h4-7H,1-3H3 \\
\hline 1749 & C6H12OOH1-4O & $\mathrm{C}(\mathrm{OO}) \mathrm{CCC}([\mathrm{O}]) \mathrm{CC}$ & InChl=1S/C6H13O3/c1-2-6(7)4-3-5-9-8/h6,8H,2-5H2,1H3 \\
\hline 1750 & CYHX13ENE & $\mathrm{C} 1 \mathrm{CC}=\mathrm{CC}=\mathrm{C} 1$ & InChl=1S/C6H8/c1-2-4-6-5-3-1/h1-4H,5-6H2 \\
\hline 1751 & $13 \mathrm{C601-4}$ & CCC1COCC1 & InChl=1S/C6H12O/c1-2-6-3-4-7-5-6/h6H,2-5H2,1H3 \\
\hline 1752 & IC6OOH3-5 & {$[\mathrm{CH} 2] \mathrm{CC}(\mathrm{C}(\mathrm{C}) \mathrm{C}) \mathrm{OO}$} & InChl=1S/C6H13O2/c1-4-6(8-7)5(2)3/h5-7H,1,4H2,2-3H3 \\
\hline 1753 & С6H12OOH1-50 & $\mathrm{C}(\mathrm{OO}) \operatorname{cccc}([0]) \mathrm{C}$ & InChl=1S/C6H13O3/c1-6(7)4-2-3-5-9-8/h6,8H,2-5H2,1H3 \\
\hline 1754 & CYHX14ENE & $\mathrm{C} 1=\mathrm{CCC}=\mathrm{CC} 1$ & $\operatorname{lnChl}=15 / \mathrm{C} 6 \mathrm{H} 8 / \mathrm{c} 1-2-4-6-5-3-1 / \mathrm{h} 1-2,5-6 \mathrm{H}, 3-4 \mathrm{H} 2$ \\
\hline 1755 & $13 \mathrm{C601-5}$ & CC1OCCC1C & $\operatorname{lnChl}=1 \mathrm{~S} / \mathrm{C} 6 \mathrm{H} 12 \mathrm{O} / \mathrm{c1}-5-3-4-7-6(5) 2 / \mathrm{h} 5-6 \mathrm{H}, 3-4 \mathrm{H} 2,1-2 \mathrm{H} 3$ \\
\hline 1756 & IC60OH4-1 & $\mathrm{OOC}(\mathrm{CC}([\mathrm{CH} 2]) \mathrm{C}) \mathrm{C}$ & InChl=1S/C6H13O2/c1-5(2)4-6(3)8-7/h5-7H,1,4H2,2-3H3 \\
\hline 1757 & $\mathrm{C} 6 \mathrm{H} 12 \mathrm{OOH} 2-10$ & $\mathrm{C}([\mathrm{O}]) \mathrm{C}(\mathrm{OO}) \mathrm{CCCC}$ & InChl=1S/C6H13O3/c1-2-3-4-6(5-7)9-8/h6,8H,2-5H2,1H3 \\
\hline 1758 & $\mathrm{C} 5 \mathrm{H} 5 \mathrm{CH} 3-1$ & $\mathrm{C} 1=\mathrm{CC}=\mathrm{C}(\mathrm{C}) \mathrm{C} 1$ & InChl=1S/C6H8/c1-6-4-2-3-5-6/h2-4H,5H2,1H3 \\
\hline 1759 & $13 \mathrm{C601-6}$ & CC1CCOCC1 & InChl=1S/C6H12O/c1-6-2-4-7-5-3-6/h6H,2-5H2,1H3 \\
\hline 1760 & $\mathrm{IC6OOH} 4-2$ & $\mathrm{OOC}(\mathrm{C}[\mathrm{C}](\mathrm{C}) \mathrm{C}) \mathrm{C}$ & $\operatorname{lnChl}=1 \mathrm{~S} / \mathrm{C} 6 \mathrm{H} 13 \mathrm{O} 2 / \mathrm{c} 1-5(2) 4-6(3) 8-7 / \mathrm{h} 6-7 \mathrm{H}, 4 \mathrm{H} 2,1-3 \mathrm{H} 3$ \\
\hline 1761 & C6H12OOH2-3O & $\mathrm{CC}(\mathrm{OO}) \mathrm{C}([\mathrm{O}]) \mathrm{CCC}$ & InChl=15/C6H13O3/c1-3-4-6/7)5(2)9-8/h5-6,8H,3-4H2,1-2H3 \\
\hline 1762 & $\mathrm{C} 5 \mathrm{H} 5 \mathrm{CH} 3-2$ & $\mathrm{C} 1=\mathrm{CC}(\mathrm{C})=\mathrm{CC} 1$ & $\operatorname{lnChl}=1 \mathrm{~S} / \mathrm{C} 6 \mathrm{H} 8 / \mathrm{c1}-6-4-2-3-5-6 / \mathrm{h} 2,4-5 \mathrm{H}, 3 \mathrm{H} 2,1 \mathrm{H} 3$ \\
\hline 1763 & $13 \mathrm{C} 602-3$ & $\operatorname{CCC1}(\mathrm{C}) \mathrm{OC} 1 \mathrm{C}$ & InChl=1S/C6H12O/c1-4-6(3)5(2)7-6/h5H,4H2,1-3H3 \\
\hline 1764 & $\mathrm{IC} 6 \mathrm{OOH} 4-3$ & $\mathrm{OOC}([\mathrm{CH}] \mathrm{C}(\mathrm{C}) \mathrm{C}) \mathrm{C}$ & InChl=1S/C6H13O2/c1-5(2)4-6(3)8-7/h4-7H,1-3H3 \\
\hline 1765 & $\mathrm{C} 6 \mathrm{H} 12 \mathrm{OOH} 2-4 \mathrm{O}$ & $\mathrm{CC}(\mathrm{OO}) \mathrm{CC}([\mathrm{O}]) \mathrm{CC}$ & $\operatorname{lnChl}=1 \mathrm{~S} / \mathrm{C} 6 \mathrm{H} 1303 / \mathrm{c} 1-3-6(7) 4-5(2) 9-8 / \mathrm{h5}-6,8 \mathrm{H}, 3-4 \mathrm{H} 2,1-2 \mathrm{H} 3$ \\
\hline 1766 & $\mathrm{C} 5 \mathrm{H} 5 \mathrm{CH} 3$ & $\mathrm{CC} 1 \mathrm{C}=\mathrm{CC}=\mathrm{C} 1$ & $\operatorname{lnChl}=15 / C 6 H 8 / c 1-6-4-2-3-5-6 / h 2-6 \mathrm{H}, 1 \mathrm{H} 3$ \\
\hline 1767 & $13 \mathrm{C6O2-4}$ & CCC1COC1C & $\operatorname{lnChl}=1 \mathrm{~S} / \mathrm{C} 6 \mathrm{H} 12 \mathrm{O} / \mathrm{c1}-3-6-4-7-5(6) 2 / \mathrm{h} 5-6 \mathrm{H}, 3-4 \mathrm{H} 2,1-2 \mathrm{H} 3$ \\
\hline 1768 & IC6OOH4-5 & $\mathrm{OOC}(\mathrm{CC}(\mathrm{C}) \mathrm{C})[\mathrm{CH} 2]$ & $\operatorname{lnChl}=1 \mathrm{~S} / \mathrm{C} 6 \mathrm{H} 13 \mathrm{O} 2 / \mathrm{c} 1-5(2) 4-6(3) 8-7 / \mathrm{h} 5-7 \mathrm{H}, 3-4 \mathrm{H} 2,1-2 \mathrm{H} 3$ \\
\hline 1769 & $\mathrm{C} 6 \mathrm{H} 12 \mathrm{OOH} 2-5 \mathrm{O}$ & $\mathrm{CC}(\mathrm{OO}) \mathrm{CcC}([0]) \mathrm{C}$ & InChl=15/C6H13O3/c1-5(7)3-4-6(2)9-8/h5-6,8H,3-4H2,1-2H3 \\
\hline 1770 & IC6D134 & $\mathrm{C}=\mathrm{C}(\mathrm{C}) \mathrm{C}=\mathrm{C}=\mathrm{C}$ & InChl=1S/C6H8/c1-4-5-6(2)3/h5H,1-2H2,3H3 \\
\hline 1771 & $13 \mathrm{C} 602-5$ & $\mathrm{CC} 1 \mathrm{C}(\mathrm{C}) \mathrm{OC} 1 \mathrm{C}$ & InChl=15/C6H12O/c1-4-5(2)7-6(4)3/h4-6H,1-3H3 \\
\hline 1772 & IC60OH5-1 & $\mathrm{OOCCCC}([\mathrm{CH} 2]) \mathrm{C}$ & InChl=15/C6H13O2/c1-6(2)4-3-5-8-7/h6-7H,1,3-5H2,2H3 \\
\hline 1773 & $\mathrm{C} 6 \mathrm{H} 12 \mathrm{OOH} 2-6 \mathrm{O}$ & $\mathrm{CC}(00) \operatorname{cccc}([0])$ & InChl=1S/C6H13O3/c1-6(9-8)4-2-3-5-7/h6,8H,2-5H2,1H3 \\
\hline 1774 & C6H9-A & $\mathrm{C}=\mathrm{CC}[\mathrm{CH}] \mathrm{C}=\mathrm{C}$ & InChl=1S/C6H9/c1-3-5-6-4-2/h3-5H,1-2,6H2 \\
\hline 1775 & $13 \mathrm{C} 6 \mathrm{O3}-4$ & $\mathrm{CCC} 1(\mathrm{CC}) \mathrm{OC} 1$ & InChl=1S/C6H12O/c1-3-6(4-2)5-7-6/h3-5H2,1-2H3 \\
\hline 1776 & IC6OOH5-2 & OOCCC[C](C)C & $\operatorname{lnChl}=15 / \mathrm{C} 6 \mathrm{H} 13 \mathrm{O} 2 / \mathrm{c1}-6(2) 4-3-5-8-7 / \mathrm{h} 7 \mathrm{H}, 3-5 \mathrm{H} 2,1-2 \mathrm{H} 3$ \\
\hline 1777 & С6H12OOH3-10 & $\mathrm{C}([\mathrm{O}]) \mathrm{CC}(\mathrm{OO}) \mathrm{CCC}$ & $\operatorname{lnChl}=1 \mathrm{~S} / \mathrm{C} 6 \mathrm{H} 13 \mathrm{O} 3 / \mathrm{c1}-2-3-6(9-8) 4-5-7 / \mathrm{h} 6,8 \mathrm{H}, 2-5 \mathrm{H} 2,1 \mathrm{H} 3$ \\
\hline 1778 & CYHX1N3J & $\mathrm{C} 1 \mathrm{CCC}=\mathrm{C}[\mathrm{CH}] 1$ & InChl=1S/C6H9/c1-2-4-6-5-3-1/h1-3H,4-6H2 \\
\hline 1779 & IC601-1A & CCCC1COC1 & InChl=1S/C6H12O/c1-2-3-6-4-7-5-6/h6H,2-5H2,1H3 \\
\hline 1780 & IC6OOH5-3 & $\mathrm{OOCC}[\mathrm{CH}] \mathrm{C}(\mathrm{C}) \mathrm{C}$ & $\operatorname{lnChl}=1 \mathrm{~S} / \mathrm{C} 6 \mathrm{H} 13 \mathrm{O} 2 / \mathrm{c} 1-6(2) 4-3-5-8-7 / \mathrm{h} 4,6-7 \mathrm{H}, 3,5 \mathrm{H} 2,1-2 \mathrm{H} 3$ \\
\hline 1781 & С6H12OOH3-2O & $\mathrm{CC}([\mathrm{O}]) \mathrm{C}(\mathrm{OO}) \mathrm{CCC}$ & InChl=15/C6H1303/c1-3-4-6(9-8)5(2)7/h5-6,8H,3-4H2,1-2H3 \\
\hline 1782 & CYHX1N4J & $\mathrm{C} 1[\mathrm{CH}] \mathrm{CC}=\mathrm{CC} 1$ & InChl=1S/C6H9/c1-2-4-6-5-3-1/h1-2,5H,3-4,6H2 \\
\hline 1783 & IC601-2 & $\operatorname{CCCC1(C)CO1}$ & $\operatorname{lnChl}=15 / C 6 H 12 \mathrm{O} / \mathrm{c1}-3-4-6(2) 5-7-6 / \mathrm{h} 3-5 \mathrm{H} 2,1-2 \mathrm{H} 3$ \\
\hline 1784 & IC6OOH5-4 & $\mathrm{OOC}[\mathrm{CH}] \mathrm{CC}(\mathrm{C}) \mathrm{C}$ & InChl=15/C6H13O2/c1-6(2)4-3-5-8-7/h3,6-7H,4-5H2,1-2H3 \\
\hline 1785 & C6H12OOH3-4O & $\operatorname{ccc}(\mathrm{OOO}) \mathrm{C}([\mathrm{O}]) \mathrm{CC}$ & InChl=1S/C6H13O3/c1-3-5(7)6(4-2)9-8/h5-6,8H,3-4H2,1-2H3 \\
\hline 1786 & HX13EN6J & {$[\mathrm{CH} 2] \mathrm{CC}=\mathrm{CC}=\mathrm{C}$} & InChl=1S/C6H9/c1-3-5-6-4-2/h3,5-6H,1-2,4H2 \\
\hline 1787 & IC6Y4 & $\mathrm{CC}(\mathrm{C}) \mathrm{CC}(=\mathrm{O}) \mathrm{C}$ & InChl=1S/C6H12O/c1-5(2)4-6(3)7/h5H,4H2,1-3H3 \\
\hline 1788 & $\mathrm{XC6OOH1-1 \textrm {A }}$ & $\mathrm{OOCC}(\mathrm{C}(\mathrm{C}) \mathrm{C})[\mathrm{CH} 2]$ & InChl=15/C6H13O2/c1-5(2)6(3)4-8-7/h5-7H,3-4H2,1-2H3 \\
\hline 1789 & С6H12OOH3-50 & $\operatorname{ccc}(00) \operatorname{cc}([0]) \mathrm{C}$ & $\operatorname{lnChl}=1 \mathrm{~S} / \mathrm{C} 6 \mathrm{H} 1303 / \mathrm{c} 1-3-6(9-8) 4-5(2) 7 / \mathrm{h5}-6,8 \mathrm{H}, 3-4 \mathrm{H} 2,1-2 \mathrm{H} 3$ \\
\hline 1790 & I3C6D13-5 & $\mathrm{C}[\mathrm{CH}] \mathrm{C}(=\mathrm{C}) \mathrm{C}=\mathrm{C}$ & InChl=1S/C6H9/c1-4-6(3)5-2/h4-5H,1,3H2,2H3 \\
\hline 1791 & NEOC5H11CHO & $\mathrm{CC}(\mathrm{C})(\mathrm{C}) \mathrm{CC}=\mathrm{O}$ & InChl=1S/C6H12O/c1-6(2,3)4-5-7/h5H,4H2,1-3H3 \\
\hline 1792 & $\mathrm{XC6OOH1-2}$ & $O O O C[C](C(C) C) C$ & InChl=1S/C6H13O2/c1-5(2)6(3)4-8-7/h5,7H,4H2,1-3H3 \\
\hline 1793 & С6H12OOH3-60 & $\operatorname{ccc}(00) \operatorname{ccc}([0])$ & InChl=1S/C6H13O3/c1-2-6(9-8)4-3-5-7/h6,8H,2-5H2,1H3 \\
\hline 1794 & I3C6D13-6 & {$[\mathrm{CH} 2] \mathrm{CC}(=\mathrm{C}) \mathrm{C}=\mathrm{C}$} & InChl=1S/C6H9/c1-4-6(3)5-2/h4H,1-3,5H2 \\
\hline 1795 & C6H13O-1 & $\mathrm{C}([\mathrm{O}]) \mathrm{CCCCC}$ & $\operatorname{lnChl}=1 \mathrm{~S} / \mathrm{C} 6 \mathrm{H} 13 \mathrm{O} / \mathrm{c} 1-2-3-4-5-6-7 / \mathrm{h} 2-6 \mathrm{H} 2,1 \mathrm{H} 3$ \\
\hline 1796 & $\mathrm{XC6OOH1-3}$ & $00 \mathrm{OOC}([\mathrm{C}](\mathrm{C}) \mathrm{C}) \mathrm{C}$ & InChl=1S/C6H13O2/c1-5(2)6(3)4-8-7/h6-7H,4H2,1-3H3 \\
\hline 1797 & C6H12OH-102-2 & $\mathrm{C}(\mathrm{O}) \mathrm{C}(\mathrm{O}[\mathrm{O}]) \mathrm{CCCC}$ & InChl=1S/C6H13O3/c1-2-3-4-6(5-7)9-8/h6-7H,2-5H2,1H3 \\
\hline 1798 & I3C6D15-3 & $\mathrm{C}=\mathrm{C}[\mathrm{C}](\mathrm{C}=\mathrm{C}) \mathrm{C}$ & InChl=1S/C6H9/c1-4-6(3)5-2/h4-5H,1-2H2,3H3 \\
\hline 1799 & C6H13O-2 & $\mathrm{CC}([\mathrm{O}]) \mathrm{CCCC}$ & InChl=1S/C6H13O/c1-3-4-5-6(2)7/h6H,3-5H2,1-2H3 \\
\hline 1800 & $\mathrm{XC6OOH1-4}$ & $\mathrm{OOCC}(\mathrm{C}([\mathrm{CH} 2]) \mathrm{C}) \mathrm{C}$ & InChl=1S/C6H13O2/c1-5(2)6(3)4-8-7/h5-7H,1,4H2,2-3H3 \\
\hline 1801 & $\mathrm{C} 6 \mathrm{H} 12 \mathrm{OH}-2 \mathrm{O} 2-1$ & $\mathrm{C}(\mathrm{O}[\mathrm{O}]) \mathrm{C}(\mathrm{O}) \mathrm{CCCC}$ & InChl=1S/C6H13O3/c1-2-3-4-6(7)5-9-8/h6-7H,2-5H2,1H3 \\
\hline 1802 & I3C6D15-4 & $\mathrm{C}=\mathrm{CC}(\mathrm{C}=\mathrm{C})[\mathrm{CH} 2]$ & $\operatorname{lnChl}=15 / \mathrm{C} 6 \mathrm{H} 9 / \mathrm{cc}-4-6(3) 5-2 / \mathrm{h} 4-6 \mathrm{H}, 1-3 \mathrm{H} 2$ \\
\hline 1803 & $\mathrm{C} 6 \mathrm{H} 13 \mathrm{O}-3$ & $\operatorname{ccc}([0]) \operatorname{ccc}$ & InChl=1S/C6H13O/c1-3-5-6(7)4-2/h6H,3-5H2,1-2H3 \\
\hline 1804 & $\mathrm{XC6OOH2-1}$ & $\mathrm{OOC}(\mathrm{C}(\mathrm{C}) \mathrm{C})([\mathrm{CH} 2]) \mathrm{C}$ & InChl=1S/C6H13O2/c1-5(2)6(3,4)8-7/h5,7H,3H2,1-2,4H3 \\
\hline 1805 & C6H12OH-2O2-3 & $\mathrm{CC}(\mathrm{O}) \mathrm{C}(\mathrm{O}[\mathrm{O}]) \mathrm{CCC}$ & $\mathrm{InChl}=1 \mathrm{~S} / \mathrm{C} 6 \mathrm{H} 13 \mathrm{O} / \mathrm{c} 1-3-4-6(9-8) 5(2) 7 / \mathrm{h} 5-7 \mathrm{H}, 3-4 \mathrm{H} 2,1-2 \mathrm{H} 3$ \\
\hline 1806 & IC6D14-1 & $\mathrm{C}=\mathrm{CCC}(=\mathrm{C})[\mathrm{CH} 2]$ & $\operatorname{lnChl}=15 / C 6 H 9 / c 1-4-5-6(2) 3 / h 4 H, 1-3,5 \mathrm{H} 2$ \\
\hline 1807 & $\mathrm{C} 6 \mathrm{H} 12 \mathrm{OH}-1 \mathrm{~J} 2$ & $\mathrm{C}(\mathrm{O})[\mathrm{CH}] \mathrm{CCCC}$ & $\operatorname{lnChl}=1 \mathrm{~S} / \mathrm{C} 6 \mathrm{H} 130 / \mathrm{c1} 1-2-3-4-5-6-7 / \mathrm{h} 5,7 \mathrm{H}, 2-4,6 \mathrm{H} 2,1 \mathrm{H} 3$ \\
\hline 1808 & $\mathrm{XC6OOH2-3}$ & $00 \mathrm{O}([\mathrm{C}](\mathrm{C}) \mathrm{C})(\mathrm{C}) \mathrm{C}$ & InChl=1S/C6H13O2/c1-5(2)6(3,4)8-7/h7H,1-4H3 \\
\hline 1809 & $\mathrm{C} 6 \mathrm{H} 12 \mathrm{OH}-3 \mathrm{O} 2-2$ & $\mathrm{CC}(\mathrm{O}[\mathrm{O}]) \mathrm{C}(\mathrm{O}) \mathrm{CCC}$ & InChl=1S/C6H13O3/c1-3-4-6(7)5(2)9-8/h5-7H,3-4H2,1-2H3 \\
\hline 1810 & XC6D13-1AR & $\mathrm{CC}(=\mathrm{C}) \mathrm{C}(=\mathrm{C})[\mathrm{CH} 2]$ & InChl=1S/C6H9/c1-5(2)6(3)4/h1-3H2,4H3 \\
\hline 1811 & $\mathrm{C} 6 \mathrm{H} 12 \mathrm{OH}-2 \mathrm{~J} 1$ & {$[\mathrm{CH} 2] \mathrm{C}(\mathrm{O}) \mathrm{CCCC}$} & InChl=1S/C6H130/c1-3-4-5-6(2)7/h6-7H,2-5H2,1H3 \\
\hline 1812 & $\mathrm{XC6OOH2-4}$ & $\mathrm{OOC}(\mathrm{C}([\mathrm{CH} 2]) \mathrm{C})(\mathrm{C}) \mathrm{C}$ & $\operatorname{lnChl}=1 \mathrm{~S} / \mathrm{C} 6 \mathrm{H} 13 \mathrm{O} 2 / \mathrm{c} 1-5(2) 6(3,4) 8-7 / \mathrm{h} 5,7 \mathrm{H}, 1 \mathrm{H} 2,2-4 \mathrm{H} 3$ \\
\hline 1813 & C6H12OH-3O2-4 & $\operatorname{ccc}(\mathrm{O}) \mathrm{C}(\mathrm{O}[\mathrm{O}]) \mathrm{CC}$ & InChl=1S/C6H13O3/c1-3-5(7)6(4-2)9-8/h5-7H,3-4H2,1-2H3 \\
\hline 1814 & IC6D13-4R & $\mathrm{C}=\mathrm{C}(\mathrm{C}) \mathrm{C}=[\mathrm{C}] \mathrm{C}$ & InChl=15/C6H9/c1-4-5-6(2)3/h5H,2H2,1,3H3 \\
\hline 1815 & $\mathrm{C} 6 \mathrm{H} 12 \mathrm{OH}-2 \mathrm{J3}$ & $\mathrm{CC}(\mathrm{O})[\mathrm{CH}] \mathrm{CCC}$ & $\operatorname{lnChl}=1 \mathrm{~S} / \mathrm{C} 6 \mathrm{H} 130 / \mathrm{c1} 1-3-4-5-6(2) 7 / \mathrm{h} 5-7 \mathrm{H}, 3-4 \mathrm{H} 2,1-2 \mathrm{H} 3$ \\
\hline 1816 & $13 \mathrm{C} 6 \mathrm{OOH} 1-2$ & $\mathrm{CC}(\mathrm{CC})[\mathrm{CH}] \mathrm{COO}$ & InChl=15/C6H1302/c1-3-6(2)4-5-8-7/h4,6-7H,3,5H2,1-2H3 \\
\hline
\end{tabular}




\begin{tabular}{|c|c|c|c|}
\hline 1817 & 13С-OH1-2O2 & $\mathrm{CCC}(\mathrm{C}(\mathrm{O}[\mathrm{O}]) \mathrm{CO}) \mathrm{C}$ & $\operatorname{lnChl}=1 \mathrm{~S} / \mathrm{C} 6 \mathrm{H} 13 \mathrm{O} 3 / \mathrm{c} 1-3-5(2) 6(4-7) 9-8 / \mathrm{h} 5-7 \mathrm{H}, 3-4 \mathrm{H} 2,1-2 \mathrm{H} 3$ \\
\hline 1818 & IC6D34-2R & $C[C](C) C=C=C$ & InChl=1S/C6H9/c1-4-5-6(2)3/h5H,1H2,2-3H3 \\
\hline 1819 & $\mathrm{C} 6 \mathrm{H} 12 \mathrm{OH}-3 \mathrm{~J} 2$ & $\mathrm{C}[\mathrm{CH}] \mathrm{C}(\mathrm{O}) \mathrm{CCC}$ & $\ln \mathrm{Chl}=1 \mathrm{~S} / \mathrm{C} 6 \mathrm{H} 13 \mathrm{O} / \mathrm{c1} 1-3-5-6(7) 4-2 / \mathrm{h} 4,6-7 \mathrm{H}, 3,5 \mathrm{H} 2,1-2 \mathrm{H} 3$ \\
\hline 1820 & 13С6OOH1-3 & $\mathrm{C}[\mathrm{C}](\mathrm{CC}) \mathrm{CCOO}$ & $\ln \mathrm{Chl}=1 \mathrm{~S} / \mathrm{C} 6 \mathrm{H} 13 \mathrm{O} 2 / \mathrm{c1}-3-6(2) 4-5-8-7 / \mathrm{h} 7 \mathrm{H}, 3-5 \mathrm{H} 2,1-2 \mathrm{H} 3$ \\
\hline 1821 & $13 \mathrm{C} 6 \mathrm{OH} 2-102$ & $\mathrm{CC}(\mathrm{C}(\mathrm{CO}[\mathrm{O}]) \mathrm{O}) \mathrm{CC}$ & $\mathrm{InChl}=1 \mathrm{~S} / \mathrm{C} 6 \mathrm{H} 13 \mathrm{O} / \mathrm{c} 1-3-5(2) 6(7) 4-9-8 / \mathrm{h} 5-7 \mathrm{H}, 3-4 \mathrm{H} 2,1-2 \mathrm{H} 3$ \\
\hline 1822 & IC6D13-1AR & $\mathrm{C}=\mathrm{C}([\mathrm{CH} 2]) \mathrm{C}=\mathrm{CC}$ & $\operatorname{lnChl}=1 \mathrm{~S} / \mathrm{C} 6 \mathrm{H} 9 / \mathrm{c} 1-4-5-6(2) 3 / \mathrm{h} 4-5 \mathrm{H}, 2-3 \mathrm{H} 2,1 \mathrm{H} 3$ \\
\hline 1823 & $\mathrm{C} 6 \mathrm{H} 12 \mathrm{OH}-3 \mathrm{J4}$ & $\mathrm{CCC}(\mathrm{O})[\mathrm{CH}] \mathrm{CC}$ & InChl=1S/C6H130/c1-3-5-6(7)4-2/h5-7H,3-4H2,1-2H3 \\
\hline 1824 & 13C6OOH1-4 & {$[\mathrm{CH} 2] \mathrm{C}(\mathrm{CC}) \mathrm{CCOO}$} & $\operatorname{lnChl}=15 / C 6 H 13 O 2 / c 1-3-6(2) 4-5-8-7 / h 6-7 \mathrm{H}, 2-5 \mathrm{H} 2,1 \mathrm{H} 3$ \\
\hline 1825 & $13 \mathrm{C} 6 \mathrm{OH} 2-302$ & $\operatorname{ccc}(\mathrm{C}(\mathrm{O}) \mathrm{C})(\mathrm{O}[\mathrm{O}]) \mathrm{C}$ & $\operatorname{lnChl}=1 \mathrm{~S} / \mathrm{C} 6 \mathrm{H} 13 \mathrm{O} 3 / \mathrm{c} 1-4-6(3,9-8) 5(2) 7 / \mathrm{h} 5,7 \mathrm{H}, 4 \mathrm{H} 2,1-3 \mathrm{H} 3$ \\
\hline 1826 & IC6D13-5R & $\mathrm{C}=\mathrm{C}(\mathrm{C}) \mathrm{C}=\mathrm{C}[\mathrm{CH} 2]$ & $\operatorname{lnChl}=1 \mathrm{~S} / \mathrm{C} 6 \mathrm{H} 9 / \mathrm{c1} 1-4-5-6(2) 3 / \mathrm{h} 4-5 \mathrm{H}, 1-2 \mathrm{H} 2,3 \mathrm{H} 3$ \\
\hline 1827 & $\mathrm{IC6OH1-2J}$ & $\mathrm{CCC}[\mathrm{C}](\mathrm{CO}) \mathrm{C}$ & InChl=1S/C6H130/c1-3-4-6(2)5-7/h7H,3-5H2,1-2H3 \\
\hline 1828 & 13C6OOH1-5 & $\mathrm{CC}([\mathrm{CH}] \mathrm{C}) \mathrm{CCOO}$ & InChl=1S/C6H13O2/c1-3-6(2)4-5-8-7/h3,6-7H,4-5H2,1-2H3 \\
\hline 1829 & 13C6OH3-202 & {$[0] \mathrm{OC}(\mathrm{C}(\mathrm{CC})(\mathrm{O}) \mathrm{C}) \mathrm{C}$} & InChl=15/C6H13O3/c1-4-6(3,7)5(2)9-8/h5,7H,4H2,1-3H3 \\
\hline 1830 & C6H101-5 & $\mathrm{C}=\mathrm{CCCC}=\mathrm{C}$ & InChl=15/C6H10/c1-3-5-6-4-2/h3-4H,1-2,5-6H2 \\
\hline 1831 & $\mathrm{IC} 6 \mathrm{OH} 2-1 \mathrm{~J}$ & $\operatorname{CCCC}(\mathrm{O})([\mathrm{CH} 2]) \mathrm{C}$ & $\operatorname{lnChl}=1 \mathrm{~S} / \mathrm{C} 6 \mathrm{H} 13 \mathrm{O} / \mathrm{c} 1-4-5-6(2,3) 7 / \mathrm{h} 7 \mathrm{H}, 2,4-5 \mathrm{H} 2,1,3 \mathrm{H} 3$ \\
\hline 1832 & 13С6OOH1-6 & $\mathrm{CC}(\mathrm{C}[\mathrm{CH} 2]) \mathrm{CCOO}$ & $\mathrm{InChl}=1 \mathrm{~S} / \mathrm{C} 6 \mathrm{H} 13 \mathrm{O} 2 / \mathrm{c} 1-3-6(2) 4-5-8-7 / \mathrm{h} 6-7 \mathrm{H}, 1,3-5 \mathrm{H} 2,2 \mathrm{H} 3$ \\
\hline 1833 & $13 \mathrm{C} 6 \mathrm{OH} 3-402$ & $\operatorname{ccc}(\mathrm{CO}[\mathrm{O}])(\mathrm{CC}) \mathrm{O}$ & $\mathrm{InChl}=1 \mathrm{~S} / \mathrm{C} 6 \mathrm{H} 13 \mathrm{O} 3 / \mathrm{c} 1-3-6(7,4-2) 5-9-8 / \mathrm{h} 7 \mathrm{H}, 3-5 \mathrm{H} 2,1-2 \mathrm{H} 3$ \\
\hline 1834 & $\mathrm{C} 6 \mathrm{H} 101-3,3$ & $\mathrm{C}=\mathrm{CC}(\mathrm{C})=\mathrm{CC}$ & $\operatorname{lnChl}=15 / C 6 H 10 / c 1-4-6(3) 5-2 / h 4-5 H, 1 H 2,2-3 \mathrm{H} 3$ \\
\hline 1835 & $\mathrm{XC6OH} 1-2 \mathrm{~J}$ & $\mathrm{OC}[\mathrm{C}](\mathrm{C}(\mathrm{C}) \mathrm{C}) \mathrm{C}$ & InChl=1S/C6H130/c1-5(2)6(3)4-7/h5,7H,4H2,1-3H3 \\
\hline 1836 & $13 \mathrm{C} 6 \mathrm{OOH} 2-1$ & $\mathrm{CCC}(\mathrm{C}(\mathrm{OO})[\mathrm{CH} 2]) \mathrm{C}$ & $\operatorname{lnChl}=15 / \mathrm{C}^{2} \mathrm{H} 13 \mathrm{O} 2 / \mathrm{c} 1-4-5(2) 6(3) 8-7 / \mathrm{h5} 5-7 \mathrm{H}, 3-4 \mathrm{H} 2,1-2 \mathrm{H} 3$ \\
\hline 1837 & $13 \mathrm{C} 6 \mathrm{OH} 4-302$ & $\mathrm{CCC}(\mathrm{O}[\mathrm{O}])(\mathrm{CO}) \mathrm{CC}$ & $\operatorname{lnChl}=15 / \mathrm{CH}_{13} \mathrm{O} / \mathrm{c} 1-3-6(4-2,5-7) 9-8 / \mathrm{h} 7 \mathrm{H}, 3-5 \mathrm{H} 2,1-2 \mathrm{H} 3$ \\
\hline 1838 & C6H10D14 & $\mathrm{C} / \mathrm{C}=\mathrm{C} / \mathrm{CC}=\mathrm{C}$ & InChl=1S/C6H10/c1-3-5-6-4-2/h3-4,6H,1,5H2,2H3 \\
\hline 1839 & $\mathrm{XC6OH2-1J}$ & $\mathrm{CC}(\mathrm{C}(\mathrm{O})([\mathrm{CH} 2]) \mathrm{C}) \mathrm{C}$ & $\operatorname{lnChl}=1 \mathrm{~S} / \mathrm{C} 6 \mathrm{H} 130 / \mathrm{c} 1-5(2) 6(3,4) 7 / \mathrm{h} 5,7 \mathrm{H}, 3 \mathrm{H} 2,1-2,4 \mathrm{H} 3$ \\
\hline 1840 & $13 \mathrm{C} 6 \mathrm{OOH} 2-3$ & $\mathrm{CC}[\mathrm{C}](\mathrm{C}(\mathrm{OO}) \mathrm{C}) \mathrm{C}$ & $\operatorname{lnChl}=15 / C 6 H 13 O 2 / c 1-4-5(2) 6(3) 8-7 / h 6-7 \mathrm{H}, 4 \mathrm{H} 2,1-3 \mathrm{H} 3$ \\
\hline 1841 & $\mathrm{IC} 6 \mathrm{OH} 1-2 \mathrm{O} 2$ & $\operatorname{cccC}(\mathrm{O}[\mathrm{O}])(\mathrm{CO}) \mathrm{C}$ & $\mathrm{InChl}=1 \mathrm{~S} / \mathrm{C} 6 \mathrm{H} 13 \mathrm{O} 3 / \mathrm{c} 1-3-4-6(2,5-7) 9-8 / \mathrm{h} 7 \mathrm{H}, 3-5 \mathrm{H} 2,1-2 \mathrm{H} 3$ \\
\hline 1842 & $\mathrm{C} 6 \mathrm{H} 10 \mathrm{D} 13$ & $\mathrm{C}=\mathrm{CC}=\mathrm{CCC}$ & InChl=1S/C6H10/c1-3-5-6-4-2/h3,5-6H,1,4H2,2H3 \\
\hline 1843 & $\mathrm{XC6OH} 2-3 \mathrm{~J}$ & $C[C](C(O)(C) C) C$ & $\operatorname{lnChl}=15 / \mathrm{C} 6 \mathrm{H} 13 \mathrm{O} / \mathrm{c} 1-5(2) 6(3,4) 7 / \mathrm{h} 7 \mathrm{H}, 1-4 \mathrm{H} 3$ \\
\hline 1844 & 13С60OH2-4 & $\operatorname{cCC}(\mathrm{C}(\mathrm{OO}) \mathrm{C})[\mathrm{CH} 2]$ & $\operatorname{lnChl}=1 \mathrm{~S} / \mathrm{C} 6 \mathrm{H} 13 \mathrm{O} 2 / \mathrm{c} 1-4-5(2) 6(3) 8-7 / \mathrm{h} 5-7 \mathrm{H}, 2,4 \mathrm{H} 2,1,3 \mathrm{H} 3$ \\
\hline 1845 & $\mathrm{IC} 6 \mathrm{OH} 2-102$ & $\operatorname{cccc}(\operatorname{co}[0])(0) \mathrm{C}$ & $\mathrm{InChl}=1 \mathrm{~S} / \mathrm{C} 6 \mathrm{H} 13 \mathrm{O} 3 / \mathrm{c} 1-3-4-6(2,7) 5-9-8 / \mathrm{h} 7 \mathrm{H}, 3-5 \mathrm{H} 2,1-2 \mathrm{H} 3$ \\
\hline 1846 & $\mathrm{C} 6 \mathrm{H} 10 \mathrm{D} 24$ & $\mathrm{CC}=\mathrm{CC}=\mathrm{CC}$ & InChl=15/C6H10/c1-3-5-6-4-2/h3-6H,1-2H3 \\
\hline 1847 & $13 \mathrm{C} 6 \mathrm{OH} 1-2 \mathrm{~J}$ & $\mathrm{OC}[\mathrm{CH}] \mathrm{C}(\mathrm{CC}) \mathrm{C}$ & $\operatorname{lnChl}=1 \mathrm{~S} / \mathrm{C} 6 \mathrm{H} 13 \mathrm{O} / \mathrm{c1} 1-3-6(2) 4-5-7 / \mathrm{h} 4,6-7 \mathrm{H}, 3,5 \mathrm{H} 2,1-2 \mathrm{H} 3$ \\
\hline 1848 & $13 \mathrm{C} 6 \mathrm{OOH} 2-5$ & $\mathrm{C}[\mathrm{CH}] \mathrm{C}(\mathrm{C}(\mathrm{OO}) \mathrm{C}) \mathrm{C}$ & $\ln \mathrm{Chl}=1 \mathrm{~S} / \mathrm{C} 6 \mathrm{H} 13 \mathrm{O} 2 / \mathrm{c} 1-4-5(2) 6(3) 8-7 / \mathrm{h} 4-7 \mathrm{H}, 1-3 \mathrm{H} 3$ \\
\hline 1849 & $\mathrm{XC6OH1-202}$ & $\mathrm{OCC}(\mathrm{C}(\mathrm{C}) \mathrm{C})(\mathrm{O}[\mathrm{O}]) \mathrm{C}$ & $\operatorname{lnChl}=1 \mathrm{~S} / \mathrm{C} 6 \mathrm{H} 13 \mathrm{O} 3 / \mathrm{c} 1-5(2) 6(3,4-7) 9-8 / \mathrm{h} 5,7 \mathrm{H}, 4 \mathrm{H} 2,1-3 \mathrm{H} 3$ \\
\hline 1850 & CYCHEXENE & $\mathrm{C} 1 \mathrm{CCC}=\mathrm{CC} 1$ & InChl=1S/C6H10/c1-2-4-6-5-3-1/h1-2H,3-6H2 \\
\hline 1851 & $13 \mathrm{C} 6 \mathrm{OH} 2-1 \mathrm{~J}$ & $\operatorname{CCC}(\mathrm{C}(\mathrm{O})[\mathrm{CH} 2]) \mathrm{C}$ & $\operatorname{lnChl}=1 \mathrm{~S} / \mathrm{C} 6 \mathrm{H} 13 \mathrm{O} / \mathrm{c} 1-4-5(2) 6(3) 7 / \mathrm{h} 5-7 \mathrm{H}, 3-4 \mathrm{H} 2,1-2 \mathrm{H} 3$ \\
\hline 1852 & NEC6-102 & $\operatorname{ccc}(\mathrm{CO}[\mathrm{O}])(\mathrm{C}) \mathrm{C}$ & InChl=1S/C6H13O2/c1-4-6(2,3)5-8-7/h4-5H2,1-3H3 \\
\hline 1853 & $\mathrm{XC6OH2-102}$ & {$[\mathrm{O}] \mathrm{OCC}(\mathrm{C}(\mathrm{C}) \mathrm{C})(\mathrm{O}) \mathrm{C}$} & $\operatorname{lnChl}=1 \mathrm{~S} / \mathrm{C} 6 \mathrm{H} 13 \mathrm{O} 3 / \mathrm{c} 1-5(2) 6(3,7) 4-9-8 / \mathrm{h} 5,7 \mathrm{H}, 4 \mathrm{H} 2,1-3 \mathrm{H} 3$ \\
\hline 1854 & 13C6D13 & $\operatorname{ccC}(=C) \mathrm{C}=\mathrm{C}$ & InChl=15/C6H10/c1-4-6(3)5-2/h4H,1,3,5H2,2H3 \\
\hline 1855 & I3C6OH2-3Ј & $\mathrm{CC}[\mathrm{C}](\mathrm{C}(\mathrm{O}) \mathrm{C}) \mathrm{C}$ & InChl=1S/C6H130/c1-4-5(2)6(3)7/h6-7H,4H2,1-3H3 \\
\hline 1856 & NEC6-302 & {$[\mathrm{O}] \mathrm{OC}(\mathrm{C}(\mathrm{C})(\mathrm{C}) \mathrm{C}) \mathrm{C}$} & $\operatorname{lnChl}=15 / C 6 H 13 O 2 / c 1-5(8-7) 6(2,3) 4 / h 5 H, 1-4 \mathrm{H} 3$ \\
\hline 1857 & $\mathrm{XC6OH2-302}$ & {$[\mathrm{O}] \mathrm{OC}(\mathrm{C}(\mathrm{O})(\mathrm{C}) \mathrm{C})(\mathrm{C}) \mathrm{C}$} & InChl=1S/C6H13O3/c1-5(2,7)6(3,4)9-8/h7H,1-4H3 \\
\hline 1858 & 13C6D15 & $\mathrm{C}=\mathrm{CC}(\mathrm{C}=\mathrm{C}) \mathrm{C}$ & InChl=15/C6H10/c1-4-6(3)5-2/h4-6H,1-2H2,3H3 \\
\hline 1859 & $13 \mathrm{C} 6 \mathrm{OH} 3-2 \mathrm{~J}$ & $\mathrm{C}[\mathrm{CH}] \mathrm{C}(\mathrm{CC})(\mathrm{O}) \mathrm{C}$ & $\operatorname{lnChl}=15 / \mathrm{C} 6 \mathrm{H} 13 \mathrm{O} / \mathrm{c1} 1-4-6(3,7) 5-2 / \mathrm{h} 4,7 \mathrm{H}, 5 \mathrm{H} 2,1-3 \mathrm{H} 3$ \\
\hline 1860 & $13 \mathrm{C6} 6-102$ & $\mathrm{CC}(\mathrm{CC}) \mathrm{CCO}[\mathrm{O}]$ & $\operatorname{lnChl}=1 \mathrm{~S} / \mathrm{C} 6 \mathrm{H} 13 \mathrm{O} 2 / \mathrm{c1}-3-6(2) 4-5-8-7 / \mathrm{h} 6 \mathrm{H}, 3-5 \mathrm{H} 2,1-2 \mathrm{H} 3$ \\
\hline 1861 & $\mathrm{IC6OH2-302R}$ & $\mathrm{CC}(\mathrm{O})(\mathrm{C}) \mathrm{C}(\mathrm{O}[\mathrm{O}]) \mathrm{CC}$ & InChl=1S/C6H13O3/c1-4-5(9-8)6(2,3)7/h5,7H,4H2,1-3H3 \\
\hline 1862 & XC6D13 & $\mathrm{CC}(=\mathrm{C}) \mathrm{C}(=\mathrm{C}) \mathrm{C}$ & $\operatorname{lnChl}=1 \mathrm{~S} / \mathrm{C} 6 \mathrm{H} 10 / \mathrm{c} 1-5(2) 6(3) 4 / \mathrm{h} 1,3 \mathrm{H} 2,2,4 \mathrm{H} 3$ \\
\hline 1863 & $13 \mathrm{C} 6 \mathrm{OH} 3-4 \mathrm{~J}$ & $\mathrm{CCC}(\mathrm{CC})(\mathrm{O})[\mathrm{CH} 2]$ & $\operatorname{lnChl}=1 \mathrm{~S} / \mathrm{C} 6 \mathrm{H} 13 \mathrm{O} / \mathrm{c} 1-4-6(3,7) 5-2 / \mathrm{h} 7 \mathrm{H}, 3-5 \mathrm{H} 2,1-2 \mathrm{H} 3$ \\
\hline 1864 & NEC6-402 & {$[0] \operatorname{OCCC}(\mathrm{C})(\mathrm{C}) \mathrm{C}$} & InChl=1S/C6H13O2/c1-6(2,3)4-5-8-7/h4-5H2,1-3H3 \\
\hline 1865 & $\mathrm{IC6OH} 3-2 \mathrm{O} 2 \mathrm{R}$ & $\mathrm{CC}(\mathrm{O}[\mathrm{O}])(\mathrm{C}) \mathrm{C}(\mathrm{O}) \mathrm{CC}$ & InChl=15/C6H13O3/c1-4-5(7)6(2,3)9-8/h5,7H,4H2,1-3H3 \\
\hline 1866 & IC6D14 & $\mathrm{C}=\mathrm{CCC}(=\mathrm{C}) \mathrm{C}$ & InChl=15/C6H10/c1-4-5-6(2)3/h4H,1-2,5H2,3H3 \\
\hline 1867 & I3C6OH4-3J & $\mathrm{CC}[\mathrm{C}](\mathrm{CO}) \mathrm{CC}$ & InChl=15/C6H130/c1-3-6(4-2)5-7/h7H,3-5H2,1-2H3 \\
\hline 1868 & 13C6-202 & $\operatorname{ccc}(\mathrm{C}(\mathrm{O}[\mathrm{O}]) \mathrm{C}) \mathrm{C}$ & $\ln \mathrm{Chl}=1 \mathrm{~S} / \mathrm{C} 6 \mathrm{H} 13 \mathrm{O} 2 / \mathrm{c} 1-4-5(2) 6(3) 8-7 / \mathrm{h} 5-6 \mathrm{H}, 4 \mathrm{H} 2,1-3 \mathrm{H} 3$ \\
\hline 1869 & $\mathrm{IC} 6 \mathrm{OH} 3-4 \mathrm{O} 2 \mathrm{R}$ & $\mathrm{CC}(\mathrm{C}) \mathrm{C}(\mathrm{O}) \mathrm{C}(\mathrm{O}[\mathrm{O}]) \mathrm{C}$ & InChl=15/C6H1303/c1-4(2)6(7)5(3)9-8/h4-7H,1-3H3 \\
\hline 1870 & IC6D13 & $\mathrm{C}=\mathrm{C}(\mathrm{C}) \mathrm{C}=\mathrm{CC}$ & InChl=15/C6H10/c1-4-5-6(2)3/h4-5H,2H2,1,3H3 \\
\hline 1871 & NEC6-3O & $\mathrm{CC}(\mathrm{C}(\mathrm{C})(\mathrm{C}) \mathrm{C})[\mathrm{O}]$ & $\operatorname{lnChl}=1 \mathrm{~S} / \mathrm{C} 6 \mathrm{H} 13 \mathrm{O} / \mathrm{c} 1-5(7) 6(2,3) 4 / \mathrm{h} 5 \mathrm{H}, 1-4 \mathrm{H} 3$ \\
\hline 1872 & $13 \mathrm{C6} 6-302$ & $\mathrm{CCC}(\mathrm{O}[\mathrm{O}])(\mathrm{CC}) \mathrm{C}$ & InChl=1S/C6H13O2/c1-4-6(3,5-2)8-7/h4-5H2,1-3H3 \\
\hline 1873 & $\mathrm{IC6OH4-302R}$ & $\mathrm{CC}(\mathrm{C}) \mathrm{C}(\mathrm{O}[\mathrm{O}]) \mathrm{C}(\mathrm{O}) \mathrm{C}$ & InChl=1S/C6H1303/c1-4(2)6(9-8)5(3)7/h4-7H,1-3H3 \\
\hline 1874 & IC6D24 & $\mathrm{CC}(\mathrm{C})=\mathrm{CC}=\mathrm{C}$ & $\operatorname{lnChl}=15 / C 6 H 10 / c 1-4-5-6(2) 3 / h 4-5 \mathrm{H}, 1 \mathrm{H} 2,2-3 \mathrm{H} 3$ \\
\hline 1875 & $13 \mathrm{C6}-10$ & {$[\mathrm{O}] \mathrm{CCC}(\mathrm{CC}) \mathrm{C}$} & $\operatorname{lnChl}=1 \mathrm{~S} / \mathrm{C} 6 \mathrm{H} 13 \mathrm{O} / \mathrm{c} 1-3-6(2) 4-5-7 / \mathrm{h} 6 \mathrm{H}, 3-5 \mathrm{H} 2,1-2 \mathrm{H} 3$ \\
\hline 1876 & $13 \mathrm{C} 6-402$ & $\operatorname{cCC}(\mathrm{CO}[\mathrm{O}]) \mathrm{CC}$ & $\ln \mathrm{Chl}=1 \mathrm{~S} / \mathrm{C} 6 \mathrm{H} 13 \mathrm{O} 2 / \mathrm{c1}-3-6 \mathrm{-6}(4-2) 5-8-7 / \mathrm{h} 6 \mathrm{H}, 3-5 \mathrm{H} 2,1-2 \mathrm{H} 3$ \\
\hline 1877 & $\mathrm{IC} 6 \mathrm{OH} 4-5 \mathrm{O} 2 \mathrm{R}$ & $\mathrm{CC}(\mathrm{C}) \mathrm{CC}(\mathrm{O}) \mathrm{CO}[\mathrm{O}]$ & InChl=1S/C6H13O3/c1-5(2)3-6(7)4-9-8/h5-7H,3-4H2,1-2H3 \\
\hline 1878 & C6H111-3 & $\mathrm{C}=\mathrm{C}[\mathrm{CH}] \mathrm{CCC}$ & InChl=15/C6H11/c1-3-5-6-4-2/h3,5H,1,4,6H2,2H3 \\
\hline 1879 & NEC6-4O & {$[\mathrm{O}] \operatorname{ccc}(\mathrm{C})(\mathrm{C}) \mathrm{C}$} & $\ln \mathrm{Chl}=15 / \mathrm{C} 6 \mathrm{H} 13 \mathrm{O} / \mathrm{c1}-6(2,3) 4-5-7 / \mathrm{h} 4-5 \mathrm{H} 2,1-3 \mathrm{H} 3$ \\
\hline 1880 & $\mathrm{x} 66-102$ & {$[0] \operatorname{Occ}(\mathrm{C}(\mathrm{C}) \mathrm{C}) \mathrm{C}$} & $\ln \mathrm{Chl}=1 \mathrm{~S} / \mathrm{C} 6 \mathrm{H} 13 \mathrm{O} 2 / \mathrm{c} 1-5(2) 6(3) 4-8-7 / \mathrm{h} 5-6 \mathrm{H}, 4 \mathrm{H} 2,1-3 \mathrm{H} 3$ \\
\hline 1881 & IC6OH5-402R & $\mathrm{CC}(\mathrm{C}) \mathrm{CC}(\mathrm{O}[\mathrm{O}]) \mathrm{CO}$ & InChl=1S/C6H13O3/c1-5(2)3-6(4-7)9-8/h5-7H,3-4H2,1-2H3 \\
\hline 1882 & C6H111-4 & $\mathrm{C}=\mathrm{CC}[\mathrm{CH}] \mathrm{CC}$ & $\operatorname{lnChl}=1 \mathrm{~S} / \mathrm{C} 6 \mathrm{H} 11 / \mathrm{cc} 1-3-5-6-4-2 / \mathrm{h} 3,6 \mathrm{H}, 1,4-5 \mathrm{H} 2,2 \mathrm{H} 3$ \\
\hline 1883 & $13 \mathrm{C} 6-20$ & $\operatorname{ccc}(C([0])) C$ & $\operatorname{lnChl}=1 \mathrm{~S} / \mathrm{C} 6 \mathrm{H} 13 \mathrm{O} / \mathrm{c} 1-4-5(2) 6(3) 7 / \mathrm{h} 5-6 \mathrm{H}, 4 \mathrm{H} 2,1-3 \mathrm{H} 3$ \\
\hline 1884 & IC6-102 & $\operatorname{cccc}(\operatorname{co}[0]) \mathrm{C}$ & $\operatorname{lnChl}=15 / C 6 H 13 O 2 / c 1-3-4-6(2) 5-8-7 / h 6 H, 3-5 \mathrm{H} 2,1-2 \mathrm{H} 3$ \\
\hline 1885 & $\mathrm{NEC6OH3-402R}$ & $\mathrm{CC}(\mathrm{C})(\mathrm{C}) \mathrm{C}(\mathrm{O}) \mathrm{CO}[\mathrm{O}]$ & InChl=15/C6H13O3/c1-6(2,3)5(7)4-9-8/h5,7H,4H2,1-3H3 \\
\hline 1886 & C6H111-5 & $\mathrm{C}=\mathrm{CCC}[\mathrm{CH}] \mathrm{C}$ & InChl=1S/C6H11/c1-3-5-6-4-2/h3-4H,1,5-6H2,2H3 \\
\hline 1887 & $13 \mathrm{C} 6-30$ & $\operatorname{ccc}(\operatorname{CC})([\mathrm{O}]) \mathrm{C}$ & $\ln \mathrm{Chl}=1 \mathrm{~S} / \mathrm{C} 6 \mathrm{H} 13 \mathrm{O} / \mathrm{c} 1-4-6(3,7) 5-2 / \mathrm{h} 4-5 \mathrm{H} 2,1-3 \mathrm{H} 3$ \\
\hline 1888 & $\mathrm{xC6-202}$ & {$[0] \mathrm{OC}(\mathrm{C}(\mathrm{C}) \mathrm{C})(\mathrm{C}) \mathrm{C}$} & $\ln \mathrm{Chl}=1 \mathrm{~S} / \mathrm{C} 6 \mathrm{H} 13 \mathrm{O} 2 / \mathrm{c} 1-5(2) 6(3,4) 8-7 / \mathrm{h} 5 \mathrm{H}, 1-4 \mathrm{H} 3$ \\
\hline 1889 & $\mathrm{NEC6OH4-302R}$ & $\mathrm{CC}(\mathrm{C})(\mathrm{C}) \mathrm{C}(\mathrm{O}[\mathrm{O}]) \mathrm{CO}$ & $\operatorname{lnChl}=1 \mathrm{~S} / \mathrm{C} 6 \mathrm{H} 13 \mathrm{O} 3 / \mathrm{c} 1-6(2,3) 5(4-7) 9-8 / \mathrm{h} 5,7 \mathrm{H}, 4 \mathrm{H} 2,1-3 \mathrm{H} 3$ \\
\hline 1890 & C6H111-6 & $\mathrm{C}=\mathrm{CCCC}[\mathrm{CH} 2]$ & InChl=15/C6H11/c1-3-5-6-4-2/h3H,1-2,4-6H2 \\
\hline 1891 & $13 \mathrm{C} 6-40$ & $\operatorname{ccc}(\mathrm{C}[\mathrm{O}]) \mathrm{CC}$ & InChl=1S/C6H130/c1-3-6(4-2)5-7/h6H,3-5H2,1-2H3 \\
\hline 1892 & IC6-202 & $\operatorname{cccc}(\mathrm{O}[\mathrm{O}])(\mathrm{C}) \mathrm{C}$ & InChI=15/C6H13O2/c1-4-5-6(2,3)8-7/h4-5H2,1-3H3 \\
\hline 1893 & $\mathrm{C} 6 \mathrm{H} 1010 \mathrm{OH} 3-4 \mathrm{O} 2$ & $\mathrm{CCC}(\mathrm{O}[\mathrm{O}]) \mathrm{C}(\mathrm{OO}) \mathrm{C}=\mathrm{C}$ & $\operatorname{lnChl}=1 \mathrm{~S} / \mathrm{C} 6 \mathrm{H} 1104 / \mathrm{c1}-3-5(9-7) 6(4-2) 10-8 / \mathrm{h} 3,5-7 \mathrm{H}, 1,4 \mathrm{H} 2,2 \mathrm{H} 3$ \\
\hline 1894 & C6H112-4 & $\mathrm{CC}=\mathrm{C}[\mathrm{CH}] \mathrm{CC}$ & $\operatorname{lnChl}=1 \mathrm{~S} / \mathrm{C} 6 \mathrm{H} 11 / \mathrm{c} 1-3-5-6-4-2 / \mathrm{h} 3,5-6 \mathrm{H}, 4 \mathrm{H} 2,1-2 \mathrm{H} 3$ \\
\hline 1895 & $\mathrm{xC6-10}$ & {$[\mathrm{O}] \mathrm{CC}(\mathrm{C}(\mathrm{C}) \mathrm{C}) \mathrm{C}$} & $\operatorname{lnChl}=1 \mathrm{~S} / \mathrm{C} 6 \mathrm{H} 13 \mathrm{O} / \mathrm{c} 1-5(2) 6(3) 4-7 / \mathrm{h} 5-6 \mathrm{H}, 4 \mathrm{H} 2,1-3 \mathrm{H} 3$ \\
\hline 1896 & IC6-302 & $\operatorname{ccc}(\mathrm{C}(\mathrm{C}) \mathrm{C}) \mathrm{O}[\mathrm{O}]$ & $\operatorname{lnChl}=15 / C 6 H 13 O 2 / c 1-4-6(8-7) 5(2) 3 / h 5-6 \mathrm{H}, 4 \mathrm{H} 2,1-3 \mathrm{H} 3$ \\
\hline 1897 & $\mathrm{C} 6 \mathrm{H} 1010 \mathrm{OH} 3-5 \mathrm{O} 2$ & $\mathrm{CC}(\mathrm{O}[\mathrm{O}]) \mathrm{CC}(\mathrm{OO}) \mathrm{C}=\mathrm{C}$ & $\operatorname{lnChl}=1 \mathrm{~S} / \mathrm{C} 6 \mathrm{H} 11 \mathrm{O} 4 / \mathrm{c} 1-3-6(10-8) 4-5(2) 9-7 / \mathrm{h} 3,5-6,8 \mathrm{H}, 1,4 \mathrm{H} 2,2 \mathrm{H} 3$ \\
\hline 1898 & C6H112-5 & $\mathrm{CC}=\mathrm{CC}[\mathrm{CH}] \mathrm{C}$ & $\operatorname{lnChl}=15 / C 6 H 11 / c 1-3-5-6-4-2 / h 3-5 \mathrm{H}, 6 \mathrm{H} 2,1-2 \mathrm{H} 3$ \\
\hline 1899 & IC6-10 & $\operatorname{cccc}(\mathrm{C}[0]) \mathrm{C}$ & InChl=1S/C6H130/c1-3-4-6(2)5-7/h6H,3-5H2,1-2H3 \\
\hline 1900 & IC6-402 & {$[0] \mathrm{OC}(\mathrm{CC}(\mathrm{C}) \mathrm{C}) \mathrm{C}$} & $\operatorname{lnChl}=1 \mathrm{~S} / \mathrm{C} 6 \mathrm{H} 13 \mathrm{O} 2 / \mathrm{c} 1-5(2) 4-6(3) 8-7 / \mathrm{h} 5-6 \mathrm{H}, 4 \mathrm{H} 2,1-3 \mathrm{H} 3$ \\
\hline 1901 & C6H1010OH3-602 & $\mathrm{C}(\mathrm{O}[\mathrm{O}]) \mathrm{CCC}(\mathrm{OO}) \mathrm{C}=\mathrm{C}$ & InChl=1S/C6H1104/c1-2-6(10-8)4-3-5-9-7/h2,6,8H,1,3-5H2 \\
\hline 1902 & C6H112-6 & $\mathrm{CC}=\mathrm{CCC}[\mathrm{CH} 2]$ & InChl=15/C6H11/c1-3-5-6-4-2/h4,6H,1,3,5H2,2H3 \\
\hline 1903 & $\mathrm{xC6}-2 \mathrm{O}$ & $\operatorname{CC}(C([0])(C) C) C$ & InChl=15/C6H13O/c1-5(2)6(3,4)7/h5H,1-4H3 \\
\hline 1904 & IC6-502 & {$[0] \operatorname{Occcc}(\mathrm{C}) \mathrm{C}$} & InChl=1S/C6H13O2/c1-6(2)4-3-5-8-7/h6H,3-5H2,1-2H3 \\
\hline 1905 & $\mathrm{C} 6 \mathrm{H} 1010 \mathrm{OH} 4-3 \mathrm{O} 2$ & $\mathrm{CCC}(\mathrm{OO}) \mathrm{C}(\mathrm{O}[\mathrm{O}]) \mathrm{C}=\mathrm{C}$ & InChl=1S/C6H11O4/c1-3-5(9-7)6(4-2)10-8/h3,5-6,8H,1,4H2,2H3 \\
\hline 1906 & C6H113-1 & $\mathrm{CCC}=\mathrm{CC}[\mathrm{CH} 2]$ & InChl=1S/C6H11/c1-3-5-6-4-2/h5-6H,1,3-4H2,2H3 \\
\hline 1907 & IC6-2O & $\operatorname{cCcC}([0])(C) C$ & $\operatorname{lnChl}=1 \mathrm{~S} / \mathrm{C} 6 \mathrm{H} 13 \mathrm{O} / \mathrm{c1}-4-5-6(2,3) 7 / \mathrm{h} 4-5 \mathrm{H} 2,1-3 \mathrm{H} 3$ \\
\hline
\end{tabular}




\begin{tabular}{|c|c|c|c|}
\hline 1908 & C6H130OH-1 & $\mathrm{C}(\mathrm{OO}) \mathrm{CCCCC}$ & $\operatorname{lnChl}=15 / \mathrm{CH} 1402 / \mathrm{c1}-2-3-4-5-6-8-7 / \mathrm{h} 7 \mathrm{H}, 2-6 \mathrm{H} 2,1 \mathrm{H} 3$ \\
\hline 1909 & $\mathrm{C} 6 \mathrm{H} 1010 \mathrm{OH} 4-502$ & $\mathrm{CC}(\mathrm{O}[\mathrm{O}]) \mathrm{C}(\mathrm{OO}) \mathrm{CC}=\mathrm{C}$ & InChl=1S/C6H1104/c1-3-4-6(10-8)5(2)9-7/h3,5-6,8H,1,4H2,2H3 \\
\hline 1910 & CHXRAD & $\mathrm{C} 1[\mathrm{CH}] \mathrm{CCCC} 1$ & InChl=1S/C6H11/c1-2-4-6-5-3-1/h1H,2-6H2 \\
\hline 1911 & IC6-30 & $\operatorname{ccc}(C(C) C)[0]$ & InChl=1S/C6H130/c1-4-6(7)5(2)3/h5-6H,4H2,1-3H3 \\
\hline 1912 & $\mathrm{C} 6 \mathrm{H} 13 \mathrm{OOH}-2$ & $\mathrm{CC}(\mathrm{OO}) \mathrm{CCCC}$ & $\mathrm{InChl}=1 \mathrm{~S} / \mathrm{C} 6 \mathrm{H} 14 \mathrm{O} 2 / \mathrm{c} 1-3-4-5-6(2) 8-7 / \mathrm{h} 6-7 \mathrm{H}, 3-5 \mathrm{H} 2,1-2 \mathrm{H} 3$ \\
\hline 1913 & $\mathrm{C} 6 \mathrm{H} 1010 \mathrm{OH} 4-6 \mathrm{O} 2$ & $\mathrm{C}(\mathrm{O}[\mathrm{O}]) \mathrm{CC}(\mathrm{OO}) \mathrm{CC}=\mathrm{C}$ & $\operatorname{lnChl}=1 \mathrm{~S} / \mathrm{C} 6 \mathrm{H} 1104 / \mathrm{c} 1-2-3-6(10-8) 4-5-9-7 / \mathrm{h} 2,6,8 \mathrm{H}, 1,3-5 \mathrm{H} 2$ \\
\hline 1914 & 13C6D1-3 & $\mathrm{C}[\mathrm{C}](\mathrm{C}=\mathrm{C}) \mathrm{CC}$ & $\operatorname{lnChl}=15 / \mathrm{C} 6 \mathrm{H} 11 / \mathrm{c} 1-4-6(3) 5-2 / \mathrm{h} 4 \mathrm{H}, 1,5 \mathrm{H} 2,2-3 \mathrm{H} 3$ \\
\hline 1915 & IC6-40 & $\mathrm{CC}(\mathrm{CC}([0]) \mathrm{C}) \mathrm{C}$ & InChl=1S/C6H13O/c1-5(2)4-6(3)7//h5-6H,4H2,1-3H3 \\
\hline 1916 & $\mathrm{C} 6 \mathrm{H} 130 \mathrm{OH}-3$ & $\mathrm{CCC}(\mathrm{OO}) \mathrm{CCC}$ & $\operatorname{lnChl}=15 / \mathrm{C} 6 \mathrm{H} 14 \mathrm{O} 2 / \mathrm{c} 1-3-5-6(4-2) 8-7 / \mathrm{h} 6-7 \mathrm{H}, 3-5 \mathrm{H} 2,1-2 \mathrm{H} 3$ \\
\hline 1917 & $\mathrm{C} 6 \mathrm{H} 1010 \mathrm{OH} 5-3 \mathrm{O} 2$ & $\mathrm{CC}(\mathrm{OO}) \mathrm{CC}(\mathrm{O}[\mathrm{O}]) \mathrm{C}=\mathrm{C}$ & InChl=15/C6H11O4/c1-3-6(10-8)4-5(2)9-7/h3,5-7H,1,4H2,2H3 \\
\hline 1918 & 13C6D1-4 & {$[\mathrm{CH} 2] \mathrm{C}(\mathrm{C}=\mathrm{C}) \mathrm{CC}$} & InChl=15/C6H11/c1-4-6(3)5-2/h4,6H,1,3,5H2,2H3 \\
\hline 1919 & IC6-50 & {$[0] \operatorname{cccc}(\mathrm{C}) \mathrm{C}$} & InChl=1S/C6H130/c1-6(2)4-3-5-7/h6H,3-5H2,1-2H3 \\
\hline 1920 & NEC6-10OH & $\operatorname{ccc}(\mathrm{COO})(\mathrm{C}) \mathrm{C}$ & InChl=15/C6H14O2/c1-4-6(2,3)5-8-7/h7H,4-5H2,1-3H3 \\
\hline 1921 & C6H1010OH5-402 & $\mathrm{CC}(\mathrm{OO}) \mathrm{C}(\mathrm{O}[\mathrm{O}]) \mathrm{CC}=\mathrm{C}$ & InChl=15/C6H1104/c1-3-4-6(10-8)5(2)9-7//h3,5-7H,1,4H2,2H3 \\
\hline 1922 & 13C6D1-5 & $\mathrm{CC}(\mathrm{C}=\mathrm{C})[\mathrm{CH}] \mathrm{C}$ & $\operatorname{lnChl}=15 / C 6 H 11 / c 1-4-6(3) 5-2 / h 4-6 \mathrm{H}, 1 \mathrm{H} 2,2-3 \mathrm{H} 3$ \\
\hline 1923 & NEC6-10 & $\operatorname{ccc}(\mathrm{C}[\mathrm{O}])(\mathrm{C}) \mathrm{C}$ & $\operatorname{lnChl}=1 \mathrm{~S} / \mathrm{C} 6 \mathrm{H} 13 \mathrm{O} / \mathrm{c} 1-4-6(2,3) 5-7 / \mathrm{h} 4-5 \mathrm{H} 2,1-3 \mathrm{H} 3$ \\
\hline 1924 & NEC6-30OH & $00 \mathrm{OC}(\mathrm{C}(\mathrm{C})(\mathrm{C}) \mathrm{C}) \mathrm{C}$ & InChl=1S/C6H14O2/c1-5(8-7)6(2,3)4/h5,7H,1-4H3 \\
\hline 1925 & C6H1010OH5-6O2 & $\mathrm{C}(\mathrm{O}[\mathrm{O}]) \mathrm{C}(\mathrm{OO}) \mathrm{CCC}=\mathrm{C}$ & $\operatorname{lnChl}=1 \mathrm{~S} / \mathrm{C} 6 \mathrm{H} 1104 / \mathrm{c} 1-2-3-4-6(10-8) 5-9-7 / \mathrm{h} 2,6,8 \mathrm{H}, 1,3-5 \mathrm{H} 2$ \\
\hline 1926 & 13C6D1-6 & $\mathrm{CC}(\mathrm{C}=\mathrm{C}) \mathrm{C}[\mathrm{CH} 2]$ & InChl=15/C6H11/c1-4-6(3)5-2/h4,6H,1-2,5H2,3H3 \\
\hline 1927 & $\mathrm{IC} 6 \mathrm{OH} 2-3 \mathrm{R}$ & $\mathrm{CC}(\mathrm{O})(\mathrm{C})[\mathrm{CH}] \mathrm{CC}$ & $\operatorname{lnChl}=15 / C 6 H 130 / c 1-4-5-6(2,3) 7 / h 5,7 \mathrm{H}, 4 \mathrm{H} 2,1-3 \mathrm{H} 3$ \\
\hline 1928 & I3C6-10OH & $\mathrm{CC}(\mathrm{CC}) \mathrm{CCOO}$ & $\operatorname{lnChl}=15 / \mathrm{C} 6 \mathrm{H} 14 \mathrm{O} 2 / \mathrm{c} 1-3-6(2) 4-5-8-7 / \mathrm{h} 6-7 \mathrm{H}, 3-5 \mathrm{H} 2,1-2 \mathrm{H} 3$ \\
\hline 1929 & C6H1010OH6-302 & $\mathrm{C}(\mathrm{OO}) \mathrm{CCC}(\mathrm{O}[\mathrm{O}]) \mathrm{C}=\mathrm{C}$ & InChl=1S/C6H1104/c1-2-6(10-8)4-3-5-9-7/h2,6-7H,1,3-5H2 \\
\hline 1930 & 13C6D2-5 & $\mathrm{C}[\mathrm{CH}] \mathrm{C}(=\mathrm{CC}) \mathrm{C}$ & InChl=15/C6H11/c1-4-6(3)5-2/h4-5H,1-3H3 \\
\hline 1931 & $\mathrm{IC} 6 \mathrm{OH} 3-2 \mathrm{R}$ & $\mathrm{C}[\mathrm{C}](\mathrm{C}) \mathrm{C}(\mathrm{O}) \mathrm{CC}$ & InChl=1S/C6H130/c1-4-6(7)5(2)3/h6-7H,4H2,1-3H3 \\
\hline 1932 & NEC6-4OOH & OOCcC(C)(C)C & $\operatorname{lnChl}=15 / C 6 H 1402 / \mathrm{c} 1-6(2,3) 4-5-8-7 / \mathrm{h} 7 \mathrm{H}, 4-5 \mathrm{H} 2,1-3 \mathrm{H} 3$ \\
\hline 1933 & C6H101OOH6-402 & $\mathrm{C}(\mathrm{OO}) \mathrm{CC}(\mathrm{O}[\mathrm{O}]) \mathrm{CC}=\mathrm{C}$ & InChl=1S/C6H1104/c1-2-3-6(10-8)4-5-9-7/h2,6-7H,1,3-5H2 \\
\hline 1934 & $13 \mathrm{C} 6 \mathrm{D2} 2-6$ & {$[\mathrm{CH} 2] \mathrm{CC}(=\mathrm{CC}) \mathrm{C}$} & $\operatorname{lnChl}=1 \mathrm{~S} / \mathrm{C} 6 \mathrm{H} 11 / \mathrm{c} 1-4-6(3) 5-2 / \mathrm{h} 5 \mathrm{H}, 1,4 \mathrm{H} 2,2-3 \mathrm{H} 3$ \\
\hline 1935 & $\mathrm{IC} 6 \mathrm{OH} 3-4 \mathrm{R}$ & $\mathrm{CC}(\mathrm{C}) \mathrm{C}(\mathrm{O})[\mathrm{CH}] \mathrm{C}$ & InChl=15/C6H13O/c1-4-6(7)5(2)3/h4-7H,1-3H3 \\
\hline 1936 & $13 \mathrm{C} 6-2 \mathrm{OOH}$ & $\operatorname{CCC}(\mathrm{C}(\mathrm{OO}) \mathrm{C}) \mathrm{C}$ & $\operatorname{lnChl}=1 \mathrm{~S} / \mathrm{C} 6 \mathrm{H} 14 \mathrm{O} 2 / \mathrm{c1}-4-5(2) 6(3) 8-7 / \mathrm{h} 5-7 \mathrm{H}, 4 \mathrm{H} 2,1-3 \mathrm{H} 3$ \\
\hline 1937 & C6H1010OH6-502 & $\mathrm{C}(\mathrm{OO}) \mathrm{C}(\mathrm{O}[\mathrm{O}]) \mathrm{CCC}=\mathrm{C}$ & $\operatorname{lnChl}=1 \mathrm{~S} / \mathrm{C} 6 \mathrm{H} 1104 / \mathrm{c1}-2-3-4-6(10-8) 5-9-7 / \mathrm{h} 2,6-7 \mathrm{H}, 1,3-5 \mathrm{H} 2$ \\
\hline 1938 & 13C6D3-1 & $\mathrm{CCC}(=\mathrm{C}) \mathrm{C}[\mathrm{CH} 2]$ & $\operatorname{lnChl}=1 \mathrm{~S} / \mathrm{C} 6 \mathrm{H} 11 / \mathrm{c} 1-4-6(3) 5-2 / \mathrm{h} 1,3-5 \mathrm{H} 2,2 \mathrm{H} 3$ \\
\hline 1939 & $\mathrm{IC} 6 \mathrm{OH} 4-3 \mathrm{R}$ & $\mathrm{CC}(\mathrm{C})[\mathrm{CH}] \mathrm{C}(\mathrm{O}) \mathrm{C}$ & $\mathrm{InChl}=1 \mathrm{~S} / \mathrm{C} 6 \mathrm{H} 130 / \mathrm{c} 1-5(2) 4-6(3) 7 / \mathrm{h} 4-7 \mathrm{H}, 1-3 \mathrm{H} 3$ \\
\hline 1940 & $13 \mathrm{C} 6-3 \mathrm{OOH}$ & $\mathrm{CCC}(\mathrm{OO})(\mathrm{CC}) \mathrm{C}$ & $\operatorname{lnChl}=15 / C 6 H 1402 / c 1-4-6(3,5-2) 8-7 / \mathrm{h} 7 \mathrm{H}, 4-5 \mathrm{H} 2,1-3 \mathrm{H} 3$ \\
\hline 1941 & $\mathrm{C} 6 \mathrm{H} 102 \mathrm{OOH} 4-5 \mathrm{O} 2$ & $\mathrm{CC}(\mathrm{O}[\mathrm{O}]) \mathrm{C}(\mathrm{OO}) \mathrm{C}=\mathrm{CC}$ & InChl=15/C6H11O4/c1-3-4-6(10-8)5(2)9-7/h3-6,8H,1-2H3 \\
\hline 1942 & XC6D1-1A & {$[\mathrm{CH} 2] \mathrm{C}(=\mathrm{C}) \mathrm{C}(\mathrm{C}) \mathrm{C}$} & $\ln \mathrm{Chl}=1 \mathrm{~S} / \mathrm{C} 6 \mathrm{H} 11 / \mathrm{c} 1-5(2) 6(3) 4 / \mathrm{h} 6 \mathrm{H}, 1-2 \mathrm{H} 2,3-4 \mathrm{H} 3$ \\
\hline 1943 & $\mathrm{IC} 6 \mathrm{OH} 4-5 \mathrm{R}$ & $\mathrm{CC}(\mathrm{C}) \mathrm{CC}(\mathrm{O})[\mathrm{CH} 2]$ & InChl=1S/C6H130/c1-5(2)4-6(3)7/h5-7H,3-4H2,1-2H3 \\
\hline 1944 & $13 \mathrm{C} 6-40 \mathrm{OH}$ & $\mathrm{CCC}(\mathrm{COO}) \mathrm{CC}$ & InChl=15/C6H14O2/c1-3-6(4-2)5-8-7/h6-7H,3-5H2,1-2H3 \\
\hline 1945 & $\mathrm{C} 6 \mathrm{H} 102 \mathrm{OOH} 4-6 \mathrm{O} 2$ & $\mathrm{C}(\mathrm{O}[\mathrm{O}]) \mathrm{CC}(\mathrm{OO}) \mathrm{C}=\mathrm{CC}$ & InChl=1S/C6H1104/c1-2-3-6(10-8)4-5-9-7/h2-3,6,8H,4-5H2,1H3 \\
\hline 1946 & 13C6D3-2 & $\mathrm{C}[\mathrm{CH}] \mathrm{C}(=\mathrm{C}) \mathrm{CC}$ & InChl=1S/C6H11/c1-4-6(3)5-2/h4H,3,5H2,1-2H3 \\
\hline 1947 & IC6OH5-4R & $\mathrm{CC}(\mathrm{C}) \mathrm{C}[\mathrm{CH}] \mathrm{CO}$ & $\operatorname{lnChl}=15 / \mathrm{C} 6 \mathrm{H} 130 / \mathrm{c} 1-6(2) 4-3-5-7 / \mathrm{h} 3,6-7 \mathrm{H}, 4-5 \mathrm{H} 2,1-2 \mathrm{H} 3$ \\
\hline 1948 & $\mathrm{XC6-10OH}$ & $\mathrm{OOCC}(\mathrm{C}(\mathrm{C}) \mathrm{C}) \mathrm{C}$ & $\operatorname{lnChl}=1 \mathrm{~S} / \mathrm{C} 6 \mathrm{H} 14 \mathrm{O} 2 / \mathrm{c} 1-5(2) 6(3) 4-8-7 / \mathrm{h} 5-7 \mathrm{H}, 4 \mathrm{H} 2,1-3 \mathrm{H} 3$ \\
\hline 1949 & C6H102OOH5-4O2 & $\mathrm{CC}(\mathrm{OO}) \mathrm{C}(\mathrm{O}[\mathrm{O}]) \mathrm{C}=\mathrm{CC}$ & $\ln C h \mathrm{l}=1 \mathrm{~S} / \mathrm{C} 6 \mathrm{H} 1104 / \mathrm{c} 1-3-4-6(10-8) 5(2) 9-7 / \mathrm{h} 3-7 \mathrm{H}, 1-2 \mathrm{H} 3$ \\
\hline 1950 & XC6D1-3 & $\mathrm{CC}(=\mathrm{C})[\mathrm{C}](\mathrm{C}) \mathrm{C}$ & $\operatorname{lnChl}=1 \mathrm{~S} / \mathrm{C} 6 \mathrm{H} 11 / \mathrm{c} 1-5(2) 6(3) 4 / \mathrm{h} 1 \mathrm{H} 2,2-4 \mathrm{H} 3$ \\
\hline 1951 & NEC6OH3-4R & $\mathrm{CC}(\mathrm{C})(\mathrm{C}) \mathrm{C}(\mathrm{O})[\mathrm{CH} 2]$ & InChl=1S/C6H130/c1-5(7)6(2,3)4/h5,7H,1H2,2-4H3 \\
\hline 1952 & IC6-10OH & $\operatorname{cccc}(\mathrm{COO}) \mathrm{C}$ & InChl=1S/C6H14O2/c1-3-4-6(2)5-8-7/h6-7H,3-5H2,1-2H3 \\
\hline 1953 & $\mathrm{C} 6 \mathrm{H} 102 \mathrm{OOH} 5-602$ & $\mathrm{C}(\mathrm{O}[\mathrm{O}]) \mathrm{C}(\mathrm{OO}) \mathrm{CC}=\mathrm{CC}$ & InChl=1S/C6H1104/c1-2-3-4-6(10-8)5-9-7/h2-3,6,8H,4-5H2,1H3 \\
\hline 1954 & XC6D1-4 & $\mathrm{CC}(=\mathrm{C}) \mathrm{C}([\mathrm{CH} 2]) \mathrm{C}$ & $\operatorname{lnChl}=15 / C 6 H 11 / c 1-5(2) 6(3) 4 / h 5 H, 1,3 \mathrm{H} 2,2,4 \mathrm{H} 3$ \\
\hline 1955 & NEC6OH4-3R & $\mathrm{CC}(\mathrm{C})(\mathrm{C})[\mathrm{CH}] \mathrm{CO}$ & InChl=1S/C6H13O/c1-6(2,3)4-5-7/h4,7H,5H2,1-3H3 \\
\hline 1956 & XC6-2OOH & $0 O C(C(C) C)(C) C$ & InChl=1S/C6H1402/c1-5(2)6(3,4)8-7/h5,7H,1-4H3 \\
\hline 1957 & $\mathrm{C} 6 \mathrm{H} 102 \mathrm{OOH} 6-402$ & $\mathrm{C}(\mathrm{OO}) \mathrm{CC}(\mathrm{O}[\mathrm{O}]) \mathrm{C}=\mathrm{CC}$ & InChl=1S/C6H1104/c1-2-3-6(10-8)4-5-9-7/h2-3,6-7H,4-5H2,1H3 \\
\hline 1958 & IC6D1-1A & $\mathrm{CCCC}(=\mathrm{C})[\mathrm{CH} 2]$ & InChl=1S/C6H11/c1-4-5-6(2)3/h2-5H2,1H3 \\
\hline 1959 & P-C6H3O2 & $\mathrm{C1} 1=0)[\mathrm{C}]=\mathrm{CC}(=0) \mathrm{C}=\mathrm{C} 1$ & $\operatorname{lnChl}=1 \mathrm{~S} / \mathrm{C} 6 \mathrm{H} 3 \mathrm{O} 2 / \mathrm{c} 7-5-1-2-6(8) 4-3-5 / \mathrm{h} 1-3 \mathrm{H}$ \\
\hline 1960 & $\mathrm{IC6}-2 \mathrm{OOH}$ & $\operatorname{cccc}(0 O)(C) C$ & $\operatorname{lnChl}=15 / C 6 H 1402 / c 1-4-5-6(2,3) 8-7 / \mathrm{h} 7 \mathrm{H}, 4-5 \mathrm{H} 2,1-3 \mathrm{H} 3$ \\
\hline 1961 & C6H102OOH6-502 & $\mathrm{C}(\mathrm{OO}) \mathrm{C}(\mathrm{O}[\mathrm{O}]) \mathrm{CC}=\mathrm{CC}$ & InChl=1S/C6H11O4/c1-2-3-4-6(10-8)5-9-7/h2-3,6-7H,4-5H2,1H3 \\
\hline 1962 & IC6D1-5 & {$[\mathrm{CH} 2] \mathrm{CCC}(=\mathrm{C}) \mathrm{C}$} & $\operatorname{lnChl}=1 \mathrm{~S} / \mathrm{C} 6 \mathrm{H} 11 / \mathrm{c} 1-4-5-6(2) 3 / \mathrm{h} 1-2,4-5 \mathrm{H} 2,3 \mathrm{H} 3$ \\
\hline 1963 & $\mathrm{O}-\mathrm{C} 6 \mathrm{H} 4 \mathrm{O} 2$ & $C 1(=0) C(=0) C=C C=C 1$ & $\operatorname{lnChl}=1 \mathrm{~S} / \mathrm{C} 6 \mathrm{H} 402 / \mathrm{c} 7-5-3-1-2-4-6(5) 8 / \mathrm{h} 1-4 \mathrm{H}$ \\
\hline 1964 & $\mathrm{IC6} 6-30 \mathrm{OH}$ & $\operatorname{ccc}(C(C) C) 00$ & InChl=1S/C6H14O2/c1-4-6(8-7)5(2)3/h5-7H,4H2,1-3H3 \\
\hline 1965 & $\mathrm{C} 6 \mathrm{H} 103 \mathrm{OOH} 1-2 \mathrm{O} 2$ & $\mathrm{C}(\mathrm{OO}) \mathrm{C}(\mathrm{O}[\mathrm{O}]) \mathrm{C}=\mathrm{CCC}$ & InChl=1S/C6H1104/c1-2-3-4-6(10-8)5-9-7/h3-4,6-7H,2,5H2,1H3 \\
\hline 1966 & IC6D1-4R & $\mathrm{C}=\mathrm{C}(\mathrm{C}) \mathrm{C}[\mathrm{CH}] \mathrm{C}$ & $\operatorname{lnChl}=1 \mathrm{~S} / \mathrm{C} 6 \mathrm{H} 11 / \mathrm{c} 1-4-5-6(2) 3 / \mathrm{h} 4 \mathrm{H}, 2,5 \mathrm{H} 2,1,3 \mathrm{H} 3$ \\
\hline 1967 & P-C6H402 & $\mathrm{C} 1(=0) \mathrm{C}=\mathrm{CC}(=0) \mathrm{C}=\mathrm{C} 1$ & $\operatorname{lnChl}=1 \mathrm{~S} / \mathrm{C} 6 \mathrm{H} 4 \mathrm{O} 2 / \mathrm{c} 7-5-1-2-6(8) 4-3-5 / \mathrm{h} 1-4 \mathrm{H}$ \\
\hline 1968 & $\mathrm{IC} 6-4 \mathrm{OOH}$ & $\mathrm{OOC}(\mathrm{CC}(\mathrm{C}) \mathrm{C}) \mathrm{C}$ & $\operatorname{lnChl}=15 / C 6 \mathrm{H} 14 \mathrm{O} 2 / \mathrm{c} 1-5(2) 4-6(3) 8-7 / \mathrm{h} 5-7 \mathrm{H}, 4 \mathrm{H} 2,1-3 \mathrm{H} 3$ \\
\hline 1969 & $\mathrm{C} 6 \mathrm{H} 103 \mathrm{OOH} 2-1 \mathrm{O} 2$ & $\mathrm{C}(\mathrm{O}[\mathrm{O}]) \mathrm{C}(\mathrm{OO}) \mathrm{C}=\mathrm{CCC}$ & InChl=1S/C6H1104/c1-2-3-4-6(10-8)5-9-7/h3-4,6,8H,2,5H2,1H3 \\
\hline 1970 & IC6D3-2R & $C[C](C) C=C C$ & $\mathrm{InChl}=1 \mathrm{~S} / \mathrm{C} 6 \mathrm{H} 11 / \mathrm{c} 1-4-5-6(2) 3 / \mathrm{h} 4-5 \mathrm{H}, 1-3 \mathrm{H} 3$ \\
\hline 1971 & MF25CJO & $\mathrm{CC} 1=\mathrm{CC}=\mathrm{C}([\mathrm{C}]=0) 01$ & $\operatorname{lnChl}=1 \mathrm{~S} / \mathrm{C} 6 \mathrm{H} 5 \mathrm{O} 2 / \mathrm{c1} 1-5-2-3-6(4-7) 8-5 / \mathrm{h} 2-3 \mathrm{H}, 1 \mathrm{H} 3$ \\
\hline 1972 & $\mathrm{IC} 6-5 \mathrm{OOH}$ & $\operatorname{oocccc}(\mathrm{C}) \mathrm{C}$ & $\mathrm{InChl}=1 \mathrm{~S} / \mathrm{C} 6 \mathrm{H} 1402 / \mathrm{c} 1-6(2) 4-3-5-8-7 / \mathrm{h} 6-7 \mathrm{H}, 3-5 \mathrm{H} 2,1-2 \mathrm{H} 3$ \\
\hline 1973 & CHX1Q2QJ & OOC1CCCCC1O[0] & InChl=1S/C6H11O4/c7-9-5-3-1-2-4-6(5)10-8/h5-7H,1-4H2 \\
\hline 1974 & IC6D4-2R & $\mathrm{C}[\mathrm{C}](\mathrm{C}) \mathrm{CC}=\mathrm{C}$ & $\operatorname{lnChl}=15 / \mathrm{C} 6 \mathrm{H} 11 / \mathrm{c} 1-4-5-6(2) 3 / \mathrm{h} 4 \mathrm{H}, 1,5 \mathrm{H} 2,2-3 \mathrm{H} 3$ \\
\hline 1975 & MF2J5CHO & {$[\mathrm{CH} 2] \mathrm{C} 1=\mathrm{CC}=\mathrm{C}(\mathrm{C}=\mathrm{O}) 01$} & $\operatorname{lnChl}=1 \mathrm{~S} / \mathrm{C} 6 \mathrm{H} 5 \mathrm{O} 2 / \mathrm{c1}-5-2-3-6(4-7) 8-5 / \mathrm{h} 2-4 \mathrm{H}, 1 \mathrm{H} 2$ \\
\hline 1976 & DCHOF2J & $\mathrm{O}=[\mathrm{C}] \mathrm{C} 1=\mathrm{CC}=\mathrm{C}(\mathrm{C}=\mathrm{O}) 01$ & InChl=1S/C6H3O3/c7-3-5-1-2-6(4-8)9-5/h1-3H \\
\hline 1977 & CHX1Q3QJ & $\mathrm{O} 0 \mathrm{C} 1 \mathrm{cccc}(\mathrm{C} 1) \mathrm{O}[\mathrm{O}]$ & InChl=1S/C6H1104/c7-9-5-2-1-3-6(4-5)10-8/h5-7H,1-4H2 \\
\hline 1978 & IC6D1-3R & $\mathrm{C}=\mathrm{C}(\mathrm{C})[\mathrm{CH}] \mathrm{CC}$ & $\operatorname{lnChl}=1 \mathrm{~S} / \mathrm{C} 6 \mathrm{H} 11 / \mathrm{c} 1-4-5-6(2) 3 / \mathrm{h} 5 \mathrm{H}, 2,4 \mathrm{H} 2,1,3 \mathrm{H} 3$ \\
\hline 1979 & $\mathrm{C} 6 \mathrm{H} 50 \mathrm{O}$ & $\mathrm{C} 1=\mathrm{C}(\mathrm{O}[\mathrm{O}]) \mathrm{C}=\mathrm{CC}=\mathrm{C} 1$ & $\operatorname{lnChl}=1 \mathrm{~S} / \mathrm{C} 6 \mathrm{H} 5 \mathrm{O} 2 / \mathrm{c} 7-8-6-4-2-1-3-5-6 / \mathrm{h} 1-5 \mathrm{H}$ \\
\hline 1980 & DCHO25F & $\mathrm{O}=\mathrm{CC} 1=\mathrm{CC}=\mathrm{C}(\mathrm{C}=\mathrm{O}) \mathrm{O} 1$ & $\operatorname{lnChl}=1 \mathrm{~S} / \mathrm{C} 6 \mathrm{H} 403 / \mathrm{C} 7-3-5-1-2-6(4-8) 9-5 / \mathrm{h} 1-4 \mathrm{H}$ \\
\hline 1981 & CHX1Q4QJ & OOC1CCC(CC1)O[O] & InChl=1S/C6H11O4/c7-9-5-1-2-6(10-8)4-3-5/h5-7H,1-4H2 \\
\hline 1982 & IC6D2-5R & $\mathrm{CC}(\mathrm{C})=\mathrm{CC}[\mathrm{CH} 2]$ & InChl=15/C6H11/c1-4-5-6(2)3/h5H,1,4H2,2-3H3 \\
\hline 1983 & $\mathrm{O}-\mathrm{OC} 6 \mathrm{H} 4 \mathrm{OH}$ & $\mathrm{OC} 1=\mathrm{CC}=\mathrm{CC}=\mathrm{C} 1[0]$ & InChl=15/C6H5O2/c7-5-3-1-2-4-6(5)8/h1-4,7H \\
\hline 1984 & H34DE1260 & $\mathrm{O}=\mathrm{CC}=\mathrm{C}=\mathrm{CC}(=\mathrm{O}) \mathrm{C}=\mathrm{O}$ & $\operatorname{lnChl}=15 / \mathrm{C} 6 \mathrm{H} 4 \mathrm{OO} / \mathrm{c} 7-4-2-1-3-6(9) 5-8 / \mathrm{h} 2-5 \mathrm{H}$ \\
\hline 1985 & IC6D3-1R & {$[\mathrm{CH} 2] \mathrm{C}(\mathrm{C}) \mathrm{C}=\mathrm{CC}$} & $\operatorname{lnChl}=15 / C 6 H 11 / c 1-4-5-6(2) 3 / h 4-6 \mathrm{H}, 2 \mathrm{H} 2,1,3 \mathrm{H} 3$ \\
\hline 1986 & $\mathrm{P}-\mathrm{OC} 6 \mathrm{H} 4 \mathrm{OH}$ & {$[\mathrm{O}] \mathrm{C} 1=\mathrm{CC}=\mathrm{C}(\mathrm{O}) \mathrm{C}=\mathrm{C} 1$} & $\operatorname{lnChl}=1 \mathrm{~S} / \mathrm{C} 6 \mathrm{H} 502 / \mathrm{c} 7-5-1-2-6(8) 4-3-5 / \mathrm{h} 1-4,7 \mathrm{H}$ \\
\hline 1987 & HMF5OJ & {$[\mathrm{O}] \mathrm{CC} 1=\mathrm{CC}=\mathrm{C}(\mathrm{C}=\mathrm{O}) 01$} & $\operatorname{lnChl}=15 / \mathrm{CHH}^{2} \mathrm{O} / \mathrm{c} 7-3-5-1-2-6(4-8) 9-5 / \mathrm{h} 1-3 \mathrm{H}, 4 \mathrm{H} 2$ \\
\hline 1988 & IC6D4-1R & {$[\mathrm{CH} 2] \mathrm{C}(\mathrm{C}) \mathrm{CC}=\mathrm{C}$} & InChl=15/C6H11/c1-4-5-6(2)3/h4,6H,1-2,5H2,3H3 \\
\hline 1989 & H34DE210 & $\mathrm{CC}=\mathrm{C}=\mathrm{CC}(=\mathrm{O}) \mathrm{C}=\mathrm{O}$ & $\operatorname{lnChl}=1 \mathrm{~S} / \mathrm{C} 6 \mathrm{H} 6 \mathrm{O} / \mathrm{c} 1-2-3-4-6(8) 5-7 / \mathrm{h} 2,4-5 \mathrm{H}, 1 \mathrm{H} 3$ \\
\hline 1990 & $\mathrm{P}-\mathrm{OOC6} 6 \mathrm{H} 4 \mathrm{OH}$ & {$[\mathrm{O}] \mathrm{OC} 1=\mathrm{CC}=\mathrm{C}(\mathrm{O}) \mathrm{C}=\mathrm{C} 1$} & $\operatorname{lnChl}=15 / C 6 H 503 / c 7-5-1-3-6(9-8) 4-2-5 / h 1-4,7 \mathrm{H}$ \\
\hline 1991 & IC6D4-3R & $\mathrm{CC}(\mathrm{C})[\mathrm{CH}] \mathrm{C}=\mathrm{C}$ & $\ln \mathrm{Chl}=1 \mathrm{~S} / \mathrm{C} 6 \mathrm{H} 11 / \mathrm{c} 1-4-5-6(2) 3 / \mathrm{h} 4-6 \mathrm{H}, 1 \mathrm{H} 2,2-3 \mathrm{H} 3$ \\
\hline 1992 & H34DE260 & $\mathrm{CC}(=\mathrm{O}) \mathrm{C}=\mathrm{C}=\mathrm{CC}=\mathrm{O}$ & InChl=1S/C6H6O2/c1-6(8)4-2-3-5-7/h3-5H,1H3 \\
\hline 1993 & $\mathrm{O}-\mathrm{OOC} 6 \mathrm{H} 4 \mathrm{OH}$ & $\mathrm{OC} 1=\mathrm{C}(\mathrm{O}[\mathrm{O}]) \mathrm{C}=\mathrm{CC}=\mathrm{C} 1$ & $\operatorname{lnChl}=15 / C 6 H 503 / c 7-5-3-1-2-4-6(5) 9-8 / h 1-4,7 \mathrm{H}$ \\
\hline 1994 & NEC6D3-1R & {$[\mathrm{CH} 2] \mathrm{C}(\mathrm{C})(\mathrm{C}) \mathrm{C}=\mathrm{C}$} & $\operatorname{lnChl}=1 \mathrm{~S} / \mathrm{C} 6 \mathrm{H} 11 / \mathrm{c} 1-5-6(2,3) 4 / \mathrm{h} 5 \mathrm{H}, 1-2 \mathrm{H} 2,3-4 \mathrm{H} 3$ \\
\hline 1995 & MF25CHO & $\mathrm{CC} 1=\mathrm{CC}=\mathrm{C}(\mathrm{C}=0) 01$ & $\operatorname{lnChl}=1 \mathrm{~S} / \mathrm{C} 6 \mathrm{H} 6 \mathrm{O} / \mathrm{c} 1-5-2-3-6(4-7) 8-5 / \mathrm{h} 2-4 \mathrm{H}, 1 \mathrm{H} 3$ \\
\hline 1996 & MF25CH2OOJ & $\mathrm{CC} 1=\mathrm{CC}=\mathrm{C}(\mathrm{CO}[\mathrm{O}]) 01$ & $\operatorname{lnChl}=15 / \mathrm{C} 6 \mathrm{H} 703 / \mathrm{c} 1-5-2-3-6(9-5) 4-8-7 / \mathrm{h} 2-3 \mathrm{H}, 4 \mathrm{H} 2,1 \mathrm{H} 3$ \\
\hline 1997 & $\mathrm{C} 6 \mathrm{H} 12 \mathrm{OOH} 1-2 \mathrm{O} 2$ & $\mathrm{C}(\mathrm{OO}) \mathrm{C}(\mathrm{O}[\mathrm{O}]) \mathrm{CCCC}$ & $\operatorname{lnChl}=1 \mathrm{~S} / \mathrm{C} 6 \mathrm{H} 13 \mathrm{O} 4 / \mathrm{c1}-2-3-4-6(10-8) 5-9-7 / \mathrm{h} 6-7 \mathrm{H}, 2-5 \mathrm{H} 2,1 \mathrm{H} 3$ \\
\hline 1998 & C6H12-1 & $\mathrm{C}=\mathrm{CCCCC}$ & $\operatorname{lnChl}=1 \mathrm{~S} / \mathrm{C} 6 \mathrm{H} 12 / \mathrm{c1}-3-5-6-4-2 / \mathrm{h} 3 \mathrm{H}, 1,4-6 \mathrm{H} 2,2 \mathrm{H} 3$ \\
\hline
\end{tabular}




\begin{tabular}{|c|c|c|c|}
\hline 1999 & $\mathrm{C} 6 \mathrm{H} 5 \mathrm{OOH}$ & $\mathrm{C} 1=\mathrm{C}(\mathrm{OO}) \mathrm{C}=\mathrm{CC}=\mathrm{C} 1$ & InChl=1S/C6H6O2/c7-8-6-4-2-1-3-5-6/h1-5,7H \\
\hline 2000 & NC6D1KET34 & $\operatorname{ccc}(\mathrm{OO}) \mathrm{C}(=0) \mathrm{C}=\mathrm{C}$ & InChl=1S/C6H1003/c1-3-5(7)6(4-2)9-8/h3,6,8H,1,4H2,2H3 \\
\hline 2001 & $\mathrm{C} 6 \mathrm{H} 12 \mathrm{OOH} 1-3 \mathrm{O} 2$ & $\mathrm{C}(\mathrm{OO}) \mathrm{CC}(\mathrm{O}[\mathrm{O}]) \mathrm{CCC}$ & $\operatorname{lnChl}=1 \mathrm{~S} / \mathrm{C} 6 \mathrm{H} 13 \mathrm{O} 4 / \mathrm{c1}-2-3-6(10-8) 4-5-9-7 / \mathrm{h6}-7 \mathrm{H}, 2-5 \mathrm{H} 2,1 \mathrm{H} 3$ \\
\hline 2002 & $\mathrm{C} 6 \mathrm{H} 12-2$ & $\mathrm{CC}=\mathrm{CCCC}$ & InChl=1S/C6H12/c1-3-5-6-4-2/h3,5H,4,6H2,1-2H3 \\
\hline 2003 & $\mathrm{O}-\mathrm{HOC} 6 \mathrm{H} 4 \mathrm{OH}$ & $\mathrm{OC} 1=\mathrm{CC}=\mathrm{CC}=\mathrm{C} 1 \mathrm{O}$ & InChl=15/C6H6O2/c7-5-3-1-2-4-6(5)8/h1-4,7-8H \\
\hline 2004 & NC6D1KET35 & $\mathrm{CC}(\mathrm{OO}) \mathrm{CC}(=\mathrm{O}) \mathrm{C}=\mathrm{C}$ & InChl=15/C6H1003/c1-3-6(7)4-5(2)9-8/h3,5,8H,1,4H2,2H3 \\
\hline 2005 & $\mathrm{C} 6 \mathrm{H} 12 \mathrm{OOH} 1-4 \mathrm{O} 2$ & $\mathrm{C}(\mathrm{OO}) \mathrm{CCC}(\mathrm{O}[\mathrm{O}]) \mathrm{CC}$ & InChl=1S/C6H13O4/c1-2-6(10-8)4-3-5-9-7/h6-7H,2-5H2,1H3 \\
\hline 2006 & $\mathrm{C} 6 \mathrm{H} 12-3$ & $\mathrm{CCC}=\mathrm{CCC}$ & InChl=1S/C6H12/c1-3-5-6-4-2/h5-6H,3-4H2,1-2H3 \\
\hline 2007 & $\mathrm{P}-\mathrm{HOC} 6 \mathrm{H} 4 \mathrm{OH}$ & $\mathrm{OC} 1=\mathrm{CC}=\mathrm{C}(\mathrm{O}) \mathrm{C}=\mathrm{C} 1$ & InChl=1S/C6H6O2/c7-5-1-2-6(8)4-3-5/h1-4,7-8H \\
\hline 2008 & NC6D1KET36 & $\mathrm{C}(\mathrm{OO}) \mathrm{CCC}(=\mathrm{O}) \mathrm{C}=\mathrm{C}$ & InChl=1S/C6H1003/c1-2-6(7)4-3-5-9-8/h2,8H,1,3-5H2 \\
\hline 2009 & C6H12OOH1-502 & $\mathrm{C}(\mathrm{OO}) \mathrm{CCCC}(\mathrm{O}[\mathrm{O}]) \mathrm{C}$ & $\operatorname{lnChl}=1 \mathrm{~S} / \mathrm{C} 6 \mathrm{H} 1304 / \mathrm{c1}-6(10-8) 4-2-3-5-9-7 / \mathrm{h} 6-7 \mathrm{H}, 2-5 \mathrm{H} 2,1 \mathrm{H} 3$ \\
\hline 2010 & $\mathrm{CHX}$ & $\mathrm{C} 1 \mathrm{CCCCC} 1$ & InChl=1S/C6H12/c1-2-4-6-5-3-1/h1-6H2 \\
\hline 2011 & DMF252OJ & $\mathrm{CC} 1=\mathrm{CC}=\mathrm{C}(\mathrm{C}[0]) 01$ & InChl=1S/C6H702/c1-5-2-3-6(4-7)8-5/h2-3H,4H2,1H3 \\
\hline 2012 & NC6D1KET43 & $\mathrm{CCC}(=0) \mathrm{C}(\mathrm{OO}) \mathrm{C}=\mathrm{C}$ & InChl=1S/C6H1003/c1-3-5(7)6(4-2)9-8/h4,6,8H,2-3H2,1H3 \\
\hline 2013 & $\mathrm{C} 6 \mathrm{H} 12 \mathrm{OOH} 2-1 \mathrm{O} 2$ & $\mathrm{C}(\mathrm{O}[\mathrm{O}]) \mathrm{C}(\mathrm{OO}) \mathrm{CCCC}$ & $\operatorname{lnChl}=1 \mathrm{~S} / \mathrm{C} 6 \mathrm{H} 13 \mathrm{O} 4 / \mathrm{c1}-2-3-4-6(10-8) 5-9-7 / \mathrm{h} 6,8 \mathrm{H}, 2-5 \mathrm{H} 2,1 \mathrm{H} 3$ \\
\hline 2014 & I3C6D1 & $\mathrm{CC}(\mathrm{C}=\mathrm{C}) \mathrm{CC}$ & InChl=15/C6H12/c1-4-6(3)5-2/h4,6H,1,5H2,2-3H3 \\
\hline 2015 & H3E1501J & $\mathrm{O}=[\mathrm{C}] \mathrm{CC}=\mathrm{CC}(=\mathrm{O}) \mathrm{C}$ & InChl=1S/C6H7O2/c1-6(8)4-2-3-5-7/h2,4H,3H2,1H3 \\
\hline 2016 & NC6D1KET45 & $\mathrm{CC}(\mathrm{OO}) \mathrm{C}(=\mathrm{O}) \mathrm{CC}=\mathrm{C}$ & InChl=15/C6H1003/c1-3-4-6(7)5(2)9-8/h3,5,8H,1,4H2,2H3 \\
\hline 2017 & $\mathrm{C} 6 \mathrm{H} 12 \mathrm{OOH} 2-3 \mathrm{O} 2$ & $\mathrm{CC}(\mathrm{OOO}) \mathrm{C}(\mathrm{O}[\mathrm{O}]) \mathrm{CCC}$ & InChl=1S/C6H13O4/c1-3-4-6(10-8)5(2)9-7/h5-7H,3-4H2,1-2H3 \\
\hline 2018 & 13C6D2 & $\operatorname{cCC}(=\mathrm{CC}) \mathrm{C}$ & InChl=1S/C6H12/c1-4-6(3)5-2/h4H,5H2,1-3H3 \\
\hline 2019 & H3E2501J & {$[\mathrm{CH} 2] \mathrm{C}(=\mathrm{O}) \mathrm{C}=\mathrm{CC}(=\mathrm{O}) \mathrm{C}$} & InChl=1S/C6H702/c1-5(7)3-4-6(2)8/h3-4H,1H2,2H3 \\
\hline 2020 & NC6D1KET46 & $\mathrm{C}(\mathrm{OO}) \mathrm{CC}(=\mathrm{O}) \mathrm{CC}=\mathrm{C}$ & $\operatorname{lnChl}=15 / \mathrm{C} 6 \mathrm{H} 1003 / \mathrm{c} 1-2-3-6(7) 4-5-9-8 / \mathrm{h} 2,8 \mathrm{H}, 1,3-5 \mathrm{H} 2$ \\
\hline 2021 & $\mathrm{C} 6 \mathrm{H} 12 \mathrm{OOH} 2-4 \mathrm{O} 2$ & $\mathrm{CC}(\mathrm{OO}) \mathrm{CC}(\mathrm{O}[\mathrm{O}]) \mathrm{CC}$ & InChl=1S/C6H13O4/c1-3-6(10-8)4-5(2)9-7/h5-7H,3-4H2,1-2H3 \\
\hline 2022 & $13 \mathrm{C} 6 \mathrm{D} 3$ & $\mathrm{CCC}(=\mathrm{C}) \mathrm{CC}$ & InChl=1S/C6H12/c1-4-6(3)5-2/h3-5H2,1-2H3 \\
\hline 2023 & H3E250 & $\mathrm{CC}(=0) \mathrm{C}=\mathrm{CC}(=0) \mathrm{C}$ & $\operatorname{lnChl}=1 \mathrm{~S} / \mathrm{C} 6 \mathrm{H} 8 \mathrm{O} 2 / \mathrm{c1}-5(7) 3-4-6(2) 8 / \mathrm{h} 3-4 \mathrm{H}, 1-2 \mathrm{H} 3$ \\
\hline 2024 & NC6D1KET53 & $\mathrm{CC}(=0) \mathrm{CC}(\mathrm{OO}) \mathrm{C}=\mathrm{C}$ & InChl=1S/C6H10O3/c1-3-6(9-8)4-5(2)7/h3,6,8H,1,4H2,2H3 \\
\hline 2025 & $\mathrm{C} 6 \mathrm{H} 12 \mathrm{OOH} 2-5 \mathrm{O} 2$ & $\mathrm{CC}(\mathrm{OO}) \mathrm{CCC}(\mathrm{O}[\mathrm{O}]) \mathrm{C}$ & InChl=15/C6H1304/c1-5(9-7)3-4-6(2)10-8/h5-7H,3-4H2,1-2H3 \\
\hline 2026 & XC6D1 & $\mathrm{CC}(=\mathrm{C}) \mathrm{C}(\mathrm{C}) \mathrm{C}$ & InChl=1S/C6H12/c1-5(2)6(3)4/h6H,1H2,2-4H3 \\
\hline 2027 & $\mathrm{CYC} 6 \mathrm{H} 724-\mathrm{OOH}$ & $\mathrm{OOC} 1 \mathrm{CC}=\mathrm{CC}=\mathrm{C} 1$ & InChl=1S/C6H8O2/c7-8-6-4-2-1-3-5-6/h1-4,6-7H,5H2 \\
\hline 2028 & NC6D1KET54 & $\mathrm{CC}(=\mathrm{O}) \mathrm{C}(\mathrm{OO}) \mathrm{CC}=\mathrm{C}$ & $\operatorname{lnChl}=1 \mathrm{~S} / \mathrm{C} 6 \mathrm{H} 1003 / \mathrm{c} 1-3-4-6(9-8) 5(2) 7 / \mathrm{h} 3,6,8 \mathrm{H}, 1,4 \mathrm{H} 2,2 \mathrm{H} 3$ \\
\hline 2029 & $\mathrm{C} 6 \mathrm{H} 12 \mathrm{OOH} 2-6 \mathrm{O} 2$ & $\mathrm{CC}(\mathrm{OO}) \operatorname{cccc}(\mathrm{O}[\mathrm{O}])$ & InChl=1S/C6H13O4/c1-66(10-8)4-2-3-5-9-7/h6,8H,2-5H2,1H3 \\
\hline 2030 & $\mathrm{XC6D2}$ & $\mathrm{CC}(=\mathrm{C}(\mathrm{C}) \mathrm{C}) \mathrm{C}$ & InChl=1S/C6H12/c1-5(2)6(3)4/h1-4H3 \\
\hline 2031 & $\mathrm{CYC} 6 \mathrm{H} 725-\mathrm{OOH}$ & $\mathrm{OOC1C}=\mathrm{CCC}=\mathrm{C} 1$ & $\operatorname{lnChl}=1 \mathrm{~S} / \mathrm{C} 6 \mathrm{H} 8 \mathrm{O} 2 / \mathrm{c} 7-8-6-4-2-1-3-5-6 / \mathrm{h} 2-7 \mathrm{H}, 1 \mathrm{H} 2$ \\
\hline 2032 & NC6D1KET56 & $\mathrm{C}(\mathrm{OO}) \mathrm{C}(=\mathrm{O}) \mathrm{CCC}=\mathrm{C}$ & $\operatorname{lnChl}=1 \mathrm{~S} / \mathrm{C} 6 \mathrm{H} 1003 / \mathrm{c} 1-2-3-4-6(7) 5-9-8 / \mathrm{h} 2,8 \mathrm{H}, 1,3-5 \mathrm{H} 2$ \\
\hline 2033 & $\mathrm{C} 6 \mathrm{H} 12 \mathrm{OOH} 3-1 \mathrm{O} 2$ & $\mathrm{C}(\mathrm{O}[\mathrm{O}]) \mathrm{CC}(\mathrm{OO}) \mathrm{CCC}$ & InChl=1S/C6H1304/c1-2-3-6(10-8)4-5-9-7/h6,8H,2-5H2,1H3 \\
\hline 2034 & IC6D1 & $\operatorname{cccC}(=\mathrm{C}) \mathrm{C}$ & InChl=1S/C6H12/c1-4-5-6(2)3/h2,4-5H2,1,3H3 \\
\hline 2035 & DMF252OH3J & $\mathrm{CC} 1=\mathrm{C}[\mathrm{CH}] \mathrm{C}(\mathrm{O})(\mathrm{C}) \mathrm{O} 1$ & InChl=1S/C6H9O2/c1-5-3-4-6(2,7)8-5/h3-4,7H,1-2H3 \\
\hline 2036 & NC6D1KET63 & $\mathrm{O}=\mathrm{CCCC}(\mathrm{OO}) \mathrm{C}=\mathrm{C}$ & $\operatorname{lnChl}=1 \mathrm{~S} / \mathrm{C} 6 \mathrm{H} 1003 / \mathrm{cc} 1-2-6(9-8) 4-3-5-7 / \mathrm{h} 2,5-6,8 \mathrm{H}, 1,3-4 \mathrm{H} 2$ \\
\hline 2037 & $\mathrm{C} 6 \mathrm{H} 12 \mathrm{OOH} 3-2 \mathrm{O} 2$ & $\mathrm{CC}(\mathrm{O}[\mathrm{O}]) \mathrm{C}(\mathrm{OO}) \mathrm{CCC}$ & InChl=1S/C6H13O4/c1-3-4-6(10-8)5(2)9-7/h5-6,8H,3-4H2,1-2H3 \\
\hline 2038 & IC6D2 & $\mathrm{CC}(\mathrm{C})=\mathrm{CCC}$ & InChl=15/C6H12/c1-4-5-6(2)3/h5H,4H2,1-3H3 \\
\hline 2039 & H2503J & $\mathrm{CC}(=\mathrm{O}) \mathrm{C}[\mathrm{CH}] \mathrm{C}(=\mathrm{O}) \mathrm{C}$ & InChl=1S/C6H9O2/c1-5(7)3-4-6(2)8/h3H,4H2,1-2H3 \\
\hline 2040 & NC6D1KET64 & $\mathrm{O}=\mathrm{CCC}(\mathrm{OO}) \mathrm{CC}=\mathrm{C}$ & InChl=1S/C6H10O3/c1-2-3-6(9-8)4-5-7/h2,5-6,8H,1,3-4H2 \\
\hline 2041 & $\mathrm{C} 6 \mathrm{H} 12 \mathrm{OOH} 3-4 \mathrm{O} 2$ & $\operatorname{CCC}(\mathrm{OO}) \mathrm{C}(\mathrm{O}[\mathrm{O}]) \mathrm{CC}$ & InChl=1S/C6H13O4/c1-3-5(9-7)6(4-2)10-8/h5-7H,3-4H2,1-2H3 \\
\hline 2042 & IC6D3 & $\mathrm{CC}(\mathrm{C}) \mathrm{C}=\mathrm{CC}$ & InChl=1S/C6H12/c1-4-5-6(2)3/h4-6H,1-3H3 \\
\hline 2043 & H3E2O5OH5J & $\mathrm{CC}(=\mathrm{O})[\mathrm{CH}] \mathrm{C}=\mathrm{C}(\mathrm{O}) \mathrm{C}$ & InChl=1S/C6H9O2/c1-5(7)3-4-6(2)8/h3-4,7H,1-2H3 \\
\hline 2044 & NC6D1KET65 & $\mathrm{O}=\mathrm{CC}(\mathrm{OO}) \mathrm{CCC}=\mathrm{C}$ & InChl=1S/C6H10O3/c1-2-3-4-6(5-7)9-8/h2,5-6,8H,1,3-4H2 \\
\hline 2045 & $\mathrm{C} 6 \mathrm{H} 12 \mathrm{OOH} 3-5 \mathrm{O} 2$ & $\operatorname{ccC}(\mathrm{OO}) \operatorname{cc}(\mathrm{O}[\mathrm{O}]) \mathrm{C}$ & InChl=1S/C6H13O4/c1-3-6(10-8)4-5(2)9-7/h5-6,8H,3-4H2,1-2H3 \\
\hline 2046 & IC6D4 & $\mathrm{CC}(\mathrm{C}) \mathrm{CC}=\mathrm{C}$ & InChl=1S/C6H12/c1-4-5-6(2)3/h4,6H,1,5H2,2-3H3 \\
\hline 2047 & CHX1VO2OJ & {$[0] \mathrm{C} 1 \mathrm{CCCCC} 1=\mathrm{O}$} & InChl=1S/C6H9O2/c7-5-3-1-2-4-6(5)8/h5H,1-4H2 \\
\hline 2048 & NC6D2KET45 & $\mathrm{CC}(\mathrm{OO}) \mathrm{C}(=\mathrm{O}) \mathrm{C}=\mathrm{CC}$ & InChl=1S/C6H10O3/c1-3-4-6/7)5(2)9-8/h3-5,8H,1-2H3 \\
\hline 2049 & $\mathrm{C} 6 \mathrm{H} 12 \mathrm{OOH} 3-6 \mathrm{O} 2$ & $\operatorname{ccc}(00) \operatorname{ccc}(0[0])$ & InChl=15/C6H13O4/c1-2-6(10-8)4-3-5-9-7/h6,8H,2-5H2,1H3 \\
\hline 2050 & NEC6D3 & $\mathrm{CC}(\mathrm{C})(\mathrm{C}) \mathrm{C}=\mathrm{C}$ & $\operatorname{lnChl}=1 \mathrm{~S} / \mathrm{C} 6 \mathrm{H} 12 / \mathrm{c} 1-5-6(2,3) 4 / \mathrm{h} 5 \mathrm{H}, 1 \mathrm{H} 2,2-4 \mathrm{H} 3$ \\
\hline 2051 & CHX1V03OJ & {$[0] \mathrm{C} 1 \mathrm{CCCC}(=0) \mathrm{C} 1$} & InChl=1S/C6H9O2/c7-5-2-1-3-6(8)4-5/h5H,1-4H2 \\
\hline 2052 & NC6D2KET46 & $\mathrm{C}(\mathrm{OO}) \mathrm{CC}(=\mathrm{O}) \mathrm{C}=\mathrm{CC}$ & InChl=1S/C6H1003/c1-2-3-6(7)4-5-9-8/h2-3,8H,4-5H2,1H3 \\
\hline 2053 & C6H11Q12-3 & $\mathrm{C}(\mathrm{OO}) \mathrm{C}(\mathrm{OO})[\mathrm{CH}] \mathrm{CCC}$ & InChl=1S/C6H13O4/c1-2-3-4-6(10-8)5-9-7/h4,6-8H,2-3,5H2,1H3 \\
\hline 2054 & C6H13-1 & {$[\mathrm{CH} 2] \mathrm{CCCCC}$} & InChl=1S/C6H13/c1-3-5-6-4-2/h1,3-6H2,2H3 \\
\hline 2055 & CHX1VO4OJ & {$[0] \mathrm{C} 1 \mathrm{CCC}(=0) \mathrm{CC} 1$} & $\operatorname{lnChl}=1 \mathrm{~S} / \mathrm{C} 6 \mathrm{H} 9 \mathrm{OO} / \mathrm{c} 7-5-1-2-6(8) 4-3-5 / \mathrm{h} 5 \mathrm{H}, 1-4 \mathrm{H} 2$ \\
\hline 2056 & NC6D2KET54 & $\mathrm{CC}(=\mathrm{O}) \mathrm{C}(\mathrm{OO}) \mathrm{C}=\mathrm{CC}$ & InChl=1S/C6H1003/c1-3-4-6(9-8)5(2)7/h3-4,6,8H,1-2H3 \\
\hline 2057 & $\mathrm{C} 6 \mathrm{H} 11 \mathrm{Q} 12-4$ & $\mathrm{C}(\mathrm{OO}) \mathrm{C}(\mathrm{OO}) \mathrm{C}[\mathrm{CH}] \mathrm{CC}$ & InChl=1S/C6H1304/c1-2-3-4-6(10-8)5-9-7/h3,6-8H,2,4-5H2,1H3 \\
\hline 2058 & C6H13-2 & $\mathrm{C}[\mathrm{CH}] \mathrm{CCCC}$ & $\operatorname{lnChl}=1 \mathrm{~S} / \mathrm{C} 6 \mathrm{H} 13 / \mathrm{c} 1-3-5-6-4-2 / \mathrm{h} 3 \mathrm{H}, 4-6 \mathrm{H} 2,1-2 \mathrm{H} 3$ \\
\hline 2059 & HX12AL1J & $\operatorname{ccccc}(=\mathrm{C}=0)[0]$ & $\operatorname{lnChl}=15 / \mathrm{C} 6 \mathrm{H} 902 / \mathrm{c} 1-2-3-4-6(8) 5-7 / \mathrm{h} 2-4 \mathrm{H} 2,1 \mathrm{H} 3$ \\
\hline 2060 & NC6D2KET56 & $\mathrm{C}(\mathrm{OO}) \mathrm{C}(=\mathrm{O}) \mathrm{CC}=\mathrm{CC}$ & InChl=1S/C6H1003/c1-2-3-4-6(7)5-9-8/h2-3,8H,4-5H2,1H3 \\
\hline 2061 & $\mathrm{C} 6 \mathrm{H} 11 \mathrm{Q} 12-5$ & $\mathrm{C}(\mathrm{OO}) \mathrm{C}(\mathrm{OO}) \mathrm{CC}[\mathrm{CH}] \mathrm{C}$ & InChl=15/C6H13O4/c1-2-3-4-6(10-8)5-9-7/h2,6-8H,3-5H2,1H3 \\
\hline 2062 & C6H13-3 & $\mathrm{CC}[\mathrm{CH}] \mathrm{CCC}$ & InChl=1S/C6H13/c1-3-5-6-4-2/h5H,3-4,6H2,1-2H3 \\
\hline 2063 & HX12ALGJ & {$[\mathrm{CH} 2] \mathrm{CCCC}(=\mathrm{O}) \mathrm{C}=\mathrm{O}$} & InChl=1S/C6H9O2/c1-2-3-4-6(8)5-7/h5H,1-4H2 \\
\hline 2064 & NC6D2KET64 & $\mathrm{O}=\mathrm{CCC}(\mathrm{OO}) \mathrm{C}=\mathrm{CC}$ & InChl=15/C6H1003/c1-2-3-6(9-8)4-5-7/h2-3,5-6,8H,4H2,1H3 \\
\hline 2065 & C6H11Q12-6 & $\mathrm{C}(\mathrm{OO}) \mathrm{C}(\mathrm{OO}) \mathrm{CCC}[\mathrm{CH} 2]$ & InChl=1S/C6H13O4/c1-2-3-4-6(10-8)5-9-7/h6-8H,1-5H2 \\
\hline 2066 & NEC6-1 & $\mathrm{CCC}([\mathrm{CH} 2])(\mathrm{C}) \mathrm{C}$ & InChl=1S/C6H13/c1-5-6(2,3)4/h2,5H2,1,3-4H3 \\
\hline 2067 & NC6D2KET65 & $\mathrm{O}=\mathrm{CC}(\mathrm{OO}) \mathrm{CC}=\mathrm{CC}$ & InChl=1S/C6H1003/c1-2-3-4-6(5-7)9-8/h2-3,5-6,8H,4H2,1H3 \\
\hline 2068 & C6H11Q13-2 & $\mathrm{C}(\mathrm{OO})[\mathrm{CH}] \mathrm{C}(\mathrm{OO}) \mathrm{CCC}$ & InChl=1S/C6H13O4/c1-2-3-6(10-8)4-5-9-7/h4,6-8H,2-3,5H2,1H3 \\
\hline 2069 & 13C6-1 & $\mathrm{CCC}(\mathrm{C}[\mathrm{CH} 2]) \mathrm{C}$ & InChl=1S/C6H13/c1-4-6(3)5-2/h6H,1,4-5H2,2-3H3 \\
\hline 2070 & HX14AL2J & $\mathrm{CCC}(=\mathrm{O}) \mathrm{CC}=\mathrm{C}[\mathrm{O}]$ & InChl=1S/C6H9O2/c1-2-6(8)4-3-5-7/h3,5H,2,4H2,1H3 \\
\hline 2071 & NC6D3KET12 & $\mathrm{O}=\mathrm{CC}(\mathrm{OO}) \mathrm{C}=\mathrm{CCC}$ & InChl=15/C6H10O3/c1-2-3-4-6(5-7)9-8/h3-6,8H,2H2,1H3 \\
\hline 2072 & C6H11Q13-4 & $\mathrm{C}(\mathrm{OO}) \mathrm{CC}(\mathrm{OO})[\mathrm{CH}] \mathrm{CC}$ & InChl=1S/C6H13O4/c1-2-3-6(10-8)4-5-9-7/h3,6-8H,2,4-5H2,1H3 \\
\hline 2073 & 13C6-2 & $\mathrm{C}[\mathrm{CH}] \mathrm{C}(\mathrm{CC}) \mathrm{C}$ & $\operatorname{lnChl}=1 \mathrm{~S} / \mathrm{C} 6 \mathrm{H} 13 / \mathrm{c} 1-4-6(3) 5-2 / \mathrm{h} 4,6 \mathrm{H}, 5 \mathrm{H} 2,1-3 \mathrm{H} 3$ \\
\hline 2074 & HX14AL6J & $[\mathrm{CH} 2] \mathrm{CC}=\mathrm{O}) \mathrm{CCC}=\mathrm{O}$ & InChl=1S/C6H9O2/c1-2-6(8)4-3-5-7/h5H,1-4H2 \\
\hline 2075 & NC6D3KET21 & $\mathrm{OOCC}(=0) \mathrm{C}=\mathrm{CCC}$ & InChl=1S/C6H1003/c1-2-3-4-6(7)5-9-8/h3-4,8H,2,5H2,1H3 \\
\hline 2076 & C6H11Q13-5 & $\mathrm{C}(\mathrm{OO}) \mathrm{CC}(\mathrm{OO}) \mathrm{C}[\mathrm{CH}] \mathrm{C}$ & InChl=1S/C6H13O4/c1-2-3-6(10-8)4-5-9-7/h2,6-8H,3-5H2,1H3 \\
\hline 2077 & $13 \mathrm{C6} 6-3$ & $\mathrm{CC}[\mathrm{C}](\mathrm{CC}) \mathrm{C}$ & InChl=1S/C6H13/c1-4-6(3)5-2/h4-5H2,1-3H3 \\
\hline 2078 & CHX1VO2Q & OOC1CCCCC1 $=0$ & InChl=1S/C6H10O3/c7-5-3-1-2-4-6(5)9-8/h6,8H,1-4H2 \\
\hline 2079 & C6H11Q13-6 & $\mathrm{C}(\mathrm{OO}) \mathrm{CC}(\mathrm{OO}) \mathrm{CC}[\mathrm{CH} 2]$ & InChl=1S/C6H13O4/c1-2-3-6(10-8)4-5-9-7/h6-8H,1-5H2 \\
\hline 2080 & $13 \mathrm{C} 6-4$ & $\mathrm{CCC}(\mathrm{CC})[\mathrm{CH} 2]$ & InChl=1S/C6H13/c1-4-6(3)5-2/h6H,3-5H2,1-2H3 \\
\hline 2081 & HX15AL6J & $\mathrm{O}=\mathrm{CCCCC}(=\mathrm{C})[\mathrm{O}]$ & InChl=1S/C6H9O2/c1-6(8)4-2-3-5-7/h5H,1-4H2 \\
\hline 2082 & CHX1VO3Q & OOC1CCCC $(=0) \mathrm{C} 1$ & InChl=15/C6H10O3/c7-5-2-1-3-6(4-5)9-8/h6,8H,1-4H2 \\
\hline 2083 & C6H11Q14-2 & $\mathrm{C}(\mathrm{OO})[\mathrm{CH}] \mathrm{CC}(\mathrm{OO}) \mathrm{CC}$ & InChl=1S/C6H1304/c1-2-6(10-8)4-3-5-9-7/h3,6-8H,2,4-5H2,1H3 \\
\hline 2084 & $\mathrm{xC6}-1$ & $\mathrm{CC}(\mathrm{C}(\mathrm{C}) \mathrm{C})[\mathrm{CH} 2]$ & InChl=15/C6H13/c1-5(2)6(3)4/h5-6H,1H2,2-4H3 \\
\hline 2085 & HX16AL1J & $\mathrm{O}=[\mathrm{C}] \mathrm{CCCCC}=\mathrm{O}$ & InChl=1S/C6H9O2/c7-5-3-1-2-4-6-8/h5H,1-4H2 \\
\hline 2086 & CHX1VO4Q & OOC1CCC( $=0) \mathrm{CC} 1$ & InChl=1S/C6H10O3/c7-5-1-3-6(9-8)4-2-5/h6,8H,1-4H2 \\
\hline 2087 & C6H11Q14-3 & $\mathrm{C}(\mathrm{OO}) \mathrm{C}[\mathrm{CH}] \mathrm{C}(\mathrm{OO}) \mathrm{CC}$ & InChl=1S/C6H13O4/c1-2-6(10-8)4-3-5-9-7/h4,6-8H,2-3,5H2,1H3 \\
\hline 2088 & $x \mathrm{CC}-2$ & $\mathrm{C}[\mathrm{C}](\mathrm{C}(\mathrm{C}) \mathrm{C}) \mathrm{C}$ & $\operatorname{lnChl}=15 / \mathrm{C} 6 \mathrm{H} 13 / \mathrm{c} 1-5(2) 6(3) 4 / \mathrm{h} 5 \mathrm{H}, 1-4 \mathrm{H} 3$ \\
\hline 2089 & HX16AL2J & $\mathrm{O}=\mathrm{CCCCC}=\mathrm{C}[\mathrm{O}]$ & $\operatorname{lnChl}=1 \mathrm{~S} / \mathrm{C} 6 \mathrm{H} 902 / \mathrm{c} 7-5-3-1-2-4-6-8 / \mathrm{h} 3,5-6 \mathrm{H}, 1-2,4 \mathrm{H} 2$ \\
\hline
\end{tabular}




\begin{tabular}{|c|c|c|c|}
\hline 2090 & C6KET12 & $\mathrm{Cl}=0) \mathrm{C}(\mathrm{OO}) \mathrm{cccc}$ & InChl=1S/C6H12O3/c1-2-3-4-6(5-7)9-8/h5-6,8H,2-4H2,1H3 \\
\hline 2091 & C6H11Q14-5 & $\mathrm{C}(\mathrm{OO}) \mathrm{CCC}(\mathrm{OO})[\mathrm{CH}] \mathrm{C}$ & InChl=1S/C6H13O4/c1-2-6(10-8)4-3-5-9-7/h2,6-8H,3-5H2,1H3 \\
\hline 2092 & IC6D1Y4-3OR & $\mathrm{C}=\mathrm{C}(\mathrm{C}) \mathrm{C}([\mathrm{O}]) \mathrm{C}(=\mathrm{O}) \mathrm{C}$ & InChl=1S/C6H9O2/c1-4(2)6(8)5(3)7/h6H,1H2,2-3H3 \\
\hline 2093 & C6KET13 & $\mathrm{C}(=0) \mathrm{CC}(\mathrm{OO}) \mathrm{CCC}$ & InChl=1S/C6H12O3/c1-2-3-6(9-8)4-5-7/h5-6,8H,2-4H2,1H3 \\
\hline 2094 & C6H11Q14-6 & $\mathrm{C}(\mathrm{OO}) \mathrm{CCC}(\mathrm{OO}) \mathrm{C}[\mathrm{CH} 2]$ & InChl=15/C6H13O4/c1-2-6(10-8)4-3-5-9-7/h6-8H,1-5H2 \\
\hline 2095 & IC6-2R & $\mathrm{C}[\mathrm{C}](\mathrm{C}) \mathrm{CCC}$ & InChl=1S/C6H13/c1-4-5-6(2)3/h4-5H2,1-3H3 \\
\hline 2096 & C6H9D14-5OOH & $\mathrm{OOCC}=\mathrm{CCC}=\mathrm{C}$ & InChl=1S/C6H1002/c1-2-3-4-5-6-8-7/h2,4-5,7H,1,3,6H2 \\
\hline 2097 & C6KET14 & $\mathrm{C}(=0) \mathrm{CCC}(\mathrm{OO}) \mathrm{CC}$ & InChl=15/C6H1203/c1-2-6(9-8)4-3-5-7/h5-6,8H,2-4H2,1H3 \\
\hline 2098 & C6H11Q15-2 & $\mathrm{C}(\mathrm{OO})[\mathrm{CH}] \mathrm{CCC}(\mathrm{OO}) \mathrm{C}$ & InChl=1S/C6H13O4/c1-6(10-8)4-2-3-5-9-7/h3,6-8H,2,4-5H2,1H3 \\
\hline 2099 & IC6-3R & $\mathrm{CC}(\mathrm{C})[\mathrm{CH}] \mathrm{CC}$ & $\operatorname{lnChl}=1 \mathrm{~S} / \mathrm{C} 6 \mathrm{H} 13 / \mathrm{c} 1-4-5-6(2) 3 / \mathrm{h5}-6 \mathrm{H}, 4 \mathrm{H} 2,1-3 \mathrm{H} 3$ \\
\hline 2100 & C6H9D15-30OH & $\mathrm{C}=\mathrm{CCC}(\mathrm{C}=\mathrm{C}) \mathrm{OO}$ & InChl=1S/C6H1002/c1-3-5-6(4-2)8-7/h3-4,6-7H,1-2,5H2 \\
\hline 2101 & C6KET15 & $\mathrm{C}(=0) \operatorname{cccc}(\mathrm{OO}) \mathrm{C}$ & InChl=1S/C6H12O3/c1-6(9-8)4-2-3-5-7/h5-6,8H,2-4H2,1H3 \\
\hline 2102 & C6H11Q15-3 & $\mathrm{C}(\mathrm{OO}) \mathrm{C}[\mathrm{CH}] \mathrm{CC}(\mathrm{OO}) \mathrm{C}$ & InChl=1S/C6H1304/c1-6(10-8)4-2-3-5-9-7/h2,6-8H,3-5H2,1H3 \\
\hline 2103 & IC6-4R & $\mathrm{CC}(\mathrm{C}) \mathrm{C}[\mathrm{CH}] \mathrm{C}$ & InChl=1S/C6H13/c1-4-5-6(2)3/h4,6H,5H2,1-3H3 \\
\hline 2104 & CYHX1EN3Q & $\mathrm{OOC1CCCC}=\mathrm{C} 1$ & InChl=1S/C6H10O2/c7-8-6-4-2-1-3-5-6/h2,4,6-7H,1,3,5H2 \\
\hline 2105 & C6KET21 & $\mathrm{C}(\mathrm{OO}) \mathrm{C}(=\mathrm{O}) \mathrm{CCCC}$ & $\operatorname{lnChl}=1 \mathrm{~S} / \mathrm{C} 6 \mathrm{H} 12 \mathrm{O} / \mathrm{c1}-2-3-4-6(7) 5-9-8 / \mathrm{h} 8 \mathrm{H}, 2-5 \mathrm{H} 2,1 \mathrm{H} 3$ \\
\hline 2106 & C6H11Q15-4 & $\mathrm{C}(\mathrm{OO}) \mathrm{CC}[\mathrm{CH}] \mathrm{C}(\mathrm{OO}) \mathrm{C}$ & InChl=1S/C6H13O4/c1-6(10-8)4-2-3-5-9-7/h4,6-8H,2-3,5H2,1H3 \\
\hline 2107 & IC6-5R & $\mathrm{CC}(\mathrm{C}) \mathrm{CC}[\mathrm{CH} 2]$ & InChl=1S/C6H13/c1-4-5-6(2)3/h6H,1,4-5H2,2-3H3 \\
\hline 2108 & CYHX1EN4Q & $\mathrm{OOC} 1 \mathrm{CCC}=\mathrm{CC} 1$ & InChl=1S/C6H1002/c7-8-6-4-2-1-3-5-6/h1-2,6-7H,3-5H2 \\
\hline 2109 & C6KET23 & $\mathrm{CC}(=0) \mathrm{C}(\mathrm{OO}) \mathrm{CCC}$ & InChl=1S/C6H12O3/c1-3-4-6(9-8)5(2)7/h6,8H,3-4H2,1-2H3 \\
\hline 2110 & C6H11Q15-6 & $\mathrm{C}(\mathrm{OO}) \mathrm{CCCC}(\mathrm{OO})[\mathrm{CH} 2]$ & InChl=1S/C6H13O4/c1-6(10-8)4-2-3-5-9-7/h6-8H,1-5H2 \\
\hline 2111 & NEC6-3R & $\mathrm{CC}(\mathrm{C})(\mathrm{C})[\mathrm{CH}] \mathrm{C}$ & InChl=1S/C6H13/c1-5-6(2,3)4/h5H,1-4H3 \\
\hline 2112 & C6KET12O & $\mathrm{C}(=0) \mathrm{C}([0]) \mathrm{CCCC}$ & $\operatorname{lnChl}=1 \mathrm{~S} / \mathrm{C} 6 \mathrm{H} 11 \mathrm{O} 2 / \mathrm{c1}-2-3-4-6(8) 5-7 / \mathrm{h} 5-6 \mathrm{H}, 2-4 \mathrm{H} 2,1 \mathrm{H} 3$ \\
\hline 2113 & C6KET24 & $\mathrm{CC}(=0) \mathrm{CC}(\mathrm{OO}) \mathrm{CC}$ & InChl=1S/C6H12O3/c1-3-6(9-8)4-5(2)7/h6,8H,3-4H2,1-2H3 \\
\hline 2114 & C6H11Q23-1 & {$[\mathrm{CH} 2] \mathrm{C}(\mathrm{OO}) \mathrm{C}(\mathrm{OO}) \mathrm{CCC}$} & InChl=1S/C6H13O4/c1-3-4-6(10-8)5(2)9-7/h5-8H,2-4H2,1H3 \\
\hline 2115 & NEC6-4R & $\mathrm{CC}(\mathrm{C})(\mathrm{C}) \mathrm{C}[\mathrm{CH} 2]$ & InChl=1S/C6H13/c1-5-6(2,3)4/h1,5H2,2-4H3 \\
\hline 2116 & C6KET130 & $\mathrm{C}(=0) \mathrm{CC}([\mathrm{O}]) \mathrm{CCC}$ & $\operatorname{lnChl}=1 \mathrm{~S} / \mathrm{C} 6 \mathrm{H} 11 \mathrm{O} 2 / \mathrm{c1}-2-3-6(8) 4-5-7 / \mathrm{h} 5-6 \mathrm{H}, 2-4 \mathrm{H} 2,1 \mathrm{H} 3$ \\
\hline 2117 & C6KET25 & $\mathrm{CC}(=0) \mathrm{CCC}(\mathrm{OO}) \mathrm{C}$ & InChl=1S/C6H12O3/c1-5(7)3-4-6(2)9-8/h6,8H,3-4H2,1-2H3 \\
\hline 2118 & $\mathrm{C} 6 \mathrm{H} 11 \mathrm{Q} 23-4$ & $\mathrm{CC}(\mathrm{OO}) \mathrm{C}(\mathrm{OO})[\mathrm{CH}] \mathrm{CC}$ & InChl=1S/C6H13O4/c1-3-4-6(10-8)5(2)9-7/h4-8H,3H2,1-2H3 \\
\hline 2119 & NC6H14 & $\mathrm{cccccc}$ & $\operatorname{lnChl=15/C6H14/c1-3-5-6-4-2/h3-6H2,1-2H3~}$ \\
\hline 2120 & C6KET14O & $\mathrm{C}(=0) \mathrm{CCC}([0]) \mathrm{CC}$ & InChl=15/C6H1102/c1-2-6(8)4-3-5-7/h5-6H,2-4H2,1H3 \\
\hline 2121 & C6KET26 & $\mathrm{CC}(=0) \operatorname{CcCC}(00)$ & $\operatorname{lnChl}=1 \mathrm{~S} / \mathrm{C} 6 \mathrm{H} 12 \mathrm{O} / \mathrm{c1}-6 \mathrm{6}(7) 4-2-3-5-9-8 / \mathrm{h} 8 \mathrm{H}, 2-5 \mathrm{H} 2,1 \mathrm{H} 3$ \\
\hline 2122 & $\mathrm{C} 6 \mathrm{H} 11 \mathrm{Q} 23-5$ & $\mathrm{CC}(\mathrm{OO}) \mathrm{C}(\mathrm{OO}) \mathrm{C}[\mathrm{CH}] \mathrm{C}$ & InChl=15/C6H13O4/c1-3-4-6(10-8)5(2)9-7/h3,5-8H,4H2,1-2H3 \\
\hline 2123 & NEC6 & $\operatorname{ccc}(\mathrm{C})(\mathrm{C}) \mathrm{C}$ & InChl=1S/C6H14/c1-5-6(2,3)4/h5H2,1-4H3 \\
\hline 2124 & C6KET150 & $\mathrm{C}(=0) \operatorname{cccc}([0]) \mathrm{C}$ & $\operatorname{lnChl}=1 \mathrm{~S} / \mathrm{C} 6 \mathrm{H} 11 \mathrm{O} 2 / \mathrm{c} 1-6(8) 4-2-3-5-7 / \mathrm{h} 5-6 \mathrm{H}, 2-4 \mathrm{H} 2,1 \mathrm{H} 3$ \\
\hline 2125 & C6KET31 & $\mathrm{C}(\mathrm{OO}) \mathrm{CC}(=\mathrm{O}) \mathrm{CCC}$ & InChl=1S/C6H12O3/c1-2-3-6(7)4-5-9-8/h8H,2-5H2,1H3 \\
\hline 2126 & $\mathrm{C} 6 \mathrm{H} 11 \mathrm{Q} 23-6$ & $\mathrm{CC}(\mathrm{OO}) \mathrm{C}(\mathrm{OO}) \mathrm{CC}[\mathrm{CH} 2]$ & InChl=1S/C6H13O4/c1-3-4-6(10-8)5(2)9-7/h5-8H,1,3-4H2,2H3 \\
\hline 2127 & $13 \mathrm{C6}$ & $\operatorname{ccc}(\operatorname{CC}) \mathrm{C}$ & InChl=1S/C6H14/c1-4-6(3)5-2/h6H,4-5H2,1-3H3 \\
\hline 2128 & C6KET210 & $C([0]) C(=0) C C C C$ & $\operatorname{lnChl}=1 \mathrm{~S} / \mathrm{C} 6 \mathrm{H} 11 \mathrm{O} 2 / \mathrm{c} 1-2-3-4-6(8) 5-7 / \mathrm{h} 2-5 \mathrm{H} 2,1 \mathrm{H} 3$ \\
\hline 2129 & C6KET32 & $\mathrm{CC}(\mathrm{OO}) \mathrm{C}(=\mathrm{O}) \mathrm{CCC}$ & InChl=1S/C6H12O3/c1-3-4-6(7)5(2)9-8/h5,8H,3-4H2,1-2H3 \\
\hline 2130 & $\mathrm{C} 6 \mathrm{H} 11 \mathrm{Q} 24-1$ & {$[\mathrm{CH} 2] \mathrm{C}(\mathrm{OO}) \mathrm{CC}(\mathrm{OO}) \mathrm{CC}$} & $\operatorname{lnChl}=1 \mathrm{~S} / \mathrm{C} 6 \mathrm{H} 13 \mathrm{O} 4 / \mathrm{c1}-3-6(10-8) 4-5(2) 9-7 / \mathrm{h} 5-8 \mathrm{H}, 2-4 \mathrm{H} 2,1 \mathrm{H} 3$ \\
\hline 2131 & $\mathrm{xC6}$ & $\operatorname{CC}(\mathrm{C}(\mathrm{C}) \mathrm{C}) \mathrm{C}$ & InChl=1S/C6H14/c1-5(2)6(3)4/h5-6H,1-4H3 \\
\hline 2132 & C6KET230 & $\mathrm{CC}(=0) \mathrm{C}([\mathrm{O}]) \mathrm{CCC}$ & $\operatorname{lnChl}=1 \mathrm{~S} / \mathrm{C} 6 \mathrm{H} 11 \mathrm{O} 2 / \mathrm{c1}-3-4-6(8) 5(2) 7 / \mathrm{h} 6 \mathrm{H}, 3-4 \mathrm{H} 2,1-2 \mathrm{H} 3$ \\
\hline 2133 & C6KET34 & $\mathrm{CCC}(=0) \mathrm{C}(\mathrm{OO}) \mathrm{CC}$ & InChl=1S/C6H12O3/c1-3-5(7)6(4-2)9-8/h6,8H,3-4H2,1-2H3 \\
\hline 2134 & $\mathrm{C} 6 \mathrm{H} 11 \mathrm{Q} 24-3$ & $\mathrm{CC}(\mathrm{OO})[\mathrm{CH}] \mathrm{C}(\mathrm{OO}) \mathrm{CC}$ & InChl=1S/C6H13O4/c1-3-6(10-8)4-5(2)9-7/h4-8H,3H2,1-2H3 \\
\hline 2135 & IC6 & $\operatorname{cccc}(\mathrm{C}) \mathrm{C}$ & $\operatorname{lnChl}=1 \mathrm{~S} / \mathrm{C} 6 \mathrm{H} 14 / \mathrm{c} 1-4-5-6(2) 3 / \mathrm{h} 6 \mathrm{H}, 4-5 \mathrm{H} 2,1-3 \mathrm{H} 3$ \\
\hline 2136 & C6KET24O & $\mathrm{CC}(=\mathrm{O}) \mathrm{CC}([\mathrm{O}]) \mathrm{CC}$ & InChl=1S/C6H11O2/c1-3-6(8)4-5(2)7/h6H,3-4H2,1-2H3 \\
\hline 2137 & C6KET35 & $\operatorname{ccC}(=0) \operatorname{cc}(\mathrm{OO}) \mathrm{C}$ & InChl=1S/C6H12O3/c1-3-6(7)4-5(2)9-8/h5,8H,3-4H2,1-2H3 \\
\hline 2138 & $\mathrm{C} 6 \mathrm{H} 11 \mathrm{Q} 24-5$ & $\mathrm{CC}(\mathrm{OO}) \mathrm{CC}(\mathrm{OO})[\mathrm{CH}] \mathrm{C}$ & InChl=1S/C6H13O4/c1-3-6(10-8)4-5(2)9-7/h3,5-8H,4H2,1-2H3 \\
\hline 2139 & $\mathrm{C} 6 \mathrm{H} 5 \mathrm{O}$ & $\mathrm{C} 1([\mathrm{O}])=\mathrm{CC}=\mathrm{CC}=\mathrm{C} 1$ & InChl=1S/C6H50/c7-6-4-2-1-3-5-6/h1-5H \\
\hline 2140 & C6KET250 & $\mathrm{CC}(=0) \operatorname{CCC}([0]) \mathrm{C}$ & $\operatorname{lnChl}=15 / \mathrm{C} 6 \mathrm{H} 1102 / \mathrm{c} 1-5(7) 3-4-6(2) 8 / \mathrm{h} 5 \mathrm{H}, 3-4 \mathrm{H} 2,1-2 \mathrm{H} 3$ \\
\hline 2141 & C6KET36 & $\mathrm{CCC}(=0) \mathrm{CCC}(\mathrm{OO})$ & $\operatorname{lnChl}=1 \mathrm{~S} / \mathrm{C} 6 \mathrm{H} 12 \mathrm{O} / \mathrm{c1}-2-6(7) 4-3-5-9-8 / \mathrm{h} 8 \mathrm{H}, 2-5 \mathrm{H} 2,1 \mathrm{H} 3$ \\
\hline 2142 & $\mathrm{C} 6 \mathrm{H} 11 \mathrm{Q} 24-6$ & $\mathrm{CC}(\mathrm{OO}) \mathrm{CC}(\mathrm{OO}) \mathrm{C}[\mathrm{CH} 2]$ & InChl=1S/C6H13O4/c1-3-6(10-8)4-5(2)9-7/h5-8H,1,3-4H2,2H3 \\
\hline 2143 & $\mathrm{C} 6 \mathrm{H} 4 \mathrm{OH}$ & $\mathrm{C} 1=[\mathrm{C}] \mathrm{C}=\mathrm{C}(\mathrm{O}) \mathrm{C}=\mathrm{C} 1$ & InChl=1S/C6H5O/c7-6-4-2-1-3-5-6/h1-2,4-5,7H \\
\hline 2144 & C6KET260 & $\mathrm{CC}(=0) \operatorname{cccc}([0])$ & $\operatorname{lnChl}=1 \mathrm{~S} / \mathrm{C} 6 \mathrm{H} 11 \mathrm{O} 2 / \mathrm{c} 1-6(8) 4-2-3-5-7 / \mathrm{h} 2-5 \mathrm{H} 2,1 \mathrm{H} 3$ \\
\hline 2145 & $\mathrm{C} 6 \mathrm{H} 11012-30 \mathrm{OH}$ & $\mathrm{C} 1 \mathrm{C}(\mathrm{O} 1) \mathrm{C}(\mathrm{OO}) \mathrm{CCC}$ & $\operatorname{lnChl}=1 \mathrm{~S} / \mathrm{C} 6 \mathrm{H} 12 \mathrm{O} / \mathrm{c} 1-2-3-5(9-7) 6-4-8-6 / \mathrm{h} 5-7 \mathrm{H}, 2-4 \mathrm{H} 2,1 \mathrm{H} 3$ \\
\hline 2146 & C6H11Q25-1 & {$[\mathrm{CH} 2] \mathrm{C}(\mathrm{OO}) \mathrm{CCC}(\mathrm{OO}) \mathrm{C}$} & InChl=1S/C6H13O4/c1-5(9-7)3-4-6(2)10-8/h5-8H,1,3-4H2,2H3 \\
\hline 2147 & DMF25CH2 & $\mathrm{C}=\mathrm{C} 1 \mathrm{C}=\mathrm{CC}(=\mathrm{C}) \mathrm{O} 1$ & InChl=1S/C6H6O/c1-5-3-4-6(2)7-5/h3-4H,1-2H2 \\
\hline 2148 & C6KET310 & $\operatorname{cccC}(=0) \operatorname{cC}([0])$ & $\operatorname{lnChl}=1 \mathrm{~S} / \mathrm{C} 6 \mathrm{H} 1102 / \mathrm{c} 1-2-3-6(8) 4-5-7 / \mathrm{h} 2-5 \mathrm{H} 2,1 \mathrm{H} 3$ \\
\hline 2149 & $\mathrm{C} 6 \mathrm{H} 11012-4 \mathrm{OOH}$ & $\mathrm{C} 1 \mathrm{C}(\mathrm{O} 1) \mathrm{CC}(\mathrm{OO}) \mathrm{CC}$ & InChl=1S/C6H12O3/c1-2-5(9-7)3-6-4-8-6/h5-7H,2-4H2,1H3 \\
\hline 2150 & $\mathrm{C} 6 \mathrm{H} 11 \mathrm{Q} 25-3$ & $\mathrm{CC}(\mathrm{OO})[\mathrm{CH}] \mathrm{CC}(\mathrm{OO}) \mathrm{C}$ & InChl=1S/C6H13O4/c1-5(9-7)3-4-6(2)10-8/h3,5-8H,4H2,1-2H3 \\
\hline 2151 & H145TE3O & $\mathrm{C}=\mathrm{CC}(=\mathrm{O}) \mathrm{C}=\mathrm{C}=\mathrm{C}$ & InChl=1S/C6H6O/c1-3-5-6(7)4-2/h4-5H,1-2H2 \\
\hline 2152 & C6KET32O & $\operatorname{cccc}(=0) c([0]) \mathrm{C}$ & $\operatorname{lnChl}=15 / C 6 H 1102 / c 1-3-4-6(8) 5(2) 7 / \mathrm{h} 5 \mathrm{H}, 3-4 \mathrm{H} 2,1-2 \mathrm{H} 3$ \\
\hline 2153 & $\mathrm{C} 6 \mathrm{H} 11012-5 \mathrm{OOH}$ & $\mathrm{C} 1 \mathrm{C}(\mathrm{O} 1) \mathrm{CCC}(\mathrm{OO}) \mathrm{C}$ & $\operatorname{lnChl}=1 \mathrm{~S} / \mathrm{C} 6 \mathrm{H} 12 \mathrm{O} / \mathrm{c} 1-5(9-7) 2-3-6-4-8-6 / \mathrm{h} 5-7 \mathrm{H}, 2-4 \mathrm{H} 2,1 \mathrm{H} 3$ \\
\hline 2154 & C6H11Q34-1 & {$[\mathrm{CH} 2] \mathrm{CC}(\mathrm{OO}) \mathrm{C}(\mathrm{OO}) \mathrm{CC}$} & InChl=1S/C6H13O4/c1-3-5(9-7)6(4-2)10-8/h5-8H,1,3-4H2,2H3 \\
\hline 2155 & H235TE1O & $\mathrm{C}=\mathrm{CC}=\mathrm{C}=\mathrm{CC}=\mathrm{O}$ & $\operatorname{lnChl}=1 \mathrm{~S} / \mathrm{C} 6 \mathrm{H} 6 \mathrm{O} / \mathrm{c1} 1-2-3-4-5-6-7 / \mathrm{h} 2-3,5-6 \mathrm{H}, 1 \mathrm{H} 2$ \\
\hline 2156 & C6KET34O & $\operatorname{ccC}([0]) \mathrm{C}(=0) \mathrm{CC}$ & InChl=1S/C6H11O2/c1-3-5(7)6(8)4-2/h5H,3-4H2,1-2H3 \\
\hline 2157 & $\mathrm{C} 6 \mathrm{H} 11012-60 \mathrm{OH}$ & $\mathrm{C} 1 \mathrm{C}(\mathrm{O} 1) \mathrm{CCCC}(\mathrm{OO})$ & InChl=1S/C6H12O3/c7-9-4-2-1-3-6-5-8-6/h6-7H,1-5H2 \\
\hline 2158 & C6H11Q34-2 & $\mathrm{C}[\mathrm{CH}] \mathrm{C}(\mathrm{OO}) \mathrm{C}(\mathrm{OO}) \mathrm{CC}$ & InChl=1S/C6H13O4/c1-3-5(9-7)6(4-2)10-8/h3,5-8H,4H2,1-2H3 \\
\hline 2159 & $\mathrm{~V} 2 \mathrm{~F}$ & $\mathrm{C}=\mathrm{CC} 1=\mathrm{CC}=\mathrm{CO} 1$ & InChl=1S/C6H6O/c1-2-6-4-3-5-7-6/h2-5H,1H2 \\
\hline 2160 & C6KET350 & $\mathrm{CC}([0]) \mathrm{CC}(=0) \mathrm{CC}$ & InChl=1S/C6H11O2/c1-3-6(8)4-5(2)7/h5H,3-4H2,1-2H3 \\
\hline 2161 & C6H11013-2OOH & $\mathrm{C} 1 \mathrm{C}(\mathrm{OO}) \mathrm{C}(\mathrm{O} 1) \mathrm{CCC}$ & InChl=1S/C6H12O3/c1-2-3-5-6(9-7)4-8-5/h5-7H,2-4H2,1H3 \\
\hline 2162 & 13C6Q12-3 & $\mathrm{C}[\mathrm{C}](\mathrm{C}(\mathrm{OO}) \mathrm{COO}) \mathrm{CC}$ & InChl=1S/C6H13O4/c1-3-5(2)6(10-8)4-9-7/h6-8H,3-4H2,1-2H3 \\
\hline 2163 & $\mathrm{C} 6 \mathrm{H} 5 \mathrm{OH}$ & $\mathrm{C} 1(\mathrm{O})=\mathrm{CC}=\mathrm{CC}=\mathrm{C} 1$ & InChl=1S/C6H6O/c7-6-4-2-1-3-5-6/h1-5,7H \\
\hline 2164 & C6KET360 & $\mathrm{C}([0]) \mathrm{CCC}(=0) \mathrm{CC}$ & InChl=1S/C6H1102/c1-2-6(8)4-3-5-7/h2-5H2,1H3 \\
\hline 2165 & C6H11013-40OH & $\mathrm{C} 1 \mathrm{CC}(\mathrm{O} 1) \mathrm{C}(\mathrm{OO}) \mathrm{CC}$ & InChl=1S/C6H12O3/c1-2-5(9-7)6-3-4-8-6/h5-7H,2-4H2,1H3 \\
\hline 2166 & $13 \mathrm{C} 6 \mathrm{Q} 12-4$ & {$[\mathrm{CH} 2] \mathrm{C}(\mathrm{C}(\mathrm{OO}) \mathrm{COO}) \mathrm{CC}$} & InChl=1S/C6H13O4/c1-3-5(2)6(10-8)4-9-7/h5-8H,2-4H2,1H3 \\
\hline 2167 & $\mathrm{C} 6 \mathrm{H} 6 \mathrm{O}-24$ & $\mathrm{C} 1=\mathrm{CC}=\mathrm{CCC} 1=\mathrm{O}$ & InChl=1S/C6H6O/c7-6-4-2-1-3-5-6/h1-4H,5H2 \\
\hline 2168 & $\mathrm{C} 6 \mathrm{H} 111 \mathrm{O} 2-3$ & $\mathrm{CCCC}(\mathrm{O}[\mathrm{O}]) \mathrm{C}=\mathrm{C}$ & $\operatorname{lnChl}=1 \mathrm{~S} / \mathrm{C} 6 \mathrm{H} 11 \mathrm{O} 2 / \mathrm{c} 1-3-5-6(4-2) 8-7 / \mathrm{h} 4,6 \mathrm{H}, 2-3,5 \mathrm{H} 2,1 \mathrm{H} 3$ \\
\hline 2169 & $\mathrm{C} 6 \mathrm{H} 11013-50 \mathrm{OH}$ & $\mathrm{C} 1 \mathrm{CC}(\mathrm{O} 1) \mathrm{CC}(\mathrm{OO}) \mathrm{C}$ & $\mathrm{InChl}=1 \mathrm{~S} / \mathrm{C} 6 \mathrm{H} 12 \mathrm{O} / \mathrm{c} 1-5(9-7) 4-6-2-3-8-6 / \mathrm{h} 5-7 \mathrm{H}, 2-4 \mathrm{H} 2,1 \mathrm{H} 3$ \\
\hline 2170 & $13 \mathrm{C} 6 \mathrm{Q} 12-5$ & $\mathrm{CC}(\mathrm{C}(\mathrm{OO}) \mathrm{COO})[\mathrm{CH}] \mathrm{C}$ & $\operatorname{lnChl}=1 \mathrm{~S} / \mathrm{C} 6 \mathrm{H} 13 \mathrm{O} 4 / \mathrm{c1} 1-3-5(2) 6(10-8) 4-9-7 / \mathrm{h} 3,5-8 \mathrm{H}, 4 \mathrm{H} 2,1-2 \mathrm{H} 3$ \\
\hline 2171 & $\mathrm{C} 6 \mathrm{H} 6 \mathrm{O}-25$ & $\mathrm{O}=\mathrm{C} 1 \mathrm{C}=\mathrm{CCC}=\mathrm{C} 1$ & InChl=1S/C6H6O/c7-6-4-2-1-3-5-6/h2-5H,1H2 \\
\hline 2172 & C6H11102-4 & $\mathrm{CCC}(\mathrm{O}[\mathrm{O}]) \mathrm{CC}=\mathrm{C}$ & InChl=1S/C6H11O2/c1-3-5-6(4-2)8-7/h3,6H,1,4-5H2,2H3 \\
\hline 2173 & C6H11013-60OH & $\mathrm{C} 1 \mathrm{CC}(\mathrm{O} 1) \mathrm{CCC}(\mathrm{OO})$ & $\operatorname{lnChl}=1 \mathrm{~S} / \mathrm{C} 6 \mathrm{H} 12 \mathrm{O} / \mathrm{c} 7-9-4-1-2-6-3-5-8-6 / \mathrm{h} 6-7 \mathrm{H}, 1-5 \mathrm{H} 2$ \\
\hline 2174 & 13C6Q12-6 & $\mathrm{CC}(\mathrm{C}(\mathrm{OO}) \mathrm{COO}) \mathrm{C}[\mathrm{CH} 2]$ & $\operatorname{lnChl}=15 / C 6 H 1304 / c 1-3-5(2) 6(10-8) 4-9-7 / h 5-8 \mathrm{H}, 1,3-4 \mathrm{H} 2,2 \mathrm{H} 3$ \\
\hline 2175 & C4D13KET & $\mathrm{C}=\mathrm{CC}=\mathrm{CC}=\mathrm{C}=\mathrm{O}$ & InChl=1S/C6H6O/c1-2-3-4-5-6-7/h2-5H,1H2 \\
\hline 2176 & C6H111O2-5 & $\mathrm{CC}(\mathrm{O}[\mathrm{O}]) \mathrm{CCC}=\mathrm{C}$ & InChl=1S/C6H1102/c1-3-4-5-6(2)8-7/h3,6H,1,4-5H2,2H3 \\
\hline 2177 & C6H11014-2OOH & $\mathrm{C} 1 \mathrm{C}(\mathrm{OO}) \mathrm{CC}(\mathrm{O} 1) \mathrm{CC}$ & InChl=1S/C6H12O3/c1-2-5-3-6(9-7)4-8-5/h5-7H,2-4H2,1H3 \\
\hline 2178 & $13 \mathrm{C} 6 \mathrm{Q} 13-2$ & $\mathrm{OOC}[\mathrm{CH}] \mathrm{C}(\mathrm{OO})(\mathrm{CC}) \mathrm{C}$ & InChl=1S/C6H13O4/c1-3-6(2,10-8)4-5-9-7/h4,7-8H,3,5H2,1-2H3 \\
\hline 2179 & C6H6O-B1 & $\mathrm{O}=\mathrm{C} 1[\mathrm{CH}] \mathrm{CC}=\mathrm{C}[\mathrm{CH}] 1$ & $\operatorname{lnChl}=1 \mathrm{~S} / \mathrm{C} 6 \mathrm{H} 6 \mathrm{O} / \mathrm{c} 7-6-4-2-1-3-5-6 / \mathrm{h} 1-2,4-5 \mathrm{H}, 3 \mathrm{H} 2$ \\
\hline 2180 & $\mathrm{C} 6 \mathrm{H} 11102-6$ & $\mathrm{C}(\mathrm{O}[\mathrm{O}]) \mathrm{CCCC}=\mathrm{C}$ & InChl=1S/C6H11O2/c1-2-3-4-5-6-8-7/h2H,1,3-6H2 \\
\hline
\end{tabular}




\begin{tabular}{|c|c|c|c|}
\hline 2181 & $\mathrm{C} 6 \mathrm{H} 11014-30 \mathrm{OH}$ & $\mathrm{C} 1 \mathrm{CC}(\mathrm{OO}) \mathrm{C}(\mathrm{O} 1) \mathrm{CC}$ & InChl=1S/C6H12O3/c1-2-5-6(9-7)3-4-8-5/h5-7H,2-4H2,1H3 \\
\hline 2182 & 13C6Q13-4 & $\mathrm{OOCCC}(\mathrm{OO})(\mathrm{CC})[\mathrm{CH} 2]$ & InChl=15/C6H13O4/c1-3-6(2,10-8)4-5-9-7/h7-8H,2-5H2,1H3 \\
\hline 2183 & CHE2104J & $\mathrm{C} 1(=\mathrm{O}) \mathrm{C}=\mathrm{C}[\mathrm{CH}] \mathrm{CC} 1$ & InChl=1S/C6H7O/c7-6-4-2-1-3-5-6/h1-2,4H,3,5H2 \\
\hline 2184 & C6H112O2-4 & $\mathrm{CCC}(\mathrm{O}[\mathrm{O}]) \mathrm{C}=\mathrm{CC}$ & $\operatorname{lnChl}=1 \mathrm{~S} / \mathrm{C} 6 \mathrm{H} 11 \mathrm{O} 2 / \mathrm{c} 1-3-5-6(4-2) 8-7 / \mathrm{h} 3,5-6 \mathrm{H}, 4 \mathrm{H} 2,1-2 \mathrm{H} 3$ \\
\hline 2185 & $\mathrm{C} 6 \mathrm{H} 11014-5 \mathrm{OOH}$ & $\mathrm{C} 1 \mathrm{CCC}(\mathrm{O} 1) \mathrm{C}(\mathrm{OO}) \mathrm{C}$ & InChl=1S/C6H1203/c1-5(9-7)6-3-2-4-8-6/h5-7H,2-4H2,1H3 \\
\hline 2186 & I3C6Q13-5 & $\mathrm{OOCCC}(\mathrm{OO})([\mathrm{CH}] \mathrm{C}) \mathrm{C}$ & InChl=1S/C6H13O4/c1-3-6(2,10-8)4-5-9-7/h3,7-8H,4-5H2,1-2H3 \\
\hline 2187 & CHE2105J & $\mathrm{C} 1(=\mathrm{O}) \mathrm{C}=\mathrm{CC}[\mathrm{CH}] \mathrm{C} 1$ & InChl=1S/C6H7O/c7-6-4-2-1-3-5-6/h2-4H,1,5H2 \\
\hline 2188 & $\mathrm{C} 6 \mathrm{H} 112 \mathrm{O} 2-5$ & $\mathrm{CC}(\mathrm{O}[\mathrm{O}]) \mathrm{CC}=\mathrm{CC}$ & InChl=1S/C6H11O2/c1-3-4-5-6(2)8-7/h3-4,6H,5H2,1-2H3 \\
\hline 2189 & C6H11014-60OH & $\mathrm{C} 1 \mathrm{CCC}(\mathrm{O} 1) \mathrm{CC}(\mathrm{OO})$ & $\operatorname{lnChl}=1 \mathrm{~S} / \mathrm{C} 6 \mathrm{H} 12 \mathrm{O} / \mathrm{c} 7-9-5-3-6-2-1-4-8-6 / \mathrm{h6}-7 \mathrm{H}, 1-5 \mathrm{H} 2$ \\
\hline 2190 & $13 \mathrm{C} 6 \mathrm{Q} 13-6$ & $\mathrm{OOCCC}(\mathrm{OO})(\mathrm{C}[\mathrm{CH} 2]) \mathrm{C}$ & $\operatorname{lnChl}=1 \mathrm{~S} / \mathrm{C} 6 \mathrm{H} 13 \mathrm{O} 4 / \mathrm{c1}-3-6(2,10-8) 4-5-9-7 / \mathrm{h} 7-8 \mathrm{H}, 1,3-5 \mathrm{H} 2,2 \mathrm{H} 3$ \\
\hline 2191 & CPROPC3H4-A & $\mathrm{C}=\mathrm{C}[\mathrm{CH}] \mathrm{C}(\mathrm{C} 1) \mathrm{C} 1(=\mathrm{O})$ & InChl=1S/C6H7O/c1-2-3-5-4-6(5)7/h2-3,5H,1,4H2 \\
\hline 2192 & $\mathrm{C} 6 \mathrm{H} 112 \mathrm{O} 2-6$ & $\mathrm{C}(\mathrm{O}[\mathrm{O}]) \mathrm{CCC}=\mathrm{CC}$ & $\operatorname{lnChl}=1 \mathrm{~S} / \mathrm{C} 6 \mathrm{H} 11 \mathrm{O} 2 / \mathrm{c} 1-2-3-4-5-6-8-7 / \mathrm{h} 2-3 \mathrm{H}, 4-6 \mathrm{H} 2,1 \mathrm{H} 3$ \\
\hline 2193 & $\mathrm{C} 6 \mathrm{H} 11015-2 \mathrm{OOH}$ & $\mathrm{C} 1 \mathrm{C}(\mathrm{OO}) \mathrm{CCC}(\mathrm{O} 1) \mathrm{C}$ & InChl=1S/C6H12O3/c1-5-2-3-6(9-7)4-8-5/h5-7H,2-4H2,1H3 \\
\hline 2194 & I3C6Q14-2 & $\mathrm{OOC}[\mathrm{CH}] \mathrm{C}(\mathrm{COO}) \mathrm{CC}$ & InChl=1S/C6H13O4/c1-2-6(5-10-8)3-4-9-7/h3,6-8H,2,4-5H2,1H3 \\
\hline 2195 & $\mathrm{C} 6 \mathrm{H} 113 \mathrm{O} 2-2$ & $\mathrm{CC}(\mathrm{O}[\mathrm{O}]) \mathrm{C}=\mathrm{CCC}$ & $\operatorname{lnChl}=15 / C 6 H 1102 / c 1-3-4-5-6(2) 8-7 / h 4-6 \mathrm{H}, 3 \mathrm{H} 2,1-2 \mathrm{H} 3$ \\
\hline 2196 & $\mathrm{C} 6 \mathrm{H} 11015-30 \mathrm{OH}$ & $\mathrm{C} 1 \mathrm{CC}(\mathrm{OOO}) \mathrm{CC}(01) \mathrm{C}$ & InChl=1S/C6H12O3/c1-5-4-6(9-7)2-3-8-5/h5-7H,2-4H2,1H3 \\
\hline 2197 & 13C6Q14-3 & OOCC[C](COO)CC & InChl=1S/C6H13O4/c1-2-6(5-10-8)3-4-9-7/h7-8H,2-5H2,1H3 \\
\hline 2198 & $\mathrm{C} 6 \mathrm{H} 113 \mathrm{O} 2-1$ & $\mathrm{C}(\mathrm{O}[\mathrm{O}]) \mathrm{CC}=\mathrm{CCC}$ & InChl=15/C6H11O2/c1-2-3-4-5-6-8-7/h3-4H,2,5-6H2,1H3 \\
\hline 2199 & $\mathrm{C} 6 \mathrm{H} 11015-40 \mathrm{OH}$ & $\mathrm{C} 1 \mathrm{CCC}(\mathrm{OO}) \mathrm{C}(\mathrm{O} 1) \mathrm{C}$ & InChl=1S/C6H12O3/c1-5-6(9-7)3-2-4-8-5/h5-7H,2-4H2,1H3 \\
\hline 2200 & I3C6Q14-5 & $\mathrm{OOCCC}(\mathrm{COO})[\mathrm{CH}] \mathrm{C}$ & InChl=1S/C6H13O4/c1-2-6(5-10-8)3-4-9-7/h2,6-8H,3-5H2,1H3 \\
\hline 2201 & DMF252J & $\mathrm{CC} 1=\mathrm{CC}=\mathrm{C}([\mathrm{CH} 2]) \mathrm{O} 1$ & InChl=1S/C6H7O/c1-5-3-4-6(2)7-5/h3-4H,1H2,2H3 \\
\hline 2202 & C6H1010OH3-4 & $\mathrm{CC}[\mathrm{CH}] \mathrm{C}(\mathrm{OO}) \mathrm{C}=\mathrm{C}$ & $\operatorname{lnChl}=15 / \mathrm{C} 6 \mathrm{H} 1102 / \mathrm{c} 1-3-5-6(4-2) 8-7 / \mathrm{h} 4-7 \mathrm{H}, 2-3 \mathrm{H} 2,1 \mathrm{H} 3$ \\
\hline 2203 & C6H11015-60OH & $\mathrm{C} 1 \mathrm{CCCC}(\mathrm{O} 1) \mathrm{C}(\mathrm{OO})$ & $\operatorname{lnChl}=15 / C 6 H 1203 / c 7-9-5-6-3-1-2-4-8-6 / h 6-7 \mathrm{H}, 1-5 \mathrm{H} 2$ \\
\hline 2204 & $13 \mathrm{C} 6 \mathrm{Q} 14-6$ & $\mathrm{OOCCC}(\mathrm{COO}) \mathrm{C}[\mathrm{CH} 2]$ & $\operatorname{lnChl}=1 \mathrm{~S} / \mathrm{C} 6 \mathrm{H} 13 \mathrm{O} 4 / \mathrm{c1}-2-6(5-10-8) 3-4-9-7 / \mathrm{h} 6-8 \mathrm{H}, 1-5 \mathrm{H} 2$ \\
\hline 2205 & DMF253J & $\mathrm{CC} 1=\mathrm{C}[\mathrm{C}]=\mathrm{C}(\mathrm{C}) \mathrm{O} 1$ & InChl=1S/C6H7O/c1-5-3-4-6(2)7-5/h3H,1-2H3 \\
\hline 2206 & C6H1010OH3-5 & $\mathrm{C}[\mathrm{CH}] \mathrm{CC}(\mathrm{OO}) \mathrm{C}=\mathrm{C}$ & InChl=1S/C6H1102/c1-3-5-6(4-2)8-7/h3-4,6-7H,2,5H2,1H3 \\
\hline 2207 & $\mathrm{C} 6 \mathrm{H} 11023-10 \mathrm{OH}$ & $\mathrm{C}(\mathrm{OO}) \mathrm{C} 1 \mathrm{C}(\mathrm{O} 1) \mathrm{CCC}$ & $\operatorname{lnChl}=1 \mathrm{~S} / \mathrm{C} 6 \mathrm{H} 12 \mathrm{O} / \mathrm{c} 1-2-3-5-6(9-5) 4-8-7 / \mathrm{h} 5-7 \mathrm{H}, 2-4 \mathrm{H} 2,1 \mathrm{H} 3$ \\
\hline 2208 & I3C6Q15-2 & $\mathrm{OOC}[\mathrm{CH}] \mathrm{C}(\mathrm{C}(\mathrm{OO}) \mathrm{C}) \mathrm{C}$ & InChl=15/C6H13O4/c1-5(3-4-9-7)6(2)10-8/h3,5-8H,4H2,1-2H3 \\
\hline 2209 & E2F2J-A & $\mathrm{C}[\mathrm{CH}] \mathrm{C} 1=\mathrm{CC}=\mathrm{CO} 1$ & $\operatorname{lnChl}=1 \mathrm{~S} / \mathrm{C} 6 \mathrm{H} 7 \mathrm{O} / \mathrm{c} 1-2-6-4-3-5-7-6 / \mathrm{h} 2-5 \mathrm{H}, 1 \mathrm{H} 3$ \\
\hline 2210 & C6H1010OH3-6 & {$[\mathrm{CH} 2] \mathrm{CCC}(\mathrm{OO}) \mathrm{C}=\mathrm{C}$} & $\operatorname{lnChl}=1 \mathrm{~S} / \mathrm{C} 6 \mathrm{H} 1102 / \mathrm{c} 1-3-5-6(4-2) 8-7 / \mathrm{h} 4,6-7 \mathrm{H}, 1-3,5 \mathrm{H} 2$ \\
\hline 2211 & $\mathrm{C} 6 \mathrm{H} 11023-40 \mathrm{OH}$ & $\mathrm{CC} 1 \mathrm{C}(\mathrm{O} 1) \mathrm{C}(\mathrm{OO}) \mathrm{CC}$ & InChl=1S/C6H12O3/c1-3-5(9-7)6-4(2)8-6/h4-7H,3H2,1-2H3 \\
\hline 2212 & I3C6Q15-3 & $0 \mathrm{OCC}[\mathrm{C}](\mathrm{C}(\mathrm{OO}) \mathrm{C}) \mathrm{C}$ & InChl=1S/C6H13O4/c1-5(3-4-9-7)6(2)10-8/h6-8H,3-4H2,1-2H3 \\
\hline 2213 & E2F2J-P & {$[\mathrm{CH} 2] \mathrm{CC} 1=\mathrm{CC}=\mathrm{CO} 1$} & $\operatorname{lnChl}=1 \mathrm{~S} / \mathrm{C} 6 \mathrm{H} 7 \mathrm{O} / \mathrm{c1}-2-6-4-3-3-5-7-6 / \mathrm{h} 3-5 \mathrm{H}, 1-2 \mathrm{H} 2$ \\
\hline 2214 & $\mathrm{C} 6 \mathrm{H} 1010 \mathrm{OH} 4-3$ & $\mathrm{CCC}(\mathrm{OO})[\mathrm{CH}] \mathrm{C}=\mathrm{C}$ & $\operatorname{lnChl}=1 \mathrm{~S} / \mathrm{C} 6 \mathrm{H} 11 \mathrm{O} 2 / \mathrm{c1}-3-5-6(4-2) 8-7 / \mathrm{h} 3,5-7 \mathrm{H}, 1,4 \mathrm{H} 2,2 \mathrm{H} 3$ \\
\hline 2215 & $\mathrm{C} 6 \mathrm{H} 11023-5 \mathrm{OOH}$ & $\mathrm{CC} 1 \mathrm{C}(\mathrm{O} 1) \mathrm{CC}(\mathrm{OO}) \mathrm{C}$ & InChl=1S/C6H12O3/c1-4(9-7)3-6-5(2)8-6/h4-7H,3H2,1-2H3 \\
\hline 2216 & $13 \mathrm{C} 6 \mathrm{Q} 15-4$ & $\mathrm{OOCCC}(\mathrm{C}(\mathrm{OO}) \mathrm{C})[\mathrm{CH} 2]$ & InChl=1S/C6H13O4/c1-5(3-4-9-7)6(2)10-8/h5-8H,1,3-4H2,2H3 \\
\hline 2217 & E2F5J & $\mathrm{CCC} 1=\mathrm{CC}=[\mathrm{C}] \mathrm{O} 1$ & InChl=1S/C6H7O/c1-2-6-4-3-5-7-6/h3-4H,2H2,1H3 \\
\hline 2218 & $\mathrm{C} 6 \mathrm{H} 1010 \mathrm{OH} 4-5$ & $\mathrm{C}[\mathrm{CH}] \mathrm{C}(\mathrm{OO}) \mathrm{CC}=\mathrm{C}$ & InChl=1S/C6H1102/c1-3-5-6(4-2)8-7/h3-4,6-7H,1,5H2,2H3 \\
\hline 2219 & $\mathrm{C} 6 \mathrm{H} 11023-60 \mathrm{OH}$ & $\operatorname{cC1C}(01) \operatorname{ccc}(00)$ & InChl=1S/C6H12O3/c1-5-6(9-5)3-2-4-8-7/h5-7H,2-4H2,1H3 \\
\hline 2220 & I3C6Q15-6 & $\mathrm{OOCCC}(\mathrm{C}(\mathrm{OO})[\mathrm{CH} 2]) \mathrm{C}$ & InChl=15/C6H13O4/c1-5(3-4-9-7)6(2)10-8/h5-8H,2-4H2,1H3 \\
\hline 2221 & H13DE106J & {$[\mathrm{CH} 2] \mathrm{CC}=\mathrm{CC}=\mathrm{C}=\mathrm{O}$} & $\operatorname{lnChl}=1 \mathrm{~S} / \mathrm{C} 6 \mathrm{H} 7 \mathrm{O} / \mathrm{c} 1-2-3-4-5-6-7 / \mathrm{h} 3-5 \mathrm{H}, 1-2 \mathrm{H} 2$ \\
\hline 2222 & $\mathrm{C} 6 \mathrm{H} 1010 \mathrm{OH} 4-6$ & {$[\mathrm{CH} 2] \mathrm{CC}(\mathrm{OO}) \mathrm{CC}=\mathrm{C}$} & InChl=15/C6H1102/c1-3-5-6(4-2)8-7/h3,6-7H,1-2,4-5H2 \\
\hline 2223 & C6H11024-10OH & $\mathrm{C}(\mathrm{OO}) \mathrm{C} 1 \mathrm{CC}(\mathrm{O} 1) \mathrm{CC}$ & InChl=1S/C6H12O3/c1-2-5-3-6(9-5)4-8-7/h5-7H,2-4H2,1H3 \\
\hline 2224 & NEC6Q11A-1B & $\mathrm{OOCC}(\mathrm{COO})(\mathrm{CC})[\mathrm{CH} 2]$ & InChl=1S/C6H1304/c1-3-6(2,4-9-7)5-10-8/h7-8H,2-5H2,1H3 \\
\hline 2225 & H25DE101J & $\mathrm{C}=\mathrm{CCC}=\mathrm{C}[\mathrm{C}]=\mathrm{O}$ & $\operatorname{lnChl}=1 \mathrm{~S} / \mathrm{C} 6 \mathrm{H} 7 \mathrm{O} / \mathrm{c1}-2-3-4-5-5-6-7 / \mathrm{h} 2,4-5 \mathrm{H}, 1,3 \mathrm{H} 2$ \\
\hline 2226 & C6H1010OH5-3 & $\mathrm{CC}(\mathrm{OO}) \mathrm{C}[\mathrm{CH}] \mathrm{C}=\mathrm{C}$ & InChl=1S/C6H1102/c1-3-4-5-6(2)8-7/h3-4,6-7H,1,5H2,2H3 \\
\hline 2227 & $\mathrm{C} 6 \mathrm{H} 11024-3 \mathrm{OOH}$ & $\mathrm{CC} 1 \mathrm{C}(\mathrm{OO}) \mathrm{C}(\mathrm{O} 1) \mathrm{CC}$ & InChl=1S/C6H12O3/c1-3-5-6(9-7)4(2)8-5/h4-7H,3H2,1-2H3 \\
\hline 2228 & $13 \mathrm{C} 6 \mathrm{Q} 16-2$ & $0 O C[\mathrm{CH}] \mathrm{C}(\mathrm{CCOO}) \mathrm{C}$ & InChl=15/C6H13O4/c1-6(2-4-9-7)3-5-10-8/h2,6-8H,3-5H2,1H3 \\
\hline 2229 & H34DE2O4J & $\mathrm{CC}=\mathrm{O}) \mathrm{C}=\mathrm{C}=[\mathrm{C}] \mathrm{C}$ & InChl=1S/C6H7O/c1-3-4-5-6(2)7/h5H,1-2H3 \\
\hline 2230 & C6H1010OH5-4 & $\mathrm{CC}(\mathrm{OO})[\mathrm{CH}] \mathrm{CC}=\mathrm{C}$ & InChl=1S/C6H1102/c1-3-4-5-6(2)8-7/h3,5-7H,1,4H2,2H3 \\
\hline 2231 & $\mathrm{C} 6 \mathrm{H} 11024-5 \mathrm{OOH}$ & $\mathrm{CC} 1 \mathrm{CC}(\mathrm{O} 1) \mathrm{C}(\mathrm{OO}) \mathrm{C}$ & InChl=15/C6H12O3/c1-4-3-6(8-4)5(2)9-7/h4-7H,3H2,1-2H3 \\
\hline 2232 & NEC6Q11A-3 & $\mathrm{OOCC}(\mathrm{COO})([\mathrm{CH}] \mathrm{C}) \mathrm{C}$ & InChl=1S/C6H13O4/c1-3-6(2,4-9-7)5-10-8/h3,7-8H,4-5H2,1-2H3 \\
\hline 2233 & H35DE2O1J & $\mathrm{C}=\mathrm{CC}=\mathrm{CC}(=\mathrm{O})[\mathrm{CH} 2]$ & InChl=1S/C6H7O/c1-3-4-5-6(2)7/h3-5H,1-2H2 \\
\hline 2234 & C6H1010OH5-6 & {$[\mathrm{CH} 2] \mathrm{C}(\mathrm{OO}) \mathrm{CCC}=\mathrm{C}$} & InChl=1S/C6H11O2/c1-3-4-5-6(2)8-7/h3,6-7H,1-2,4-5H2 \\
\hline 2235 & $\mathrm{C} 6 \mathrm{H} 11024-60 \mathrm{OH}$ & $\mathrm{CC} 1 \mathrm{CC}(\mathrm{O} 1) \mathrm{CC}(\mathrm{OO})$ & InChl=1S/C6H12O3/c1-5-4-6(9-5)2-3-8-7/h5-7H,2-4H2,1H3 \\
\hline 2236 & I3C6Q16-3 & $\mathrm{OOCC}[\mathrm{C}](\mathrm{CCOO}) \mathrm{C}$ & InChl=1S/C6H13O4/c1-6(2-4-9-7)3-5-10-8/h7-8H,2-5H2,1H3 \\
\hline 2237 & H45DE2O3J & $\mathrm{C}=\mathrm{C}=\mathrm{C}[\mathrm{CH}] \mathrm{C}(=\mathrm{O}) \mathrm{C}$ & $\operatorname{lnChl}=1 \mathrm{~S} / \mathrm{C} 6 \mathrm{H} 7 \mathrm{O} / \mathrm{c1}-3-4-5-6(2) 7 / \mathrm{h} 4-5 \mathrm{H}, 1 \mathrm{H} 2,2 \mathrm{H} 3$ \\
\hline 2238 & C6H1010OH6-3 & $\mathrm{C}(\mathrm{OO}) \mathrm{CC}[\mathrm{CH}] \mathrm{C}=\mathrm{C}$ & InChl=1S/C6H1102/c1-2-3-4-5-6-8-7/h2-3,7H,1,4-6H2 \\
\hline 2239 & $\mathrm{C} 6 \mathrm{H} 11025-10 \mathrm{OH}$ & $\mathrm{C}(\mathrm{OO}) \mathrm{C} 1 \mathrm{CCC}(\mathrm{O} 1) \mathrm{C}$ & InChl=1S/C6H12O3/c1-5-2-3-6(9-5)4-8-7/h5-7H,2-4H2,1H3 \\
\hline 2240 & NEC6Q11A-4 & $\mathrm{OOCC}(\mathrm{COO})(\mathrm{C}[\mathrm{CH} 2]) \mathrm{C}$ & InChl=1S/C6H13O4/c1-3-6(2,4-9-7)5-10-8/h7-8H,1,3-5H2,2H3 \\
\hline 2241 & CYC6H724-OJ & {$[\mathrm{O}] \mathrm{C} 1 \mathrm{CC}=\mathrm{CC}=\mathrm{C} 1$} & $\operatorname{lnChl}=1 \mathrm{~S} / \mathrm{C} 6 \mathrm{H} 7 \mathrm{O} / \mathrm{c} 7-6-4-2-1-3-5-6 / \mathrm{h} 1-4,6 \mathrm{H}, 5 \mathrm{H} 2$ \\
\hline 2242 & $\begin{array}{ll}\mathrm{C} 6 \mathrm{H} 1010 \mathrm{OH} 6-4 \\
\end{array}$ & $\mathrm{C}(\mathrm{OO}) \mathrm{C}[\mathrm{CH}] \mathrm{CC}=\mathrm{C}$ & InChl=1S/C6H1102/c1-2-3-4-5-6-8-7/h2,4,7H,1,3,5-6H2 \\
\hline 2243 & $\mathrm{C} 6 \mathrm{H} 11025-30 \mathrm{OH}$ & $\mathrm{CC} 1 \mathrm{C}(\mathrm{OO}) \mathrm{CC}(\mathrm{O} 1) \mathrm{C}$ & InChl=1S/C6H12O3/c1-4-3-6(9-7)5(2)8-4/h4-7H,3H2,1-2H3 \\
\hline 2244 & I3C6Q16-4 & $\mathrm{OOCCC}(\mathrm{CCOO})[\mathrm{CH} 2]$ & InChl=15/C6H13O4/c1-6(2-4-9-7)3-5-10-8/h6-8H,1-5H2 \\
\hline 2245 & CYC6H725-OJ & {$[\mathrm{O}] \mathrm{C} 1 \mathrm{C}=\mathrm{CCC}=\mathrm{C} 1$} & InChl=1S/C6H7O/c7-6-4-2-1-3-5-6/h2-6H,1H2 \\
\hline 2246 & $\mathrm{C} 6 \mathrm{H} 1010 \mathrm{OH} 6-5$ & $\mathrm{C}(\mathrm{OO})[\mathrm{CH}] \mathrm{CCC}=\mathrm{C}$ & InChl=15/C6H1102/c1-2-3-4-5-6-8-7/h2,5,7H,1,3-4,6H2 \\
\hline 2247 & $\mathrm{C} 6 \mathrm{H} 11034-10 \mathrm{OH}$ & $\mathrm{C}(\mathrm{OO}) \mathrm{CC} 1 \mathrm{C}(\mathrm{O} 1) \mathrm{CC}$ & InChl=1S/C6H12O3/c1-2-5-6(9-5)3-4-8-7/h5-7H,2-4H2,1H3 \\
\hline 2248 & NEC6Q13-1A & $\mathrm{OOCC}(\mathrm{C}(\mathrm{OO}) \mathrm{C})([\mathrm{CH} 2]) \mathrm{C}$ & InChl=1S/C6H13O4/c1-5(10-8)6(2,3)4-9-7/h5,7-8H,2,4H2,1,3H3 \\
\hline 2249 & C5D14CHO & $\mathrm{C}=\mathrm{CCC}=\mathrm{CC}=\mathrm{O}$ & InChl=1S/C6H8O/c1-2-3-4-5-6-7/h2,4-6H,1,3H2 \\
\hline 2250 & $\mathrm{C} 6 \mathrm{H} 102 \mathrm{OOH} 4-5$ & $\mathrm{C}[\mathrm{CH}] \mathrm{C}(\mathrm{OO}) \mathrm{C}=\mathrm{CC}$ & InChl=1S/C6H11O2/c1-3-5-6(4-2)8-7/h3-7H,1-2H3 \\
\hline 2251 & C6H11034-2OOH & $\mathrm{CC}(\mathrm{OO}) \mathrm{C} 1 \mathrm{C}(\mathrm{O} 1) \mathrm{CC}$ & InChl=1S/C6H12O3/c1-3-5-6(8-5)4(2)9-7/h4-7H,3H2,1-2H3 \\
\hline 2252 & 13C6Q23-1 & $\mathrm{OOC}(\mathrm{C}(\mathrm{OO})(\mathrm{CC}) \mathrm{C})[\mathrm{CH} 2]$ & InChl=1S/C6H13O4/c1-4-6(3,10-8)5(2)9-7/h5,7-8H,2,4H2,1,3H3 \\
\hline 2253 & $\mathrm{CC} 31 \mathrm{M} 2 \mathrm{CHO}$ & $\mathrm{CC}(\mathrm{C} 1 \mathrm{C}=\mathrm{C} 1 \mathrm{C})=\mathrm{O}$ & $\operatorname{lnChl}=1 \mathrm{~S} / \mathrm{C} 6 \mathrm{H} 8 \mathrm{O} / \mathrm{c1} 1-4-3-6(4) 5(2) 7 / \mathrm{h} 3,6 \mathrm{H}, 1-2 \mathrm{H} 3$ \\
\hline 2254 & $\mathrm{C} 6 \mathrm{H} 102 \mathrm{OOH} 4-6$ & {$[\mathrm{CH} 2] \mathrm{CC}(\mathrm{OO}) \mathrm{C}=\mathrm{CC}$} & InChl=1S/C6H11O2/c1-3-5-6(4-2)8-7/h3,5-7H,2,4H2,1H3 \\
\hline 2255 & NEC6KET1-1A & $\mathrm{CC}(\mathrm{C}=0)(\mathrm{CC}) \mathrm{COO}$ & InChl=15/C6H1203/c1-3-6(2,4-7)5-9-8/h4,8H,3,5H2,1-2H3 \\
\hline 2256 & NEC6Q13-4 & $\mathrm{OOCC}(\mathrm{C}(\mathrm{OO})[\mathrm{CH} 2])(\mathrm{C}) \mathrm{C}$ & InChl=1S/C6H13O4/c1-5(10-8)6(2,3)4-9-7/h5,7-8H,1,4H2,2-3H3 \\
\hline 2257 & DMF24 & $\mathrm{CC} 1=\mathrm{CC}(\mathrm{C})=\mathrm{CO} 1$ & InChl=1S/C6H8O/c1-5-3-6(2)7-4-5/h3-4H,1-2H3 \\
\hline 2258 & C6H102OOH5-4 & $\mathrm{CC}(\mathrm{OO})[\mathrm{CH}] \mathrm{C}=\mathrm{CC}$ & InChl=1S/C6H11O2/c1-3-4-5-6(2)8-7/h3-7H,1-2H3 \\
\hline 2259 & NEC6KET1-3 & $\mathrm{OOCC}(\mathrm{C}(\mathrm{C}=\mathrm{O})(\mathrm{C}) \mathrm{C}) \mathrm{C}$ & InChl=1S/C6H12O3/c1-5(9-8)6(2,3)4-7/h4-5,8H,1-3H3 \\
\hline 2260 & $13 \mathrm{C} 6 \mathrm{Q} 23-4$ & $\mathrm{OOC}(\mathrm{C}(\mathrm{OO})(\mathrm{CC})[\mathrm{CH} 2]) \mathrm{C}$ & InChl=1S/C6H13O4/c1-4-6(3,10-8)5(2)9-7/h5,7-8H,3-4H2,1-2H3 \\
\hline 2261 & DMF24BCAR & $\mathrm{CC} 1 \mathrm{OC}=\mathrm{C}(\mathrm{C})[\mathrm{C}] 1$ & InChl=1S/C6H8O/c1-5-3-6(2)7-4-5/h4,6H,1-2H3 \\
\hline 2262 & C6H102OOH5-6 & {$[\mathrm{CH} 2] \mathrm{C}(\mathrm{OO}) \mathrm{CC}=\mathrm{CC}$} & InChl=1S/C6H1102/c1-3-4-5-6(2)8-7/h3-4,6-7H,2,5H2,1H3 \\
\hline 2263 & NEC6KET1-4 & $\mathrm{OOCCC}(\mathrm{C}=\mathrm{O})(\mathrm{C}) \mathrm{C}$ & $\operatorname{lnChl}=1 \mathrm{~S} / \mathrm{C} 6 \mathrm{H} 12 \mathrm{O} 3 / \mathrm{c} 1-6(2,5-7) 3-4-9-8 / \mathrm{h} 5,8 \mathrm{H}, 3-4 \mathrm{H} 2,1-2 \mathrm{H3}$ \\
\hline 2264 & NEC6Q14-1A & $\mathrm{OOCCC}(\mathrm{COO})([\mathrm{CH} 2]) \mathrm{C}$ & InChl=15/C6H13O4/c1-6(2,5-10-8)3-4-9-7/h7-8H,1,3-5H2,2H3 \\
\hline 2265 & DMF25 & $\mathrm{CC} 1=\mathrm{CC}=\mathrm{C}(\mathrm{C}) 01$ & InChl=1S/C6H8O/c1-5-3-4-6(2)7-5/h3-4H,1-2H3 \\
\hline 2266 & C6H102OOH6-4 & $\mathrm{C}(\mathrm{OO}) \mathrm{C}[\mathrm{CH}] \mathrm{C}=\mathrm{CC}$ & InChl=15/C6H11O2/c1-2-3-4-5-6-8-7/h2-4,7H,5-6H2,1H3 \\
\hline 2267 & NECGKET3-1 & $\operatorname{Oocc}(C(=)) C)(C) C$ & $\operatorname{lnChl}=1 \mathrm{~S} / \mathrm{C} 6 \mathrm{H} 12 \mathrm{O} 3 / \mathrm{c} 1-5(7) 6(2,3) 4-9-8 / \mathrm{h} 8 \mathrm{H}, 4 \mathrm{H} 2,1-3 \mathrm{H} 3$ \\
\hline 2268 & $13 \mathrm{C} 6 \mathrm{Q} 23-5$ & $\mathrm{OOC}(\mathrm{C}(\mathrm{OO})([\mathrm{CH}] \mathrm{C}) \mathrm{C}) \mathrm{C}$ & InChl=15/C6H13O4/c1-4-6(3,10-8)5(2)9-7/h4-5,7-8H,1-3H3 \\
\hline 2269 & DMF25ACAR & $\mathrm{CC} 1=\mathrm{CC}(\mathrm{C})[\mathrm{C}] \mathrm{O} 1$ & InChl=1S/C6H8O/c1-5-3-6(2)7-4-5/h3,5H,1-2H3 \\
\hline 2270 & C6H102OOH6-5 & $\mathrm{C}(\mathrm{OO})[\mathrm{CH}] \mathrm{CC}=\mathrm{CC}$ & $\ln \mathrm{Chl}=1 \mathrm{~S} / \mathrm{C} 6 \mathrm{H} 11 \mathrm{O} 2 / \mathrm{c1}-2-3-4-4-5-6-8-7 / \mathrm{h} 2-3,5,7 \mathrm{H}, 4,6 \mathrm{H} 2,1 \mathrm{H} 3$ \\
\hline 2271 & NEC6KET3-4 & $0 O C C(=0) C(C)(C) C$ & $\operatorname{lnChl}=15 / C 6 H 12 \mathrm{O} / \mathrm{c} 1-6(2,3) 5(7) 4-9-8 / \mathrm{h} 8 \mathrm{H}, 4 \mathrm{H} 2,1-3 \mathrm{H} 3$ \\
\hline
\end{tabular}




\begin{tabular}{|c|c|c|c|}
\hline 2272 & NEC6Q14-3 & $\mathrm{OOC}[\mathrm{CH}] \mathrm{C}(\mathrm{COO})(\mathrm{C}) \mathrm{C}$ & $\operatorname{lnChl}=1 \mathrm{~S} / \mathrm{C} 6 \mathrm{H} 1304 / \mathrm{c} 1-6(2,5-10-8) 3-4-9-7 / \mathrm{h} 3,7-8 \mathrm{H}, 4-5 \mathrm{H} 2,1-2 \mathrm{H} 3$ \\
\hline 2273 & DMF25BCAR & $\mathrm{CC} 1=\mathrm{C}[\mathrm{C}] \mathrm{C}(\mathrm{C}) 01$ & InChl=15/C6H8O/c1-5-3-4-6(2)7-5/h3,6H,1-2H3 \\
\hline 2274 & C6H1030OH1-2 & $\mathrm{C}(\mathrm{OO})[\mathrm{CH}] \mathrm{C}=\mathrm{CCC}$ & $\operatorname{lnChl}=1 \mathrm{~S} / \mathrm{C} 6 \mathrm{H} 11 \mathrm{O} 2 / \mathrm{c1}-2-3-4-5-6-8-7 / \mathrm{h} 3-5,7 \mathrm{H}, 2,6 \mathrm{H} 2,1 \mathrm{H} 3$ \\
\hline 2275 & NEC6KET4-1 & $\mathrm{OOCC}(\mathrm{CC}=\mathrm{O})(\mathrm{C}) \mathrm{C}$ & InChl=1S/C6H12O3/c1-6(2,3-4-7)5-9-8/h4,8H,3,5H2,1-2H3 \\
\hline 2276 & $13 \mathrm{C6Q23-6}$ & $\mathrm{OOC}(\mathrm{C}(\mathrm{OO})(\mathrm{C}[\mathrm{CH} 2]) \mathrm{C}) \mathrm{C}$ & InChl=1S/C6H13O4/c1-4-6(3,10-8)5(2)9-7/h5,7-8H,1,4H2,2-3H3 \\
\hline 2277 & DMF25OPB1 & $C /[C]=C / C([C]=0) C$ & $\operatorname{lnChl}=1 \mathrm{~S} / \mathrm{C} 6 \mathrm{H} 8 \mathrm{O} / \mathrm{c} 1-3-4-6(2) 5-7 / \mathrm{h} 4,6 \mathrm{H}, 1-2 \mathrm{H} 3$ \\
\hline 2278 & $\mathrm{C} 6 \mathrm{H} 103 \mathrm{OOH} 2-1$ & {$[\mathrm{CH} 2] \mathrm{C}(\mathrm{OO}) \mathrm{C}=\mathrm{CCC}$} & $\operatorname{lnChl}=1 \mathrm{~S} / \mathrm{C} 6 \mathrm{H} 11 \mathrm{O} 2 / \mathrm{c} 1-3-4-5-5-6(2) 8-7 / \mathrm{h} 4-7 \mathrm{H}, 2-3 \mathrm{H} 2,1 \mathrm{H} 3$ \\
\hline 2279 & NEC6KET4-3 & $\mathrm{OOC}(\mathrm{C}(\mathrm{C})(\mathrm{C}) \mathrm{C}) \mathrm{C}=\mathrm{O}$ & $\operatorname{lnChl}=1 \mathrm{~S} / \mathrm{C} 6 \mathrm{H} 12 \mathrm{O} 3 / \mathrm{c} 1-6(2,3) 5(4-7) 9-8 / \mathrm{h} 4-5,8 \mathrm{H}, 1-3 \mathrm{H} 3$ \\
\hline 2280 & NEC6Q34-1 & $\mathrm{OOCC}(\mathrm{C}([\mathrm{CH} 2])(\mathrm{C}) \mathrm{C}) \mathrm{OO}$ & InChl=1S/C6H13O4/c1-6(2,3)5(10-8)4-9-7/h5,7-8H,1,4H2,2-3H3 \\
\hline 2281 & DMF250PB2 & $C / C([O])=C / C=[C] / C$ & $\operatorname{lnChl}=1 \mathrm{~S} / \mathrm{C} 6 \mathrm{H} 8 \mathrm{O} / \mathrm{c} 1-3-4-5-6(2) 7 / \mathrm{h} 4-5 \mathrm{H}, 1-2 \mathrm{H} 3$ \\
\hline 2282 & $\mathrm{CHXO2J}$ & [0]0C1CCCCC1 & $\operatorname{lnChl}=15 / C 6 \mathrm{H} 1102 / \mathrm{c} 7-8-6-4-2-1-3-5-6 / \mathrm{h} 6 \mathrm{H}, 1-5 \mathrm{H} 2$ \\
\hline 2283 & 13С6KET1-2 & $\operatorname{ccc}(C(C=0) 00) c$ & InChl=1S/C6H12O3/c1-3-5(2)6(4-7)9-8/h4-6,8H,3H2,1-2H3 \\
\hline 2284 & 13C6Q24-1 & $\mathrm{OOCC}(\mathrm{C}(\mathrm{OO})[\mathrm{CH} 2]) \mathrm{CC}$ & InChl=15/C6H13O4/c1-3-6(4-9-7)5(2)10-8/h5-8H,2-4H2,1H3 \\
\hline 2285 & DMF34 & $\mathrm{C} 1=\mathrm{C}(\mathrm{C}) \mathrm{C}(\mathrm{C})=\mathrm{CO} 1$ & $\operatorname{lnChl}=1 \mathrm{~S} / \mathrm{C} 6 \mathrm{H} 8 \mathrm{O} / \mathrm{c1}-5-3-7-4-6(5) 2 / \mathrm{h} 3-4 \mathrm{H}, 1-2 \mathrm{H} 3$ \\
\hline 2286 & $\mathrm{CH} \times 1 \mathrm{Q}_{2 \mathrm{~J}}$ & $\mathrm{OOC1}[\mathrm{CH}] \mathrm{CCCC} 1$ & InChl=1S/C6H11O2/c7-8-6-4-2-1-3-5-6/h4,6-7H,1-3,5H2 \\
\hline 2287 & I3C6KET1-3 & $\mathrm{CC}(\mathrm{OO})(\mathrm{CC}) \mathrm{CC}=\mathrm{O}$ & InChl=1S/C6H12O3/c1-3-6(2,9-8)4-5-7/h5,8H,3-4H2,1-2H3 \\
\hline 2288 & I3C6Q24-3 & $\mathrm{OOC}[\mathrm{C}](\mathrm{C}(\mathrm{OO}) \mathrm{C}) \mathrm{CC}$ & InChl=1S/C6H13O4/c1-3-6(4-9-7)5(2)10-8/h5,7-8H,3-4H2,1-2H3 \\
\hline 2289 & DMF34ACAR & $\mathrm{CC} 1=\mathrm{CO}[\mathrm{C}] \mathrm{C} 1 \mathrm{C}$ & $\operatorname{lnChl}=1 \mathrm{~S} / \mathrm{C} 6 \mathrm{H} 8 \mathrm{O} / \mathrm{c} 1-5-3-7-4-6(5) 2 / \mathrm{h} 3,6 \mathrm{H}, 1-2 \mathrm{H} 3$ \\
\hline 2290 & CHX1Q3J & $\mathrm{OOC1C[CH]CCC1}$ & InChl=1S/C6H11O2/c7-8-6-4-2-1-3-5-6/h2,6-7H,1,3-5H2 \\
\hline 2291 & I3C6KET1-4 & OOCC(CC=0)CC & 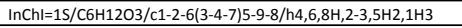 \\
\hline 2292 & $13 \mathrm{C} 6 \mathrm{Q} 24-5$ & $\mathrm{OOCC}(\mathrm{C}(\mathrm{OO}) \mathrm{C})[\mathrm{CH}] \mathrm{C}$ & InChl=1S/C6H13O4/c1-3-6(4-9-7)5(2)10-8/h3,5-8H,4H2,1-2H3 \\
\hline 2293 & E2F & $\mathrm{CCC} 1=\mathrm{CC}=\mathrm{CO} 1$ & $\operatorname{lnChl}=15 / \mathrm{C} 6 \mathrm{H} 8 \mathrm{O} / \mathrm{c1} 1-2-6-4-3-5-7-6 / \mathrm{h} 3-5 \mathrm{H}, 2 \mathrm{H} 2,1 \mathrm{H} 3$ \\
\hline 2294 & $\mathrm{CH} \times 1 \mathrm{Q} 4 \mathrm{~J}$ & $\mathrm{OOC1CC}[\mathrm{CH}] \mathrm{CC} 1$ & $\operatorname{lnChl}=15 / C 6 H 1102 / c 7-8-6-4-2-1-3-5-6 / h 1,6-7 \mathrm{H}, 2-5 \mathrm{H} 2$ \\
\hline 2295 & I3C6KET1-5 & $\mathrm{CC}(\mathrm{C}(\mathrm{OO}) \mathrm{C}) \mathrm{CC}=\mathrm{O}$ & InChl=1S/C6H12O3/c1-5(3-4-7)6(2)9-8/h4-6,8H,3H2,1-2H3 \\
\hline 2296 & IC6Q11A-2 & $\mathrm{CCC}[\mathrm{C}](\mathrm{COO}) \mathrm{COO}$ & InChl=1S/C6H13O4/c1-2-3-6(4-9-7)5-10-8/h7-8H,2-5H2,1H3 \\
\hline 2297 & H23DE1O & $\mathrm{CCC}=\mathrm{C}=\mathrm{CC}=\mathrm{O}$ & InChl=1S/C6H8O/c1-2-3-4-5-6-7/h3,5-6H,2H2,1H3 \\
\hline 2298 & XCGKET1-1A & $\mathrm{OOCC}(\mathrm{C}(\mathrm{C}) \mathrm{C}) \mathrm{C}=\mathrm{O}$ & $\operatorname{lnChl}=1 \mathrm{~S} / \mathrm{C} 6 \mathrm{H} 12 \mathrm{O} 3 / \mathrm{c} 1-5(2) 6(3-7) 4-9-8 / \mathrm{h} 3,5-6,8 \mathrm{H}, 4 \mathrm{H} 2,1-2 \mathrm{H} 3$ \\
\hline 2299 & I3C6Q24-6 & $\mathrm{OOCC}(\mathrm{C}(\mathrm{OO}) \mathrm{C}) \mathrm{C}[\mathrm{CH} 2]$ & InChl=15/C6H13O4/c1-3-6(4-9-7)5(2)10-8/h5-8H,1,3-4H2,2H3 \\
\hline 2300 & IC6KET4-10 & $\mathrm{CC}(\mathrm{C}[\mathrm{O}]) \mathrm{CC}(=\mathrm{O}) \mathrm{C}$ & $\operatorname{lnChl}=1 \mathrm{~S} / \mathrm{C} 6 \mathrm{H} 11 \mathrm{O} 2 / \mathrm{c} 1-5(4-7) 3-6(2) 8 / \mathrm{h} 5 \mathrm{H}, 3-4 \mathrm{H} 2,1-2 \mathrm{H} 3$ \\
\hline 2301 & I3С6KET1-6 & $\mathrm{OOCCC}(\mathrm{CC}=\mathrm{O}) \mathrm{C}$ & $\operatorname{lnChl}=15 / \mathrm{C} 6 \mathrm{H} 12 \mathrm{O} / \mathrm{c} 1-6(2-4-7) 3-5-9-8 / \mathrm{h} 4,6,8 \mathrm{H}, 2-3,5 \mathrm{H} 2,1 \mathrm{H} 3$ \\
\hline 2302 & IC6Q11A-3 & $\mathrm{CC}[\mathrm{CH}] \mathrm{C}(\mathrm{COO}) \mathrm{COO}$ & $\operatorname{lnChl}=1 \mathrm{~S} / \mathrm{C} 6 \mathrm{H} 13 \mathrm{O} 4 / \mathrm{c} 1-2-3-6(4-9-7) 5-10-8 / \mathrm{h} 3,6-8 \mathrm{H}, 2,4-5 \mathrm{H} 2,1 \mathrm{H} 3$ \\
\hline 2303 & H34DE2O & $\mathrm{CC}=\mathrm{C}=\mathrm{CC}(=\mathrm{O}) \mathrm{C}$ & $\operatorname{lnChl}=1 \mathrm{~S} / \mathrm{C} 6 \mathrm{H} 8 \mathrm{O} / \mathrm{c} 1-3-4-5-6(2) 7 / \mathrm{h} 3,5 \mathrm{H}, 1-2 \mathrm{H} 3$ \\
\hline 2304 & IC6KET4-2O & $\mathrm{CC}(=0) \mathrm{CC}([\mathrm{O}])(\mathrm{C}) \mathrm{C}$ & $\operatorname{lnChl}=1 \mathrm{~S} / \mathrm{C} 6 \mathrm{H} 1102 / \mathrm{c} 1-5(7) 4-6(2,3) 8 / \mathrm{h} 4 \mathrm{H} 2,1-3 \mathrm{H} 3$ \\
\hline 2305 & XC6KET1-2 & $\mathrm{OOC}(\mathrm{C}(\mathrm{C}) \mathrm{C})(\mathrm{C}=\mathrm{O}) \mathrm{C}$ & $\operatorname{lnChl}=1 \mathrm{~S} / \mathrm{C} 6 \mathrm{H} 12 \mathrm{O} 3 / \mathrm{c} 1-5(2) 6(3,4-7) 9-8 / \mathrm{h} 4-5,8 \mathrm{H}, 1-3 \mathrm{H} 3$ \\
\hline 2306 & I3C6Q25-1 & $\mathrm{OOC}(\mathrm{C}(\mathrm{C}(\mathrm{OO}) \mathrm{C}) \mathrm{C})[\mathrm{CH} 2]$ & InChl=15/C6H13O4/c1-4(5(2)9-7)6(3)10-8/h4-8H,2H2,1,3H3 \\
\hline 2307 & H35DE2O & $\mathrm{C}=\mathrm{CC}=\mathrm{CC}(=\mathrm{O}) \mathrm{C}$ & $\operatorname{lnChl}=15 / C 6 H 80 / c 1-3-4-5-6(2) 7 / h 3-5 \mathrm{H}, 1 \mathrm{H} 2,2 \mathrm{H} 3$ \\
\hline 2308 & IC6KET4-30 & $\mathrm{CC}(\mathrm{C}(\mathrm{C}(=) \mathrm{O}) \mathrm{C})[0]) \mathrm{C}$ & InChl=1S/C6H1102/c1-4(2)6(8)5(3)7/h4,6H,1-3H3 \\
\hline 2309 & I3C6KET2-1 & $\mathrm{CC}(\mathrm{C}(=0) \mathrm{COO}) \mathrm{CC}$ & InChl=1S/C6H12O3/c1-3-5(2)6(7)4-9-8/h5,8H,3-4H2,1-2H3 \\
\hline 2310 & IC6Q11A-4 & $\mathrm{C}[\mathrm{CH}] \mathrm{CC}(\mathrm{COO}) \mathrm{COO}$ & InChl=15/C6H13O4/c1-2-3-6(4-9-7)5-10-8/h2,6-8H,3-5H2,1H3 \\
\hline 2311 & H45DE3O & $\mathrm{CCC}(=\mathrm{O}) \mathrm{C}=\mathrm{C}=\mathrm{C}$ & $\operatorname{lnChl}=1 \mathrm{~S} / \mathrm{C} 6 \mathrm{H} 8 \mathrm{O} / \mathrm{c1}-3-5-6(7) 4-2 / \mathrm{h} 5 \mathrm{H}, 1,4 \mathrm{H} 2,2 \mathrm{H} 3$ \\
\hline 2312 & IC6KET4-5O & {$[\mathrm{O}] \mathrm{CC}(=\mathrm{O}) \mathrm{CC}(\mathrm{C}) \mathrm{C}$} & $\operatorname{lnChl}=1 \mathrm{~S} / \mathrm{C} 6 \mathrm{H} 11 \mathrm{O} 2 / \mathrm{c} 1-5(2) 3-6(8) 4-7 / \mathrm{h} 5 \mathrm{H}, 3-4 \mathrm{H} 2,1-2 \mathrm{H} 3$ \\
\hline 2313 & XС6KET1-3 & $\mathrm{O}=\mathrm{CC}(\mathrm{C}(\mathrm{OO})(\mathrm{C}) \mathrm{C}) \mathrm{C}$ & $\operatorname{lnChl}=1 \mathrm{~S} / \mathrm{C} 6 \mathrm{H} 12 \mathrm{O} 3 / \mathrm{c} 1-5(4-7) 6(2,3) 9-8 / \mathrm{h} 4-5,8 \mathrm{H}, 1-3 \mathrm{H} 3$ \\
\hline 2314 & I3C6Q25-3 & $\mathrm{OOC}([\mathrm{C}](\mathrm{C}(\mathrm{OO}) \mathrm{C}) \mathrm{C}) \mathrm{C}$ & $\operatorname{lnChl}=15 / \mathrm{C} 6 \mathrm{H} 13 \mathrm{O} 4 / \mathrm{c} 1-4(5(2) 9-7) 6(3) 10-8 / \mathrm{h} 5-8 \mathrm{H}, 1-3 \mathrm{H} 3$ \\
\hline 2315 & IC6KET5-10 & $\mathrm{CC}(\mathrm{C}[\mathrm{O}]) \mathrm{CCC}=\mathrm{O}$ & $\operatorname{lnChl}=1 \mathrm{~S} / \mathrm{C} 6 \mathrm{H} 11 \mathrm{O} 2 / \mathrm{c} 1-6(5-8) 3-2-4-7 / \mathrm{h} 4,6 \mathrm{H}, 2-3,5 \mathrm{H} 2,1 \mathrm{H} 3$ \\
\hline 2316 & 13С6KET2-3 & $\operatorname{ccc}(\mathrm{C}(=0) \mathrm{C})(\mathrm{OO}) \mathrm{C}$ & $\operatorname{lnChl}=15 / C 6 \mathrm{H} 12 \mathrm{O} 3 / \mathrm{c1}-4-6(3,9-8) 5(2) 7 / \mathrm{h} 8 \mathrm{H}, 4 \mathrm{H} 2,1-3 \mathrm{H} 3$ \\
\hline 2317 & IC6Q11A-5 & {$[\mathrm{CH} 2] \mathrm{CCC}(\mathrm{COO}) \mathrm{COO}$} & $\ln \mathrm{Chl}=15 / \mathrm{C} 6 \mathrm{H} 13 \mathrm{O} 4 / \mathrm{c1}-2-3-6(4-9-7) 5-10-8 / \mathrm{h} 6-8 \mathrm{H}, 1-5 \mathrm{H} 2$ \\
\hline 2318 & IC6KET5-2O & $\mathrm{O}=\mathrm{CCCC}([\mathrm{O}])(\mathrm{C}) \mathrm{C}$ & $\operatorname{lnChl}=1 \mathrm{~S} / \mathrm{C} 6 \mathrm{H} 11 \mathrm{O} 2 / \mathrm{c1}-6(2,8) 4-3-5-7 / \mathrm{h} 5 \mathrm{H}, 3-4 \mathrm{H} 2,1-2 \mathrm{H} 3$ \\
\hline 2319 & XC6KET1-4 & $\mathrm{CC}(\mathrm{C}(\mathrm{C}=0) \mathrm{C}) \mathrm{COO}$ & $\operatorname{lnChl}=15 / \mathrm{C} 6 \mathrm{H} 12 \mathrm{O} / \mathrm{c} 1-5(3-7) 6(2) 4-9-8 / \mathrm{h} 3,5-6,8 \mathrm{H}, 4 \mathrm{H} 2,1-2 \mathrm{H} 3$ \\
\hline 2320 & $13 \mathrm{C} 6 \mathrm{Q} 25-4$ & $\mathrm{OOC}(\mathrm{C}(\mathrm{C}(\mathrm{OO}) \mathrm{C})[\mathrm{CH} 2]) \mathrm{C}$ & InChl=1S/C6H13O4/c1-4(5(2)9-7)6(3)10-8/h4-8H,1H2,2-3H3 \\
\hline 2321 & C6H9D14-5OR & {$[\mathrm{O}] \mathrm{CC}=\mathrm{CCC}=\mathrm{C}$} & InChl=1S/C6H9O/c1-2-3-4-5-6-7/h2,4-5H,1,3,6H2 \\
\hline 2322 & IC6KET5-30 & $\mathrm{O}=\mathrm{CCC}(\mathrm{C}(\mathrm{C}) \mathrm{C})[\mathrm{O}]$ & $\operatorname{lnChl}=15 / C 6 H 1102 / c 1-5(2) 6(8) 3-4-7 / h 4-6 \mathrm{H}, 3 \mathrm{H} 2,1-2 \mathrm{H} 3$ \\
\hline 2323 & 13С6KET2-4 & $\mathrm{OOCC}(\mathrm{C}(=\mathrm{O}) \mathrm{C}) \mathrm{CC}$ & InChl=15/C6H12O3/c1-3-6(4-9-8)5(2)7/h6,8H,3-4H2,1-2H3 \\
\hline 2324 & IC6Q12-1A & $\operatorname{CCCC}(\mathrm{COO})(\mathrm{OO})[\mathrm{CH} 2]$ & $\operatorname{lnChl}=1 \mathrm{~S} / \mathrm{C} 6 \mathrm{H} 1304 / \mathrm{c} 1-3-4-6(2,10-8) 5-9-7 / \mathrm{h} 7-8 \mathrm{H}, 2-5 \mathrm{H} 2,1 \mathrm{H} 3$ \\
\hline 2325 & C6H9D15-3OR & $\mathrm{C}=\mathrm{CCC}(\mathrm{C}=\mathrm{C})[\mathrm{O}]$ & $\operatorname{lnChl}=15 / \mathrm{C} 6 \mathrm{H} 9 \mathrm{O} / \mathrm{c} 1-3-5-6(7) 4-2 / \mathrm{h} 3-4,6 \mathrm{H}, 1-2,5 \mathrm{H} 2$ \\
\hline 2326 & IC6KET5-4O & $\mathrm{O}=\mathrm{CC}(\mathrm{CC}(\mathrm{C}) \mathrm{C})[\mathrm{O}]$ & $\operatorname{lnChl}=1 \mathrm{~S} / \mathrm{C} 6 \mathrm{H} 11 \mathrm{O} 2 / \mathrm{c1}-5(2) 3-6(8) 4-7 / \mathrm{h} 4-6 \mathrm{H}, 3 \mathrm{H} 2,1-2 \mathrm{H} 3$ \\
\hline 2327 & 13С6KET2-5 & $\mathrm{OOC}(\mathrm{C}(\mathrm{C}(=\mathrm{O}) \mathrm{C}) \mathrm{C}) \mathrm{C}$ & $\operatorname{lnChl}=1 \mathrm{~S} / \mathrm{C} 6 \mathrm{H} 12 \mathrm{O} 3 / \mathrm{c} 1-4(5(2) 7) 6(3) 9-8 / \mathrm{h} 4,6,8 \mathrm{H}, 1-3 \mathrm{H} 3$ \\
\hline 2328 & I3C6Q34-1 & $\mathrm{OOCC}(\mathrm{OO})(\mathrm{C}[\mathrm{CH} 2]) \mathrm{CC}$ & $\operatorname{lnChl}=15 / C 6 \mathrm{H} 1304 / c 1-3-6(4-2,10-8) 5-9-7 / h 7-8 \mathrm{H}, 1,3-5 \mathrm{H} 2,2 \mathrm{H} 3$ \\
\hline 2329 & DMF252H3J & $\mathrm{CC} 1=\mathrm{C}[\mathrm{CH}] \mathrm{C}(\mathrm{C}) \mathrm{O} 1$ & $\operatorname{lnChl}=15 / C 6 H 9 O / c 1-5-3-4-6(2) 7-5 / \mathrm{h} 3-5 \mathrm{H}, 1-2 \mathrm{H} 3$ \\
\hline 2330 & NEC6KET1-1AO & $\operatorname{ccc}(\mathrm{C}=\mathrm{O})(\mathrm{C}[\mathrm{O}]) \mathrm{C}$ & $\ln \mathrm{Chl}=1 \mathrm{~S} / \mathrm{C} 6 \mathrm{H} 11 \mathrm{O} 2 / \mathrm{c} 1-3-6(2,4-7) 5-8 / \mathrm{h} 4 \mathrm{H}, 3,5 \mathrm{H} 2,1-2 \mathrm{H} 3$ \\
\hline 2331 & 13С6КET2-6 & $\mathrm{CC}(\mathrm{C}(=) \mathrm{O}) \mathrm{C}) \mathrm{COO}$ & $\operatorname{lnChl}=1 \mathrm{~S} / \mathrm{C} 6 \mathrm{H} 12 \mathrm{O} / \mathrm{c} 1-5(6(2) 7) 3-4-9-8 / \mathrm{h} 5,8 \mathrm{H}, 3-4 \mathrm{H} 2,1-2 \mathrm{H} 3$ \\
\hline 2332 & IC6Q12-3 & $\mathrm{CC}[\mathrm{CH}] \mathrm{C}(\mathrm{COO})(\mathrm{OO}) \mathrm{C}$ & InChl=15/C6H13O4/c1-3-4-6(2,10-8)5-9-7/h4,7-8H,3,5H2,1-2H3 \\
\hline 2333 & DMF253H2J & $\mathrm{CC} 1=\mathrm{CC}[\mathrm{C}](\mathrm{C}) 01$ & InChl=1S/C6H9O/c1-5-3-4-6(2)7-5/h3H,4H2,1-2H3 \\
\hline 2334 & NEC6KET1-3O & $\mathrm{O}=\mathrm{CC}(\mathrm{C}([\mathrm{OO}]) \mathrm{C})(\mathrm{C}) \mathrm{C}$ & InChl=1S/C6H11O2/c1-5(8)6(2,3)4-7/h4-5H,1-3H3 \\
\hline 2335 & I3C6KET4-1 & $\operatorname{CCC}(\mathrm{C}=0) \mathrm{CCOO}$ & $\operatorname{lnChl}=1 \mathrm{~S} / \mathrm{C} 6 \mathrm{H} 12 \mathrm{O} / \mathrm{c} 1-2-6(5-7) 3-4-9-8 / \mathrm{h} 5-6,8 \mathrm{H}, 2-4 \mathrm{H} 2,1 \mathrm{H} 3$ \\
\hline 2336 & $13 \mathrm{C} 6 \mathrm{Q} 34-2$ & $\mathrm{OOCC}(\mathrm{OO})([\mathrm{CH}] \mathrm{C}) \mathrm{CC}$ & InChl=1S/C6H13O4/c1-3-6(4-2,10-8)5-9-7/h3,7-8H,4-5H2,1-2H3 \\
\hline 2337 & H4E2O1J & $\mathrm{CC}=\mathrm{CCC}(=\mathrm{O})[\mathrm{CH} 2]$ & $\operatorname{lnChl}=1 \mathrm{~S} / \mathrm{C} 6 \mathrm{H} 9 \mathrm{O} / \mathrm{c} 1-3-4-5-6(2) 7 / \mathrm{h} 3-4 \mathrm{H}, 2,5 \mathrm{H} 2,1 \mathrm{H} 3$ \\
\hline 2338 & NEC6KET1-4O & {$[\mathrm{O}] \mathrm{CCC}(\mathrm{C}=\mathrm{O})(\mathrm{C}) \mathrm{C}$} & $\operatorname{lnChl}=1 \mathrm{~S} / \mathrm{C} 6 \mathrm{H} 11 \mathrm{O} 2 / \mathrm{c1} 1-6(2,5-8) 3-4-7 / \mathrm{h} 5 \mathrm{H}, 3-4 \mathrm{H} 2,1-2 \mathrm{H} 3$ \\
\hline 2339 & I3C6KET4-2 & $\operatorname{ccc}(\mathrm{C}(\mathrm{OO}) \mathrm{c}) \mathrm{C}=0$ & InChl=1S/C6H12O3/c1-3-6(4-7)5(2)9-8/h4-6,8H,3H2,1-2H3 \\
\hline 2340 & IC6Q12-4 & $\mathrm{C}[\mathrm{CH}] \mathrm{CC}(\mathrm{COO})(\mathrm{OO}) \mathrm{C}$ & InChl=1S/C6H13O4/c1-3-4-6(2,10-8)5-9-7/h3,7-8H,4-5H2,1-2H3 \\
\hline 2341 & H4E2O3J & $\mathrm{CC}(=\mathrm{O})[\mathrm{CH}] \mathrm{C}=\mathrm{CC}$ & InChl=1S/C6H9O/c1-3-4-5-6(2)7/h3-5H,1-2H3 \\
\hline 2342 & NEC6KET3-10 & {$[0] \mathrm{CC}(\mathrm{C}(=\mathrm{O}) \mathrm{C})(\mathrm{C}) \mathrm{C}$} & InChl=15/C6H11O2/c1-5(8)6(2,3)4-7/h4H2,1-3H3 \\
\hline 2343 & I3C6KET4-3 & $\operatorname{ccc}(\mathrm{C}=0)(\mathrm{OO}) \mathrm{CC}$ & InChl=1S/C6H12O3/c1-3-6(4-2,5-7)9-8/h5,8H,3-4H2,1-2H3 \\
\hline 2344 & IC6Q12-5 & {$[\mathrm{CH} 2] \mathrm{CCC}(\mathrm{COO})(\mathrm{OO}) \mathrm{C}$} & InChl=15/C6H13O4/c1-3-4-6(2,10-8)5-9-7/h7-8H,1,3-5H2,2H3 \\
\hline 2345 & H4E2O5J & $C[C]=C C C(=0) C$ & $\operatorname{lnChl}=15 / C 6 H 90 / c 1-3-4-5-6(2) 7 / h 4 H, 5 H 2,1-2 \mathrm{H} 3$ \\
\hline 2346 & NEC6KET3-4O & {$[0] C C(=O) C(C)(C) C$} & $\operatorname{lnChl}=1 \mathrm{~S} / \mathrm{C} 6 \mathrm{H} 1102 / \mathrm{c} 1-6(2,3) 5(8) 4-7 / \mathrm{h} 4 \mathrm{H} 2,1-3 \mathrm{H} 3$ \\
\hline 2347 & IC6KET1-1A & $\operatorname{cccc}(\operatorname{COO}) \mathrm{C}=0$ & $\ln \mathrm{Chl}=15 / \mathrm{C} 6 \mathrm{H} 12 \mathrm{O} 3 / \mathrm{c} 1-2-3-6(4-7) 5-9-8 / \mathrm{h} 4,6,8 \mathrm{H}, 2-3,5 \mathrm{H} 2,1 \mathrm{H} 3$ \\
\hline 2348 & IC6Q13-1A & $\mathrm{OOCC}(\mathrm{C}(\mathrm{OO}) \mathrm{CC})[\mathrm{CH} 2]$ & $\operatorname{lnChl}=1 \mathrm{~S} / \mathrm{C} 6 \mathrm{H} 13 \mathrm{O} 4 / \mathrm{c} 1-3-6(10-8) 5(2) 4-9-7 / \mathrm{h} 5-8 \mathrm{H}, 2-4 \mathrm{H} 2,1 \mathrm{H} 3$ \\
\hline 2349 & H5E2O4J & $\mathrm{CC}(=\mathrm{O}) \mathrm{C}[\mathrm{CH}] \mathrm{C}=\mathrm{C}$ & $\operatorname{lnChl}=1 \mathrm{~S} / \mathrm{C} 6 \mathrm{H} 9 \mathrm{O} / \mathrm{c} 1-3-4-5-6(2) 7 / \mathrm{h} 3-4 \mathrm{H}, 1,5 \mathrm{H} 2,2 \mathrm{H} 3$ \\
\hline 2350 & NEC6KET4-10 & {$[\mathrm{O}] \mathrm{CC}(\mathrm{CC}=\mathrm{O})(\mathrm{C}) \mathrm{C}$} & $\operatorname{lnChl}=1 \mathrm{~S} / \mathrm{C} 6 \mathrm{H} 11 \mathrm{O} 2 / \mathrm{c1} 1-6(2,5-8) 3-4-7 / \mathrm{h} 4 \mathrm{H}, 3,5 \mathrm{H} 2,1-2 \mathrm{H} 3$ \\
\hline 2351 & IC6KET1-2 & $\mathrm{CC}(\mathrm{C}=\mathrm{O})(\mathrm{OO}) \mathrm{CCC}$ & InChl=1S/C6H12O3/c1-3-4-6(2,5-7)9-8/h5,8H,3-4H2,1-2H3 \\
\hline 2352 & IC6Q13-2 & $\mathrm{OOC}[\mathrm{C}](\mathrm{C}(\mathrm{OO}) \mathrm{CC}) \mathrm{C}$ & InChl=1S/C6H13O4/c1-3-6(10-8)5(2)4-9-7/h6-8H,3-4H2,1-2H3 \\
\hline 2353 & $\mathrm{IC} 3 \mathrm{H} 6 \mathrm{COC} 2 \mathrm{H} 3$ & {$[\mathrm{CH} 2] \mathrm{C}(\mathrm{C}) \mathrm{C}(=\mathrm{O}) \mathrm{C}=\mathrm{C}$} & InChl=1S/C6H9O/c1-4-6(7)5(2)3/h4-5H,1-2H2,3H3 \\
\hline 2354 & NEC6KET4-30 & $\mathrm{O}=\mathrm{CC}(\mathrm{C}(\mathrm{C})(\mathrm{C}) \mathrm{C})[\mathrm{O}]$ & InChl=1S/C6H11O2/c1-6(2,3)5(8)4-7/h4-5H,1-3H3 \\
\hline 2355 & IC6KET1-3 & $\operatorname{ccc}(C(C=0) C) 00$ & InChl=1S/C6H12O3/c1-3-6(9-8)5(2)4-7/h4-6,8H,3H2,1-2H3 \\
\hline 2356 & IC6Q13-4 & $\mathrm{OOCC}(\mathrm{C}(\mathrm{OO})[\mathrm{CH}] \mathrm{C}) \mathrm{C}$ & InChl=1S/C6H13O4/c1-3-6(10-8)5(2)4-9-7/h3,5-8H,4H2,1-2H3 \\
\hline 2357 & TСC3H6COC2H3 & $C[C](C) C(=O) C=C$ & $\operatorname{lnChl}=1 \mathrm{~S} / \mathrm{C} 6 \mathrm{H} 9 \mathrm{O} / \mathrm{c1}-4-6(7) 5(2) 3 / \mathrm{h} 4 \mathrm{H}, 1 \mathrm{H} 2,2-3 \mathrm{H} 3$ \\
\hline 2358 & I3C6KET1-20 & $\operatorname{CCC}(\mathrm{C}(\mathrm{C}=\mathrm{O})[0]) \mathrm{C}$ & $\operatorname{lnChl}=1 \mathrm{~S} / \mathrm{C} 6 \mathrm{H} 11 \mathrm{O} 2 / \mathrm{c1}-3-5(2) 6(8) 4-7 / \mathrm{h} 4-6 \mathrm{H}, 3 \mathrm{H} 2,1-2 \mathrm{H} 3$ \\
\hline 2359 & IC6KET1-4 & $\mathrm{OOC}(\mathrm{CC}(\mathrm{C}=0) \mathrm{C}) \mathrm{C}$ & InChl=1S/C6H12O3/c1-5(4-7)3-6(2)9-8/h4-6,8H,3H2,1-2H3 \\
\hline 2360 & IC6Q13-5 & $\mathrm{OOCC}(\mathrm{C}(\mathrm{OO}) \mathrm{C}[\mathrm{CH} 2]) \mathrm{C}$ & InChl=1S/C6H13O4/c1-3-6(10-8)5(2)4-9-7/h5-8H,1,3-4H2,2H3 \\
\hline 2361 & CYHX1N3OJ & {$[\mathrm{O}] \mathrm{C} 1 \mathrm{CCCC}=\mathrm{C} 1$} & $\operatorname{lnChl}=15 / C 6 H 9 O / c 7-6-4-2-1-3-5-6 / h 2,4,6 \mathrm{H}, 1,3,5 \mathrm{H} 2$ \\
\hline 2362 & I3C6KET1-30 & $\mathrm{CC}(\mathrm{CC})(\mathrm{CC}=0)[0]$ & $\operatorname{lnChl}=1 \mathrm{~S} / \mathrm{C} 6 \mathrm{H} 11 \mathrm{O} 2 / \mathrm{c1} 1-3-6(2,8) 4-5-7 / \mathrm{h} 5 \mathrm{H}, 3-4 \mathrm{H} 2,1-2 \mathrm{H} 3$ \\
\hline
\end{tabular}




\begin{tabular}{|c|c|c|c|}
\hline 2363 & IC6KET1-5 & $\operatorname{oocccc}(\mathrm{C}=0) \mathrm{C}$ & InChl=15/C6H12O3/c1-6(5-7)3-2-4-9-8/h5-6,8H,2-4H2,1H3 \\
\hline 2364 & IC6Q14-1A & $\mathrm{OOCC}(\mathrm{CC}(\mathrm{OO}) \mathrm{C})[\mathrm{CH} 2]$ & InChl=15/C6H13O4/c1-5(4-9-7)3-6(2)10-8/h5-8H,1,3-4H2,2H3 \\
\hline 2365 & CYHX1N4OJ & {$[0] \mathrm{C} 1 \mathrm{CCC}=\mathrm{CC} 1$} & InChl=15/C6H9O/c7-6-4-2-1-3-5-6/h1-2,6H,3-5H2 \\
\hline 2366 & I3C6KET1-40 & $\mathrm{O}=\mathrm{CCC}(\mathrm{C}[\mathrm{O}]) \mathrm{CC}$ & $\operatorname{lnChl}=1 \mathrm{~S} / \mathrm{C} 6 \mathrm{H} 11 \mathrm{O} 2 / \mathrm{c1}-2-6(5-8) 3-4-7 / \mathrm{h} 4,6 \mathrm{H}, 2-3,5 \mathrm{H} 2,1 \mathrm{H} 3$ \\
\hline 2367 & IC6KET3-1 & $\mathrm{CC}(\mathrm{C}(=0) \mathrm{CC}) \mathrm{COO}$ & InChl=1S/C6H12O3/c1-3-6(7)5(2)4-9-8/h5,8H,3-4H2,1-2H3 \\
\hline 2368 & IC6Q14-2 & $\mathrm{OOC}[\mathrm{C}](\mathrm{CC}(\mathrm{OO}) \mathrm{C}) \mathrm{C}$ & InChl=15/C6H13O4/c1-5(4-9-7)3-6(2)10-8/h6-8H,3-4H2,1-2H3 \\
\hline 2369 & HX2N1AL6J & {$[\mathrm{CH} 2] \mathrm{CCC}=\mathrm{CC}=\mathrm{O}$} & InChl=1S/C6H9O/c1-2-3-4-5-6-7/h4-6H,1-3H2 \\
\hline 2370 & I3C6KET1-50 & $\mathrm{CC}(\mathrm{C}([\mathrm{O}]) \mathrm{C}) \mathrm{CC}=0$ & InChl=1S/C6H11O2/c1-5(3-4-7)6(2)8/h4-6H,3H2,1-2H3 \\
\hline 2371 & IC6KET3-2 & $\operatorname{ccC}(=0) C(O O)(C) C$ & InChl=1S/C6H12O3/c1-4-5(7)6(2,3)9-8/h8H,4H2,1-3H3 \\
\hline 2372 & IC6Q14-3 & $\mathrm{OOCC}([\mathrm{CH}] \mathrm{C}(\mathrm{OO}) \mathrm{C}) \mathrm{C}$ & InChl=1S/C6H13O4/c1-5(4-9-7)3-6(2)10-8/h3,5-8H,4H2,1-2H3 \\
\hline 2373 & 13С6KET1-60 & {$[\mathrm{O}] \mathrm{CCC}(\mathrm{CC}=\mathrm{O}) \mathrm{C}$} & $\operatorname{lnChl}=1 \mathrm{~S} / \mathrm{C} 6 \mathrm{H} 11 \mathrm{O} 2 / \mathrm{c1}-6(2-4-7) 3-5-8 / \mathrm{h} 4,6 \mathrm{H}, 2-3,5 \mathrm{H} 2,1 \mathrm{H} 3$ \\
\hline 2374 & IC6KET3-4 & $\mathrm{OOC}(\mathrm{C}(=0) \mathrm{C}(\mathrm{C}) \mathrm{C}) \mathrm{C}$ & InChl=1S/C6H12O3/c1-4(2)6(7)5(3)9-8/h4-5,8H,1-3H3 \\
\hline 2375 & IC6Q14-5 & $\mathrm{OOCC}(\mathrm{CC}(\mathrm{OO})[\mathrm{CH} 2]) \mathrm{C}$ & InChl=1S/C6H13O4/c1-5(4-9-7)3-6(2)10-8/h5-8H,2-4H2,1H3 \\
\hline 2376 & CHX1V04J & $\mathrm{O}=\mathrm{C} 1 \mathrm{CC}[\mathrm{CH}] \mathrm{CC} 1$ & InChl=1S/C6H9O/c7-6-4-2-1-3-5-6/h1H,2-5H2 \\
\hline 2377 & I3С6KET2-10 & $\operatorname{CCC}(\mathrm{C}(=0) \mathrm{C}[\mathrm{O}]) \mathrm{C}$ & $\operatorname{lnChl}=1 \mathrm{~S} / \mathrm{C} 6 \mathrm{H} 11 \mathrm{O} 2 / \mathrm{c1}-3-5(2) 6(8) 4-7 / \mathrm{h} 5 \mathrm{H}, 3-4 \mathrm{H} 2,1-2 \mathrm{H} 3$ \\
\hline 2378 & IC6KET3-5 & $00 \operatorname{ccc}(=0) \mathrm{C}(\mathrm{C}) \mathrm{C}$ & InChl=1S/C6H12O3/c1-5(2)6(7)3-4-9-8/h5,8H,3-4H2,1-2H3 \\
\hline 2379 & IC6Q15-1A & $\mathrm{OOCCCC}(\mathrm{COO})[\mathrm{CH} 2]$ & InChl=15/C6H13O4/c1-6(5-10-8)3-2-4-9-7/h6-8H,1-5H2 \\
\hline 2380 & HX1N5AL6J & $\mathrm{C}=\mathrm{CCCC}(=\mathrm{C})[0]$ & $\operatorname{lnChl}=1 \mathrm{~S} / \mathrm{C} 6 \mathrm{H} 9 \mathrm{O} / \mathrm{c1}-3-4-5-6(2) 7 / \mathrm{h} 3 \mathrm{H}, 1-2,4-5 \mathrm{H} 2$ \\
\hline 2381 & I3C6KET2-30 & $\operatorname{ccc}(\mathrm{C}(=)) \mathrm{C})([\mathrm{O}]) \mathrm{C}$ & InChl=1S/C6H1102/c1-4-6(3,8)5(2)7/h4H2,1-3H3 \\
\hline 2382 & IC6KET4-1 & OOCC $(\mathrm{CC}(=0) \mathrm{C}) \mathrm{C}$ & InChl=1S/C6H12O3/c1-5(4-9-8)3-6(2)7/h5,8H,3-4H2,1-2H3 \\
\hline 2383 & IC6Q15-2 & OOCCC[C](COO)C & InChl=1S/C6H13O4/c1-6(5-10-8)3-2-4-9-7/h7-8H,2-5H2,1H3 \\
\hline 2384 & I3C6KET2-4O & $\operatorname{ccc}(\mathrm{C}(=0) \mathrm{C}) \mathrm{C}[\mathrm{O}]$ & $\operatorname{lnChl}=15 / \mathrm{C} 6 \mathrm{H} 1102 / \mathrm{c} 1-3-6(4-7) 5(2) 8 / \mathrm{h} 6 \mathrm{H}, 3-4 \mathrm{H} 2,1-2 \mathrm{H} 3$ \\
\hline 2385 & IC6KET4-2 & $\mathrm{OOC}(\mathrm{CC}(=\mathrm{O}) \mathrm{C})(\mathrm{C}) \mathrm{C}$ & $\operatorname{lnChl}=1 \mathrm{~S} / \mathrm{C} 6 \mathrm{H} 12 \mathrm{O} 3 / \mathrm{c} 1-5(7) 4-6(2,3) 9-8 / \mathrm{h} 8 \mathrm{H}, 4 \mathrm{H} 2,1-3 \mathrm{H} 3$ \\
\hline 2386 & IC6Q15-3 & $\mathrm{OOCC}[\mathrm{CH}] \mathrm{C}(\mathrm{COO}) \mathrm{C}$ & InChl=1S/C6H13O4/c1-6(5-10-8)3-2-4-9-7/h3,6-8H,2,4-5H2,1H3 \\
\hline 2387 & HX5ENAL1J & $\mathrm{C}=\mathrm{CCCC}[\mathrm{C}]=\mathrm{O}$ & $\operatorname{lnChl}=1 \mathrm{~S} / \mathrm{C} 6 \mathrm{H} 9 \mathrm{O} / \mathrm{c1}-2-3-4-5-6-7 / \mathrm{h} 2 \mathrm{H}, 1,3-5 \mathrm{H} 2$ \\
\hline 2388 & I3C6KET2-50 & $\mathrm{CC}(\mathrm{C}(\mathrm{C}(=\mathrm{O}) \mathrm{C}) \mathrm{C})[0]$ & InChl=1S/C6H11O2/c1-4(5(2)7)6(3)8/h4-5H,1-3H3 \\
\hline 2389 & IC6KET4-3 & $0 O C(C(=0) C) C(C) C$ & InChl=1S/C6H12O3/c1-4(2)6(9-8)5(3)7/h4,6,8H,1-3H3 \\
\hline 2390 & IC6Q15-4 & $\mathrm{OOC}[\mathrm{CH}] \mathrm{CC}(\mathrm{COO}) \mathrm{C}$ & InChl=15/C6H13O4/c1-6(5-10-8)3-2-4-9-7/h2,6-8H,3-5H2,1H3 \\
\hline 2391 & HX5ENAL2J & $\mathrm{C}=\mathrm{CCCC}=\mathrm{C}[\mathrm{O}]$ & $\operatorname{lnChl}=1 \mathrm{~S} / \mathrm{C} 6 \mathrm{H} 9 \mathrm{O} / \mathrm{c} 1-2-3-4-5-6-7 / \mathrm{h} 2,5-6 \mathrm{H}, 1,3-4 \mathrm{H} 2$ \\
\hline 2392 & I3C6KET2-60 & $\mathrm{CC}(\mathrm{C}(=) \mathrm{O}) \mathrm{CC}[\mathrm{O}]$ & $\operatorname{lnChl}=1 \mathrm{~S} / \mathrm{C} 6 \mathrm{H} 11 \mathrm{O} 2 / \mathrm{c} 1-5(3-4-7) 6(2) 8 / \mathrm{h} 5 \mathrm{H}, 3-4 \mathrm{H} 2,1-2 \mathrm{H} 3$ \\
\hline 2393 & IC6KET4-5 & $\mathrm{OOCC}(=0) \operatorname{cc}(\mathrm{C}) \mathrm{C}$ & InChl=1S/C6H12O3/c1-5(2)3-6(7)4-9-8/h5,8H,3-4H2,1-2H3 \\
\hline 2394 & IC6Q23-1 & $\operatorname{CCC}(\mathrm{C}(\mathrm{OO})([\mathrm{CH} 2]) \mathrm{C}) \mathrm{OO}$ & InChl=1S/C6H1304/c1-4-5(9-7)6(2,3)10-8/h5,7-8H,2,4H2,1,3H3 \\
\hline 2395 & HX5ENAL3J & $\mathrm{C}=\mathrm{CC}[\mathrm{CH}] \mathrm{CC}=\mathrm{O}$ & $\operatorname{lnChl}=1 \mathrm{~S} / \mathrm{C} 6 \mathrm{H} 9 \mathrm{O} / \mathrm{c} 1-2-3-4-5-6-7 / \mathrm{h} 2,4,6 \mathrm{H}, 1,3,5 \mathrm{H} 2$ \\
\hline 2396 & XC6KET1-1AO & {$[0] \operatorname{CC}(\mathrm{C}(\mathrm{C}) \mathrm{C}) \mathrm{C}=\mathrm{O}$} & $\operatorname{lnChl}=1 \mathrm{~S} / \mathrm{C} 6 \mathrm{H} 11 \mathrm{O} 2 / \mathrm{c} 1-5(2) 6(3-7) 4-8 / \mathrm{h} 3,5-6 \mathrm{H}, 4 \mathrm{H} 2,1-2 \mathrm{H} 3$ \\
\hline 2397 & IC6KET5-1 & $\mathrm{OOCC}(\mathrm{CCC}=0) \mathrm{C}$ & InChl=1S/C6H12O3/c1-6(5-9-8)3-2-4-7/h4,6,8H,2-3,5H2,1H3 \\
\hline 2398 & IC6Q23-4 & $\mathrm{C}[\mathrm{CH}] \mathrm{C}(\mathrm{C}(\mathrm{OO})(\mathrm{C}) \mathrm{C}) \mathrm{OO}$ & InChl=1S/C6H1304/c1-4-5(9-7)6(2,3)10-8/h4-5,7-8H,1-3H3 \\
\hline 2399 & HX5ENAL4J & $\mathrm{C}=\mathrm{C}[\mathrm{CH}] \mathrm{CCC}=\mathrm{O}$ & InChl=15/C6H9O/c1-2-3-4-5-6-7/h2-3,6H,1,4-5H2 \\
\hline 2400 & I3C6KET4-10 & {$[\mathrm{O}] \mathrm{CCC}(\mathrm{C}=0) \mathrm{CCC}$} & $\operatorname{lnChl}=1 \mathrm{~S} / \mathrm{C} 6 \mathrm{H} 11 \mathrm{O} 2 / \mathrm{c1}-2-6(5-8) 3-4-7 / \mathrm{h} 5-6 \mathrm{H}, 2-4 \mathrm{H} 2,1 \mathrm{H} 3$ \\
\hline 2401 & IC6KET5-2 & $\mathrm{OOC}(\mathrm{CCC}=\mathrm{O})(\mathrm{C}) \mathrm{C}$ & InChl=1S/C6H12O3/c1-6(2,9-8)4-3-5-7/h5,8H,3-4H2,1-2H3 \\
\hline 2402 & IC6Q23-5 & {$[\mathrm{CH} 2] \mathrm{CC}(\mathrm{C}(\mathrm{OO})(\mathrm{C}) \mathrm{C}) \mathrm{OO}$} & InChl=1S/C6H13O4/c1-4-5(9-7)6(2,3)10-8/h5,7-8H,1,4H2,2-3H3 \\
\hline 2403 & C6D2Y1-1R & $\mathrm{CCCC}=\mathrm{C}[\mathrm{C}](=\mathrm{O})$ & InChl=1S/C6H9O/c1-2-3-4-5-6-7/h4-5H,2-3H2,1H3 \\
\hline 2404 & XC6KET1-2O & $\mathrm{O}=\mathrm{CC}(\mathrm{C}(\mathrm{C}) \mathrm{C})([\mathrm{O}]) \mathrm{C}$ & InChl=1S/C6H11O2/c1-5(2)6(3,8)4-7/h4-5H,1-3H3 \\
\hline 2405 & IC6KET5-3 & $\mathrm{OOC}(\mathrm{C}(\mathrm{C}) \mathrm{C}) \mathrm{CC}=\mathrm{O}$ & InChl=1S/C6H12O3/c1-5(2)6(9-8)3-4-7/h4-6,8H,3H2,1-2H3 \\
\hline 2406 & IC6Q24-1 & $\mathrm{OOC}(\mathrm{CC}(\mathrm{OO})([\mathrm{CH} 2]) \mathrm{C}) \mathrm{C}$ & InChl=1S/C6H1304/c1-5(9-7)4-6(2,3)10-8/h5,7-8H,2,4H2,1,3H3 \\
\hline 2407 & IC6D34-2OR & $\mathrm{CC}([\mathrm{O}])(\mathrm{C}) \mathrm{C}=\mathrm{C}=\mathrm{C}$ & InChl=1S/C6H9O/c1-4-5-6(2,3)7/h5H,1H2,2-3H3 \\
\hline 2408 & I3C6KET4-2O & $\operatorname{ccc}(C([0])) C) C=0$ & InChl=1S/C6H11O2/c1-3-6(4-7)5(2)8/h4-6H,3H2,1-2H3 \\
\hline 2409 & IC6KET5-4 & $\mathrm{OOC}(\mathrm{CC}(\mathrm{C}) \mathrm{C}) \mathrm{C}=\mathrm{O}$ & InChl=1S/C6H12O3/c1-5(2)3-6(4-7)9-8/h4-6,8H,3H2,1-2H3 \\
\hline 2410 & IC6Q24-3 & $\mathrm{OOC}([\mathrm{CH}] \mathrm{C}(\mathrm{OO})(\mathrm{C}) \mathrm{C}) \mathrm{C}$ & InChl=1S/C6H1304/c1-5(9-7)4-6(2,3)10-8/h4-5,7-8H,1-3H3 \\
\hline 2411 & IC6D1Y4-3R & $\mathrm{C}=\mathrm{C}(\mathrm{C})[\mathrm{CH}] \mathrm{C}(=\mathrm{O}) \mathrm{C}$ & $\operatorname{lnChl}=1 \mathrm{~S} / \mathrm{C} 6 \mathrm{H} 9 \mathrm{O} / \mathrm{c1}-5(2) 4-6(3) 7 / \mathrm{h} 4 \mathrm{H}, 1 \mathrm{H} 2,2-3 \mathrm{H} 3$ \\
\hline 2412 & XC6KET1-30 & $0=C C(C([0])(C) C) C$ & InChl=15/C6H1102/c1-5(4-7)6(2,3)8/h4-5H,1-3H3 \\
\hline 2413 & I3C6O23-60OH & CC1(CCOO)OC1C & InChl=15/C6H12O3/c1-5-6(2,9-5)3-4-8-7/h5,7H,3-4H2,1-2H3 \\
\hline 2414 & IC6Q24-5 & $\mathrm{OOC}(\mathrm{CC}(\mathrm{OO})(\mathrm{C}) \mathrm{C})[\mathrm{CH} 2]$ & InChl=1S/C6H13O4/c1-5(9-7)4-6(2,3)10-8/h5,7-8H,1,4H2,2-3H3 \\
\hline 2415 & IC3H7COC2H3 & $C C(C) C(=0) C=C$ & InChl=1S/C6H10O/c1-4-6(7)5(2)3/h4-5H,1H2,2-3H3 \\
\hline 2416 & I3C6KET4-30 & $\operatorname{ccc}(\mathrm{C}=0)(\mathrm{CC})[\mathrm{O}]$ & InChl=1S/C6H11O2/c1-3-6(8,4-2)5-7/h5H,3-4H2,1-2H3 \\
\hline 2417 & 13C6O24-10OH & CCC1COC1COO & InChl=1S/C6H12O3/c1-2-5-3-8-6(5)4-9-7/h5-7H,2-4H2,1H3 \\
\hline 2418 & IC6Q25-1 & $\mathrm{OOCCCC}(\mathrm{OO})([\mathrm{CH} 2]) \mathrm{C}$ & InChl=1S/C6H13O4/c1-6(2,10-8)4-3-5-9-7/h7-8H,1,3-5H2,2H3 \\
\hline 2419 & ETES1 & $\mathrm{C}=\mathrm{CC}(\mathrm{O} 1) \mathrm{C} 1 \mathrm{CC}$ & $\operatorname{lnChl}=1 \mathrm{~S} / \mathrm{C} 6 \mathrm{H} 100 / \mathrm{c} 1-3-5-6(4-2) 7-5 / \mathrm{h} 3,5-6 \mathrm{H}, 1,4 \mathrm{H} 2,2 \mathrm{H} 3$ \\
\hline 2420 & XC6KET1-4O & {$[\mathrm{O}] \mathrm{CC}(\mathrm{C}(\mathrm{C}=\mathrm{O}) \mathrm{C}) \mathrm{C}$} & InChl=1S/C6H11O2/c1-5(3-7)6(2)4-8/h3,5-6H,4H2,1-2H3 \\
\hline 2421 & 13C6O24-30OH & $\operatorname{ccc} 1(00) \operatorname{coc} 1 \mathrm{C}$ & InChl=1S/C6H12O3/c1-3-6(9-7)4-8-5(6)2/h5,7H,3-4H2,1-2H3 \\
\hline 2422 & XC6Q11A-2 & $\mathrm{OOC}[\mathrm{C}](\mathrm{C}(\mathrm{C}) \mathrm{C}) \mathrm{COO}$ & InChl=1S/C6H13O4/c1-5(2)6(3-9-7)4-10-8/h5,7-8H,3-4H2,1-2H3 \\
\hline 2423 & MVOX & $\mathrm{C}=\mathrm{CC}(\mathrm{O} 1) \mathrm{CC} 1 \mathrm{C}$ & $\operatorname{lnChl}=1 \mathrm{~S} / \mathrm{C} 6 \mathrm{H} 100 / \mathrm{c} 1-3-6-4-5(2) 7-6 / \mathrm{h} 3,5-6 \mathrm{H}, 1,4 \mathrm{H} 2,2 \mathrm{H} 3$ \\
\hline 2424 & IC6KET1-1AO & $\operatorname{cccc}(\mathrm{C}=0) \mathrm{C}[\mathrm{O}]$ & InChl=1S/C6H11O2/c1-2-3-6(4-7)5-8/h4,6H,2-3,5H2,1H3 \\
\hline 2425 & I3C6O24-50OH & $\mathrm{CC}(\mathrm{C} 1 \mathrm{COC} 1 \mathrm{C}) \mathrm{OO}$ & InChl=15/C6H12O3/c1-4-6(3-8-4)5(2)9-7/h4-7H,3H2,1-2H3 \\
\hline 2426 & IC6Q25-3 & $\mathrm{OOCC}[\mathrm{CH}] \mathrm{C}(\mathrm{OO})(\mathrm{C}) \mathrm{C}$ & InChl=1S/C6H13O4/c1-6(2,10-8)4-3-5-9-7/h4,7-8H,3,5H2,1-2H3 \\
\hline 2427 & VTHF & $\mathrm{C}=\mathrm{CC} 1 \mathrm{CCCO} 1$ & InChl=15/C6H10O/c1-2-6-4-3-5-7-6/h2,6H,1,3-5H2 \\
\hline 2428 & IC6KET1-2O & $\mathrm{CC}(\mathrm{C}=\mathrm{O})(\mathrm{CCC})[\mathrm{O}]$ & $\operatorname{lnChl}=1 \mathrm{~S} / \mathrm{C} 6 \mathrm{H} 11 \mathrm{O} 2 / \mathrm{c} 1-3-4-6(2,8) 5-7 / \mathrm{h} 5 \mathrm{H}, 3-4 \mathrm{H} 2,1-2 \mathrm{H} 3$ \\
\hline 2429 & $13 \mathrm{C} 6024-60 \mathrm{OH}$ & CC1OCC1CCOO & InChl=1S/C6H12O3/c1-5-6(4-8-5)2-3-9-7/h5-7H,2-4H2,1H3 \\
\hline 2430 & $\mathrm{XC6Q11 \textrm {A } - 3}$ & $00 \mathrm{OOC}([\mathrm{C}](\mathrm{C}) \mathrm{C}) \mathrm{COO}$ & InChl=1S/C6H13O4/c1-5(2)6(3-9-7)4-10-8/h6-8H,3-4H2,1-2H3 \\
\hline 2431 & EDHF & $\mathrm{CCC} 1 \mathrm{C}=\mathrm{CCO} 1$ & InChl=1S/C6H100/c1-2-6-4-3-5-7-6/h3-4,6H,2,5H2,1H3 \\
\hline 2432 & IC6KET1-30 & $\operatorname{ccc}(\mathrm{C}(\mathrm{C}=\mathrm{O}) \mathrm{C})[\mathrm{O}]$ & InChl=1S/C6H11O2/c1-3-6(8)5(2)4-7/h4-6H,3H2,1-2H3 \\
\hline 2433 & I3C6O25-10OH & CC1C(COO)OC1C & InChl=1S/C6H12O3/c1-4-5(2)9-6(4)3-8-7/h4-7H,3H2,1-2H3 \\
\hline 2434 & IC6Q25-4 & $\mathrm{OOC}[\mathrm{CH}] \mathrm{CC}(\mathrm{OO})(\mathrm{C}) \mathrm{C}$ & InChl=1S/C6H13O4/c1-6(2,10-8)4-3-5-9-7/h3,7-8H,4-5H2,1-2H3 \\
\hline 2435 & CHXYO12 & $\mathrm{C} 1 \mathrm{CCC} 2 \mathrm{C}(\mathrm{C} 1) \mathrm{O} 2$ & InChl=15/C6H100/c1-2-4-6-5(3-1)7-6/h5-6H,1-4H2 \\
\hline 2436 & IC6KET1-4O & $\mathrm{CC}(\mathrm{C}=0) \mathrm{CC}([0]) \mathrm{C}$ & InChl=15/C6H1102/c1-5(4-7)3-6(2)8/h4-6H,3H2,1-2H3 \\
\hline 2437 & I3C6O25-30OH & $0 O C 1(C) C(C) O C 1 C$ & InChl=15/C6H12O3/c1-4-6(3,9-7)5(2)8-4/h4-5,7H,1-3H3 \\
\hline 2438 & XC6Q11A-4 & $\mathrm{OOCC}(\mathrm{C}([\mathrm{CH} 2]) \mathrm{C}) \mathrm{COO}$ & InChl=15/C6H13O4/c1-5(2)6(3-9-7)4-10-8/h5-8H,1,3-4H2,2H3 \\
\hline 2439 & CHXYO14 & C1CC2OC1CC2 & InChl=1S/C6H100/c1-2-6-4-3-5(1)7-6/h5-6H,1-4H2 \\
\hline 2440 & IC6KET1-5O & $\mathrm{CC}(\mathrm{C}=0) \mathrm{CCC}[\mathrm{O}]$ & $\operatorname{lnChl}=1 \mathrm{~S} / \mathrm{C} 6 \mathrm{H} 11 \mathrm{O} 2 / \mathrm{c1}-6(5-8) 3-2-4-7 / \mathrm{h} 5-6 \mathrm{H}, 2-4 \mathrm{H} 2,1 \mathrm{H} 3$ \\
\hline 2441 & $13 \mathrm{C} 6 \mathrm{O} 25-4 \mathrm{OOH}$ & OOCC1C(C)OC1C & InChl=15/C6H12O3/c1-4-6(3-8-7)5(2)9-4/h4-7H,3H2,1-2H3 \\
\hline 2442 & IC6Q34-1 & $\mathrm{OOC}(\mathrm{C}(\mathrm{OO}) \mathrm{C}) \mathrm{C}([\mathrm{CH} 2]) \mathrm{C}$ & InChl=1S/C6H13O4/c1-4(2)6(10-8)5(3)9-7/h4-8H,1H2,2-3H3 \\
\hline 2443 & HEX5ENAL & $\mathrm{C}=\mathrm{CCCCC}=\mathrm{O}$ & $\operatorname{lnChl}=1 \mathrm{~S} / \mathrm{C} 6 \mathrm{H} 10 \mathrm{O} / \mathrm{c1}-2-3-4-5-6-7 / \mathrm{h} 2,6 \mathrm{H}, 1,3-5 \mathrm{H} 2$ \\
\hline 2444 & IC6KET3-10 & {$[\mathrm{O}] \mathrm{CC}(\mathrm{C}(=\mathrm{O}) \mathrm{CC}) \mathrm{C}$} & $\operatorname{lnChl}=1 \mathrm{~S} / \mathrm{C} 6 \mathrm{H} 11 \mathrm{O} 2 / \mathrm{c} 1-3-6(8) 5(2) 4-7 / \mathrm{h} 5 \mathrm{H}, 3-4 \mathrm{H} 2,1-2 \mathrm{H} 3$ \\
\hline 2445 & I3C6O34-10OH & $\mathrm{CCC1}(\mathrm{CCOO}) \mathrm{CO} 1$ & InChl=1S/C6H12O3/c1-2-6(5-8-6)3-4-9-7/h7H,2-5H2,1H3 \\
\hline 2446 & XC6Q12-1A & $\mathrm{OOCC}(\mathrm{C}(\mathrm{C}) \mathrm{C})(\mathrm{OO})[\mathrm{CH} 2]$ & InChl=1S/C6H13O4/c1-5(2)6(3,10-8)4-9-7/h5,7-8H,3-4H2,1-2H3 \\
\hline 2447 & C6D2Y1 & $\mathrm{CCCC}=\mathrm{CC}(=0)$ & InChl=1S/C6H10O/c1-2-3-4-5-6-7/h4-6H,2-3H2,1H3 \\
\hline 2448 & IC6KET3-2O & $\operatorname{ccC}(=0) C([O])(C) C$ & $\operatorname{lnChl}=1 \mathrm{~S} / \mathrm{C} 6 \mathrm{H} 1102 / \mathrm{c} 1-4-5(7) 6(2,3) 8 / \mathrm{h} 4 \mathrm{H} 2,1-3 \mathrm{H} 3$ \\
\hline 2449 & IC6011A-20OH & $\operatorname{cccc1}(00) \operatorname{COC} 1$ & InChl=1S/C6H12O3/c1-2-3-6(9-7)4-8-5-6/h7H,2-5H2,1H3 \\
\hline 2450 & IC6Q34-2 & $0 O C(C(O O) C)[C](C) C$ & InChl=1S/C6H13O4/c1-4(2)6(10-8)5(3)9-7/h5-8H,1-3H3 \\
\hline 2451 & IC6D2Y4 & $\mathrm{CC}(\mathrm{C})=\mathrm{CC}(=\mathrm{O}) \mathrm{C}$ & InChl=1S/C6H100/c1-5(2)4-6(3)7/h4H,1-3H3 \\
\hline 2452 & IC6KET3-4O & $\mathrm{CC}(\mathrm{C}(=\mathrm{O}) \mathrm{C}([\mathrm{O}]) \mathrm{C}) \mathrm{C}$ & InChl=1S/C6H11O2/c1-4(2)6(8)5(3)7/h4-5H,1-3H3 \\
\hline 2453 & 13С6034-20OH & $\mathrm{CC}(\mathrm{C} 1(\mathrm{CC}) \mathrm{CO} 1) \mathrm{OO}$ & InChl=15/C6H12O3/c1-3-6(4-8-6)5(2)9-7/h5,7H,3-4H2,1-2H3 \\
\hline
\end{tabular}




\begin{tabular}{|c|c|c|c|}
\hline 2454 & $\mathrm{XC6Q12-3}$ & $00 c c([C](C) C)(O O) C$ & InChl=15/C6H13O4/c1-5(2)6(3,10-8)4-9-7/h7-8H,4H2,1-3Н3 \\
\hline 2455 & NC3H7COC2H4S & $\operatorname{CCCC}(=0)[\mathrm{CH}] \mathrm{C}$ & InChl=1S/C6H11O/c1-3-5-6(7)4-2/h4H,3,5H2,1-2H3 \\
\hline 2456 & IC6KET3-50 & {$[\mathrm{O}] \mathrm{CCC}(=\mathrm{O}) \mathrm{C}(\mathrm{C}) \mathrm{C}$} & $\operatorname{lnChl}=1 \mathrm{~S} / \mathrm{C} 6 \mathrm{H} 11 \mathrm{O} 2 / \mathrm{c} 1-5(2) 6(8) 3-4-7 / \mathrm{h} 5 \mathrm{H}, 3-4 \mathrm{H} 2,1-2 \mathrm{H} 3$ \\
\hline 2457 & IC6011A-30OH & $\mathrm{CCC}(\mathrm{C} 1 \mathrm{COC} 1) \mathrm{OO}$ & $\operatorname{lnChl}=1 \mathrm{~S} / \mathrm{C} 6 \mathrm{H} 12 \mathrm{O} / \mathrm{c} 1-2-6(9-7) 5-3-8-4-5 / \mathrm{h} 5-7 \mathrm{H}, 2-4 \mathrm{H} 2,1 \mathrm{H} 3$ \\
\hline 2458 & IC6Q34-5 & $\mathrm{OOC}(\mathrm{C}(\mathrm{OO})[\mathrm{CH} 2]) \mathrm{C}(\mathrm{C}) \mathrm{C}$ & InChl=15/C6H13O4/c1-4(2)6(10-8)5(3)9-7/h4-8H,3H2,1-2H3 \\
\hline 2459 & IC5H11CO & $\mathrm{CC}(\mathrm{C}) \mathrm{CC}[\mathrm{C}]=0$ & $\operatorname{lnChl}=1 \mathrm{~S} / \mathrm{C} 6 \mathrm{H} 11 \mathrm{O} / \mathrm{c} 1-6(2) 4-3-5-7 / \mathrm{h} 6 \mathrm{H}, 3-4 \mathrm{H} 2,1-2 \mathrm{H} 3$ \\
\hline 2460 & C6H11-1D30OH & $\mathrm{C}=\mathrm{CC}(\mathrm{OO}) \mathrm{CCC}$ & InChl=1S/C6H12O2/c1-3-5-6(4-2)8-7/h4,6-7H,2-3,5H2,1H3 \\
\hline 2461 & IC6O11A-40OH & $\mathrm{OOC}(\mathrm{CC} 1 \mathrm{COC} 1) \mathrm{C}$ & InChl=1S/C6H12O3/c1-5(9-7)2-6-3-8-4-6/h5-7H,2-4H2,1H3 \\
\hline 2462 & $\mathrm{XC6Q12-4}$ & $\mathrm{OOCC}(\mathrm{C}([\mathrm{CH} 2]) \mathrm{C})(\mathrm{OO}) \mathrm{C}$ & InChl=1S/C6H13O4/c1-5(2)6(3,10-8)4-9-7/h5,7-8H,1,4H2,2-3H3 \\
\hline 2463 & C6H11-1D30 & $\mathrm{C}=\mathrm{CC}([\mathrm{O}]) \mathrm{CCC}$ & InChl=1S/C6H110/c1-3-5-6(7)4-2/h4,6H,2-3,5H2,1H3 \\
\hline 2464 & $\mathrm{C} 6 \mathrm{H} 11-1 \mathrm{D} 40 \mathrm{OH}$ & $\mathrm{C}=\mathrm{CCC}(\mathrm{OO}) \mathrm{CC}$ & $\operatorname{lnChl}=1 \mathrm{~S} / \mathrm{C} 6 \mathrm{H} 12 \mathrm{O} / \mathrm{cc1}-3-5-6(4-2) 8-7 / \mathrm{h} 3,6-7 \mathrm{H}, 1,4-5 \mathrm{H} 2,2 \mathrm{H} 3$ \\
\hline 2465 & IC6011A-50OH & OOCCCC1COC1 & $\operatorname{lnChl}=1 \mathrm{~S} / \mathrm{C} 6 \mathrm{H} 12 \mathrm{O} / \mathrm{c} 7-9-3-1-2-6-4-8-5-6 / \mathrm{h} 6-7 \mathrm{H}, 1-5 \mathrm{H} 2$ \\
\hline 2466 & IC6Q35-1 & $\mathrm{OOCCC}(\mathrm{C}([\mathrm{CH} 2]) \mathrm{C}) \mathrm{OO}$ & InChl=1S/C6H13O4/c1-5(2)6(10-8)3-4-9-7/h5-8H,1,3-4H2,2H3 \\
\hline 2467 & C6H11-1D40 & $\mathrm{C}=\mathrm{CCC}([\mathrm{O}]) \mathrm{CC}$ & InChl=1S/C6H110/c1-3-5-6(7)4-2/h3,6H,1,4-5H2,2H3 \\
\hline 2468 & $\mathrm{C} 6 \mathrm{H} 11-1 \mathrm{D} 5 \mathrm{OOH}$ & $\mathrm{C}=\mathrm{CCCC}(\mathrm{OO}) \mathrm{C}$ & $\operatorname{lnChl}=1 \mathrm{~S} / \mathrm{C} 6 \mathrm{H} 12 \mathrm{O} 2 / \mathrm{c} 1-3-4-5-6(2) 8-7 / \mathrm{h} 3,6-7 \mathrm{H}, 1,4-5 \mathrm{H} 2,2 \mathrm{H} 3$ \\
\hline 2469 & IC6O12-1AOOH & CC1(CCC)OC100 & InChl=1S/C6H12O3/c1-3-4-6(2)5(8-6)9-7/h5,7H,3-4H2,1-2H3 \\
\hline 2470 & XC6Q13-1A & $\mathrm{OOCC}(\mathrm{C}(\mathrm{OO})(\mathrm{C}) \mathrm{C})[\mathrm{CH} 2]$ & InChl=1S/C6H13O4/c1-5(4-9-7)6(2,3)10-8/h5,7-8H,1,4H2,2-3H3 \\
\hline 2471 & C6H11-1D50 & $\mathrm{C}=\mathrm{CCCC}([0]) \mathrm{C}$ & $\operatorname{lnChl}=1 \mathrm{~S} / \mathrm{C} 6 \mathrm{H} 110 / \mathrm{c} 1-3-4-5-6(2) 7 / \mathrm{h} 3,6 \mathrm{H}, 1,4-5 \mathrm{H} 2,2 \mathrm{H} 3$ \\
\hline 2472 & C6H11-1D60OH & $\mathrm{C}=\mathrm{Ccccc}(\mathrm{OO})$ & InChl=1S/C6H12O2/c1-2-3-4-5-6-8-7/h2,7H,1,3-6H2 \\
\hline 2473 & $\mathrm{IC} 6012-300 \mathrm{H}$ & $\operatorname{ccc}(\mathrm{C} 1(\mathrm{C}) \mathrm{CO} 1) 0 \mathrm{O}$ & InChl=15/C6H12O3/c1-3-5(9-7)6(2)4-8-6/h5,7H,3-4H2,1-2H3 \\
\hline 2474 & IC6Q35-2 & $00 \mathrm{occ}([\mathrm{C}](\mathrm{C}) \mathrm{C}) \mathrm{OO}$ & InChl=1S/C6H13O4/c1-5(2)6(10-8)3-4-9-7/h6-8H,3-4H2,1-2H3 \\
\hline 2475 & C6H11-1D6O & $\mathrm{C}=\mathrm{CCCCC}([0])$ & InChl=1S/C6H110/c1-2-3-4-5-6-7/h2H,1,3-6H2 \\
\hline 2476 & $\mathrm{C} 6 \mathrm{H} 11-2 \mathrm{D} 10 \mathrm{OH}$ & $\mathrm{C}(\mathrm{OO}) \mathrm{C}=\mathrm{CCCC}$ & InChl=1S/C6H12O2/c1-2-3-4-5-6-8-7/h4-5,7H,2-3,6H2,1H3 \\
\hline 2477 & $\mathrm{IC} 6012-40 \mathrm{OH}$ & $\mathrm{CC}(\mathrm{OO}) \mathrm{CC} 1(\mathrm{C}) \mathrm{CO} 1$ & InChl=1S/C6H12O3/c1-5(9-7)3-6(2)4-8-6/h5,7H,3-4H2,1-2H3 \\
\hline 2478 & XC6Q13-2 & $\mathrm{OOC}[\mathrm{C}](\mathrm{C}(\mathrm{OO})(\mathrm{C}) \mathrm{C}) \mathrm{C}$ & InChl=1S/C6H1304/c1-5(4-9-7)6(2,3)10-8/h7-8H,4H2,1-3H3 \\
\hline 2479 & C6H11-2D1O & $C([0]) \mathrm{C}=\mathrm{CCCC}$ & InChl=1S/C6H110/c1-2-3-4-5-6-7/h4-5H,2-3,6H2,1H3 \\
\hline 2480 & $\mathrm{C} 6 \mathrm{H} 11-2 \mathrm{D} 4 \mathrm{OOH}$ & $\mathrm{CC}=\mathrm{CC}(\mathrm{OO}) \mathrm{CC}$ & InChl=15/C6H12O2/c1-3-5-6(4-2)8-7/h3,5-7H,4H2,1-2H3 \\
\hline 2481 & $\mathrm{IC} 6012-50 \mathrm{OH}$ & $00 \mathrm{CCCC} 1(\mathrm{C}) \mathrm{CO} 1$ & InChl=15/C6H12O3/c1-6(5-8-6)3-2-4-9-7/h7H,2-5H2,1H3 \\
\hline 2482 & IC6Q35-4 & $\mathrm{OOC}[\mathrm{CH}] \mathrm{C}(\mathrm{C}(\mathrm{C}) \mathrm{C}) \mathrm{OO}$ & InChl=15/C6H13O4/c1-5(2)6(10-8)3-4-9-7/h3,5-8H,4H2,1-2H3 \\
\hline 2483 & C6H11-2D4O & $\mathrm{CC}=\mathrm{CC}([\mathrm{O}]) \mathrm{CC}$ & $\operatorname{lnChl}=1 \mathrm{~S} / \mathrm{C} 6 \mathrm{H} 110 / \mathrm{c} 1-3-5-6(7) 4-2 / \mathrm{h} 3,5-6 \mathrm{H}, 4 \mathrm{H} 2,1-2 \mathrm{H} 3$ \\
\hline 2484 & $\mathrm{C} 6 \mathrm{H} 11-2 \mathrm{D} 5 \mathrm{OOH}$ & $\mathrm{CC}=\mathrm{CCC}(\mathrm{OO}) \mathrm{C}$ & InChl=1S/C6H12O2/c1-3-4-5-6(2)8-7/h3-4,6-7H,5H2,1-2H3 \\
\hline 2485 & IC6O13-1AOOH & CCC1OCC1COO & InChl=1S/C6H12O3/c1-2-6-5(3-8-6)4-9-7/h5-7H,2-4H2,1H3 \\
\hline 2486 & $\mathrm{XC6Q13-4}$ & $\mathrm{OOCC}(\mathrm{C}(\mathrm{OO})([\mathrm{CH} 2]) \mathrm{C}) \mathrm{C}$ & InChl=1S/C6H1304/c1-5(4-9-7)6(2,3)10-8/h5,7-8H,2,4H2,1,3H3 \\
\hline 2487 & C6H11-2D5O & $\mathrm{CC}=\mathrm{CCC}([0]) \mathrm{C}$ & InChl=1S/C6H110/c1-3-4-5-6(2)7/h3-4,6H,5H2,1-2H3 \\
\hline 2488 & $\mathrm{C} 6 \mathrm{H} 11-2 \mathrm{D} 6 \mathrm{OOH}$ & $\mathrm{CC}=\mathrm{CCCC}(\mathrm{OO})$ & InChl=15/C6H12O2/c1-2-3-4-5-6-8-7/h2-3,7H,4-6H2,1H3 \\
\hline 2489 & NEC6O11A-1BOOH & $\operatorname{ccC} 1(\mathrm{COO}) \operatorname{COC} 1$ & InChl=15/C6H12O3/c1-2-6(5-9-7)3-8-4-6/h7H,2-5H2,1H3 \\
\hline 2490 & IC6Q45-1 & $\mathrm{OOCC}(\mathrm{CC}([\mathrm{CH} 2]) \mathrm{C}) \mathrm{OO}$ & InChl=15/C6H13O4/c1-5(2)3-6(10-8)4-9-7/h5-8H,1,3-4H2,2H3 \\
\hline 2491 & C6H11-2D60 & $\mathrm{CC}=\mathrm{CCCC}([0])$ & $\operatorname{lnChl}=1 \mathrm{~S} / \mathrm{C} 6 \mathrm{H} 11 \mathrm{O} / \mathrm{c1}-2-3-4-5-6-7 / \mathrm{h} 2-3 \mathrm{H}, 4-6 \mathrm{H} 2,1 \mathrm{H} 3$ \\
\hline 2492 & C6H11-3D10OH & $\mathrm{C}(\mathrm{OO}) \mathrm{CC}=\mathrm{CCC}$ & InChl=1S/C6H12O2/c1-2-3-4-5-6-8-7/h3-4,7H,2,5-6H2,1H3 \\
\hline 2493 & $\mathrm{IC} 6013-20 \mathrm{OH}$ & $\mathrm{CC} 1(\mathrm{COC} 1 \mathrm{CC}) 00$ & InChl=15/C6H12O3/c1-3-5-6(2,9-7)4-8-5/h5,7H,3-4H2,1-2H3 \\
\hline 2494 & XC6Q14-1A & $\mathrm{OOCC}(\mathrm{C}(\mathrm{COO}) \mathrm{C})[\mathrm{CH} 2]$ & InChl=1S/C6H13O4/c1-5(3-9-7)6(2)4-10-8/h5-8H,1,3-4H2,2H3 \\
\hline 2495 & C6H11-3D10 & $\mathrm{CCC}=\mathrm{CCC}([0])$ & $\operatorname{lnChl}=1 \mathrm{~S} / \mathrm{C} 6 \mathrm{H} 110 / \mathrm{c1} 1-2-3-4-5-6-7 / \mathrm{h} 3-4 \mathrm{H}, 2,5-6 \mathrm{H} 2,1 \mathrm{H} 3$ \\
\hline 2496 & $\mathrm{C} 6 \mathrm{H} 11-3 \mathrm{D} 2 \mathrm{OOH}$ & $\mathrm{CC}(\mathrm{OO}) \mathrm{C}=\mathrm{CCC}$ & InChl=1S/C6H12O2/c1-3-4-5-6(2)8-7/h4-7H,3H2,1-2H3 \\
\hline 2497 & NEC6011A-30OH & $\mathrm{OOC}(\mathrm{C} 1(\mathrm{C}) \operatorname{coc} 1) \mathrm{C}$ & InChl=1S/C6H12O3/c1-5(9-7)6(2)3-8-4-6/h5,7H,3-4H2,1-2H3 \\
\hline 2498 & IC6Q45-2 & $00 \mathrm{OCC}(\mathrm{C}[\mathrm{C}](\mathrm{C}) \mathrm{C}) 00$ & InChl=1S/C6H13O4/c1-5(2)3-6(10-8)4-9-7/h6-8H,3-4H2,1-2H3 \\
\hline 2499 & NC5H11CO & $\operatorname{ccccc}[C]=0$ & InChl=1S/C6H110/c1-2-3-4-5-6-7/h2-5H2,1H3 \\
\hline 2500 & NEC6D3-10OH & $\mathrm{OOCC}(\mathrm{C}=\mathrm{C})(\mathrm{C}) \mathrm{C}$ & InChl=1S/C6H12O2/c1-4-6(2,3)5-8-7/h4,7H,1,5H2,2-3H3 \\
\hline 2501 & $\mathrm{IC} 6 \mathrm{O} 13-40 \mathrm{OH}$ & $\mathrm{CC}(\mathrm{C} 10 \mathrm{CC} 1 \mathrm{C}) 00$ & InChl=1S/C6H12O3/c1-4-3-8-6(4)5(2)9-7/h4-7H,3H2,1-2H3 \\
\hline 2502 & $\mathrm{XC6Q14-2}$ & $\mathrm{OOC}[\mathrm{C}](\mathrm{C}(\mathrm{COO}) \mathrm{C}) \mathrm{C}$ & InChl=1S/C6H13O4/c1-5(3-9-7)6(2)4-10-8/h5,7-8H,3-4H2,1-2H3 \\
\hline 2503 & NC5H10CHO-1 & $\mathrm{CCCC}[\mathrm{CH}] \mathrm{C}=\mathrm{O}$ & InChl=15/C6H110/c1-2-3-4-5-6-7/h5-6H,2-4H2,1H3 \\
\hline 2504 & I3C6D1-30OH & $\mathrm{CC}(\mathrm{C}=\mathrm{C})(\mathrm{OO}) \mathrm{CC}$ & InChl=1S/C6H12O2/c1-4-6(3,5-2)8-7/h4,7H,1,5H2,2-3H3 \\
\hline 2505 & NEC6011A-40OH & $00 \mathrm{CCC} 1(\mathrm{C}) \operatorname{coc} 1$ & InChl=15/C6H12O3/c1-6(2-3-9-7)4-8-5-6/h7H,2-5H2,1H3 \\
\hline 2506 & IC6Q45-3 & $\mathrm{OOCC}([\mathrm{CH}] \mathrm{C}(\mathrm{C}) \mathrm{C}) \mathrm{OO}$ & InChl=15/C6H13O4/c1-5(2)3-6(10-8)4-9-7/h3,5-8H,4H2,1-2H3 \\
\hline 2507 & $\mathrm{NC5H} 10 \mathrm{CHO}-2$ & $\mathrm{CCC}[\mathrm{CH}] \mathrm{CC}=\mathrm{O}$ & InChl=1S/C6H110/c1-2-3-4-5-6-7/h4,6H,2-3,5H2,1H3 \\
\hline 2508 & 13C6D1-40OH & $\operatorname{ccc}(\mathrm{C}=\mathrm{C}) \mathrm{COO}$ & InChl=1S/C6H12O2/c1-3-6(4-2)5-8-7/h3,6-7H,1,4-5H2,2H3 \\
\hline 2509 & $\mathrm{IC} 6013-50 \mathrm{OH}$ & CC1COC1CCOO & InChl=1S/C6H12O3/c1-5-4-8-6(5)2-3-9-7/h5-7H,2-4H2,1H3 \\
\hline 2510 & $\mathrm{XC6Q23-1}$ & $\mathrm{OOC}(\mathrm{C}(\mathrm{OO})(\mathrm{C}) \mathrm{C})([\mathrm{CH} 2]) \mathrm{C}$ & InChl=1S/C6H1304/c1-5(2,9-7)6(3,4)10-8/h7-8H,1H2,2-4H3 \\
\hline 2511 & $\mathrm{NC5H} 10 \mathrm{CHO}-3$ & $\mathrm{CC}[\mathrm{CH}] \mathrm{CCC}=\mathrm{O}$ & InChl=1S/C6H110/c1-2-3-4-5-6-7/h3,6H,2,4-5H2,1H3 \\
\hline 2512 & $13 \mathrm{C} 6 \mathrm{D} 1-5 \mathrm{OOH}$ & $\mathrm{OOC}(\mathrm{C}(\mathrm{C}=\mathrm{C}) \mathrm{C}) \mathrm{C}$ & $\operatorname{lnChl}=1 \mathrm{~S} / \mathrm{C} 6 \mathrm{H} 12 \mathrm{O} 2 / \mathrm{c1}-4-5(2) 6(3) 8-7 / \mathrm{h} 4-7 \mathrm{H}, 1 \mathrm{H} 2,2-3 \mathrm{H} 3$ \\
\hline 2513 & NEC6013-1AOOH & $\mathrm{CC} 1(\mathrm{COO}) \operatorname{COC} 1 \mathrm{C}$ & $\operatorname{lnChl}=1 \mathrm{~S} / \mathrm{C} 6 \mathrm{H} 12 \mathrm{O} 3 / \mathrm{c} 1-5-6(2,3-8-5) 4-9-7 / \mathrm{h} 5,7 \mathrm{H}, 3-4 \mathrm{H} 2,1-2 \mathrm{H} 3$ \\
\hline 2514 & $13 \mathrm{C} 6 \mathrm{OOH} 1-2 \mathrm{O} 2$ & $\mathrm{CC}(\mathrm{C}(\mathrm{O}[\mathrm{O}]) \mathrm{COO}) \mathrm{CC}$ & InChl=15/C6H13O4/c1-3-5(2)6(10-8)4-9-7/h5-7H,3-4H2,1-2H3 \\
\hline 2515 & NC5H10CHO-4 & $\mathrm{C}[\mathrm{CH}] \mathrm{CCCC}=\mathrm{O}$ & InChl=1S/C6H11O/c1-2-3-4-5-6-7/h2,6H,3-5H2,1H3 \\
\hline 2516 & I3C6D1-60OH & $\mathrm{CC}(\mathrm{C}=\mathrm{C}) \mathrm{CCOO}$ & InChl=15/C6H12O2/c1-3-6(2)4-5-8-7/h3,6-7H,1,4-5H2,2H3 \\
\hline 2517 & IC6O14-1AOOH & OOCC1COC(C1)C & InChl=1S/C6H12O3/c1-5-2-6(3-8-5)4-9-7/h5-7H,2-4H2,1H3 \\
\hline 2518 & $13 \mathrm{C} 6 \mathrm{OOH} 1-3 \mathrm{O} 2$ & $00 \mathrm{OCC}(\mathrm{O}[\mathrm{O}])(\mathrm{CC}) \mathrm{C}$ & InChl=1S/C6H13O4/c1-3-6(2,10-8)4-5-9-7/h7H,3-5H2,1-2H3 \\
\hline 2519 & $\mathrm{NC5H} 10 \mathrm{CHO}-5$ & {$[\mathrm{CH} 2] \mathrm{CCCCC}=\mathrm{O}$} & InChl=1S/C6H11O/c1-2-3-4-5-6-7/h6H,1-5H2 \\
\hline 2520 & 13C6D2-10OH & $\mathrm{CC}(=\mathrm{CCOO}) \mathrm{CC}$ & InChl=1S/C6H12O2/c1-3-6(2)4-5-8-7/h4,7H,3,5H2,1-2H3 \\
\hline 2521 & NEC6O13-40OH & $00 \mathrm{cc} 10 \mathrm{OCC} 1(\mathrm{C}) \mathrm{C}$ & $\operatorname{lnChl}=1 \mathrm{~S} / \mathrm{C} 6 \mathrm{H} 12 \mathrm{O} / \mathrm{c} 1-6(2) 4-8-5(6) 3-9-7 / \mathrm{h} 5,7 \mathrm{H}, 3-4 \mathrm{H} 2,1-2 \mathrm{H} 3$ \\
\hline 2522 & $13 \mathrm{C} 6 \mathrm{OOH} 1-4 \mathrm{O} 2$ & OOCCC(CO[O])CC & InChl=1S/C6H13O4/c1-2-6(5-10-8)3-4-9-7/h6-7H,2-5H2,1H3 \\
\hline 2523 & $13 \mathrm{C} 6 \mathrm{D} 1-30$ & $\mathrm{CC}(\mathrm{C}=\mathrm{C})(\mathrm{CC})[\mathrm{O}]$ & InChl=1S/C6H11O/c1-4-6(3,7)5-2/h4H,1,5H2,2-3H3 \\
\hline 2524 & I3C6D2-40OH & $\mathrm{OOCC}(=\mathrm{CC}) \mathrm{CC}$ & InChl=15/C6H12O2/c1-3-6(4-2)5-8-7/h3,7H,4-5H2,1-2H3 \\
\hline 2525 & IC6O14-20OH & $\mathrm{OOC} 1(\mathrm{C}) \operatorname{coc}(\mathrm{C} 1) \mathrm{C}$ & $\operatorname{lnChl}=1 \mathrm{~S} / \mathrm{C} 6 \mathrm{H} 12 \mathrm{O} 3 / \mathrm{c} 1-5-3-6(2,9-7) 4-8-5 / \mathrm{h} 5,7 \mathrm{H}, 3-4 \mathrm{H} 2,1-2 \mathrm{H} 3$ \\
\hline 2526 & $13 \mathrm{C} 6 \mathrm{OOH} 1-5 \mathrm{O} 2$ & OOCCC $(\mathrm{C}(\mathrm{O}[\mathrm{O}]) \mathrm{C}) \mathrm{C}$ & InChl=15/C6H13O4/c1-5(3-4-9-7)6(2)10-8/h5-7H,3-4H2,1-2H3 \\
\hline 2527 & 13C6D1-40 & $\operatorname{ccc}(\mathrm{C}=\mathrm{C}) \mathrm{C}[\mathrm{O}]$ & InChl=15/C6H110/c1-3-6(4-2)5-7/h3,6H,1,4-5H2,2H3 \\
\hline 2528 & I3C6D2-50OH & $O O C(C(=C C) C) C$ & InChl=1S/C6H12O2/c1-4-5(2)6(3)8-7/h4,6-7H,1-3H3 \\
\hline 2529 & NEC6014-1AOOH & $00 \mathrm{OCC} 1(\mathrm{C}) \operatorname{cocc} 1$ & InChl=15/C6H12O3/c1-6(5-9-7)2-3-8-4-6/h7H,2-5H2,1H3 \\
\hline 2530 & $13 \mathrm{C} 6 \mathrm{OOH} 1-6 \mathrm{OO}$ & OOCCC(CCO[O])C & InChl=1S/C6H13O4/c1-6(2-4-9-7)3-5-10-8/h6-7H,2-5H2,1H3 \\
\hline 2531 & I3C6D1-50 & $\operatorname{CC}(C([0]) C) C=C$ & InChl=1S/C6H110/c1-4-5(2)6(3)7/h4-6H,1H2,2-3H3 \\
\hline 2532 & $13 \mathrm{C} 6 \mathrm{D} 2-60 \mathrm{OH}$ & $\mathrm{CC}(=\mathrm{CC}) \mathrm{CCOO}$ & $\operatorname{lnChl}=1 \mathrm{~S} / \mathrm{C} 6 \mathrm{H} 12 \mathrm{O} 2 / \mathrm{c} 1-3-6(2) 44-5-8-7 / \mathrm{h} 3,7 \mathrm{H}, 4-5 \mathrm{H} 2,1-2 \mathrm{H} 3$ \\
\hline 2533 & $\mathrm{IC} 6014-300 \mathrm{H}$ & $00 \mathrm{C} 1 \mathrm{C}(\mathrm{C}) \operatorname{coc} 1 \mathrm{C}$ & InChl=1S/C6H12O3/c1-4-3-8-5(2)6(4)9-7/h4-7H,3H2,1-2H3 \\
\hline 2534 & $13 \mathrm{C} 6 \mathrm{OOH} 2-102$ & $\mathrm{CC}(\mathrm{C}(\mathrm{OO}) \mathrm{CO}[\mathrm{O}]) \mathrm{CC}$ & InChl=1S/C6H13O4/c1-3-5(2)6(10-8)4-9-7/h5-6,8H,3-4H2,1-2H3 \\
\hline 2535 & $13 \mathrm{C} 6 \mathrm{D} 1-60$ & $\mathrm{CC}(\mathrm{C}=\mathrm{C}) \mathrm{CC}[\mathrm{O}]$ & InChl=1S/C6H110/c1-3-6(2)4-5-7/h3,6H,1,4-5H2,2H3 \\
\hline 2536 & I3C6D3-10OH & $\mathrm{CCC}(=\mathrm{C}) \mathrm{CCOO}$ & InChl=1S/C6H12O2/c1-3-6(2)4-5-8-7/h7H,2-5H2,1H3 \\
\hline 2537 & NEC6O14-30OH & $00 \mathrm{C} 1 \operatorname{cocc} 1(\mathrm{c}) \mathrm{C}$ & $\operatorname{lnChl}=1 \mathrm{~S} / \mathrm{C} 6 \mathrm{H} 12 \mathrm{O} 3 / \mathrm{c} 1-6(2) 4-8-3-5(6) 9-7 / \mathrm{h} 5,7 \mathrm{H}, 3-4 \mathrm{H} 2,1-2 \mathrm{H} 3$ \\
\hline 2538 & $13 \mathrm{C} 6 \mathrm{OOH} 2-3 \mathrm{O} 2$ & $\mathrm{OOC}(\mathrm{C}(\mathrm{O}[\mathrm{O}])(\mathrm{CC}) \mathrm{C}) \mathrm{C}$ & InChl=15/C6H1304/c1-4-6(3,10-8)5(2)9-7/h5,7H,4H2,1-3H3 \\
\hline 2539 & $13 \mathrm{C} 6 \mathrm{D} 2-50$ & $\mathrm{CC}=\mathrm{C}(\mathrm{C}([\mathrm{O}]) \mathrm{C}) \mathrm{C}$ & InChl=15/C6H110/c1-4-5(2)6(3)7/h4,6H,1-3H3 \\
\hline 2540 & $13 \mathrm{C6D} 3-2 \mathrm{OOH}$ & $\mathrm{CC}(\mathrm{C}(=\mathrm{C}) \mathrm{CC}) \mathrm{OO}$ & InChl=1S/C6H12O2/c1-4-5(2)6(3)8-7/h6-7H,2,4H2,1,3H3 \\
\hline 2541 & $\mathrm{IC} 6014-50 \mathrm{OH}$ & $\mathrm{CC} 1 \mathrm{CC}(\mathrm{OC} 1) \mathrm{COO}$ & InChl=1S/C6H12O3/c1-5-2-6(4-9-7)8-3-5/h5-7H,2-4H2,1H3 \\
\hline 2542 & $13 \mathrm{C} 6 \mathrm{OOH} 2-4 \mathrm{O} 2$ & {$[0] \mathrm{OCC}(\mathrm{C}(\mathrm{OO}) \mathrm{C}) \mathrm{CC}$} & InChl=1S/C6H13O4/c1-3-6(4-9-7)5(2)10-8/h5-6,8H,3-4H2,1-2H3 \\
\hline 2543 & 13C6D2-60 & $\mathrm{CC}(=\mathrm{CC}) \mathrm{CC}[\mathrm{O}]$ & InChl=1S/C6H11O/c1-3-6(2)4-5-7/h3H,4-5H2,1-2H3 \\
\hline 2544 & XC6D1-1AOOH & $\mathrm{OOCC}(=\mathrm{C}) \mathrm{C}(\mathrm{C}) \mathrm{C}$ & InChl=1S/C6H12O2/c1-5(2)6(3)4-8-7/h5,7H,3-4H2,1-2H3 \\
\hline
\end{tabular}




\begin{tabular}{|c|c|c|c|}
\hline 2545 & NEC6034-10OH & oocc $(\mathrm{C} 1 \mathrm{OC} 1)(\mathrm{C}) \mathrm{C}$ & InChl=1S/C6H12O3/c1-6(2,4-9-7)5-3-8-5/h5,7H,3-4H2,1-2H3 \\
\hline 2546 & 13С60OH2-502 & $\mathrm{OOC}(\mathrm{C}(\mathrm{C}(\mathrm{O}[\mathrm{O}]) \mathrm{C}) \mathrm{C}) \mathrm{C}$ & InChl=1S/C6H13O4/c1-4(5(2)9-7)6(3)10-8/h4-7H,1-3H3 \\
\hline 2547 & $13 \mathrm{C} 6 \mathrm{D} 3-10$ & {$[\mathrm{O}] \mathrm{CCC}(=\mathrm{C}) \mathrm{CC}$} & InChl=1S/C6H110/c1-3-6(2)4-5-7/h2-5H2,1H3 \\
\hline 2548 & $\mathrm{XC6D1-30OH}$ & $\mathrm{OOC}(\mathrm{C}(=\mathrm{C}) \mathrm{C})(\mathrm{C}) \mathrm{C}$ & $\operatorname{lnChl}=1 \mathrm{~S} / \mathrm{C} 6 \mathrm{H} 12 \mathrm{O} 2 / \mathrm{c} 1-5(2) 6(3,4) 8-7 / \mathrm{h} 7 \mathrm{H}, 1 \mathrm{H} 2,2-4 \mathrm{H} 3$ \\
\hline 2549 & IC6O15-1AOOH & Oocc1CcCoc1 & InChl=1S/C6H12O3/c7-9-5-6-2-1-3-8-4-6/h6-7H,1-5H2 \\
\hline 2550 & NEC6OOH1-1AO2 & $\mathrm{OOCC}(\mathrm{CO}[\mathrm{O}])(\mathrm{CC}) \mathrm{C}$ & InChl=1S/C6H13O4/c1-3-6(2,4-9-7)5-10-8/h7H,3-5H2,1-2H3 \\
\hline 2551 & $13 \mathrm{C} 6 \mathrm{D} 3-2 \mathrm{O}$ & $\operatorname{cCC}(=\mathrm{C}) \mathrm{C}([\mathrm{O}]) \mathrm{C}$ & InChl=15/C6H110/c1-4-5(2)6(3)7/h6H,2,4H2,1,3H3 \\
\hline 2552 & $\mathrm{XC6D1-40OH}$ & $\mathrm{CC}(\mathrm{C}(=\mathrm{C}) \mathrm{C}) \mathrm{COO}$ & InChl=1S/C6H12O2/c1-5(2)6(3)4-8-7/h6-7H,1,4H2,2-3H3 \\
\hline 2553 & $\mathrm{IC} 6 \mathrm{O} 15-2 \mathrm{OOH}$ & $\mathrm{OOC} 1(\mathrm{C}) \mathrm{CCCOC} 1$ & InChl=1S/C6H12O3/c1-6(9-7)3-2-4-8-5-6/h7H,2-5H2,1H3 \\
\hline 2554 & $13 \mathrm{C} 6 \mathrm{OOH} 2-6 \mathrm{O} 2$ & {$[0] \mathrm{OCCC}(\mathrm{C}(\mathrm{OO}) \mathrm{C}) \mathrm{C}$} & InChl=1S/C6H1304/c1-5(3-4-9-7)6(2)10-8/h5-6,8H,3-4H2,1-2H3 \\
\hline 2555 & XC6D1-1AO & {$[\mathrm{O}] \mathrm{CC}(=\mathrm{C}) \mathrm{C}(\mathrm{C}) \mathrm{C}$} & InChl=1S/C6H11O/c1-5(2)6(3)4-7/h5H,3-4H2,1-2H3 \\
\hline 2556 & $\mathrm{XC6D2-10OH}$ & $\mathrm{OOCC}(=\mathrm{C}(\mathrm{C}) \mathrm{C}) \mathrm{C}$ & InChl=1S/C6H12O2/c1-5(2)6(3)4-8-7/h7H,4H2,1-3H3 \\
\hline 2557 & $\mathrm{IC} 6 \mathrm{O} 15-30 \mathrm{OH}$ & ooc1Ccocc1C & InChl=1S/C6H12O3/c1-5-4-8-3-2-6(5)9-7/h5-7H,2-4H2,1H3 \\
\hline 2558 & NEC6OOH1-302 & $\mathrm{OOCC}(\mathrm{C}(\mathrm{O}[\mathrm{O}]) \mathrm{C})(\mathrm{C}) \mathrm{C}$ & InChl=1S/C6H13O4/c1-5(10-8)6(2,3)4-9-7/h5,7H,4H2,1-3H3 \\
\hline 2559 & XC6D1-30 & $\mathrm{CC}(=\mathrm{C}) \mathrm{C}([\mathrm{O}])(\mathrm{C}) \mathrm{C}$ & $\operatorname{lnChl}=1 \mathrm{~S} / \mathrm{C} 6 \mathrm{H} 11 \mathrm{O} / \mathrm{c} 1-5(2) 6(3,4) 7 / \mathrm{h} 1 \mathrm{H} 2,2-4 \mathrm{H} 3$ \\
\hline 2560 & IC6D1-1AOOH & $\operatorname{cccc}(=\mathrm{C}) \operatorname{coo}$ & InChl=1S/C6H12O2/c1-3-4-6(2)5-8-7/h7H,2-5H2,1H3 \\
\hline 2561 & $\mathrm{IC} 6015-40 \mathrm{OH}$ & $\mathrm{CC} 1 \mathrm{CC}(\mathrm{COC} 1) \mathrm{OO}$ & InChl=15/C6H1203/c1-5-2-6(9-7)4-8-3-5/h5-7H,2-4H2,1H3 \\
\hline 2562 & $13 \mathrm{C} 6 \mathrm{OOH} 3-102$ & {$[\mathrm{O}] \mathrm{OCCC}(\mathrm{OO})(\mathrm{CC}) \mathrm{C}$} & InChl=1S/C6H13O4/c1-3-6(2,10-8)4-5-9-7/h8H,3-5H2,1-2H3 \\
\hline 2563 & IC6D1-1AO & $\operatorname{cccC}(=\mathrm{C}) \mathrm{C}[\mathrm{O}]$ & InChl=1S/C6H11O/c1-3-4-6(2)5-7/h2-5H2,1H3 \\
\hline 2564 & IC6D1-30OH & $\operatorname{ccc}(C(=) C) C) 00$ & InChl=1S/C6H12O2/c1-4-6(8-7)5(2)3/h6-7H,2,4H2,1,3H3 \\
\hline 2565 & $\mathrm{IC} 6 \mathrm{O} 23-10 \mathrm{OH}$ & $\mathrm{CC} 1(\mathrm{COO}) \mathrm{OC} 1 \mathrm{CC}$ & InChl=1S/C6H12O3/c1-3-5-6(2,9-5)4-8-7/h5,7H,3-4H2,1-2H3 \\
\hline 2566 & NEC6OOH1-402 & {$[0] \mathrm{OCCC}(\mathrm{COO})(\mathrm{C}) \mathrm{C}$} & InChl=1S/C6H13O4/c1-6(2,5-10-8)3-4-9-7/h8H,3-5H2,1-2H3 \\
\hline 2567 & C6H11013-6 & $\mathrm{C} 1 \mathrm{CC}(\mathrm{O} 1) \mathrm{CC}[\mathrm{CH} 2]$ & InChl=1S/C6H110/c1-2-3-6-4-5-7-6/h6H,1-5H2 \\
\hline 2568 & $\mathrm{IC6D1-40OH}$ & $\mathrm{CC}(\mathrm{OO}) \mathrm{CC}(=\mathrm{C}) \mathrm{C}$ & InChl=1S/C6H12O2/c1-5(2)4-6(3)8-7/h6-7H,1,4H2,2-3H3 \\
\hline 2569 & $\mathrm{IC} 6023-40 \mathrm{OH}$ & $00 \mathrm{C}(\mathrm{C} 1 \mathrm{OC} 1(\mathrm{C}) \mathrm{C}) \mathrm{C}$ & InChl=15/C6H1203/c1-4(9-7)5-6(2,3)8-5/h4-5,7H,1-3H3 \\
\hline 2570 & 13С-6OOH3-2O2 & {$[\mathrm{O}] \mathrm{OC}(\mathrm{C}(\mathrm{OO})(\mathrm{CC}) \mathrm{C}) \mathrm{C}$} & InChl=1S/C6H13O4/c1-4-6(3,10-8)5(2)9-7/h5,8H,4H2,1-3H3 \\
\hline 2571 & C6H11014-6 & $\mathrm{C} 1 \mathrm{CCC}(\mathrm{O} 1) \mathrm{C}[\mathrm{CH} 2]$ & InChl=1S/C6H110/c1-2-6-4-3-5-7-6/h6H,1-5H2 \\
\hline 2572 & IC6D1-50OH & OOCCCC $(=\mathrm{C}) \mathrm{C}$ & InChl=15/C6H12O2/c1-6(2)4-3-5-8-7/h7H,1,3-5H2,2H3 \\
\hline 2573 & $\mathrm{IC} 6 \mathrm{O} 23-50 \mathrm{OH}$ & $00 \mathrm{CcC} 1 \mathrm{OC} 1(\mathrm{C}) \mathrm{C}$ & InChl=15/C6H12O3/c1-6(2)5(9-6)3-4-8-7/h5,7H,3-4H2,1-2H3 \\
\hline 2574 & NEC6OOH3-102 & {$[\mathrm{O}] \mathrm{OCC}(\mathrm{C}(\mathrm{OO}) \mathrm{C})(\mathrm{C}) \mathrm{C}$} & InChl=1S/C6H13O4/c1-5(10-8)6(2,3)4-9-7/h5,8H,4H2,1-3H3 \\
\hline 2575 & C6H11015-6 & $\mathrm{C} 1 \mathrm{CCCC}(\mathrm{O} 1)[\mathrm{CH} 2]$ & InChl=1S/C6H110/c1-6-4-2-3-5-7-6/h6H,1-5H2 \\
\hline 2576 & IC6D2-10OH & $\mathrm{CC}(=\mathrm{CCC}) \mathrm{COO}$ & InChl=1S/C6H12O2/c1-3-4-6(2)5-8-7/h4,7H,3,5H2,1-2H3 \\
\hline 2577 & $\mathrm{IC} 6 \mathrm{O} 24-10 \mathrm{OH}$ & $\mathrm{CC} 1(\mathrm{COO}) \mathrm{CC}(\mathrm{O} 1) \mathrm{C}$ & InChl=1S/C6H12O3/c1-5-3-6(2,9-5)4-8-7/h5,7H,3-4H2,1-2H3 \\
\hline 2578 & $13 \mathrm{C} 6 \mathrm{OOH} 3-402$ & {$[\mathrm{O}] \mathrm{OCC}(\mathrm{OO})(\mathrm{CC}) \mathrm{CC}$} & InChl=1S/C6H13O4/c1-3-6(4-2,10-8)5-9-7/h8H,3-5H2,1-2H3 \\
\hline 2579 & C6H11023-1 & {$[\mathrm{CH} 2] \mathrm{C} 1 \mathrm{C}(\mathrm{O} 1) \mathrm{CCC}$} & InChl=15/C6H110/c1-3-4-6-5(2)7-6/h5-6H,2-4H2,1H3 \\
\hline 2580 & $\mathrm{IC} 6 \mathrm{D} 2-4 \mathrm{OOH}$ & $\mathrm{OOC}(\mathrm{C}=\mathrm{C}(\mathrm{C}) \mathrm{C}) \mathrm{C}$ & InChl=1S/C6H12O2/c1-5(2)4-6(3)8-7/h4,6-7H,1-3H3 \\
\hline 2581 & $\mathrm{IC} 6 \mathrm{O} 24-30 \mathrm{OH}$ & $00 \mathrm{C} 1 \mathrm{C}(\mathrm{C}) \mathrm{OC} 1(\mathrm{C}) \mathrm{C}$ & InChl=15/C6H12O3/c1-4-5(9-7)6(2,3)8-4/h4-5,7H,1-3H3 \\
\hline 2582 & NEC6OOH3-402 & {$[0] \mathrm{OCC}(\mathrm{C}(\mathrm{C})(\mathrm{C}) \mathrm{C}) 00$} & InChl=1S/C6H13O4/c1-6(2,3)5(10-8)4-9-7/h5,8H,4H2,1-3H3 \\
\hline 2583 & C6H11023-6 & $\mathrm{CC} 1 \mathrm{C}(\mathrm{O} 1) \mathrm{CC}[\mathrm{CH} 2]$ & InChl=1S/C6H110/c1-3-4-6-5(2)7-6/h5-6H,1,3-4H2,2H3 \\
\hline 2584 & IC6D2-50OH & OOCCC $=C(C) C$ & InChl=1S/C6H12O2/c1-6(2)4-3-5-8-7/h4,7H,3,5H2,1-2H3 \\
\hline 2585 & IC6O24-50OH & OOCC1CC(O1)(C)C & InChl=1S/C6H12O3/c1-6(2)3-5(9-6)4-8-7/h5,7H,3-4H2,1-2H3 \\
\hline 2586 & $13 \mathrm{C} 6 \mathrm{OOH} 4-102$ & {$[0] \mathrm{OCCC}(\mathrm{COO}) \mathrm{CC}$} & InChl=1S/C6H13O4/c1-2-6(5-10-8)3-4-9-7/h6,8H,2-5H2,1H3 \\
\hline 2587 & C6H11024-1 & {$[\mathrm{CH} 2] \mathrm{C} 1 \mathrm{CC}(\mathrm{O} 1) \mathrm{CC}$} & InChl=15/C6H110/c1-3-6-4-5(2)7-6/h5-6H,2-4H2,1H3 \\
\hline 2588 & IC6D3-10OH & $\mathrm{CC}(\mathrm{C}=\mathrm{CC}) \mathrm{COO}$ & InChl=1S/C6H12O2/c1-3-4-6(2)5-8-7/h3-4,6-7H,5H2,1-2H3 \\
\hline 2589 & $\mathrm{IC} 6025-10 \mathrm{OH}$ & $00 \mathrm{cc} 1(\mathrm{C}) \mathrm{ccc} 01$ & $\operatorname{lnChl}=1 \mathrm{~S} / \mathrm{C} 6 \mathrm{H} 12 \mathrm{O} / \mathrm{c} 1-6(5-9-7) 3-2-4-8-6 / \mathrm{h} 7 \mathrm{H}, 2-5 \mathrm{H} 2,1 \mathrm{H} 3$ \\
\hline 2590 & NEC6OOH4-102 & $00 \mathrm{ccc}(\mathrm{CO}[\mathrm{O}])(\mathrm{C}) \mathrm{C}$ & InChl=1S/C6H1304/c1-6(2,5-10-8)3-4-9-7/h7H,3-5H2,1-2H3 \\
\hline 2591 & C6H11024-6 & $\mathrm{CC} 1 \mathrm{CC}(\mathrm{O} 1) \mathrm{C}[\mathrm{CH} 2]$ & $\operatorname{lnChl}=1 \mathrm{~S} / \mathrm{C} 6 \mathrm{H} 110 / \mathrm{c1} 1-3-6-4-5(2) 7-6 / \mathrm{h} 5-6 \mathrm{H}, 1,3-4 \mathrm{H} 2,2 \mathrm{H} 3$ \\
\hline 2592 & IC6D3-20OH & $\mathrm{OOC}(\mathrm{C}=\mathrm{CC})(\mathrm{C}) \mathrm{C}$ & InChl=1S/C6H12O2/c1-4-5-6(2,3)8-7/h4-5,7H,1-3H3 \\
\hline 2593 & IC6O25-30OH & $00 \mathrm{C} 1 \mathrm{CcOC} 1(\mathrm{c}) \mathrm{C}$ & $\operatorname{lnChl}=1 \mathrm{~S} / \mathrm{C} 6 \mathrm{H} 12 \mathrm{O} / \mathrm{c} 1-6(2) 5(9-7) 3-4-8-6 / \mathrm{h} 5,7 \mathrm{H}, 3-4 \mathrm{H} 2,1-2 \mathrm{H} 3$ \\
\hline 2594 & $13 \mathrm{C} 6 \mathrm{OOH} 4-2 \mathrm{O} 2$ & $\mathrm{OOCC}(\mathrm{C}(\mathrm{O}[\mathrm{O}]) \mathrm{C}) \mathrm{CC}$ & InChl=1S/C6H13O4/c1-3-6(4-9-7)5(2)10-8/h5-7H,3-4H2,1-2H3 \\
\hline 2595 & C6H11034-1 & $\mathrm{CCC} 1 \mathrm{C}(\mathrm{O} 1) \mathrm{C}[\mathrm{CH} 2]$ & InChl=15/C6H110/c1-3-5-6(4-2)7-5/h5-6H,1,3-4H2,2H3 \\
\hline 2596 & IC6D3-50OH & $\mathrm{OOCC}=\mathrm{CC}(\mathrm{C}) \mathrm{C}$ & InChl=15/C6H12O2/c1-6(2)4-3-5-8-7/h3-4,6-7H,5H2,1-2H3 \\
\hline 2597 & $\mathrm{IC} 6 \mathrm{O} 25-40 \mathrm{OH}$ & $00 \mathrm{OC} 1 \operatorname{coc}(\mathrm{C} 1)(\mathrm{C}) \mathrm{C}$ & InChl=15/C6H12O3/c1-6(2)3-5(9-7)4-8-6/h5,7H,3-4H2,1-2H3 \\
\hline 2598 & NEC6OOH4-3O2 & $00 \mathrm{OCC}(\mathrm{C}(\mathrm{C})(\mathrm{C}) \mathrm{C}) \mathrm{O}[\mathrm{O}]$ & InChl=15/C6H13O4/c1-6(2,3)5(10-8)4-9-7/h5,7H,4H2,1-3H3 \\
\hline 2599 & C6Y2-1J & {$[\mathrm{CH} 2] \mathrm{C}(=\mathrm{O}) \mathrm{CCCC}$} & InChl=1S/C6H110/c1-3-4-5-6(2)7/h2-5H2,1H3 \\
\hline 2600 & IC6D4-10OH & $\mathrm{OOCC}(\mathrm{CC}=\mathrm{C}) \mathrm{C}$ & InChl=1S/C6H12O2/c1-3-4-6(2)5-8-7/h3,6-7H,1,4-5H2,2H3 \\
\hline 2601 & $\mathrm{IC} 6 \mathrm{O} 34-10 \mathrm{OH}$ & $\mathrm{CC}(\mathrm{C} 1 \mathrm{OC} 1 \mathrm{C}) \mathrm{COO}$ & InChl=1S/C6H12O3/c1-4(3-8-7)6-5(2)9-6/h4-7H,3H2,1-2H3 \\
\hline 2602 & $13 \mathrm{C} 6 \mathrm{OOH} 4-3 \mathrm{O} 2$ & $\mathrm{OOCC}(\mathrm{O}[\mathrm{O}])(\mathrm{CC}) \mathrm{CC}$ & InChl=1S/C6H13O4/c1-3-6(4-2,10-8)5-9-7/h7H,3-5H2,1-2H3 \\
\hline 2603 & C6Y3-6J & {$[\mathrm{CH} 2] \mathrm{CCC}(=\mathrm{O}) \mathrm{CC}$} & $\operatorname{lnChl}=1 \mathrm{~S} / \mathrm{C} 6 \mathrm{H} 11 \mathrm{O} / \mathrm{c} 1-3-5-6(7) 4-2 / \mathrm{h} 1,3-5 \mathrm{H} 2,2 \mathrm{H} 3$ \\
\hline 2604 & IC6D4-2OOH & $\mathrm{OOC}(\mathrm{CC}=\mathrm{C})(\mathrm{C}) \mathrm{C}$ & InChl=1S/C6H12O2/c1-4-5-6(2,3)8-7/h4,7H,1,5H2,2-3H3 \\
\hline 2605 & IC6O34-20OH & $\mathrm{OOC}(\mathrm{C} 1 \mathrm{OC} 1 \mathrm{C})(\mathrm{C}) \mathrm{C}$ & InChl=1S/C6H12O3/c1-4-5(8-4)6(2,3)9-7/h4-5,7H,1-3H3 \\
\hline 2606 & IC6OOH1-1AO2 & $\operatorname{cccc}(\mathrm{CO}[0]) \mathrm{COO}$ & InChl=1S/C6H13O4/c1-2-3-6(4-9-7)5-10-8/h6-7H,2-5H2,1H3 \\
\hline 2607 & C6Y3-1J & {$[\mathrm{CH} 2] \mathrm{CC}(=0) \mathrm{CCC}$} & $\operatorname{lnChl}=1 \mathrm{~S} / \mathrm{C} 6 \mathrm{H} 11 \mathrm{O} / \mathrm{c} 1-3-5-6(7) 4-2 / \mathrm{h} 2-5 \mathrm{H} 2,1 \mathrm{H} 3$ \\
\hline 2608 & IC6D4-30OH & $\mathrm{OOC}(\mathrm{C}(\mathrm{C}) \mathrm{C}) \mathrm{C}=\mathrm{C}$ & $\operatorname{lnChl}=15 / C 6 H 12 \mathrm{O} 2 / \mathrm{c1}-4-6(8-7) 5(2) 3 / \mathrm{h} 4-7 \mathrm{H}, 1 \mathrm{H} 2,2-3 \mathrm{H} 3$ \\
\hline 2609 & $\mathrm{IC} 6 \mathrm{O} 34-50 \mathrm{OH}$ & $00 \mathrm{CC} 10 \mathrm{C} 1 \mathrm{C}(\mathrm{C}) \mathrm{C}$ & InChl=15/C6H12O3/c1-4(2)6-5(9-6)3-8-7/h4-7H,3H2,1-2H3 \\
\hline 2610 & IC6OOH1-202 & $\operatorname{cccc}(\mathrm{COO})(\mathrm{O}[\mathrm{O}]) \mathrm{C}$ & InChl=15/C6H13O4/c1-3-4-6(2,10-8)5-9-7/h7H,3-5H2,1-2H3 \\
\hline 2611 & IC6D1-4OR & $\mathrm{C}=\mathrm{C}(\mathrm{C}) \mathrm{CC}([\mathrm{O}]) \mathrm{C}$ & InChl=1S/C6H11O/c1-5(2)4-6(3)7/h6H,1,4H2,2-3H3 \\
\hline 2612 & $\mathrm{C} 6 \mathrm{H} 13 \mathrm{O} 2-1$ & $\mathrm{C}(\mathrm{O}[\mathrm{O}]) \mathrm{CCCCC}$ & InChl=1S/C6H13O2/c1-2-3-4-5-6-8-7/h2-6H2,1H3 \\
\hline 2613 & $\mathrm{IC} 6 \mathrm{O} 35-10 \mathrm{OH}$ & $\mathrm{CC}(\mathrm{C} 1 \mathrm{CCO} 1) \mathrm{COO}$ & InChl=1S/C6H12O3/c1-5(4-9-7)6-2-3-8-6/h5-7H,2-4H2,1H3 \\
\hline 2614 & $\mathrm{IC} 6 \mathrm{OOH} 1-3 \mathrm{O} 2$ & $\mathrm{OOCC}(\mathrm{C}(\mathrm{O}[\mathrm{O}]) \mathrm{CC}) \mathrm{C}$ & InChl=1S/C6H13O4/c1-3-6(10-8)5(2)4-9-7/h5-7H,3-4H2,1-2H3 \\
\hline 2615 & IC6D3-2OR & $\mathrm{CC}([\mathrm{O}])(\mathrm{C}) \mathrm{C}=\mathrm{CC}$ & InChl=1S/C6H110/c1-4-5-6(2,3)7/h4-5H,1-3H3 \\
\hline 2616 & $\mathrm{C} 6 \mathrm{H} 13 \mathrm{O} 2-2$ & $\mathrm{CC}(\mathrm{O}[\mathrm{O}]) \mathrm{CCCC}$ & $\operatorname{lnChl}=15 / C 6 H 1302 / c 1-3-4-5-6(2) 8-7 / \mathrm{h} 6 \mathrm{H}, 3-5 \mathrm{H} 2,1-2 \mathrm{H} 3$ \\
\hline 2617 & IC6O35-20OH & $\mathrm{OOC}(\mathrm{C} 1 \mathrm{CCO})(\mathrm{C}) \mathrm{C}$ & $\operatorname{lnChl}=15 / \mathrm{C} 6 \mathrm{H} 12 \mathrm{O} / \mathrm{c} 1-6(2,9-7) 5-3-4-8-5 / \mathrm{h} 5,7 \mathrm{H}, 3-4 \mathrm{H} 2,1-2 \mathrm{H} 3$ \\
\hline 2618 & IC6OOH1-402 & $00 \mathrm{OCC}(\mathrm{CC}(\mathrm{O}[\mathrm{O}]) \mathrm{C}) \mathrm{C}$ & InChl=15/C6H13O4/c1-5(4-9-7)3-6(2)10-8/h5-7H,3-4H2,1-2H3 \\
\hline 2619 & NEOC5H11CO & $\mathrm{CC}(\mathrm{C})(\mathrm{C}) \mathrm{C}[\mathrm{C}]=\mathrm{O}$ & InChl=1S/C6H110/c1-6(2,3)4-5-7/h4H2,1-3H3 \\
\hline 2620 & $\mathrm{C} 6 \mathrm{H} 13 \mathrm{O} 2-3$ & $\mathrm{CCC}(\mathrm{O}[\mathrm{O}]) \mathrm{CCC}$ & InChl=1S/C6H13O2/c1-3-5-6(4-2)8-7/h6H,3-5H2,1-2H3 \\
\hline 2621 & $\mathrm{IC} 6 \mathrm{O} 35-40 \mathrm{OH}$ & $00 \mathrm{C} 1 \operatorname{coc} 1 \mathrm{C}(\mathrm{C}) \mathrm{C}$ & InChl=1S/C6H12O3/c1-4(2)6-5(9-7)3-8-6/h4-7H,3H2,1-2H3 \\
\hline 2622 & IC6OOH1-502 & {$[0] \mathrm{OCCCC}(\mathrm{COO}) \mathrm{C}$} & InChl=1S/C6H1304/c1-66(5-10-8)3-2-4-9-7/h6,8H,2-5H2,1H3 \\
\hline 2623 & IC6D1-30R & $\mathrm{C}=\mathrm{C}(\mathrm{C}) \mathrm{C}([\mathrm{O}]) \mathrm{CC}$ & InChl=1S/C6H110/c1-4-6(7)5(2)3/h6H,2,4H2,1,3H3 \\
\hline 2624 & $\mathrm{C} 6 \mathrm{H} 12 \mathrm{OOH} 1-2$ & $\mathrm{C}(\mathrm{OO})[\mathrm{CH}] \mathrm{CCCC}$ & InChl=1S/C6H13O2/c1-2-3-4-5-6-8-7/h5,7H,2-4,6H2,1H3 \\
\hline 2625 & $\mathrm{IC} 6 \mathrm{O} 45-10 \mathrm{OH}$ & OOCC(CC1CO1)C & InChl=1S/C6H12O3/c1-5(3-9-7)2-6-4-8-6/h5-7H,2-4H2,1H3 \\
\hline 2626 & $\mathrm{IC} 6 \mathrm{OOH} 2-102$ & $\operatorname{cccc}(\mathrm{CO}[\mathrm{O}])(\mathrm{OO}) \mathrm{C}$ & InChl=1S/C6H1304/c1-3-4-6(2,10-8)5-9-7/h8H,3-5H2,1-2H3 \\
\hline 2627 & IC6D4-3OR & $\mathrm{CC}(\mathrm{C}) \mathrm{C}([\mathrm{O}]) \mathrm{C}=\mathrm{C}$ & InChl=1S/C6H110/c1-4-6(7)5(2)3/h4-6H,1H2,2-3H3 \\
\hline 2628 & $\mathrm{C} 6 \mathrm{H} 12 \mathrm{OOH} 1-3$ & $\mathrm{C}(\mathrm{OO}) \mathrm{C}[\mathrm{CH}] \mathrm{CCC}$ & $\operatorname{lnChl}=1 \mathrm{~S} / \mathrm{C} 6 \mathrm{H} 13 \mathrm{O} 2 / \mathrm{c} 1-2-3-4-5-6-8-7 / \mathrm{h} 4,7 \mathrm{H}, 2-3,5-6 \mathrm{H} 2,1 \mathrm{H} 3$ \\
\hline 2629 & IC6O45-20OH & OOC(CC1OC1)(C)C & InChl=15/C6H12O3/c1-6(2,9-7)3-5-4-8-5/h5,7H,3-4H2,1-2H3 \\
\hline 2630 & $1 \mathrm{C} 60 \mathrm{OH} 2-302$ & $\operatorname{ccc}(\mathrm{C}(\mathrm{OO})(\mathrm{C}) \mathrm{C}) \mathrm{O}[\mathrm{O}]$ & InChl=15/C6H13O4/c1-4-5(9-7)6(2,3)10-8/h5,8H,4H2,1-3H3 \\
\hline 2631 & IC6Y4-1R & {$[\mathrm{CH} 2] \mathrm{C}(\mathrm{C}) \mathrm{CC}(=\mathrm{O}) \mathrm{C}$} & $\operatorname{lnChl}=1 \mathrm{~S} / \mathrm{C} 6 \mathrm{H} 11 \mathrm{O} / \mathrm{c} 1-5(2) 4-6(3) 7 / \mathrm{h} 5 \mathrm{H}, 1,4 \mathrm{H} 2,2-3 \mathrm{H} 3$ \\
\hline 2632 & $\mathrm{C} 6 \mathrm{H} 12 \mathrm{OOH} 1-4$ & $\mathrm{C}(\mathrm{OO}) \mathrm{CC}[\mathrm{CH}] \mathrm{CC}$ & InChl=15/C6H13O2/c1-2-3-4-5-6-8-7/h3,7H,2,4-6H2,1H3 \\
\hline 2633 & IC6O45-30OH & $\mathrm{OOC}(\mathrm{C} 1 \mathrm{OC} 1) \mathrm{C}(\mathrm{C}) \mathrm{C}$ & InChl=15/C6H12O3/c1-4(2)6(9-7)5-3-8-5/h4-7H,3H2,1-2H3 \\
\hline 2634 & $\mathrm{IC} 6 \mathrm{OOH} 2-402$ & {$[\mathrm{O}] \mathrm{OC}(\mathrm{CC}(\mathrm{OO})(\mathrm{C}) \mathrm{C}) \mathrm{C}$} & InChl=1S/C6H1304/c1-5(9-7)4-6(2,3)10-8/h5,8H,4H2,1-3H3 \\
\hline 2635 & IC6Y4-2R & $\mathrm{C}[\mathrm{C}](\mathrm{C}) \mathrm{CC}(=\mathrm{O}) \mathrm{C}$ & InChl=1S/C6H110/c1-5(2)4-6(3)7/h4H2,1-3H3 \\
\hline
\end{tabular}




\begin{tabular}{|c|c|c|c|}
\hline 2636 & $\mathrm{C} 6 \mathrm{H} 12 \mathrm{OOH} 1-5$ & $\mathrm{C}(\mathrm{OO}) \mathrm{CCC}[\mathrm{CH}] \mathrm{C}$ & InChl=1S/C6H13O2/c1-2-3-4-5-6-8-7/h2,7H,3-6H2,1H3 \\
\hline 2637 & $13 \mathrm{C} 6 \mathrm{O12}-3 \mathrm{OOH}$ & $\mathrm{CC}(\mathrm{C} 1 \mathrm{CO} 1)(\mathrm{OO}) \mathrm{CC}$ & $\operatorname{lnChl}=15 / \mathrm{C} 6 \mathrm{H} 12 \mathrm{O} / \mathrm{c} 1-3-6(2,9-7) 5-4-8-5 / \mathrm{h} 5,7 \mathrm{H}, 3-4 \mathrm{H} 2,1-2 \mathrm{H} 3$ \\
\hline 2638 & $\mathrm{IC6OOH2-502}$ & {$[0] \mathrm{OCCCC}(\mathrm{OO})(\mathrm{C}) \mathrm{C}$} & InChl=1S/C6H13O4/c1-6(2,10-8)4-3-5-9-7/h8H,3-5H2,1-2H3 \\
\hline 2639 & IC6Y4-3R & $\mathrm{CC}(\mathrm{C})[\mathrm{CH}] \mathrm{C}(=\mathrm{O}) \mathrm{C}$ & InChl=1S/C6H110/c1-5(2)4-6(3)7/h4-5H,1-3H3 \\
\hline 2640 & C6H12OOH2-1 & {$[\mathrm{CH} 2] \mathrm{C}(\mathrm{OO}) \mathrm{CCCC}$} & $\operatorname{lnChl}=1 \mathrm{~S} / \mathrm{C} 6 \mathrm{H} 13 \mathrm{O} 2 / \mathrm{c1} 1-3-4-5-6(2) 8-7 / \mathrm{h} 6-7 \mathrm{H}, 2-5 \mathrm{H} 2,1 \mathrm{H} 3$ \\
\hline 2641 & 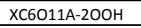 & $\mathrm{OOC} 1(\operatorname{COC} 1) \mathrm{C}(\mathrm{C}) \mathrm{C}$ & $\operatorname{lnChl}=15 / \mathrm{C} 6 \mathrm{H} 12 \mathrm{O} / \mathrm{c} 1-5(2) 6(9-7) 3-8-4-6 / \mathrm{h} 5,7 \mathrm{H}, 3-4 \mathrm{H} 2,1-2 \mathrm{H3}$ \\
\hline 2642 & $\mathrm{IC} 60 \mathrm{OH} 3-102$ & {$[0] \mathrm{OCC}(\mathrm{C}(\mathrm{OO}) \mathrm{CC}) \mathrm{C}$} & InChl=1S/C6H13O4/c1-3-6(10-8)5(2)4-9-7/h5-6,8H,3-4H2,1-2H3 \\
\hline 2643 & IC6Y4-5R & $\mathrm{CC}(\mathrm{C}) \mathrm{CC}(=\mathrm{O})[\mathrm{CH} 2]$ & InChl=1S/C6H11O/c1-5(2)4-6(3)7/h5H,3-4H2,1-2H3 \\
\hline 2644 & $\mathrm{C} 6 \mathrm{H} 12 \mathrm{OOH} 2-3$ & $\mathrm{CC}(\mathrm{OO})[\mathrm{CH}] \mathrm{CCC}$ & InChl=15/C6H13O2/c1-3-4-5-6(2)8-7/h5-7H,3-4H2,1-2H3 \\
\hline 2645 & $13 \mathrm{C} 6 \mathrm{O} 12-4 \mathrm{OOH}$ & $\mathrm{CCC}(\mathrm{C} 1 \mathrm{CO} 1) \mathrm{COO}$ & $\operatorname{lnChl}=1 \mathrm{~S} / \mathrm{C} 6 \mathrm{H} 12 \mathrm{O} / \mathrm{c} 1-2-5(3-9-7) 6-4-8-6 / \mathrm{h} 5-7 \mathrm{H}, 2-4 \mathrm{H} 2,1 \mathrm{H} 3$ \\
\hline 2646 & $\mathrm{IC} 60 \mathrm{OH} 3-2 \mathrm{O} 2$ & $\operatorname{ccc}(\mathrm{C}(\mathrm{O}[\mathrm{O}])(\mathrm{C}) \mathrm{C}) \mathrm{OO}$ & InChl=1S/C6H13O4/c1-4-5(9-7)6(2,3)10-8/h5,7H,4H2,1-3H3 \\
\hline 2647 & NEC6D3-1OR & {$[O] C C(C)(C) C=C$} & InChl=1S/C6H11O/c1-4-6(2,3)5-7/h4H,1,5H2,2-3H3 \\
\hline 2648 & $\mathrm{C} 6 \mathrm{H} 12 \mathrm{OOH} 2-4$ & $\mathrm{CC}(\mathrm{OO}) \mathrm{C}[\mathrm{CH}] \mathrm{CC}$ & InChl=1S/C6H13O2/c1-3-4-5-6(2)8-7/h4,6-7H,3,5H2,1-2H3 \\
\hline 2649 & XC6O11A-30OH & $\mathrm{OOC}(\mathrm{C} 1 \mathrm{COC} 1)(\mathrm{C}) \mathrm{C}$ & $\operatorname{lnChl}=1 \mathrm{~S} / \mathrm{C} 6 \mathrm{H} 12 \mathrm{O} / \mathrm{c} 1-6(2,9-7) 5-3-8-4-5 / \mathrm{h} 5,7 \mathrm{H}, 3-4 \mathrm{H} 2,1-2 \mathrm{H} 3$ \\
\hline 2650 & $\mathrm{IC} 6 \mathrm{OOH} 3-402$ & $\mathrm{OOC}(\mathrm{C}(\mathrm{O}[\mathrm{O}]) \mathrm{C}) \mathrm{C}(\mathrm{C}) \mathrm{C}$ & InChl=1S/C6H13O4/c1-4(2)6(10-8)5(3)9-7/h4-6,8H,1-3H3 \\
\hline 2651 & NEOC5H1OCHO-1 & {$[\mathrm{CH} 2] \mathrm{C}(\mathrm{C})(\mathrm{C}) \mathrm{CC}=\mathrm{O}$} & InChl=1S/C6H11O/c1-6(2,3)4-5-7/h5H,1,4H2,2-3H3 \\
\hline 2652 & $\mathrm{C} 6 \mathrm{H} 12 \mathrm{OOH} 2-5$ & $\mathrm{CC}(\mathrm{OO}) \mathrm{CC}[\mathrm{CH}] \mathrm{C}$ & $\mathrm{InChl}=1 \mathrm{~S} / \mathrm{C} 6 \mathrm{H} 13 \mathrm{O} 2 / \mathrm{c} 1-3-4-5-6(2) 8-7 / \mathrm{h} 3,6-7 \mathrm{H}, 4-5 \mathrm{H} 2,1-2 \mathrm{H} 3$ \\
\hline 2653 & $13 \mathrm{C} 6 \mathrm{O} 12-5 \mathrm{OOH}$ & $\mathrm{OOC}(\mathrm{C}(\mathrm{C} 1 \mathrm{OC} 1) \mathrm{C}) \mathrm{C}$ & $\operatorname{lnChl}=1 \mathrm{~S} / \mathrm{C} 6 \mathrm{H} 12 \mathrm{O} 3 / \mathrm{c} 1-4(5(2) 9-7) 6-3-8-6 / \mathrm{h} 4-7 \mathrm{H}, 3 \mathrm{H} 2,1-2 \mathrm{H} 3$ \\
\hline 2654 & $\mathrm{IC6OOH} 3-5 \mathrm{O} 2$ & {$[0] \operatorname{Occc}(\mathrm{C}(\mathrm{C}) \mathrm{C}) 0 \mathrm{O}$} & InChl=1S/C6H13O4/c1-5(2)6(10-8)3-4-9-7/h5-6,8H,3-4H2,1-2H3 \\
\hline 2655 & NEOC5H1OCHO-3 & $\mathrm{CC}(\mathrm{C})(\mathrm{C})[\mathrm{CH}] \mathrm{C}=\mathrm{O}$ & $\mathrm{InChl}=1 \mathrm{~S} / \mathrm{C} 6 \mathrm{H} 110 / \mathrm{c} 1-6(2,3) 4-5-7 / \mathrm{h} 4-5 \mathrm{H}, 1-3 \mathrm{H} 3$ \\
\hline 2656 & $\mathrm{C} 6 \mathrm{H} 12 \mathrm{OOH} 2-6$ & $\mathrm{CC}(\mathrm{OO}) \mathrm{CCC}[\mathrm{CH} 2]$ & $\operatorname{lnChl}=1 \mathrm{~S} / \mathrm{C} 6 \mathrm{H} 13 \mathrm{O} 2 / \mathrm{c} 1-3-4-5-6(2) 8-7 / \mathrm{h} 6-7 \mathrm{H}, 1,3-5 \mathrm{H} 2,2 \mathrm{H} 3$ \\
\hline 2657 & XC6O11A-40OH & OOCC(C1COC1)C & InChl=1S/C6H12O3/c1-5(2-9-7)6-3-8-4-6/h5-7H,2-4H2,1H3 \\
\hline 2658 & $\mathrm{IC} 60 \mathrm{OH} 4-102$ & {$[0] \mathrm{OCC}(\mathrm{CC}(\mathrm{OO}) \mathrm{C}) \mathrm{C}$} & InChl=1S/C6H13O4/c1-5(4-9-7)3-6(2)10-8/h5-6,8H,3-4H2,1-2H3 \\
\hline 2659 & $\mathrm{IC5H} 11 \mathrm{CHO}$ & $\mathrm{CC}(\mathrm{C}) \mathrm{CCC}=\mathrm{O}$ & InChl=1S/C6H12O/c1-6(2)4-3-5-7/h5-6H,3-4H2,1-2H3 \\
\hline 2660 & C6H12OOH3-1 & {$[\mathrm{CH} 2] \mathrm{CC}(\mathrm{OO}) \mathrm{CCC}$} & $\operatorname{lnChl}=1 \mathrm{~S} / \mathrm{C} 6 \mathrm{H} 13 \mathrm{O} 2 / \mathrm{c1}-3-5-5-6(4-2) 8-7 / \mathrm{h} 6-7 \mathrm{H}, 2-5 \mathrm{H} 2,1 \mathrm{H} 3$ \\
\hline 2661 & $13 \mathrm{C} 6 \mathrm{O12}-6 \mathrm{OOH}$ & $\mathrm{CC}(\mathrm{C} 1 \mathrm{CO} 1) \mathrm{CCOO}$ & InChl=1S/C6H12O3/c1-5(2-3-9-7)6-4-8-6/h5-7H,2-4H2,1H3 \\
\hline 2662 & $\mathrm{IC6OOH} 4-2 \mathrm{O} 2$ & $\mathrm{OOC}(\mathrm{CC}(\mathrm{O}[\mathrm{O}])(\mathrm{C}) \mathrm{C}) \mathrm{C}$ & InChl=1S/C6H13O4/c1-5(9-7)4-6(2,3)10-8/h5,7H,4H2,1-3H3 \\
\hline 2663 & C6H12O1-2 & $\mathrm{C} 1 \mathrm{C}(01) \mathrm{CCCC}$ & $\operatorname{lnChl}=1 \mathrm{~S} / \mathrm{C} 6 \mathrm{H} 12 \mathrm{O} / \mathrm{c1}-2-3-4-6-5-7-6 / \mathrm{h} 6 \mathrm{H}, 2-5 \mathrm{H} 2,1 \mathrm{H} 3$ \\
\hline 2664 & $\mathrm{C} 6 \mathrm{H} 12 \mathrm{OOH} 3-2$ & $\mathrm{C}[\mathrm{CH}] \mathrm{C}(\mathrm{OO}) \mathrm{CCC}$ & $\operatorname{lnChl}=1 \mathrm{~S} / \mathrm{C} 6 \mathrm{H} 1302 / \mathrm{cc} 1-3-5-6(4-2) 8-7 / \mathrm{h} 4,6-7 \mathrm{H}, 3,5 \mathrm{H} 2,1-2 \mathrm{H} 3$ \\
\hline 2665 & XC6O12-1AOOH & OOCC1 $(\mathrm{OC} 1) \mathrm{C}(\mathrm{C}) \mathrm{C}$ & $\operatorname{lnChl}=1 \mathrm{~S} / \mathrm{C} 6 \mathrm{H} 12 \mathrm{O} 3 / \mathrm{c} 1-5(2) 6(3-8-6) 4-9-7 / \mathrm{h} 5,7 \mathrm{H}, 3-4 \mathrm{H} 2,1-2 \mathrm{H} 3$ \\
\hline 2666 & $\mathrm{IC6OOH} 4-302$ & {$[0] O C(C(O O) C) C(C) C$} & $\operatorname{lnChl}=15 / \mathrm{C} 6 \mathrm{H} 13 \mathrm{O} 4 / \mathrm{c1}-4(2) 6(10-8) 5(3) 9-7 / \mathrm{h} 4-7 \mathrm{H}, 1-3 \mathrm{H} 3$ \\
\hline 2667 & C6H1201-3 & $\mathrm{C} 1 \mathrm{CC}(\mathrm{O} 1) \mathrm{CCC}$ & InChl=1S/C6H12O/c1-2-3-6-4-5-7-6/h6H,2-5H2,1H3 \\
\hline 2668 & $\mathrm{C} 6 \mathrm{H} 12 \mathrm{OOH} 3-4$ & $\mathrm{CCC}(\mathrm{OO})[\mathrm{CH}] \mathrm{CC}$ & $\operatorname{lnChl}=15 / \mathrm{C} 6 \mathrm{H} 13 \mathrm{O} 2 / \mathrm{c} 1-3-5-6(4-2) 8-7 / \mathrm{h} 5-7 \mathrm{H}, 3-4 \mathrm{H} 2,1-2 \mathrm{H} 3$ \\
\hline 2669 & $13 \mathrm{C} 6013-2 \mathrm{OOH}$ & CC1(CC)OCC100 & $\operatorname{lnChl}=15 / \mathrm{C} 6 \mathrm{H} 12 \mathrm{O} 3 / \mathrm{c} 1-3-6(2) 5(9-7) 4-8-6 / \mathrm{h} 5,7 \mathrm{H}, 3-4 \mathrm{H} 2,1-2 \mathrm{H} 3$ \\
\hline 2670 & XC60OH1-1AO2 & $00 \mathrm{CC}(\mathrm{C}(\mathrm{C}) \mathrm{C}) \mathrm{CO}[\mathrm{O}]$ & InChl=1S/C6H13O4/c1-5(2)6(3-9-7)4-10-8/h5-7H,3-4H2,1-2H3 \\
\hline 2671 & C6H12O1-4 & $\mathrm{C} 1 \mathrm{CCC}(\mathrm{O} 1) \mathrm{CC}$ & $\operatorname{lnChl}=1 \mathrm{~S} / \mathrm{C} 6 \mathrm{H} 12 \mathrm{O} / \mathrm{c1}-2-6-4-3-5-7-6 / \mathrm{h} 6 \mathrm{H}, 2-5 \mathrm{H} 2,1 \mathrm{H} 3$ \\
\hline 2672 & C6H12OOH3-5 & $\mathrm{CCC}(\mathrm{OO}) \mathrm{C}[\mathrm{CH}] \mathrm{C}$ & $\operatorname{lnChl}=1 \mathrm{~S} / \mathrm{C} 6 \mathrm{H} 13 \mathrm{O} 2 / \mathrm{c} 1-3-5-6(4-2) 8-7 / \mathrm{h} 3,6-7 \mathrm{H}, 4-5 \mathrm{H} 2,1-2 \mathrm{H} 3$ \\
\hline 2673 & XC6012-30OH & $\mathrm{OOC}(\mathrm{C} 1(\mathrm{C}) \mathrm{OC} 1)(\mathrm{C}) \mathrm{C}$ & InChl=1S/C6H12O3/c1-5(2,9-7)6(3)4-8-6/h7H,4H2,1-3H3 \\
\hline 2674 & $\mathrm{IC} 60 \mathrm{OH} 4-5 \mathrm{O} 2$ & {$[0] \mathrm{OCC}(\mathrm{CC}(\mathrm{C}) \mathrm{C}) \mathrm{OO}$} & InChl=1S/C6H13O4/c1-5(2)3-6(10-8)4-9-7/h5-6,8H,3-4H2,1-2H3 \\
\hline 2675 & C6H12O1-5 & $\mathrm{C} 1 \mathrm{CCCC}(\mathrm{O} 1) \mathrm{C}$ & $\operatorname{lnChl}=1 \mathrm{~S} / \mathrm{C} 6 \mathrm{H} 12 \mathrm{O} / \mathrm{c1}-6-4-2-3-5-7-6 / \mathrm{h} 6 \mathrm{H}, 2-5 \mathrm{H} 2,1 \mathrm{H} 3$ \\
\hline 2676 & C6H12OOH3-6 & $\mathrm{CCC}(\mathrm{OO}) \mathrm{CC}[\mathrm{CH} 2]$ & $\mathrm{InChl}=1 \mathrm{~S} / \mathrm{C} 6 \mathrm{H} 13 \mathrm{O} 2 / \mathrm{c} 1-3-5-6(4-2) 8-7 / \mathrm{h} 6-7 \mathrm{H}, 1,3-5 \mathrm{H} 2,2 \mathrm{H} 3$ \\
\hline 2677 & $13 \mathrm{C} 6 \mathrm{O13}-4 \mathrm{OOH}$ & $\mathrm{CCC1}(\mathrm{COO}) \mathrm{CCO} 1$ & InChl=1S/C6H12O3/c1-2-6(5-9-7)3-4-8-6/h7H,2-5H2,1H3 \\
\hline 2678 & $\mathrm{XC6OOH1-202}$ & $\mathrm{OOCC}(\mathrm{C}(\mathrm{C}) \mathrm{C})(\mathrm{O}[\mathrm{O}]) \mathrm{C}$ & $\operatorname{lnChl}=1 \mathrm{~S} / \mathrm{C} 6 \mathrm{H} 13 \mathrm{O} 4 / \mathrm{c} 1-5(2) 6(3,10-8) 4-9-7 / \mathrm{h} 5,7 \mathrm{H}, 4 \mathrm{H} 2,1-3 \mathrm{H} 3$ \\
\hline 2679 & $\mathrm{C} 6 \mathrm{H} 12 \mathrm{O} 2-3$ & $\mathrm{CC1C}(01) \mathrm{CCC}$ & $\operatorname{lnChl}=15 / \mathrm{C} 6 \mathrm{H} 12 \mathrm{O} / \mathrm{c} 1-3-4-6-5(2) 7-6 / \mathrm{h} 5-6 \mathrm{H}, 3-4 \mathrm{H} 2,1-2 \mathrm{H} 3$ \\
\hline 2680 & $13 \mathrm{C} 6 \mathrm{OOH} 2-6$ & {$[\mathrm{CH} 2] \mathrm{CC}(\mathrm{C}(\mathrm{OO}) \mathrm{C}) \mathrm{C}$} & $\operatorname{lnChl}=1 \mathrm{~S} / \mathrm{C} 6 \mathrm{H} 13 \mathrm{O} 2 / \mathrm{c} 1-4-5(2) 6(3) 8-7 / \mathrm{h} 5-7 \mathrm{H}, 1,4 \mathrm{H} 2,2-3 \mathrm{H} 3$ \\
\hline 2681 & $\mathrm{XC6012-40OH}$ & $\mathrm{CC}(\mathrm{C} 1(\mathrm{C}) \mathrm{CO} 1) \mathrm{COO}$ & InChl=1S/C6H12O3/c1-5(3-9-7)6(2)4-8-6/h5,7H,3-4H2,1-2H3 \\
\hline 2682 & IC6OOH5-102 & OOCCCC(CO[O])C & InChl=1S/C6H13O4/c1-6(5-10-8)3-2-4-9-7/h6-7H,2-5H2,1H3 \\
\hline 2683 & C6H12O2-4 & $\mathrm{CC} 1 \mathrm{CC}(01) \mathrm{CC}$ & InChl=15/C6H12O/c1-3-6-4-5(2)7-6/h5-6H,3-4H2,1-2H3 \\
\hline 2684 & 13С6OOH3-1 & $\mathrm{CCC}(\mathrm{OO})(\mathrm{C}[\mathrm{CH} 2]) \mathrm{C}$ & InChl=1S/C6H13O2/c1-4-6(3,5-2)8-7/h7H,1,4-5H2,2-3H3 \\
\hline 2685 & $13 \mathrm{C} 6 \mathrm{O} 13-5 \mathrm{OOH}$ & $\mathrm{OOC}(\mathrm{C} 1(\mathrm{C}) \mathrm{CCO} 1) \mathrm{C}$ & $\operatorname{lnChl}=1 \mathrm{~S} / \mathrm{C} 6 \mathrm{H} 12 \mathrm{O} 3 / \mathrm{c} 1-5(9-7) 6(2) 3-4-8-6 / \mathrm{h} 5,7 \mathrm{H}, 3-4 \mathrm{H} 2,1-2 \mathrm{H} 3$ \\
\hline 2686 & $\mathrm{XC6OOH1-302}$ & $\mathrm{OOCC}(\mathrm{C}(\mathrm{O}[\mathrm{O}])(\mathrm{C}) \mathrm{C}) \mathrm{C}$ & InChl=1S/C6H13O4/c1-5(4-9-7)6(2,3)10-8/h5,7H,4H2,1-3H3 \\
\hline 2687 & $\mathrm{C} 6 \mathrm{H} 12 \mathrm{O} 2-5$ & $\mathrm{CC} 1 \mathrm{CCC}(\mathrm{O} 1) \mathrm{C}$ & $\ln C h \mathrm{l}=1 \mathrm{~S} / \mathrm{C} 6 \mathrm{H} 12 \mathrm{O} / \mathrm{c} 1-5-3-4-6(2) 7-5 / \mathrm{h} 5-6 \mathrm{H}, 3-4 \mathrm{H} 2,1-2 \mathrm{H} 3$ \\
\hline 2688 & $13 \mathrm{C} 6 \mathrm{OOH} 3-2$ & $\mathrm{C}[\mathrm{CH}] \mathrm{C}(\mathrm{OO})(\mathrm{CC}) \mathrm{C}$ & $\operatorname{lnChl}=1 \mathrm{~S} / \mathrm{C} 6 \mathrm{H} 13 \mathrm{O} 2 / \mathrm{c} 1-4-6(3,5-2) 8-7 / \mathrm{h} 4,7 \mathrm{H}, 5 \mathrm{H} 2,1-3 \mathrm{H} 3$ \\
\hline 2689 & XC6O13-1AOOH & OOCC1COC1(C)C & $\operatorname{lnChl}=15 / \mathrm{C} 6 \mathrm{H} 12 \mathrm{O} / \mathrm{c} 1-6(2) 5(3-8-6) 4-9-7 / \mathrm{h} 5,7 \mathrm{H}, 3-4 \mathrm{H} 2,1-2 \mathrm{H} 3$ \\
\hline 2690 & $\mathrm{IC} 6 \mathrm{OOH} 5-2 \mathrm{O} 2$ & OOCCCC(O[O])(C)C & $\operatorname{lnChl}=1 \mathrm{~S} / \mathrm{C} 6 \mathrm{H} 13 \mathrm{O} 4 / \mathrm{c} 1-6(2,10-8) 4-3-5-9-7 / \mathrm{h} 7 \mathrm{H}, 3-5 \mathrm{H} 2,1-2 \mathrm{H} 3$ \\
\hline 2691 & $\mathrm{C} 6 \mathrm{H} 12 \mathrm{O}-4$ & $\mathrm{CCC1C}(01) \mathrm{CC}$ & $\operatorname{lnChl}=1 \mathrm{~S} / \mathrm{C} 6 \mathrm{H} 12 \mathrm{O} / \mathrm{c} 1-3-5-6(4-2) 7-5 / \mathrm{h} 5-6 \mathrm{H}, 3-4 \mathrm{H} 2,1-2 \mathrm{H} 3$ \\
\hline 2692 & $13 \mathrm{C} 6 \mathrm{OOH} 3-4$ & $\mathrm{CCC}(\mathrm{OO})(\mathrm{CC})[\mathrm{CH} 2]$ & $\operatorname{lnChl}=15 / C 6 \mathrm{H} 13 \mathrm{O} 2 / \mathrm{c1}-4-6(3,5-2) 8-7 / \mathrm{h} 7 \mathrm{H}, 3-5 \mathrm{H} 2,1-2 \mathrm{H} 3$ \\
\hline 2693 & $13 \mathrm{C} 6013-60 \mathrm{OH}$ & $\mathrm{CC1}(\mathrm{CCOO}) \mathrm{CCO} 1$ & $\operatorname{lnChl}=1 \mathrm{~S} / \mathrm{C} 6 \mathrm{H} 12 \mathrm{O} 3 / \mathrm{c} 1-6(2-4-8-6) 3-5-9-7 / \mathrm{h} 7 \mathrm{H}, 2-5 \mathrm{H} 2,1 \mathrm{H} 3$ \\
\hline 2694 & $\mathrm{XC6OOH1-402}$ & $\mathrm{OOCC}(\mathrm{C}(\mathrm{CO}[\mathrm{O}]) \mathrm{C}) \mathrm{C}$ & $\operatorname{lnChl}=1 \mathrm{~S} / \mathrm{C} 6 \mathrm{H} 13 \mathrm{O} 4 / \mathrm{c} 1-5(3-9-7) 6(2) 4-10-8 / \mathrm{h} 5-7 \mathrm{H}, 3-4 \mathrm{H} 2,1-2 \mathrm{H} 3$ \\
\hline 2695 & $\mathrm{NC5H} 11 \mathrm{CHO}$ & $\mathrm{CCCCCC}=0$ & InChl=1S/C6H12O/c1-2-3-4-5-6-7/h6H,2-5H2,1H3 \\
\hline 2696 & $13 \mathrm{C} 6 \mathrm{OOH} 4-1$ & $\mathrm{CCC}(\mathrm{COO}) \mathrm{C}[\mathrm{CH} 2]$ & InChl=15/C6H13O2/c1-3-6(4-2)5-8-7/h6-7H,1,3-5H2,2H3 \\
\hline 2697 & XC6013-20OH & $00 \mathrm{O} 1(\mathrm{C}) \operatorname{coc} 1(\mathrm{C}) \mathrm{C}$ & InChl=1S/C6H12O3/c1-5(2)6(3,9-7)4-8-5/h7H,4H2,1-3H3 \\
\hline 2698 & IC60OH5-302 & $00 \mathrm{OCC}(\mathrm{C}(\mathrm{C}) \mathrm{C}) \mathrm{O}[\mathrm{O}]$ & $\ln \mathrm{Chl}=1 \mathrm{~S} / \mathrm{C} 6 \mathrm{H} 13 \mathrm{O} 4 / \mathrm{c} 1-5(2) 6(10-8) 3-4-9-7 / \mathrm{h} 5-7 \mathrm{H}, 3-4 \mathrm{H} 2,1-2 \mathrm{H3}$ \\
\hline 2699 & $\mathrm{C} 6 \mathrm{Y} 2$ & $\mathrm{CC}(=0) \mathrm{CCCC}$ & $\operatorname{lnChl}=1 \mathrm{~S} / \mathrm{C} 6 \mathrm{H} 12 \mathrm{O} / \mathrm{c} 1-3-4-5-6(2) 7 / \mathrm{h} 3-5 \mathrm{H} 2,1-2 \mathrm{H} 3$ \\
\hline 2700 & $13 \mathrm{C} 6 \mathrm{OOH} 4-2$ & $\mathrm{C}[\mathrm{CH}] \mathrm{C}(\mathrm{COO}) \mathrm{CC}$ & InChl=1S/C6H13O2/c1-3-6(4-2)5-8-7/h3,6-7H,4-5H2,1-2H3 \\
\hline 2701 & $13 \mathrm{C} 6 \mathrm{O} 14-2 \mathrm{OOH}$ & CCC1COCC10O & $\mathrm{InChl}=1 \mathrm{~S} / \mathrm{C} 6 \mathrm{H} 12 \mathrm{O} / \mathrm{c} 1-2-5-3-8-4-6(5) 9-7 / \mathrm{h} 5-7 \mathrm{H}, 2-4 \mathrm{H} 2,1 \mathrm{H} 3$ \\
\hline 2702 & $\mathrm{XC6OOH2-102}$ & {$[0] \mathrm{OCC}(\mathrm{C}(\mathrm{C}) \mathrm{C})(\mathrm{OO}) \mathrm{C}$} & $\operatorname{lnChl}=1 \mathrm{~S} / \mathrm{C} 6 \mathrm{H} 13 \mathrm{O} 4 / \mathrm{c} 1-5(2) 6(3,10-8) 4-9-7 / \mathrm{h} 5,8 \mathrm{H}, 4 \mathrm{H} 2,1-3 \mathrm{H} 3$ \\
\hline 2703 & IC601-3 & CCC1OCC1C & InChl=1S/C6H12O/c1-3-6-5(2)4-7-6/h5-6H,3-4H2,1-2H3 \\
\hline 2704 & $13 \mathrm{C} 6 \mathrm{OOH} 4-3$ & $\mathrm{CC}[\mathrm{C}](\mathrm{COO}) \mathrm{CC}$ & InChl=1S/C6H13O2/c1-3-6(4-2)5-8-7/h7H,3-5H2,1-2H3 \\
\hline 2705 & $\mathrm{XC6013-40 \textrm {OH }}$ & $\mathrm{CC1}(\mathrm{COO}) \mathrm{OCC} 1 \mathrm{C}$ & $\operatorname{lnChl}=15 / \mathrm{C} 6 \mathrm{H} 12 \mathrm{O} 3 / \mathrm{c} 1-5-3-8-6(5,2) 4-9-7 / \mathrm{h} 5,7 \mathrm{H}, 3-4 \mathrm{H} 2,1-2 \mathrm{H} 3$ \\
\hline 2706 & IC6OOH5-4O2 & $\mathrm{OOCC}(\mathrm{CC}(\mathrm{C}) \mathrm{C}) \mathrm{O}[\mathrm{O}]$ & InChl=15/C6H13O4/c1-5(2)3-6(10-8)4-9-7/h5-7H,3-4H2,1-2H3 \\
\hline 2707 & IC601-4 & $\mathrm{CC} 1 \mathrm{OCC}(\mathrm{C} 1) \mathrm{C}$ & InChl=15/C6H12O/c1-5-3-6(2)7-4-5/h5-6H,3-4H2,1-2H3 \\
\hline 2708 & IC6OOH1-1A & $\mathrm{CCCC}(\mathrm{COO})[\mathrm{CH} 2]$ & $\operatorname{lnChl}=1 \mathrm{~S} / \mathrm{C} 6 \mathrm{H} 13 \mathrm{O} 2 / \mathrm{c1}-3-4-4-6(2) 5-8-7 / \mathrm{h} 6-7 \mathrm{H}, 2-5 \mathrm{H} 2,1 \mathrm{H} 3$ \\
\hline 2709 & $13 \mathrm{C} 6 \mathrm{O} 14-3 \mathrm{OOH}$ & $\operatorname{ccC1}(00) \operatorname{COCC1}$ & $\operatorname{lnChl}=1 \mathrm{~S} / \mathrm{C} 6 \mathrm{H} 12 \mathrm{O} / \mathrm{c} 1-2-6(9-7) 3-4-8-5-6 / \mathrm{h} 7 \mathrm{H}, 2-5 \mathrm{H} 2,1 \mathrm{H} 3$ \\
\hline 2710 & $\mathrm{XC6OOH2-302}$ & $\mathrm{OOC}(\mathrm{C}(\mathrm{O}[\mathrm{O}])(\mathrm{C}) \mathrm{C})(\mathrm{C}) \mathrm{C}$ & InChl=1S/C6H13O4/c1-5(2,9-7)6(3,4)10-8/h7H,1-4H3 \\
\hline 2711 & IC601-5 & CC1CCCOC1 & $\operatorname{lnChl}=1 \mathrm{~S} / \mathrm{C} 6 \mathrm{H} 12 \mathrm{O} / \mathrm{c} 1-6-3-2-4-7-5-6 / \mathrm{h} 6 \mathrm{H}, 2-5 \mathrm{H} 2,1 \mathrm{H} 3$ \\
\hline 2712 & IC6OOH1-2 & $\mathrm{CCC}[\mathrm{C}](\mathrm{COO}) \mathrm{C}$ & $\operatorname{lnChl}=15 / C 6 H 13 O 2 / c 1-3-4-6(2) 5-8-7 / h 7 H, 3-5 \mathrm{H} 2,1-2 \mathrm{H} 3$ \\
\hline 2713 & XC6O14-1AOOH & CC1COCC1COO & $\mathrm{InChl}=1 \mathrm{~S} / \mathrm{C} 6 \mathrm{H} 12 \mathrm{O} / \mathrm{c} 1-5-2-8-3-6(5) 4-9-7 / \mathrm{h} 5-7 \mathrm{H}, 2-4 \mathrm{H} 2,1 \mathrm{H} 3$ \\
\hline 2714 & $\mathrm{XC6OOH2-402}$ & {$[\mathrm{O}] \mathrm{OCC}(\mathrm{C}(\mathrm{OO})(\mathrm{C}) \mathrm{C}) \mathrm{C}$} & $\operatorname{lnChl}=1 \mathrm{~S} / \mathrm{C} 6 \mathrm{H} 13 \mathrm{O} 4 / \mathrm{c} 1-5(4-9-7) 6(2,3) 10-8 / \mathrm{h} 5,8 \mathrm{H}, 4 \mathrm{H} 2,1-3 \mathrm{H} 3$ \\
\hline 2715 & IC6O2-3 & $\operatorname{ccc} 10 \mathrm{OC} 1(\mathrm{C}) \mathrm{C}$ & InChl=1S/C6H12O/c1-4-5-6(2,3)7-5/h5H,4H2,1-3H3 \\
\hline 2716 & $\mathrm{IC} 6 \mathrm{OOH} 1-3$ & $\mathrm{CC}[\mathrm{CH}] \mathrm{C}(\mathrm{COO}) \mathrm{C}$ & InChl=1S/C6H13O2/c1-3-4-6(2)5-8-7/h4,6-7H,3,5H2,1-2H3 \\
\hline 2717 & $13 \mathrm{C} 6 \mathrm{O} 14-5 \mathrm{OOH}$ & $\mathrm{OOC}(\mathrm{C} 1 \mathrm{COCC} 1) \mathrm{C}$ & InChl=1S/C6H12O3/c1-5(9-7)6-2-3-8-4-6/h5-7H,2-4H2,1H3 \\
\hline 2718 & HX5ENAL4OJ & $\mathrm{O}=\mathrm{CCCC}(\mathrm{C}=\mathrm{C})[\mathrm{O}]$ & $\operatorname{lnChl}=1 \mathrm{~S} / \mathrm{C} 6 \mathrm{H} 902 / \mathrm{cc} 1-2-6(8) 4-3-5-7 / \mathrm{h} 2,5-6 \mathrm{H}, 1,3-4 \mathrm{H} 2$ \\
\hline 2719 & IC6O2-4 & $\mathrm{CC} 1 \mathrm{CC}(\mathrm{O} 1)(\mathrm{C}) \mathrm{C}$ & InChl=1S/C6H12O/c1-5-4-6(2,3)7-5/h5H,4H2,1-3H3 \\
\hline 2720 & IC6OOH1-4 & $\mathrm{C}[\mathrm{CH}] \mathrm{CC}(\mathrm{COO}) \mathrm{C}$ & InChl=1S/C6H13O2/c1-3-4-6(2)5-8-7/h3,6-7H,4-5H2,1-2H3 \\
\hline 2721 & XC6014-20OH & $\mathrm{CC} 1(\operatorname{COCC} 1 \mathrm{C}) 0 \mathrm{O}$ & $\operatorname{lnChl}=15 / \mathrm{C} 6 \mathrm{H} 12 \mathrm{O} / \mathrm{c} 1-5-3-8-4-6(5,2) 9-7 / \mathrm{h} 5,7 \mathrm{H}, 3-4 \mathrm{H} 2,1-2 \mathrm{H} 3$ \\
\hline 2722 & CYHX1N4QJ & {$[\mathrm{O}] \mathrm{OC1CCC}=\mathrm{CC} 1$} & InChl=1S/C6H9O2/c7-8-6-4-2-1-3-5-6/h1-2,6H,3-5H2 \\
\hline 2723 & IC6O2-5 & $\mathrm{CC1}(\mathrm{C}) \mathrm{CCCO} 1$ & $\operatorname{lnChl}=1 \mathrm{~S} / \mathrm{C} 6 \mathrm{H} 12 \mathrm{O} / \mathrm{c1}-6(2) 4-3-5-7-6 / \mathrm{h} 3-5 \mathrm{H} 2,1-2 \mathrm{H} 3$ \\
\hline 2724 & IC6OOH1-5 & {$[\mathrm{CH} 2] \mathrm{CCC}(\mathrm{COO}) \mathrm{C}$} & $\operatorname{lnChl}=15 / \mathrm{C} 6 \mathrm{H} 13 \mathrm{O} 2 / \mathrm{c} 1-3-4-6(2) 5-8-7 / \mathrm{h} 6-7 \mathrm{H}, 1,3-5 \mathrm{H} 2,2 \mathrm{H} 3$ \\
\hline 2725 & $13 \mathrm{C} 6 \mathrm{O} 14-6 \mathrm{OOH}$ & OOCCC1COCC1 & $\operatorname{lnChl}=15 / \mathrm{C} 6 \mathrm{H} 12 \mathrm{O} / \mathrm{c} 7-9-4-2-6-1-3-8-5-6 / \mathrm{h} 6-7 \mathrm{H}, 1-5 \mathrm{H} 2$ \\
\hline 2726 & CYHX1N3QJ & {$[0] \mathrm{OC1CCCC}=\mathrm{C} 1$} & $\operatorname{lnChl}=15 / C 6 H 902 / c 7-8-6-4-2-1-3-5-6 / \mathrm{h2}, 4,6 \mathrm{H}, 1,3,5 \mathrm{H} 2$ \\
\hline
\end{tabular}




\begin{tabular}{|c|c|c|c|}
\hline 2727 & C6Y1D2-60OJ & {$[\mathrm{O}] \mathrm{OCCCC}=\mathrm{CC}=\mathrm{O}$} & InChl=1S/C6H9O3/c7-5-3-1-2-4-6-9-8/h1,3,5H,2,4,6H2 \\
\hline 2728 & C6Y1D2-60O-4J & $\mathrm{OOCCC}=\mathrm{CC}=\mathrm{C}[\mathrm{O}]$ & InChl=15/C6H9O3/c7-5-3-1-2-4-6-9-8/h1-3,5,8H,4,6H2 \\
\hline 2729 & C6Y15Q6-4J & $\mathrm{OOCC}(=\mathrm{CCCC}=0)[\mathrm{O}]$ & InChl=1S/C6H9O4/c7-4-2-1-3-6(8)5-10-9/h3-4,9H,1-2,5H2 \\
\hline 2730 & C6Y15Q6-4QJ & $\mathrm{OOCC}(=\mathrm{O}) \mathrm{C}(\mathrm{CCC}=\mathrm{O}) \mathrm{O}[\mathrm{O}]$ & InChl=1S/C6H9O6/c7-3-1-2-6(12-10)5(8)4-11-9/h3,6,9H,1-2,4H2 \\
\hline 2731 & C6Y156-4Q & $\mathrm{O}=\operatorname{CCCC}(\mathrm{C}(=0) \mathrm{C}=0) 00$ & InChl=15/C6H805/c7-3-1-2-6(11-10)5(9)4-8/h3-4,6,10H,1-2H2 \\
\hline 2732 & C6Y15-60OJ & {$[\mathrm{O}] \mathrm{OCC}(=\mathrm{O}) \mathrm{CCCC}=0$} & InChl=1S/C6H9O4/c7-4-2-1-3-6(8)5-10-9/h4H,1-3,5H2 \\
\hline 2733 & C6D2Y1Q6-4QJ & {$[\mathrm{O}] \mathrm{OC}(\mathrm{C}=\mathrm{CC}=\mathrm{O}) \mathrm{CCOO}$} & InChl=1S/C6H9O5/c7-4-1-2-6(11-9)3-5-10-8/h1-2,4,6,8H,3,5H2 \\
\hline 2734 & C6D2Y16Q4 & $\mathrm{O}=\mathrm{CC}=\mathrm{CC}(\mathrm{CC}=0) 00$ & InChl=1S/C6H8O4/c7-4-1-2-6(10-9)3-5-8/h1-2,4-6,9H,3H2 \\
\hline 2735 & $\mathrm{C} 6 \mathrm{H} 11-3 \mathrm{D} 2 \mathrm{O}$ & $\mathrm{CCC}=\mathrm{CC}([0]) \mathrm{C}$ & $\mathrm{InChl}=1 \mathrm{~S} / \mathrm{C} 6 \mathrm{H} 110 / \mathrm{c} 1-3-4-5-6(2) 7 / \mathrm{h} 4-6 \mathrm{H}, 3 \mathrm{H} 2,1-2 \mathrm{H} 3$ \\
\hline 2736 & C7H5 & {$[\mathrm{CH}]=\mathrm{C}=\mathrm{C} 1 \mathrm{C}=\mathrm{CC}=\mathrm{C} 1$} & InChl=1S/C7H5/c1-2-7-5-3-4-6-7/h1,3-6H \\
\hline 2737 & $\mathrm{C} 7 \mathrm{H} 15-4 \mathrm{O}$ & $\operatorname{cccc}([0]) \operatorname{ccc}$ & InChl=1S/C7H15O/c1-3-5-7(8)6-4-2/h7H,3-6H2,1-2H3 \\
\hline 2738 & $\mathrm{C} 7 \mathrm{H} 14 \mathrm{OOH} 4-1$ & $\mathrm{CCCC}(\mathrm{OO}) \mathrm{CC}[\mathrm{CH} 2]$ & $\operatorname{lnChl}=1 \mathrm{~S} / \mathrm{C} 7 \mathrm{H} 15 \mathrm{O} 2 / \mathrm{c1}-3-5-7(9-8) 6-4-2 / \mathrm{h} 7-8 \mathrm{H}, 1,3-6 \mathrm{H} 2,2 \mathrm{H} 3$ \\
\hline 2739 & C7H14OOH2-10 & $c([0]) c(00) \operatorname{ccccc}$ & InChl=1S/C7H15O3/c1-2-3-4-5-7(6-8)10-9/h7,9H,2-6H2,1H3 \\
\hline 2740 & $\mathrm{C} 7 \mathrm{H} 6$ & $\mathrm{C}=\mathrm{C}=\mathrm{C} 1 \mathrm{C}=\mathrm{CC}=\mathrm{C} 1$ & InChl=1S/C7H6/c1-2-7-5-3-4-6-7/h3-6H,1H2 \\
\hline 2741 & $\mathrm{C} 7 \mathrm{H} 14 \mathrm{OH}-1 \mathrm{~J} 2$ & $\mathrm{C}(\mathrm{O})[\mathrm{CH}] \mathrm{CCCCC}$ & InChl=15/C7H15O/c1-2-3-4-5-6-7-8/h6,8H,2-5,7H2,1H3 \\
\hline 2742 & $\mathrm{C} 7 \mathrm{H} 14 \mathrm{OOH} 4-2$ & $\mathrm{CCCC}(\mathrm{OO}) \mathrm{C}[\mathrm{CH}] \mathrm{C}$ & InChl=1S/C7H15O2/c1-3-5-7(9-8)6-4-2/h3,7-8H,4-6H2,1-2H3 \\
\hline 2743 & $\mathrm{C} 7 \mathrm{H} 14 \mathrm{OOH} 2-3 \mathrm{O}$ & $\mathrm{CC}(\mathrm{OO}) \mathrm{C}([\mathrm{O}]) \mathrm{CCCC}$ & InChl=1S/C7H1503/c1-3-4-5-7(8)6(2)10-9/h6-7,9H,3-5H2,1-2H3 \\
\hline 2744 & $\mathrm{C} 6 \mathrm{H} 5 \mathrm{CH} 2$ & $\mathrm{c1cc}([\mathrm{CH} 2]) \operatorname{ccc} 1$ & $\mathrm{InChl}=1 \mathrm{~S} / \mathrm{C} 7 \mathrm{H} 7 / \mathrm{c1}-7-5-3-2-4-6-7 / \mathrm{h} 2-6 \mathrm{H}, 1 \mathrm{H} 2$ \\
\hline 2745 & C7H14OH-2J1 & {$[\mathrm{CH} 2] \mathrm{C}(\mathrm{O}) \mathrm{CCCCC}$} & InChl=1S/C7H15O/c1-3-4-5-6-7(2)8/h7-8H,2-6H2,1H3 \\
\hline 2746 & $\mathrm{C} 7 \mathrm{H} 14 \mathrm{OOH} 4-3$ & $\mathrm{CCCC}(\mathrm{OO})[\mathrm{CH}] \mathrm{CC}$ & InChl=15/C7H15O2/c1-3-5-7(9-8)6-4-2/h5,7-8H,3-4,6H2,1-2H3 \\
\hline 2747 & $\mathrm{C} 7 \mathrm{H} 14 \mathrm{OOH} 2-4 \mathrm{O}$ & $\mathrm{CC}(\mathrm{OO}) \mathrm{CC}([0]) \mathrm{CCC}$ & InChl=1S/C7H1503/c1-3-4-7(8)5-6(2)10-9/h6-7,9H,3-5H2,1-2H3 \\
\hline 2748 & $\mathrm{C} 6 \mathrm{H} 4 \mathrm{CH} 3$ & Cc1c[c]ccc1 & InChl=1S/C7H7/c1-7-5-3-2-4-6-7/h2-3,5-6H,1H3 \\
\hline 2749 & $\mathrm{C} 7 \mathrm{H} 14 \mathrm{OH}-2 \mathrm{~J} 3$ & $\mathrm{CC}(\mathrm{O})[\mathrm{CH}] \mathrm{CCCC}$ & InChl=1S/C7H15O/c1-3-4-5-6-7(2)8/h6-8H,3-5H2,1-2H3 \\
\hline 2750 & C7H15OOH-1-2 & OOCC(C)cccc & InChl=1S/C7H16O2/c1-3-4-5-7(2)6-9-8/h7-8H,3-6H2,1-2H3 \\
\hline 2751 & $\mathrm{C} 7 \mathrm{H} 14 \mathrm{OOH} 2-5 \mathrm{O}$ & $\mathrm{CC}(\mathrm{OO}) \mathrm{CCC}([\mathrm{O}]) \mathrm{CC}$ & InChl=1S/C7H1503/c1-3-7(8)5-4-6(2)10-9/h6-7,9H,3-5H2,1-2H3 \\
\hline 2752 & $\mathrm{C} 6 \mathrm{H} 5 \mathrm{CH} 3$ & $\operatorname{c1cc}(\mathrm{C}) \mathrm{ccc} 1$ & InChl=1S/C7H8/c1-7-5-3-2-4-6-7/h2-6H,1H3 \\
\hline 2753 & $\mathrm{C} 7 \mathrm{H} 14 \mathrm{OH}-3 \mathrm{~J} 2$ & $\mathrm{C}[\mathrm{CH}] \mathrm{C}(\mathrm{O}) \mathrm{CCCC}$ & $\operatorname{lnChl}=1 \mathrm{~S} / \mathrm{C} 7 \mathrm{H} 15 \mathrm{O} / \mathrm{c1} 1-3-5-6-7(8) 4-2 / \mathrm{h} 4,7-8 \mathrm{H}, 3,5-6 \mathrm{H} 2,1-2 \mathrm{H} 3$ \\
\hline 2754 & C7H15OOH-2-2 & $\mathrm{CC}(\mathrm{C})(\mathrm{OO}) \mathrm{CCCC}$ & InChl=1S/C7H16O2/c1-4-5-6-7(2,3)9-8/h8H,4-6H2,1-3H3 \\
\hline 2755 & $\mathrm{C} 7 \mathrm{H} 14 \mathrm{OOH} 2-6 \mathrm{O}$ & $\mathrm{CC}(\mathrm{OO}) \operatorname{cccc}([\mathrm{O}]) \mathrm{C}$ & InChl=1S/C7H15O3/c1-6(8)4-3-5-7(2)10-9/h6-7,9H,3-5H2,1-2H3 \\
\hline 2756 & DMCPDR & $\mathrm{CC} 1=\mathrm{CC}=\mathrm{C}(\mathrm{C})[\mathrm{CH}] 1$ & $\operatorname{lnChl}=1 \mathrm{~S} / \mathrm{C} 7 \mathrm{H} 9 / \mathrm{c1} 1-6-3-4-7(2) 5-6 / \mathrm{h} 3-5 \mathrm{H}, 1-2 \mathrm{H} 3$ \\
\hline 2757 & $\mathrm{C} 7 \mathrm{H} 14 \mathrm{OH}-3 \mathrm{J4}$ & $\mathrm{CCC}(\mathrm{O})[\mathrm{CH}] \mathrm{CCC}$ & InChl=15/C7H15O/c1-3-5-6-7(8)4-2/h6-8H,3-5H2,1-2H3 \\
\hline 2758 & C7H15OOH-3-2 & $\mathrm{CC}(\mathrm{C}) \mathrm{C}(\mathrm{OO}) \mathrm{CCC}$ & InChl=15/C7H1602/c1-4-5-7(9-8)6(2)3/h6-8H,4-5H2,1-3H3 \\
\hline 2759 & C7H14OOH3-1O & $\mathrm{C}([\mathrm{O}]) \mathrm{CC}(\mathrm{OO}) \mathrm{CCCC}$ & InChl=1S/C7H1503/c1-2-3-4-7(10-9)5-6-8/h7,9H,2-6H2,1H3 \\
\hline 2760 & DMCPD & $\mathrm{CC} 1=\mathrm{CC}=\mathrm{C}(\mathrm{C}) \mathrm{C} 1$ & $\operatorname{lnChl}=15 / C 7 H 10 / c 1-6-3-4-7(2) 5-6 / h 3-4 \mathrm{H}, 5 \mathrm{H} 2,1-2 \mathrm{H} 3$ \\
\hline 2761 & C7H14OH-4J3 & $\mathrm{CC}[\mathrm{CH}] \mathrm{C}(\mathrm{O}) \mathrm{CCC}$ & $\operatorname{lnChl}=1 \mathrm{~S} / \mathrm{C} 7 \mathrm{H} 15 \mathrm{O} / \mathrm{c1} 1-3-5-7(8) 6-4-2 / \mathrm{h} 5,7-8 \mathrm{H}, 3-4,6 \mathrm{H} 2,1-2 \mathrm{H} 3$ \\
\hline 2762 & $\mathrm{C7H} 15 \mathrm{OOH}-4-2$ & $\mathrm{CC}(\mathrm{C}) \mathrm{CC}(\mathrm{OO}) \mathrm{CC}$ & InChl=1S/C7H16O2/c1-4-7(9-8)5-6(2)3/h6-8H,4-5H2,1-3H3 \\
\hline 2763 & $\mathrm{C} 7 \mathrm{H} 14 \mathrm{OOH} 3-2 \mathrm{O}$ & $\mathrm{CC}([\mathrm{O}]) \mathrm{C}(\mathrm{OO}) \mathrm{CCCC}$ & InChl=1S/C7H1503/c1-3-4-5-7(10-9)6(2)8/h6-7,9H,3-5H2,1-2H3 \\
\hline 2764 & C7H111-5,3,6P & {$[\mathrm{CH}]=\mathrm{CCC}(\mathrm{C}) \mathrm{C}=\mathrm{C}$} & InChl=1S/C7H11/c1-4-6-7(3)5-2/h1,4-5,7H,2,6H2,3H3 \\
\hline 2765 & I24C7OH1-2R & $O C[C](C) C C(C) C$ & InChl=15/C7H15O/c1-6(2)4-7(3)5-8/h6,8H,4-5H2,1-3H3 \\
\hline 2766 & C7H15OOH-5-2 & $\mathrm{CC}(\mathrm{C}) \mathrm{CCC}(\mathrm{OO}) \mathrm{C}$ & InChl=15/C7H1602/c1-6(2)4-5-7(3)9-8/h6-8H,4-5H2,1-3H3 \\
\hline 2767 & C7H14OOH3-4O & $\mathrm{CCC}(\mathrm{OO}) \mathrm{C}([\mathrm{O}]) \mathrm{CCC}$ & InChl=1S/C7H15O3/c1-3-5-6(8)7(4-2)10-9/h6-7,9H,3-5H2,1-2H3 \\
\hline 2768 & C7H111-5,1P & {$[\mathrm{CH}]=\mathrm{CCCC}=\mathrm{CC}$} & InChl=1S/C7H11/c1-3-5-7-6-4-2/h1,3-4,6H,5,7H2,2H3 \\
\hline 2769 & I24C7OH2-1R & {$[\mathrm{CH} 2] \mathrm{C}(\mathrm{O})(\mathrm{C}) \mathrm{CC}(\mathrm{C}) \mathrm{C}$} & $\operatorname{lnChl}=1 \mathrm{~S} / \mathrm{C} 7 \mathrm{H} 15 \mathrm{O} / \mathrm{c} 1-6(2) 5-7(3,4) 8 / \mathrm{h} 6,8 \mathrm{H}, 3,5 \mathrm{H} 2,1-2,4 \mathrm{H} 3$ \\
\hline 2770 & $\mathrm{C} 7 \mathrm{H} 15 \mathrm{OOH}-6-2$ & $\mathrm{CC}(\mathrm{C}) \mathrm{CCCCOO}$ & InChl=1S/C7H16O2/c1-7(2)5-3-4-6-9-8/h7-8H,3-6H2,1-2H3 \\
\hline 2771 & C7H14OOH3-5O & $\operatorname{CCC}(00) \operatorname{CC}([0]) \operatorname{CC}$ & InChl=1S/C7H1503/c1-3-6(8)5-7(4-2)10-9/h6-7,9H,3-5H2,1-2H3 \\
\hline 2772 & H15DE2M-T & $\mathrm{C}=[\mathrm{C}] \mathrm{CCC}(=\mathrm{C}) \mathrm{C}$ & InChl=15/C7H11/c1-4-5-6-7(2)3/h1-2,5-6H2,3H3 \\
\hline 2773 & $124 \mathrm{C} 7 \mathrm{OH} 2-3 \mathrm{R}$ & $\mathrm{CC}(\mathrm{O})(\mathrm{C})[\mathrm{CH}] \mathrm{C}(\mathrm{C}) \mathrm{C}$ & InChl=1S/C7H15O/c1-6(2)5-7(3,4)8/h5-6,8H,1-4H3 \\
\hline 2774 & C7H15OOH-1 & $\mathrm{C}(\mathrm{OO}) \mathrm{CCCCCC}$ & InChl=1S/C7H16O2/c1-2-3-4-5-6-7-9-8/h8H,2-7H2,1H3 \\
\hline 2775 & $\mathrm{C7H140OH3-6O}$ & $\operatorname{ccc}(00) \operatorname{ccc}([0]) \mathrm{C}$ & InChl=1S/C7H1503/c1-3-7(10-9)5-4-6(2)8/h6-7,9H,3-5H2,1-2H3 \\
\hline 2776 & I24C7D13-1AR & $\mathrm{C}=\mathrm{C}([\mathrm{CH} 2]) \mathrm{C}=\mathrm{C}(\mathrm{C}) \mathrm{C}$ & $\mathrm{InChl}=1 \mathrm{~S} / \mathrm{C} 7 \mathrm{H} 11 / \mathrm{c} 1-6(2) 5-7(3) 4 / \mathrm{h} 5 \mathrm{H}, 1-2 \mathrm{H} 2,3-4 \mathrm{H} 3$ \\
\hline 2777 & 124C7OH3-2R & $\mathrm{C}[\mathrm{C}](\mathrm{C}) \mathrm{C}(\mathrm{O}) \mathrm{C}(\mathrm{C}) \mathrm{C}$ & InChl=1S/C7H15O/c1-5(2)7(8)6(3)4/h5,7-8H,1-4H3 \\
\hline 2778 & $\mathrm{C} 7 \mathrm{H} 15 \mathrm{OOH}-2$ & $\mathrm{CC}(\mathrm{OO}) \mathrm{CCCCC}$ & InChl=15/C7H1602/c1-3-4-5-6-7(2)9-8/h7-8H,3-6H2,1-2H3 \\
\hline 2779 & C7H14OOH3-7O & $\operatorname{ccc}(00) \operatorname{cccc}([0])$ & InChl=1S/C7H15O3/c1-2-7(10-9)5-3-4-6-8/h7,9H,2-6H2,1H3 \\
\hline 2780 & 124C7D14-3R & $\mathrm{C}=\mathrm{C}(\mathrm{C})[\mathrm{CH}] \mathrm{C}(\mathrm{C})=\mathrm{C}$ & $\mathrm{InChl}=1 \mathrm{~S} / \mathrm{C} 7 \mathrm{H} 11 / \mathrm{c1}-6(2) 5-7(3) 4 / \mathrm{h} 5 \mathrm{H}, 1,3 \mathrm{H} 2,2,4 \mathrm{H} 3$ \\
\hline 2781 & NEC7OH3-4R & $\mathrm{CC}(\mathrm{C})(\mathrm{C}) \mathrm{C}(\mathrm{O})[\mathrm{CH}] \mathrm{C}$ & InChl=1S/C7H15O/c1-5-6(8)7(2,3)4/h5-6,8H,1-4H3 \\
\hline 2782 & $\mathrm{C} 7 \mathrm{H} 15 \mathrm{OOH}-3$ & $\operatorname{ccc}(00) \operatorname{cccc}$ & InChl=1S/C7H16O2/c1-3-5-6-7(4-2)9-8/h7-8H,3-6H2,1-2H3 \\
\hline 2783 & $\mathrm{C} 7 \mathrm{H} 14 \mathrm{OOH} 4-1 \mathrm{O}$ & $\operatorname{cccc}(00) \operatorname{ccc}([0])$ & InChl=1S/C7H15O3/c1-2-4-7(10-9)5-3-6-8/h7,9H,2-6H2,1H3 \\
\hline 2784 & 124C7D13 & $\mathrm{C}=\mathrm{C}(\mathrm{C}) \mathrm{C}=\mathrm{C}(\mathrm{C}) \mathrm{C}$ & $\operatorname{lnChl}=15 / \mathrm{C} 7 \mathrm{H} 12 / \mathrm{c} 1-6(2) 5-7(3) 4 / \mathrm{h} 5 \mathrm{H}, 1 \mathrm{H} 2,2-4 \mathrm{H} 3$ \\
\hline 2785 & $\mathrm{NEC7OH4-3R}$ & $\mathrm{CC}(\mathrm{C})(\mathrm{C})[\mathrm{CH}] \mathrm{C}(\mathrm{O}) \mathrm{C}$ & $\operatorname{lnChl}=1 \mathrm{~S} / \mathrm{C} 7 \mathrm{H} 15 \mathrm{O} / \mathrm{c} 1-6(8) 5-7(2,3) 4 / \mathrm{h5}-6,8 \mathrm{H}, 1-4 \mathrm{H} 3$ \\
\hline 2786 & $\mathrm{C} 7 \mathrm{H} 15 \mathrm{OOH}-4$ & $\operatorname{cccc}(00) \operatorname{ccc}$ & $\operatorname{lnChl}=1 \mathrm{~S} / \mathrm{C} 7 \mathrm{H} 1602 / \mathrm{c} 1-3-5-7(9-8) 6-4-2 / \mathrm{h} 7-8 \mathrm{H}, 3-6 \mathrm{H} 2,1-2 \mathrm{H} 3$ \\
\hline 2787 & $\mathrm{C} 7 \mathrm{H} 14 \mathrm{OOH} 4-2 \mathrm{O}$ & $\operatorname{cccc}(00) \operatorname{cc}([0]) \mathrm{C}$ & InChl=1S/C7H1503/c1-3-4-7(10-9)5-6(2)8/h6-7,9H,3-5H2,1-2H3 \\
\hline 2788 & 124C7D14 & $\mathrm{C}=\mathrm{C}(\mathrm{C}) \mathrm{CC}(\mathrm{C})=\mathrm{C}$ & InChl=1S/C7H12/c1-6(2)5-7(3)4/h1,3,5H2,2,4H3 \\
\hline 2789 & NEC7OH4-5R & $\mathrm{CC}(\mathrm{C})(\mathrm{C}) \mathrm{CC}(\mathrm{O})[\mathrm{CH} 2]$ & InChl=15/C7H15O/c1-6(8)5-7(2,3)4/h6,8H,1,5H2,2-4H3 \\
\hline 2790 & $\mathrm{OOC} 6 \mathrm{H} 4 \mathrm{OCH} 3$ & $\operatorname{COc} 1 \mathrm{c}(\mathrm{O}[\mathrm{O}]) \operatorname{cccc} 1$ & InChl=1S/C7H7O3/c1-9-6-4-2-3-5-7(6)10-8/h2-5H,1H3 \\
\hline 2791 & $\mathrm{C} 7 \mathrm{H} 14 \mathrm{OOH} 4-3 \mathrm{O}$ & $\operatorname{cccc}(00) C([0]) \operatorname{cc}$ & InChl=1S/C7H15O3/c1-3-5-7(10-9)6(8)4-2/h6-7,9H,3-5H2,1-2H3 \\
\hline 2792 & C7H13-1-2 & $\mathrm{C}=\mathrm{C}(\mathrm{C})[\mathrm{CH}] \mathrm{CCC}$ & $\mathrm{InChl}=1 \mathrm{~S} / \mathrm{C} 7 \mathrm{H} 13 / \mathrm{c1} 1-4-5-6-7(2) 3 / \mathrm{h} 6 \mathrm{H}, 2,4-5 \mathrm{H} 2,1,3 \mathrm{H} 3$ \\
\hline 2793 & NEC7OH5-4R & $\mathrm{CC}(\mathrm{C})(\mathrm{C}) \mathrm{C}[\mathrm{CH}] \mathrm{CO}$ & InChl=15/C7H15O/c1-7(2,3)5-4-6-8/h4,8H,5-6H2,1-3H3 \\
\hline 2794 & $\mathrm{C} 6 \mathrm{H} 5 \mathrm{OCH} 2 \mathrm{OO}$ & {$[\mathrm{O}] \mathrm{OCOC} 1=\mathrm{CC}=\mathrm{CC}=\mathrm{C} 1$} & InChl=1S/C7H7O3/c8-10-6-9-7-4-2-1-3-5-7/h1-5H,6H2 \\
\hline 2795 & $\mathrm{C} 7 \mathrm{H} 14 \mathrm{OH}-102-2$ & $\mathrm{C}(\mathrm{O}) \mathrm{C}(\mathrm{O}[\mathrm{O}]) \mathrm{CCCCC}$ & InChl=1S/C7H15O3/c1-2-3-4-5-7(6-8)10-9/h7-8H,2-6H2,1H3 \\
\hline 2796 & C7H131-3 & $\mathrm{C}=\mathrm{C}[\mathrm{CH}] \mathrm{CCCC}$ & $\operatorname{lnChl}=1 \mathrm{~S} / \mathrm{C} 7 \mathrm{H} 13 / \mathrm{c} 1-3-5-7-6-4-2 / \mathrm{h} 3,5 \mathrm{H}, 1,4,6-7 \mathrm{H} 2,2 \mathrm{H} 3$ \\
\hline 2797 & $\mathrm{HOC} 6 \mathrm{H} 4 \mathrm{CO}$ & $C 1=C C([C]=O)=C C(O)=C 1$ & InChl=1S/C7H5O2/c8-5-6-2-1-3-7(9)4-6/h1-4,9H \\
\hline 2798 & $\mathrm{HOC} 6 \mathrm{H} 4 \mathrm{CH} 2 \mathrm{OO}$ & $\mathrm{C} 1=\mathrm{CC}(\mathrm{CO}[0])=\mathrm{CC}(\mathrm{O})=\mathrm{C} 1$ & $\mathrm{InChl}=1 \mathrm{~S} / \mathrm{C} 7 \mathrm{H} 7 \mathrm{O} / \mathrm{c} 8-7-3-1-2-6(4-7) 5-10-9 / \mathrm{h} 1-4,8 \mathrm{H}, 5 \mathrm{H} 2$ \\
\hline 2799 & $\mathrm{C} 7 \mathrm{H} 14 \mathrm{OH}-2 \mathrm{O} 2-1$ & $\mathrm{C}(\mathrm{O}[\mathrm{O}]) \mathrm{C}(\mathrm{O}) \mathrm{cCcCC}$ & InChl=1S/C7H15O3/c1-2-3-4-5-7(8)6-10-9/h7-8H,2-6H2,1H3 \\
\hline 2800 & C7H131-4 & $\mathrm{C}=\mathrm{CC}[\mathrm{CH}] \mathrm{CCC}$ & InChl=1S/C7H13/c1-3-5-7-6-4-2/h3,7H,1,4-6H2,2H3 \\
\hline 2801 & $\mathrm{HOC} 6 \mathrm{H} 4 \mathrm{CHO}$ & $\mathrm{C} 1=\mathrm{CC}(\mathrm{C}=\mathrm{O})=\mathrm{CC}(\mathrm{O})=\mathrm{C} 1$ & InChl=15/C7H6O2/c8-5-6-2-1-3-7(9)4-6/h1-5,9H \\
\hline 2802 & C6H5OCH2OOH & $\mathrm{OOCOC} 1=\mathrm{CC}=\mathrm{CC}=\mathrm{C} 1$ & $\operatorname{lnChl}=15 / \mathrm{C} 7 \mathrm{H} 803 / \mathrm{c} 8-10-6-9-7-4-2-1-3-5-7 / \mathrm{h} 1-5,8 \mathrm{H}, 6 \mathrm{H} 2$ \\
\hline 2803 & C7H14OH-2O2-3 & $\mathrm{CC}(\mathrm{O}) \mathrm{C}(\mathrm{O}[\mathrm{O}]) \mathrm{CCCC}$ & InChl=15/C7H1503/c1-3-4-5-7(10-9)6(2)8/h6-8H,3-5H2,1-2H3 \\
\hline 2804 & C7H131-5 & $\mathrm{C}=\mathrm{CCC}[\mathrm{CH}] \mathrm{CC}$ & InChl=1S/C7H13/c1-3-5-7-6-4-2/h3,6H,1,4-5,7H2,2H3 \\
\hline 2805 & $\mathrm{HOC} 6 \mathrm{H} 4 \mathrm{OCH} 2$ & {$[\mathrm{CH} 2] \mathrm{Oc} 1 \mathrm{c}(\mathrm{O}) \mathrm{cccc} 1$} & $\operatorname{lnChl}=1 \mathrm{~S} / \mathrm{C} 7 \mathrm{H} 702 / \mathrm{c1}-9-7-5-3-2-4-6(7) 8 / \mathrm{h} 2-5,8 \mathrm{H}, 1 \mathrm{H} 2$ \\
\hline 2806 & $\mathrm{HOC} 6 \mathrm{H} 4 \mathrm{CH} 2 \mathrm{OOH}$ & $\mathrm{C} 1=\mathrm{CC}(\mathrm{COO})=\mathrm{CC}(\mathrm{O})=\mathrm{C} 1$ & $\mathrm{InChl}=1 \mathrm{~S} / \mathrm{C} 7 \mathrm{H} 8 \mathrm{O} 3 / \mathrm{c} 8-7-3-1-2-6(4-7) 5-10-9 / \mathrm{h} 1-4,8-9 \mathrm{H}, 5 \mathrm{H} 2$ \\
\hline 2807 & $\mathrm{C} 7 \mathrm{H} 14 \mathrm{OH}-3 \mathrm{O} 2-2$ & $\mathrm{CC}(\mathrm{O}[\mathrm{O}]) \mathrm{C}(\mathrm{O}) \mathrm{CCCC}$ & InChl=15/C7H15O3/c1-3-4-5-7(8)6(2)10-9/h6-8H,3-5H2,1-2H3 \\
\hline 2808 & C7H131-6 & $\mathrm{C}=\mathrm{CCCC}[\mathrm{CH}] \mathrm{C}$ & $\operatorname{lnChl}=1 \mathrm{~S} / \mathrm{C} 7 \mathrm{H} 13 / \mathrm{c1} 1-3-5-7-6-4-2 / \mathrm{h} 3-4 \mathrm{H}, 1,5-7 \mathrm{H} 2,2 \mathrm{H} 3$ \\
\hline 2809 & $\mathrm{OC} 6 \mathrm{H} 4 \mathrm{OCH} 3$ & $\operatorname{COc1c([0])\operatorname {cccc}1}$ & InChl=1S/C7H7O2/c1-9-7-5-3-2-4-6(7)8/h2-5H,1H3 \\
\hline 2810 & C7KET1-1-2 & $\mathrm{O}=\mathrm{CC}(\mathrm{COO}) \mathrm{CCCC}$ & InChl=1S/C7H14O3/c1-2-3-4-7(5-8)6-10-9/h5,7,9H,2-4,6H2,1H3 \\
\hline 2811 & $\mathrm{C} 7 \mathrm{H} 14 \mathrm{OH}-3 \mathrm{O} 2-4$ & $\operatorname{ccc}(\mathrm{O}) \mathrm{C}(\mathrm{O}[\mathrm{O}]) \mathrm{CCC}$ & InChl=15/C7H15O3/c1-3-5-7(10-9)6(8)4-2/h6-8H,3-5H2,1-2H3 \\
\hline 2812 & C7H131-7 & $\mathrm{C}=\mathrm{CCCCC}[\mathrm{CH} 2]$ & InChl=1S/C7H13/c1-3-5-7-6-4-2/h3H,1-2,4-7H2 \\
\hline 2813 & HOC6H4CYCH $2 \mathrm{O}$ & $\mathrm{OC} 1=\mathrm{C}[\mathrm{CH}] \mathrm{C}=\mathrm{CC} 12 \mathrm{CO} 2$ & InChl=1S/C7H7O2/c8-6-3-1-2-4-7(6)5-9-7/h1-4,8H,5H2 \\
\hline 2814 & C7KET1-2-2 & $\mathrm{O}=\mathrm{CC}(\mathrm{C})(\mathrm{OO}) \mathrm{CCCC}$ & InChl=15/C7H14O3/c1-3-4-5-7(2,6-8)10-9/h6,9H,3-5H2,1-2H3 \\
\hline 2815 & C7H14OH-4O2-3 & $\mathrm{CCC}(\mathrm{O}[\mathrm{O}]) \mathrm{C}(\mathrm{O}) \mathrm{CCC}$ & InChl=15/C7H1503/c1-3-5-6(8)7(4-2)10-9/h6-8H,3-5H2,1-2H3 \\
\hline 2816 & $\begin{array}{cc}\mathrm{CH} 132-4 \\
\end{array}$ & $\mathrm{CC}=\mathrm{C}[\mathrm{CH}] \mathrm{CCC}$ & InChl=1S/C7H13/c1-3-5-7-6-4-2/h3,5,7H,4,6H2,1-2H3 \\
\hline 2817 & E25CJOF & $\mathrm{CCC} 1=\mathrm{CC}=\mathrm{C}([\mathrm{C}]=0) 01$ & InChl=15/C7H702/c1-2-6-3-4-7(5-8)9-6/h3-4H,2H2,1H3 \\
\hline
\end{tabular}




\begin{tabular}{|c|c|c|c|}
\hline 2818 & C7KET1-3-2 & $\mathrm{O}=\mathrm{CC}(\mathrm{C}) \mathrm{C}(\mathrm{OO}) \mathrm{CCC}$ & InChl=1S/C7H14O3/c1-3-4-7(10-9)6(2)5-8/h5-7,9H,3-4H2,1-2H3 \\
\hline 2819 & 124C7OH1-2O2R & $\mathrm{OCC}(\mathrm{O}[\mathrm{O}])(\mathrm{C}) \mathrm{CC}(\mathrm{C}) \mathrm{C}$ & InChl=1S/C7H15O3/c1-6(2)4-7(3,5-8)10-9/h6,8H,4-5H2,1-3H3 \\
\hline 2820 & C7H132-5 & $\mathrm{CC}=\mathrm{CC}[\mathrm{CH}] \mathrm{CC}$ & InChl=1S/C7H13/c1-3-5-7-6-4-2/h3,5-6H,4,7H2,1-2H3 \\
\hline 2821 & E2JF5CHO-A & $\mathrm{C}[\mathrm{CH}] \mathrm{C} 1=\mathrm{CC}=\mathrm{C}(\mathrm{C}=\mathrm{O}) \mathrm{O} 1$ & InChl=1S/C7H7O2/c1-2-6-3-4-7(5-8)9-6/h2-5H,1H3 \\
\hline 2822 & C7KET1-4-2 & $\mathrm{O}=\mathrm{CC}(\mathrm{C}) \mathrm{CC}(\mathrm{OO}) \mathrm{CC}$ & InChl=1S/C7H14O3/c1-3-7(10-9)4-6(2)5-8/h5-7,9H,3-4H2,1-2H3 \\
\hline 2823 & 124C7OH2-102R & {$[\mathrm{O}] \mathrm{OCC}(\mathrm{O})(\mathrm{C}) \mathrm{CC}(\mathrm{C}) \mathrm{C}$} & InChl=15/C7H15O3/c1-6(2)4-7(3,8)5-10-9/h6,8H,4-5H2,1-3H3 \\
\hline 2824 & C7H132-6 & $\mathrm{CC}=\mathrm{CCC}[\mathrm{CH}] \mathrm{C}$ & $\operatorname{lnChl}=1 \mathrm{~S} / \mathrm{C} 7 \mathrm{H} 13 / \mathrm{c} 1-3-5-7-6-4-2 / \mathrm{h} 3-5 \mathrm{H}, 6-7 \mathrm{H} 2,1-2 \mathrm{H} 3$ \\
\hline 2825 & $\mathrm{C} 6 \mathrm{H} 5 \mathrm{CH} 2 \mathrm{OO}$ & $\mathrm{c1c}(\mathrm{CO}[0]) \operatorname{cccc} 1$ & InChl=1S/C7H7O2/c8-9-6-7-4-2-1-3-5-7/h1-5H,6H2 \\
\hline 2826 & C7KET1-5-2 & $\mathrm{O}=\mathrm{CC}(\mathrm{C}) \mathrm{CCC}(\mathrm{OO}) \mathrm{C}$ & $\operatorname{lnChl}=1 \mathrm{~S} / \mathrm{C} 7 \mathrm{H} 14 \mathrm{O} / \mathrm{c} 1-6(5-8) 3-4-7(2) 10-9 / \mathrm{h} 5-7,9 \mathrm{H}, 3-4 \mathrm{H} 2,1-2 \mathrm{H3}$ \\
\hline 2827 & 124C7OH2-302R & $\mathrm{CC}(\mathrm{O})(\mathrm{C}) \mathrm{C}(\mathrm{O}[\mathrm{O}]) \mathrm{C}(\mathrm{C}) \mathrm{C}$ & InChl=15/C7H15O3/c1-5(2)6(10-9)7(3,4)8/h5-6,8H,1-4H3 \\
\hline 2828 & C7H132-7 & $\mathrm{CC}=\mathrm{CCCC}[\mathrm{CH} 2]$ & InChl=1S/C7H13/c1-3-5-7-6-4-2/h4,6H,1,3,5,7H2,2H3 \\
\hline 2829 & $\mathrm{HOC} 6 \mathrm{H} 4 \mathrm{CH} 2 \mathrm{O}$ & {$[0] \operatorname{cc1} 1 \mathrm{ccc}(0) \mathrm{ccc} 1$} & InChl=1S/C7H7O2/c8-5-6-1-3-7(9)4-2-6/h1-4,9H,5H2 \\
\hline 2830 & C7KET3-1-2 & $\operatorname{Oocc}(\mathrm{C}) \mathrm{C}(=0) \mathrm{CCC}$ & InChl=1S/C7H14O3/c1-3-4-7(8)6(2)5-10-9/h6,9H,3-5H2,1-2H3 \\
\hline 2831 & 124C7OH3-2O2R & $\mathrm{CC}(\mathrm{O}[\mathrm{O}])(\mathrm{C}) \mathrm{C}(\mathrm{O}) \mathrm{C}(\mathrm{C}) \mathrm{C}$ & InChl=1S/C7H15O3/c1-5(2)6(8)7(3,4)10-9/h5-6,8H,1-4H3 \\
\hline 2832 & C7H133-1 & {$[\mathrm{CH} 2] \mathrm{CC}=\mathrm{CCCC}$} & InChl=1S/C7H13/c1-3-5-7-6-4-2/h5,7H,1,3-4,6H2,2H3 \\
\hline 2833 & $\mathrm{O}-\mathrm{O} 2 \mathrm{C} 6 \mathrm{H} 4 \mathrm{CH} 3$ & $\mathrm{C} 1(\mathrm{C})=\mathrm{C}(\mathrm{O}[\mathrm{O}]) \mathrm{C}=\mathrm{CC}=\mathrm{C} 1$ & InChl=1S/C7H7O2/c1-6-4-2-3-5-7(6)9-8/h2-5H,1H3 \\
\hline 2834 & C7KET3-2-2 & $\mathrm{CC}(\mathrm{C})(\mathrm{OO}) \mathrm{C}(=0) \mathrm{CCC}$ & InChl=1S/C7H14O3/c1-4-5-6(8)7(2,3)10-9/h9H,4-5H2,1-3H3 \\
\hline 2835 & NEC7OH3-4O2R & $\mathrm{CC}(\mathrm{C})(\mathrm{C}) \mathrm{C}(\mathrm{O}) \mathrm{C}(\mathrm{O}[\mathrm{O}]) \mathrm{C}$ & InChl=1S/C7H15O3/c1-5(10-9)6(8)7(2,3)4/h5-6,8H,1-4H3 \\
\hline 2836 & $\begin{array}{lll}\text { C7H133-5 } \\
\end{array}$ & $\mathrm{CCC}=\mathrm{C}[\mathrm{CH}] \mathrm{CC}$ & $\operatorname{lnChl}=1 \mathrm{~S} / \mathrm{C} 7 \mathrm{H} 13 / \mathrm{c} 1-3-5-7-6-4-2 / \mathrm{h} 5-7 \mathrm{H}, 3-4 \mathrm{H} 2,1-2 \mathrm{H} 3$ \\
\hline 2837 & $\mathrm{P}-\mathrm{O} 2 \mathrm{C} 6 \mathrm{H} 4 \mathrm{CH} 3$ & {$[0] \mathrm{OC} 1=\mathrm{CC}=\mathrm{C}(\mathrm{C}) \mathrm{C}=\mathrm{C} 1$} & InChl=1S/C7H7O2/c1-6-2-4-7(9-8)5-3-6/h2-5H,1H3 \\
\hline 2838 & C7KET3-4-2 & $\mathrm{CC}(\mathrm{C}) \mathrm{C}(=0) \mathrm{C}(\mathrm{OO}) \mathrm{CC}$ & InChl=1S/C7H14O3/c1-4-6(10-9)7(8)5(2)3/h5-6,9H,4H2,1-3H3 \\
\hline 2839 & NEC7OH4-302R & $\operatorname{CC}(\mathrm{C})(\mathrm{C}) \mathrm{C}(\mathrm{O}[\mathrm{O}]) \mathrm{C}(\mathrm{O}) \mathrm{C}$ & InChl=1S/C7H15O3/c1-5(8)6(10-9)7(2,3)4/h5-6,8H,1-4H3 \\
\hline 2840 & C7H133-6 & $\mathrm{CCC}=\mathrm{CC}[\mathrm{CH}] \mathrm{C}$ & InChl=1S/C7H13/c1-3-5-7-6-4-2/h3,6-7H,4-5H2,1-2H3 \\
\hline 2841 & $\mathrm{M}-\mathrm{O} 2 \mathrm{C} 6 \mathrm{H} 4 \mathrm{CH} 3$ & {$[0] \mathrm{OC} 1=\mathrm{CC}(\mathrm{C})=\mathrm{CC}=\mathrm{C} 1$} & $\operatorname{lnChl}=15 / C 7 H 7 O 2 / c 1-6-3-2-4-7(5-6) 9-8 / \mathrm{h} 2-5 \mathrm{H}, 1 \mathrm{H} 3$ \\
\hline 2842 & C7KET3-5-2 & $\mathrm{CC}(\mathrm{C}) \mathrm{C}(=\mathrm{O}) \mathrm{CC}(\mathrm{OO}) \mathrm{C}$ & InChl=1S/C7H14O3/c1-5(2)7(8)4-6(3)10-9/h5-6,9H,4H2,1-3H3 \\
\hline 2843 & NEC7OH4-5O2R & $\mathrm{CC}(\mathrm{C})(\mathrm{C}) \mathrm{CC}(\mathrm{O}) \mathrm{CO}[\mathrm{O}]$ & InChl=1S/C7H15O3/c1-7(2,3)4-6(8)5-10-9/h6,8H,4-5H2,1-3H3 \\
\hline 2844 & C7H133-7 & $\mathrm{CCC}=\mathrm{CCC}[\mathrm{CH} 2]$ & InChl=1S/C7H13/c1-3-5-7-6-4-2/h6-7H,1,3-5H2,2H3 \\
\hline 2845 & СНОССН3С 3Н3СО1-5 & $\mathrm{O}=[\mathrm{C}] / \mathrm{C}=\mathrm{C} / \mathrm{C}=\mathrm{C}(\mathrm{C}) / \mathrm{C}=\mathrm{O}$ & InChl=1S/C7H7O2/c1-7(6-9)4-2-3-5-8/h2-4,6H,1H3 \\
\hline 2846 & C7KET3-6-2 & $\mathrm{CC}(\mathrm{C}) \mathrm{C}(=0) \mathrm{CCCOO}$ & InChl=1S/C7H14O3/c1-6(2)7(8)4-3-5-10-9/h6,9H,3-5H2,1-2H3 \\
\hline 2847 & NEC7OH5-4O2R & $\mathrm{CC}(\mathrm{C})(\mathrm{C}) \mathrm{CC}(\mathrm{O}[\mathrm{O}]) \mathrm{CO}$ & InChl=1S/C7H15O3/c1-7(2,3)4-6(5-8)10-9/h6,8H,4-5H2,1-3H3 \\
\hline 2848 & 124C7D1-3R & $\mathrm{C}=\mathrm{C}(\mathrm{C})[\mathrm{CH}] \mathrm{C}(\mathrm{C}) \mathrm{C}$ & InChl=1S/C7H13/c1-6(2)5-7(3)4/h5,7H,1H2,2-4H3 \\
\hline 2849 & $\mathrm{HOC} 6 \mathrm{H} 4 \mathrm{OCH} 3$ & $\operatorname{COc1c}(0) \operatorname{cccc} 1$ & InChl=1S/C7H8O2/c1-9-7-5-3-2-4-6(7)8/h2-5,8H,1H3 \\
\hline 2850 & C7KET4-1-2 & $\mathrm{OOCC}(\mathrm{C}) \mathrm{CC}(=0) \mathrm{CC}$ & InChl=15/C7H14O3/c1-3-7(8)4-6(2)5-10-9/h6,9H,3-5H2,1-2H3 \\
\hline 2851 & $\mathrm{HOC} 6 \mathrm{H} 4 \mathrm{OCH} 2 \mathrm{OO}$ & {$[0] \mathrm{OCOC} 1=\mathrm{CC}=\mathrm{CC}=\mathrm{C} 10$} & InChl=1S/C7H704/c8-6-3-1-2-4-7(6)10-5-11-9/h1-4,8H,5H2 \\
\hline 2852 & 124C7D2-4R & $\mathrm{CC}(\mathrm{C})=\mathrm{C}[\mathrm{C}](\mathrm{C}) \mathrm{C}$ & $\operatorname{lnChl}=1 \mathrm{~S} / \mathrm{C} 7 \mathrm{H} 13 / \mathrm{c} 1-6(2) 5-7(3) 4 / \mathrm{h} 5 \mathrm{H}, 1-4 \mathrm{H} 3$ \\
\hline 2853 & E25CHOF & $\mathrm{CCC} 1=\mathrm{CC}=\mathrm{C}(\mathrm{C}=0) 01$ & InChl=1S/C7H8O2/c1-2-6-3-4-7(5-8)9-6/h3-5H,2H2,1H3 \\
\hline 2854 & C7KET4-2-2 & $\mathrm{CC}(\mathrm{C})(\mathrm{OO}) \mathrm{CC}(=0) \mathrm{CC}$ & InChl=1S/C7H14O3/c1-4-6(8)5-7(2,3)10-9/h9H,4-5H2,1-3H3 \\
\hline 2855 & $\mathrm{HOC} 6 \mathrm{H} 4 \mathrm{OCH} 2 \mathrm{OOH}$ & $\mathrm{OOCOC} 1=\mathrm{CC}=\mathrm{CC}=\mathrm{C} 10$ & InChl=1S/C7H8O4/c8-6-3-1-2-4-7(6)10-5-11-9/h1-4,8-9H,5H2 \\
\hline 2856 & I24C7D1-4R & $\mathrm{C}=\mathrm{C}(\mathrm{C}) \mathrm{C}[\mathrm{C}](\mathrm{C}) \mathrm{C}$ & InChl=1S/C7H13/c1-6(2)5-7(3)4/h1,5H2,2-4H3 \\
\hline 2857 & СНОССН3СЗ 3 З & $\mathrm{O}=\mathrm{CC}=\mathrm{CC}=\mathrm{C}(\mathrm{C}=\mathrm{O}) \mathrm{C}$ & $\operatorname{lnChl}=1 \mathrm{~S} / \mathrm{C} 7 \mathrm{H} 8 \mathrm{O} 2 / \mathrm{c1}-7(6-9) 4-2-3-5-8 / \mathrm{h} 2-6 \mathrm{H}, 1 \mathrm{H} 3$ \\
\hline 2858 & C7KET4-3-2 & $\mathrm{CC}(\mathrm{C}) \mathrm{C}(\mathrm{OO}) \mathrm{C}(=\mathrm{O}) \mathrm{CC}$ & $\operatorname{lnChl}=1 \mathrm{~S} / \mathrm{C} 7 \mathrm{H} 14 \mathrm{O} 3 / \mathrm{c1}-4-6(8) 7(10-9) 5(2) 3 / \mathrm{h} 5,7,9 \mathrm{H}, 4 \mathrm{H} 2,1-3 \mathrm{H} 3$ \\
\hline 2859 & C7OOH1-0O1-2 & OOCC(CO[0])CCCC & InChl=1S/C7H15O4/c1-2-3-4-7(5-10-8)6-11-9/h7-8H,2-6H2,1H3 \\
\hline 2860 & NEC7D4-4R & $\mathrm{CC}(\mathrm{C})(\mathrm{C}) \mathrm{C}[\mathrm{C}]=\mathrm{C}$ & $\operatorname{lnChl}=1 \mathrm{~S} / \mathrm{C} 7 \mathrm{H} 13 / \mathrm{cc} 1-5-6-7(2,3) 4 / \mathrm{h} 1,6 \mathrm{H} 2,2-4 \mathrm{H} 3$ \\
\hline 2861 & $\mathrm{BZCOOH}$ & c1c(COO)cccc1 & InChl=1S/C7H8O2/c8-9-6-7-4-2-1-3-5-7/h1-5,8H,6H2 \\
\hline 2862 & C7KET4-5-2 & $\mathrm{CC}(\mathrm{C}) \mathrm{CC}(=0) \mathrm{C}(\mathrm{OO}) \mathrm{C}$ & InChl=1S/C7H14O3/c1-5(2)4-7(8)6(3)10-9/h5-6,9H,4H2,1-3H3 \\
\hline 2863 & $\mathrm{C} 7 \mathrm{OOH} 1-0 \mathrm{OO2-2}$ & $\mathrm{OOCC}(\mathrm{C})(\mathrm{O}[\mathrm{O}]) \mathrm{CCCC}$ & InChl=1S/C7H15O4/c1-3-4-5-7(2,11-9)6-10-8/h8H,3-6H2,1-2H3 \\
\hline 2864 & NEC7D4-3R & $\mathrm{CC}(\mathrm{C})(\mathrm{C}) \mathrm{C}=\mathrm{C}[\mathrm{CH} 2]$ & InChl=1S/C7H13/c1-5-6-7(2,3)4/h5-6H,1H2,2-4H3 \\
\hline 2865 & HM25EFOJ & $\mathrm{CCC} 1=\mathrm{CC}=\mathrm{C}(\mathrm{C}[\mathrm{O}]) \mathrm{O} 1$ & InChl=1S/C7H9O2/c1-2-6-3-4-7(5-8)9-6/h3-4H,2,5H2,1H3 \\
\hline 2866 & C7KET4-6-2 & $\mathrm{CC}(\mathrm{C}) \mathrm{CC}(=0) \mathrm{CCOO}$ & InChl=1S/C7H14O3/c1-6(2)5-7(8)3-4-10-9/h6,9H,3-5H2,1-2H3 \\
\hline 2867 & C7OOH1-OO3-2 & $\mathrm{OOCC}(\mathrm{C}) \mathrm{C}(\mathrm{O}[\mathrm{O}]) \mathrm{CCC}$ & InChl=1S/C7H1504/c1-3-4-7(11-9)6(2)5-10-8/h6-8H,3-5H2,1-2H3 \\
\hline 2868 & NEC7D3-4R & $\mathrm{CC}(\mathrm{C})(\mathrm{C}) \mathrm{C}=[\mathrm{C}] \mathrm{C}$ & InChl=1S/C7H13/c1-5-6-7(2,3)4/h6H,1-4H3 \\
\hline 2869 & C7Y24 & $\mathrm{CC}(=0) \mathrm{CC}(=0) \mathrm{CCC}$ & InChl=1S/C7H12O2/c1-3-4-7(9)5-6(2)8/h3-5H2,1-2H3 \\
\hline 2870 & C7KET5-1-2 & $\mathrm{OOCC}(\mathrm{C}) \mathrm{CCC}(=0) \mathrm{C}$ & InChl=1S/C7H14O3/c1-6(5-10-9)3-4-7(2)8/h6,9H,3-5H2,1-2H3 \\
\hline 2871 & C7OOH1-0O4-2 & $\mathrm{OOCC}(\mathrm{C}) \mathrm{CC}(\mathrm{O}[\mathrm{O}]) \mathrm{CC}$ & InChl=1S/C7H1504/c1-3-7(11-9)4-6(2)5-10-8/h6-8H,3-5H2,1-2H3 \\
\hline 2872 & 124C7D1-5R & $\mathrm{C}=\mathrm{C}(\mathrm{C}) \mathrm{CC}(\mathrm{C})[\mathrm{CH} 2]$ & InChl=1S/C7H13/c1-6(2)5-7(3)4/h6H,1,3,5H2,2,4H3 \\
\hline 2873 & C7Y13 & $\mathrm{C}(=0) \mathrm{CC}(=0) \mathrm{CCCC}$ & $\operatorname{lnChl}=1 \mathrm{~S} / \mathrm{C} 7 \mathrm{H} 12 \mathrm{O} 2 / \mathrm{c1}-2-3-4-7(9) 5-6-8 / \mathrm{h} 6 \mathrm{H}, 2-5 \mathrm{H} 2,1 \mathrm{H} 3$ \\
\hline 2874 & C7KET5-2-2 & $\operatorname{CC}(\mathrm{C})(\mathrm{OO}) \mathrm{CCC}(=\mathrm{O}) \mathrm{C}$ & InChl=1S/C7H1403/c1-6(8)4-5-7(2,3)10-9/h9H,4-5H2,1-3H3 \\
\hline 2875 & $\mathrm{C} 70 \mathrm{OH} 1-005-2$ & $\mathrm{OOCC}(\mathrm{C}) \operatorname{ccc}(\mathrm{O}[\mathrm{O}]) \mathrm{C}$ & InChl=1S/C7H1504/c1-6(5-10-8)3-4-7(2)11-9/h6-8H,3-5H2,1-2H3 \\
\hline 2876 & 124C7D2-5R & $\mathrm{CC}(\mathrm{C})=\mathrm{CC}(\mathrm{C})[\mathrm{CH} 2]$ & $\operatorname{lnChl}=1 \mathrm{~S} / \mathrm{C} 7 \mathrm{H} 13 / \mathrm{c} 1-6(2) 5-7(3) 4 / \mathrm{h} 5-6 \mathrm{H}, 1 \mathrm{H} 2,2-4 \mathrm{H} 3$ \\
\hline 2877 & C7Y35 & $\operatorname{cCC}(=0) C C(=0) C C$ & InChl=1S/C7H12O2/c1-3-6(8)5-7(9)4-2/h3-5H2,1-2H3 \\
\hline 2878 & C7KET5-3-2 & $\mathrm{CC}(\mathrm{C}) \mathrm{C}(\mathrm{OO}) \mathrm{CC}(=\mathrm{O}) \mathrm{C}$ & InChl=1S/C7H14O3/c1-5(2)7(10-9)4-6(3)8/h5,7,9H,4H2,1-3H3 \\
\hline 2879 & $\mathrm{C} 7 \mathrm{OOH} 2-\mathrm{OO1}-2$ & {$[\mathrm{O}] \mathrm{OCC}(\mathrm{C})(\mathrm{OO}) \mathrm{CcCC}$} & InChl=1S/C7H15O4/c1-3-4-5-7(2,11-9)6-10-8/h9H,3-6H2,1-2H3 \\
\hline 2880 & NEC7D3-1R & {$[\mathrm{CH} 2] \mathrm{C}(\mathrm{C})(\mathrm{C}) \mathrm{C}=\mathrm{CC}$} & InChl=1S/C7H13/c1-5-6-7(2,3)4/h5-6H,2H2,1,3-4H3 \\
\hline 2881 & C7KET12O & $\mathrm{C}(=\mathrm{O}) \mathrm{C}([\mathrm{O}]) \mathrm{CCCCC}$ & InChl=1S/C7H13O2/c1-2-3-4-5-7(9)6-8/h6-7H,2-5H2,1H3 \\
\hline 2882 & C7KET5-4-2 & $\mathrm{CC}(\mathrm{C}) \mathrm{CC}(\mathrm{OO}) \mathrm{C}(=\mathrm{O}) \mathrm{C}$ & InChl=1S/C7H14O3/c1-5(2)4-7(10-9)6(3)8/h5,7,9H,4H2,1-3H3 \\
\hline 2883 & $\mathrm{C} 7 \mathrm{OOH} 2-0 \mathrm{O}-2$ & $\mathrm{CC}(\mathrm{C})(\mathrm{OO}) \mathrm{C}(\mathrm{O}[\mathrm{O}]) \mathrm{CCC}$ & InChl=1S/C7H1504/c1-4-5-6(10-8)7(2,3)11-9/h6,9H,4-5H2,1-3H3 \\
\hline 2884 & NEC7D4-1R & {$[\mathrm{CH} 2] \mathrm{C}(\mathrm{C})(\mathrm{C}) \mathrm{CC}=\mathrm{C}$} & $\operatorname{lnChl}=1 \mathrm{~S} / \mathrm{C} 7 \mathrm{H} 13 / \mathrm{c} 1-5-6-7(2,3) 4 / \mathrm{h} 5 \mathrm{H}, 1-2,6 \mathrm{H} 2,3-4 \mathrm{H} 3$ \\
\hline 2885 & C7KET13O & $\mathrm{C}(=0) \mathrm{CC}([0]) \mathrm{CCCC}$ & InChl=1S/C7H13O2/c1-2-3-4-7(9)5-6-8/h6-7H,2-5H2,1H3 \\
\hline 2886 & C7KET5-6-2 & $\mathrm{CC}(\mathrm{C}) \mathrm{CCC}(=\mathrm{O}) \mathrm{COO}$ & InChl=1S/C7H14O3/c1-6(2)3-4-7(8)5-10-9/h6,9H,3-5H2,1-2H3 \\
\hline 2887 & $\mathrm{C} 7 \mathrm{OOH} 2-0 \mathrm{OO}-2$ & $\mathrm{CC}(\mathrm{C})(\mathrm{OO}) \mathrm{CC}(\mathrm{O}[\mathrm{O}]) \mathrm{CC}$ & InChl=1S/C7H1504/c1-4-6(10-8)5-7(2,3)11-9/h6,9H,4-5H2,1-3H3 \\
\hline 2888 & I24C7D1-1AR & $\mathrm{C}=\mathrm{C}([\mathrm{CH} 2]) \mathrm{CC}(\mathrm{C}) \mathrm{C}$ & InChl=1S/C7H13/c1-6(2)5-7(3)4/h7H,1-2,5H2,3-4H3 \\
\hline 2889 & C7KET14O & $\mathrm{C}(=0) \operatorname{ccC}([0]) \mathrm{CCC}$ & InChl=1S/C7H13O2/c1-2-4-7(9)5-3-6-8/h6-7H,2-5H2,1H3 \\
\hline 2890 & C7KET6-2-2 & $\mathrm{CC}(\mathrm{C})(\mathrm{OO}) \mathrm{CCCC}=\mathrm{O}$ & InChl=1S/C7H14O3/c1-7(2,10-9)5-3-4-6-8/h6,9H,3-5H2,1-2H3 \\
\hline 2891 & $\mathrm{C} 7 \mathrm{OOH} 2-0 \mathrm{OO}-2$ & $\mathrm{CC}(\mathrm{C})(\mathrm{OO}) \mathrm{CCC}(\mathrm{O}[\mathrm{O}]) \mathrm{C}$ & InChl=1S/C7H15O4/c1-6(10-8)4-5-7(2,3)11-9/h6,9H,4-5H2,1-3H3 \\
\hline 2892 & C7H14-1-2 & $\mathrm{C}=\mathrm{C}(\mathrm{C}) \mathrm{CCCC}$ & $\operatorname{lnChl}=15 / C 7 H 14 / c 1-4-5-6-7(2) 3 / h 2,4-6 \mathrm{H} 2,1,3 \mathrm{H} 3$ \\
\hline 2893 & C7KET150 & $\mathrm{C}(=\mathrm{O}) \operatorname{cccc}([0]) \mathrm{CC}$ & $\operatorname{lnChl}=1 \mathrm{~S} / \mathrm{C} 7 \mathrm{H} 13 \mathrm{O} 2 / \mathrm{c} 1-2-7(9) 5-3-4-6-8 / \mathrm{h} 6-7 \mathrm{H}, 2-5 \mathrm{H} 2,1 \mathrm{H} 3$ \\
\hline 2894 & C7KET6-3-2 & $\mathrm{CC}(\mathrm{C}) \mathrm{C}(\mathrm{OO}) \mathrm{CCC}=\mathrm{O}$ & InChl=1S/C7H14O3/c1-6(2)7(10-9)4-3-5-8/h5-7,9H,3-4H2,1-2H3 \\
\hline 2895 & $\mathrm{C} 7 \mathrm{OOH} 2-0 \mathrm{O} 6-2$ & $\mathrm{CC}(\mathrm{C})(\mathrm{OO}) \mathrm{CCCCO}[\mathrm{O}]$ & InChl=1S/C7H1504/c1-7(2,11-9)5-3-4-6-10-8/h9H,3-6H2,1-2H3 \\
\hline 2896 & C7H14-2-2 & $\mathrm{CC}(\mathrm{C})=\mathrm{CCCC}$ & InChl=1S/C7H14/c1-4-5-6-7(2)3/h6H,4-5H2,1-3H3 \\
\hline 2897 & C7KET210 & $C([0]) C(=0) C C C C C$ & $\operatorname{lnChl}=1 \mathrm{~S} / \mathrm{C} 7 \mathrm{H} 13 \mathrm{O} / \mathrm{c} 1-2-3-4-5-7(9) 6-8 / \mathrm{h} 2-6 \mathrm{H} 2,1 \mathrm{H} 3$ \\
\hline 2898 & C7KET6-4-2 & $\mathrm{CC}(\mathrm{C}) \mathrm{CC}(\mathrm{OO}) \mathrm{CC}=\mathrm{O}$ & $\mathrm{InChl}=1 \mathrm{~S} / \mathrm{C} 7 \mathrm{H} 14 \mathrm{OO} / \mathrm{c1}-6(2) 5-7(10-9) 3-4-8 / \mathrm{h} 4,6-7,9 \mathrm{H}, 3,5 \mathrm{H} 2,1-2 \mathrm{H} 3$ \\
\hline 2899 & С70OH3-001-2 & {$[\mathrm{O}] \mathrm{OCC}(\mathrm{C}) \mathrm{C}(\mathrm{OO}) \mathrm{CCC}$} & InChl=15/C7H15O4/c1-3-4-7(11-9)6(2)5-10-8/h6-7,9H,3-5H2,1-2H3 \\
\hline 2900 & C7H14-3-2 & $\mathrm{CC}(\mathrm{C}) \mathrm{C}=\mathrm{CCC}$ & InChl=1S/C7H14/c1-4-5-6-7(2)3/h5-7H,4H2,1-3H3 \\
\hline 2901 & C7KET23O & $\mathrm{CC}(=0) \mathrm{C}([0]) \mathrm{CCCC}$ & InChl=1S/C7H13O2/c1-3-4-5-7(9)6(2)8/h7H,3-5H2,1-2H3 \\
\hline 2902 & C7KET6-5-2 & $\mathrm{CC}(\mathrm{C}) \mathrm{CCC}(\mathrm{OO}) \mathrm{C}=\mathrm{O}$ & InChl=1S/C7H14O3/c1-6(2)3-4-7(5-8)10-9/h5-7,9H,3-4H2,1-2H3 \\
\hline 2903 & $\mathrm{C} 7 \mathrm{OOH} 3-\mathrm{OO2}-2$ & $\mathrm{CC}(\mathrm{C})(\mathrm{O}[\mathrm{O}]) \mathrm{C}(\mathrm{OO}) \mathrm{CCC}$ & InChl=1S/C7H1504/c1-4-5-6(10-8)7(2,3)11-9/h6,8H,4-5H2,1-3H3 \\
\hline 2904 & C7H14-4-2 & $\mathrm{CC}(\mathrm{C}) \mathrm{CC}=\mathrm{CC}$ & InChl=1S/C7H14/c1-4-5-6-7(2)3/h4-5,7H,6H2,1-3H3 \\
\hline 2905 & C7KET24O & $\mathrm{CC}(=0) \mathrm{CC}([0]) \mathrm{CCC}$ & InChl=1S/C7H13O2/c1-3-4-7(9)5-6(2)8/h7H,3-5H2,1-2H3 \\
\hline 2906 & $\mathrm{C} 701-10 \mathrm{OH} 2$ & $01 \mathrm{CC}(\mathrm{C} 1)(\mathrm{OO}) \mathrm{CCCC}$ & $\operatorname{lnChl}=1 \mathrm{~S} / \mathrm{C} 7 \mathrm{H} 14 \mathrm{O} / \mathrm{c} 1-2-3-4-7(10-8) 5-9-6-7 / \mathrm{h} 8 \mathrm{H}, 2-6 \mathrm{H} 2,1 \mathrm{H} 3$ \\
\hline 2907 & C7OOH3-0O4-2 & $\mathrm{CC}(\mathrm{C}) \mathrm{C}(\mathrm{OO}) \mathrm{C}(\mathrm{O}[\mathrm{O}]) \mathrm{CC}$ & InChl=1S/C7H1504/c1-4-6(10-8)7(11-9)5(2)3/h5-7,9H,4H2,1-3H3 \\
\hline 2908 & C7H14-5-2 & $\mathrm{CC}(\mathrm{C}) \mathrm{CCC}=\mathrm{C}$ & InChl=1S/C7H14/c1-4-5-6-7(2)3/h4,7H,1,5-6H2,2-3H3 \\
\hline
\end{tabular}




\begin{tabular}{|c|c|c|c|}
\hline 2909 & C7KET250 & $\mathrm{CC}(=\mathrm{O}) \mathrm{CCC}([\mathrm{O}]) \mathrm{CC}$ & $\ln \mathrm{Chl}=1 \mathrm{~S} / \mathrm{C} 7 \mathrm{H} 13 \mathrm{O} 2 / \mathrm{c1}-3-7(9) 5-4-6(2) 8 / \mathrm{h} 7 \mathrm{H}, 3-5 \mathrm{H} 2,1-2 \mathrm{H} 3$ \\
\hline 2910 & C7O2-30OH1 & $00 \mathrm{OCC}(\mathrm{C})(01) \mathrm{C} 1 \mathrm{CCC}$ & InChl=1S/C7H14O3/c1-3-4-6-7(2,10-6)5-9-8/h6,8H,3-5H2,1-2H3 \\
\hline 2911 & С7ООНЗ-005-2 & $\mathrm{CC}(\mathrm{C}) \mathrm{C}(\mathrm{OO}) \mathrm{CC}(\mathrm{O}[\mathrm{O}]) \mathrm{C}$ & InChl=1S/C7H1504/c1-5(2)7(11-9)4-6(3)10-8/h5-7,9H,4H2,1-3H3 \\
\hline 2912 & C7H14-1 & $\mathrm{C}=\mathrm{CCCCCC}$ & InChl=1S/C7H14/c1-3-5-7-6-4-2/h3H,1,4-7H2,2H3 \\
\hline 2913 & C7KET260 & $\mathrm{CC}(=0) \operatorname{CCCC}([0]) \mathrm{C}$ & InChl=15/C7H13O2/c1-6(8)4-3-5-7(2)9/h6H,3-5H2,1-2H3 \\
\hline 2914 & $\mathrm{C} 703-40 \mathrm{OH} 1$ & $\mathrm{OOCC}(\mathrm{C}) \mathrm{c}(\mathrm{O} 1) \mathrm{C} 1 \mathrm{CC}$ & $\operatorname{lnChl}=1 \mathrm{~S} / \mathrm{C} 7 \mathrm{H} 1403 / \mathrm{c} 1-3-6-7(10-6) 5(2) 4-9-8 / \mathrm{h} 5-8 \mathrm{H}, 3-4 \mathrm{H} 2,1-2 \mathrm{H} 3$ \\
\hline 2915 & $\mathrm{C} 70 \mathrm{OH} 3-006-2$ & $\mathrm{CC}(\mathrm{C}) \mathrm{C}(\mathrm{OO}) \mathrm{CCCO}[\mathrm{O}]$ & $\operatorname{lnChl}=15 / C 7 H 1504 / c 1-6(2) 7(11-9) 4-3-5-10-8 / h 6-7,9 \mathrm{H}, 3-5 \mathrm{H} 2,1-2 \mathrm{H} 3$ \\
\hline 2916 & C7H14-2 & $\mathrm{CC}=\mathrm{CCCCC}$ & $\operatorname{lnChl}=1 \mathrm{~S} / \mathrm{C} 7 \mathrm{H} 14 / \mathrm{c1} 1-3-5-7-6-4-2 / \mathrm{h} 3,5 \mathrm{H}, 4,6-7 \mathrm{H} 2,1-2 \mathrm{H} 3$ \\
\hline 2917 & C7KET310 & $\mathrm{C}([\mathrm{O}]) \mathrm{CC}(=\mathrm{O}) \mathrm{CCCC}$ & $\operatorname{lnChl}=1 \mathrm{~S} / \mathrm{C} 7 \mathrm{H} 13 \mathrm{O} 2 / \mathrm{c} 1-2-3-4-7(9) 5-6-8 / \mathrm{h} 2-6 \mathrm{H} 2,1 \mathrm{H} 3$ \\
\hline 2918 & $\mathrm{C} 7 \mathrm{O} 4-5 \mathrm{OOH} 2$ & $\mathrm{CC}(\mathrm{C})(\mathrm{OO}) \mathrm{CC}(\mathrm{O} 1) \mathrm{C} 1 \mathrm{C}$ & InChl=1S/C7H14O3/c1-5-6(9-5)4-7(2,3)10-8/h5-6,8H,4H2,1-3H3 \\
\hline 2919 & $\mathrm{C7OOH4-001-2}$ & {$[\mathrm{O}] \mathrm{OCC}(\mathrm{C}) \mathrm{CC}(\mathrm{OO}) \mathrm{CC}$} & $\ln \mathrm{Chl}=1 \mathrm{~S} / \mathrm{C} 7 \mathrm{H} 15 \mathrm{O} 4 / \mathrm{c} 1-3-7(11-9) 4-6(2) 5-10-8 / \mathrm{h} 6-7,9 \mathrm{H}, 3-5 \mathrm{H} 2,1-2 \mathrm{H} 3$ \\
\hline 2920 & $\mathrm{C} 7 \mathrm{H} 14-3$ & $\mathrm{CCC}=\mathrm{CCCC}$ & $\operatorname{lnChl}=1 \mathrm{~S} / \mathrm{C} 7 \mathrm{H} 14 / \mathrm{c1}-3-5-7-6-4-2 / \mathrm{h} 5,7 \mathrm{H}, 3-4,6 \mathrm{H} 2,1-2 \mathrm{H} 3$ \\
\hline 2921 & C7KET32O & $\operatorname{CC}([0]) C(=0) \operatorname{CcCC}$ & InChl=15/C7H13O2/c1-3-4-5-7(9)6(2)8/h6H,3-5H2,1-2H3 \\
\hline 2922 & $\mathrm{C} 7 \mathrm{O} 5-60 \mathrm{OH} 3$ & $\mathrm{CC}(\mathrm{C}) \mathrm{C}(\mathrm{OO}) \mathrm{CC}(\mathrm{O} 1) \mathrm{C} 1$ & InChl=1S/C7H14O3/c1-5(2)7(10-8)3-6-4-9-6/h5-8H,3-4H2,1-2H3 \\
\hline 2923 & $\mathrm{C} 7 \mathrm{OOH} 4-002-2$ & $\mathrm{CC}(\mathrm{C})(\mathrm{O}[\mathrm{O}]) \mathrm{CC}(\mathrm{OO}) \mathrm{CC}$ & InChl=1S/C7H15O4/c1-4-6(10-8)5-7(2,3)11-9/h6,8H,4-5H2,1-3H3 \\
\hline 2924 & 124C7D1 & $\mathrm{C}=\mathrm{C}(\mathrm{C}) \mathrm{CC}(\mathrm{C}) \mathrm{C}$ & InChl=1S/C7H14/c1-6(2)5-7(3)4/h7H,1,5H2,2-4H3 \\
\hline 2925 & C7KET34O & $\mathrm{CCC}(=0) \mathrm{C}([0]) \mathrm{CCC}$ & $\operatorname{lnChl}=15 / \mathrm{C} 7 \mathrm{H} 13 \mathrm{O} 2 / \mathrm{c} 1-3-5-7(9) 6(8) 4-2 / \mathrm{h} 7 \mathrm{H}, 3-5 \mathrm{H} 2,1-2 \mathrm{H} 3$ \\
\hline 2926 & $\mathrm{C} 701-10 \mathrm{OH} 3$ & $01 \mathrm{CC}(\mathrm{C} 1) \mathrm{C}(\mathrm{OO}) \mathrm{CCC}$ & $\operatorname{lnChl}=1 \mathrm{~S} / \mathrm{C} 7 \mathrm{H} 14 \mathrm{OO} / \mathrm{c1}-2-3-7(10-8) 6-4-9-5-6 / \mathrm{h} 6-8 \mathrm{H}, 2-5 \mathrm{H} 2,1 \mathrm{H} 3$ \\
\hline 2927 & $\mathrm{C} 7 \mathrm{OOH} 4-003-2$ & $\mathrm{CC}(\mathrm{C}) \mathrm{C}(\mathrm{O}[\mathrm{O}]) \mathrm{C}(\mathrm{OO}) \mathrm{CC}$ & InChl=1S/C7H1504/c1-4-6(10-8)7(11-9)5(2)3/h5-8H,4H2,1-3H3 \\
\hline 2928 & 124C7D2 & $\mathrm{CC}(\mathrm{C})=\mathrm{CC}(\mathrm{C}) \mathrm{C}$ & InChl=1S/C7H14/c1-6(2)5-7(3)4/h5-6H,1-4H3 \\
\hline 2929 & C7KET350 & $\mathrm{CCC}(=0) \mathrm{CC}([0]) \mathrm{CC}$ & InChl=1S/C7H13O2/c1-3-6(8)5-7(9)4-2/h6H,3-5H2,1-2H3 \\
\hline 2930 & $\mathrm{C} 7 \mathrm{O} 2-30 \mathrm{OH} 4$ & $\mathrm{CC}(\mathrm{C})(\mathrm{O} 1) \mathrm{C} 1 \mathrm{C}(00) \mathrm{CC}$ & InChl=1S/C7H14O3/c1-4-5(10-8)6-7(2,3)9-6/h5-6,8H,4H2,1-3H3 \\
\hline 2931 & $\mathrm{C} 70 \mathrm{OH} 4-005-2$ & $\mathrm{CC}(\mathrm{C}) \mathrm{CC}(\mathrm{OO}) \mathrm{C}(\mathrm{O}[0]) \mathrm{C}$ & InChl=1S/C7H1504/c1-5(2)4-7(11-9)6(3)10-8/h5-7,9H,4H2,1-3H3 \\
\hline 2932 & NEC7D3 & $\mathrm{CC}(\mathrm{C})(\mathrm{C}) \mathrm{C}=\mathrm{CC}$ & InChl=1S/C7H14/c1-5-6-7(2,3)4/h5-6H,1-4H3 \\
\hline 2933 & C7KET360 & $\operatorname{cCC}(=0) \operatorname{ccc}([0]) \mathrm{C}$ & InChl=15/C7H13O2/c1-3-7(9)5-4-6(2)8/h6H,3-5H2,1-2H3 \\
\hline 2934 & $\mathrm{C} 703-40 \mathrm{OH} 2$ & $\mathrm{CC}(\mathrm{C})(\mathrm{OO}) \mathrm{C}(\mathrm{O} 1) \mathrm{C} 1 \mathrm{CC}$ & InChI=15/C7H14O3/c1-4-5-6(9-5)7(2,3)10-8/h5-6,8H,4H2,1-3H3 \\
\hline 2935 & $\mathrm{C} 70 \mathrm{OH} 4-006-2$ & $\mathrm{CC}(\mathrm{C}) \mathrm{CC}(\mathrm{OO}) \mathrm{CCO}[\mathrm{O}]$ & $\operatorname{lnChl}=15 / \mathrm{C} 7 \mathrm{H} 15 \mathrm{O} 4 / \mathrm{c} 1-6(2) 5-7(11-9) 3-4-10-8 / \mathrm{h} 6-7,9 \mathrm{H}, 3-5 \mathrm{H} 2,1-2 \mathrm{H} 3$ \\
\hline 2936 & NEC7D4 & $\mathrm{CC}(\mathrm{C})(\mathrm{C}) \mathrm{CC}=\mathrm{C}$ & InChl=1S/C7H14/c1-5-6-7(2,3)4/h5H,1,6H2,2-4H3 \\
\hline 2937 & C7KET370 & $\operatorname{CCC}(=0) \operatorname{CCCC}([0])$ & InChl=1S/C7H13O2/c1-2-7(9)5-3-4-6-8/h2-6H2,1H3 \\
\hline 2938 & $\mathrm{C} 7 \mathrm{O} 4-50 \mathrm{OH} 3$ & $\mathrm{CC}(\mathrm{C}) \mathrm{C}(\mathrm{OO}) \mathrm{C}(\mathrm{O} 1) \mathrm{C} 1 \mathrm{C}$ & InChl=1S/C7H14O3/c1-4(2)6(10-8)7-5(3)9-7/h4-8H,1-3H3 \\
\hline 2939 & C70OH5-001-2 & {$[\mathrm{O}] \mathrm{OCC}(\mathrm{C}) \mathrm{CCC}(\mathrm{OO}) \mathrm{C}$} & $\operatorname{lnChl}=15 / C 7 H 15 O 4 / c 1-6(5-10-8) 3-4-7(2) 11-9 / h 6-7,9 H, 3-5 \mathrm{H} 2,1-2 \mathrm{H} 3$ \\
\hline 2940 & C7H15-2A & {$[\mathrm{CH} 2] \mathrm{C}(\mathrm{C}) \mathrm{CCCC}$} & InChl=1S/C7H15/c1-4-5-6-7(2)3/h7H,2,4-6H2,1,3H3 \\
\hline 2941 & C7KET410 & $\operatorname{CCCC}(=0) \operatorname{ccc}([0])$ & InChl=1S/C7H13O2/c1-2-4-7(9)5-3-6-8/h2-6H2,1H3 \\
\hline 2942 & $\mathrm{C} 7 \mathrm{O5}-60 \mathrm{OH} 4$ & $\mathrm{CC}(\mathrm{C}) \mathrm{CC}(\mathrm{OO}) \mathrm{C}(\mathrm{O} 1) \mathrm{C} 1$ & InChl=1S/C7H14O3/c1-5(2)3-6(10-8)7-4-9-7/h5-8H,3-4H2,1-2H3 \\
\hline 2943 & $\mathrm{C} 7 \mathrm{OOH} 5-002-2$ & $\mathrm{CC}(\mathrm{C})(\mathrm{O}[\mathrm{O}]) \mathrm{CCC}(\mathrm{OO}) \mathrm{C}$ & InChl=1S/C7H1504/c1-6(10-8)4-5-7(2,3)11-9/h6,8H,4-5H2,1-3H3 \\
\hline 2944 & C7H15-2B & $\mathrm{C}[\mathrm{C}](\mathrm{C}) \mathrm{CCCC}$ & InChl=1S/C7H15/c1-4-5-6-7(2)3/h4-6H2,1-3H3 \\
\hline 2945 & C7KET42O & $\operatorname{cccC}(=0) \operatorname{cc}([0]) C$ & InChl=15/C7H13O2/c1-3-4-7(9)5-6(2)8/h6H,3-5H2,1-2H3 \\
\hline 2946 & $\mathrm{C} 7 \mathrm{O} 1-10 \mathrm{OH} 4$ & $\mathrm{O} 1 \mathrm{CC}(\mathrm{C} 1) \mathrm{CC}(\mathrm{OO}) \mathrm{CC}$ & InChl=1S/C7H14O3/c1-2-7(10-8)3-6-4-9-5-6/h6-8H,2-5H2,1H3 \\
\hline 2947 & C7OOH5-003-2 & $\mathrm{CC}(\mathrm{C}) \mathrm{C}(\mathrm{O}[\mathrm{O}]) \mathrm{CC}(\mathrm{OO}) \mathrm{C}$ & InChl=1S/C7H1504/c1-5(2)7(11-9)4-6(3)10-8/h5-8H,4H2,1-3H3 \\
\hline 2948 & C7H15-2C & $\mathrm{CC}(\mathrm{C})[\mathrm{CH}] \mathrm{CCC}$ & $\operatorname{lnChl}=1 \mathrm{~S} / \mathrm{C} 7 \mathrm{H} 15 / \mathrm{c} 1-4-5-6-7(2) 3 / \mathrm{h} 6-7 \mathrm{H}, 4-5 \mathrm{H} 2,1-3 \mathrm{H} 3$ \\
\hline 2949 & C7KET43O & $\operatorname{CCCC}(=0) C([0]) C C$ & $\operatorname{lnChl}=15 / \mathrm{C} 7 \mathrm{H} 13 \mathrm{O} 2 / \mathrm{c} 1-3-5-7(9) 6(8) 4-2 / \mathrm{h} 6 \mathrm{H}, 3-5 \mathrm{H} 2,1-2 \mathrm{H} 3$ \\
\hline 2950 & $\mathrm{C} 7 \mathrm{O2}-30 \mathrm{OH} 5$ & $\mathrm{CC}(\mathrm{C})(\mathrm{O} 1) \mathrm{C} 1 \mathrm{CC}(\mathrm{OO}) \mathrm{C}$ & InChl=1S/C7H14O3/c1-5(10-8)4-6-7(2,3)9-6/h5-6,8H,4H2,1-3H3 \\
\hline 2951 & C70OH5-004-2 & $\mathrm{CC}(\mathrm{C}) \mathrm{CC}(\mathrm{O}[\mathrm{O}]) \mathrm{C}(\mathrm{OO}) \mathrm{C}$ & InChl=1S/C7H15O4/c1-5(2)4-7(11-9)6(3)10-8/h5-8H,4H2,1-3H3 \\
\hline 2952 & C7H15-2D & $\mathrm{CC}(\mathrm{C}) \mathrm{C}[\mathrm{CH}] \mathrm{CC}$ & InChl=1S/C7H15/c1-4-5-6-7(2)3/h5,7H,4,6H2,1-3H3 \\
\hline 2953 & C7OOH1-ENE1 & $\mathrm{C}=\mathrm{C}(\mathrm{COO}) \mathrm{CCCC}$ & $\operatorname{lnChl}=1 \mathrm{~S} / \mathrm{C} 7 \mathrm{H} 14 \mathrm{O} 2 / \mathrm{c1}-3-4-5-5-7(2) 6-9-8 / \mathrm{h} 8 \mathrm{H}, 2-6 \mathrm{H} 2,1 \mathrm{H} 3$ \\
\hline 2954 & $\mathrm{C} 7 \mathrm{O3}-40 \mathrm{OH} 5$ & $\mathrm{CC}(\mathrm{C}) \mathrm{C}(\mathrm{O} 1) \mathrm{C} 1 \mathrm{C}(\mathrm{OO}) \mathrm{C}$ & InChl=1S/C7H14O3/c1-4(2)6-7(9-6)5(3)10-8/h4-8H,1-3H3 \\
\hline 2955 & $\mathrm{C} 70 \mathrm{OH} 5-006-2$ & $\mathrm{CC}(\mathrm{C}) \mathrm{CCC}(\mathrm{OO}) \mathrm{CO}[\mathrm{O}]$ & $\operatorname{lnChl}=15 / C 7 H 1504 / c 1-6(2) 3-4-7(11-9) 5-10-8 / h 6-7,9 \mathrm{H}, 3-5 \mathrm{H} 2,1-2 \mathrm{H} 3$ \\
\hline 2956 & C7H15-2E & $\mathrm{CC}(\mathrm{C}) \mathrm{CC}[\mathrm{CH}] \mathrm{C}$ & InChl=1S/C7H15/c1-4-5-6-7(2)3/h4,7H,5-6H2,1-3H3 \\
\hline 2957 & C7OOH2-ENE3 & $\mathrm{CC}(\mathrm{OO})(\mathrm{C}) \mathrm{C}=\mathrm{CCC}$ & InChl=1S/C7H1402/c1-4-5-6-7(2,3)9-8/h5-6,8H,4H2,1-3H3 \\
\hline 2958 & $\mathrm{C} 704-50 \mathrm{OH} 6$ & $\mathrm{CC}(\mathrm{C}) \mathrm{CC}(01) \mathrm{C} 1 \mathrm{COO}$ & InChl=1S/C7H14O3/c1-5(2)3-6-7(10-6)4-9-8/h5-8H,3-4H2,1-2H3 \\
\hline 2959 & $\begin{array}{cc}\mathrm{C} 70 \mathrm{OH} 6-002-2 \\
\end{array}$ & $\mathrm{CC}(\mathrm{C})(\mathrm{O}[\mathrm{O}]) \mathrm{CCCCOO}$ & InChl=1S/C7H1504/c1-7(2,11-9)5-3-4-6-10-8/h8H,3-6H2,1-2H3 \\
\hline 2960 & $\mathrm{C} 7 \mathrm{H} 15-2 \mathrm{~F}$ & $\mathrm{CC}(\mathrm{C}) \mathrm{CCC}[\mathrm{CH} 2]$ & $\operatorname{lnChl}=1 \mathrm{~S} / \mathrm{C} 7 \mathrm{H} 15 / \mathrm{c} 1-4-5-6-7(2) 3 / \mathrm{h} 7 \mathrm{H}, 1,4-6 \mathrm{H} 2,2-3 \mathrm{H} 3$ \\
\hline 2961 & C7OOH3-ENE1 & $\mathrm{C}=\mathrm{C}(\mathrm{C}) \mathrm{C}(\mathrm{OO}) \mathrm{CCC}$ & $\operatorname{lnChl}=15 / C 7 H 1402 / c 1-4-5-7(9-8) 6(2) 3 / \mathrm{h} 7-8 \mathrm{H}, 2,4-5 \mathrm{H} 2,1,3 \mathrm{H} 3$ \\
\hline 2962 & C7O1-10OH5 & $01 \mathrm{cc}(\mathrm{C} 1) \operatorname{ccc}(\mathrm{OO}) \mathrm{C}$ & $\operatorname{lnChl}=15 / C 7 H 1403 / c 1-6(10-8) 2-3-7-4-9-5-7 / h 6-8 \mathrm{H}, 2-5 \mathrm{H} 2,1 \mathrm{H} 3$ \\
\hline 2963 & C70OH6-003-2 & $\mathrm{CC}(\mathrm{C}) \mathrm{C}(\mathrm{O}[\mathrm{O}]) \mathrm{CCCOO}$ & InChl=1S/C7H1504/c1-6(2)7(11-9)4-3-5-10-8/h6-8H,3-5H2,1-2H3 \\
\hline 2964 & C7H15-1 & {$[\mathrm{CH} 2] \mathrm{CCCCCC}$} & InChl=1S/C7H15/c1-3-5-7-6-4-2/h1,3-7H2,2H3 \\
\hline 2965 & C7OOH4-ENE1 & $\mathrm{C}=\mathrm{C}(\mathrm{C}) \mathrm{CC}(\mathrm{OO}) \mathrm{CC}$ & InChl=15/C7H14O2/c1-4-7(9-8)5-6(2)3/h7-8H,2,4-5H2,1,3H3 \\
\hline 2966 & $\mathrm{C} 7 \mathrm{O} 2-40 \mathrm{OH} 1$ & $00 \mathrm{OCC}(\mathrm{C})(\mathrm{O} 1) \mathrm{CC} 1 \mathrm{CC}$ & InChl=1S/C7H14O3/c1-3-6-4-7(2,10-6)5-9-8/h6,8H,3-5H2,1-2H3 \\
\hline 2967 & $\mathrm{C} 7 \mathrm{OOH} 6-004-2$ & $\mathrm{CC}(\mathrm{C}) \mathrm{CC}(\mathrm{O}[\mathrm{O}]) \mathrm{CCOO}$ & InChl=1S/C7H1504/c1-6(2)5-7(11-9)3-4-10-8/h6-8H,3-5H2,1-2H3 \\
\hline 2968 & C7H15-2 & $\mathrm{C}[\mathrm{CH}] \mathrm{CCCCC}$ & InChl=1S/C7H15/c1-3-5-7-6-4-2/h3H,4-7H2,1-2H3 \\
\hline 2969 & C7OOH5-ENE1 & $\mathrm{C}=\mathrm{C}(\mathrm{C}) \mathrm{CCC}(\mathrm{OO}) \mathrm{C}$ & $\operatorname{lnChl}=15 / C 7 H 1402 / c 1-6(2) 4-5-7(3) 9-8 / h 7-8 \mathrm{H}, 1,4-5 \mathrm{H} 2,2-3 \mathrm{H} 3$ \\
\hline 2970 & C7O3-40OH6 & $\mathrm{CC}(\mathrm{C}) \mathrm{C}(01) \mathrm{C} 1 \mathrm{CCOO}$ & InChl=1S/C7H14O3/c1-5(2)7-6(10-7)3-4-9-8/h5-8H,3-4H2,1-2H3 \\
\hline 2971 & C70OH6-005-2 & $\mathrm{CC}(\mathrm{C}) \mathrm{CCC}(\mathrm{O}[\mathrm{O}]) \mathrm{COO}$ & InChl=1S/C7H1504/c1-6(2)3-4-7(11-9)5-10-8/h6-8H,3-5H2,1-2H3 \\
\hline 2972 & C7H15-3 & $\mathrm{CC}[\mathrm{CH}] \mathrm{CCCC}$ & $\operatorname{lnChl}=1 \mathrm{~S} / \mathrm{C} 7 \mathrm{H} 15 / \mathrm{c1}-3-5-7-6-4-2 / \mathrm{h} 5 \mathrm{H}, 3-4,6-7 \mathrm{H} 2,1-2 \mathrm{H} 3$ \\
\hline 2973 & C7OOH6-ENE1 & $\mathrm{C}=\mathrm{C}(\mathrm{C}) \mathrm{CCCCOO}$ & InChl=1S/C7H14O2/c1-7(2)5-3-4-6-9-8/h8H,1,3-6H2,2H3 \\
\hline 2974 & $\mathrm{C} 7 \mathrm{O} 4-60 \mathrm{OH} 1$ & $\mathrm{OOCC}(\mathrm{C}) \mathrm{CC}(01) \mathrm{CC} 1$ & $\operatorname{lnChl}=1 \mathrm{~S} / \mathrm{C} 7 \mathrm{H} 14 \mathrm{OO} / \mathrm{c} 1-6(5-10-8) 4-7-2-3-9-7 / \mathrm{h} 6-8 \mathrm{H}, 2-5 \mathrm{H} 2,1 \mathrm{H} 3$ \\
\hline 2975 & $\mathrm{C} 70 \mathrm{OH} 10 \mathrm{OH} 1-\mathrm{C}$ & $\mathrm{OOCC}(\mathrm{COO})[\mathrm{CH}] \mathrm{CCC}$ & $\operatorname{lnChl}=15 / C 7 \mathrm{H} 1504 / c 1-2-3-4-7(5-10-8) 6-11-9 / h 4,7-9 \mathrm{H}, 2-3,5-6 \mathrm{H} 2,1 \mathrm{H} 3$ \\
\hline 2976 & C7H15-4 & $\mathrm{CCC}[\mathrm{CH}] \mathrm{CCC}$ & InChl=1S/C7H15/c1-3-5-7-6-4-2/h7H,3-6H2,1-2H3 \\
\hline 2977 & C7OOH1-ENE2 & $\operatorname{OOCC}(\mathrm{C})=\mathrm{CCCC}$ & InChl=15/C7H14O2/c1-3-4-5-7(2)6-9-8/h5,8H,3-4,6H2,1-2H3 \\
\hline 2978 & $\mathrm{C} 7 \mathrm{O1}-2 \mathrm{OOH} 1$ & OOCC1(CO1)CCCC & $\operatorname{lnChl}=1 \mathrm{~S} / \mathrm{C} 7 \mathrm{H} 1403 / \mathrm{c} 1-2-3-4-7(5-9-7) 6-10-8 / \mathrm{h} 8 \mathrm{H}, 2-6 \mathrm{H} 2,1 \mathrm{H} 3$ \\
\hline 2979 & С70OH2OOH3-A & {$[\mathrm{CH} 2] \mathrm{C}(\mathrm{C})(\mathrm{OO}) \mathrm{C}(\mathrm{OO}) \mathrm{CCC}$} & $\operatorname{lnChl}=15 / C 7 H 1504 / c 1-4-5-6(10-8) 7(2,3) 11-9 / h 6,8-9 \mathrm{H}, 2,4-5 \mathrm{H} 2,1,3 \mathrm{H} 3$ \\
\hline 2980 & 124C7-2R & $\mathrm{C}[\mathrm{C}](\mathrm{C}) \mathrm{CC}(\mathrm{C}) \mathrm{C}$ & $\operatorname{lnChl}=15 / C 7 H 15 / c 1-6(2) 5-7(3) 4 / h 6 H, 5 H 2,1-4 H 3$ \\
\hline 2981 & C7OOH2-ENE4 & $\mathrm{CC}(\mathrm{C})(\mathrm{OO}) \mathrm{CC}=\mathrm{CC}$ & $\operatorname{lnChl}=1 \mathrm{~S} / \mathrm{C} 7 \mathrm{H} 1402 / \mathrm{C} 1-4-5-6-7(2,3) 9-8 / \mathrm{h} 4-5,8 \mathrm{H}, 6 \mathrm{H} 2,1-3 \mathrm{H} 3$ \\
\hline 2982 & $\mathrm{C} 7 \mathrm{O2}-4 \mathrm{OOH} 3$ & $\mathrm{CC}(\mathrm{C})(\mathrm{O} 1) \mathrm{C}(\mathrm{OO}) \mathrm{C} 1 \mathrm{CC}$ & InChl=1S/C7H14O3/c1-4-5-6(10-8)7(2,3)9-5/h5-6,8H,4H2,1-3H3 \\
\hline 2983 & C70OH1OOH3-A & $\mathrm{OOCC}([\mathrm{CH} 2]) \mathrm{C}(\mathrm{OO}) \mathrm{CCC}$ & InChl=1S/C7H15O4/c1-3-4-7(11-9)6(2)5-10-8/h6-9H,2-5H2,1H3 \\
\hline 2984 & NEC7-4R & $\mathrm{CC}(\mathrm{C})(\mathrm{C}) \mathrm{C}[\mathrm{CH}] \mathrm{C}$ & $\operatorname{lnChl}=15 / C 7 H 15 / c 1-5-6-7(2,3) 4 / h 5 H, 6 \mathrm{H} 2,1-4 \mathrm{H} 3$ \\
\hline 2985 & C7OOH3-ENE2 & $\mathrm{CC}(\mathrm{C})=\mathrm{C}(\mathrm{OO}) \mathrm{CCC}$ & InChl=15/C7H14O2/c1-4-5-7(9-8)6(2)3/h8H,4-5H2,1-3H3 \\
\hline 2986 & C7O3-50OH1 & $\mathrm{OOCC}(\mathrm{C}) \mathrm{C}(\mathrm{O} 1) \mathrm{CC} 1 \mathrm{C}$ & $\operatorname{lnChl}=1 \mathrm{~S} / \mathrm{C} 7 \mathrm{H} 1403 / \mathrm{C} 1-5(4-9-8) 7-3-6(2) 10-7 / \mathrm{h} 5-8 \mathrm{H}, 3-4 \mathrm{H} 2,1-2 \mathrm{H} 3$ \\
\hline 2987 & C70OH4OOH1-A & {$[\mathrm{CH} 2] \mathrm{C}(\mathrm{COO}) \mathrm{CC}(\mathrm{OO}) \mathrm{CC}$} & InChl=1S/C7H15O4/c1-3-7(11-9)4-6(2)5-10-8/h6-9H,2-5H2,1H3 \\
\hline 2988 & 124C7-1R & {$[\mathrm{CH} 2] \mathrm{C}(\mathrm{C}) \mathrm{CC}(\mathrm{C}) \mathrm{C}$} & InChl=1S/C7H15/c1-6(2)5-7(3)4/h6-7H,1,5H2,2-4H3 \\
\hline 2989 & C7OOH4-ENE2 & $\mathrm{CC}(\mathrm{C})=\mathrm{CC}(\mathrm{OO}) \mathrm{CC}$ & InChl=1S/C7H1402/c1-4-7(9-8)5-6(2)3/h5,7-8H,4H2,1-3H3 \\
\hline 2990 & $\mathrm{C} 7 \mathrm{O} 4-60 \mathrm{OH} 2$ & $\mathrm{CC}(\mathrm{C})(\mathrm{OO}) \mathrm{CC}(\mathrm{O} 1) \mathrm{CC} 1$ & InChl=1S/C7H14O3/c1-7(2,10-8)5-6-3-4-9-6/h6,8H,3-5H2,1-2H3 \\
\hline 2991 & C70OH5OOH1-A & {$[\mathrm{CH} 2] \mathrm{C}(\mathrm{COO}) \mathrm{CCC}(\mathrm{OO}) \mathrm{C}$} & InChl=1S/C7H1504/c1-6(5-10-8)3-4-7(2)11-9/h6-9H,1,3-5H2,2H3 \\
\hline 2992 & 124C7-3R & $\mathrm{CC}(\mathrm{C})[\mathrm{CH}] \mathrm{C}(\mathrm{C}) \mathrm{C}$ & InChl=1S/C7H15/c1-6(2)5-7(3)4/h5-7H,1-4H3 \\
\hline 2993 & C7OOH5-ENE2 & $\mathrm{CC}(\mathrm{C})=\mathrm{CCC}(\mathrm{OO}) \mathrm{C}$ & InChl=1S/C7H1402/c1-6(2)4-5-7(3)9-8/h4,7-8H,5H2,1-3H3 \\
\hline 2994 & $\mathrm{C} 7 \mathrm{O1}-2 \mathrm{OOH} 3$ & $01 \mathrm{CC} 1(\mathrm{C}) \mathrm{C}(\mathrm{OO}) \mathrm{CCC}$ & $\operatorname{lnChl}=1 \mathrm{~S} / \mathrm{C} 7 \mathrm{H} 1403 / \mathrm{c} 1-3-4-6(10-8) 7(2) 5-9-7 / \mathrm{h} 6,8 \mathrm{H}, 3-5 \mathrm{H} 2,1-2 \mathrm{H} 3$ \\
\hline 2995 & C70OH2OOH6-D & $\mathrm{CC}(\mathrm{C})(\mathrm{OO}) \mathrm{C}[\mathrm{CH}] \mathrm{CCOO}$ & $\operatorname{lnChl}=15 / C 7 H 1504 / c 1-7(2,11-9) 5-3-4-6-10-8 / h 3,8-9 \mathrm{H}, 4-6 \mathrm{H} 2,1-2 \mathrm{H} 3$ \\
\hline 2996 & NEC7-3R & $\mathrm{CC}(\mathrm{C})(\mathrm{C})[\mathrm{CH}] \mathrm{CC}$ & $\operatorname{lnChl}=15 / \mathrm{C} 7 \mathrm{H} 15 / \mathrm{c} 1-5-6-7(2,3) 4 / \mathrm{h} 6 \mathrm{H}, 5 \mathrm{H} 2,1-4 \mathrm{H} 3$ \\
\hline 2997 & C7OOH6-ENE2 & $\mathrm{CC}(\mathrm{C})=\mathrm{CCCCOO}$ & $\operatorname{lnChl}=15 / \mathrm{C} 7 \mathrm{H} 14 \mathrm{O} 2 / \mathrm{c} 1-7(2) 5-3-4-6-9-8 / \mathrm{h} 5,8 \mathrm{H}, 3-4,6 \mathrm{H} 2,1-2 \mathrm{H} 3$ \\
\hline 2998 & $\mathrm{C} 7 \mathrm{O2}-4 \mathrm{OOH} 5$ & $\mathrm{CC}(\mathrm{C})(\mathrm{O} 1) \mathrm{CC} 1 \mathrm{C}(\mathrm{OO}) \mathrm{C}$ & InChl=1S/C7H14O3/c1-5(10-8)6-4-7(2,3)9-6/h5-6,8H,4H2,1-3H3 \\
\hline 2999 & C70OH1OOH2-D & $\mathrm{OOCC}(\mathrm{C})(\mathrm{OO}) \mathrm{C}[\mathrm{CH}] \mathrm{CC}$ & InChl=15/C7H15O4/c1-3-4-5-7(2,11-9)6-10-8/h4,8-9H,3,5-6H2,1-2H3 \\
\hline
\end{tabular}




\begin{tabular}{|c|c|c|c|}
\hline 3000 & NEC7-5R & $\mathrm{CC}(\mathrm{C})(\mathrm{C}) \mathrm{CC}[\mathrm{CH} 2]$ & InChl=1S/C7H15/c1-5-6-7(2,3)4/h1,5-6H2,2-4H3 \\
\hline 3001 & C7OOH1-ENE3 & $\mathrm{OOCC}(\mathrm{C}) \mathrm{C}=\mathrm{CCC}$ & InChl=1S/C7H14O2/c1-3-4-5-7(2)6-9-8/h4-5,7-8H,3,6H2,1-2H3 \\
\hline 3002 & $\mathrm{C} 7 \mathrm{O}-5 \mathrm{OOH} 2$ & $\mathrm{CC}(\mathrm{C})(\mathrm{OO}) \mathrm{C}(\mathrm{O} 1) \mathrm{CC} 1 \mathrm{C}$ & InChl=1S/C7H1403/c1-5-4-6(9-5)7(2,3)10-8/h5-6,8H,4H2,1-3H3 \\
\hline 3003 & С70OH2OOH3-Е & $\mathrm{CC}(\mathrm{C})(\mathrm{OO}) \mathrm{C}(\mathrm{OO}) \mathrm{C}[\mathrm{CH}] \mathrm{C}$ & $\operatorname{lnChl}=1 \mathrm{~S} / \mathrm{C} 7 \mathrm{H} 15 \mathrm{O} 4 / \mathrm{c} 1-4-5-6(10-8) 7(2,3) 11-9 / \mathrm{h} 4,6,8-9 \mathrm{H}, 5 \mathrm{H} 2,1-3 \mathrm{H} 3$ \\
\hline 3004 & C7H16-2 & $\mathrm{CC}(\mathrm{C}) \mathrm{CCCC}$ & InChl=1S/C7H16/c1-4-5-6-7(2)3/h7H,4-6H2,1-3H3 \\
\hline 3005 & C7OOH2-ENE5 & $\mathrm{CC}(\mathrm{C})(\mathrm{OO}) \mathrm{CCC}=\mathrm{C}$ & InChl=1S/C7H14O2/c1-4-5-6-7(2,3)9-8/h4,8H,1,5-6H2,2-3H3 \\
\hline 3006 & $\mathrm{C} 7 \mathrm{O} 4-60 \mathrm{OH} 3$ & $\mathrm{CC}(\mathrm{C}) \mathrm{C}(\mathrm{OO}) \mathrm{C}(\mathrm{O} 1) \mathrm{CC} 1$ & InChl=1S/C7H14O3/c1-5(2)7(10-8)6-3-4-9-6/h5-8H,3-4H2,1-2H3 \\
\hline 3007 & C7OOH3OOH2-D & $\mathrm{CC}(\mathrm{C})(\mathrm{OO}) \mathrm{C}(\mathrm{OO})[\mathrm{CH}] \mathrm{CC}$ & InChl=1S/C7H15O4/c1-4-5-6(10-8)7(2,3)11-9/h5-6,8-9H,4H2,1-3H3 \\
\hline 3008 & NC7H16 & $\operatorname{ccccccc}$ & InChl=1S/C7H16/c1-3-5-7-6-4-2/h3-7H2,1-2H3 \\
\hline 3009 & C7OOH3-ENE3 & $\mathrm{CC}(\mathrm{C}) \mathrm{C}(\mathrm{OO})=\mathrm{CCC}$ & InChl=1S/C7H14O2/c1-4-5-7(9-8)6(2)3/h5-6,8H,4H2,1-3H3 \\
\hline 3010 & $\mathrm{C} 7 \mathrm{O} 1-2 \mathrm{OOH} 4$ & $01 \mathrm{CC} 1(\mathrm{C}) \mathrm{CC}(\mathrm{OO}) \mathrm{CC}$ & InChl=1S/C7H14O3/c1-3-6(10-8)4-7(2)5-9-7/h6,8H,3-5H2,1-2H33 \\
\hline 3011 & $\mathrm{C} 70 \mathrm{OH} 4 \mathrm{OOH} 1-\mathrm{C}$ & $\mathrm{OOCC}(\mathrm{C})[\mathrm{CH}] \mathrm{C}(\mathrm{OO}) \mathrm{CC}$ & InChl=1S/C7H15O4/c1-3-7(11-9)4-6(2)5-10-8/h4,6-9H,3,5H2,1-2H3 \\
\hline 3012 & $\mathrm{C} 6 \mathrm{H} 5 \mathrm{CO}$ & $C 1=C C([C]=0)=C C=C 1$ & $\operatorname{lnChl}=1 \mathrm{~S} / \mathrm{C} 7 \mathrm{H} 5 \mathrm{O} / \mathrm{c} 8-6-7-4-2-1-3-5-7 / \mathrm{h} 1-5 \mathrm{H}$ \\
\hline 3013 & C7OOH4-ENE3 & $\mathrm{CC}(\mathrm{C}) \mathrm{C}=\mathrm{C}(\mathrm{OO}) \mathrm{CC}$ & InChl=1S/C7H1402/c1-4-7(9-8)5-6(2)3/h5-6,8H,4H2,1-3H3 \\
\hline 3014 & $\mathrm{C} 7 \mathrm{O2}-40 \mathrm{OH} 6$ & $\mathrm{CC}(\mathrm{C})(\mathrm{O} 1) \mathrm{CC} 1 \mathrm{CCOO}$ & $\operatorname{lnChl}=1 \mathrm{~S} / \mathrm{C} 7 \mathrm{H} 14 \mathrm{OO} / \mathrm{c} 1-7(2) 5-6(10-7) 3-4-9-8 / \mathrm{h} 6,8 \mathrm{H}, 3-5 \mathrm{H} 2,1-2 \mathrm{H3}$ \\
\hline 3015 & C70OH1OOH5-C & $\mathrm{OOCC}(\mathrm{C})[\mathrm{CH}] \mathrm{CC}(\mathrm{OO}) \mathrm{C}$ & InChl=1S/C7H15O4/c1-6(5-10-8)3-4-7(2)11-9/h3,6-9H,4-5H2,1-2H3 \\
\hline 3016 & $\mathrm{C} 6 \mathrm{H} 5 \mathrm{CHO}$ & $\mathrm{c} 1 \mathrm{c}(\mathrm{C}=0) \mathrm{ccccc} 1$ & $\operatorname{lnChl}=1 \mathrm{~S} / \mathrm{C} 7 \mathrm{H} 6 \mathrm{O} / \mathrm{c} 8-6-7-4-2-1-3-5-7 / \mathrm{h} 1-6 \mathrm{H}$ \\
\hline 3017 & C7OOH5-ENE3 & $\mathrm{CC}(\mathrm{C}) \mathrm{C}=\mathrm{CC}(\mathrm{OO}) \mathrm{C}$ & InChl=1S/C7H14O2/c1-6(2)4-5-7(3)9-8/h4-8H,1-3H3 \\
\hline 3018 & $\mathrm{C} 703-50 \mathrm{OH} 4$ & $\mathrm{CC}(\mathrm{C}) \mathrm{C}(\mathrm{O} 1) \mathrm{C}(\mathrm{OO}) \mathrm{C} 1 \mathrm{C}$ & InChl=1S/C7H14O3/c1-4(2)6-7(10-8)5(3)9-6/h4-8H,1-3H3 \\
\hline 3019 & C7OOH6OOH3-A & {$[\mathrm{CH} 2] \mathrm{C}(\mathrm{C}) \mathrm{C}(\mathrm{OO}) \mathrm{CCCOO}$} & InChl=1S/C7H1504/c1-6(2)7(11-9)4-3-5-10-8/h6-9H,1,3-5H2,2H3 \\
\hline 3020 & $\mathrm{OC} 6 \mathrm{H} 4 \mathrm{CH} 2$ & $\mathrm{C}=\mathrm{C} 1 \mathrm{C}=\mathrm{CC}=\mathrm{CC} 1=\mathrm{O}$ & $\operatorname{lnChl}=1 \mathrm{~S} / \mathrm{C} 7 \mathrm{H} 6 \mathrm{O} / \mathrm{c1}-6-4-2-3-5-7(6) 8 / \mathrm{h} 2-5 \mathrm{H}, 1 \mathrm{H} 2$ \\
\hline 3021 & C7OOH6-ENE3 & $\mathrm{CC}(\mathrm{C}) \mathrm{C}=\mathrm{CCCOO}$ & InChl=1S/C7H14O2/c1-7(2)5-3-4-6-9-8/h3,5,7-8H,4,6H2,1-2H3 \\
\hline 3022 & $\mathrm{C} 7 \mathrm{O} 4-60 \mathrm{OH} 5$ & $\mathrm{CC}(\mathrm{C}) \mathrm{CC}(\mathrm{O} 1) \mathrm{C}(\mathrm{OO}) \mathrm{C} 1$ & InChl=1S/C7H1403/c1-5(2)3-6-7(10-8)4-9-6/h5-8H,3-4H2,1-2H3 \\
\hline 3023 & $\mathrm{C} 70 \mathrm{OH} 10 \mathrm{OH} 2-\mathrm{C}$ & $\mathrm{OOCC}(\mathrm{C})(\mathrm{OO})[\mathrm{CH}] \mathrm{CCC}$ & InChl=15/C7H15O4/c1-3-4-5-7(2,11-9)6-10-8/h5,8-9H,3-4,6H2,1-2H3 \\
\hline 3024 & $\mathrm{C} 6 \mathrm{H} 5 \mathrm{OCH} 2$ & [CH2]Oc1ccccc1 & InChl=15/C7H70/c1-8-7-5-3-2-4-6-7/h2-6H,1H2 \\
\hline 3025 & C7OOH1-ENE4 & $\mathrm{OOCC}(\mathrm{C}) \mathrm{CC}=\mathrm{CC}$ & InChl=15/C7H1402/c1-3-4-5-7(2)6-9-8/h3-4,7-8H,5-6H2,1-2H3 \\
\hline 3026 & C7O1-30OH1 & $01 \mathrm{C}(\mathrm{OO}) \mathrm{C}(\mathrm{C}) \mathrm{C} 1 \mathrm{CCC}$ & InChl=1S/C7H14O3/c1-3-4-6-5(2)7(9-6)10-8/h5-8H,3-4H2,1-2H3 \\
\hline 3027 & $\mathrm{C7OOH} 2 \mathrm{OOH} 4-\mathrm{F}$ & $\mathrm{CC}(\mathrm{C})(\mathrm{OO}) \mathrm{CC}(\mathrm{OO}) \mathrm{C}[\mathrm{CH} 2]$ & InChl=1S/C7H15O4/c1-4-6(10-8)5-7(2,3)11-9/h6,8-9H,1,4-5H2,2-3H3 \\
\hline 3028 & $\mathrm{C} 6 \mathrm{H} 4 \mathrm{OCH} 3$ & $\operatorname{coc} 1[\mathrm{c}] \operatorname{cccc} 1$ & InChl=1S/C7H70/c1-8-7-5-3-2-4-6-7/h2-5H,1H3 \\
\hline 3029 & C7OOH3-ENE4 & $\mathrm{CC}(\mathrm{C}) \mathrm{C}(\mathrm{OO}) \mathrm{C}=\mathrm{CC}$ & $\operatorname{lnChl}=1 \mathrm{~S} / \mathrm{C} 7 \mathrm{H} 14 \mathrm{O} 2 / \mathrm{c1} 1-4-5-7(9-8) 6(2) 3 / \mathrm{h} 4-8 \mathrm{H}, 1-3 \mathrm{H} 3$ \\
\hline 3030 & $\mathrm{C} 7 \mathrm{O} 2-5 \mathrm{OOH} 3$ & $\mathrm{CC}(\mathrm{O} 1)(\mathrm{C}) \mathrm{C}(\mathrm{OO}) \mathrm{CC} 1 \mathrm{C}$ & InChl=1S/C7H14O3/c1-5-4-6(10-8)7(2,3)9-5/h5-6,8H,4H2,1-3H3 \\
\hline 3031 & С7ООН $30 \mathrm{OH} 4-\mathrm{B}$ & $\mathrm{C}[\mathrm{C}] \mathrm{C}) \mathrm{C}(\mathrm{OO}) \mathrm{C}(\mathrm{OO}) \mathrm{CC}$ & InChl=1S/C7H15O4/c1-4-6(10-8)7(11-9)5(2)3/h6-9H,4H2,1-3H3 \\
\hline 3032 & $\mathrm{C} 6 \mathrm{H} 5 \mathrm{CYCH} 2 \mathrm{O}$ & $\mathrm{C} 1=\mathrm{CC} 2(\mathrm{CO} 2) \mathrm{C}=\mathrm{C}[\mathrm{CH}] 1$ & InChl=1S/C7H7O/c1-2-4-7(5-3-1)6-8-7/h1-5H,6H2 \\
\hline 3033 & C7OOH4-ENE4 & $\mathrm{CC}(\mathrm{C}) \mathrm{CC}(\mathrm{OO})=\mathrm{CC}$ & InChl=1S/C7H14O2/c1-4-7(9-8)5-6(2)3/h4,6,8H,5H2,1-3H3 \\
\hline 3034 & $\mathrm{C} 7 \mathrm{O}-5 \mathrm{OOH} 6$ & $\mathrm{CC}(\mathrm{C}) \mathrm{C}(\mathrm{O} 1) \mathrm{CC} 1 \mathrm{COO}$ & InChl=1S/C7H1403/c1-5(2)7-3-6(10-7)4-9-8/h5-8H,3-4H2,1-2H3 \\
\hline 3035 & C7OOH4OOH3-A & {$[\mathrm{CH} 2] \mathrm{C}(\mathrm{C}) \mathrm{C}(\mathrm{OO}) \mathrm{C}(\mathrm{OO}) \mathrm{CC}$} & InChl=1S/C7H1504/c1-4-6(10-8)7(11-9)5(2)3/h5-9H,2,4H2,1,3H3 \\
\hline 3036 & $\mathrm{HOC} 6 \mathrm{H} 4 \mathrm{CH} 2$ & $\mathrm{C} 1=\mathrm{CC}([\mathrm{CH} 2])=\mathrm{CC}(\mathrm{O})=\mathrm{C} 1$ & InChl=15/C7H7O/c1-6-3-2-4-7(8)5-6/h2-5,8H,1H2 \\
\hline 3037 & C7OOH5-ENE4 & $\mathrm{CC}(\mathrm{C}) \mathrm{CC}=\mathrm{C}(\mathrm{OO}) \mathrm{C}$ & InChl=1S/C7H14O2/c1-6(2)4-5-7(3)9-8/h5-6,8H,4H2,1-3H3 \\
\hline 3038 & $\mathrm{C} 701-30 \mathrm{OH} 2$ & $01 \mathrm{Cc}(\mathrm{C})(\mathrm{OO}) \mathrm{C} 1 \mathrm{CCC}$ & InChl=15/C7H14O3/c1-3-4-6-7(2,10-8)5-9-6/h6,8H,3-5H2,1-2H3 \\
\hline 3039 & C7OOH5OOH2-D & $\mathrm{CC}(\mathrm{C})(\mathrm{OO}) \mathrm{C}[\mathrm{CH}] \mathrm{C}(\mathrm{OO}) \mathrm{C}$ & InChl=15/C7H15O4/c1-6(10-8)4-5-7(2,3)11-9/h4,6,8-9H,5H2,1-3H3 \\
\hline 3040 & $\mathrm{OC} 6 \mathrm{H} 4 \mathrm{CH} 3$ & $\mathrm{CC} 1=\mathrm{CC}=\mathrm{CC}=\mathrm{C} 1[\mathrm{O}]$ & InChl=1S/C7H70/c1-6-4-2-3-5-7(6)8/h2-5H,1H3 \\
\hline 3041 & C7OOH6-ENE4 & $\mathrm{CC}(\mathrm{C}) \mathrm{CC}=\mathrm{CCOO}$ & InChl=15/C7H14O2/c1-7(2)5-3-4-6-9-8/h3-4,7-8H,5-6H2,1-2H3 \\
\hline 3042 & $\mathrm{C} 7 \mathrm{O} 2-5 \mathrm{OOH} 4$ & $\mathrm{CC}(\mathrm{C})(\mathrm{O} 1) \mathrm{CC}(\mathrm{OO}) \mathrm{C} 1 \mathrm{C}$ & InChl=1S/C7H14O3/c1-5-6(10-8)4-7(2,3)9-5/h5-6,8H,4H2,1-3H3 \\
\hline 3043 & C7OOH6OOH3-E & $\mathrm{CC}(\mathrm{C}) \mathrm{C}(\mathrm{OO}) \mathrm{C}[\mathrm{CH}] \mathrm{COO}$ & InChl=1S/C7H15O4/c1-6(2)7(11-9)4-3-5-10-8/h3,6-9H,4-5H2,1-2H3 \\
\hline 3044 & $\mathrm{C} 6 \mathrm{H} 5 \mathrm{CHOH}$ & $\mathrm{C} 1=\mathrm{CC}([\mathrm{CH}] \mathrm{O})=\mathrm{CC}=\mathrm{C} 1$ & InChl=1S/C7H70/c8-6-7-4-2-1-3-5-7/h1-6,8H \\
\hline 3045 & C7OOH1-ENE5 & $\mathrm{OOCC}(\mathrm{C}) \mathrm{CCC}=\mathrm{C}$ & InChl=1S/C7H14O2/c1-3-4-5-7(2)6-9-8/h3,7-8H,1,4-6H2,2H3 \\
\hline 3046 & $\mathrm{C} 7 \mathrm{O}-60 \mathrm{OH} 4$ & $\mathrm{CC}(\mathrm{C}) \mathrm{C}(\mathrm{O} 1) \mathrm{C}(\mathrm{OO}) \mathrm{CC} 1$ & InChl=1S/C7H1403/c1-5(2)7-6(10-8)3-4-9-7/h5-8H,3-4H2,1-2H3 \\
\hline 3047 & C70OH1OOH2-A & OOCC([CH2])(OO)CCCC & InChl=1S/C7H1504/c1-3-4-5-7(2,11-9)6-10-8/h8-9H,2-6H2,1H3 \\
\hline 3048 & $\mathrm{C} 6 \mathrm{H} 5 \mathrm{CH} 2 \mathrm{O}$ & $\mathrm{C} 1=\mathrm{CC}(\mathrm{C}[\mathrm{O}])=\mathrm{CC}=\mathrm{C} 1$ & $\operatorname{lnChl}=15 / C 7 H 70 / c 8-6-7-4-2-1-3-5-7 / h 1-5 \mathrm{H}, 6 \mathrm{H} 2$ \\
\hline 3049 & C7OOH3-ENE5 & $\mathrm{CC}(\mathrm{C}) \mathrm{C}(\mathrm{OO}) \mathrm{CC}=\mathrm{C}$ & $\operatorname{lnChl}=1 \mathrm{~S} / \mathrm{C} 7 \mathrm{H} 14 \mathrm{O} 2 / \mathrm{c} 1-4-5-7(9-8) 6(2) 3 / \mathrm{h} 4,6-8 \mathrm{H}, 1,5 \mathrm{H} 2,2-3 \mathrm{H} 3$ \\
\hline 3050 & $\mathrm{C} 701-30 \mathrm{OH} 4$ & $01 \mathrm{CC}(\mathrm{C}) \mathrm{C} 1 \mathrm{C}(\mathrm{OOO}) \mathrm{CC}$ & InChl=1S/C7H14O3/c1-3-6(10-8)7-5(2)4-9-7/h5-8H,3-4H2,1-2H3 \\
\hline 3051 & C7OOH2OOH5-C & $\mathrm{CC}(\mathrm{C})(\mathrm{OO})[\mathrm{CH}] \mathrm{CC}(\mathrm{OO}) \mathrm{C}$ & InChl=1S/C7H15O4/c1-6(10-8)4-5-7(2,3)11-9/h5-6,8-9H,4H2,1-3H3 \\
\hline 3052 & $\mathrm{P}-\mathrm{OC} 6 \mathrm{H} 4 \mathrm{CH} 3$ & $\mathrm{CC} 1=\mathrm{CC}=\mathrm{C}([\mathrm{O}]) \mathrm{C}=\mathrm{C} 1$ & InChl=1S/C7H70/c1-6-2-4-7(8)5-3-6/h2-5H,1H3 \\
\hline 3053 & C7OOH4-ENE5 & $\mathrm{CC}(\mathrm{C}) \mathrm{CC}(\mathrm{OO}) \mathrm{C}=\mathrm{C}$ & InChl=1S/C7H14O2/c1-4-7(9-8)5-6(2)3/h4,6-8H,1,5H2,2-3H3 \\
\hline 3054 & $\mathrm{C} 7 \mathrm{O} 2-60 \mathrm{OH} 4$ & $\mathrm{CC}(\mathrm{C})(\mathrm{O} 1) \mathrm{CC}(\mathrm{OO}) \mathrm{CC} 1$ & InChl=1S/C7H1403/c1-7(2)5-6(10-8)3-4-9-7/h6,8H,3-5H2,1-2H3 \\
\hline 3055 & $\mathrm{C} 7 \mathrm{OOH} 3 \mathrm{OOH} 4-\mathrm{F}$ & $\mathrm{CC}(\mathrm{C}) \mathrm{C}(\mathrm{OO}) \mathrm{C}(\mathrm{OO}) \mathrm{C}[\mathrm{CH} 2]$ & InChl=1S/C7H1504/c1-4-6(10-8)7(11-9)5(2)3/h5-9H,1,4H2,2-3H3 \\
\hline 3056 & $\mathrm{M}-\mathrm{OC} 6 \mathrm{H} 4 \mathrm{CH} 3$ & {$[0] \mathrm{C} 1=\mathrm{CC}(\mathrm{C})=\mathrm{CC}=\mathrm{C} 1$} & InChl=1S/C7H7O/c1-6-3-2-4-7(8)5-6/h2-5H,1H3 \\
\hline 3057 & C7OOH5-ENE5 & $\operatorname{CC}(\mathrm{C}) \operatorname{ccc}(\mathrm{OO})=\mathrm{C}$ & InChl=1S/C7H1402/c1-6(2)4-5-7(3)9-8/h6,8H,3-5H2,1-2H3 \\
\hline 3058 & $\mathrm{C} 7 \mathrm{O}-60 \mathrm{OH} 5$ & $\mathrm{CC}(\mathrm{C}) \mathrm{C}(\mathrm{O} 1) \mathrm{CC}(\mathrm{OO}) \mathrm{C} 1$ & InChl=1S/C7H1403/c1-5(2)7-3-6(10-8)4-9-7/h5-8H,3-4H2,1-2H3 \\
\hline 3059 & C7OOH4OOH3-E & $\mathrm{CC}(\mathrm{C}) \mathrm{C}(\mathrm{OO}) \mathrm{C}(\mathrm{OO})[\mathrm{CH}] \mathrm{C}$ & InChl=1S/C7H1504/c1-4-6(10-8)7(11-9)5(2)3/h4-9H,1-3H3 \\
\hline 3060 & $\mathrm{C} 6 \mathrm{H} 5 \mathrm{OCH} 3$ & COc1ccccc1 & $\operatorname{lnChl}=1 \mathrm{~S} / \mathrm{C} 7 \mathrm{H} 8 \mathrm{O} / \mathrm{c1} 1-8-7-5-3-2-4-6-7 / \mathrm{h} 2-6 \mathrm{H}, 1 \mathrm{H} 3$ \\
\hline 3061 & C7OOH6-ENE5 & $\mathrm{CC}(\mathrm{C}) \mathrm{CCC}=\mathrm{COO}$ & $\operatorname{lnChl}=15 / C 7 H 1402 / c 1-7(2) 5-3-4-6-9-8 / \mathrm{h} 4,6-8 \mathrm{H}, 3,5 \mathrm{H} 2,1-2 \mathrm{H} 3$ \\
\hline 3062 & C7O1-30OH5 & $01 \mathrm{CC}(\mathrm{C}) \mathrm{C} 1 \mathrm{CC}(\mathrm{OOO}) \mathrm{c}$ & InChl=1S/C7H1403/c1-5-4-9-7(5)3-6(2)10-8/h5-8H,3-4H2,1-2H3 \\
\hline 3063 & C70OH5OOH3-A & {$[\mathrm{CH} 2] \mathrm{C}(\mathrm{C}) \mathrm{C}(\mathrm{OO}) \mathrm{CC}(\mathrm{OO}) \mathrm{C}$} & InChl=1S/C7H15O4/c1-5(2)7(11-9)4-6(3)10-8/h5-9H,1,4H2,2-3H3 \\
\hline 3064 & CHDE24106M & $\mathrm{C} 1=\mathrm{CC}=\mathrm{CC}(\mathrm{C}) \mathrm{C} 1(=0)$ & InChl=1S/C7H8O/c1-6-4-2-3-5-7(6)8/h2-6H,1H3 \\
\hline 3065 & C7H13-1D30OH & $\mathrm{C}=\mathrm{CC}(\mathrm{OO}) \mathrm{CCCC}$ & InChl=1S/C7H14O2/c1-3-5-6-7(4-2)9-8/h4,7-8H,2-3,5-6H2,1H3 \\
\hline 3066 & $\mathrm{C} 7 \mathrm{O} 1-30 \mathrm{OH} 6$ & O1CC(C)C1CcCOO & InChl=1S/C7H14O3/c1-6-5-9-7(6)3-2-4-10-8/h6-8H,2-5H2,1H3 \\
\hline 3067 & C7OOH6OOH4-B & $\mathrm{C}[\mathrm{C}](\mathrm{C}) \mathrm{CC}(\mathrm{OO}) \mathrm{CCOO}$ & InChl=1S/C7H15O4/c1-6(2)5-7(11-9)3-4-10-8/h7-9H,3-5H2,1-2H3 \\
\hline 3068 & HPT145TE3O & $\mathrm{C}=\mathrm{CC}(=\mathrm{O}) \mathrm{C}=\mathrm{C}=\mathrm{CC}$ & InChl=1S/C7H8O/c1-3-5-6-7(8)4-2/h3-4,6H,2H2,1H3 \\
\hline 3069 & $\mathrm{C} 7 \mathrm{H} 13-1 \mathrm{D} 4 \mathrm{OOH}$ & $\mathrm{C}=\mathrm{CCC}(\mathrm{OO}) \mathrm{CCC}$ & InChl=1S/C7H14O2/c1-3-5-7(9-8)6-4-2/h3,7-8H,1,4-6H2,2H3 \\
\hline 3070 & C7O1-40OH1 & $\mathrm{OOC}(\mathrm{O} 1) \mathrm{C}(\mathrm{C}) \mathrm{CC} 1 \mathrm{CC}$ & InChl=1S/C7H14O3/c1-3-6-4-5(2)7(9-6)10-8/h5-8H,3-4H2,1-2H3 \\
\hline 3071 & C7OOH3OOH6-D & $\mathrm{CC}(\mathrm{C}) \mathrm{C}(\mathrm{OO})[\mathrm{CH}] \mathrm{CCOO}$ & $\operatorname{lnChl}=15 / C 7 H 1504 / c 1-6(2) 7(11-9) 4-3-5-10-8 / \mathrm{h} 4,6-9 \mathrm{H}, 3,5 \mathrm{H} 2,1-2 \mathrm{H} 3$ \\
\hline 3072 & HPT346TE2O & $\mathrm{C}=\mathrm{CC}=\mathrm{C}=\mathrm{CC}(=\mathrm{O}) \mathrm{C}$ & $\operatorname{lnChl}=1 \mathrm{~S} / \mathrm{C} 7 \mathrm{H} 8 \mathrm{O} / \mathrm{c} 1-3-4-5-6-7(2) 8 / \mathrm{h} 3-4,6 \mathrm{H}, 1 \mathrm{H} 2,2 \mathrm{H} 3$ \\
\hline 3073 & C7H13-1D50OH & $\mathrm{C}=\mathrm{CCCC}(\mathrm{OO}) \mathrm{CC}$ & InChl=15/C7H14O2/c1-3-5-6-7(4-2)9-8/h3,7-8H,1,4-6H2,2H3 \\
\hline 3074 & $\mathrm{C} 701-40 \mathrm{OH} 2$ & $01 \mathrm{Cc}(\mathrm{C})(00) \mathrm{CC} 1 \mathrm{Cc}$ & InChl=1S/C7H14O3/c1-3-6-4-7(2,10-8)5-9-6/h6,8H,3-5H2,1-2H3 \\
\hline 3075 & $\mathrm{C} 7 \mathrm{OOH} 4 \mathrm{OOH} 5-\mathrm{C}$ & $\mathrm{CC}(\mathrm{C})[\mathrm{CH}] \mathrm{C}(\mathrm{OO}) \mathrm{C}(\mathrm{OO}) \mathrm{C}$ & InChl=1S/C7H15O4/c1-5(2)4-7(11-9)6(3)10-8/h4-9H,1-3H3 \\
\hline 3076 & M2V5F & $\mathrm{C}=\mathrm{CC} 1=\mathrm{CC}=\mathrm{C}(\mathrm{C}) \mathrm{O} 1$ & InChl=1S/C7H8O/c1-3-7-5-4-6(2)8-7/h3-5H,1H2,2H3 \\
\hline 3077 & $\mathrm{C} 7 \mathrm{H} 13-1 \mathrm{D} 6 \mathrm{OOH}$ & $\mathrm{C}=\mathrm{CCCCC}(\mathrm{OO}) \mathrm{C}$ & InChl=1S/C7H14O2/c1-3-4-5-6-7(2)9-8/h3,7-8H,1,4-6H2,2H3 \\
\hline 3078 & $\mathrm{C} 7 \mathrm{O} 1-40 \mathrm{OH} 3$ & O1CC(C)C(OO)C1CC & InChl=1S/C7H14O3/c1-3-6-7(10-8)5(2)4-9-6/h5-8H,3-4H2,1-2H3 \\
\hline 3079 & $\mathrm{C} 7 \mathrm{OOH} 5 \mathrm{OOH} 4-\mathrm{B}$ & $\mathrm{C}[\mathrm{C}] \mathrm{C}) \mathrm{CC}(\mathrm{OO}) \mathrm{C}(\mathrm{OO}) \mathrm{C}$ & InChl=1S/C7H15O4/c1-5(2)4-7(11-9)6(3)10-8/h6-9H,4H2,1-3H3 \\
\hline 3080 & $\mathrm{HOC} 6 \mathrm{H} 4 \mathrm{CH} 3$ & $\mathrm{C} 1=\mathrm{CC}(\mathrm{C})=\mathrm{CC}(\mathrm{O})=\mathrm{C} 1$ & InChl=1S/C7H8O/c1-6-3-2-4-7(8)5-6/h2-5,8H,1H3 \\
\hline 3081 & $\mathrm{C} 7 \mathrm{H} 13-2 \mathrm{D} 10 \mathrm{OH}$ & $\mathrm{C}(\mathrm{OO}) \mathrm{C}=\mathrm{CCCCC}$ & InChl=1S/C7H14O2/c1-2-3-4-5-6-7-9-8/h5-6,8H,2-4,7H2,1H3 \\
\hline 3082 & C7O1-50OH1 & OOCC1COC(C)CC1 & InChl=1S/C7H14O3/c1-6-2-3-7(4-9-6)5-10-8/h6-8H,2-5H2,1H3 \\
\hline 3083 & C7OOH6OOH5-C & $\mathrm{CC}(\mathrm{C})[\mathrm{CH}] \mathrm{CC}(\mathrm{OO}) \mathrm{COO}$ & InChl=15/C7H15O4/c1-6(2)3-4-7(11-9)5-10-8/h3,6-9H,4-5H2,1-2H3 \\
\hline 3084 & $\mathrm{C} 6 \mathrm{H} 5 \mathrm{CH} 2 \mathrm{OH}$ & $\mathrm{C} 1=\mathrm{CC}(\mathrm{CO})=\mathrm{CC}=\mathrm{C} 1$ & InChl=1S/C7H8O/c8-6-7-4-2-1-3-5-7/h1-5,8H,6H2 \\
\hline 3085 & C7H13-2D4OOH & $\mathrm{CC}=\mathrm{CC}(\mathrm{OO}) \mathrm{CCC}$ & InChl=1S/C7H14O2/c1-3-5-7(9-8)6-4-2/h3,5,7-8H,4,6H2,1-2H3 \\
\hline 3086 & C7O1-50OH3 & $01 \mathrm{CC}(\mathrm{C}) \mathrm{C}(\mathrm{OO}) \mathrm{CC} 1 \mathrm{C}$ & InChl=1S/C7H14O3/c1-5-4-9-6(2)3-7(5)10-8/h5-8H,3-4H2,1-2H3 \\
\hline 3087 & С70ОН10OH3-Е & $\mathrm{OOCC}(\mathrm{C}) \mathrm{C}(\mathrm{OOO}) \mathrm{C}[\mathrm{CH}] \mathrm{C}$ & InChl=15/C7H15O4/c1-3-4-7(11-9)6(2)5-10-8/h3,6-9H,4-5H2,1-2H3 \\
\hline 3088 & CHE2104J5M & $\mathrm{C} 1=\mathrm{C}[\mathrm{CH}] \mathrm{C}(\mathrm{C}) \mathrm{CC} 1(=\mathrm{O})$ & InChl=1S/C7H9O/c1-6-3-2-4-7(8)55-6/h2-4,6H,5H2,1H3 \\
\hline 3089 & C7H13-2D5OOH & $\mathrm{CC}=\mathrm{CCC}(\mathrm{OO}) \mathrm{CC}$ & InChl=1S/C7H14O2/c1-3-5-6-7(4-2)9-8/h3,5,7-8H,4,6H2,1-2H3 \\
\hline 3090 & C7KET12 & $C(=0) C(O O) C C C C C$ & InChl=1S/C7H14O3/c1-2-3-4-5-7(6-8)10-9/h6-7,9H,2-5H2,1H3 \\
\hline
\end{tabular}




\begin{tabular}{|c|c|c|c|}
\hline 3091 & $\mathrm{C7OOH} 50 \mathrm{OH} 4-\mathrm{F}$ & $\mathrm{CC}(\mathrm{C}) \mathrm{CC}(\mathrm{OO}) \mathrm{C}(\mathrm{OO})[\mathrm{CH} 2]$ & InChl=15/C7H15O4/c1-5(2)4-7(11-9)6(3)10-8/h5-9H,3-4H2,1-2H3 \\
\hline 3092 & CHE2104J6M & $\mathrm{C} 1=\mathrm{C}[\mathrm{CH}] \mathrm{CC}(\mathrm{C}) \mathrm{C} 1(=\mathrm{O})$ & InChl=15/C7H9O/c1-6-4-2-3-5-7(6)8/h2-3,5-6H,4H2,1H3 \\
\hline 3093 & C7H13-2D6OOH & $\mathrm{CC}=\mathrm{CCCC}(\mathrm{OO}) \mathrm{C}$ & $\operatorname{lnChl}=1 \mathrm{~S} / \mathrm{C} 7 \mathrm{H} 14 \mathrm{O} 2 / \mathrm{c1}-3-4-5-6-7(2) 9-8 / \mathrm{h} 3-4,7-8 \mathrm{H}, 5-6 \mathrm{H} 2,1-2 \mathrm{H} 3$ \\
\hline 3094 & C7KET13 & $\mathrm{C}(=\mathrm{O}) \mathrm{CC}(\mathrm{OO}) \mathrm{CCCC}$ & InChl=1S/C7H14O3/c1-2-3-4-7(10-9)5-6-8/h6-7,9H,2-5H2,1H3 \\
\hline 3095 & C7OOH10OH4-B & $\mathrm{OOC}[\mathrm{C}](\mathrm{C}) \mathrm{CC}(\mathrm{OO}) \mathrm{CC}$ & InChl=1S/C7H1504/c1-3-7(11-9)4-6(2)5-10-8/h7-9H,3-5H2,1-2H3 \\
\hline 3096 & HPT35DE2O1J & $[\mathrm{CH} 2] \mathrm{C}=\mathrm{O}) \mathrm{C}=\mathrm{CC}=\mathrm{CC}$ & InChl=1S/C7H9O/c1-3-4-5-6-7(2)8/h3-6H,2H2,1H3 \\
\hline 3097 & C7H13-2D70OH & $\mathrm{CC}=\mathrm{CCCCC}(\mathrm{OO})$ & InChl=1S/C7H1402/c1-2-3-4-5-6-7-9-8/h2-3,8H,4-7H2,1H3 \\
\hline 3098 & C7KET14 & $\mathrm{C}(=0) \mathrm{CCC}(\mathrm{OO}) \mathrm{CCC}$ & InChl=1S/C7H14O3/c1-2-4-7(10-9)5-3-6-8/h6-7,9H,2-5H2,1H3 \\
\hline 3099 & C7OOH5OOH6-D & $\mathrm{CC}(\mathrm{C}) \mathrm{C}[\mathrm{CH}] \mathrm{C}(\mathrm{OO}) \mathrm{COO}$ & InChl=1S/C7H15O4/c1-6(2)3-4-7(11-9)5-10-8/h4,6-9H,3,5H2,1-2H3 \\
\hline 3100 & HPT45DE2O3J & $\mathrm{CC}(=\mathrm{O})[\mathrm{CH}] \mathrm{C}=\mathrm{C}=\mathrm{CC}$ & InChl=1S/C7H9O/c1-3-4-5-6-7(2)8/h3,5-6H,1-2H3 \\
\hline 3101 & C7H13-3D10OH & $\mathrm{C}(\mathrm{OO}) \mathrm{CC}=\mathrm{CCCC}$ & InChl=1S/C7H1402/c1-2-3-4-5-6-7-9-8/h4-5,8H,2-3,6-7H2,1H3 \\
\hline 3102 & C7KET15 & $\mathrm{C}(=0) \operatorname{cccc}(\mathrm{OO}) \mathrm{CC}$ & InChl=1S/C7H14O3/c1-2-7(10-9)5-3-4-6-8/h6-7,9H,2-5H2,1H3 \\
\hline 3103 & $\mathrm{C7OOH10OH4-F}$ & $\mathrm{OOCC}(\mathrm{C}) \mathrm{CC}(\mathrm{OO}) \mathrm{C}[\mathrm{CH} 2]$ & InChl=1S/C7H1504/c1-3-7(11-9)4-6(2)5-10-8/h6-9H,1,3-5H2,2H3 \\
\hline 3104 & HPT46DE3O2J & $\mathrm{C}[\mathrm{CH}] \mathrm{C}(=\mathrm{O}) \mathrm{C}=\mathrm{CC}=\mathrm{C}$ & InChl=1S/C7H9O/c1-3-5-6-7(8)4-2/h3-6H,1H2,2H3 \\
\hline 3105 & C7H13-3D2OOH & $\mathrm{CC}(\mathrm{OO}) \mathrm{C}=\mathrm{CCCC}$ & InChl=1S/C7H14O2/c1-3-4-5-6-7(2)9-8/h5-8H,3-4H2,1-2H3 \\
\hline 3106 & C7KET21 & $\mathrm{C}(\mathrm{OO}) \mathrm{C}(=0) \mathrm{CCCCC}$ & InChl=1S/C7H14O3/c1-2-3-4-5-7(8)6-10-9/h9H,2-6H2,1H3 \\
\hline 3107 & C7H14OOH1-2O2 & $\mathrm{C}(\mathrm{OO}) \mathrm{C}(\mathrm{O}[\mathrm{O}]) \mathrm{CCCCC}$ & InChl=1S/C7H1504/c1-2-3-4-5-7(11-9)6-10-8/h7-8H,2-6H2,1H3 \\
\hline 3108 & HPT56DE304J & $\mathrm{CCC}(=0)[\mathrm{CH}] \mathrm{C}=\mathrm{C}=\mathrm{C}$ & $\operatorname{lnChl}=1 \mathrm{~S} / \mathrm{C} 7 \mathrm{H} 9 \mathrm{O} / \mathrm{c} 1-3-5-6-7(8) 4-2 / \mathrm{h} 5-6 \mathrm{H}, 1,4 \mathrm{H} 2,2 \mathrm{H} 3$ \\
\hline 3109 & C7H13-3D5OOH & $\mathrm{CCC}=\mathrm{CC}(\mathrm{OO}) \mathrm{CC}$ & InChl=1S/C7H14O2/c1-3-5-6-7(4-2)9-8/h5-8H,3-4H2,1-2H3 \\
\hline 3110 & C7KET23 & $\mathrm{CC}(=\mathrm{O}) \mathrm{C}(\mathrm{OOO}) \mathrm{CCCC}$ & InChl=1S/C7H14O3/c1-3-4-5-7(10-9)6(2)8/h7,9H,3-5H2,1-2H3 \\
\hline 3111 & $\mathrm{C}$ C7H14OOH1-302 & $\mathrm{C}(\mathrm{OO}) \mathrm{CC}(\mathrm{O}[\mathrm{O}]) \mathrm{CCCC}$ & InChl=1S/C7H1504/c1-2-3-4-7(11-9)5-6-10-8/h7-8H,2-6H2,1H3 \\
\hline 3112 & M25JEF-A & $\mathrm{CC} 1=\mathrm{CC}=\mathrm{C}([\mathrm{CH}] \mathrm{C}) \mathrm{O} 1$ & InChl=1S/C7H9O/c1-3-7-5-4-6(2)8-7/h3-5H,1-2H3 \\
\hline 3113 & $\mathrm{C} 7 \mathrm{H} 13-3 \mathrm{D} 6 \mathrm{OOH}$ & $\mathrm{CCC}=\mathrm{CCC}(\mathrm{OO}) \mathrm{C}$ & InChl=1S/C7H14O2/c1-3-4-5-6-7(2)9-8/h4-5,7-8H,3,6H2,1-2H3 \\
\hline 3114 & C7KET24 & $\mathrm{CC}(=\mathrm{O}) \mathrm{CC}(\mathrm{OO}) \mathrm{CCC}$ & InChl=1S/C7H14O3/c1-3-4-7(10-9)5-6(2)8/h7,9H,3-5H2,1-2H3 \\
\hline 3115 & C7H14OOH1-402 & $\mathrm{C}(\mathrm{OO}) \mathrm{CCC}(\mathrm{O}[\mathrm{O}]) \mathrm{CCC}$ & InChl=1S/C7H1504/c1-2-4-7(11-9)5-3-6-10-8/h7-8H,2-6H2,1H3 \\
\hline 3116 & M2E5JF-P & $\mathrm{CC} 1=\mathrm{CC}=\mathrm{C}(\mathrm{C}[\mathrm{CH} 2]) \mathrm{O} 1$ & $\operatorname{lnChl}=1 \mathrm{~S} / \mathrm{C} 7 \mathrm{H} 90 / \mathrm{c} 1-3-7-5-4-6(2) 8-7 / \mathrm{h} 4-5 \mathrm{H}, 1,3 \mathrm{H} 2,2 \mathrm{H} 3$ \\
\hline 3117 & $\mathrm{C7H13-3D70OH}$ & $\mathrm{CCC}=\mathrm{CCCC}(\mathrm{OO})$ & $\operatorname{lnChl}=1 \mathrm{~S} / \mathrm{C} 7 \mathrm{H} 14 \mathrm{O} 2 / \mathrm{c} 1-2-3-4-5-6-7-9-8 / \mathrm{h} 3-4,8 \mathrm{H}, 2,5-7 \mathrm{H} 2,1 \mathrm{H} 3$ \\
\hline 3118 & C7KET25 & $\mathrm{CC}(=0) \mathrm{CCC}(\mathrm{OO}) \mathrm{CC}$ & InChl=1S/C7H14O3/c1-3-7(10-9)5-4-6(2)8/h7,9H,3-5H2,1-2H3 \\
\hline 3119 & C7H14OOH1-502 & $\mathrm{C}(\mathrm{OO}) \mathrm{CCCC}(\mathrm{O}[\mathrm{O}]) \mathrm{CC}$ & InChl=1S/C7H1504/c1-2-7(11-9)5-3-4-6-10-8/h7-8H,2-6H2,1H3 \\
\hline 3120 & M2JE5F & {$[\mathrm{CH} 2] \mathrm{C} 1=\mathrm{CC}=\mathrm{C}(\mathrm{CC}) \mathrm{O} 1$} & $\operatorname{lnChl}=1 \mathrm{~S} / \mathrm{C} 7 \mathrm{H} 9 \mathrm{O} / \mathrm{c1}-3-7-5-4-6(2) 8-7 / \mathrm{h} 4-5 \mathrm{H}, 2-3 \mathrm{H} 2,1 \mathrm{H} 3$ \\
\hline 3121 & I24C7D1-1AOOH & $\mathrm{C}=\mathrm{C}(\mathrm{COO}) \mathrm{C} C(\mathrm{C}) \mathrm{C}$ & InChl=1S/C7H1402/c1-6(2)4-7(3)5-9-8/h6,8H,3-5H2,1-2H3 \\
\hline 3122 & C7KET26 & $\mathrm{CC}(=0) \operatorname{cccc}(\mathrm{OO}) \mathrm{C}$ & InChl=1S/C7H14O3/c1-6(8)4-3-5-7(2)10-9/h7,9H,3-5H2,1-2H3 \\
\hline 3123 & $\mathrm{C} 7 \mathrm{H} 14 \mathrm{OOH} 2-102$ & $\mathrm{C}(\mathrm{O}[\mathrm{O}]) \mathrm{C}(\mathrm{OO}) \mathrm{CCCCC}$ & InChl=1S/C7H1504/c1-2-3-4-5-7(11-9)6-10-8/h7,9H,2-6H2,1H3 \\
\hline 3124 & 124C7D13Y5-5R & $\mathrm{C}=\mathrm{C}(\mathrm{C}) \mathrm{C}=\mathrm{C}(\mathrm{C})[\mathrm{C}]=\mathrm{O}$ & $\operatorname{lnChl}=1 \mathrm{~S} / \mathrm{C} 7 \mathrm{H} 9 \mathrm{O} / \mathrm{c1}-6(2) 4-7(3) 5-8 / \mathrm{h} 4 \mathrm{H}, 1 \mathrm{H} 2,2-3 \mathrm{H} 3$ \\
\hline 3125 & 124C7D1-30OH & $\mathrm{C}=\mathrm{C}(\mathrm{C}) \mathrm{C}(\mathrm{OO}) \mathrm{C}(\mathrm{C}) \mathrm{C}$ & InChl=1S/C7H14O2/c1-5(2)7(9-8)6(3)4/h6-8H,1H2,2-4H3 \\
\hline 3126 & C7KET31 & $\mathrm{C}(\mathrm{OO}) \mathrm{CC}(=\mathrm{O}) \mathrm{CCCC}$ & InChl=1S/C7H14O3/c1-2-3-4-7(8)5-6-10-9/h9H,2-6H2,1H3 \\
\hline 3127 & $\mathrm{C} 7 \mathrm{H} 14 \mathrm{OOH} 2-3 \mathrm{O} 2$ & $\mathrm{CC}(\mathrm{OO}) \mathrm{C}(\mathrm{O}[\mathrm{O}]) \mathrm{CCCC}$ & InChl=1S/C7H1504/c1-3-4-5-7(11-9)6(2)10-8/h6-8H,3-5H2,1-2H3 \\
\hline 3128 & I24C7D13Y1A-1AR & $C=C([C]=O) C=C(C) C$ & InChl=1S/C7H9O/c1-6(2)4-7(3)5-8/h4H,3H2,1-2H3 \\
\hline 3129 & I24C7D2-10OH & $\mathrm{C}(\mathrm{OO}) \mathrm{C}(\mathrm{C})=\mathrm{CC}(\mathrm{C}) \mathrm{C}$ & InChl=1S/C7H1402/c1-6(2)4-7(3)5-9-8/h4,6,8H,5H2,1-3H3 \\
\hline 3130 & C7KET32 & $\mathrm{CC}(\mathrm{OO}) \mathrm{C}(=\mathrm{O}) \mathrm{CCCC}$ & InChl=15/C7H14O3/c1-3-4-5-7(8)6(2)10-9/h6,9H,3-5H2,1-2H3 \\
\hline 3131 & $\mathrm{C} 7 \mathrm{H} 14 \mathrm{OOH} 2-4 \mathrm{O} 2$ & $\mathrm{CC}(\mathrm{OO}) \mathrm{CC}(\mathrm{O}[\mathrm{O}]) \mathrm{CCC}$ & InChl=1S/C7H15O4/c1-3-4-7(11-9)5-6(2)10-8/h6-8H,3-5H2,1-2H33 \\
\hline 3132 & HPT34DE2O & $\mathrm{CC}(=0) \mathrm{C}=\mathrm{C}=\mathrm{CCC}$ & InChl=1S/C7H10O/c1-3-4-5-6-7(2)8/h4,6H,3H2,1-2H3 \\
\hline 3133 & I24C7D2-5OOH & $\mathrm{CC}(\mathrm{C})=\mathrm{CC}(\mathrm{C}) \mathrm{COO}$ & InChl=1S/C7H1402/c1-6(2)4-7(3)5-9-8/h4,7-8H,5H2,1-3H3 \\
\hline 3134 & C7KET34 & $\operatorname{cCC}(=0) C(O O) C C C$ & InChl=1S/C7H14O3/c1-3-5-7(10-9)6(8)4-2/h7,9H,3-5H2,1-2H3 \\
\hline 3135 & $\mathrm{C} 7 \mathrm{H} 140 \mathrm{OH} 2-5 \mathrm{O} 2$ & $\mathrm{CC}(00) \mathrm{CCC}(\mathrm{O}[0]) \mathrm{CC}$ & InChl=1S/C7H15O4/c1-3-7(11-9)5-4-6(2)10-8/h6-8H,3-5H2,1-2H3 \\
\hline 3136 & HPT45DE3O & $\mathrm{CC}=\mathrm{C}=\mathrm{CC}(=\mathrm{O}) \mathrm{CC}$ & InChl=1S/C7H100/c1-3-5-6-7(8)4-2/h3,6H,4H2,1-2H3 \\
\hline 3137 & NEC7D3-10OH & $\mathrm{C}(\mathrm{OO}) \mathrm{C}(\mathrm{C})(\mathrm{C}) \mathrm{C}=\mathrm{CC}$ & InChl=1S/C7H1402/c1-4-5-7(2,3)6-9-8/h4-5,8H,6H2,1-3H3 \\
\hline 3138 & C7KET35 & $\mathrm{CCC}(=0) \mathrm{CC}(\mathrm{OO}) \mathrm{CC}$ & InChl=1S/C7H14O3/c1-3-6(8)5-7(4-2)10-9/h7,9H,3-5H2,1-2H3 \\
\hline 3139 & $\mathrm{C} 7 \mathrm{H} 14 \mathrm{OOH} 2-6 \mathrm{O} 2$ & $\mathrm{CC}(\mathrm{OO}) \mathrm{CCCC}(\mathrm{O}[\mathrm{O}]) \mathrm{C}$ & InChl=1S/C7H1504/c1-6(10-8)4-3-5-7(2)11-9/h6-8H,3-5H2,1-2H3 \\
\hline 3140 & M2E5F & $\mathrm{CC} 1=\mathrm{CC}=\mathrm{C}(\mathrm{CC}) \mathrm{O} 1$ & InChl=1S/C7H100/c1-3-7-5-4-6(2)8-7/h4-5H,3H2,1-2H3 \\
\hline 3141 & C7H15OO-1-2 & {$[0] \mathrm{OCC}(\mathrm{C}) \mathrm{CCCC}$} & InChl=15/C7H15O2/c1-3-4-5-7(2)6-9-8/h7H,3-6H2,1-2H3 \\
\hline 3142 & C7KET36 & $\mathrm{CCC}(=0) \operatorname{ccc}(\mathrm{OO}) \mathrm{C}$ & InChl=1S/C7H14O3/c1-3-7(8)5-4-6(2)10-9/h6,9H,3-5H2,1-2H3 \\
\hline 3143 & C7H14OOH3-102 & $\mathrm{C}(\mathrm{O}[\mathrm{O}]) \mathrm{CC}(\mathrm{OO}) \mathrm{CCCC}$ & InChl=1S/C7H1504/c1-2-3-4-7(11-9)5-6-10-8/h7,9H,2-6H2,1H3 \\
\hline 3144 & 124C7D13Y5 & $\mathrm{C}=\mathrm{C}(\mathrm{C}) \mathrm{C}=\mathrm{C}(\mathrm{C}) \mathrm{C}=\mathrm{O}$ & InChl=1S/C7H100/c1-6(2)4-7(3)5-8/h4-5H,1H2,2-3H3 \\
\hline 3145 & C7H15OO-2-2 & $\mathrm{CC}(\mathrm{C})(\mathrm{O}[\mathrm{O}]) \mathrm{CCCC}$ & InChl=1S/C7H1502/c1-4-5-6-7(2,3)9-8/h4-6H2,1-3H3 \\
\hline 3146 & C7KET37 & $\operatorname{ccC}(=0) \operatorname{cccc}(00)$ & InChl=1S/C7H1403/c1-2-7(8)5-3-4-6-10-9/h9H,2-6H2,1H3 \\
\hline 3147 & $\mathrm{C}$ C7H14OOH3-2O2 & $\mathrm{CC}(\mathrm{O}[0]) \mathrm{C}(\mathrm{OO}) \mathrm{CCCC}$ & InChl=1S/C7H15O4/c1-3-4-5-7(11-9)6(2)10-8/h6-7,9H,3-5H2,1-2H3 \\
\hline 3148 & I24C7D13Y1A & $\mathrm{C}=\mathrm{C}(\mathrm{C}=\mathrm{O}) \mathrm{C}=\mathrm{C}(\mathrm{C}) \mathrm{C}$ & InChl=1S/C7H100/c1-6(2)4-7(3)5-8/h4-5H,3H2,1-2H3 \\
\hline 3149 & C7H15OO-3-2 & $\mathrm{CC}(\mathrm{C}) \mathrm{C}(\mathrm{O}[\mathrm{O}]) \mathrm{CCC}$ & InChl=1S/C7H1502/c1-4-5-7(9-8)6(2)3/h6-7H,4-5H2,1-3H3 \\
\hline 3150 & C7KET41 & $\mathrm{C}(\mathrm{OO}) \mathrm{CCC}(=0) \mathrm{CCC}$ & InChl=1S/C7H14O3/c1-2-4-7(8)5-3-6-10-9/h9H,2-6H2,1H3 \\
\hline 3151 & C7H14OOH3-402 & $\mathrm{CCC}(\mathrm{OO}) \mathrm{C}(\mathrm{O}[\mathrm{O}]) \mathrm{CCC}$ & $\operatorname{lnChl}=1 \mathrm{~S} / \mathrm{C} 7 \mathrm{H} 1504 / \mathrm{c} 1-3-5-7(11-9) 6(4-2) 10-8 / \mathrm{h} 6-8 \mathrm{H}, 3-5 \mathrm{H} 2,1-2 \mathrm{H} 3$ \\
\hline 3152 & H4E2O3J5M & $\mathrm{CC}(=\mathrm{O})[\mathrm{CH}] \mathrm{C}=\mathrm{C}(\mathrm{C}) \mathrm{C}$ & InChl=1S/C7H11O/c1-6(2)4-5-7(3)8/h4-5H,1-3H3 \\
\hline 3153 & C7H15OO-4-2 & $\mathrm{CC}(\mathrm{C}) \mathrm{CC}(\mathrm{O}[\mathrm{O}]) \mathrm{CC}$ & InChl=15/C7H1502/c1-4-7(9-8)5-6(2)3/h6-7H,4-5H2,1-3H3 \\
\hline 3154 & C7KET42 & $\mathrm{CC}(\mathrm{OO}) \mathrm{CC}(=0) \mathrm{CCC}$ & InChl=1S/C7H14O3/c1-3-4-7(8)5-6(2)10-9/h6,9H,3-5H2,1-2H3 \\
\hline 3155 & C7H14OOH3-5O2 & $\mathrm{CCC}(\mathrm{OO}) \mathrm{CC}(\mathrm{O}[\mathrm{O}]) \mathrm{CC}$ & InChl=1S/C7H1504/c1-3-6(10-8)5-7(4-2)11-9/h6-8H,3-5H2,1-2H3 \\
\hline 3156 & H5E2O4J5M & $\mathrm{CC}(=\mathrm{O}) \mathrm{C}[\mathrm{CH}] \mathrm{C}(\mathrm{C})=\mathrm{C}$ & InChl=1S/C7H110/c1-6(2)4-5-7(3)8/h4H,1,5H2,2-3H3 \\
\hline 3157 & C7H15OO-5-2 & $\mathrm{CC}(\mathrm{C}) \mathrm{CCC}(\mathrm{O}[\mathrm{O}]) \mathrm{C}$ & InChl=1S/C7H15O2/c1-6(2)4-5-7(3)9-8/h6-7H,4-5H2,1-3H3 \\
\hline 3158 & C7KET43 & $\operatorname{ccc}(00) C(=0) \operatorname{ccc}$ & InChl=1S/C7H1403/c1-3-5-6(8)7(4-2)10-9/h7,9H,3-5H2,1-2H3 \\
\hline 3159 & $\mathrm{C}$ C7H14OOH3-6O2 & $\operatorname{ccc}(00) \operatorname{ccc}(\mathrm{O}[\mathrm{O}]) \mathrm{C}$ & InChl=15/C7H15O4/c1-3-7(11-9)5-4-6(2)10-8/h6-7,9H,3-5H2,1-2H3 \\
\hline 3160 & TMF2253J & $\mathrm{CC} 1=\mathrm{C}[\mathrm{CH}] \mathrm{C}(\mathrm{C})(\mathrm{C}) \mathrm{O} 1$ & InChl=1S/C7H11O/c1-6-4-5-7(2,3)8-6/h4-5H,1-3H3 \\
\hline 3161 & C7H15OO-6-2 & $\mathrm{CC}(\mathrm{C}) \mathrm{CCCCO}[\mathrm{O}]$ & InChl=1S/C7H15O2/c1-7(2)5-3-4-6-9-8/h7H,3-6H2,1-2H3 \\
\hline 3162 & $\mathrm{C} 7 \mathrm{H} 13 \mathrm{O} 12-30 \mathrm{OH}$ & $\mathrm{C} 1 \mathrm{C}(\mathrm{O} 1) \mathrm{C}(\mathrm{OO}) \mathrm{CCCC}$ & InChl=1S/C7H14O3/c1-2-3-4-6(10-8)7-5-9-7/h6-8H,2-5H2,1H3 \\
\hline 3163 & C7H14OOH3-702 & $\operatorname{ccc}(00) \operatorname{cccc}(O[O])$ & InChl=1S/C7H1504/c1-2-7(11-9)5-3-4-6-10-8/h7,9H,2-6H2,1H3 \\
\hline 3164 & 124C7D13-5OR & $\mathrm{C}=\mathrm{C}(\mathrm{C}) \mathrm{C}=\mathrm{C}(\mathrm{C}) \mathrm{C}[\mathrm{O}]$ & InChl=1S/C7H110/c1-6(2)4-7(3)5-8/h4H,1,5H2,2-3H3 \\
\hline 3165 & C7OOH1-2A & $\mathrm{OOCC}([\mathrm{CH} 2]) \mathrm{CCCC}$ & InChl=15/C7H15O2/c1-3-4-5-7(2)6-9-8/h7-8H,2-6H2,1H3 \\
\hline 3166 & $\mathrm{C} 7 \mathrm{H} 13 \mathrm{O} 12-4 \mathrm{OOH}$ & $\mathrm{C} 1 \mathrm{C}(\mathrm{O} 1) \mathrm{CC}(\mathrm{OO}) \mathrm{CCC}$ & InChl=1S/C7H14O3/c1-2-3-6(10-8)4-7-5-9-7/h6-8H,2-5H2,1H3 \\
\hline 3167 & $\mathrm{C} 7 \mathrm{H} 14 \mathrm{OOH} 4-102$ & $\operatorname{cccc}(00) \operatorname{ccc}(O[O])$ & InChl=1S/C7H15O4/c1-2-4-7(11-9)5-3-6-10-8/h7,9H,2-6H2,1H3 \\
\hline 3168 & I24C7D13OH5-5AR & $\mathrm{C}=\mathrm{C}(\mathrm{C}) \mathrm{C}=\mathrm{C}([\mathrm{CH} 2]) \mathrm{CO}$ & $\operatorname{lnChl}=1 \mathrm{~S} / \mathrm{C} 7 \mathrm{H} 11 \mathrm{O} / \mathrm{c} 1-6(2) 4-7(3) 5-8 / \mathrm{h} 4,8 \mathrm{H}, 1,3,5 \mathrm{H} 2,2 \mathrm{H} 3$ \\
\hline 3169 & $\mathrm{C} 7 \mathrm{OOH} 1-2 \mathrm{~B}$ & $0 O C[C](C) C C C C$ & $\operatorname{lnChl}=1 \mathrm{~S} / \mathrm{C} 7 \mathrm{H} 15 \mathrm{O} 2 / \mathrm{c} 1-3-4-5-7(2) 6-9-8 / \mathrm{h} 8 \mathrm{H}, 3-6 \mathrm{H} 2,1-2 \mathrm{H} 3$ \\
\hline 3170 & $\mathrm{C} 7 \mathrm{H} 13 \mathrm{O} 12-5 \mathrm{OOH}$ & $\mathrm{C} 1 \mathrm{C}(\mathrm{O} 1) \mathrm{CCC}(\mathrm{OO}) \mathrm{CC}$ & InChl=1S/C7H14O3/c1-2-6(10-8)3-4-7-5-9-7/h6-8H,2-5H2,1H3 \\
\hline 3171 & $\mathrm{C} 7 \mathrm{H} 14 \mathrm{OOH} 4-2 \mathrm{O} 2$ & $\operatorname{cccc}(\mathrm{OO}) \operatorname{cc}(\mathrm{O}[\mathrm{O}]) \mathrm{C}$ & InChl=1S/C7H15O4/c1-3-4-7(11-9)5-6(2)10-8/h6-7,9H,3-5H2,1-2H3 \\
\hline 3172 & I24C7D13-1AOR & $\mathrm{C}=\mathrm{C}(\mathrm{C}[\mathrm{O}]) \mathrm{C}=\mathrm{C}(\mathrm{C}) \mathrm{C}$ & InChl=1S/C7H110/c1-6(2)4-7(3)5-8/h4H,3,5H2,1-2H3 \\
\hline 3173 & $\mathrm{C7OOH} 1-2 \mathrm{C}$ & $\mathrm{OOCC}(\mathrm{C})[\mathrm{CH}] \mathrm{CCC}$ & InChl=1S/C7H15O2/c1-3-4-5-7(2)6-9-8/h5,7-8H,3-4,6H2,1-2H3 \\
\hline 3174 & $\mathrm{C} 7 \mathrm{H} 13 \mathrm{O} 12-60 \mathrm{OH}$ & $\mathrm{C} 1 \mathrm{C}(\mathrm{O} 1) \mathrm{CCCC}(\mathrm{OO}) \mathrm{C}$ & InChl=15/C7H1403/c1-6(10-8)3-2-4-7-5-9-7/h6-8H,2-5H2,1H3 \\
\hline 3175 & C7H14OOH4-3O2 & $\operatorname{cccc}(\mathrm{OO}) \mathrm{C}(\mathrm{O}[\mathrm{O}]) \mathrm{CC}$ & InChl=1S/C7H1504/c1-3-5-7(11-9)6(4-2)10-8/h6-7,9H,3-5H2,1-2H3 \\
\hline 3176 & 124C7D13OH5 & $\mathrm{C}=\mathrm{C}(\mathrm{C}) \mathrm{C}=\mathrm{C}(\mathrm{C}) \mathrm{CO}$ & InChl=1S/C7H12O/c1-6(2)4-7(3)5-8/h4,8H,1,5H2,2-3H3 \\
\hline 3177 & C7OOH1-2D & $\mathrm{OOCC}(\mathrm{C}) \mathrm{C}[\mathrm{CH}] \mathrm{CC}$ & InChl=1S/C7H15O2/c1-3-4-5-7(2)6-9-8/h4,7-8H,3,5-6H2,1-2H3 \\
\hline 3178 & $\mathrm{C} 7 \mathrm{H} 13 \mathrm{O} 13-2 \mathrm{OOH}$ & $\mathrm{C} 1 \mathrm{C}(\mathrm{OO}) \mathrm{C}(\mathrm{O} 1) \mathrm{CCCC}$ & InChl=1S/C7H14O3/c1-2-3-4-6-7(10-8)5-9-6/h6-8H,2-5H2,1H3 \\
\hline 3179 & C7H13Q12-3 & $\mathrm{C}(\mathrm{OO}) \mathrm{C}(\mathrm{OO})[\mathrm{CH}] \mathrm{CCCC}$ & InChl=15/C7H15O4/c1-2-3-4-5-7(11-9)6-10-8/h5,7-9H,2-4,6H2,1H3 \\
\hline 3180 & C7H13-1D3O & $\mathrm{C}=\mathrm{CC}([\mathrm{O}]) \mathrm{CCCC}$ & InChl=1S/C7H13O/c1-3-5-6-7(8)4-2/h4,7H,2-3,5-6H2,1H3 \\
\hline 3181 & C7OOH1-2E & $\mathrm{OOCC}(\mathrm{C}) \mathrm{CC}[\mathrm{CH}] \mathrm{C}$ & InChl=1S/C7H15O2/c1-3-4-5-7(2)6-9-8/h3,7-8H,4-6H2,1-2H3 \\
\hline
\end{tabular}




\begin{tabular}{|c|c|c|c|}
\hline 3182 & C7H13013-40OH & $\mathrm{C} 1 \mathrm{CC}(\mathrm{O} 1) \mathrm{C}(\mathrm{OO}) \mathrm{CCC}$ & InChl=1S/C7H14O3/c1-2-3-7(10-8)6-4-5-9-6/h6-8H,2-5H2,1H3 \\
\hline 3183 & C7H13Q12-4 & $\mathrm{C}(\mathrm{OO}) \mathrm{C}(\mathrm{OO}) \mathrm{C}[\mathrm{CH}] \mathrm{CCC}$ & InChl=1S/C7H15O4/c1-2-3-4-5-7(11-9)6-10-8/h4,7-9H,2-3,5-6H2,1H3 \\
\hline 3184 & C7H13-2D4O & $\mathrm{CC}=\mathrm{CC}([\mathrm{O}]) \mathrm{CCC}$ & InChl=1S/C7H13O/c1-3-5-7(8)6-4-2/h3,5,7H,4,6H2,1-2H3 \\
\hline 3185 & $\mathrm{C} 7 \mathrm{OOH} 2-2 \mathrm{~A}$ & {$[\mathrm{CH} 2] \mathrm{C}(\mathrm{C})(\mathrm{OO}) \mathrm{CCCC}$} & $\operatorname{lnChl}=1 \mathrm{~S} / \mathrm{C} 7 \mathrm{H} 1502 / \mathrm{O} 1-4-5-6-7(2,3) 9-8 / \mathrm{h} 8 \mathrm{H}, 2,4-6 \mathrm{H} 2,1,3 \mathrm{H} 3$ \\
\hline 3186 & C7H13013-50OH & $\mathrm{C} 1 \mathrm{CC}(01) \mathrm{CC}(\mathrm{OO}) \mathrm{CC}$ & InChl=1S/C7H1403/c1-2-6(10-8)5-7-3-4-9-7/h6-8H,2-5H2,1H3 \\
\hline 3187 & C7H13Q12-5 & $\mathrm{C}(\mathrm{OO}) \mathrm{C}(\mathrm{OO}) \mathrm{CC}[\mathrm{CH}] \mathrm{CC}$ & InChl=15/C7H15O4/c1-2-3-4-5-7(11-9)6-10-8/h3,7-9H,2,4-6H2,1H3 \\
\hline 3188 & C7H13-3D5O & $\mathrm{CCC}=\mathrm{CC}([0]) \mathrm{CC}$ & $\operatorname{lnChl}=1 \mathrm{~S} / \mathrm{C} 7 \mathrm{H} 130 / \mathrm{c1} 1-3-5-6-7(8) 4-2 / \mathrm{h} 5-7 \mathrm{H}, 3-4 \mathrm{H} 2,1-2 \mathrm{H} 3$ \\
\hline 3189 & $\mathrm{C} 7 \mathrm{OOH} 2-2 \mathrm{C}$ & $\mathrm{CC}(\mathrm{C})(\mathrm{OO})[\mathrm{CH}] \mathrm{CCC}$ & InChl=1S/C7H15O2/c1-4-5-6-7(2,3)9-8/h6,8H,4-5H2,1-3H3 \\
\hline 3190 & C7H13013-60OH & $\mathrm{C} 1 \mathrm{CC}(01) \mathrm{CCC}(\mathrm{OO}) \mathrm{C}$ & InChl=1S/C7H14O3/c1-6(10-8)2-3-7-4-5-9-7/h6-8H,2-5H2,1H3 \\
\hline 3191 & C7H13Q12-6 & $\mathrm{C}(\mathrm{OO}) \mathrm{C}(\mathrm{OO}) \mathrm{CCC}[\mathrm{CH}] \mathrm{C}$ & InChl=1S/C7H1504/c1-2-3-4-5-7(11-9)6-10-8/h2,7-9H,3-6H2,1H3 \\
\hline 3192 & 124C7D1-3OR & $\mathrm{C}=\mathrm{C}(\mathrm{C}) \mathrm{C}([0]) \mathrm{C}(\mathrm{C}) \mathrm{C}$ & $\operatorname{lnChl}=1 \mathrm{~S} / \mathrm{C} 7 \mathrm{H} 13 \mathrm{O} / \mathrm{c1}-5(2) 7(8) 6(3) 4 / \mathrm{h} 6-7 \mathrm{H}, 1 \mathrm{H} 2,2-4 \mathrm{H} 3$ \\
\hline 3193 & $\mathrm{C7OOH} 2-2 \mathrm{D}$ & $\mathrm{CC}(\mathrm{C})(\mathrm{OO}) \mathrm{C}[\mathrm{CH}] \mathrm{CC}$ & InChl=1S/C7H15O2/c1-4-5-6-7(2,3)9-8/h5,8H,4,6H2,1-3H3 \\
\hline 3194 & C7H13013-70OH & $\mathrm{C} 1 \mathrm{CC}(01) \operatorname{cccc}(00)$ & InChl=1S/C7H14O3/c8-10-5-2-1-3-7-4-6-9-7/h7-8H,1-6H2 \\
\hline 3195 & C7H13Q13-2 & $\mathrm{C}(\mathrm{OO})[\mathrm{CH}] \mathrm{C}(\mathrm{OO}) \mathrm{CCCC}$ & $\operatorname{lnChl}=15 / C 7 H 15 O 4 / c 1-2-3-4-7(11-9) 5-6-10-8 / \mathrm{h} 5,7-9 \mathrm{H}, 2-4,6 \mathrm{H} 2,1 \mathrm{H3}$ \\
\hline 3196 & 124C7D2-4OR & $\mathrm{CC}(\mathrm{C})=\mathrm{CC}([\mathrm{O}])(\mathrm{C}) \mathrm{C}$ & $\operatorname{lnChl}=1 \mathrm{~S} / \mathrm{C} 7 \mathrm{H} 130 / \mathrm{c} 1-6(2) 5-7(3,4) 8 / \mathrm{h} 5 \mathrm{H}, 1-4 \mathrm{H} 3$ \\
\hline 3197 & $\mathrm{C} 70 \mathrm{OH} 2-2 \mathrm{E}$ & $\mathrm{CC}(\mathrm{C})(\mathrm{OO}) \mathrm{CC}[\mathrm{CH}] \mathrm{C}$ & InChl=1S/C7H15O2/c1-4-5-6-7(2,3)9-8/h4,8H,5-6H2,1-3H3 \\
\hline 3198 & C7H13O14-2OOH & $\mathrm{C} 1 \mathrm{C}(00) \mathrm{CC}(01) \mathrm{CCC}$ & InChl=15/C7H14O3/c1-2-3-6-4-7(10-8)5-9-6/h6-8H,2-5H2,1H3 \\
\hline 3199 & C7H13Q13-4 & $\mathrm{C}(\mathrm{OO}) \mathrm{CC}(\mathrm{OO})[\mathrm{CH}] \mathrm{CCC}$ & InChl=1S/C7H15O4/c1-2-3-4-7(11-9)5-6-10-8/h4,7-9H,2-3,5-6H2,1H3 \\
\hline 3200 & NEC7Y4-3R & $\mathrm{CC}(\mathrm{C})(\mathrm{C})[\mathrm{CH}] \mathrm{C}(=\mathrm{O}) \mathrm{C}$ & InChl=1S/C7H130/c1-6(8)5-7(2,3)4/h5H,1-4H3 \\
\hline 3201 & $\mathrm{C} 7 \mathrm{OOH} 2-2 \mathrm{~F}$ & $\mathrm{CC}(\mathrm{C})(\mathrm{OO}) \mathrm{CCC}[\mathrm{CH} 2]$ & InChl=1S/C7H15O2/c1-4-5-6-7(2,3)9-8/h8H,1,4-6H2,2-3H3 \\
\hline 3202 & C7H13014-30OH & $\mathrm{C} 1 \mathrm{CC}(\mathrm{OO}) \mathrm{C}(01) \mathrm{CCC}$ & InChl=1S/C7H14O3/c1-2-3-6-7(10-8)4-5-9-6/h6-8H,2-5H2,1H3 \\
\hline 3203 & $\mathrm{C} 7 \mathrm{H} 13 \mathrm{Q} 13-5$ & $\mathrm{C}(\mathrm{OO}) \mathrm{CC}(\mathrm{OO}) \mathrm{C}[\mathrm{CH}] \mathrm{CC}$ & InChl=15/C7H15O4/c1-2-3-4-7(11-9)5-6-10-8/h3,7-9H,2,4-6H2,1H3 \\
\hline 3204 & NEC7Y4-5R & $\mathrm{CC}(\mathrm{C})(\mathrm{C}) \mathrm{CC}(=\mathrm{O})[\mathrm{CH} 2]$ & InChl=1S/C7H13O/c1-6(8)5-7(2,3)4/h1,5H2,2-4H3 \\
\hline 3205 & $\mathrm{C} 7 \mathrm{OOH} 3-2 \mathrm{~A}$ & {$[\mathrm{CH} 2] \mathrm{C}(\mathrm{C}) \mathrm{C}(\mathrm{OO}) \mathrm{CCC}$} & InChl=1S/C7H15O2/c1-4-5-7(9-8)6(2)3/h6-8H,2,4-5H2,1,3H3 \\
\hline 3206 & $\mathrm{C} 7 \mathrm{H} 13014-5 \mathrm{OOH}$ & $\mathrm{C} 1 \mathrm{CCC}(\mathrm{O} 1) \mathrm{C}(\mathrm{OO}) \mathrm{CC}$ & InChl=1S/C7H14O3/c1-2-6(10-8)7-4-3-5-9-7/h6-8H,2-5H2,1H3 \\
\hline 3207 & C7H13Q13-6 & $\mathrm{C}(\mathrm{OO}) \mathrm{CC}(\mathrm{OO}) \mathrm{CC}[\mathrm{CH}] \mathrm{C}$ & InChl=1S/C7H1504/c1-2-3-4-7(11-9)5-6-10-8/h2,7-9H,3-6H2,1H3 \\
\hline 3208 & 124C7D1-4OR & $\mathrm{C}=\mathrm{C}(\mathrm{C}) \mathrm{CC}([\mathrm{O}])(\mathrm{C}) \mathrm{C}$ & InChl=1S/C7H130/c1-6(2)5-7(3,4)8/h1,5H2,2-4H3 \\
\hline 3209 & C7OOH3-2B & $\mathrm{C}[\mathrm{C}](\mathrm{C}) \mathrm{C}(\mathrm{OO}) \mathrm{CCC}$ & InChl=15/C7H1502/c1-4-5-7(9-8)6(2)3/h7-8H,4-5H2,1-3H3 \\
\hline 3210 & C7H13014-60OH & $\mathrm{C} 1 \mathrm{CCC}(\mathrm{O} 1) \mathrm{CC}(\mathrm{OO}) \mathrm{C}$ & InChl=15/C7H14O3/c1-6(10-8)5-7-3-2-4-9-7/h6-8H,2-5H2,1H3 \\
\hline 3211 & C7H13Q13-7 & $\mathrm{C}(\mathrm{OO}) \mathrm{CC}(\mathrm{OO}) \mathrm{CCC}[\mathrm{CH} 2]$ & $\operatorname{lnChl}=1 \mathrm{~S} / \mathrm{C} 7 \mathrm{H} 1504 / \mathrm{C} 1-2-3-4-7(11-9) 5-6-10-8 / \mathrm{h} 7-9 \mathrm{H}, 1-6 \mathrm{H} 2$ \\
\hline 3212 & NEC7D4-3OR & $\operatorname{CC}(\mathrm{C})(\mathrm{C}) \mathrm{C}([\mathrm{O}]) \mathrm{C}=\mathrm{C}$ & InChl=1S/C7H13O/c1-5-6(8)7(2,3)4/h5-6H,1H2,2-4H3 \\
\hline 3213 & C7OOH3-2D & $\mathrm{CC}(\mathrm{C}) \mathrm{C}(\mathrm{OO})[\mathrm{CH}] \mathrm{CC}$ & InChl=1S/C7H15O2/c1-4-5-7(9-8)6(2)3/h5-8H,4H2,1-3H3 \\
\hline 3214 & C7H13014-70OH & $\mathrm{C} 1 \mathrm{CCC}(\mathrm{O} 1) \mathrm{CCC}(\mathrm{OO})$ & InChl=1S/C7H14O3/c8-10-6-2-4-7-3-1-5-9-7/h7-8H,1-6H2 \\
\hline 3215 & C7H13Q14-2 & $\mathrm{C}(\mathrm{OO})[\mathrm{CH}] \mathrm{CC}(\mathrm{OO}) \mathrm{CCC}$ & $\operatorname{lnChl}=15 / C 7 H 1504 / c 1-2-4-7(11-9) 5-3-6-10-8 / h 3,7-9 \mathrm{H}, 2,4-6 \mathrm{H} 2,1 \mathrm{H3}$ \\
\hline 3216 & C7H14O1-1-2 & O1CC(C1)CCCC & $\operatorname{lnChl}=1 \mathrm{~S} / \mathrm{C} 7 \mathrm{H} 14 \mathrm{O} / \mathrm{c1} 1-2-3-4-7-5-8-6-7 / \mathrm{h} 7 \mathrm{H}, 2-6 \mathrm{H} 2,1 \mathrm{H} 3$ \\
\hline 3217 & C7OOH3-2E & $\mathrm{CC}(\mathrm{C}) \mathrm{C}(\mathrm{OO}) \mathrm{C}[\mathrm{CH}] \mathrm{C}$ & InChl=1S/C7H1502/c1-4-5-7(9-8)6(2)3/h4,6-8H,5H2,1-3H3 \\
\hline 3218 & $\mathrm{C} 7 \mathrm{H} 13015-2 \mathrm{OOH}$ & $\mathrm{C} 1 \mathrm{C}(\mathrm{OO}) \mathrm{CCC}(01) \mathrm{CC}$ & InChl=1S/C7H14O3/c1-2-6-3-4-7(10-8)5-9-6/h6-8H,2-5H2,1H3 \\
\hline 3219 & C7H13Q14-3 & $\mathrm{C}(\mathrm{OO}) \mathrm{C}[\mathrm{CH}] \mathrm{C}(\mathrm{OO}) \mathrm{CCC}$ & $\operatorname{lnChl}=15 / \mathrm{C} 7 \mathrm{H} 15 \mathrm{O} 4 / \mathrm{c} 1-2-4-7(11-9) 5-3-6-10-8 / \mathrm{h} 5,7-9 \mathrm{H}, 2-4,6 \mathrm{H2}, 1 \mathrm{H3}$ \\
\hline 3220 & C7H14O1-2-2 & $01 \mathrm{CC} 1(\mathrm{C}) \mathrm{CCCC}$ & InChl=1S/C7H14O/c1-3-4-5-7(2)6-8-7/h3-6H2,1-2H3 \\
\hline 3221 & C7OOH3-2F & $\mathrm{CC}(\mathrm{C}) \mathrm{C}(\mathrm{OO}) \mathrm{CC}[\mathrm{CH} 2]$ & InChl=15/C7H15O2/c1-4-5-7(9-8)6(2)3/h6-8H,1,4-5H2,2-3H3 \\
\hline 3222 & $\mathrm{C} 7 \mathrm{H} 13 \mathrm{O} 15-3 \mathrm{OOH}$ & $\mathrm{C} 1 \mathrm{CC}(\mathrm{OO}) \mathrm{CC}(01) \mathrm{CC}$ & InChl=15/C7H14O3/c1-2-6-5-7(10-8)3-4-9-6/h6-8H,2-5H2,1H3 \\
\hline 3223 & C7H13Q14-5 & $\mathrm{C}(\mathrm{OO}) \mathrm{CCC}(\mathrm{OO})[\mathrm{CH}] \mathrm{CC}$ & InChl=1S/C7H15O4/c1-2-4-7(11-9)5-3-6-10-8/h4,7-9H,2-3,5-6H2,1H3 \\
\hline 3224 & C7H14O1-3-2 & O1CC(C)C1CCC & InChl=1S/C7H14O/c1-3-4-7-6(2)5-8-7/h6-7H,3-5H2,1-2H3 \\
\hline 3225 & $\mathrm{C} 7 \mathrm{OOH} 4-2 \mathrm{~A}$ & {$[\mathrm{CH} 2] \mathrm{C}(\mathrm{C}) \mathrm{CC}(\mathrm{OO}) \mathrm{CC}$} & InChl=1S/C7H15O2/c1-4-7(9-8)5-6(2)3/h6-8H,2,4-5H2,1,3H3 \\
\hline 3226 & C7H13015-40OH & $\mathrm{C} 1 \mathrm{CCC}(\mathrm{OO}) \mathrm{C}(\mathrm{O} 1) \mathrm{CC}$ & InChl=1S/C7H1403/c1-2-6-7(10-8)4-3-5-9-6/h6-8H,2-5H2,1H3 \\
\hline 3227 & C7H13Q14-6 & $\mathrm{C}(\mathrm{OO}) \mathrm{CCC}(\mathrm{OO}) \mathrm{C}[\mathrm{CH}] \mathrm{C}$ & InChl=1S/C7H1504/c1-2-4-7(11-9)5-3-6-10-8/h2,7-9H,3-6H2,1H3 \\
\hline 3228 & C7H14O1-4-2 & O1CC(C)CC1CC & InChl=1S/C7H14O/c1-3-7-4-6(2)5-8-7/h6-7H,3-5H2,1-2H3 \\
\hline 3229 & $\mathrm{C} 7 \mathrm{OOH} 4-2 \mathrm{~B}$ & $\mathrm{C}[\mathrm{C}](\mathrm{C}) \mathrm{CC}(\mathrm{OO}) \mathrm{CC}$ & InChl=1S/C7H1502/c1-4-7(9-8)5-6(2)3/h7-8H,4-5H2,1-3H3 \\
\hline 3230 & C7H13015-60OH & $\mathrm{C} 1 \mathrm{cccc}(\mathrm{O} 1) \mathrm{C}(\mathrm{OO}) \mathrm{C}$ & InChl=1S/C7H14O3/c1-6(10-8)7-4-2-3-5-9-7/h6-8H,2-5H2,1H3 \\
\hline 3231 & C7H13Q14-7 & $\mathrm{C}(\mathrm{OO}) \mathrm{CCC}(\mathrm{OO}) \mathrm{CC}[\mathrm{CH} 2]$ & InChl=1S/C7H1504/c1-2-4-7(11-9)5-3-6-10-8/h7-9H,1-6H2 \\
\hline 3232 & C7H1401-5-2 & O1CC(C)CCC1C & InChl=1S/C7H14O/c1-6-3-4-7(2)8-5-6/h6-7H,3-5H2,1-2H3 \\
\hline 3233 & $\mathrm{C} 7 \mathrm{OOH} 4-2 \mathrm{C}$ & $\mathrm{CC}(\mathrm{C})[\mathrm{CH}] \mathrm{C}(\mathrm{OO}) \mathrm{CC}$ & InChl=1S/C7H15O2/c1-4-7(9-8)5-6(2)3/h5-8H,4H2,1-3H3 \\
\hline 3234 & $\mathrm{C} 7 \mathrm{H} 13015-70 \mathrm{OH}$ & $\mathrm{C} 1 \mathrm{CCCC}(\mathrm{O} 1) \mathrm{CC}(\mathrm{OO})$ & InChl=1S/C7H14O3/c8-10-6-4-7-3-1-2-5-9-7/h7-8H,1-6H2 \\
\hline 3235 & C7H13Q15-2 & $\mathrm{C}(\mathrm{OO})[\mathrm{CH}] \mathrm{CCC}(\mathrm{OO}) \mathrm{CC}$ & InChl=15/C7H15O4/c1-2-7(11-9)5-3-4-6-10-8/h4,7-9H,2-3,5-6H2,1H3 \\
\hline 3236 & C7H14O2-3-2 & $\mathrm{CC}(\mathrm{C})(\mathrm{O} 1) \mathrm{C} 1 \mathrm{CCC}$ & InChl=1S/C7H140/c1-4-5-6-7(2,3)8-6/h6H,4-5H2,1-3H3 \\
\hline 3237 & $\mathrm{C} 7 \mathrm{OOH} 4-2 \mathrm{E}$ & $\mathrm{CC}(\mathrm{C}) \mathrm{CC}(\mathrm{OO})[\mathrm{CH}] \mathrm{C}$ & InChl=1S/C7H1502/c1-4-7(9-8)5-6(2)3/h4,6-8H,5H2,1-3H3 \\
\hline 3238 & $\mathrm{C} 7 \mathrm{H} 13 \mathrm{O} 23-10 \mathrm{OH}$ & $\mathrm{C}(\mathrm{OOO}) \mathrm{C} 1 \mathrm{C}(\mathrm{O} 1) \mathrm{CCCC}$ & InChl=1S/C7H14O3/c1-2-3-4-6-7(10-6)5-9-8/h6-8H,2-5H2,1H3 \\
\hline 3239 & C7H13Q15-3 & $\mathrm{C}(\mathrm{OO}) \mathrm{C}[\mathrm{CH}] \mathrm{CC}(\mathrm{OO}) \mathrm{CC}$ & InChl=1S/C7H15O4/c1-2-7(11-9)5-3-4-6-10-8/h3,7-9H,2,4-6H2,1H3 \\
\hline 3240 & C7H14O2-4-2 & $\mathrm{CC}(\mathrm{C})(\mathrm{O} 1) \mathrm{CC} 1 \mathrm{CC}$ & InChl=1S/C7H140/c1-4-6-5-7(2,3)8-6/h6H,4-5H2,1-3H3 \\
\hline 3241 & $\mathrm{C} 7 \mathrm{OOH} 4-2 \mathrm{~F}$ & $\mathrm{CC}(\mathrm{C}) \mathrm{CC}(\mathrm{OO}) \mathrm{C}[\mathrm{CH} 2]$ & $\operatorname{lnChl}=15 / C 7 H 1502 / c 1-4-7(9-8) 5-6(2) 3 / h 6-8 \mathrm{H}, 1,4-5 \mathrm{H} 2,2-3 \mathrm{H} 3$ \\
\hline 3242 & C7H13O23-40OH & $\mathrm{CC} 1 \mathrm{C}(01) \mathrm{C}(\mathrm{OO}) \mathrm{CCC}$ & $\operatorname{lnChl}=1 \mathrm{~S} / \mathrm{C} 7 \mathrm{H} 14 \mathrm{OO} / \mathrm{c} 1-3-4-6(10-8) 7-5(2) 9-7 / \mathrm{h} 5-8 \mathrm{H}, 3-4 \mathrm{H} 2,1-2 \mathrm{H} 3$ \\
\hline 3243 & C7H13Q15-4 & $\mathrm{C}(\mathrm{OO}) \mathrm{CC}[\mathrm{CH}] \mathrm{C}(\mathrm{OO}) \mathrm{CC}$ & $\operatorname{lnChl}=15 / C 7 H 1504 / c 1-2-7(11-9) 5-3-4-6-10-8 / h 5,7-9 H, 2-4,6 \mathrm{H2}, 1 \mathrm{H3}$ \\
\hline 3244 & C7H14O2-5-2 & $\mathrm{CC}(\mathrm{C})(\mathrm{O} 1) \mathrm{CCC} 1 \mathrm{C}$ & InChl=1S/C7H140/c1-6-4-5-7(2,3)8-6/h6H,4-5H2,1-3H3 \\
\hline 3245 & C7OOH5-2A & {$[\mathrm{CH} 2] \mathrm{C}(\mathrm{C}) \mathrm{CCC}(\mathrm{OO}) \mathrm{C}$} & $\operatorname{lnChl}=15 / C 7 H 1502 / c 1-6(2) 4-5-7(3) 9-8 / \mathrm{h} 6-8 \mathrm{H}, 1,4-5 \mathrm{H} 2,2-3 \mathrm{H} 3$ \\
\hline 3246 & C7H13O23-50OH & $\mathrm{CC} 1 \mathrm{C}(01) \mathrm{CC}(00) \mathrm{CC}$ & InChl=1S/C7H14O3/c1-3-6(10-8)4-7-5(2)9-7/h5-8H,3-4H2,1-2H3 \\
\hline 3247 & C7H13Q15-6 & $\mathrm{C}(\mathrm{OO}) \mathrm{CCCC}(\mathrm{OO})[\mathrm{CH}] \mathrm{C}$ & InChl=1S/C7H1504/c1-2-7(11-9)5-3-4-6-10-8/h2,7-9H,3-6H2,1H3 \\
\hline 3248 & C7H1402-6-2 & $\mathrm{CC}(\mathrm{C})(\mathrm{O} 1) \mathrm{CCCC1}$ & InChl=1S/C7H14O/c1-7(2)5-3-4-6-8-7/h3-6H2,1-2H3 \\
\hline 3249 & C7OOH5-2B & $\mathrm{C}[\mathrm{C}](\mathrm{C}) \mathrm{CCC}(\mathrm{OO}) \mathrm{C}$ & InChl=1S/C7H15O2/c1-6(2)4-5-7(3)9-8/h7-8H,4-5H2,1-3H3 \\
\hline 3250 & C7H13O23-60OH & $\mathrm{CC1C}(01) \mathrm{CCC}(\mathrm{OO}) \mathrm{C}$ & InChl=1S/C7H1403/c1-5(10-8)3-4-7-6(2)9-7/h5-8H,3-4H2,1-2H3 \\
\hline 3251 & C7H13Q15-7 & $\mathrm{C}(\mathrm{OO}) \mathrm{CCCC}(\mathrm{OO}) \mathrm{C}[\mathrm{CH} 2]$ & InChl=1S/C7H1504/c1-2-7(11-9)5-3-4-6-10-8/h7-9H,1-6H2 \\
\hline 3252 & C7H1403-4-2 & $\mathrm{CC}(\mathrm{C}) \mathrm{C}(01) \mathrm{C} 1 \mathrm{CC}$ & InChl=1S/C7H140/c1-4-6-7(8-6)5(2)3/h5-7H,4H2,1-3H3 \\
\hline 3253 & $\mathrm{C} 7 \mathrm{OOH}-2 \mathrm{C}$ & $\mathrm{CC}(\mathrm{C})[\mathrm{CH}] \mathrm{CC}(\mathrm{OO}) \mathrm{C}$ & InChl=1S/C7H15O2/c1-6(2)4-5-7(3)9-8/h4,6-8H,5H2,1-3H3 \\
\hline 3254 & C7H13023-70OH & $\mathrm{CC1C}(01) \operatorname{CCCC}(00)$ & InChl=1S/C7H14O3/c1-6-7(10-6)4-2-3-5-9-8/h6-8H,2-5H2,1H3 \\
\hline 3255 & C7H13Q23-1 & {$[\mathrm{CH} 2] \mathrm{C}(\mathrm{OO}) \mathrm{C}(\mathrm{OO}) \mathrm{CCCC}$} & InChl=1S/C7H15O4/c1-3-4-5-7(11-9)6(2)10-8/h6-9H,2-5H2,1H3 \\
\hline 3256 & C7H14O3-5-2 & $\mathrm{CC}(\mathrm{C}) \mathrm{C}(\mathrm{O} 1) \mathrm{CC} 1 \mathrm{C}$ & InChl=1S/C7H140/c1-5(2)7-4-6(3)8-7/h5-7H,4H2,1-3H3 \\
\hline 3257 & C7OOH5-2D & $\mathrm{CC}(\mathrm{C}) \mathrm{C}[\mathrm{CH}] \mathrm{C}(\mathrm{OO}) \mathrm{C}$ & InChl=15/C7H15O2/c1-6(2)4-5-7(3)9-8/h5-8H,4H2,1-3H3 \\
\hline 3258 & C7H13O24-10OH & $\mathrm{C}(\mathrm{OOO}) \mathrm{C} 1 \mathrm{CC}(01) \mathrm{CCC}$ & InChl=1S/C7H14O3/c1-2-3-6-4-7(10-6)5-9-8/h6-8H,2-5H2,1H3 \\
\hline 3259 & C7H13Q23-4 & $\mathrm{CC}(\mathrm{OO}) \mathrm{C}(\mathrm{OO})[\mathrm{CH}] \mathrm{CCC}$ & InChl=1S/C7H1504/c1-3-4-5-7(11-9)6(2)10-8/h5-9H,3-4H2,1-2H3 \\
\hline 3260 & C7H1403-6-2 & $\mathrm{CC}(\mathrm{C}) \mathrm{C}(01) \mathrm{CCC} 1$ & InChl=1S/C7H14O/c1-6(2)7-4-3-5-8-7/h6-7H,3-5H2,1-2H3 \\
\hline 3261 & C7OOH5-2F & $\mathrm{OOC}(\mathrm{CCC}(\mathrm{C}) \mathrm{C})[\mathrm{CH} 2]$ & InChl=1S/C7H15O2/c1-6(2)4-5-7(3)9-8/h6-8H,3-5H2,1-2H3 \\
\hline 3262 & $\mathrm{C} 7 \mathrm{H} 13 \mathrm{O} 24-3 \mathrm{OOH}$ & $\mathrm{CC} 1 \mathrm{C}(\mathrm{OO}) \mathrm{C}(01) \mathrm{CCC}$ & InChl=1S/C7H1403/c1-3-4-6-7(10-8)5(2)9-6/h5-8H,3-4H2,1-2H3 \\
\hline 3263 & C7H13Q23-5 & $\mathrm{CC}(\mathrm{OO}) \mathrm{C}(\mathrm{OO}) \mathrm{C}[\mathrm{CH}] \mathrm{CC}$ & InChl=1S/C7H15O4/c1-3-4-5-7(11-9)6(2)10-8/h4,6-9H,3,5H2,1-2H3 \\
\hline 3264 & C7H14O4-5-2 & $\mathrm{CC}(\mathrm{C}) \mathrm{CC}(\mathrm{O} 1) \mathrm{C1C}$ & InChl=1S/C7H140/c1-5(2)4-7-6(3)8-7/h5-7H,4H2,1-3H3 \\
\hline 3265 & C7OOH6-2B & $\mathrm{C}[\mathrm{C}](\mathrm{C}) \mathrm{CCCCOO}$ & InChl=1S/C7H15O2/c1-7(2)5-3-4-6-9-8/h8H,3-6H2,1-2H3 \\
\hline 3266 & C7H13O24-50OH & $\mathrm{CC} 1 \mathrm{CC}(\mathrm{O} 1) \mathrm{C}(\mathrm{OO}) \mathrm{CC}$ & InChl=15/C7H14O3/c1-3-6(10-8)7-4-5(2)9-7/h5-8H,3-4H2,1-2H3 \\
\hline 3267 & C7H13Q23-6 & $\mathrm{CC}(\mathrm{OO}) \mathrm{C}(\mathrm{OO}) \mathrm{CC}[\mathrm{CH}] \mathrm{C}$ & InChl=15/C7H15O4/c1-3-4-5-7(11-9)6(2)10-8/h3,6-9H,4-5H2,1-2H3 \\
\hline 3268 & C7H1404-6-2 & $\mathrm{CC}(\mathrm{C}) \mathrm{CC}(01) \mathrm{CC} 1$ & InChl=1S/C7H14O/c1-6(2)5-7-3-4-8-7/h6-7H,3-5H2,1-2H3 \\
\hline 3269 & C7OOH6-2C & $\mathrm{CC}(\mathrm{C})[\mathrm{CH}] \mathrm{CCCOO}$ & InChl=1S/C7H15O2/c1-7(2)5-3-4-6-9-8/h5,7-8H,3-4,6H2,1-2H3 \\
\hline 3270 & C7H13O24-6OOH & $\mathrm{CC} 1 \mathrm{CC}(\mathrm{O} 1) \mathrm{CC}(\mathrm{OO}) \mathrm{C}$ & InChl=1S/C7H1403/c1-5-3-7(9-5)4-6(2)10-8/h5-8H,3-4H2,1-2H3 \\
\hline 3271 & C7H13Q23-7 & $\mathrm{CC}(\mathrm{OO}) \mathrm{C}(\mathrm{OO}) \mathrm{CCC}[\mathrm{CH} 2]$ & InChl=1S/C7H1504/c1-3-4-5-7(11-9)6(2)10-8/h6-9H,1,3-5H2,2H3 \\
\hline 3272 & C7H1405-6-2 & $\mathrm{CC}(\mathrm{C}) \mathrm{CCC}(\mathrm{O} 1) \mathrm{C} 1$ & InChl=1S/C7H14O/c1-6(2)3-4-7-5-8-7/h6-7H,3-5H2,1-2H3 \\
\hline
\end{tabular}




\begin{tabular}{|c|c|c|c|}
\hline 3273 & C7OOH6-2D & $\mathrm{CC}(\mathrm{C}) \mathrm{C}[\mathrm{CH}] \mathrm{CCOO}$ & $\operatorname{lnChl}=15 / \mathrm{C} 7 \mathrm{H} 15 \mathrm{O} 2 / \mathrm{c} 1-7(2) 5-3-4-6-9-8 / \mathrm{h} 3,7-8 \mathrm{H}, 4-6 \mathrm{H} 2,1-2 \mathrm{H} 3$ \\
\hline 3274 & C7H13024-70OH & $\operatorname{cC} 1 \mathrm{CC}(01) \operatorname{ccc}(00)$ & InChl=1S/C7H14O3/c1-6-5-7(10-6)3-2-4-9-8/h6-8H,2-5H2,1H3 \\
\hline 3275 & C7H13Q24-1 & {$[\mathrm{CH} 2] \mathrm{C}(\mathrm{OO}) \mathrm{CC}(\mathrm{OO}) \mathrm{CCC}$} & InChl=1S/C7H15O4/c1-3-4-7(11-9)5-6(2)10-8/h6-9H,2-5H2,1H3 \\
\hline 3276 & C7H1401-2 & $\mathrm{C} 1 \mathrm{C}(\mathrm{O} 1) \mathrm{CCCCC}$ & $\operatorname{lnChl}=1 \mathrm{~S} / \mathrm{C} 7 \mathrm{H} 14 \mathrm{O} / \mathrm{c1} 1-2-3-4-5-7-6-8-7 / \mathrm{h} 7 \mathrm{H}, 2-6 \mathrm{H} 2,1 \mathrm{H} 3$ \\
\hline 3277 & C7OOH6-2E & $\mathrm{CC}(\mathrm{C}) \mathrm{CC}[\mathrm{CH}] \mathrm{COO}$ & $\mathrm{InChl}=1 \mathrm{~S} / \mathrm{C} 7 \mathrm{H} 15 \mathrm{O} 2 / \mathrm{c1}-7(2) 5-3-4-6-9-8 / \mathrm{h} 4,7-8 \mathrm{H}, 3,5-6 \mathrm{H} 2,1-2 \mathrm{H} 3$ \\
\hline 3278 & $\mathrm{C} 7 \mathrm{H} 13 \mathrm{O} 25-10 \mathrm{OH}$ & $\mathrm{C}(\mathrm{OO}) \mathrm{C1CCC}(\mathrm{O} 1) \mathrm{CC}$ & $\operatorname{lnChl}=1 \mathrm{~S} / \mathrm{C} 7 \mathrm{H} 14 \mathrm{OO} / \mathrm{c1}-2-6-3-4-7(10-6) 5-9-8 / \mathrm{h} 6-8 \mathrm{H}, 2-5 \mathrm{H} 2,1 \mathrm{H} 3$ \\
\hline 3279 & C7H13Q24-3 & $\mathrm{CC}(\mathrm{OO})[\mathrm{CH}] \mathrm{C}(\mathrm{OO}) \mathrm{CCC}$ & InChl=1S/C7H1504/c1-3-4-7(11-9)5-6(2)10-8/h5-9H,3-4H2,1-2H3 \\
\hline 3280 & C7H1401-3 & $\mathrm{C} 1 \mathrm{CC}(\mathrm{O} 1) \mathrm{CCCC}$ & $\operatorname{lnChl}=1 \mathrm{~S} / \mathrm{C} 7 \mathrm{H} 14 \mathrm{O} / \mathrm{c1} 1-2-3-4-7-5-6-8-7 / \mathrm{h} 7 \mathrm{H}, 2-6 \mathrm{H} 2,1 \mathrm{H} 3$ \\
\hline 3281 & C7H15-102 & $\mathrm{C}(\mathrm{O}[\mathrm{O}]) \mathrm{CCCCCC}$ & $\operatorname{lnChl}=1 \mathrm{~S} / \mathrm{C} 7 \mathrm{H} 15 \mathrm{O} 2 / \mathrm{c1} 1-2-3-4-5-6-7-9-8 / \mathrm{h} 2-7 \mathrm{H} 2,1 \mathrm{H} 3$ \\
\hline 3282 & $\mathrm{C} 7 \mathrm{H} 13 \mathrm{O} 25-3 \mathrm{OOH}$ & $\mathrm{CC} 1 \mathrm{C}(\mathrm{OO}) \mathrm{CC}(01) \mathrm{CC}$ & $\operatorname{lnChl}=1 \mathrm{~S} / \mathrm{C} 7 \mathrm{H} 14 \mathrm{OO} / \mathrm{c} 1-3-6-4-7(10-8) 5(2) 9-6 / \mathrm{h} 5-8 \mathrm{H}, 3-4 \mathrm{H} 2,1-2 \mathrm{H} 3$ \\
\hline 3283 & C7H13Q24-5 & $\mathrm{CC}(\mathrm{OO}) \mathrm{CC}(\mathrm{OO})[\mathrm{CH}] \mathrm{CC}$ & $\operatorname{lnChl}=15 / C 7 H 1504 / c 1-3-4-7(11-9) 5-6(2) 10-8 / h 4,6-9 \mathrm{H}, 3,5 \mathrm{H} 2,1-2 \mathrm{H} 3$ \\
\hline 3284 & C7H1401-4 & $\mathrm{C} 1 \mathrm{CCC}(\mathrm{O} 1) \mathrm{CCC}$ & $\operatorname{lnChl}=1 \mathrm{~S} / \mathrm{C} 7 \mathrm{H} 14 \mathrm{O} / \mathrm{c1}-2-4-7-5-3-6-8-7 / \mathrm{h} 7 \mathrm{H}, 2-6 \mathrm{H} 2,1 \mathrm{H} 3$ \\
\hline 3285 & C7H15-2O2 & $\mathrm{CC}(\mathrm{O}[\mathrm{O}]) \mathrm{CCCCC}$ & $\operatorname{lnChl}=15 / C 7 H 1502 / c 1-3-4-5-6-7(2) 9-8 / h 7 H, 3-6 \mathrm{H} 2,1-2 \mathrm{H} 3$ \\
\hline 3286 & C7H13O25-40OH & $\mathrm{CC} 1 \mathrm{CC}(\mathrm{OO}) \mathrm{C}(\mathrm{O} 1) \mathrm{CC}$ & InChl=1S/C7H14O3/c1-3-6-7(10-8)4-5(2)9-6/h5-8H,3-4H2,1-2H3 \\
\hline 3287 & C7H13Q24-6 & $\mathrm{CC}(\mathrm{OO}) \mathrm{CC}(\mathrm{OO}) \mathrm{C}[\mathrm{CH}] \mathrm{C}$ & $\operatorname{lnChl}=15 / \mathrm{C} 7 \mathrm{H} 15 \mathrm{O} 4 / \mathrm{c} 1-3-4-7(11-9) 5-6(2) 10-8 / \mathrm{h} 3,6-9 \mathrm{H}, 4-5 \mathrm{H} 2,1-2 \mathrm{H} 3$ \\
\hline 3288 & C7H1401-5 & $\mathrm{C} 1 \mathrm{CCCC}(01) \mathrm{CC}$ & $\operatorname{lnChl}=1 \mathrm{~S} / \mathrm{C} 7 \mathrm{H} 14 \mathrm{O} / \mathrm{c1} 1-2-7-5-3-4-6-8-7 / \mathrm{h} 7 \mathrm{H}, 2-6 \mathrm{H} 2,1 \mathrm{H} 3$ \\
\hline 3289 & C7H15-302 & $\mathrm{CCC}(\mathrm{O}[\mathrm{O}]) \mathrm{CCCC}$ & $\operatorname{lnChl}=15 / C 7 H 15 O 2 / c 1-3-5-6-7(4-2) 9-8 / h 7 H, 3-6 \mathrm{H} 2,1-2 \mathrm{H} 3$ \\
\hline 3290 & C7H13O25-60OH & $\mathrm{CC} 1 \mathrm{CCC}(\mathrm{O} 1) \mathrm{C}(\mathrm{OO}) \mathrm{C}$ & $\operatorname{lnChl}=1 \mathrm{~S} / \mathrm{C} 7 \mathrm{H} 14 \mathrm{OO} / \mathrm{c} 1-5-3-4-7(9-5) 6(2) 10-8 / \mathrm{h} 5-8 \mathrm{H}, 3-4 \mathrm{H} 2,1-2 \mathrm{H} 3$ \\
\hline 3291 & C7H13Q24-7 & $\mathrm{CC}(\mathrm{OO}) \mathrm{CC}(\mathrm{OO}) \mathrm{CC}[\mathrm{CH} 2]$ & InChl=1S/C7H1504/c1-3-4-7(11-9)5-6(2)10-8/h6-9H,1,3-5H2,2H3 \\
\hline 3292 & C7H14O2-3 & $\mathrm{CC1C}(\mathrm{O} 1) \mathrm{CCCC}$ & InChl=1S/C7H14O/c1-3-4-5-7-6(2)8-7/h6-7H,3-5H2,1-2H3 \\
\hline 3293 & $\mathrm{C} 7 \mathrm{H} 15-4 \mathrm{O} 2$ & $\operatorname{cccc}(0[0]) \operatorname{ccc}$ & InChl=1S/C7H15O2/c1-3-5-7(9-8)6-4-2/h7H,3-6H2,1-2H3 \\
\hline 3294 & $\mathrm{C} 7 \mathrm{H} 13025-70 \mathrm{OH}$ & $\operatorname{cC} 1 \mathrm{CCC}(01) \mathrm{CC}(00)$ & InChl=1S/C7H14O3/c1-6-2-3-7(10-6)4-5-9-8/h6-8H,2-5H2,1H3 \\
\hline 3295 & C7H13Q25-1 & {$[\mathrm{CH} 2] \mathrm{C}(\mathrm{OO}) \mathrm{CCC}(\mathrm{OO}) \mathrm{CC}$} & InChl=1S/C7H1504/c1-3-7(11-9)5-4-6(2)10-8/h6-9H,2-5H2,1H3 \\
\hline 3296 & C7H1402-4 & $\mathrm{CC} 1 \mathrm{CC}(\mathrm{O} 1) \mathrm{CCC}$ & InChl=1S/C7H140/c1-3-4-7-5-6(2)8-7/h6-7H,3-5H2,1-2H3 \\
\hline 3297 & C7H14OOH1-2 & $\mathrm{C}(\mathrm{OO})[\mathrm{CH}] \mathrm{CCCCC}$ & InChl=1S/C7H15O2/c1-2-3-4-5-6-7-9-8/h6,8H,2-5,7H2,1H3 \\
\hline 3298 & C7H13026-10OH & $\mathrm{c}(\mathrm{OO}) \mathrm{C1CCCC}(\mathrm{O} 1) \mathrm{C}$ & InChl=1S/C7H1403/c1-6-3-2-4-7(10-6)5-9-8/h6-8H,2-5H2,1H3 \\
\hline 3299 & C7H13Q25-3 & $\mathrm{CC}(\mathrm{OO})[\mathrm{CH}] \mathrm{CC}(\mathrm{OO}) \mathrm{CC}$ & 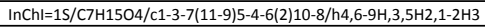 \\
\hline 3300 & C7H1402-5 & $\mathrm{CC} 1 \mathrm{CCC}(01) \mathrm{CC}$ & InChl=1S/C7H14O/c1-3-7-5-4-6(2)8-7/h6-7H,3-5H2,1-2H3 \\
\hline 3301 & C7H14OOH1-3 & $\mathrm{C}(\mathrm{OO}) \mathrm{C}[\mathrm{CH}] \mathrm{CCCC}$ & $\operatorname{lnChl}=15 / C 7 H 1502 / c 1-2-3-4-5-6-7-9-8 / h 5,8 \mathrm{H}, 2-4,6-7 \mathrm{H} 2,1 \mathrm{H} 3$ \\
\hline 3302 & C7H13O26-30OH & $\mathrm{CC} 1 \mathrm{C}(0 \mathrm{O}) \mathrm{CCC}(\mathrm{O} 1) \mathrm{C}$ & $\operatorname{lnChl}=1 \mathrm{~S} / \mathrm{C} 7 \mathrm{H} 14 \mathrm{OO} / \mathrm{c} 1-5-3-4-7(10-8) 6(2) 9-5 / \mathrm{h} 5-8 \mathrm{H}, 3-4 \mathrm{H} 2,1-2 \mathrm{H} 3$ \\
\hline 3303 & C7H13Q25-4 & $\mathrm{CC}(\mathrm{OO}) \mathrm{C}[\mathrm{CH}] \mathrm{C}(\mathrm{OO}) \mathrm{CC}$ & InChl=1S/C7H15O4/c1-3-7(11-9)5-4-6(2)10-8/h5-9H,3-4H2,1-2H3 \\
\hline 3304 & C7H1402-6 & $\mathrm{CC} 1 \mathrm{CCCC}(\mathrm{O} 1) \mathrm{C}$ & InChl=1S/C7H14O/c1-6-4-3-5-7(2)8-6/h6-7H,3-5H2,1-2H3 \\
\hline 3305 & C7H14OOH1-4 & $\mathrm{C}(\mathrm{OO}) \mathrm{CC}[\mathrm{CH}] \mathrm{CCC}$ & $\operatorname{lnChl}=15 / \mathrm{C} 7 \mathrm{H} 15 \mathrm{O} 2 / \mathrm{c1} 1-2-3-4-5-6-7-9-8 / \mathrm{h} 4,8 \mathrm{H}, 2-3,5-7 \mathrm{H} 2,1 \mathrm{H} 3$ \\
\hline 3306 & $\mathrm{C} 7 \mathrm{H} 13 \mathrm{O} 26-4 \mathrm{OOH}$ & $\mathrm{CC} 1 \mathrm{CC}(0 \mathrm{O}) \mathrm{CC}(\mathrm{O} 1) \mathrm{C}$ & InChl=1S/C7H14O3/c1-5-3-7(10-8)4-6(2)9-5/h5-8H,3-4H2,1-2H3 \\
\hline 3307 & C7H13Q25-6 & $\mathrm{CC}(\mathrm{OO}) \mathrm{CCC}(\mathrm{OO})[\mathrm{CH}] \mathrm{C}$ & $\operatorname{lnChl}=15 / C 7 H 1504 / c 1-3-7(11-9) 5-4-6(2) 10-8 / h 3,6-9 \mathrm{H}, 4-5 \mathrm{H} 2,1-2 \mathrm{H} 3$ \\
\hline 3308 & C7H14O3-4 & $\mathrm{CCC1C}(\mathrm{O} 1) \mathrm{CCC}$ & InChl=1S/C7H14O/c1-3-5-7-6(4-2)8-7/h6-7H,3-5H2,1-2H3 \\
\hline 3309 & C7H14OOH1-5 & $\mathrm{C}(\mathrm{OO}) \mathrm{CCC}[\mathrm{CH}] \mathrm{CC}$ & $\operatorname{lnChl}=1 \mathrm{~S} / \mathrm{C} 7 \mathrm{H} 1502 / \mathrm{C} 1-2-3-4-5-6-7-9-8 / \mathrm{h} 3,8 \mathrm{H}, 2,4-7 \mathrm{H} 2,1 \mathrm{H3}$ \\
\hline 3310 & C7H13034-10OH & $\mathrm{C}(\mathrm{OO}) \mathrm{CC} 1 \mathrm{C}(\mathrm{O} 1) \mathrm{CCC}$ & InChl=1S/C7H14O3/c1-2-3-6-7(10-6)4-5-9-8/h6-8H,2-5H2,1H3 \\
\hline 3311 & C7H13Q25-7 & $\mathrm{CC}(\mathrm{OO}) \mathrm{CCC}(\mathrm{OO}) \mathrm{C}[\mathrm{CH} 2]$ & InChl=1S/C7H1504/c1-3-7(11-9)5-4-6(2)10-8/h6-9H,1,3-5H2,2H3 \\
\hline 3312 & C7H1403-5 & $\mathrm{CCC1CC}(01) \mathrm{CC}$ & InChl=1S/C7H14O/c1-3-6-5-7(4-2)8-6/h6-7H,3-5H2,1-2H3 \\
\hline 3313 & $\mathrm{C} 7 \mathrm{H} 14 \mathrm{OOH} 2-1$ & {$[\mathrm{CH} 2] \mathrm{C}(\mathrm{OO}) \mathrm{CCCCC}$} & $\operatorname{lnChl}=15 / \mathrm{C} 7 \mathrm{H} 15 \mathrm{O} 2 / \mathrm{c} 1-3-4-5-6-7(2) 9-8 / \mathrm{h} 7-8 \mathrm{H}, 2-6 \mathrm{H} 2,1 \mathrm{H} 3$ \\
\hline 3314 & C7H13034-20OH & $\mathrm{CC}(00) \mathrm{C} 1 \mathrm{C}(\mathrm{O} 1) \mathrm{CCC}$ & InChl=1S/C7H14O3/c1-3-4-6-7(9-6)5(2)10-8/h5-8H,3-4H2,1-2H3 \\
\hline 3315 & C7H13Q26-1 & $\mathrm{CC}(\mathrm{OO}) \mathrm{CCCC}(\mathrm{OO})[\mathrm{CH} 2]$ & InChl=1S/C7H1504/c1-6(10-8)4-3-5-7(2)11-9/h6-9H,1,3-5H2,2H3 \\
\hline 3316 & NEC7Y4 & $\mathrm{CC}(\mathrm{C})(\mathrm{C}) \mathrm{CC}(=0) \mathrm{C}$ & $\operatorname{lnChl}=1 \mathrm{~S} / \mathrm{C} 7 \mathrm{H} 14 \mathrm{O} / \mathrm{c1}-6(8) 5-7(2,3) 4 / \mathrm{h} 5 \mathrm{H} 2,1-4 \mathrm{H} 3$ \\
\hline 3317 & $\mathrm{C} 7 \mathrm{H} 14 \mathrm{OOH} 2-3$ & $\mathrm{CC}(\mathrm{OO})[\mathrm{CH}] \mathrm{CCCC}$ & InChl=1S/C7H15O2/c1-3-4-5-6-7(2)9-8/h6-8H,3-5H2,1-2H3 \\
\hline 3318 & $\mathrm{C} 7 \mathrm{H} 13 \mathrm{O} 34-5 \mathrm{OOH}$ & $\operatorname{ccc} 1 \mathrm{C}(\mathrm{O} 1) \mathrm{C}(\mathrm{OO}) \mathrm{CC}$ & InChl=1S/C7H14O3/c1-3-5-7(9-5)6(4-2)10-8/h5-8H,3-4H2,1-2H3 \\
\hline 3319 & C7H13Q26-3 & $\mathrm{CC}(\mathrm{OO}) \mathrm{CC}[\mathrm{CH}] \mathrm{C}(\mathrm{OO}) \mathrm{C}$ & $\operatorname{lnChl}=1 \mathrm{~S} / \mathrm{C} 7 \mathrm{H} 15 \mathrm{O} 4 / \mathrm{c} 1-6(10-8) 4-3-5-7(2) 11-9 / \mathrm{h} 4,6-9 \mathrm{H}, 3,5 \mathrm{H} 2,1-2 \mathrm{H} 3$ \\
\hline 3320 & C7H15O-1-2 & {$[0] \mathrm{CC}(\mathrm{C}) \mathrm{CCCC}$} & $\operatorname{lnChl}=1 \mathrm{~S} / \mathrm{C} 7 \mathrm{H} 15 \mathrm{O} / \mathrm{c1} 1-3-4-5-7(2) 6-8 / \mathrm{h} 7 \mathrm{H}, 3-6 \mathrm{H} 2,1-2 \mathrm{H} 3$ \\
\hline 3321 & $\mathrm{C} 7 \mathrm{H} 14 \mathrm{OOH} 2-4$ & $\mathrm{CC}(\mathrm{OO}) \mathrm{C}[\mathrm{CH}] \mathrm{CCC}$ & InChl=1S/C7H15O2/c1-3-4-5-6-7(2)9-8/h5,7-8H,3-4,6H2,1-2H3 \\
\hline 3322 & $\mathrm{C} 7 \mathrm{H} 13 \mathrm{O} 34-60 \mathrm{OH}$ & $\operatorname{ccc} 1 \mathrm{C}(01) \operatorname{cc}(00) \mathrm{C}$ & InChl=1S/C7H14O3/c1-3-6-7(9-6)4-5(2)10-8/h5-8H,3-4H2,1-2H3 \\
\hline 3323 & C7H13Q26-4 & $\mathrm{CC}(\mathrm{OO}) \mathrm{C}[\mathrm{CH}] \mathrm{CC}(\mathrm{OO}) \mathrm{C}$ & $\operatorname{lnChl}=15 / C 7 H 1504 / c 1-6(10-8) 4-3-5-7(2) 11-9 / h 3,6-9 \mathrm{H}, 4-5 \mathrm{H} 2,1-2 \mathrm{H} 3$ \\
\hline 3324 & $\mathrm{C} 7 \mathrm{H} 15 \mathrm{O}-2-2$ & $\mathrm{CC}(\mathrm{C})([\mathrm{O}]) \mathrm{CCCC}$ & $\operatorname{lnChl}=1 \mathrm{~S} / \mathrm{C} 7 \mathrm{H} 15 \mathrm{O} / \mathrm{c1} 1-4-5-6-7(2,3) 8 / \mathrm{h} 4-6 \mathrm{H} 2,1-3 \mathrm{H} 3$ \\
\hline 3325 & $\mathrm{C} 7 \mathrm{H} 14 \mathrm{OOH} 2-5$ & $\mathrm{CC}(\mathrm{OO}) \mathrm{CC}[\mathrm{CH}] \mathrm{CC}$ & $\operatorname{lnChl}=1 \mathrm{~S} / \mathrm{C} 7 \mathrm{H} 15 \mathrm{O} 2 / \mathrm{c1}-3-4-5-6-7(2) 9-8 / \mathrm{h} 4,7-8 \mathrm{H}, 3,5-6 \mathrm{H} 2,1-2 \mathrm{H} 3$ \\
\hline 3326 & $\mathrm{C} 7 \mathrm{H} 13034-70 \mathrm{OH}$ & $\operatorname{ccc1c}(01) \operatorname{ccc}(00)$ & $\operatorname{lnChl}=15 / C 7 H 1403 / c 1-2-6-7(10-6) 4-3-5-9-8 / h 6-8 \mathrm{H}, 2-5 \mathrm{H} 2,1 \mathrm{H} 3$ \\
\hline 3327 & C7H13Q34-1 & {$[\mathrm{CH} 2] \mathrm{CC}(\mathrm{OO}) \mathrm{C}(\mathrm{OO}) \mathrm{CCC}$} & InChl=1S/C7H15O4/c1-3-5-7(11-9)6(4-2)10-8/h6-9H,2-5H2,1H3 \\
\hline 3328 & $\mathrm{C} 7 \mathrm{H} 15 \mathrm{O}-3-2$ & $\mathrm{CC}(\mathrm{C}) \mathrm{C}([\mathrm{O}]) \mathrm{CCC}$ & $\operatorname{lnChl}=1 \mathrm{~S} / \mathrm{C} 7 \mathrm{H} 15 \mathrm{O} / \mathrm{c} 1-4-5-7(8) 6(2) 3 / \mathrm{h} 6-7 \mathrm{H}, 4-5 \mathrm{H} 2,1-3 \mathrm{H} 3$ \\
\hline 3329 & $\mathrm{C} 7 \mathrm{H} 14 \mathrm{OOH} 2-6$ & $\mathrm{CC}(\mathrm{OO}) \mathrm{CCC}[\mathrm{CH}] \mathrm{C}$ & InChl=15/C7H15O2/c1-3-4-5-6-7(2)9-8/h3,7-8H,4-6H2,1-2H3 \\
\hline 3330 & $\mathrm{C} 7 \mathrm{H} 13 \mathrm{O} 35-10 \mathrm{OH}$ & $\operatorname{cCC} 1 \mathrm{CC}(01) \mathrm{CC}(00)$ & InChl=15/C7H14O3/c1-2-6-5-7(10-6)3-4-9-8/h6-8H,2-5H2,1H3 \\
\hline 3331 & C7H13Q34-2 & $\mathrm{C}[\mathrm{CH}] \mathrm{C}(\mathrm{OO}) \mathrm{C}(\mathrm{OO}) \mathrm{CCC}$ & $\operatorname{lnChl}=1 \mathrm{~S} / \mathrm{C} 7 \mathrm{H} 15 \mathrm{O} 4 / \mathrm{c} 1-3-5-7(11-9) 6(4-2) 10-8 / \mathrm{h} 4,6-9 \mathrm{H}, 3,5 \mathrm{H} 2,1-2 \mathrm{H} 3$ \\
\hline 3332 & C7H15O-4-2 & $\mathrm{CC}(\mathrm{C}) \mathrm{CC}([\mathrm{O}]) \mathrm{CC}$ & InChl=15/C7H15O/c1-4-7(8)5-6(2)3/h6-7H,4-5H2,1-3H3 \\
\hline 3333 & $\mathrm{C} 7 \mathrm{H} 14 \mathrm{OOH} 3-1$ & {$[\mathrm{CH} 2] \mathrm{CC}(\mathrm{OO}) \mathrm{CCCC}$} & $\operatorname{lnChl}=15 / C 7 H 1502 / c 1-3-5-6-7(4-2) 9-8 / h 7-8 \mathrm{H}, 2-6 \mathrm{H} 2,1 \mathrm{H} 3$ \\
\hline 3334 & C7H13O35-2OOH & $\mathrm{CCC1CC}(01) \mathrm{C}(00) \mathrm{C}$ & InChl=1S/C7H14O3/c1-3-6-4-7(9-6)5(2)10-8/h5-8H,3-4H2,1-2H3 \\
\hline 3335 & C7H13Q34-5 & $\mathrm{CCC}(\mathrm{OO}) \mathrm{C}(\mathrm{OO})[\mathrm{CH}] \mathrm{CC}$ & InChl=1S/C7H1504/c1-3-5-7(11-9)6(4-2)10-8/h5-9H,3-4H2,1-2H3 \\
\hline 3336 & $\mathrm{C} 7 \mathrm{H} 15 \mathrm{O}-5-2$ & $\mathrm{CC}(\mathrm{C}) \mathrm{CCC}([\mathrm{OO}]) \mathrm{C}$ & $\operatorname{lnChl}=1 \mathrm{~S} / \mathrm{C} 7 \mathrm{H} 15 \mathrm{O} / \mathrm{c} 1-6(2) 4-5-7(3) 8 / \mathrm{h} 6-7 \mathrm{H}, 4-5 \mathrm{H} 2,1-3 \mathrm{H} 3$ \\
\hline 3337 & С7H140OH3-2 & $\mathrm{C}[\mathrm{CH}] \mathrm{C}(\mathrm{OO}) \mathrm{CCCC}$ & $\operatorname{lnChl=1S/C7H15O2/c1-3-5-6-7(4-2)9-8/h4,7-8H,3,5-6H2,1-2H3~}$ \\
\hline 3338 & $\mathrm{C} 7 \mathrm{H} 13 \mathrm{O} 35-4 \mathrm{OOH}$ & $\mathrm{CCC1C}(00) \mathrm{C}(01) \mathrm{CC}$ & InChl=1S/C7H14O3/c1-3-5-7(10-8)6(4-2)9-5/h5-8H,3-4H2,1-2H3 \\
\hline 3339 & C7H13Q34-6 & $\mathrm{CCC}(\mathrm{OO}) \mathrm{C}(\mathrm{OO}) \mathrm{C}[\mathrm{CH}] \mathrm{C}$ & $\operatorname{lnChl}=1 \mathrm{~S} / \mathrm{C} 7 \mathrm{H} 15 \mathrm{O} 4 / \mathrm{c} 1-3-5-7(11-9) 6(4-2) 10-8 / \mathrm{h} 3,6-9 \mathrm{H}, 4-5 \mathrm{H} 2,1-2 \mathrm{H} 3$ \\
\hline 3340 & C7H15O-6-2 & $\mathrm{CC}(\mathrm{C}) \mathrm{CCCC}[\mathrm{O}]$ & $\operatorname{lnChl}=15 / C 7 H 150 / c 1-7(2) 5-3-4-6-8 / h 7 H, 3-6 \mathrm{H} 2,1-2 \mathrm{H} 3$ \\
\hline 3341 & $\mathrm{C} 7 \mathrm{H} 14 \mathrm{OOH} 3-4$ & $\mathrm{CCC}(\mathrm{OO})[\mathrm{CH}] \mathrm{CCC}$ & InChl=1S/C7H15O2/c1-3-5-6-7(4-2)9-8/h6-8H,3-5H2,1-2H3 \\
\hline 3342 & $\mathrm{C} 7 \mathrm{H} 140 \mathrm{OH} 1-2 \mathrm{O}$ & $\mathrm{C}(\mathrm{OO}) \mathrm{C}([\mathrm{O}]) \mathrm{CCCCC}$ & $\operatorname{lnChl}=1 \mathrm{~S} / \mathrm{C} 7 \mathrm{H} 15 \mathrm{O} / \mathrm{c1}-2-3-4-5-7(8) 6-10-9 / \mathrm{h} 7,9 \mathrm{H}, 2-6 \mathrm{H} 2,1 \mathrm{H} 3$ \\
\hline 3343 & C7H13Q34-7 & $\mathrm{CCC}(\mathrm{OO}) \mathrm{C}(\mathrm{OO}) \mathrm{CC}[\mathrm{CH} 2]$ & InChl=1S/C7H1504/c1-3-5-7(11-9)6(4-2)10-8/h6-9H,1,3-5H2,2H3 \\
\hline 3344 & C7H15-10 & $\mathrm{C}([0]) \operatorname{cccccc}$ & $\ln \mathrm{Chl}=1 \mathrm{~S} / \mathrm{C} 7 \mathrm{H} 15 \mathrm{O} / \mathrm{c1}-2-3-4-5-6-7-8 / \mathrm{h} 2-7 \mathrm{H} 2,1 \mathrm{H} 3$ \\
\hline 3345 & С7H140OH3-5 & $\mathrm{CCC}(\mathrm{OO}) \mathrm{C}[\mathrm{CH}] \mathrm{CC}$ & InChl=1S/C7H15O2/c1-3-5-6-7(4-2)9-8/h5,7-8H,3-4,6H2,1-2H3 \\
\hline 3346 & C7H14OOH1-3O & $\mathrm{C}(\mathrm{OO}) \mathrm{CC}([\mathrm{O}]) \mathrm{CCCC}$ & InChl=15/C7H15O3/c1-2-3-4-7(8)5-6-10-9/h7,9H,2-6H2,1H3 \\
\hline 3347 & C7H13Q35-1 & $\mathrm{CCC}(\mathrm{OO}) \mathrm{CC}(\mathrm{OO}) \mathrm{C}[\mathrm{CH} 2]$ & InChl=1S/C7H1504/c1-3-6(10-8)5-7(4-2)11-9/h6-9H,1,3-5H2,2H3 \\
\hline 3348 & $\begin{array}{cc}\mathrm{CH} 15-2 \mathrm{O} \\
\end{array}$ & $\mathrm{CC}([\mathrm{O}]) \mathrm{CCCCC}$ & $\operatorname{lnChl}=1 \mathrm{~S} / \mathrm{C} 7 \mathrm{H} 15 \mathrm{O} / \mathrm{c1} 1-3-4-5-6-7(2) 8 / \mathrm{h} 7 \mathrm{H}, 3-6 \mathrm{H} 2,1-2 \mathrm{H} 3$ \\
\hline 3349 & C7H140OH3-6 & $\mathrm{CCC}(\mathrm{OO}) \mathrm{CC}[\mathrm{CH}] \mathrm{C}$ & $\operatorname{lnChl}=15 / C 7 H 15 O 2 / c 1-3-5-6-7(4-2) 9-8 / h 3,7-8 \mathrm{H}, 4-6 \mathrm{H} 2,1-2 \mathrm{H} 3$ \\
\hline 3350 & C7H14OOH1-4O & $\mathrm{C}(\mathrm{OO}) \mathrm{CCC}([\mathrm{O}]) \mathrm{CCC}$ & $\operatorname{lnChl}=1 \mathrm{~S} / \mathrm{C} 7 \mathrm{H} 1503 / \mathrm{c} 1-2-4-7(8) 5-3-6-10-9 / \mathrm{h} 7,9 \mathrm{H}, 2-6 \mathrm{H} 2,1 \mathrm{H} 3$ \\
\hline 3351 & C7H13Q35-2 & $\mathrm{CCC}(\mathrm{OO}) \mathrm{CC}(\mathrm{OO})[\mathrm{CH}] \mathrm{C}$ & $\operatorname{lnChl}=1 \mathrm{~S} / \mathrm{C} 7 \mathrm{H} 15 \mathrm{O} 4 / \mathrm{c} 1-3-6(10-8) 5-7(4-2) 11-9 / \mathrm{h} 3,6-9 \mathrm{H}, 4-5 \mathrm{H} 2,1-2 \mathrm{H} 3$ \\
\hline 3352 & C7H15-30 & $\operatorname{ccc}([0]) \operatorname{cccc}$ & $\operatorname{lnChl}=1 \mathrm{~S} / \mathrm{C} 7 \mathrm{H} 15 \mathrm{O} / \mathrm{c} 1-3-5-6-7(8) 4-2 / \mathrm{h} 7 \mathrm{H}, 3-6 \mathrm{H} 2,1-2 \mathrm{H} 3$ \\
\hline 3353 & $\mathrm{C} 7 \mathrm{H} 14 \mathrm{OOH} 3-7$ & $\mathrm{CCC}(\mathrm{OO}) \mathrm{CCC}[\mathrm{CH} 2]$ & InChl=1S/C7H15O2/c1-3-5-6-7(4-2)9-8/h7-8H,1,3-6H2,2H3 \\
\hline 3354 & C7H14OOH1-5O & $\mathrm{C}(\mathrm{OO}) \mathrm{CCCC}([0]) \mathrm{CC}$ & InChl=1S/C7H15O3/c1-2-7(8)5-3-4-6-10-9/h7,9H,2-6H2,1H3 \\
\hline 3355 & C7H13Q35-4 & $\mathrm{CCC}(\mathrm{OO})[\mathrm{CH}] \mathrm{C}(\mathrm{OO}) \mathrm{CC}$ & InChl=1S/C7H1504/c1-3-6(10-8)5-7(4-2)11-9/h5-9H,3-4H2,1-2H3 \\
\hline 3356 & $\mathrm{C} 6 \mathrm{H} 4 \mathrm{C} 2 \mathrm{H}$ & $\mathrm{CHCC} 1=[\mathrm{C}] \mathrm{C}=\mathrm{CC}=\mathrm{C} 1$ & $\operatorname{lnChl}=15 / \mathrm{C} 8 \mathrm{H} 5 / \mathrm{c} 1-2-8-6-4-3-5-7-8 / \mathrm{h} 1,3-6 \mathrm{H}$ \\
\hline 3357 & $\mathrm{HOC} 6 \mathrm{H} 4 \mathrm{C} 2 \mathrm{H} 4-\mathrm{S}$ & $\mathrm{C}[\mathrm{CH}] \mathrm{C} 1=\mathrm{CC}=\mathrm{CC}=\mathrm{C} 10$ & InChl=1S/C8H9O/c1-2-7-5-3-4-6-8(7)9/h2-6,9H,1H3 \\
\hline 3358 & IC8KET3-40 & $\mathrm{CC}(\mathrm{C})(\mathrm{C}) \mathrm{C}(=\mathrm{O}) \mathrm{C}([\mathrm{O}])(\mathrm{C}) \mathrm{C}$ & InChl=1S/C8H1502/c1-7(2,3)6(9)8(4,5)10/h1-5H3 \\
\hline 3359 & $\mathrm{IC} 8015-40 \mathrm{OH}$ & $\mathrm{C}(\mathrm{O} 1) \mathrm{C}(\mathrm{C})(\mathrm{C}) \mathrm{CC}(\mathrm{OO})(\mathrm{C}) \mathrm{C} 1$ & $\operatorname{lnChl}=1 \mathrm{~S} / \mathrm{C} 8 \mathrm{H} 1603 / \mathrm{C} 1-7(2) 4-8(3,11-9) 6-10-5-7 / \mathrm{h} 9 \mathrm{H}, 4-6 \mathrm{H} 2,1-3 \mathrm{H} 3$ \\
\hline 3360 & $\mathrm{C} 6 \mathrm{H} 5 \mathrm{C} 2 \mathrm{H}$ & 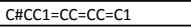 & $\operatorname{lnChl}=15 / \mathrm{C} 8 \mathrm{H} 6 / \mathrm{c} 1-2-8-6-4-3-5-7-8 / \mathrm{h} 1,3-7 \mathrm{H}$ \\
\hline 3361 & $\mathrm{OCH} 3 \mathrm{C} 6 \mathrm{H} 3 \mathrm{CH} 3$ & $\mathrm{CC} 1=\mathrm{CC}=\mathrm{C}(\mathrm{C}) \mathrm{C}=\mathrm{C} 1[\mathrm{O}]$ & $\operatorname{lnChl}=1 \mathrm{~S} / \mathrm{C} 8 \mathrm{H} 9 \mathrm{O} / \mathrm{c1} 1-6-3-4-7(2) 8(9) 5-6 / \mathrm{h} 3-5 \mathrm{H}, 1-2 \mathrm{H} 3$ \\
\hline 3362 & IC8KET3-50 & $\mathrm{CC}(\mathrm{C})(\mathrm{C}) \mathrm{C}(=\mathrm{O}) \mathrm{C}(\mathrm{C}) \mathrm{C}[\mathrm{O}]$ & $\operatorname{lnChl}=1 \mathrm{~S} / \mathrm{C} 8 \mathrm{H} 15 \mathrm{O} 2 / \mathrm{c} 1-6(5-9) 7(10) 8(2,3) 4 / \mathrm{h} 6 \mathrm{H}, 5 \mathrm{H} 2,1-4 \mathrm{H} 3$ \\
\hline 3363 & IC8O15-5AOOH & $\mathrm{C}(\mathrm{O} 1) \mathrm{C}(\mathrm{C})(\mathrm{C}) \mathrm{CC}(\mathrm{COO}) \mathrm{C} 1$ & InChl=1S/C8H16O3/c1-8(2)3-7(5-11-9)4-10-6-8/h7,9H,3-6H2,1-2h \\
\hline
\end{tabular}




\begin{tabular}{|c|c|c|c|}
\hline 3364 & $\mathrm{C} 6 \mathrm{H}_{4} \mathrm{C} 2 \mathrm{H} 3$ & $\mathrm{C}=\mathrm{CC} 1=\mathrm{CC}=\mathrm{CC}=[\mathrm{C}] 1$ & InChl=1S/C8H7/c1-2-8-6-4-3-5-7-8/h2-6H,1H2 \\
\hline 3365 & $\mathrm{HOC6H} 4 \mathrm{C} 2 \mathrm{H} 5$ & CCc1c(0)cccc1 & InChl=1S/C8H10O/c1-2-7-5-3-4-6-8(7)9/h3-6,9H,2H2,1H3 \\
\hline 3366 & IC8KET5-10 & $\mathrm{C}([0]) \mathrm{C}(\mathrm{C})(\mathrm{C}) \mathrm{CC}(\mathrm{C}) \mathrm{C}=0$ & InChl=15/C8H15O2/c1-7(5-9)4-8(2,3)6-10/h5,7H,4,6H2,1-3H3 \\
\hline 3367 & $\mathrm{IC8O34-10OH}$ & $\mathrm{C}(\mathrm{OO}) \mathrm{C}(\mathrm{C})(\mathrm{C}) \mathrm{C}(\mathrm{O} 1) \mathrm{C} 1(\mathrm{C}) \mathrm{C}$ & $\operatorname{lnChl}=1 \mathrm{~S} / \mathrm{C} 8 \mathrm{H} 1603 / \mathrm{c} 1-7(2,5-10-9) 6-8(3,4) 11-6 / \mathrm{h} 6,9 \mathrm{H}, 5 \mathrm{H} 2,1-4 \mathrm{H} 3$ \\
\hline 3368 & $\mathrm{C} 6 \mathrm{H} 5 \mathrm{CCH} 2$ & $C 1=C C([C]=C)=C C=C 1$ & InChl=1S/C8H7/c1-2-8-6-4-3-5-7-8/h3-7H,1H2 \\
\hline 3369 & $\mathrm{CH} 3 \mathrm{C} 6 \mathrm{H} 4 \mathrm{OCH} 3$ & $\operatorname{coc} 1 \mathrm{c}(\mathrm{C}) \operatorname{cccc} 1$ & $\operatorname{lnChl}=1 \mathrm{~S} / \mathrm{C} 8 \mathrm{H} 100 / \mathrm{c} 1-7-5-3-4-6-8(7) 9-2 / \mathrm{h} 3-6 \mathrm{H}, 1-2 \mathrm{H} 3$ \\
\hline 3370 & IC8KET5-30 & $\mathrm{CC}(\mathrm{C})(\mathrm{C}) \mathrm{C}([\mathrm{O}]) \mathrm{C}(\mathrm{C}) \mathrm{C}=\mathrm{O}$ & $\operatorname{lnChl}=1 \mathrm{~S} / \mathrm{C} 8 \mathrm{H} 15 \mathrm{O} 2 / \mathrm{c1}-6(5-9) 7(10) 8(2,3) 4 / \mathrm{h} 5-7 \mathrm{H}, 1-4 \mathrm{H} 3$ \\
\hline 3371 & $\mathrm{IC} 8034-50 \mathrm{OH}$ & $\mathrm{CC}(\mathrm{C})(\mathrm{C}) \mathrm{C}(\mathrm{O} 1) \mathrm{C} 1(\mathrm{C}) \mathrm{COO}$ & InChl=1S/C8H1603/c1-7(2,3)6-8(4,11-6)5-10-9/h6,9H,5H2,1-4H3 \\
\hline 3372 & $\mathrm{C} 6 \mathrm{H} 5 \mathrm{CHCH}$ & $\mathrm{C} 1=\mathrm{CC}(\mathrm{C}=[\mathrm{CH}])=\mathrm{CC}=\mathrm{C} 1$ & $\operatorname{lnChl}=15 / \mathrm{C} 8 \mathrm{H} 7 / \mathrm{c1} 1-2-8-6-4-3-5-7-8 / \mathrm{h} 1-7 \mathrm{H}$ \\
\hline 3373 & $\mathrm{HOCH} 3 \mathrm{C} 6 \mathrm{H} 3 \mathrm{CH} 3$ & $\mathrm{C} 1=\mathrm{C}(\mathrm{O}) \mathrm{C}(\mathrm{C})=\mathrm{CC}=\mathrm{C} 1 \mathrm{C}$ & $\operatorname{lnChl}=1 \mathrm{~S} / \mathrm{C} 8 \mathrm{H} 100 / \mathrm{c1}-6-3-4-7(2) 8(9) 5-6 / \mathrm{h} 3-5,9 \mathrm{H}, 1-2 \mathrm{H} 3$ \\
\hline 3374 & IC8KET5-4O & $\mathrm{CC}(\mathrm{C})(\mathrm{C}) \mathrm{CC}([\mathrm{O}])(\mathrm{C}) \mathrm{C}=\mathrm{O}$ & InChl=1S/C8H15O2/c1-7(2,3)5-8(4,10)6-9/h6H,5H2,1-4H3 \\
\hline 3375 & $\mathrm{IC8O35-100 \textrm {H }}$ & $\mathrm{C}(\mathrm{OO}) \mathrm{C}(\mathrm{C})(\mathrm{C}) \mathrm{C}(\mathrm{O} 1) \mathrm{C}(\mathrm{C}) \mathrm{C} 1$ & InChl=15/C8H16O3/c1-6-4-10-7(6)8(2,3)5-11-9/h6-7,9H,4-5H2,1-3H3 \\
\hline 3376 & P-XYLYLE & $\mathrm{C}=\mathrm{c} 1 \mathrm{ccc}(=\mathrm{C}) \mathrm{cc} 1$ & $\operatorname{lnChl}=15 / C 8 H 8 / c 1-7-3-5-8(2) 6-4-7 / h 3-6 \mathrm{H}, 1-2 \mathrm{H} 2$ \\
\hline 3377 & $\mathrm{C} 8 \mathrm{H} 131-5,3-4, \mathrm{TAO}$ & $\mathrm{C}=\mathrm{CC}(\mathrm{C}) \mathrm{C}(\mathrm{C})([0]) \mathrm{C}=\mathrm{C}$ & InChl=1S/C8H13O/c1-5-7(3)8(4,9)6-2/h5-7H,1-2H2,3-4H3 \\
\hline 3378 & IC8KET5-5AO & $\mathrm{CC}(\mathrm{C})(\mathrm{C}) \mathrm{CC}(\mathrm{C}[\mathrm{O}]) \mathrm{C}=\mathrm{O}$ & $\operatorname{lnChl}=15 / \mathrm{C} 8 \mathrm{H} 15 \mathrm{O} 2 / \mathrm{c1} 1-8(2,3) 4-7(5-9) 6-10 / \mathrm{h} 5,7 \mathrm{H}, 4,6 \mathrm{H} 2,1-3 \mathrm{H} 3$ \\
\hline 3379 & $\mathrm{IC} 8035-40 \mathrm{OH}$ & $\mathrm{CC}(\mathrm{C})(\mathrm{C}) \mathrm{C}(\mathrm{O} 1) \mathrm{C}(\mathrm{OO})(\mathrm{C}) \mathrm{C} 1$ & InChl=15/C8H1603/c1-7(2,3)6-8(4,11-9)5-10-6/h6,9H,5H2,1-4H3 \\
\hline 3380 & O-XYLYLE & $\mathrm{C}=\mathrm{c} 1 \mathrm{cccccc} 1=\mathrm{C}$ & $\operatorname{lnChl}=15 / C 8 H 8 / c 1-7-5-3-4-6-8(7) 2 / h 3-6 \mathrm{H}, 1-2 \mathrm{H} 2$ \\
\hline 3381 & C8H131-5,3,TAO & $\mathrm{C}=\mathrm{CC}(\mathrm{C})([\mathrm{O}]) \mathrm{CC}=\mathrm{CC}$ & InChl=1S/C8H13O/c1-4-6-7-8(3,9)5-2/h4-6H,2,7H2,1,3H3 \\
\hline 3382 & IC8D3-10OH & $C(O O) C(C)(C) C=C(C) C$ & InChl=1S/C8H16O2/c1-7(2)5-8(3,4)6-10-9/h5,9H,6H2,1-4H3 \\
\hline 3383 & IC8O35-5АOOH & $\mathrm{CC}(\mathrm{C})(\mathrm{C}) \mathrm{C}(\mathrm{O} 1) \mathrm{C}(\mathrm{COO}) \mathrm{C} 1$ & InChl=1S/C8H1603/c1-8(2,3)7-6(4-10-7)5-11-9/h6-7,9H,4-5H2,1-3H3 \\
\hline 3384 & BZCYBUT & $\mathrm{c} 1 \mathrm{ccc} 2 \mathrm{c}(\mathrm{c} 1) \mathrm{cc} 2$ & InChl=1S/C8H8/c1-2-4-8-6-5-7(8)3-1/h1-4H,5-6H2 \\
\hline 3385 & C8H131-5,3,SAO & $\mathrm{C}=\mathrm{CC}(\mathrm{C}) \mathrm{C}([\mathrm{O}]) \mathrm{C}=\mathrm{CC}$ & InChl=1S/C8H130/c1-4-6-8(9)7(3)5-2/h4-8H,2H2,1,3H3 \\
\hline 3386 & IC8D3-5OOH & $\mathrm{CC}(\mathrm{C})(\mathrm{C}) \mathrm{C}=\mathrm{C}(\mathrm{C}) \mathrm{COO}$ & InChl=15/C8H1602/c1-7(6-10-9)5-8(2,3)4/h5,9H,6H2,1-4H3 \\
\hline 3387 & $\mathrm{IC8O45-10OH}$ & $\mathrm{C}(\mathrm{OO}) \mathrm{C}(\mathrm{C})(\mathrm{C}) \mathrm{CC}(\mathrm{O} 1)(\mathrm{C}) \mathrm{C} 1$ & InChl=1S/C8H16O3/c1-7(2,5-11-9)4-8(3)6-10-8/h9H,4-6H2,1-3H3 \\
\hline 3388 & CH3C7H5 & $\mathrm{C}=\mathrm{C}=\mathrm{C} 1 \mathrm{C}=\mathrm{CC}(=\mathrm{C} 1) \mathrm{C}$ & InChl=15/C8H8/c1-3-8-5-4-7(2)6-8/h4-6H,1H2,2H3 \\
\hline 3389 & C8H131-5,3,PAO & $\mathrm{C}=\mathrm{CC}(\mathrm{C}) \mathrm{CC}=\mathrm{CC}[\mathrm{O}]$ & InChl=1S/C8H130/c1-3-8(2)6-4-5-7-9/h3-5,8H,1,6-7H2,2H3 \\
\hline 3390 & IC8D4-10OH & $\mathrm{C}(\mathrm{OO}) \mathrm{C}(\mathrm{C})(\mathrm{C}) \mathrm{CC}(\mathrm{C})=\mathrm{C}$ & InChl=1S/C8H16O2/c1-7(2)5-8(3,4)6-10-9/h9H,1,5-6H2,2-4H3 \\
\hline 3391 & $\mathrm{IC} 8045-30 \mathrm{OH}$ & $\mathrm{CC}(\mathrm{C})(\mathrm{C}) \mathrm{C}(\mathrm{OO}) \mathrm{C}(\mathrm{O} 1)(\mathrm{C}) \mathrm{C} 1$ & InChl=15/C8H16O3/c1-7(2,3)6(11-9)8(4)5-10-8/h6,9H,5H2,1-4H3 \\
\hline 3392 & $\mathrm{C} 6 \mathrm{H} 5 \mathrm{C} 2 \mathrm{H} 3$ & $\mathrm{C} 1=\mathrm{CC}(\mathrm{C}=\mathrm{C})=\mathrm{CC}=\mathrm{C} 1$ & InChl=1S/C8H8/c1-2-8-6-4-3-5-7-8/h2-7H,1H2 \\
\hline 3393 & $\mathrm{C} 8 \mathrm{H} 132-6, \mathrm{SAO}$ & $\mathrm{CC}=\mathrm{CCC}([\mathrm{O}]) \mathrm{C}=\mathrm{CC}$ & InChl=1S/C8H130/c1-3-5-7-8(9)6-4-2/h3-6,8H,7H2,1-2H3 \\
\hline 3394 & IC8D4-30OH & $\mathrm{CC}(\mathrm{C})(\mathrm{C}) \mathrm{C}(\mathrm{OOO}) \mathrm{C}(\mathrm{C})=\mathrm{C}$ & $\operatorname{lnChl}=1 \mathrm{~S} / \mathrm{C} 8 \mathrm{H} 16 \mathrm{O} / \mathrm{c} 1-6(2) 7(10-9) 8(3,4) 5 / \mathrm{h} 7,9 \mathrm{H}, 1 \mathrm{H} 2,2-5 \mathrm{H} 3$ \\
\hline 3395 & IC8O45-5AOOH & $\mathrm{CC}(\mathrm{C})(\mathrm{C}) \mathrm{CC}(\mathrm{O} 1)(\mathrm{COO}) \mathrm{C} 1$ & InChl=1S/C8H1603/c1-7(2,3)4-8(5-10-8)6-11-9/h9H,4-6H2,1-3H3 \\
\hline 3396 & $\mathrm{CH} 3 \mathrm{C} 6 \mathrm{H} 3 \mathrm{CH} 3$ & $\mathrm{CC} 1=[\mathrm{C}] \mathrm{C}=\mathrm{C}(\mathrm{C}) \mathrm{C}=\mathrm{C} 1$ & InChl=1S/C8H9/c1-7-3-5-8(2)6-4-7/h3-5H,1-2H3 \\
\hline 3397 & $\mathrm{C} 8 \mathrm{H} 132-6, \mathrm{PAO}$ & $\mathrm{CC}=\mathrm{CCCC}=\mathrm{CC}[\mathrm{O}]$ & InChl=15/C8H13O/c1-2-3-4-5-6-7-8-9/h2-3,6-7H,4-5,8H2,1H3 \\
\hline 3398 & IC8D4-5OOH & $\mathrm{CC}(\mathrm{C})(\mathrm{C}) \mathrm{CC}(\mathrm{COO})=\mathrm{C}$ & $\operatorname{lnChl}=15 / \mathrm{C} 8 \mathrm{H} 1602 / \mathrm{c1}-7(6-10-9) 5-8(2,3) 4 / \mathrm{h} 9 \mathrm{H}, 1,5-6 \mathrm{H} 2,2-4 \mathrm{H} 3$ \\
\hline 3399 & IC8055A-10OH & $\mathrm{C}(\mathrm{OO}) \mathrm{C}(\mathrm{C})(\mathrm{C}) \mathrm{CC}(\mathrm{CO} 1) \mathrm{C} 1$ & InChl=15/C8H1603/c1-8(2,6-11-9)3-7-4-10-5-7/h7,9H,3-6H2,1-2H3 \\
\hline 3400 & $\mathrm{O}-\mathrm{CH} 3 \mathrm{C} 6 \mathrm{H} 4 \mathrm{CH} 2$ & $\mathrm{CC} 1=\mathrm{CC}=\mathrm{CC}=\mathrm{C} 1[\mathrm{CH} 2]$ & InChl=15/C8H9/c1-7-5-3-4-6-8(7)2/h3-6H,1H2,2H3 \\
\hline 3401 & H15DE25DM-AO & $\mathrm{C}=\mathrm{C}(\mathrm{C}) \mathrm{CCC}(=\mathrm{C}) \mathrm{C}[\mathrm{O}]$ & InChl=15/C8H130/c1-7(2)4-5-8(3)6-9/h1,3-6H2,2H3 \\
\hline 3402 & IC8-102R & $\mathrm{C}(\mathrm{O}[\mathrm{O}]) \mathrm{C}(\mathrm{C})(\mathrm{C}) \mathrm{CC}(\mathrm{C}) \mathrm{C}$ & InChl=15/C8H1702/c1-7(2)5-8(3,4)6-10-9/h7H,5-6H2,1-4H3 \\
\hline 3403 & IC8055A-30OH & $\mathrm{CC}(\mathrm{C})(\mathrm{C}) \mathrm{C}(\mathrm{OO}) \mathrm{C}(\mathrm{CO} 1) \mathrm{C} 1$ & InChl=15/C8H16O3/c1-8(2,3)7(11-9)6-4-10-5-6/h6-7,9H,4-5H2,1-3H3 \\
\hline 3404 & P-CH3C6H4CH2 & $\mathrm{CC} 1=\mathrm{CC}=\mathrm{C}([\mathrm{CH} 2]) \mathrm{C}=\mathrm{C} 1$ & InChl=1S/C8H9/c1-7-3-5-8(2)6-4-7/h3-6H,1H2,2H3 \\
\hline 3405 & H15DE25DM-SO & $\mathrm{C}=\mathrm{C}(\mathrm{C}) \mathrm{CC}(\mathrm{C}(=\mathrm{C}) \mathrm{C})[\mathrm{O}]$ & InChl=1S/C8H13O/c1-6(2)5-8(9)7(3)4/h8H,1,3,5H2,2,4H3 \\
\hline 3406 & IC8-302R & $\mathrm{CC}(\mathrm{C})(\mathrm{C}) \mathrm{C}(\mathrm{O}[\mathrm{O}]) \mathrm{C}(\mathrm{C}) \mathrm{C}$ & InChl=1S/C8H1702/c1-6(2)7(10-9)8(3,4)5/h6-7H,1-5H3 \\
\hline 3407 & IC8O55A-40OH & $\mathrm{CC}(\mathrm{C})(\mathrm{C}) \mathrm{CC}(\mathrm{OO})(\mathrm{CO} 1) \mathrm{C} 1$ & InChl=1S/C8H1603/c1-7(2,3)4-8(11-9)5-10-6-8/h9H,4-6H2,1-3H3 \\
\hline 3408 & $\mathrm{C} 6 \mathrm{H} 5 \mathrm{CHCH} 3$ & $\mathrm{C} 1=\mathrm{CC}([\mathrm{CH}] \mathrm{C})=\mathrm{CC}=\mathrm{C} 1$ & $\operatorname{lnChl}=1 \mathrm{~S} / \mathrm{C} 8 \mathrm{H} 9 / \mathrm{c1}-2-8-6-4-3-5-7-8 / \mathrm{h} 2-7 \mathrm{H}, 1 \mathrm{H} 3$ \\
\hline 3409 & IC8D301-1A & $01 \mathrm{CC}(\mathrm{C} 1)(\mathrm{C}) \mathrm{C}=\mathrm{C}(\mathrm{C}) \mathrm{C}$ & InChl=1S/C8H14O/c1-7(2)4-8(3)5-9-6-8/h4H,5-6H2,1-3H3 \\
\hline 3410 & IC8-402R & $\mathrm{CC}(\mathrm{C})(\mathrm{C}) \mathrm{CC}(\mathrm{O}[\mathrm{O}])(\mathrm{C}) \mathrm{C}$ & $\operatorname{lnChl}=15 / \mathrm{C} 8 \mathrm{H} 1702 / \mathrm{c} 1-7(2,3) 6-8(4,5) 10-9 / \mathrm{h} 6 \mathrm{H} 2,1-5 \mathrm{H} 3$ \\
\hline 3411 & IC8OH3-402R & $\mathrm{CC}(\mathrm{C})(\mathrm{C}) \mathrm{C}(\mathrm{O}) \mathrm{C}(\mathrm{O}[\mathrm{O}])(\mathrm{C}) \mathrm{C}$ & InChl=1S/C8H1703/c1-7(2,3)6(9)8(4,5)11-10/h6,9H,1-5H3 \\
\hline 3412 & $\mathrm{C} 6 \mathrm{H} 5 \mathrm{CH} 2 \mathrm{CH} 2$ & $\mathrm{C} 1=\mathrm{CC}(\mathrm{C}[\mathrm{CH} 2])=\mathrm{CC}=\mathrm{C} 1$ & InChl=1S/C8H9/c1-2-8-6-4-3-5-7-8/h3-7H,1-2H2 \\
\hline 3413 & IC8D401-1A & $01 \mathrm{CC}(\mathrm{C} 1)(\mathrm{C}) \mathrm{CC}(\mathrm{C})=\mathrm{C}$ & InChl=1S/C8H14O/c1-7(2)4-8(3)5-9-6-8/h1,4-6H2,2-3H3 \\
\hline 3414 & IC8-502R & $\mathrm{CC}(\mathrm{C})(\mathrm{C}) \mathrm{CC}(\mathrm{C}) \mathrm{CO}[\mathrm{O}]$ & InChl=15/C8H1702/c1-7(6-10-9)5-8(2,3)4/h7H,5-6H2,1-4H3 \\
\hline 3415 & IC8OH4-3O2R & $\mathrm{CC}(\mathrm{C})(\mathrm{C}) \mathrm{C}(\mathrm{O}[\mathrm{O}]) \mathrm{C}(\mathrm{O})(\mathrm{C}) \mathrm{C}$ & InChl=1S/C8H1703/c1-7(2,3)6(11-10)8(4,5)9/h6,9H,1-5H3 \\
\hline 3416 & $\mathrm{O}-\mathrm{XYL}$ & $\mathrm{CC} 1=\mathrm{C}(\mathrm{C}) \mathrm{C}=\mathrm{CC}=\mathrm{C} 1$ & InChl=1S/C8H10/c1-7-5-3-4-6-8(7)2/h3-6H,1-2H3 \\
\hline 3417 & IC8D3-5OR & $\mathrm{CC}(\mathrm{C})(\mathrm{C}) \mathrm{C}=\mathrm{C}(\mathrm{C}) \mathrm{C}[\mathrm{O}]$ & $\operatorname{lnChl}=1 \mathrm{~S} / \mathrm{C} 8 \mathrm{H} 15 \mathrm{O} / \mathrm{c1} 1-7(6-9) 5-8(2,3) 4 / \mathrm{h} 5 \mathrm{H}, 6 \mathrm{H} 2,1-4 \mathrm{H} 3$ \\
\hline 3418 & IC8OOH1-1AR & $\mathrm{C}(\mathrm{OO}) \mathrm{C}([\mathrm{CH} 2])(\mathrm{C}) \mathrm{CC}(\mathrm{C}) \mathrm{C}$ & InChl=15/C8H1702/c1-7(2)5-8(3,4)6-10-9/h7,9H,3,5-6H2,1-2,4H3 \\
\hline 3419 & $\mathrm{IC} 8 \mathrm{OH} 4-5 \mathrm{O} 2 \mathrm{R}$ & $\mathrm{CC}(\mathrm{C})(\mathrm{C}) \mathrm{CC}(\mathrm{O})(\mathrm{C}) \mathrm{CO}[\mathrm{O}]$ & InChl=1S/C8H17O3/c1-7(2,3)5-8(4,9)6-11-10/h9H,5-6H2,1-4H3 \\
\hline 3420 & P-XYL & $\mathrm{CC} 1=\mathrm{CC}=\mathrm{C}(\mathrm{C}) \mathrm{C}=\mathrm{C} 1$ & InChl=1S/C8H10/c1-7-3-5-8(2)6-4-7/h3-6H,1-2H3 \\
\hline 3421 & IC8D4-3OR & $\mathrm{CC}(\mathrm{C})(\mathrm{C}) \mathrm{C}([\mathrm{O}]) \mathrm{C}(\mathrm{C})=\mathrm{C}$ & $\operatorname{lnChl}=1 \mathrm{~S} / \mathrm{C} 8 \mathrm{H} 15 \mathrm{O} / \mathrm{c1} 1-6(2) 7(9) 8(3,4) 5 / \mathrm{h} 7 \mathrm{H}, 1 \mathrm{H} 2,2-5 \mathrm{H} 3$ \\
\hline 3422 & IC8OOH1-3R & $\mathrm{C}(\mathrm{OO}) \mathrm{C}(\mathrm{C})(\mathrm{C})[\mathrm{CH}] \mathrm{C}(\mathrm{C}) \mathrm{C}$ & $\operatorname{lnChl}=1 \mathrm{~S} / \mathrm{C} 8 \mathrm{H} 1702 / \mathrm{c1}-7(2) 5-8(3,4) 6-10-9 / \mathrm{h} 5,7,9 \mathrm{H}, 6 \mathrm{H} 2,1-4 \mathrm{H} 3$ \\
\hline 3423 & IC8OH5-4O2R & $\mathrm{CC}(\mathrm{C})(\mathrm{C}) \mathrm{CC}(\mathrm{O}[\mathrm{O}])(\mathrm{C}) \mathrm{CO}$ & InChl=15/C8H1703/c1-7(2,3)5-8(4,6-9)11-10/h9H,5-6H2,1-4H3 \\
\hline 3424 & $\mathrm{C} 6 \mathrm{H} 5 \mathrm{C} 2 \mathrm{H} 5$ & $\mathrm{C} 1=\mathrm{CC}(\mathrm{CC})=\mathrm{CC}=\mathrm{C} 1$ & $\operatorname{lnChl}=1 \mathrm{~S} / \mathrm{C} 8 \mathrm{H} 10 / \mathrm{cc} 1-2-8-6-4-3-5-7-8 / \mathrm{h} 3-7 \mathrm{H}, 2 \mathrm{H} 2,1 \mathrm{H} 3$ \\
\hline 3425 & IC8D4-5OR & $\mathrm{CC}(\mathrm{C})(\mathrm{C}) \mathrm{CC}(\mathrm{C}[\mathrm{O}])=\mathrm{C}$ & InChl=1S/C8H15O/c1-7(6-9)5-8(2,3)4/h1,5-6H2,2-4H3 \\
\hline 3426 & IC8OOH1-4R & $\mathrm{C}(\mathrm{OO}) \mathrm{C}(\mathrm{C})(\mathrm{C}) \mathrm{C}[\mathrm{C}](\mathrm{C}) \mathrm{C}$ & InChl=15/C8H1702/c1-7(2)5-8(3,4)6-10-9/h9H,5-6H2,1-4H3 \\
\hline 3427 & O-XYLSECO2 & {$[0] \mathrm{OCC} 1=\mathrm{C}(\mathrm{COO}) \mathrm{C}=\mathrm{CC}=\mathrm{C} 1$} & InChl=1S/C8H9O4/c9-11-5-7-3-1-2-4-8(7)6-12-10/h1-4,9H,5-6H2 \\
\hline 3428 & C8H131-5,3-4,TA & $\mathrm{C}=\mathrm{C}[\mathrm{C}](\mathrm{C}) \mathrm{C}(\mathrm{C}) \mathrm{C}=\mathrm{C}$ & $\operatorname{lnChl}=15 / C 8 H 13 / c 1-5-7(3) 8(4) 6-2 / h 5-7 \mathrm{H}, 1-2 \mathrm{H} 2,3-4 \mathrm{H} 3$ \\
\hline 3429 & IC8Y3-1R & {$[\mathrm{CH} 2] \mathrm{C}(\mathrm{C})(\mathrm{C}) \mathrm{C}(=\mathrm{O}) \mathrm{C}(\mathrm{C}) \mathrm{C}$} & InChl=1S/C8H15O/c1-6(2)7(9)8(3,4)5/h6H,3H2,1-2,4-5H3 \\
\hline 3430 & IC8OOH1-5R & $\mathrm{C}(\mathrm{OO}) \mathrm{C}(\mathrm{C})(\mathrm{C}) \mathrm{CC}(\mathrm{C})[\mathrm{CH} 2]$ & InChl=1S/C8H1702/c1-7(2)5-8(3,4)6-10-9/h7,9H,1,5-6H2,2-4H3 \\
\hline 3431 & IC8D3OOH1-1AO2R & $\mathrm{OOCC}(\mathrm{CO}[\mathrm{O}])(\mathrm{C}) \mathrm{C}=\mathrm{C}(\mathrm{C}) \mathrm{C}$ & InChl=15/C8H15O4/c1-7(2)4-8(3,5-11-9)6-12-10/h4,9H,5-6H2,1-3H3 \\
\hline 3432 & $\mathrm{C} 8 \mathrm{H} 131-5,3, \mathrm{TA}$ & $\mathrm{C}=\mathrm{C}[\mathrm{C}](\mathrm{C}) \mathrm{CC}=\mathrm{CC}$ & InChl=1S/C8H13/c1-4-6-7-8(3)5-2/h4-6H,2,7H2,1,3H3 \\
\hline 3433 & IC8Y3-4R & $\mathrm{CC}(\mathrm{C})(\mathrm{C}) \mathrm{C}(=\mathrm{O})[\mathrm{C}](\mathrm{C}) \mathrm{C}$ & $\operatorname{lnChl}=1 \mathrm{~S} / \mathrm{C} 8 \mathrm{H} 15 \mathrm{O} / \mathrm{c} 1-6(2) 7(9) 8(3,4) 5 / \mathrm{h} 1-5 \mathrm{H} 3$ \\
\hline 3434 & IC8OOH3-1R & {$[\mathrm{CH} 2] \mathrm{C}(\mathrm{C})(\mathrm{C}) \mathrm{C}(\mathrm{OO}) \mathrm{C}(\mathrm{C}) \mathrm{C}$} & InChl=15/C8H1702/c1-6(2)7(10-9)8(3,4)5/h6-7,9H,3H2,1-2,4-5H3 \\
\hline 3435 & IC8D4OOH1-1AO2R & $\mathrm{OOCC}(\mathrm{CO}[\mathrm{O}])(\mathrm{C}) \mathrm{CC}(\mathrm{C})=\mathrm{C}$ & InChl=15/C8H15O4/c1-7(2)4-8(3,5-11-9)6-12-10/h9H,1,4-6H2,2-3H3 \\
\hline 3436 & $\mathrm{C} 8 \mathrm{H} 131-5,3, \mathrm{SA}$ & $\mathrm{C}=\mathrm{CC}(\mathrm{C})[\mathrm{CH}] \mathrm{C}=\mathrm{CC}$ & $\operatorname{lnChl}=1 \mathrm{~S} / \mathrm{C} 8 \mathrm{H} 13 / \mathrm{c} 1-4-6-7-8(3) 5-2 / \mathrm{h} 4-8 \mathrm{H}, 2 \mathrm{H} 2,1,3 \mathrm{H} 3$ \\
\hline 3437 & IC8Y3-5R & $\mathrm{CC}(\mathrm{C})(\mathrm{C}) \mathrm{C}(=\mathrm{O}) \mathrm{C}(\mathrm{C})[\mathrm{CH} 2]$ & InChl=15/C8H15O/c1-6(2)7(9)8(3,4)5/h6H,1H2,2-5H3 \\
\hline 3438 & IC8OOH3-4R & $\mathrm{CC}(\mathrm{C})(\mathrm{C}) \mathrm{C}(\mathrm{OO})[\mathrm{C}](\mathrm{C}) \mathrm{C}$ & InChl=15/C8H1702/c1-6(2)7(10-9)8(3,4)5/h7,9H,1-5H3 \\
\hline 3439 & IC8OOH1-1AO2R & $\mathrm{C}(\mathrm{OO}) \mathrm{C}(\mathrm{CO}[\mathrm{O}])(\mathrm{C}) \mathrm{CC}(\mathrm{C}) \mathrm{C}$ & $\operatorname{lnChl}=15 / \mathrm{C} 8 \mathrm{H} 1704 / \mathrm{c} 1-7(2) 4-8(3,5-11-9) 6-12-10 / \mathrm{h} 7,9 \mathrm{H}, 4-6 \mathrm{H} 2,1-3 \mathrm{H} 3$ \\
\hline 3440 & $\mathrm{C} 8 \mathrm{H} 131-5,3, \mathrm{PA}$ & {$[\mathrm{CH} 2] \mathrm{C}=\mathrm{CCC}(\mathrm{C}) \mathrm{C}=\mathrm{C}$} & InChl=1S/C8H13/c1-4-6-7-8(3)5-2/h4-6,8H,1-2,7H2,3H3 \\
\hline 3441 & IC801-1A & $\mathrm{C}(\mathrm{O} 1) \mathrm{C}(\mathrm{C} 1)(\mathrm{C}) \mathrm{CC}(\mathrm{C}) \mathrm{C}$ & InChl=1S/C8H16O/c1-7(2)4-8(3)5-9-6-8/h7H,4-6H2,1-3H3 \\
\hline 3442 & IC8OOH3-5R & $\mathrm{CC}(\mathrm{C})(\mathrm{C}) \mathrm{C}(\mathrm{OO}) \mathrm{C}(\mathrm{C})[\mathrm{CH} 2]$ & InChl=1S/C8H1702/c1-6(2)7(10-9)8(3,4)5/h6-7,9H,1H2,2-5H3 \\
\hline 3443 & IC8OOH1-302R & $\mathrm{C}(\mathrm{OO}) \mathrm{C}(\mathrm{C})(\mathrm{C}) \mathrm{C}(\mathrm{O}[\mathrm{O}]) \mathrm{C}(\mathrm{C}) \mathrm{C}$ & InChl=15/C8H17O4/c1-6(2)7(12-10)8(3,4)5-11-9/h6-7,9H,5H2,1-4H3 \\
\hline 3444 & $\mathrm{C} 8 \mathrm{H} 132-6, \mathrm{SA}$ & $\mathrm{CC}=\mathrm{C}[\mathrm{CH}] \mathrm{CC}=\mathrm{CC}$ & $\operatorname{lnChl}=1 \mathrm{~S} / \mathrm{C} 8 \mathrm{H} 13 / \mathrm{cc} 1-3-5-7-8-6-4-2 / \mathrm{h} 3-7 \mathrm{H}, 8 \mathrm{H} 2,1-2 \mathrm{H} 3$ \\
\hline 3445 & IC801-3 & $\mathrm{C}(\mathrm{O} 1) \mathrm{C}(\mathrm{C})(\mathrm{C}) \mathrm{C} 1 \mathrm{C}(\mathrm{C}) \mathrm{C}$ & InChl=1S/C8H16O/c1-6(2)7-8(3,4)5-9-7/h6-7H,5H2,1-4H3 \\
\hline 3446 & IC8OOH4-1R & {$[\mathrm{CH} 2] \mathrm{C}(\mathrm{C})(\mathrm{C}) \mathrm{CC}(\mathrm{OO})(\mathrm{C}) \mathrm{C}$} & $\operatorname{lnChl}=1 \mathrm{~S} / \mathrm{C} 8 \mathrm{H} 1702 / \mathrm{c1}-7(2,3) 6-8(4,5) 10-9 / \mathrm{h} 9 \mathrm{H}, 1,6 \mathrm{H} 2,2-5 \mathrm{H} 3$ \\
\hline 3447 & IC8OOH1-402R & $\mathrm{C}(\mathrm{OO}) \mathrm{C}(\mathrm{C})(\mathrm{C}) \mathrm{CC}(\mathrm{O}[\mathrm{O}])(\mathrm{C}) \mathrm{C}$ & $\operatorname{lnChl}=1 \mathrm{~S} / \mathrm{C} 8 \mathrm{H} 1704 / \mathrm{c} 1-7(2,6-11-9) 5-8(3,4) 12-10 / \mathrm{h} 9 \mathrm{H}, 5-6 \mathrm{H} 2,1-4 \mathrm{H} 3$ \\
\hline 3448 & $\mathrm{C} 8 \mathrm{H} 132-6, \mathrm{PA}$ & {$[\mathrm{CH} 2] \mathrm{C}=\mathrm{CCCC}=\mathrm{CC}$} & InChl=1S/C8H13/c1-3-5-7-8-6-4-2/h3-6H,1,7-8H2,2H3 \\
\hline 3449 & IC801-4 & $\mathrm{C}(\mathrm{O} 1) \mathrm{C}(\mathrm{C})(\mathrm{C}) \mathrm{CC} 1(\mathrm{C}) \mathrm{C}$ & $\operatorname{lnChl}=15 / \mathrm{C} 8 \mathrm{H} 160 / \mathrm{c} 1-7(2) 5-8(3,4) 9-6-7 / \mathrm{h5}-6 \mathrm{H} 2,1-4 \mathrm{H} 3$ \\
\hline 3450 & $\mathrm{IC} 8 \mathrm{OOH} 4-3 \mathrm{R}$ & $\mathrm{CC}(\mathrm{C})(\mathrm{C})[\mathrm{CH}] \mathrm{C}(\mathrm{OO})(\mathrm{C}) \mathrm{C}$ & $\operatorname{lnChl}=1 \mathrm{~S} / \mathrm{C} 8 \mathrm{H} 1702 / \mathrm{c} 1-7(2,3) 6-8(4,5) 10-9 / \mathrm{h} 6,9 \mathrm{H}, 1-5 \mathrm{H} 3$ \\
\hline 3451 & IC8OOH1-502R & $\mathrm{C}(\mathrm{OO}) \mathrm{C}(\mathrm{C})(\mathrm{C}) \mathrm{CC}(\mathrm{C}) \mathrm{CO}[\mathrm{O}]$ & InChl=15/C8H1704/c1-7(5-11-9)4-8(2,3)6-12-10/h7,10H,4-6H2,1-3H3 \\
\hline 3452 & H15DE25DM-S & $\mathrm{C}=\mathrm{C}(\mathrm{C})[\mathrm{CH}] \mathrm{CC}(=\mathrm{C}) \mathrm{C}$ & InChl=1S/C8H13/c1-7(2)5-6-8(3)4/h5H,1,3,6H2,2,4H3 \\
\hline 3453 & IC801-5 & $\mathrm{C}(\mathrm{O} 1) \mathrm{C}(\mathrm{C})(\mathrm{C}) \mathrm{CC}(\mathrm{C}) \mathrm{C} 1$ & InChl=1S/C8H16O/c1-7-4-8(2,3)6-9-5-7/h7H,4-6H2,1-3H3 \\
\hline 3454 & IC8OOH4-5R & $\mathrm{CC}(\mathrm{C})(\mathrm{C}) \mathrm{CC}(\mathrm{OO})(\mathrm{C})[\mathrm{CH} 2]$ & InChl=15/C8H1702/c1-7(2,3)6-8(4,5)10-9/h9H,4,6H2,1-3,5H3 \\
\hline
\end{tabular}




\begin{tabular}{|c|c|c|c|}
\hline 3455 & ІС8OOH3-102R & $\mathrm{C}(\mathrm{O}[\mathrm{O}]) \mathrm{C}(\mathrm{C})(\mathrm{C}) \mathrm{C}(\mathrm{OO}) \mathrm{C}(\mathrm{C}) \mathrm{C}$ & $\operatorname{lnChl}=15 / \mathrm{C} 8 \mathrm{H} 1704 / \mathrm{c1}-6(2) 7(12-10) 8(3,4) 5-11-9 / \mathrm{h6}-7,10 \mathrm{H}, 5 \mathrm{H} 2,1-4 \mathrm{H} 3$ \\
\hline 3456 & H15DE25DM-A & $\mathrm{C}=\mathrm{C}([\mathrm{CH} 2]) \mathrm{CCC}(=\mathrm{C}) \mathrm{C}$ & $\operatorname{lnChl}=1 \mathrm{~S} / \mathrm{C} 8 \mathrm{H} 13 / \mathrm{c} 1-7(2) 5-6-8(3) 4 / \mathrm{h} 1-3,5-6 \mathrm{H} 2,4 \mathrm{H} 3$ \\
\hline 3457 & IC803-4 & $\mathrm{CC}(\mathrm{C})(\mathrm{C}) \mathrm{C}(\mathrm{O} 1) \mathrm{C} 1(\mathrm{C}) \mathrm{C}$ & InChl=1S/C8H16O/c1-7(2,3)6-8(4,5)9-6/h6H,1-5H3 \\
\hline 3458 & $\mathrm{IC} 8 \mathrm{OOH} 5-1 \mathrm{R}$ & {$[\mathrm{CH} 2] \mathrm{C}(\mathrm{C})(\mathrm{C}) \mathrm{CC}(\mathrm{C}) \mathrm{COO}$} & InChl=1S/C8H1702/c1-7(6-10-9)5-8(2,3)4/h7,9H,2,5-6H2,1,3-4H3 \\
\hline 3459 & IC8OOH3-4O2R & $\mathrm{CC}(\mathrm{C})(\mathrm{C}) \mathrm{C}(\mathrm{OO}) \mathrm{C}(\mathrm{O}[\mathrm{O}])(\mathrm{C}) \mathrm{C}$ & $\operatorname{lnChl}=1 \mathrm{~S} / \mathrm{C} 8 \mathrm{H} 17 \mathrm{O} 4 / \mathrm{c} 1-7(2,3) 6(11-9) 8(4,5) 12-10 / \mathrm{h} 6,9 \mathrm{H}, 1-5 \mathrm{H} 3$ \\
\hline 3460 & C8H141-5,3-4 & $C=C C(C) C(C) C=C$ & $\operatorname{lnChl}=1 \mathrm{~S} / \mathrm{C} 8 \mathrm{H} 14 / \mathrm{c} 1-5-7(3) 8(4) 6-2 / \mathrm{h5}-8 \mathrm{H}, 1-2 \mathrm{H} 2,3-4 \mathrm{H} 3$ \\
\hline 3461 & IC803-5 & $\mathrm{CC}(\mathrm{C})(\mathrm{C}) \mathrm{C}(\mathrm{O} 1) \mathrm{C}(\mathrm{C}) \mathrm{C} 1$ & InChl=1S/C8H160/c1-6-5-9-7(6)8(2,3)4/h6-7H,5H2,1-4H3 \\
\hline 3462 & IC8OOH5-3R & $\mathrm{CC}(\mathrm{C})(\mathrm{C})[\mathrm{CH}] \mathrm{C}(\mathrm{C}) \mathrm{COO}$ & InChl=1S/C8H1702/c1-7(6-10-9)5-8(2,3)4/h5,7,9H,6H2,1-4H3 \\
\hline 3463 & IC8OOH3-502R & $\mathrm{CC}(\mathrm{C})(\mathrm{C}) \mathrm{C}(\mathrm{OO}) \mathrm{C}(\mathrm{C}) \mathrm{CO}[\mathrm{O}]$ & InChl=15/C8H1704/c1-6(5-11-9)7(12-10)8(2,3)4/h6-7,10H,5H2,1-4H3 \\
\hline 3464 & $\mathrm{C} 8 \mathrm{H} 141-5,3$ & $\mathrm{C}=\mathrm{CC}(\mathrm{C}) \mathrm{CC}=\mathrm{CC}$ & InChl=1S/C8H14/c1-4-6-7-8(3)5-2/h4-6,8H,2,7H2,1,3H3 \\
\hline 3465 & IC804-5 & $\mathrm{CC}(\mathrm{C})(\mathrm{C}) \mathrm{CC}(\mathrm{O} 1)(\mathrm{C}) \mathrm{C} 1$ & $\operatorname{lnChl}=15 / \mathrm{C} 8 \mathrm{H} 160 / \mathrm{c} 1-7(2,3) 5-8(4) 6-9-8 / \mathrm{h} 5-6 \mathrm{H} 2,1-4 \mathrm{H} 3$ \\
\hline 3466 & IC8OOH5-4R & $\mathrm{CC}(\mathrm{C})(\mathrm{C}) \mathrm{C}[\mathrm{C}](\mathrm{C}) \mathrm{COO}$ & $\operatorname{lnChl}=1 \mathrm{~S} / \mathrm{C} 8 \mathrm{H} 1702 / \mathrm{c} 1-7(6-10-9) 5-8(2,3) 4 / \mathrm{h} 9 \mathrm{H}, 5-6 \mathrm{H} 2,1-4 \mathrm{H} 3$ \\
\hline 3467 & IC8OOH4-102R & $\mathrm{C}(\mathrm{O}[\mathrm{O}]) \mathrm{C}(\mathrm{C})(\mathrm{C}) \mathrm{CC}(\mathrm{OO})(\mathrm{C}) \mathrm{C}$ & InChl=15/C8H17O4/c1-7(2,6-11-9)5-8(3,4)12-10/h10H,5-6H2,1-4H3 \\
\hline 3468 & C8H142-6 & $\mathrm{CC}=\mathrm{CCCC}=\mathrm{CC}$ & $\operatorname{lnChl}=1 \mathrm{~S} / \mathrm{C} 8 \mathrm{H} 14 / \mathrm{c} 1-3-5-7-8-6-4-2 / \mathrm{h} 3-6 \mathrm{H}, 7-8 \mathrm{H} 2,1-2 \mathrm{H} 3$ \\
\hline 3469 & IC8O5-5A & $\mathrm{CC}(\mathrm{C})(\mathrm{C}) \mathrm{CC}(\mathrm{CO} 1) \mathrm{C} 1$ & InChl=1S/C8H160/c1-8(2,3)4-7-5-9-6-7/h7H,4-6H2,1-3H3 \\
\hline 3470 & IC8OOH5-5AR & $\mathrm{CC}(\mathrm{C})(\mathrm{C}) \mathrm{CC}([\mathrm{CH} 2]) \mathrm{COO}$ & InChl=1S/C8H1702/c1-7(6-10-9)5-8(2,3)4/h7,9H,1,5-6H2,2-4H3 \\
\hline 3471 & $\mathrm{IC} 80 \mathrm{OH} 4-3 \mathrm{O} 2 \mathrm{R}$ & $\mathrm{CC}(\mathrm{C})(\mathrm{C}) \mathrm{C}(\mathrm{O}[\mathrm{O}]) \mathrm{C}(\mathrm{OO})(\mathrm{C}) \mathrm{C}$ & $\operatorname{lnChl}=1 \mathrm{~S} / \mathrm{c} 8 \mathrm{H} 1704 / \mathrm{c} 1-7(2,3) 6(11-9) 8(4,5) 12-10 / \mathrm{h} 6,10 \mathrm{H}, 1-5 \mathrm{H} 3$ \\
\hline 3472 & H15DE25DM & $\mathrm{C}=\mathrm{C}(\mathrm{C}) \mathrm{CCC}(=\mathrm{C}) \mathrm{C}$ & $\operatorname{lnChl}=1 \mathrm{~S} / \mathrm{C} 8 \mathrm{H} 14 / \mathrm{c} 1-7(2) 5-6-8(3) 4 / \mathrm{h} 1,3,5-6 \mathrm{H} 2,2,4 \mathrm{H} 3$ \\
\hline 3473 & IC8Y3 & $\mathrm{CC}(\mathrm{C})(\mathrm{C}) \mathrm{C}(=0) \mathrm{C}(\mathrm{C}) \mathrm{C}$ & $\operatorname{lnChl}=1 \mathrm{~S} / \mathrm{C} 8 \mathrm{H} 16 \mathrm{O} / \mathrm{c1}-6(2) 7(9) 8(3,4) 5 / \mathrm{h} 6 \mathrm{H}, 1-5 \mathrm{H} 3$ \\
\hline 3474 & $\mathrm{IC} 80 \mathrm{OH} 1$ & $\mathrm{C}(\mathrm{OO}) \mathrm{C}(\mathrm{C})(\mathrm{C}) \mathrm{CC}(\mathrm{C}) \mathrm{C}$ & InChl=1S/C8H18O2/c1-7(2)5-8(3,4)6-10-9/h7,9H,5-6H2,1-4H3 \\
\hline 3475 & IC8OOH4-5O2R & $\mathrm{CC}(\mathrm{C})(\mathrm{C}) \mathrm{CC}(\mathrm{OO})(\mathrm{C}) \mathrm{CO}[\mathrm{O}]$ & $\operatorname{lnChl}=15 / \mathrm{C} 8 \mathrm{H} 1704 / \mathrm{c} 1-7(2,3) 5-8(4,12-10) 6-11-9 / \mathrm{h} 10 \mathrm{H}, 5-6 \mathrm{H} 2,1-4 \mathrm{H} 3$ \\
\hline 3476 & IC8D3-5R & $\mathrm{CC}(\mathrm{C})(\mathrm{C}) \mathrm{C}=\mathrm{C}(\mathrm{C})[\mathrm{CH} 2]$ & InChl=1S/C8H15/c1-7(2)6-8(3,4)5/h6H,1H2,2-5H3 \\
\hline 3477 & $\mathrm{IC} 8 \mathrm{OH} 3-4 \mathrm{R}$ & $\mathrm{CC}(\mathrm{C})(\mathrm{C}) \mathrm{C}(\mathrm{O})[\mathrm{C}](\mathrm{C}) \mathrm{C}$ & InChl=15/C8H17O/c1-6(2)7(9)8(3,4)5/h7,9H,1-5H3 \\
\hline 3478 & $\mathrm{IC} 80 \mathrm{OH} 3$ & $\mathrm{CC}(\mathrm{C})(\mathrm{C}) \mathrm{C}(\mathrm{OO}) \mathrm{C}(\mathrm{C}) \mathrm{C}$ & InChl=15/C8H18O2/c1-6(2)7(10-9)8(3,4)5/h6-7,9H,1-5H3 \\
\hline 3479 & IC8OOH5-102R & $\mathrm{C}(\mathrm{O}[\mathrm{O}]) \mathrm{C}(\mathrm{C})(\mathrm{C}) \mathrm{CC}(\mathrm{C}) \mathrm{COO}$ & InChl=15/C8H1704/c1-7(5-11-9)4-8(2,3)6-12-10/h7,9H,4-6H2,1-3H3 \\
\hline 3480 & IC8D4-5R & $\mathrm{CC}(\mathrm{C})(\mathrm{C}) \mathrm{CC}([\mathrm{CH} 2])=\mathrm{C}$ & InChl=15/C8H15/c1-7(2)6-8(3,4)5/h1-2,6H2,3-5H3 \\
\hline 3481 & IC8OH4-3R & $\mathrm{CC}(\mathrm{C})(\mathrm{C})[\mathrm{CH}] \mathrm{C}(\mathrm{O})(\mathrm{C}) \mathrm{C}$ & InChl=1S/C8H17O/c1-7(2,3)6-8(4,5)9/h6,9H,1-5H3 \\
\hline 3482 & $\mathrm{IC} 80 \mathrm{OH} 4$ & $\mathrm{CC}(\mathrm{C})(\mathrm{C}) \mathrm{CC}(\mathrm{OO})(\mathrm{C}) \mathrm{C}$ & InChl=15/C8H18O2/c1-7(2,3)6-8(4,5)10-9/h9H,6H2,1-5H3 \\
\hline 3483 & IC8OOH5-3O2R & $\mathrm{CC}(\mathrm{C})(\mathrm{C}) \mathrm{C}(\mathrm{O}[\mathrm{O}]) \mathrm{C}(\mathrm{C}) \mathrm{COO}$ & $\operatorname{lnChl}=1 \mathrm{~S} / \mathrm{C} 8 \mathrm{H} 1704 / \mathrm{c} 1-6(5-11-9) 7(12-10) 8(2,3) 4 / \mathrm{h} 6-7,9 \mathrm{H}, 5 \mathrm{H} 2,1-4 \mathrm{H} 3$ \\
\hline 3484 & IC8D4-1R & {$[\mathrm{CH} 2] \mathrm{C}(\mathrm{C})(\mathrm{C}) \mathrm{CC}(\mathrm{C})=\mathrm{C}$} & $\operatorname{lnChl}=1 \mathrm{~S} / \mathrm{C} 8 \mathrm{H} 15 / \mathrm{c} 1-7(2) 6-8(3,4) 5 / \mathrm{h} 1,3,6 \mathrm{H} 2,2,4-5 \mathrm{H} 3$ \\
\hline 3485 & $\mathrm{IC} 8 \mathrm{OH} 4-5 \mathrm{R}$ & $\mathrm{CC}(\mathrm{C})(\mathrm{C}) \mathrm{CC}(\mathrm{O})([\mathrm{CH} 2]) \mathrm{C}$ & InChl=15/C8H170/c1-7(2,3)6-8(4,5)9/h9H,4,6H2,1-3,5H3 \\
\hline 3486 & IC8OOH5 & $\mathrm{CC}(\mathrm{C})(\mathrm{C}) \mathrm{CC}(\mathrm{C}) \mathrm{COO}$ & InChl=1S/C8H1802/c1-7(6-10-9)5-8(2,3)4/h7,9H,5-6H2,1-4H3 \\
\hline 3487 & IC8OOH5-4O2R & $\mathrm{CC}(\mathrm{C})(\mathrm{C}) \mathrm{CC}(\mathrm{O}[\mathrm{O}])(\mathrm{C}) \mathrm{COO}$ & $\operatorname{lnChl}=1 \mathrm{~S} / \mathrm{C} 8 \mathrm{H} 1704 / \mathrm{c} 1-7(2,3) 5-8(4,12-10) 6-11-9 / \mathrm{h} 9 \mathrm{H}, 5-6 \mathrm{H} 2,1-4 \mathrm{H} 3$ \\
\hline 3488 & IC8D3-1R & {$[\mathrm{CH} 2] \mathrm{C}(\mathrm{C})(\mathrm{C}) \mathrm{C}=\mathrm{C}(\mathrm{C}) \mathrm{C}$} & $\operatorname{lnChl}=1 \mathrm{~S} / \mathrm{C} 8 \mathrm{H} 15 / \mathrm{c1}-7(2) 6-8(3,4) 5 / \mathrm{h} 6 \mathrm{H}, 3 \mathrm{H} 2,1-2,4-5 \mathrm{H} 3$ \\
\hline 3489 & IC8OH5-4R & $\mathrm{CC}(\mathrm{C})(\mathrm{C}) \mathrm{C}[\mathrm{C}](\mathrm{C}) \mathrm{CO}$ & $\operatorname{lnChl}=1 \mathrm{~S} / \mathrm{C} 8 \mathrm{H} 170 / \mathrm{c1} 1-7(6-9) 5-8(2,3) 4 / \mathrm{h} 9 \mathrm{H}, 5-6 \mathrm{H} 2,1-4 \mathrm{H} 3$ \\
\hline 3490 & O-XYLKET & $\mathrm{O}=\mathrm{CC} 1=\mathrm{C}(\mathrm{COO}) \mathrm{C}=\mathrm{CC}=\mathrm{C} 1$ & $\operatorname{lnChl}=15 / C 8 H 803 / c 9-5-7-3-1-2-4-8(7) 6-11-10 / h 1-5,10 \mathrm{H}, 6 \mathrm{H} 2$ \\
\hline 3491 & IC8OOH5-5AO2R & $\mathrm{CC}(\mathrm{C})(\mathrm{C}) \mathrm{CC}(\mathrm{CO}[\mathrm{O}]) \mathrm{COO}$ & $\operatorname{lnChl}=15 / C 8 H 1704 / c 1-8(2,3) 4-7(5-11-9) 6-12-10 / h 7,9 H, 4-6 \mathrm{H} 2,1-3 \mathrm{H} 3$ \\
\hline 3492 & IC8D3 & $\mathrm{CC}(\mathrm{C})(\mathrm{C}) \mathrm{C}=\mathrm{C}(\mathrm{C}) \mathrm{C}$ & InChl=1S/C8H16/c1-7(2)6-8(3,4)5/h6H,1-5H3 \\
\hline 3493 & IC8-10R & $C([0]) C(C)(C) C C(C) C$ & $\operatorname{lnChl}=15 / C 8 H 170 / c 1-7(2) 5-8(3,4) 6-9 / h 7 H, 5-6 \mathrm{H} 2,1-4 \mathrm{H} 3$ \\
\hline 3494 & $\mathrm{CH} 3 \mathrm{C} 6 \mathrm{H} 4 \mathrm{OCH} 2 \mathrm{OO}$ & {$[\mathrm{O}] \mathrm{OCOC} 1=\mathrm{CC}=\mathrm{CC}=\mathrm{C} 1 \mathrm{C}$} & InChl=1S/C8H9O3/c1-7-4-2-3-5-8(7)10-6-11-9/h2-5H,6H2,1H3 \\
\hline 3495 & IC8Q11A-1BR & $\mathrm{C}(\mathrm{OO}) \mathrm{C}(\mathrm{COO})([\mathrm{CH} 2]) \mathrm{CC}(\mathrm{C}) \mathrm{C}$ & $\mathrm{InChl}=1 \mathrm{~S} / \mathrm{C} 8 \mathrm{H} 1704 / \mathrm{c} 1-7(2) 4-8(3,5-11-9) 6-12-10 / \mathrm{h} 7,9-10 \mathrm{H}, 3-6 \mathrm{H} 2,1-2 \mathrm{H} 3$ \\
\hline 3496 & IC8D4 & $\mathrm{CC}(\mathrm{C})(\mathrm{C}) \mathrm{CC}(\mathrm{C})=\mathrm{C}$ & $\operatorname{lnChl}=1 \mathrm{~S} / \mathrm{C} 8 \mathrm{H} 16 / \mathrm{c1}-7(2) 6-8(3,4) 5 / \mathrm{h} 1,6 \mathrm{H} 2,2-5 \mathrm{H} 3$ \\
\hline 3497 & IC8-3OR & $\mathrm{CC}(\mathrm{C})(\mathrm{C}) \mathrm{C}([\mathrm{O}]) \mathrm{C}(\mathrm{C}) \mathrm{C}$ & $\operatorname{lnChl}=15 / \mathrm{C} 8 \mathrm{H} 170 / \mathrm{c} 1-6(2) 7(9) 8(3,4) 5 / \mathrm{h} 6-7 \mathrm{H}, 1-5 \mathrm{H} 3$ \\
\hline 3498 & $\mathrm{CH} 3 \mathrm{C} 6 \mathrm{H} 4 \mathrm{OCH} 2 \mathrm{OOH}$ & $\mathrm{OOCOC1}=\mathrm{CC}=\mathrm{CC}=\mathrm{C} 1 \mathrm{C}$ & InChl=1S/C8H1003/c1-7-4-2-3-5-8(7)10-6-11-9/h2-5,9H,6H2,1H3 \\
\hline 3499 & IC8Q11A-3R & $\mathrm{C}(\mathrm{OO}) \mathrm{C}(\mathrm{COO})(\mathrm{C})[\mathrm{CH}] \mathrm{C}(\mathrm{C}) \mathrm{C}$ & InChl=15/C8H1704/c1-7(2)4-8(3,5-11-9)6-12-10/h4,7,9-10H,5-6H2,1-3H3 \\
\hline 3500 & IC8-1R & {$[\mathrm{CH} 2] \mathrm{C}(\mathrm{C})(\mathrm{C}) \mathrm{CC}(\mathrm{C}) \mathrm{C}$} & InChl=15/C8H17/c1-7(2)6-8(3,4)5/h7H,3,6H2,1-2,4-5H3 \\
\hline 3501 & IC8-4OR & $\mathrm{CC}(\mathrm{C})(\mathrm{C}) \mathrm{CC}([\mathrm{O}])(\mathrm{C}) \mathrm{C}$ & InChl=1S/C8H170/c1-7(2,3)6-8(4,5)9/h6H2,1-5H3 \\
\hline 3502 & IC8D3KET1-1A & $\operatorname{OOOCC}(\mathrm{C}=0)(\mathrm{C}) \mathrm{C}=\mathrm{C}(\mathrm{C}) \mathrm{C}$ & InChl=1S/C8H14O3/c1-7(2)4-8(3,5-9)6-11-10/h4-5,10H,6H2,1-3H3 \\
\hline 3503 & IC8Q11A-4R & $\mathrm{C}(\mathrm{OO}) \mathrm{C}(\mathrm{COO})(\mathrm{C}) \mathrm{C}[\mathrm{C}](\mathrm{C}) \mathrm{C}$ & InChl=1S/C8H1704/c1-7(2)4-8(3,5-11-9)6-12-10/h9-10H,4-6H2,1-3H3 \\
\hline 3504 & IC8-3R & $\mathrm{CC}(\mathrm{C})(\mathrm{C})[\mathrm{CH}] \mathrm{C}(\mathrm{C}) \mathrm{C}$ & $\operatorname{lnChl}=1 \mathrm{~S} / \mathrm{C} 8 \mathrm{H} 17 / \mathrm{c1} 1-7(2) 6-8(3,4) 5 / \mathrm{h} 6-7 \mathrm{H}, 1-5 \mathrm{H} 3$ \\
\hline 3505 & IC8-5OR & $\mathrm{CC}(\mathrm{C})(\mathrm{C}) \mathrm{CC}(\mathrm{C}) \mathrm{C}[\mathrm{O}]$ & $\operatorname{lnChl}=15 / \mathrm{C} 8 \mathrm{H} 170 / \mathrm{c} 1-7(6-9) 5-8(2,3) 4 / \mathrm{h} 7 \mathrm{H}, 5-6 \mathrm{H} 2,1-4 \mathrm{H} 3$ \\
\hline 3506 & IC8D4KET1-1A & $00 c c(C=0)(C) \operatorname{cc}(C)=C$ & $\operatorname{lnChl}=1 \mathrm{~S} / \mathrm{C} 8 \mathrm{H} 14 \mathrm{O} 3 / \mathrm{c} 1-7(2) 4-8(3,5-9) 6-11-10 / \mathrm{h} 5,10 \mathrm{H}, 1,4,6 \mathrm{H} 2,2-3 \mathrm{H} 3$ \\
\hline 3507 & IC8Q11A-5R & $\mathrm{C}(\mathrm{OO}) \mathrm{C}(\mathrm{COO})(\mathrm{C}) \mathrm{CC}(\mathrm{C})[\mathrm{CH} 2]$ & $\operatorname{lnChl}=15 / \mathrm{C} 8 \mathrm{H} 1704 / \mathrm{c} 1-7(2) 4-8(3,5-11-9) 6-12-10 / \mathrm{h} 7,9-10 \mathrm{H}, 1,4-6 \mathrm{H} 2,2-3 \mathrm{H} 3$ \\
\hline 3508 & IC8-4R & $\mathrm{CC}(\mathrm{C})(\mathrm{C}) \mathrm{C}[\mathrm{C}](\mathrm{C}) \mathrm{C}$ & $\mathrm{InChl}=1 \mathrm{~S} / \mathrm{C} 8 \mathrm{H} 17 / \mathrm{c} 1-7(2) 6-8(3,4) 5 / \mathrm{h} 6 \mathrm{H} 2,1-5 \mathrm{H} 3$ \\
\hline 3509 & $\mathrm{CHOC6H4CO}$ & $\mathrm{O}=[\mathrm{C}] \mathrm{C} 1=\mathrm{C}(\mathrm{C}=\mathrm{O}) \mathrm{C}=\mathrm{CC}=\mathrm{C} 1$ & InChl=1S/C8H502/c9-5-7-3-1-2-4-8(7)6-10/h1-5H \\
\hline 3510 & IC8KET1-1A & $\mathrm{O}=\mathrm{CC}(\mathrm{COO})(\mathrm{C}) \mathrm{CC}(\mathrm{C}) \mathrm{C}$ & $\operatorname{lnChl}=1 \mathrm{~S} / \mathrm{C} 8 \mathrm{H} 16 \mathrm{O} 3 / \mathrm{c} 1-7(2) 4-8(3,5-9) 6-11-10 / \mathrm{h} 5,7,10 \mathrm{H}, 4,6 \mathrm{H} 2,1-3 \mathrm{H} 3$ \\
\hline 3511 & IC8Q13-1AR & $\mathrm{C}(\mathrm{OO}) \mathrm{C}([\mathrm{CH} 2])(\mathrm{C}) \mathrm{C}(\mathrm{OO}) \mathrm{C}(\mathrm{C}) \mathrm{C}$ & $\operatorname{lnChl}=15 / \mathrm{C} 8 \mathrm{H} 1704 / \mathrm{c} 1-6(2) 7(12-10) 8(3,4) 5-11-9 / h 6-7,9-10 \mathrm{H}, 3,5 \mathrm{H} 2,1-2,4 \mathrm{H} 3$ \\
\hline 3512 & IC8-5R & $\mathrm{CC}(\mathrm{C})(\mathrm{C}) \mathrm{CC}(\mathrm{C})[\mathrm{CH} 2]$ & InChl=1S/C8H17/c1-7(2)6-8(3,4)5/h7H,1,6H2,2-5H3 \\
\hline 3513 & $\mathrm{CHOC6H} 4 \mathrm{CHO}$ & $\mathrm{O}=\mathrm{CC} 1=\mathrm{C}(\mathrm{C}=\mathrm{O}) \mathrm{C}=\mathrm{CC}=\mathrm{C} 1$ & InChl=1S/C8H6O2/c9-5-7-3-1-2-4-8(7)6-10/h1-6H \\
\hline 3514 & IC8KET1-3 & $\mathrm{O}=\mathrm{CC}(\mathrm{C})(\mathrm{C}) \mathrm{C}(\mathrm{OO}) \mathrm{C}(\mathrm{C}) \mathrm{C}$ & $\operatorname{lnChl}=1 \mathrm{~S} / \mathrm{C} 8 \mathrm{H} 16 \mathrm{O} / \mathrm{c1}-6(2) 7(11-10) 8(3,4) 5-9 / \mathrm{h} 5-7,10 \mathrm{H}, 1-4 \mathrm{H} 3$ \\
\hline 3515 & IC8Q13-4R & $\mathrm{C}(\mathrm{OO}) \mathrm{C}(\mathrm{C})(\mathrm{C}) \mathrm{C}(\mathrm{OO})[\mathrm{C}](\mathrm{C}) \mathrm{C}$ & InChl=15/c8H1704/c1-6(2)7(12-10)8(3,4)5-11-9/h7,9-10H,5H2,1-4H3 \\
\hline 3516 & IC8 & $\mathrm{CC}(\mathrm{C})(\mathrm{C}) \mathrm{CC}(\mathrm{C}) \mathrm{C}$ & InChl=1S/C8H18/c1-7(2)6-8(3,4)5/h7H,6H2,1-5H3 \\
\hline 3517 & CHOC6H4CH2OJ & $\mathrm{O}=\mathrm{CC} 1=\mathrm{C}(\mathrm{C}[\mathrm{O}]) \mathrm{C}=\mathrm{CC}=\mathrm{C} 1$ & InChl=15/C8H702/c9-5-7-3-1-2-4-8(7)6-10/h1-5H,6H2 \\
\hline 3518 & IC8KET1-4 & $\mathrm{O}=\mathrm{CC}(\mathrm{C})(\mathrm{C}) \mathrm{CC}(\mathrm{OO})(\mathrm{C}) \mathrm{C}$ & InChl=1S/C8H16O3/c1-7(2,6-9)5-8(3,4)11-10/h6,10H,5H2,1-4H3 \\
\hline 3519 & IC8Q13-5R & $\mathrm{C}(\mathrm{OO}) \mathrm{C}(\mathrm{C})(\mathrm{C}) \mathrm{C}(\mathrm{OO}) \mathrm{C}(\mathrm{C})[\mathrm{CH} 2]$ & $\operatorname{lnChl}=15 / \mathrm{C} 8 \mathrm{H} 1704 / \mathrm{c} 1-6(2) 7(12-10) 8(3,4) 5-11-9 / \mathrm{h} 6-7,9-10 \mathrm{H}, 1,5 \mathrm{H} 2,2-4 \mathrm{H} 3$ \\
\hline 3520 & $\mathrm{C} 6 \mathrm{H} 5 \mathrm{CCO}$ & $\mathrm{O}=\mathrm{C}=[\mathrm{C}] \mathrm{C} 1=\mathrm{CC}=\mathrm{CC}=\mathrm{C} 1$ & InChl=1S/C8H5O/c9-7-6-8-4-2-1-3-5-8/h1-5H \\
\hline 3521 & $\mathrm{O}-\mathrm{CH} 3 \mathrm{C} 6 \mathrm{H} 4 \mathrm{CH} 2 \mathrm{OO}$ & $\mathrm{CC} 1=\mathrm{C}(\mathrm{CO}[\mathrm{O}]) \mathrm{C}=\mathrm{CC}=\mathrm{C} 1$ & InChl=1S/C8H9O2/c1-7-4-2-3-5-8(7)6-10-9/h2-5H,6H2,1H3 \\
\hline 3522 & IC8KET1-5 & $\mathrm{O}=\mathrm{CC}(\mathrm{C})(\mathrm{C}) \mathrm{CC}(\mathrm{C}) \mathrm{COO}$ & InChl=15/C8H16O3/c1-7(5-11-10)4-8(2,3)6-9/h6-7,10H,4-5H2,1-3H3 \\
\hline 3523 & IC8Q14-1AR & $\mathrm{C}(\mathrm{OO}) \mathrm{C}([\mathrm{CH} 2])(\mathrm{C}) \mathrm{CC}(\mathrm{OO})(\mathrm{C}) \mathrm{C}$ & InChl=1S/C8H1704/c1-7(2,6-11-9)5-8(3,4)12-10/h9-10H,1,5-6H2,2-4H3 \\
\hline 3524 & $\mathrm{C} 8 \mathrm{H} 6 \mathrm{O}$ & $\mathrm{C} 12=\mathrm{CC}=\mathrm{CC}=\mathrm{C} 10 \mathrm{C}=\mathrm{C} 2$ & InChl=15/C8H6O/c1-2-4-8-7(3-1)5-6-9-8/h1-6H \\
\hline 3525 & P-CH3C6H4CH2OO & $\mathrm{CC} 1=\mathrm{CC}=\mathrm{C}(\mathrm{CO}[\mathrm{O}]) \mathrm{C}=\mathrm{C} 1$ & InChl=1S/C8H9O2/c1-7-2-4-8(5-3-7)6-10-9/h2-5H,6H2,1H3 \\
\hline 3526 & IC8KET3-1 & $c(00) c(c)(C) C(=0) c(C) c$ & InChl=1S/C8H16O3/c1-6(2)7(9)8(3,4)5-11-10/h6,10H,5H2,1-4H3 \\
\hline 3527 & IC8Q14-3R & $\mathrm{C}(\mathrm{OO}) \mathrm{C}(\mathrm{C})(\mathrm{C})[\mathrm{CH}] \mathrm{C}(\mathrm{OO})(\mathrm{C}) \mathrm{C}$ & InChl=1S/C8H1704/c1-7(2,6-11-9)5-8(3,4)12-10/h5,9-10H,6H2,1-4H3 \\
\hline 3528 & $\mathrm{C} 6 \mathrm{H} 5 \mathrm{CHCO}$ & $\mathrm{O}=\mathrm{C}=\mathrm{CC} 1=\mathrm{CC}=\mathrm{CC}=\mathrm{C} 1$ & InChl=1S/C8H6O/c9-7-6-8-4-2-1-3-5-8/h1-6H \\
\hline 3529 & $\mathrm{O} 2 \mathrm{CH} 3 \mathrm{C} 6 \mathrm{H} 3 \mathrm{CH} 3$ & $\mathrm{CC} 1=\mathrm{CC}(\mathrm{O}[\mathrm{O}])=\mathrm{C}(\mathrm{C}) \mathrm{C}=\mathrm{C} 1$ & InChl=1S/C8H9O2/c1-6-3-4-7(2)8(5-6)10-9/h3-5H,1-2H3 \\
\hline 3530 & IC8KET3-4 & $\mathrm{CC}(\mathrm{C})(\mathrm{C}) \mathrm{C}(=\mathrm{O}) \mathrm{C}(\mathrm{OO})(\mathrm{C}) \mathrm{C}$ & InChl=1S/C8H16O3/c1-7(2,3)6(9)8(4,5)11-10/h10H,1-5H3 \\
\hline 3531 & IC8Q14-5R & $\mathrm{C}(\mathrm{OO}) \mathrm{C}(\mathrm{C})(\mathrm{C}) \mathrm{CC}(\mathrm{OO})(\mathrm{C})[\mathrm{CH} 2]$ & 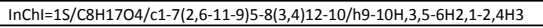 \\
\hline 3532 & $\mathrm{C} 8 \mathrm{H} 7 \mathrm{O}-2$ & $\mathrm{c} 1 \mathrm{c} 2 \mathrm{O}[\mathrm{CH}] \mathrm{Cc} 2 \mathrm{ccc} 1$ & $\operatorname{lnChl}=1 \mathrm{~S} / \mathrm{C} 8 \mathrm{H} 7 \mathrm{O} / \mathrm{c1} 1-2-4-8-7(3-1) 5-6-9-8 / \mathrm{h} 1-4,6 \mathrm{H}, 5 \mathrm{H} 2$ \\
\hline 3533 & O-XYLQOOH & {$[\mathrm{C}] \mathrm{C} 1=\mathrm{C}(\mathrm{COO}) \mathrm{C}=\mathrm{CC}=\mathrm{C} 1$} & $\operatorname{lnChl}=1 \mathrm{~S} / \mathrm{C} 8 \mathrm{H} 902 / \mathrm{c} 1-7-4-2-3-5-8(7) 6-10-9 / \mathrm{h} 2-5,9 \mathrm{H}, 1,6 \mathrm{H} 2$ \\
\hline 3534 & IC8KET3-5 & $\mathrm{CC}(\mathrm{C})(\mathrm{C}) \mathrm{C}(=\mathrm{O}) \mathrm{C}(\mathrm{C}) \mathrm{COO}$ & InChl=1S/C8H16O3/c1-6(5-11-10)7(9)8(2,3)4/h6,10H,5H2,1-4H3 \\
\hline 3535 & IC8Q15-1AR & $\mathrm{C}(\mathrm{OO}) \mathrm{C}([\mathrm{CH} 2])(\mathrm{C}) \mathrm{CC}(\mathrm{C}) \mathrm{COO}$ & InChl=15/C8H1704/c1-7(5-11-9)4-8(2,3)6-12-10/h7,9-10H,2,4-6H2,1,3H3 \\
\hline 3536 & $\mathrm{C} 8 \mathrm{H} 7 \mathrm{O}-1$ & $\mathrm{c} 1 \mathrm{c} 2 \mathrm{OC}[\mathrm{CH}] \mathrm{c} 2 \mathrm{ccc} 1$ & InChl=1S/C8H70/c1-2-4-8-7(3-1)5-6-9-8/h1-5H,6H2 \\
\hline 3537 & $\mathrm{C} 6 \mathrm{H} 5 \mathrm{CHOOCH} 3$ & $\mathrm{CC}(\mathrm{O}[0]) \mathrm{C} 1 \mathrm{C}=\mathrm{CC}=\mathrm{CC}=1$ & $\operatorname{lnChl}=1 \mathrm{~S} / \mathrm{C} 8 \mathrm{H} 902 / \mathrm{c} 1-7(10-9) 8-5-3-2-4-6-8 / \mathrm{h} 2-7 \mathrm{H}, 1 \mathrm{H} 3$ \\
\hline 3538 & IC8KET5-1 & $C(00) C(C)(C) C C(C) C=0$ & InChl=15/C8H16O3/c1-7(5-9)4-8(2,3)6-11-10/h5,7,10H,4,6H2,1-3H3 \\
\hline 3539 & IC8Q15-3R & $\mathrm{C}(\mathrm{OO}) \mathrm{C}(\mathrm{C})(\mathrm{C})[\mathrm{CH}] \mathrm{C}(\mathrm{C}) \mathrm{COO}$ & $\operatorname{lnChl}=15 / \mathrm{C} 8 \mathrm{H} 1704 / \mathrm{c} 1-7(5-11-9) 4-8(2,3) 6-12-10 / \mathrm{h} 4,7,9-10 \mathrm{H}, 5-6 \mathrm{H} 2,1-3 \mathrm{H} 3$ \\
\hline 3540 & $\mathrm{CH} 3 \mathrm{C} 6 \mathrm{H} 4 \mathrm{CO}$ & $\mathrm{CC} 1=\mathrm{CC}=\mathrm{C}([\mathrm{C}]=\mathrm{O}) \mathrm{C}=\mathrm{C} 1$ & InChl=1S/C8H7O/c1-7-2-4-8(6-9)5-3-7/h2-5H,1H3 \\
\hline 3541 & $\mathrm{C} 6 \mathrm{H} 5 \mathrm{CH} 2 \mathrm{CH} 2 \mathrm{OO}$ & {$[0] \mathrm{OCCC} 1 \mathrm{C}=\mathrm{CC}=\mathrm{CC}=1$} & InChl=1S/C8H9O2/c9-10-7-6-8-4-2-1-3-5-8/h1-5H,6-7H2 \\
\hline 3542 & IC8KET5-3 & $\mathrm{CC}(\mathrm{C})(\mathrm{C}) \mathrm{C}(\mathrm{OO}) \mathrm{C}(\mathrm{C}) \mathrm{C}=0$ & $\operatorname{lnChl}=1 \mathrm{~S} / \mathrm{C} 8 \mathrm{H} 16 \mathrm{O} / \mathrm{c1}-6(5-9) 7(11-10) 8(2,3) 4 / \mathrm{h} 5-7,10 \mathrm{H}, 1-4 \mathrm{H} 3$ \\
\hline 3543 & IC8Q15-4R & $\mathrm{C}(\mathrm{OO}) \mathrm{C}(\mathrm{C})(\mathrm{C}) \mathrm{C}[\mathrm{C}](\mathrm{C}) \mathrm{COO}$ & InChl=15/C8H1704/c1-7(5-11-9)4-8(2,3)6-12-10/h9-10H,4-6H2,1-3H3 \\
\hline 3544 & $\mathrm{CHOC6H} 4 \mathrm{CH} 2$ & {$[\mathrm{C}] \mathrm{C} 1=\mathrm{C}(\mathrm{C}=\mathrm{O}) \mathrm{C}=\mathrm{CC}=\mathrm{C} 1$} & InChl=1S/C8H7O/c1-7-4-2-3-5-8(7)6-9/h2-6H,1H2 \\
\hline 3545 & $\mathrm{O}-\mathrm{CH} 3 \mathrm{C} 6 \mathrm{H} 4 \mathrm{CH} 2 \mathrm{OOH}$ & $\mathrm{CC} 1=\mathrm{C}(\mathrm{COO}) \mathrm{C}=\mathrm{CC}=\mathrm{C} 1$ & $\operatorname{lnChl}=15 / C 8 H 1002 / c 1-7-4-2-3-5-8(7) 6-10-9 / h 2-5,9 H, 6 \mathrm{H} 2,1 \mathrm{H} 3$ \\
\hline
\end{tabular}




\begin{tabular}{|c|c|c|c|}
\hline 3546 & IC8KETS-4 & $\mathrm{CC}(\mathrm{C})(\mathrm{C}) \mathrm{CC}(\mathrm{OO})(\mathrm{C}) \mathrm{C}=\mathrm{O}$ & InChl=1S/C8H16O3/c1-7(2,3)5-8(4,6-9)11-10/h6,10H,5H2,1-4H3 \\
\hline 3547 & IC8Q15-5AR & $\mathrm{C}(\mathrm{OO}) \mathrm{C}(\mathrm{C})(\mathrm{C}) \mathrm{CC}([\mathrm{CH} 2]) \mathrm{COO}$ & InChl=15/C8H1704/c1-7(5-11-9)4-8(2,3)6-12-10/h7,9-10H,1,4-6H2,2-3H3 \\
\hline 3548 & $\mathrm{C} 6 \mathrm{H} 5 \mathrm{CH} 2 \mathrm{CO}$ & $\mathrm{O}=[\mathrm{C}] \mathrm{CC} 1=\mathrm{CC}=\mathrm{CC}=\mathrm{C} 1$ & InChl=1S/C8H7O/c9-7-6-8-4-2-1-3-5-8/h1-5H,6H2 \\
\hline 3549 & P-CH3C6H4CH2OOH & $\mathrm{CC} 1=\mathrm{CC}=\mathrm{C}(\mathrm{COO}) \mathrm{C}=\mathrm{C} 1$ & InChl=1S/C8H10O2/c1-7-2-4-8(5-3-7)6-10-9/h2-5,9H,6H2,1H3 \\
\hline 3550 & IC8KET5-5A & $\mathrm{CC}(\mathrm{C})(\mathrm{C}) \mathrm{CC}(\mathrm{COO}) \mathrm{C}=\mathrm{O}$ & InChl=1S/C8H16O3/c1-8(2,3)4-7(5-9)6-11-10/h5,7,10H,4,6H2,1-3H3 \\
\hline 3551 & IC8Q34-1R & {$[\mathrm{CH} 2] \mathrm{C}(\mathrm{C})(\mathrm{C}) \mathrm{C}(\mathrm{OO}) \mathrm{C}(\mathrm{OO})(\mathrm{C}) \mathrm{C}$} & InChl=1S/C8H1704/c1-7(2,3)6(11-9)8(4,5)12-10/h6,9-10H,1H2,2-5H3 \\
\hline 3552 & $\mathrm{C} 6 \mathrm{H} 5 \mathrm{COCH} 2$ & {$[\mathrm{CH} 2] \mathrm{C}(\mathrm{C} 1=\mathrm{CC}=\mathrm{CC}=\mathrm{C} 1)=\mathrm{O}$} & InChl=1S/C8H7O/c1-7(9)8-5-3-2-4-6-8/h2-6H,1H2 \\
\hline 3553 & IC4H7OOIC4H7 & $\mathrm{CC}(=\mathrm{C}) \operatorname{COOCC}(=\mathrm{C}) \mathrm{C}$ & $\operatorname{lnChl}=15 / \mathrm{C} 8 \mathrm{H} 14 \mathrm{O} 2 / \mathrm{c} 1-7(2) 5-9-10-6-8(3) 4 / \mathrm{h} 1,3,5-6 \mathrm{H} 2,2,4 \mathrm{H} 3$ \\
\hline 3554 & IC8011A-1BOOH & $\mathrm{C}(\mathrm{OO}) \mathrm{C}(\mathrm{CO} 1)(\mathrm{C} 1) \mathrm{CC}(\mathrm{C}) \mathrm{C}$ & InChl=1S/C8H1603/c1-7(2)3-8(6-11-9)4-10-5-8/h7,9H,3-6H2,1-2H3 \\
\hline 3555 & IC8Q34-5R & $\mathrm{CC}(\mathrm{C})(\mathrm{C}) \mathrm{C}(\mathrm{OO}) \mathrm{C}(\mathrm{OO})(\mathrm{C})[\mathrm{CH} 2]$ & InChl=1S/C8H1704/c1-7(2,3)6(11-9)8(4,5)12-10/h6,9-10H,4H2,1-3,5H3 \\
\hline 3556 & $\mathrm{C} 8 \mathrm{H} 8 \mathrm{O}$ & c1c20ccc2ccc1 & $\operatorname{lnChl}=1 \mathrm{~S} / \mathrm{C} 8 \mathrm{H} 80 / \mathrm{c1} 1-2-4-8-7(3-1) 5-6-9-8 / \mathrm{h} 1-4 \mathrm{H}, 5-6 \mathrm{H} 2$ \\
\hline 3557 & IC8D3-102R & {$[\mathrm{O}] \mathrm{OCC}(\mathrm{C})(\mathrm{C}) \mathrm{C}=\mathrm{C}(\mathrm{C}) \mathrm{C}$} & InChl=1S/C8H15O2/c1-7(2)5-8(3,4)6-10-9/h5H,6H2,1-4H3 \\
\hline 3558 & IC8011A-30OH & $\mathrm{CC}(\mathrm{CO} 1)(\mathrm{C} 1) \mathrm{C}(\mathrm{OO}) \mathrm{C}(\mathrm{C}) \mathrm{C}$ & InChl=15/C8H16O3/c1-6(2)7(11-9)8(3)4-10-5-8/h6-7,9H,4-5H2,1-3H3 \\
\hline 3559 & IC8Q35-1R & {$[\mathrm{CH} 2] \mathrm{C}(\mathrm{C})(\mathrm{C}) \mathrm{C}(\mathrm{OO}) \mathrm{C}(\mathrm{C}) \mathrm{COO}$} & $\operatorname{lnChl}=15 / \mathrm{C} 8 \mathrm{H} 1704 / \mathrm{c1}-6(5-11-9) 7(12-10) 8(2,3) 4 / \mathrm{h} 6-7,9-10 \mathrm{H}, 2,5 \mathrm{H} 2,1,3-4 \mathrm{H} 3$ \\
\hline 3560 & $\mathrm{HOC} 6 \mathrm{H}_{4} \mathrm{C} 2 \mathrm{H} 3$ & $\mathrm{OC} 1=\mathrm{CC}=\mathrm{CC}=\mathrm{C} 1 \mathrm{C}=\mathrm{C}$ & InChl=1S/C8H8O/c1-2-7-5-3-4-6-8(7)9/h2-6,9H,1H2 \\
\hline 3561 & IC8D4-102R & {$[0] \operatorname{OCC}(\mathrm{C})(\mathrm{C}) \mathrm{CC}(\mathrm{C})=\mathrm{C}$} & InChl=1S/C8H15O2/c1-7(2)5-8(3,4)6-10-9/h1,5-6H2,2-4H3 \\
\hline 3562 & IC8O11A-40OH & $\mathrm{CC}(\mathrm{CO} 1)(\mathrm{C} 1) \mathrm{CC}(\mathrm{OO})(\mathrm{C}) \mathrm{C}$ & InChl=15/C8H1603/c1-7(2,11-9)4-8(3)5-10-6-8/h9H,4-6H2,1-3H3 \\
\hline 3563 & IC8Q35-4R & $\mathrm{CC}(\mathrm{C})(\mathrm{C}) \mathrm{C}(\mathrm{OO})[\mathrm{C}](\mathrm{C}) \mathrm{COO}$ & InChl=1S/C8H1704/c1-6(5-11-9)7(12-10)8(2,3)4/h7,9-10H,5H2,1-4H3 \\
\hline 3564 & $\mathrm{CH} 3 \mathrm{C} 6 \mathrm{H} 4 \mathrm{CHO}$ & $\mathrm{CC} 1=\mathrm{CC}=\mathrm{C}(\mathrm{C}=\mathrm{O}) \mathrm{C}=\mathrm{C} 1$ & InChl=1S/C8H8O/c1-7-2-4-8(6-9)5-3-7/h2-6H,1H3 \\
\hline 3565 & IC8D3OOH1-1AR & $\mathrm{OOCC}([\mathrm{CH} 2])(\mathrm{C}) \mathrm{C}=\mathrm{C}(\mathrm{C}) \mathrm{C}$ & InChl=1S/C8H15O2/c1-7(2)5-8(3,4)6-10-9/h5,9H,3,6H2,1-2,4H3 \\
\hline 3566 & IC8011A-50OH & $\mathrm{CC}(\mathrm{CO} 1)(\mathrm{C} 1) \mathrm{CC}(\mathrm{C}) \mathrm{COO}$ & InChl=1S/C8H1603/c1-7(4-11-9)3-8(2)5-10-6-8/h7,9H,3-6H2,1-2H3 \\
\hline 3567 & IC8Q35-5AR & $\mathrm{CC}(\mathrm{C})(\mathrm{C}) \mathrm{C}(\mathrm{OO}) \mathrm{C}([\mathrm{CH} 2]) \mathrm{COO}$ & $\operatorname{lnChl}=15 / \mathrm{C} 8 \mathrm{H} 1704 / \mathrm{c} 1-6(5-11-9) 7(12-10) 8(2,3) 4 / \mathrm{h} 6-7,9-10 \mathrm{H}, 1,5 \mathrm{H} 2,2-4 \mathrm{H} 3$ \\
\hline 3568 & O-XYLET & $\mathrm{C} 12=\mathrm{C}(\mathrm{COC} 2) \mathrm{C}=\mathrm{CC}=\mathrm{C} 1$ & InChl=1S/C8H8O/c1-2-4-8-6-9-5-7(8)3-1/h1-4H,5-6H2 \\
\hline 3569 & IC8D4OOH1-1AR & $\mathrm{OOCC}([\mathrm{CH} 2])(\mathrm{C}) \mathrm{CC}(\mathrm{C})=\mathrm{C}$ & InChl=1S/C8H15O2/c1-7(2)5-8(3,4)6-10-9/h9H,1,3,5-6H2,2,4H3 \\
\hline 3570 & IC8O13-1AOOH & $\mathrm{C}(\mathrm{O} 1) \mathrm{C}(\mathrm{COO})(\mathrm{C}) \mathrm{C} 1 \mathrm{C}(\mathrm{C}) \mathrm{C}$ & InChl=15/C8H16O3/c1-6(2)7-8(3,4-10-7)5-11-9/h6-7,9H,4-5H2,1-3H3 \\
\hline 3571 & IC8Q45-1R & {$[\mathrm{CH} 2] \mathrm{C}(\mathrm{C})(\mathrm{C}) \mathrm{CC}(\mathrm{OO})(\mathrm{C}) \mathrm{COO}$} & InChl=1S/C8H1704/c1-7(2,3)5-8(4,12-10)6-11-9/h9-10H,1,5-6H2,2-4H3 \\
\hline 3572 & XYLMETHIDE & $\mathrm{CC} 1=\mathrm{CC}=\mathrm{CC}(\mathrm{C} 1=\mathrm{C})=0$ & $\operatorname{lnChl}=1 \mathrm{~S} / \mathrm{C} 8 \mathrm{H} 8 \mathrm{O} / \mathrm{c1} 1-6-4-3-5-8(9) 7(6) 2 / \mathrm{h} 3-5 \mathrm{H}, 2 \mathrm{H} 2,1 \mathrm{H} 3$ \\
\hline 3573 & IC8D4OOH1-3R & $\mathrm{OOCC}(\mathrm{C})(\mathrm{C})[\mathrm{CH}] \mathrm{C}(\mathrm{C})=\mathrm{C}$ & InChl=15/C8H15O2/c1-7(2)5-8(3,4)6-10-9/h5,9H,1,6H2,2-4H3 \\
\hline 3574 & $\mathrm{IC} 8013-40 \mathrm{OH}$ & $\mathrm{C}(\mathrm{O} 1) \mathrm{C}(\mathrm{C})(\mathrm{C}) \mathrm{C} 1 \mathrm{C}(\mathrm{OO})(\mathrm{C}) \mathrm{C}$ & InChl=15/C8H1603/c1-7(2)5-10-6(7)8(3,4)11-9/h6,9H,5H2,1-4H3 \\
\hline 3575 & IC8Q45-3R & $\mathrm{CC}(\mathrm{C})(\mathrm{C})[\mathrm{CH}] \mathrm{C}(\mathrm{OO})(\mathrm{C}) \mathrm{COO}$ & InChl=1S/C8H1704/c1-7(2,3)5-8(4,12-10)6-11-9/h5,9-10H,6H2,1-4H3 \\
\hline 3576 & $\mathrm{C} 6 \mathrm{H} 5 \mathrm{CH} 2 \mathrm{CHO}$ & $\mathrm{O}=\mathrm{CCC} 1=\mathrm{CC}=\mathrm{CC}=\mathrm{C} 1$ & $\operatorname{lnChl}=1 \mathrm{~S} / \mathrm{C} 8 \mathrm{H} 8 \mathrm{O} / \mathrm{c} 9-7-6-8-4-2-1-3-5-8 / \mathrm{h} 1-5,7 \mathrm{H}, 6 \mathrm{H} 2$ \\
\hline 3577 & IC8O13-50OH & $\mathrm{C}(\mathrm{O} 1) \mathrm{C}(\mathrm{C})(\mathrm{C}) \mathrm{C} 1 \mathrm{C}(\mathrm{C}) \mathrm{COO}$ & InChl=15/C8H16O3/c1-6(4-11-9)7-8(2,3)5-10-7/h6-7,9H,4-5H2,1-3H3 \\
\hline 3578 & IC8Q45-5AR & $\mathrm{CC}(\mathrm{C})(\mathrm{C}) \mathrm{CC}(\mathrm{OO})([\mathrm{CH} 2]) \mathrm{COO}$ & InChl=1S/C8H1704/c1-7(2,3)5-8(4,12-10)6-11-9/h9-10H,4-6H2,1-3H3 \\
\hline 3579 & $\mathrm{HOC} 6 \mathrm{H} 4 \mathrm{C} 2 \mathrm{H} 4$ & {$[\mathrm{CH} 2] \mathrm{Cc} 1 \mathrm{c}(\mathrm{O}) \mathrm{cccc} 1$} & InChl=1S/C8H9O/c1-2-7-5-3-4-6-8(7)9/h3-6,9H,1-2H2 \\
\hline 3580 & IC8KET1-1AO & $\mathrm{O}=\mathrm{CC}(\mathrm{C}[\mathrm{O}])(\mathrm{C}) \mathrm{CC}(\mathrm{C}) \mathrm{C}$ & $\operatorname{lnChl}=1 \mathrm{~S} / \mathrm{C} 8 \mathrm{H} 15 \mathrm{O} 2 / \mathrm{c1}-7(2) 4-8(3,5-9) 6-10 / \mathrm{h} 5,7 \mathrm{H}, 4,6 \mathrm{H} 2,1-3 \mathrm{H} 3$ \\
\hline 3581 & IC8O14-1AOOH & $\mathrm{C}(\mathrm{O} 1) \mathrm{C}(\mathrm{COO})(\mathrm{C}) \mathrm{CC} 1(\mathrm{C}) \mathrm{C}$ & InChl=1S/C8H1603/c1-7(2)4-8(3,5-10-7)6-11-9/h9H,4-6H2,1-3H3 \\
\hline 3582 & IC8Q55A-1R & {$[\mathrm{CH} 2] \mathrm{C}(\mathrm{C})(\mathrm{C}) \mathrm{CC}(\mathrm{COO}) \mathrm{COO}$} & InChl=15/C8H1704/c1-8(2,3)4-7(5-11-9)6-12-10/h7,9-10H,1,4-6H2,2-3H3 \\
\hline 3583 & $\mathrm{CH} 3 \mathrm{C} 6 \mathrm{H} 4 \mathrm{OCH} 2$ & {$[\mathrm{CH} 2] \mathrm{Oc} 1 \mathrm{c}(\mathrm{C}) \mathrm{cccc} 1$} & $\operatorname{lnChl}=1 \mathrm{~S} / \mathrm{C} 8 \mathrm{H} 90 / \mathrm{c} 1-7-5-3-4-6-8(7) 9-2 / \mathrm{h} 3-6 \mathrm{H}, 2 \mathrm{H} 2,1 \mathrm{H} 3$ \\
\hline 3584 & IC8KET1-30 & $\mathrm{O}=\mathrm{CC}(\mathrm{C})(\mathrm{C}) \mathrm{C}([\mathrm{O}]) \mathrm{C}(\mathrm{C}) \mathrm{C}$ & InChl=15/C8H15O2/c1-6(2)7(10)8(3,4)5-9/h5-7H,1-4H3 \\
\hline 3585 & IC8O14-30OH & $\mathrm{C}(\mathrm{O} 1) \mathrm{C}(\mathrm{C})(\mathrm{C}) \mathrm{C}(\mathrm{OO}) \mathrm{C} 1(\mathrm{C}) \mathrm{C}$ & InChl=15/C8H1603/c1-7(2)5-10-8(3,4)6(7)11-9/h6,9H,5H2,1-4H3 \\
\hline 3586 & IC8Q55A-3R & $\mathrm{CC}(\mathrm{C})(\mathrm{C})[\mathrm{CH}] \mathrm{C}(\mathrm{COO}) \mathrm{COO}$ & $\operatorname{lnChl}=15 / \mathrm{C} 8 \mathrm{H} 1704 / \mathrm{c} 1-8(2,3) 4-7(5-11-9) 6-12-10 / \mathrm{h} 4,7,9-10 \mathrm{H}, 5-6 \mathrm{H} 2,1-3 \mathrm{H} 3$ \\
\hline 3587 & $\mathrm{CH} 2 \mathrm{C} 6 \mathrm{H} 4 \mathrm{OCH} 3$ & $\operatorname{COc} 1 \mathrm{c}([\mathrm{CH} 2]) \operatorname{cccc} 1$ & InChl=1S/C8H9O/c1-7-5-3-4-6-8/7)9-2/h3-6H,1H2,2H3 \\
\hline 3588 & IC8KET1-4O & $\mathrm{O}=\mathrm{CC}(\mathrm{C})(\mathrm{C}) \mathrm{CC}([\mathrm{O}])(\mathrm{C}) \mathrm{C}$ & InChl=1S/C8H15O2/c1-7(2,6-9)5-8(3,4)10/h6H,5H2,1-4H3 \\
\hline 3589 & IC8O14-50OH & $\mathrm{C}(\mathrm{O} 1) \mathrm{C}(\mathrm{C})(\mathrm{C}) \mathrm{CC} 1(\mathrm{C}) \mathrm{COO}$ & InChl=1S/C8H1603/c1-7(2)4-8(3,6-11-9)10-5-7/h9H,4-6H2,1-3H3 \\
\hline 3590 & IC8Q55A-4R & $\mathrm{CC}(\mathrm{C})(\mathrm{C}) \mathrm{C}[\mathrm{C}](\mathrm{COO}) \mathrm{COO}$ & InChl=1S/C8H17O4/c1-8(2,3)4-7(5-11-9)6-12-10/h9-10H,4-6H2,1-3H3 \\
\hline 3591 & $\mathrm{CH} 3 \mathrm{C}_{6} \mathrm{H} 4 \mathrm{CH} 2 \mathrm{O}$ & $\mathrm{CC} 1=\mathrm{CC}=\mathrm{CC}=\mathrm{C} 1 \mathrm{C}[\mathrm{O}]$ & InChl=1S/C8H9O/c1-7-4-2-3-5-8(7)6-9/h2-5H,6H2,1H3 \\
\hline 3592 & IC8KET1-5O & $\mathrm{O}=\mathrm{CC}(\mathrm{C})(\mathrm{C}) \mathrm{CC}(\mathrm{C}) \mathrm{C}[\mathrm{O}]$ & InChl=1S/C8H15O2/c1-7(5-9)4-8(2,3)6-10/h6-7H,4-5H2,1-3H3 \\
\hline 3593 & IC8O15-1AOOH & $\mathrm{C}(\mathrm{O} 1) \mathrm{C}(\mathrm{COO})(\mathrm{C}) \mathrm{CC}(\mathrm{C}) \mathrm{C} 1$ & InChl=1S/C8H1603/c1-7-3-8(2,6-11-9)5-10-4-7/h7,9H,3-6H2,1-2H3 \\
\hline 3594 & $\mathrm{C} 6 \mathrm{H} 5 \mathrm{CCOH}$ & OcCc1ccccc1 & $\operatorname{lnChl}=15 / C 8 H 100 / c 9-7-6-8-4-2-1-3-5-8 / h 1-5,9 H, 6-7 \mathrm{H} 2$ \\
\hline 3595 & $\mathrm{CH} 3 \mathrm{C} 6 \mathrm{H} 4 \mathrm{CYCH} 2 \mathrm{O}$ & $\mathrm{CC} 1=\mathrm{C}[\mathrm{CH}] \mathrm{C}=\mathrm{CC} 12 \mathrm{CO} 2$ & InChl=1S/C8H9O/c1-7-4-2-3-5-8(7)6-9-8/h2-5H,6H2,1H3 \\
\hline 3596 & IC8KET3-10 & $C([0]) C(C)(C) C(=0) C(C) C$ & InChl=1S/C8H15O2/c1-6(2)7(10)8(3,4)5-9/h6H,5H2,1-4H3 \\
\hline 3597 & $\mathrm{IC} 8015-300 \mathrm{H}$ & $\mathrm{C}(\mathrm{O} 1) \mathrm{C}(\mathrm{C})(\mathrm{C}) \mathrm{C}(\mathrm{OO}) \mathrm{C}(\mathrm{C}) \mathrm{C} 1$ & InChl=15/C8H16O3/c1-6-4-10-5-8(2,3)7(6)11-9/h6-7,9H,4-5H2,1-3H3 \\
\hline 3598 & $\mathrm{C} 6 \mathrm{H} 5 \mathrm{CJCOH}$ & $\mathrm{OC}[\mathrm{CH}] \mathrm{C} 1 \mathrm{cccccc} 1$ & InChl=1S/C8H9O/c9-7-6-8-4-2-1-3-5-8/h1-6,9H,7H2 \\
\hline 3599 & $\mathrm{C} 6 \mathrm{H} 5 \mathrm{CCJOH}$ & $\mathrm{O}[\mathrm{CH}] \mathrm{Cc} 1 \mathrm{cccccc} 1$ & InChl=1S/C8H9O/c9-7-6-8-4-2-1-3-5-8/h1-5,7,9H,6H2 \\
\hline 3600 & $\mathrm{C} 6 \mathrm{H} 5 \mathrm{C}(\mathrm{OOJ}) \mathrm{COH}$ & $\mathrm{OCC}(\operatorname{c1ccccc} 1) \mathrm{O}[\mathrm{O}]$ & InChl=1S/C8H9O3/c9-6-8(11-10)7-4-2-1-3-5-7/h1-5,8-9H,6H2 \\
\hline 3601 & $\mathrm{C9H7}$ & {$[\mathrm{CH}] 1 \mathrm{C}=\mathrm{CC} 2=\mathrm{CC}=\mathrm{CC}=\mathrm{C} 12$} & InChl=1S/C9H7/c1-2-5-9-7-3-6-8(9)4-1/h1-7H \\
\hline 3602 & O-XYLCH2 & $\mathrm{C} 1=\mathrm{C}(\mathrm{C}) \mathrm{C}([\mathrm{CH} 2])=\mathrm{CC}=\mathrm{C} 1 \mathrm{C}$ & InChl=1S/C9H11/c1-7-4-5-8(2)9(3)6-7/h4-6H,2H2,1,3H3 \\
\hline 3603 & $0-X Y L C O$ & $\mathrm{C} 1=\mathrm{C}(\mathrm{C}) \mathrm{C}([\mathrm{C}]=\mathrm{O})=\mathrm{CC}=\mathrm{C} 1 \mathrm{C}$ & InChl=1S/C9H9O/c1-7-3-4-9(6-10)8(2)5-7/h3-5H,1-2H3 \\
\hline 3604 & CHOTOLCH2O & $\mathrm{CC} 1=\mathrm{CC}(\mathrm{C}=\mathrm{O})=\mathrm{C}(\mathrm{C}[\mathrm{O}]) \mathrm{C}=\mathrm{C} 1$ & InChl=1S/C9H9O2/c1-7-2-3-8(5-10)9(4-7)6-11/h2-4,6H,5H2,1H3 \\
\hline 3605 & $\mathrm{C} 6 \mathrm{H} 5 \mathrm{CH} 2 \mathrm{CCH}$ & C\#CCc1ccccc1 & $\operatorname{lnChl}=1 \mathrm{~S} / \mathrm{C} 9 \mathrm{H} 8 / \mathrm{c1} 1-2-6-9-7-4-3-5-8-9 / \mathrm{h} 1,3-5,7-8 \mathrm{H}, 6 \mathrm{H} 2$ \\
\hline 3606 & P-XYLCH2 & $\mathrm{C} 1=\mathrm{C}(\mathrm{C}) \mathrm{C}(\mathrm{C})=\mathrm{CC}=\mathrm{C} 1[\mathrm{CH} 2]$ & InChl=1S/C9H11/c1-7-4-5-8(2)9(3)6-7/h4-6H,1H2,2-3H3 \\
\hline 3607 & PCHOTOLCH2 & {$[\mathrm{CH} 2] \mathrm{C} 1=\mathrm{CC}=\mathrm{C}(\mathrm{C}=\mathrm{O}) \mathrm{C}=\mathrm{C} 1 \mathrm{C}$} & InChl=1S/C9H9O/c1-7-3-4-9(6-10)5-8(7)2/h3-6H,1H2,2H3 \\
\hline 3608 & $\mathrm{O}-\mathrm{XYLCH} 2 \mathrm{OO}$ & $\mathrm{C} 1=\mathrm{C}(\mathrm{C}) \mathrm{C}(\mathrm{CO}[\mathrm{O}])=\mathrm{CC}=\mathrm{C} 1 \mathrm{C}$ & InChl=1S/C9H1102/c1-7-3-4-9(6-11-10)8(2)5-7/h3-5H,6H2,1-2H3 \\
\hline 3609 & IND & $\mathrm{C} 1=\mathrm{CC}=\mathrm{C} 2 \mathrm{CC}=\mathrm{C} C 2=\mathrm{C} 1$ & InChl=1S/C9H8/c1-2-5-9-7-3-6-8(9)4-1/h1-6H,7H2 \\
\hline 3610 & $\begin{array}{ll}\mathrm{CH} 3 \mathrm{C} 6 \mathrm{H} 4 \mathrm{CHCH} 3 \\
\mathrm{n}\end{array}$ & $\mathrm{CC} 1=\mathrm{C}([\mathrm{CH}] \mathrm{C}) \mathrm{C}=\mathrm{CC}=\mathrm{C} 1$ & InChl=1S/C9H11/c1-3-9-7-5-4-6-8(9)2/h3-7H,1-2H3 \\
\hline 3611 & P-XYLCO & $\mathrm{C} 1=\mathrm{C}(\mathrm{C}) \mathrm{C}(\mathrm{C})=\mathrm{CC}=\mathrm{C} 1[\mathrm{C}]=\mathrm{O}$ & InChl=1S/C9H9O/c1-7-3-4-9(6-10)5-8(7)2/h3-5H,1-2H3 \\
\hline 3612 & P-XYLCH2OO & $\mathrm{C} 1=\mathrm{C}(\mathrm{C}) \mathrm{C}(\mathrm{C})=\mathrm{CC}=\mathrm{C} 1 \mathrm{CO}[\mathrm{O}]$ & InChl=1S/C9H1102/c1-7-3-4-9(6-11-10)5-8(7)2/h3-5H,6H2,1-2H3 \\
\hline 3613 & T135MBJ & {$[\mathrm{CH} 2] \mathrm{C} 1=\mathrm{CC}(\mathrm{C})=\mathrm{CC}(\mathrm{C})=\mathrm{C} 1$} & InChl=1S/C9H11/c1-7-4-8(2)6-9(3)5-7/h4-6H,1H2,2-3H3 \\
\hline 3614 & $\mathrm{C} 2 \mathrm{H} 3 \mathrm{C} 6 \mathrm{H} 4 \mathrm{CH} 2 \mathrm{O}$ & $\mathrm{C}=\mathrm{CC} 1=\mathrm{C}(\mathrm{C}[\mathrm{O}]) \mathrm{C}=\mathrm{CC}=\mathrm{C} 1$ & InChl=1S/C9H9O/c1-2-8-5-3-4-6-9(8)7-10/h2-6H,1,7H2 \\
\hline 3615 & $\mathrm{O}-\mathrm{CH} 2 \mathrm{TOLCH} 2 \mathrm{OOH}$ & $\mathrm{CC} 1=\mathrm{CC}=\mathrm{C}(\mathrm{COO}) \mathrm{C}([\mathrm{CH} 2])=\mathrm{C} 1$ & InChl=15/C9H1102/c1-7-3-4-9(6-11-10)8(2)5-7/h3-5,10H,2,6H2,1H3 \\
\hline 3616 & T124MBZ & $\mathrm{CC} 1=\mathrm{C}(\mathrm{C}) \mathrm{C}=\mathrm{C}(\mathrm{C}) \mathrm{C}=\mathrm{C} 1$ & InChl=1S/C9H12/c1-7-4-5-8(2)9(3)6-7/h4-6H,1-3H3 \\
\hline 3617 & O-XYLCHO & $C 1=C(C) C(C=0)=C C=C 1 C$ & InChl=1S/C9H100/c1-7-3-4-9(6-10)8(2)5-7/h3-6H,1-2H3 \\
\hline 3618 & CH3A1CHOOCH3 & $\mathrm{CC} 1=\mathrm{C}(\mathrm{C}(\mathrm{C}) \mathrm{O}[\mathrm{O}]) \mathrm{C}=\mathrm{CC}=\mathrm{C} 1$ & InChl=1S/C9H1102/c1-7-5-3-4-6-9(7)8(2)11-10/h3-6,8H,1-2H3 \\
\hline 3619 & $\mathrm{CH} 2 \mathrm{C} 6 \mathrm{H} 4 \mathrm{C} 2 \mathrm{H} 3$ & {$[\mathrm{CH} 2] \mathrm{C} 1=\mathrm{C}(\mathrm{C}=\mathrm{C}) \mathrm{C}=\mathrm{CC}=\mathrm{C} 1$} & InChl=1S/C9H9/c1-3-9-7-5-4-6-8(9)2/h3-7H,1-2H2 \\
\hline 3620 & T135MBZ & $\mathrm{CC} 1=\mathrm{CC}(\mathrm{C})=\mathrm{CC}(\mathrm{C})=\mathrm{C} 1$ & InChl=15/C9H12/c1-7-4-8(2)6-9(3)5-7/h4-6H,1-3H3 \\
\hline 3621 & P-XYLCHO & $C 1=C(C) C(C)=C C=C 1 C=0$ & InChl=1S/C9H100/c1-7-3-4-9(6-10)5-8(7)2/h3-6H,1-2H3 \\
\hline 3622 & $\mathrm{O}-\mathrm{XYLCH} 2 \mathrm{OOH}$ & $\mathrm{C} 1=\mathrm{C}(\mathrm{C}) \mathrm{C}(\mathrm{COOH})=\mathrm{CC}=\mathrm{C} 1 \mathrm{C}$ & InChl=15/C9H12O2/c1-7-3-4-9(6-11-10)8(2)5-7/h3-5,10H,6H2,1-2H3 \\
\hline 3623 & $\mathrm{CH} 3 \mathrm{C} 6 \mathrm{H} 4 \mathrm{C} 2 \mathrm{H} 5$ & CCc1ccccc1C & InChl=1S/C9H12/c1-3-9-7-5-4-6-8(9)2/h4-7H,3H2,1-2H3 \\
\hline 3624 & CH3TOLETCOC & $\mathrm{CC} 1=\mathrm{CC} 2=\mathrm{C}(\mathrm{COC} 2) \mathrm{C}=\mathrm{C} 1$ & $\operatorname{lnChl}=15 / \mathrm{C} 9 \mathrm{H} 100 / c 1-7-2-3-8-5-10-6-9(8) 4-7 / \mathrm{h} 2-4 \mathrm{H}, 5-6 \mathrm{H} 2,1 \mathrm{H} 3$ \\
\hline 3625 & P-XYLCH2OOH & $\mathrm{C} 1=\mathrm{C}(\mathrm{C}) \mathrm{C}(\mathrm{C})=\mathrm{CC}=\mathrm{C} 1 \mathrm{COO}$ & InChl=15/C9H12O2/c1-7-3-4-9(6-11-10)5-8(7)2/h3-5,10H,6H2,1-2H3 \\
\hline 3626 & P-MEXYLYLE & $\mathrm{C}=\mathrm{c} 1 \mathrm{ccc}(=\mathrm{C}) \mathrm{c}(\mathrm{c} 1) \mathrm{C}$ & InChl=1S/C9H10/c1-7-4-5-8(2)9(3)6-7/h4-6H,1-2H2,3H3 \\
\hline 3627 & $\mathrm{C9H} 6 \mathrm{O}$ & $\mathrm{C} 1=\mathrm{CC}=\mathrm{CC} 2=\mathrm{C} 1 \mathrm{C}(=\mathrm{O}) \mathrm{C}(=\mathrm{C} 2)$ & InChl=1S/C9H6O/c10-9-6-5-7-3-1-2-4-8(7)9/h1-6H \\
\hline 3628 & $\mathrm{O}-\mathrm{XYLCH} 2 \mathrm{O}$ & $\mathrm{C} 1=\mathrm{C}(\mathrm{C}) \mathrm{C}(\mathrm{C}[\mathrm{O}])=\mathrm{CC}=\mathrm{C} 1 \mathrm{C}$ & $\operatorname{lnChl}=15 / \mathrm{C} 9 \mathrm{H} 110 / \mathrm{c} 1-7-3-4-9(6-10) 8(2) 5-7 / \mathrm{h} 3-5 \mathrm{H}, 6 \mathrm{H} 2,1-2 \mathrm{H} 3$ \\
\hline 3629 & TMBKET & $\mathrm{O}=\mathrm{CC} 1=\mathrm{C}(\mathrm{COO}) \mathrm{C}=\mathrm{C}(\mathrm{C}) \mathrm{C}=\mathrm{C} 1$ & InChl=15/C9H1003/c1-7-2-3-8(5-10)9(4-7)6-12-11/h2-5,11H,6H2,1H3 \\
\hline 3630 & MEXYLYLE & $\operatorname{cc} 1 \mathrm{ccc}(=\mathrm{C}) \mathrm{c}(=\mathrm{C}) \mathrm{c} 1$ & InChl=1S/C9H10/c1-7-4-5-8(2)9(3)6-7/h4-6H,2-3H2,1H3 \\
\hline 3631 & $\mathrm{C} 2 \mathrm{H} 3 \mathrm{C} 6 \mathrm{H} 4 \mathrm{CO}$ & $\mathrm{C}=\mathrm{CC} 1=\mathrm{C}([\mathrm{C}]=0) \mathrm{C}=\mathrm{CC}=\mathrm{C} 1$ & $\operatorname{lnChl}=15 / C 9 H 70 / c 1-2-8-5-3-4-6-9(8) 7-10 / \mathrm{h} 2-6 \mathrm{H}, 1 \mathrm{H} 2$ \\
\hline 3632 & P-XYLCH2O & $\mathrm{C} 1=\mathrm{C}(\mathrm{C}) \mathrm{C}(\mathrm{C})=\mathrm{CC}=\mathrm{C} 1 \mathrm{C}[\mathrm{O}]$ & $\operatorname{lnChl}=15 / \mathrm{C} 9 \mathrm{H} 110 / \mathrm{c} 1-7-3-4-9(6-10) 5-8(7) 2 / \mathrm{h} 3-5 \mathrm{H}, 6 \mathrm{H} 2,1-2 \mathrm{H} 3$ \\
\hline 3633 & $\mathrm{O} 2 \mathrm{CH} 2 \mathrm{TOLCH} 2 \mathrm{OOH}$ & $\mathrm{CC} 1=\mathrm{CC}(\mathrm{COO})=\mathrm{C}(\mathrm{CO}[\mathrm{O}]) \mathrm{C}=\mathrm{C} 1$ & InChl=1S/C9H1104/c1-7-2-3-8(5-12-10)9(4-7)6-13-11/h2-4,11H,5-6H2,1H3 \\
\hline 3634 & MEBZCYBUT & Cc1ccc2c(c1)cc2 & InChl=1S/C9H10/c1-7-2-3-8-4-5-9(8)6-7/h2-3,6H,4-5H2,1H3 \\
\hline 3635 & $\mathrm{C9H7O}$ & {$[\mathrm{O}] \mathrm{C} 1 \mathrm{C}=\mathrm{CC} 2=\mathrm{C} 1 \mathrm{C}=\mathrm{CC}=\mathrm{C} 2$} & InChl=1S/C9H7O/c10-9-6-5-7-3-1-2-4-8(7)9/h1-6,9H \\
\hline 3636 & CHOTOLCO & $\mathrm{CC} 1=\mathrm{CC}(\mathrm{C}=\mathrm{O})=\mathrm{C}([\mathrm{C}]=0) \mathrm{C}=\mathrm{C} 1$ & InChl=1S/C9H7O2/c1-7-2-3-8(5-10)9(4-7)6-11/h2-4,6H,1H3 \\
\hline
\end{tabular}




\begin{tabular}{|c|c|c|c|}
\hline 3637 & $\mathrm{CH} 3 \mathrm{C}_{6} \mathrm{H} 4 \mathrm{C} 2 \mathrm{H} 3$ & $\mathrm{CC} 1=\mathrm{C}(\mathrm{C}=\mathrm{C}) \mathrm{C}=\mathrm{CC}=\mathrm{C} 1$ & $\mathrm{C} \mathrm{Chl}=1 \mathrm{~S} / \mathrm{C} 9 \mathrm{H} 10 / \mathrm{c} 1-3-9-7-5-4-6-8(9) 2 / \mathrm{h} 3-7 \mathrm{H}, 1 \mathrm{H} 2,2 \mathrm{H} 3$ \\
\hline 3638 & $\mathrm{C} 2 \mathrm{H} 3 \mathrm{C} 6 \mathrm{H} 4 \mathrm{CHO}$ & $\mathrm{C}=\mathrm{CC} 1=\mathrm{C}(\mathrm{C}=\mathrm{O}) \mathrm{C}=\mathrm{CC}=\mathrm{C} 1$ & InChl=1S/C9H8O/c1-2-8-5-3-4-6-9(8)7-10/h2-7H,1H2 \\
\hline 3639 & CHOTOLCHO & $\mathrm{CC} 1=\mathrm{CC}(\mathrm{C}=0)=\mathrm{C}(\mathrm{C}=0) \mathrm{C}=\mathrm{C} 1$ & InChl=1S/C9H8O2/c1-7-2-3-8(5-10)9(4-7)6-11/h2-6H,1H3 \\
\hline 3640 & NAPHTHYNE12 & $\mathrm{c} 1 \mathrm{ccc} 2 \mathrm{c}(\mathrm{c} 1) \mathrm{CHCC}=\mathrm{C} 2$ & $\operatorname{lnChl}=1 \mathrm{~S} / \mathrm{C} 10 \mathrm{H} 6 / \mathrm{c} 1-2-6-10-8-4-3-7-9(10) 5-1 / \mathrm{h} 1-3,5-7 \mathrm{H}$ \\
\hline 3641 & NAPHV & $\mathrm{C} 1=[\mathrm{C}] \mathrm{C}=\mathrm{CC} 2=\mathrm{C} 1 \mathrm{C}=\mathrm{C}(\mathrm{C}=\mathrm{C} 2)$ & $\begin{array}{ll}\operatorname{lnChl}=1 \mathrm{~S} / \mathrm{C} 10 \mathrm{H} 7 / \mathrm{c} 1-2-6-10-8-4-3-7-9(10) 5-1 / \mathrm{h} 1-3,5-8 \mathrm{H} \\
\end{array}$ \\
\hline 3642 & $\mathrm{C9H6CH3}$ & $\mathrm{C}[\mathrm{C}] 1 \mathrm{C}=\mathrm{Cc} 2 \mathrm{c} 1 \mathrm{cccc} 2$ & $\operatorname{lnChl}=15 / C 10 \mathrm{H} 9 / \mathrm{c} 1-8-6-7-9-4-2-3-5-10(8) 9 / \mathrm{h} 2-7 \mathrm{H}, 1 \mathrm{H} 3$ \\
\hline 3643 & $\mathrm{C} 10 \mathrm{H} 10$ & $\mathrm{C} 1 \mathrm{C}=\mathrm{CC} 2 \mathrm{CCC}=\mathrm{CC}=2 \mathrm{C}=1$ & InChl=1S/C10H10/c1-2-6-10-8-4-3-7-9(10)5-1/h1-3,5-7H,4,8H2 \\
\hline 3644 & NAPHTHYNE23 & $\mathrm{c} 1 \mathrm{ccc} 2 \mathrm{c}(\mathrm{c} 1) \mathrm{C}=\mathrm{C}=\mathrm{C}=\mathrm{C} 2$ & InChl=1S/C10H6/c1-2-6-10-8-4-3-7-9(10)5-1/h1-2,5-8H \\
\hline 3645 & $\mathrm{C} 9 \mathrm{H} 7 \mathrm{CH} 2$ & {$[\mathrm{CH} 2] \mathrm{C} 1 \mathrm{C}=\mathrm{Cc} 2 \mathrm{c} 1 \mathrm{cccc} 2$} & InChl=1S/C10H9/c1-8-6-7-9-4-2-3-5-10(8)9/h2-8H,1H2 \\
\hline 3646 & NAPHO & {$[0] \mathrm{C} 1=\mathrm{CC}=\mathrm{CC} 2 \mathrm{C}=\mathrm{CC}=\mathrm{CC}=21$} & $\operatorname{lnChl}=1 \mathrm{~S} / \mathrm{C} 10 \mathrm{H} 70 / \mathrm{c} 11-10-7-3-5-8-4-1-2-6-9(8) 10 / \mathrm{h} 1-7 \mathrm{H}$ \\
\hline 3647 & $\mathrm{O}-\mathrm{C} 2 \mathrm{HC} 6 \mathrm{H} 4 \mathrm{C} 2 \mathrm{H}$ & C\#Cc1 1сcccc1C\#C & $\operatorname{lnChl}=15 / \mathrm{C} 10 \mathrm{H} 6 / \mathrm{c} 1-3-9-7-5-6-8-10(9) 4-2 / \mathrm{h1} 1-2,5-8 \mathrm{H}$ \\
\hline 3648 & $\mathrm{C} 1 \mathrm{OH} 9$ & {$[\mathrm{CH}] 1 \mathrm{CC}=\mathrm{CC} 2 \mathrm{C}=\mathrm{CC}=\mathrm{CC}=21$} & InChl=15/C10H9/c1-2-6-10-8-4-3-7-9(10)5-1/h1-3,5-8H,4H2 \\
\hline 3649 & NAPHOH & $\mathrm{C} 1=\mathrm{CC}(\mathrm{C}=\mathrm{CC}=\mathrm{C} 2)=\mathrm{C} 2 \mathrm{C}=\mathrm{C} 1$ & InChl=1S/C10H80/c11-10-7-3-5-8-4-1-2-6-9(8)10/h1-7,11H \\
\hline 3650 & $\mathrm{INDCH}$ & {$[\mathrm{CH}]=\mathrm{C} 1 \mathrm{C}=\mathrm{Cc} 2 \mathrm{c} 1 \mathrm{cccc} 2$} & InChl=1S/C10H7/c1-8-6-7-9-4-2-3-5-10(8)9/h1-7H \\
\hline 3651 & $\mathrm{C9H7CH3}$ & $\mathrm{CC} 1 \mathrm{C}=\mathrm{Cc} 2 \mathrm{c} 1 \mathrm{cccc} 2$ & InChl=1S/C10H10/c1-8-6-7-9-4-2-3-5-10(8)9/h2-8H,1H3 \\
\hline 3652 & NAPH- & {$[\mathrm{C}] 1=\mathrm{CC}=\mathrm{CC} 2=\mathrm{C} 1 \mathrm{C}=\mathrm{C}(\mathrm{C}=\mathrm{C} 2)$} & InChl=1S/C10H7/c1-2-6-10-8-4-3-7-9(10)5-1/h1-7H \\
\hline 3653 & NAPH & $\mathrm{C} 1 \mathrm{C}=\mathrm{CC} 2 \mathrm{C}=\mathrm{CC}=\mathrm{CC}=2 \mathrm{C}=1$ & InChl=1S/C10H8/c1-2-6-10-8-4-3-7-9(10)5-1/h1-8H \\
\hline 3654 & $\mathrm{~A} 1 \mathrm{C} 4 \mathrm{H} 5$ & $\mathrm{C} 1=\mathrm{CC}=\mathrm{CC}=\mathrm{C} 1 \mathrm{C}=\mathrm{CC}=\mathrm{C}$ & $\operatorname{lnChl}=1 \mathrm{~S} / \mathrm{C} 10 \mathrm{H} 10 / \mathrm{c1}-2-3-7-10-8-5-4-6-9-10 / \mathrm{h} 2-9 \mathrm{H}, 1 \mathrm{H} 2$ \\
\hline 3655 & $\mathrm{~A} 2 \mathrm{CH} 2$ & $\mathrm{C}=\mathrm{C} 1 \mathrm{C}=\mathrm{C}[\mathrm{CH}] \mathrm{c} 2 \mathrm{ccccc} 12$ & InChl=1S/C11H9/c1-9-5-4-7-10-6-2-3-8-11(9)10/h2-8H,1H2 \\
\hline 3656 & $\mathrm{~A} 2 \mathrm{CH} 3$ & Cc1cccc2 $2 \operatorname{ccccc} 12$ & InChl=1S/C11H10/c1-9-5-4-7-10-6-2-3-8-11(9)10/h2-8H,1H3 \\
\hline 3657 & $\mathrm{~A} 2 \mathrm{OHCH} 3$ & Cc1c2ccccc $2 \operatorname{ccc} 10$ & InChl=1S/C11H100/c1-8-10-5-3-2-4-9(10)6-7-11(8)12/h2-7,12H,1H3 \\
\hline 3658 & $\mathrm{M}-\mathrm{O} 2 \mathrm{C} 6 \mathrm{H} 4 \mathrm{~A} 1 \mathrm{CH} 3$ & Cc1cc(cc2ccccc12)O[O] & $\operatorname{lnChl}=15 / C 11 H 902 / c 1-8-6-10(13-12) 7-9-4-2-3-5-11(8) 9 / h 2-7 \mathrm{H}, 1 \mathrm{H3}$ \\
\hline 3659 & $\mathrm{~A} 2 \mathrm{CH} 2 \mathrm{O}$ & $\mathrm{c} 1 \mathrm{ccc} 2 \mathrm{c}(\mathrm{c} 1) \operatorname{cccc} 2 \mathrm{C}[\mathrm{O}]$ & InChl=1S/C11H9o/c12-8-10-6-3-5-9-4-1-2-7-11(9)10/h1-7H,8H2 \\
\hline 3660 & $\mathrm{~A} 2 \mathrm{CHO}$ & $\operatorname{c1ccc2c}(\mathrm{c} 1) \operatorname{cccc} 2 \mathrm{C}=\mathrm{O}$ & InChl=1S/C11H80/c12-8-10-6-3-5-9-4-1-2-7-11(9)10/h1-8H \\
\hline 3661 & C11H9O-LIN & $\operatorname{Cc} 1 \operatorname{cccc}(\mathrm{C}=\mathrm{C}[\mathrm{C}]=0) \mathrm{c} 1 \mathrm{C}=0$ & $\operatorname{lnChl}=15 / C 11 \mathrm{H} 902 / \mathrm{c} 1-9-4-2-5-10(6-3-7-12) 11(9) 8-13 / \mathrm{h} 2-6,8 \mathrm{H}, 1 \mathrm{H} 3$ \\
\hline 3662 & $\mathrm{O} 2 \mathrm{~A} 2 \mathrm{CH} 3$ & Cc1cccc2cccc(c12)O[O] & $\operatorname{lnChl}=1 \mathrm{~S} / \mathrm{C} 11 \mathrm{H} 902 / \mathrm{c} 1-8-4-2-5-9-6-3-7-10(13-12) 11(8) 9 / \mathrm{h} 2-7 \mathrm{H}, 1 \mathrm{H} 3$ \\
\hline 3663 & $\mathrm{~A} 2 \mathrm{CHOH}$ & $\mathrm{c} 1 \mathrm{ccc} 2 \mathrm{c}(\mathrm{c} 1) \mathrm{cccc} 2[\mathrm{CH}] \mathrm{O}$ & $\operatorname{lnChl=1S/C11H9O/c12-8-10-6-3-5-9-4-1-2-7-11(9)10/h1-8,12H~}$ \\
\hline 3664 & C6H3A1CH3 & $\mathrm{CC} 1=\mathrm{C} 2 \mathrm{C}=\mathrm{C}=\mathrm{CC}=\mathrm{C} 2[\mathrm{CH}] \mathrm{C}=\mathrm{C} 1$ & $\operatorname{lnChl}=1 \mathrm{~S} / \mathrm{C} 11 \mathrm{Hg} / \mathrm{c} 1-9-5-4-7-10-6-2-3-8-11(9) 10 / \mathrm{h} 2,4-8 \mathrm{H}, 1 \mathrm{H} 3$ \\
\hline 3665 & $\mathrm{OA} 2 \mathrm{CH} 3$ & $\operatorname{Cc1cccc2ccc(\operatorname {cc}12)[0]}$ & InChl=1S/C11H9o/c1-8-3-2-4-9-5-6-10(12)7-11(8)9/h2-7H,1H3 \\
\hline 3666 & $\mathrm{~A} 2 \mathrm{CH} 2 \mathrm{OO}$ & $\mathrm{c} 1 \mathrm{ccc} 2 \mathrm{c}(\mathrm{c} 1) \mathrm{cccc} 2 \mathrm{CO}[\mathrm{O}]$ & $\operatorname{lnChl}=15 / C 11 H 902 / c 12-13-8-10-6-3-5-9-4-1-2-7-11(9) 10 / h 1-7 \mathrm{H}, 8 \mathrm{H} 2$ \\
\hline 3667 & $\mathrm{~A} 2 \mathrm{CO}$ & $\operatorname{c1ccc} 2 c(\mathrm{c} 1) \operatorname{cccc} 2[\mathrm{C}]=0$ & InChl=1S/C11H70/c12-8-10-6-3-5-9-4-1-2-7-11(9)10/h1-7H \\
\hline 3668 & $\mathrm{C} 6 \mathrm{H} 4 \mathrm{~A} 1 \mathrm{CH} 3$ & $\mathrm{CC} 1=\mathrm{C}=\mathrm{C}[\mathrm{CH}] \mathrm{c} 2 \mathrm{ccccc} 12$ & InChl=1S/C11H9/c1-9-5-4-7-10-6-2-3-8-11(9)10/h2-4,6-8H,1H3 \\
\hline 3669 & $\mathrm{OC6H4A1CH2}$ & $\mathrm{C}=\mathrm{C} 1 \mathrm{c} 2 \operatorname{ccccc} 2 \mathrm{C}=\mathrm{CC} 1=\mathrm{O}$ & InChl=1S/C11H8O/c1-8-10-5-3-2-4-9(10)6-7-11(8)12/h2-7H,1H2 \\
\hline 3670 & $\mathrm{~A} 2 \mathrm{CH} 2 \mathrm{OOH}$ & 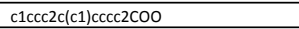 & $\operatorname{lnChl}=1 \mathrm{~S} / \mathrm{C} 11 \mathrm{H} 1002 / \mathrm{c} 12-13-8-10-6-3-5-9-4-1-2-7-11(9) 10 / \mathrm{h} 1-7,12 \mathrm{H}, 8 \mathrm{H} 2$ \\
\hline 3671 & $\mathrm{~A} 2 \mathrm{OCH} 3$ & Cc1c2 $\operatorname{ccccc} 2 \operatorname{ccc} 1[0]$ & InChl=1S/C11H9O/c1-8-10-5-3-2-4-9(10)6-7-11(8)12/h2-7H,1H3 \\
\hline 3672 & $\mathrm{HOA} 2 \mathrm{CH} 3$ & Cc1cccc2ccc(cc12)O & $\operatorname{lnChl}=1 \mathrm{~S} / \mathrm{C} 11 \mathrm{H} 100 / \mathrm{c} 1-8-3-2-4-9-5-6-10(12) 7-11(8) 9 / \mathrm{h} 2-7,12 \mathrm{H}, 1 \mathrm{H} 3$ \\
\hline 3673 & $\mathrm{O}-\mathrm{O} 2 \mathrm{C} 6 \mathrm{H} 4 \mathrm{~A} 1 \mathrm{CH} 3$ & Cc1c2ccccc2ccc10[0] & $\operatorname{lnChl}=1 \mathrm{~S} / \mathrm{C} 11 \mathrm{H} 902 / \mathrm{c1}-8-10-5-3-2-4-9(10) 6-7-11(8) 13-12 / \mathrm{h} 2-7 \mathrm{H}, 1 \mathrm{H} 3$ \\
\hline 3674 & CHOCCH3ACO1-5 & $\mathrm{c} 1 \mathrm{ccc} 2 \mathrm{c}(\mathrm{c} 1) \operatorname{ccc}(\mathrm{c} 2 \mathrm{c}[0]) 0$ & InChl=1S/C11H9O2/c12-7-10-9-4-2-1-3-8(9)5-6-11(10)13/h1-6,13H,7H2 \\
\hline 3675 & $\mathrm{C} 14 \mathrm{H} 11$ & $\operatorname{c1ccc}(\operatorname{cc} 1) \mathrm{C}=[\mathrm{C}] \mathrm{c} 1 \mathrm{ccccc} 1$ & $\operatorname{lnChl}=15 / C 14 H 11 / c 1-3-7-13(8-4-1) 11-12-14-9-5-2-6-10-14 / h 1-11 \mathrm{H}$ \\
\hline 3676 & $\mathrm{C} 14 \mathrm{H} 14$ & c1ccc(cc1)(Ccc1ccccc1 & InChl=1S/C14H14/c1-3-7-13(8-4-1)11-12-14-9-5-2-6-10-14/h1-10H,11-12H2 \\
\hline 3677 & $\mathrm{C} 14 \mathrm{H} 12 \mathrm{OOH}$ & $\mathrm{c} 1 \mathrm{ccc}(\mathrm{cc} 1)[\mathrm{CH}] \mathrm{C}(\mathrm{c} 1 \mathrm{ccccc} 1) \mathrm{OO}$ & $\mathrm{InChl}=1 \mathrm{~S} / \mathrm{C} 14 \mathrm{H} 1302 / \mathrm{c} 15-16-14(13-9-5-2-6-10-13) 11-12-7-3-1-4-8-12 / \mathrm{h} 1-11,14-15 \mathrm{H}$ \\
\hline 3678 & $\mathrm{C} 14 \mathrm{H} 12 \mathrm{O} 2 \mathrm{H}-102$ & 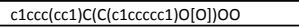 & 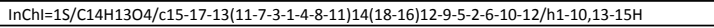 \\
\hline 3679 & $\mathrm{C} 14 \mathrm{H} 12$ & $\mathrm{c} 1 \mathrm{ccc}(\mathrm{cc} 1) \mathrm{C}=\mathrm{Cc} 1 \mathrm{ccccc} 1$ & $\operatorname{lnChl}=1 S / C 14 H 12 / c 1-3-7-13(8-4-1) 11-12-14-9-5-2-6-10-14 / h 1-12 \mathrm{H}$ \\
\hline 3680 & $\mathrm{C} 14 \mathrm{H} 13 \mathrm{O}$ & $\mathrm{c1ccc}(\mathrm{cc} 1) \operatorname{cc}(\mathrm{c} 1 \mathrm{ccccc} 1)[0]$ & InChl=1S/C14H130/c15-14(13-9-5-2-6-10-13)11-12-7-3-1-4-8-12/h1-10,14H,11H2 \\
\hline 3681 & $\mathrm{C} 14 \mathrm{H} 130 \mathrm{OH}$ & $\mathrm{c1ccc}(\mathrm{cc} 1) \mathrm{cC}(\mathrm{c} 1 \mathrm{ccccc} 1) 00$ & InChl=1S/C14H1402/c15-16-14(13-9-5-2-6-10-13)11-12-7-3-1-4-8-12/h1-10,14-15H,11H2 \\
\hline 3682 & $\mathrm{C} 14 \mathrm{H} 13$ & $\mathrm{c} 1 \mathrm{ccc}(\mathrm{cc} 1)[\mathrm{CH}] \mathrm{Cc} 1 \mathrm{ccccc} 1$ & InChl=1S/C14H13/c1-3-7-13(8-4-1)11-12-14-9-5-2-6-10-14/h1-11H,12H2 \\
\hline 3683 & $\mathrm{C} 14 \mathrm{H} 130 \mathrm{O}$ & $\mathrm{c1ccc}(\mathrm{cc} 1) \mathrm{cc}(\mathrm{c} 1 \mathrm{ccccc} 1) \mathrm{O}[\mathrm{O}]$ & $\operatorname{lnChl}=15 / C 14 H 1302 / c 15-16-14(13-9-5-2-6-10-13) 11-12-7-3-1-4-8-12 / h 1-10,14 \mathrm{H}, 11 \mathrm{H} 2$ \\
\hline 3684 & $\mathrm{C} 14 \mathrm{H} 11 \mathrm{O}-1 \mathrm{O} 2 \mathrm{H}$ & $\operatorname{c1ccc}(\operatorname{cc} 1) \mathrm{C}(=0) \mathrm{C}(\operatorname{c1} 1 \mathrm{ccccc} 1) 00$ & $\operatorname{lnChl}=15 / C 14 H 1203 / c 15-13(11-7-3-1-4-8-11) 14(17-16) 12-9-5-2-6-10-12 / h 1-10,14,16 \mathrm{H}$ \\
\hline 3685 & P-XYLDIMERJ & $\mathrm{C}[\mathrm{C}] 1 \mathrm{C}=\mathrm{CC}(=\mathrm{CCc} 2 \mathrm{ccc}(\mathrm{C}) \mathrm{cc} 2) \mathrm{C}=\mathrm{C} 1$ & InChl=15/C16H17/c1-13-3-7-15(8-4-13)11-12-16-9-5-14(2)6-10-16/h3-11H,12H2,1-2H3 \\
\hline 3686 & O-XYLDIMERJ & Cc1ccccc1[CH]Cc1ccccc1C & $\operatorname{lnChl}=15 / C 16 \mathrm{H} 17 / \mathrm{c} 1-13-7-3-5-9-15(13) 11-12-16-10-6-4-8-14(16) 2 / \mathrm{h} 3-11 \mathrm{H}, 12 \mathrm{H} 2,1-2 \mathrm{H} 3$ \\
\hline 3687 & P-XYLDIMER & $\operatorname{Cc1} 1 \mathrm{ccc}(\mathrm{cc} 1) \operatorname{ccc} 1 \mathrm{ccc}(\mathrm{C}) \mathrm{cc} 1$ & 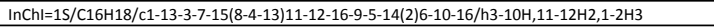 \\
\hline 3688 & O-XYLDIMER & Cc1ccccc1CCc1 1ccccc1C & InChl=1S/C16H18/c1-13-7-3-5-9-15(13)11-12-16-10-6-4-8-14(16)2/h3-10H,11-12H2,1-2H3 \\
\hline 3689 & R-TMBDIMER & $\mathrm{CC} 1=\mathrm{C}[\mathrm{C}](\mathrm{C}) \mathrm{C}(=\mathrm{CCc} 2 \mathrm{ccc}(\mathrm{C}) \mathrm{cc} 2 \mathrm{C}) \mathrm{C}=\mathrm{C} 1$ & 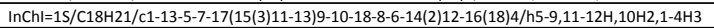 \\
\hline 3690 & R-TMB135DIMER & $\mathrm{CC} 1=\mathrm{CC}(=\mathrm{CC}(=\mathrm{CCc} 2 \mathrm{cc}(\mathrm{C}) \mathrm{cc}(\mathrm{C}) \mathrm{c} 2)[\mathrm{CH}] 1) \mathrm{C}$ & InChl=1S/C18H21/c1-13-7-14(2)10-17(9-13)5-6-18-11-15(3)8-16(4)12-18/h5,7-12H,6H2,1-4H3 \\
\hline 3691 & TMBDIMER & $\mathrm{Cc} 1 \mathrm{ccc}(\mathrm{CCc} 2 \operatorname{ccc}(\mathrm{C}) \mathrm{cc} 2 \mathrm{C}) \mathrm{c}(\mathrm{C}) \mathrm{c} 1$ & 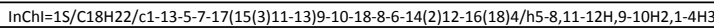 \\
\hline 3692 & TMB135DIMER & $\operatorname{Cc1} 1 \mathrm{cc}(\mathrm{C}) \mathrm{cc}(\mathrm{CCc} 2 \mathrm{cc}(\mathrm{C}) \mathrm{cc}(\mathrm{C}) \mathrm{c} 2) \mathrm{c1}$ & $\operatorname{lnChl}=1 \mathrm{~S} / \mathrm{C} 18 \mathrm{H} 22 / \mathrm{c1}-13-7-14(2) 10-17(9-13) 5-6-18-11-15(3) 8-16(4) 12-18 / \mathrm{h} 7-12 \mathrm{H}, 5-6 \mathrm{H} 2,1-4 \mathrm{H} 3$ \\
\hline 3693 & C7H6-1 & $\mathrm{CACC1}=\mathrm{CC}=\mathrm{CC} 1$ & InChl=1S/C7H6/c1-2-7-5-3-4-6-7/h1,3-5H,6H2 \\
\hline 3694 & CHXD13-5M & $\mathrm{CC} 1 \mathrm{CC}=\mathrm{CC}=\mathrm{C} 1$ & InChl=1S/C7H10/c1-7-5-3-2-4-6-7/h2-5,7H,6H2,1H3 \\
\hline 3695 & CHXD14-3M & $\mathrm{CC} 1 \mathrm{C}=\mathrm{CCC}=\mathrm{C} 1$ & InChl=1S/C7H10/c1-7-5-3-2-4-6-7/h3-7H,2H2,1H3 \\
\hline 3696 & $\mathrm{C} 12 \mathrm{H} 10$ & 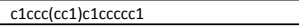 & $\operatorname{lnChl}=15 / \mathrm{C} 12 \mathrm{H} 10 / \mathrm{c} 1-3-7-11(8-4-1) 12-9-5-2-6-10-12 / \mathrm{h} 1-10 \mathrm{H}$ \\
\hline 3697 & $\mathrm{C} 12 \mathrm{H9}$ & $\mathrm{C} 1=\mathrm{CC}=\mathrm{C}([\mathrm{C}]=\mathrm{C} 1) \mathrm{c} 1 \mathrm{ccccc} 1$ & $\operatorname{lnChl}=1 \mathrm{~S} / \mathrm{C} 12 \mathrm{H} / / \mathrm{c} 1-3-7-11(8-4-1) 12-9-5-2-6-10-12 / \mathrm{h} 1-9 \mathrm{H}$ \\
\hline 3698 & TRIPHENYLENE & $\mathrm{c} 1 \mathrm{ccc} 2 \mathrm{c}(\mathrm{c} 1) \mathrm{c} 1 \mathrm{ccccc} 1 \mathrm{c} 1 \mathrm{c} 2 \mathrm{cccc} 1$ & 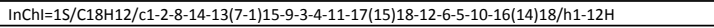 \\
\hline 3699 & ISOC9H9 & {$[\mathrm{CH} 2] \mathrm{C}(=\mathrm{C}) \mathrm{c} 1 \mathrm{ccccc} 1$} & InChl=1S/C9H9/c1-8(2)9-6-4-3-5-7-9/h3-7H,1-2H2 \\
\hline 3700 & $\mathrm{IC12H12- \textrm {A }}$ & $\mathrm{C}=\mathrm{C}=\mathrm{CCC}(=\mathrm{C}) \mathrm{c} 1 \mathrm{ccccc} 1$ & $\operatorname{lnChl}=1 \mathrm{~S} / \mathrm{C} 12 \mathrm{H} 12 / \mathrm{c} 1-3-4-8-11(2) 12-9-6-5-7-10-12 / \mathrm{h} 4-7,9-10 \mathrm{H}, 1-2,8 \mathrm{H} 2$ \\
\hline 3701 & IC12H12-P & $\mathrm{CHCCCC}(=\mathrm{C}) \mathrm{c} 1 \mathrm{cccccc} 1$ & InChl=1S/C12H12/c1-3-4-8-11(2)12-9-6-5-7-10-12/h1,5-7,9-10H,2,4,8H2 \\
\hline 3702 & C12H9-PM & $\mathrm{C} 1=\mathrm{CC}=\mathrm{C}(\mathrm{C}=[\mathrm{C}] 1) \mathrm{c} 1 \mathrm{ccccc} 1$ & InChl=1S/C12H9/c1-3-7-11(8-4-1)12-9-5-2-6-10-12/h1-5,7-10H \\
\hline 3703 & NAPHCHCCH3-1 & $\mathrm{C}[\mathrm{C}]=\mathrm{Cc} 1 \mathrm{ccccc} 2 \mathrm{c} 1 \mathrm{cccc} 2$ & $\operatorname{lnChl}=1 \mathrm{~S} / \mathrm{C} 13 \mathrm{H} 11 / \mathrm{c} 1-2-6-11-8-5-9-12-7-3-4-10-13(11) 12 / \mathrm{h} 3-10 \mathrm{H}, 1 \mathrm{H} 3$ \\
\hline 3704 & NAPHCCCH3-1 & CC\#Cc1 1cccc2c1cccc2 & $\operatorname{lnChl}=1 \mathrm{~S} / \mathrm{C} 13 \mathrm{H} 10 / \mathrm{c} 1-2-6-11-8-5-9-12-7-3-4-10-13(11) 12 / \mathrm{h} 3-5,7-10 \mathrm{H}, 1 \mathrm{H} 3$ \\
\hline 3705 & NAPHCHCCH2-1 & $\mathrm{C}=\mathrm{C}=\mathrm{Cc} 1 \mathrm{ccccc} 2 \mathrm{c} 1 \mathrm{ccccc} 2$ & InChl=1S/C13H10/c1-2-6-11-8-5-9-12-7-3-4-10-13(11)12/h3-10H,1H2 \\
\hline 3706 & $\mathrm{~A} 2 \mathrm{C} 2 \mathrm{H}-1$ & C\#Cc1cccc2 $2 \operatorname{cccc} 12$ & $\operatorname{lnChl}=1 \mathrm{~S} / \mathrm{C} 12 \mathrm{H} 8 / \mathrm{c1}-2-10-7-5-8-11-6-3-4-9-12(10) 11 / \mathrm{h} 1,3-9 \mathrm{H}$ \\
\hline 3707 & A2CYC5 & $\mathrm{C} 1=\mathrm{Cc} 2 \mathrm{c}(\mathrm{C} 1) \mathrm{c} 1 \mathrm{ccccc} 1 \mathrm{cc} 2$ & $\operatorname{lnChl}=15 / C 13 H 10 / c 1-2-6-12-10(4-1) 8-9-11-5-3-7-13(11) 12 / h 1-6,8-9 H, 7 \mathrm{H} 2$ \\
\hline 3708 & ISOC13H11 & {$[\mathrm{CH} 2] \mathrm{C}(=\mathrm{C}) \mathrm{c} 1 \mathrm{cccc} 2 \mathrm{c} 1 \mathrm{cccc} 2$} & InChl=1S/C13H11/c1-10(2)12-9-5-7-11-6-3-4-8-13(11)12/h3-9H,1-2H2 \\
\hline 3709 & NAPHCH2CCH2-1 & $\mathrm{C}=[\mathrm{C}] \mathrm{cc} 1 \mathrm{cccc} 2 \mathrm{c} 1 \mathrm{cccc} 2$ & InChl=1S/C13H11/c1-2-6-11-8-5-9-12-7-3-4-10-13(11)12/h3-5,7-10H,1,6H2 \\
\hline 3710 & NAPHCH2CCH-1 & C\#CCc1cccc2c1cccc2 & InChl=1S/C13H10/c1-2-6-11-8-5-9-12-7-3-4-10-13(11)12/h1,3-5,7-10H,6H2 \\
\hline 3711 & NAPHC3H4 & $\mathrm{C}=\mathrm{CC}=\mathrm{C} 1 \mathrm{C}=\mathrm{C}[\mathrm{CH}] \mathrm{c} 2 \mathrm{c} 1 \mathrm{cccc} 2$ & InChl=1S/C13H11/c1-2-6-11-8-5-9-12-7-3-4-10-13(11)12/h2-10H,1H2 \\
\hline 3712 & A2R5 & $\mathrm{c} 1 \mathrm{cc} 2 \mathrm{cccc} 3 \mathrm{c} 2 \mathrm{c}(\mathrm{c} 1) \mathrm{C}=\mathrm{C} 3$ & InChl=1S/C12H8/c1-3-9-4-2-6-11-8-7-10(5-1)12(9)11/h1-8H \\
\hline 3713 & $\mathrm{C} 6 \mathrm{H} 5 \mathrm{C} 3 \mathrm{H} 4$ & $\mathrm{C}=\mathrm{CC}=\mathrm{C} 1[\mathrm{CH}] \mathrm{C}=\mathrm{CC}=\mathrm{C} 1$ & InChl=1S/C9H9/c1-2-6-9-7-4-3-5-8-9/h2-8H,1H2 \\
\hline 3714 & CYC7RAD & $\mathrm{C} 1=\mathrm{CC}=\mathrm{CC}=\mathrm{C}[\mathrm{CH}] 1$ & $\operatorname{lnChl}=1 \mathrm{~S} / \mathrm{C} 7 \mathrm{H} 7 / \mathrm{c} 1-2-4-6-7-5-3-1 / \mathrm{h} 1-7 \mathrm{H}$ \\
\hline 3715 & CYC7TRIENE & $\mathrm{C} 1=\mathrm{CC}=\mathrm{CC}=\mathrm{CC} 1$ & $\operatorname{lnChl}=1 \mathrm{~S} / \mathrm{C} 7 \mathrm{H} 8 / \mathrm{c} 1-2-4-6-7-5-3-1 / \mathrm{h} 1-6 \mathrm{H}, 7 \mathrm{H} 2$ \\
\hline 3716 & C5H6-L & $\mathrm{CC}=\mathrm{CCHC}$ & InChl=1S/C5H6/c1-3-5-4-2/h1,4-5H,2H3 \\
\hline 3717 & IC6-1R & $\mathrm{CCCC}([\mathrm{CH} 2]) \mathrm{C}$ & InChl=1S/C6H13/c1-4-5-6(2)3/h6H,2,4-5H2,1,3H3 \\
\hline 3718 & PHENYLA2-2 & 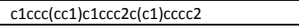 & $\operatorname{lnChl}=1 \mathrm{~S} / \mathrm{C} 16 \mathrm{H} 12 / \mathrm{c} 1-2-6-13(7-3-1) 16-11-10-14-8-4-5-9-15(14) 12-16 / \mathrm{h} 1-12 \mathrm{H}$ \\
\hline 3719 & PHENYLA2-1 & 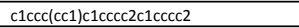 & InChl=1S/C16H12/c1-2-7-13(8-3-1)16-12-6-10-14-9-4-5-11-15(14)16/h1-12H \\
\hline 3720 & ANTHRACENE & $\mathrm{c1} 1 \mathrm{ccc} 2 \mathrm{c}(\mathrm{c} 1) \operatorname{cc} 1 \mathrm{c}(\mathrm{c} 2) \mathrm{cccc} 1$ & $\operatorname{lnChl}=1 \mathrm{~S} / \mathrm{C} 14 \mathrm{H} 10 / \mathrm{c} 1-2-6-12-10-14-8-4-3-7-13(14) 9-11(12) 5-1 / \mathrm{h} 1-10 \mathrm{H}$ \\
\hline 3721 & PYRENE & c1cc2ccc3c4c2c(c1)ccc4ccc3 & 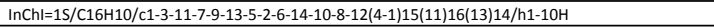 \\
\hline 3722 & RPYRENE1 & $\mathrm{c} 1 \mathrm{cc} 2 \mathrm{ccc} 3 \mathrm{c} 4 \mathrm{c} 2 \mathrm{c}(\mathrm{c} 1) \mathrm{ccc} 4=[\mathrm{c}] \mathrm{C}=\mathrm{C} 3$ & InChl=15/C16H9/c1-3-11-7-9-13-5-2-6-14-10-8-12(4-1)15(11)16(13)14/h1-5,7-10H \\
\hline 3723 & ALLYLNAPH-1 & $\mathrm{C}=\mathrm{CCc} 1 \mathrm{cccc} 2 \mathrm{c} 1 \mathrm{cccc} 2$ & InChl=1S/C13H12/c1-2-6-11-8-5-9-12-7-3-4-10-13(11)12/h2-5,7-10H,1,6H2 \\
\hline 3724 & $\mathrm{~A} 2 \mathrm{C} 2 \mathrm{HV}$ & C\#Cc1 1ccc2c(c1)cccc2 & InChl=1S/C12H8/c1-2-10-7-8-11-5-3-4-6-12(11)9-10/h1,3-9H \\
\hline 3725 & ALLYLNAPH-2 & $\mathrm{C}=\mathrm{CCc} 1 \mathrm{ccc} 2 \mathrm{c}(\mathrm{c} 1) \operatorname{cccc} 2$ & $\operatorname{lnChl}=15 / \mathrm{C} 13 \mathrm{H} 12 / \mathrm{c} 1-2-5-11-8-9-12-6-3-4-7-13(12) 10-11 / \mathrm{h} 2-4,6-10 \mathrm{H}, 1,5 \mathrm{H} 2$ \\
\hline 3726 & CHRYSENE & 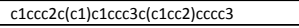 & $\operatorname{lnChl}=15 / C 18 H 12 / c 1-3-7-15-13(5-1) 9-11-18-16-8-4-2-6-14(16) 10-12-17(15) 18 / h 1-12 \mathrm{H}$ \\
\hline 3727 & C7H11D16-3R & $\mathrm{C}=\mathrm{C}[\mathrm{CH}] \mathrm{CCC}=\mathrm{C}$ & $\operatorname{lnChl}=1 \mathrm{~S} / \mathrm{C} 7 \mathrm{H} 11 / \mathrm{c} 1-3-5-7-6-4-2 / \mathrm{h} 3-5 \mathrm{H}, 1-2,6-7 \mathrm{H} 2$ \\
\hline
\end{tabular}




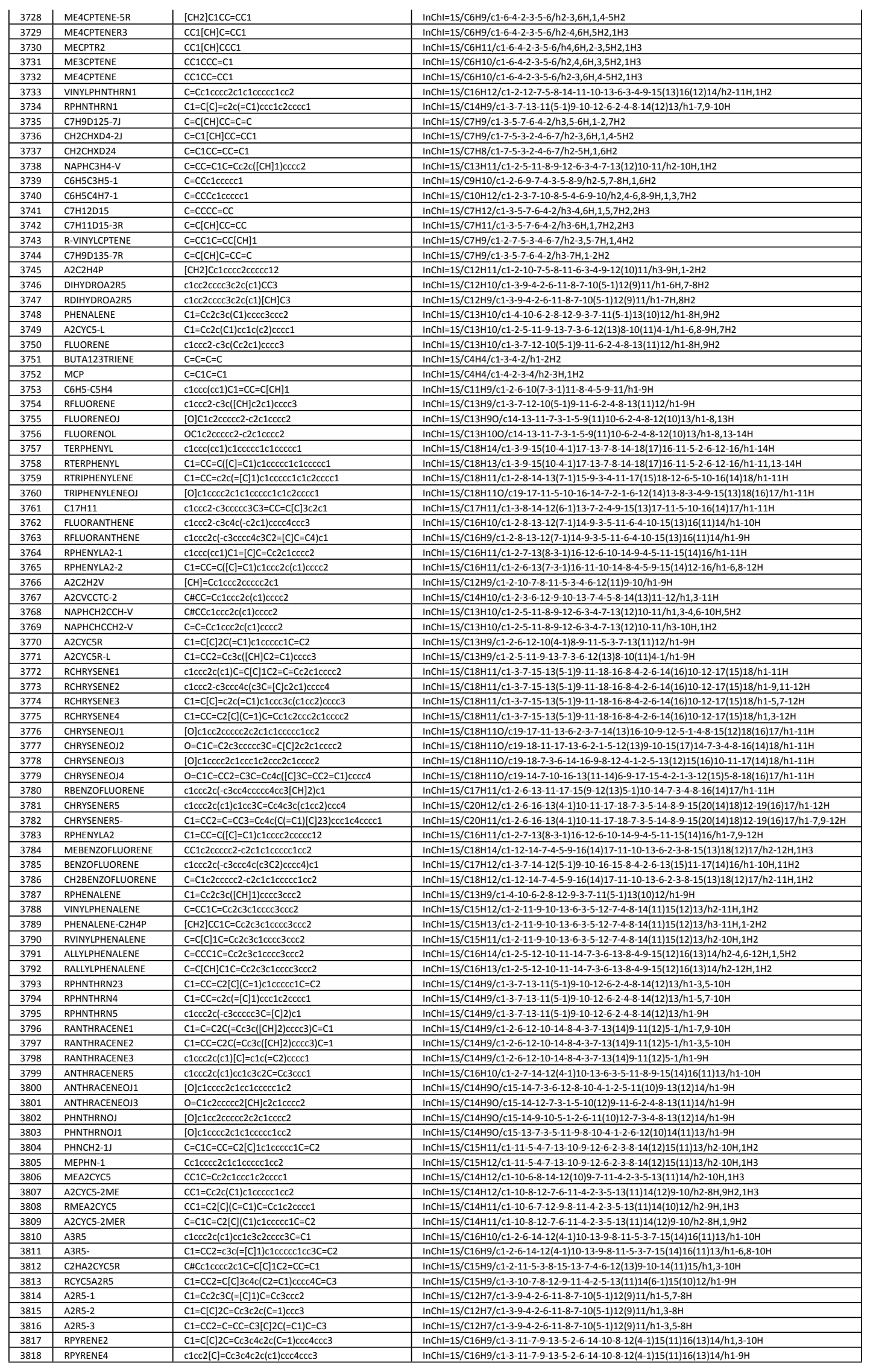




\begin{tabular}{|c|c|c|c|}
\hline 3819 & PYRENEOJ1 & {$[0] c 1 c c 2 c c c c 3 c 2 c 2 c 1 c c c c 2 c c 3$} & InChl=1S/C16H9o/c17-14-9-12-5-1-3-10-7-8-11-4-2-6-13(14)16(11)15(10) \\
\hline 3820 & PYRENEOJ2 & $\mathrm{O}=\mathrm{C} 1 \mathrm{C}=\mathrm{C} 2 \mathrm{C}=\mathrm{Cc} 3 \mathrm{c} 4[\mathrm{C}] 2 \mathrm{C}(=\mathrm{C} 1) \mathrm{C}=\mathrm{C} c 4 \mathrm{ccc} 3$ & InChl=1S/C16H9O/c17-14-8-12-6-4-10-2-1-3-11-5-7-13(9-14)16(12)15(10)11/h1-9H \\
\hline 3821 & A4R5 & $\mathrm{c} 1 \mathrm{cc} 2 \mathrm{ccc} 3 \mathrm{c} 4 \mathrm{c} 2 \mathrm{c}(\mathrm{c} 1) \mathrm{cc} 1 \mathrm{c} 4 \mathrm{c}(\mathrm{C}=\mathrm{C} 1) \mathrm{cc} 3$ & 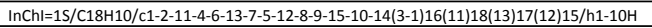 \\
\hline 3822 & PHNTHRNC5OJ & 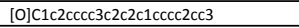 & InChl=1S/C15H9o/c16-15-11-5-1-3-9-7-8-10-4-2-6-12(15)14(10)13(9)11/h1-8,15H \\
\hline 3823 & A2CTCCVC-2 & $\mathrm{C}=\mathrm{CC} \# \mathrm{Cc} 1 \mathrm{ccc} 2 \mathrm{c}(\mathrm{c} 1) \operatorname{cccc} 2$ & $\operatorname{lnChl}=1 \mathrm{~S} / \mathrm{C} 14 \mathrm{H} 10 / \mathrm{c} 1-2-3-6-12-9-10-13-7-4-5-8-14(13) 11-12 / \mathrm{h} 2,4-5,7-11 \mathrm{H}, 1 \mathrm{H} 2$ \\
\hline 3824 & MEFLUORENE & CC1c2 2cccce2-c2c1cccc2 & InChl=1S/C14H12/c1-10-11-6-2-4-8-13(11)14-9-5-3-7-12(10)14/h2-10H,1H3 \\
\hline 3825 & CH2FLUROENE & $\mathrm{C}=\mathrm{C} 1 \mathrm{c} 2 \mathrm{ccccc} 2-\mathrm{c} 2 \mathrm{c} 1 \mathrm{ccccc} 2$ & $\operatorname{lnChl}=1 \mathrm{~S} / \mathrm{C} 14 \mathrm{H} 10 / \mathrm{c1}-10-11-6-2-4-8-13(11) 14-9-5-3-7-12(10) 14 / \mathrm{h} 2-9 \mathrm{H}, 1 \mathrm{H} 2$ \\
\hline 3826 & A2R5- & $\mathrm{c} 1 \mathrm{cc} 2 \mathrm{cccc} 3 \mathrm{c} 2 \mathrm{c}(\mathrm{c} 1)[\mathrm{c}]=\mathrm{C} 3$ & InChl=1S/C12H7/c1-3-9-4-2-6-11-8-7-10(5-1)12(9)11/h1-7H \\
\hline 3827 & A2DIR5 & $\mathrm{C} 1=\mathrm{Cc} 2 \mathrm{c} 3 \mathrm{c} 1 \mathrm{ccc} 1 \mathrm{c} 3 \mathrm{c}(\mathrm{cc} 2) \mathrm{C}=\mathrm{C} 1$ & InChl=1S/C14H8/c1-2-10-7-8-12-4-3-11-6-5-9(1)13(10)14(11)12/h1-8H \\
\hline 3828 & A2R5CHCH-2 & {$[\mathrm{CH}]=\mathrm{C} c 1 \mathrm{cc} 2 \mathrm{C}=\mathrm{Cc} 3 \mathrm{c} 2 \mathrm{c}(\mathrm{c} 1) \mathrm{ccc} 3$} & $\operatorname{lnChl=15/C14H9/c1-2-10-8-12-5-3-4-11-6-7-13(9-10)14(11)12/h1-9H~}$ \\
\hline 3829 & C2H3A2R5-2 & $\mathrm{C}=\mathrm{CC} 1=\mathrm{C} c 2 \mathrm{c} 3 \mathrm{C}(=[\mathrm{C}] 1) \mathrm{C}=\mathrm{C} c 3 \mathrm{ccc} 2$ & $\operatorname{lnChl}=1 \mathrm{~S} / \mathrm{C} 14 \mathrm{H} 9 / \mathrm{c1}-2-10-8-12-5-3-4-11-6-7-13(9-10) 14(11) 12 / \mathrm{h} 2-8 \mathrm{H}, 1 \mathrm{H} 2$ \\
\hline 3830 & C4H3A2R5-2 & $\mathrm{CHCC}=\mathrm{Cc} 1 \mathrm{cc} 2 \mathrm{C}=\mathrm{Cc} 3 \mathrm{c} 2 \mathrm{c}(\mathrm{c} 1) \mathrm{ccc} 3$ & 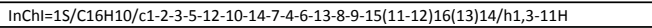 \\
\hline 3831 & A2R5CHCH-1 & {$[\mathrm{CH}]=\mathrm{Cc} 1 \mathrm{ccc} 2 \mathrm{c} 3 \mathrm{c} 1 \mathrm{C}=\mathrm{Cc} 3 \mathrm{ccc} 2$} & InChl=1S/C14H9/c1-2-10-6-7-11-4-3-5-12-8-9-13(10)14(11)12/h1-9H \\
\hline 3832 & C2H3A2R5-1 & $\mathrm{C}=\mathrm{CC} 1=\mathrm{C}=\mathrm{Cc} 2 \mathrm{c} 3[\mathrm{C}] 1 \mathrm{C}=\mathrm{Cc} 3 \mathrm{ccc} 2$ & InChl=1S/C14H9/c1-2-10-6-7-11-4-3-5-12-8-9-13(10)14(11)12/h2-5,7-9H,1H2 \\
\hline 3833 & C4H3A2R5 & $\mathrm{CHCC}=\mathrm{Cc} 1 \mathrm{ccc} 2 \mathrm{c} 3 \mathrm{c} 1 \mathrm{C}=\mathrm{Cc} 3 \mathrm{ccc} 2$ & $\operatorname{lnChl}=15 / C 16 H 10 / c 1-2-3-5-12-8-9-13-6-4-7-14-10-11-15(12) 16(13) 14 / h 1,3-11 \mathrm{H}$ \\
\hline 3834 & MEA2R5 & $\mathrm{CC} 1=\mathrm{Cc} 2 \mathrm{c} 3 \mathrm{c} 1 \mathrm{cccc} 3 \mathrm{ccc} 2$ & InChl=1S/C13H10/c1-9-8-11-6-2-4-10-5-3-7-12(9)13(10)11/h2-8H,1H3 \\
\hline 3835 & RMEA2R5 & $\mathrm{C}=\mathrm{C} 1[\mathrm{CH}] \mathrm{c} 2 \mathrm{c} 3 \mathrm{c} 1 \mathrm{cccc} 3 \mathrm{ccc} 2$ & $\operatorname{lnChl}=1 \mathrm{~S} / \mathrm{C} 13 \mathrm{H} 9 / \mathrm{c} 1-9-8-11-6-2-4-10-5-3-7-12(9) 13(10) 11 / \mathrm{h} 2-8 \mathrm{H}, 1 \mathrm{H} 2$ \\
\hline 3836 & IC7D124 & $\mathrm{C}=\mathrm{C}=\mathrm{CCC}(=\mathrm{C}) \mathrm{C}$ & $\ln C h \mathrm{l}=1 \mathrm{~S} / \mathrm{C} 7 \mathrm{H} 10 / \mathrm{c} 1-4-5-6-7(2) 3 / \mathrm{h} 5 \mathrm{H}, 1-2,6 \mathrm{H} 2,3 \mathrm{H} 3$ \\
\hline 3837 & IC7D1T5 & $\mathrm{CHCCCC}(=\mathrm{C}) \mathrm{C}$ & InChl=1S/C7H10/c1-4-5-6-7(2)3/h1H,2,5-6H2,3H3 \\
\hline 3838 & $\mathrm{~A} 2 \mathrm{C} 2 \mathrm{HR}$ & $\mathrm{CHCC} 1=\mathrm{C}=\mathrm{C}[\mathrm{CH}] \mathrm{c} 2 \mathrm{c} 1 \mathrm{cccc} 2$ & InChl=1S/C12H7/c1-2-10-7-5-8-11-6-3-4-9-12(10)11/h1,3-6,8-9H \\
\hline 3839 & C2H3A2R5- & $\mathrm{C}=\mathrm{CC} 1=[\mathrm{C}] \mathrm{c} 2 \mathrm{c} 3 \mathrm{c} 1 \mathrm{cccc} 3 \mathrm{ccc} 2$ & $\operatorname{lnChl}=15 / \mathrm{C} 14 \mathrm{H} 9 / \mathrm{c} 1-2-10-9-12-7-3-5-11-6-4-8-13(10) 14(11) 12 / \mathrm{h} 2-8 \mathrm{H}, 1 \mathrm{H} 2$ \\
\hline 3840 & CPT-3CH2-1R & $\mathrm{C}=\mathrm{C} 1 \mathrm{C}[\mathrm{CH}] \mathrm{CC} 1$ & InChl=1S/C6H9/c1-6-4-2-3-5-6/h2H,1,3-5H2 \\
\hline 3841 & 124C7D14-1R & $\mathrm{CC}(=\mathrm{C}) \mathrm{CC}(=\mathrm{C})[\mathrm{CH} 2]$ & InChl=1S/C7H11/c1-6(2)5-7(3)4/h1-3,5H2,4H3 \\
\hline 3842 & ME1CPANE-4CH2-1R & $\mathrm{C}[\mathrm{C}] 1 \mathrm{CCC}(=\mathrm{C}) \mathrm{C} 1$ & $\operatorname{lnChl}=1 \mathrm{~S} / \mathrm{C} 7 \mathrm{H} 11 / \mathrm{c} 1-6-3-4-7(2) 5-6 / \mathrm{h} 1,3-5 \mathrm{H} 2,2 \mathrm{H} 3$ \\
\hline 3843 & A2R5-MER & $\mathrm{C}=\mathrm{C} 1 \mathrm{C}=\mathrm{C} c 2 \mathrm{c} 3[\mathrm{C}] 1 \mathrm{C}=\mathrm{C} c 3 \mathrm{ccc} 2$ & InChl=1S/C13H9/c1-9-5-6-10-3-2-4-11-7-8-12(9)13(10)11/h2-8H,1H2 \\
\hline 3844 & A2R5-ME & $\mathrm{Cc} 1 \mathrm{ccc} 2 \mathrm{c} 3 \mathrm{c} 1 \mathrm{C}=\mathrm{Cc} 3 \mathrm{ccc} 2$ & InChl=1S/C13H10/c1-9-5-6-10-3-2-4-11-7-8-12(9)13(10)11/h2-8H,1H3 \\
\hline 3845 & CYC5A2R5 & $\mathrm{C} 1=\mathrm{Cc} 2 \mathrm{c}(\mathrm{C} 1) \mathrm{c} 1 \mathrm{cccc} 3 \mathrm{c} 1 \mathrm{c}(\mathrm{c} 2) \mathrm{C}=\mathrm{C} 3$ & InChl=1S/C15H10/c1-3-10-7-8-12-9-11-4-2-5-13(11)14(6-1)15(10)12/h1-4,6-9H,5H2 \\
\hline 3846 & A2C2HR-CH2J & $\mathrm{CHC}[\mathrm{C}] 1 \mathrm{C}(=\mathrm{C}) \mathrm{C}=\mathrm{Cc} 2 \mathrm{c} 1 \mathrm{cccc} 2$ & $\operatorname{lnChl}=1 \mathrm{~S} / \mathrm{C} 13 \mathrm{H} 9 / \mathrm{c1}-3-12-10(2) 8-9-11-6-4-5-7-13(11) 12 / \mathrm{h} 1,4-9 \mathrm{H}, 2 \mathrm{H} 2$ \\
\hline 3847 & $\mathrm{~A} 2 \mathrm{C} 2 \mathrm{HL}-\mathrm{CH} 2 \mathrm{~J}$ & $\mathrm{C \# CC} 1=\mathrm{C}[\mathrm{C}] 2 \mathrm{C}(=\mathrm{CC}=\mathrm{CC} 2=\mathrm{C}) \mathrm{C}=\mathrm{C} 1$ & InChl=1S/C13H9/c1-3-11-7-8-12-6-4-5-10(2)13(12)9-11/h1,4-9H,2H2 \\
\hline 3848 & ME-A2C2HR & C\#Cc1cccc2c1cccc2C & InChl=1S/C13H10/c1-3-11-7-5-8-12-10(2)6-4-9-13(11)12/h1,4-9H,2H3 \\
\hline 3849 & ME-A2C2HL & C\#Cc1ccc(c2c1cccc2)c & InChl=1S/C13H10/c1-3-11-9-8-10(2)12-6-4-5-7-13(11)12/h1,4-9H,2H3 \\
\hline 3850 & A2R5CHCCH3-1 & $\mathrm{C}[\mathrm{C}]=\mathrm{Cc} 1 \mathrm{ccc} 2 \mathrm{c} 3 \mathrm{c} 1 \mathrm{C}=\mathrm{Cc} 3 \mathrm{ccc} 2$ & $\operatorname{lnChl}=15 / C 15 H 11 / c 1-2-4-11-7-8-12-5-3-6-13-9-10-14(11) 15(12) 13 / h 3-10 \mathrm{H}, 1 \mathrm{H} 3$ \\
\hline 3851 & A2R5CCCH3-1 & 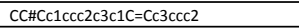 & InChl=1S/C15H10/c1-2-4-11-7-8-12-5-3-6-13-9-10-14(11)15(12)13/h3,5-10H,1H3 \\
\hline 3852 & A2R5CHCCH2-1 & $\mathrm{C}=\mathrm{C}=\mathrm{Cc} 1 \mathrm{ccc} 2 \mathrm{c} 3 \mathrm{c} 1 \mathrm{C}=\mathrm{Cc} 3 \mathrm{ccc} 2$ & $\operatorname{lnChl}=15 / C 15 H 10 / c 1-2-4-11-7-8-12-5-3-6-13-9-10-14(11) 15(12) 13 / h 3-10 \mathrm{H}, 1 \mathrm{H} 2$ \\
\hline 3853 & A2R5IC3H4-1R & {$[\mathrm{CH} 2] \mathrm{C}(=\mathrm{C}) \mathrm{c} 1 \mathrm{ccc} 2 \mathrm{c} 3 \mathrm{c} 1 \mathrm{C}=\mathrm{Cc} 3 \mathrm{ccc} 2$} & InChl=1S/C15H11/c1-10(2)13-8-6-11-4-3-5-12-7-9-14(13)15(11)12/h3-9H,1-2H2 \\
\hline 3854 & $\mathrm{~A} 2 \mathrm{R} 5 \mathrm{CH} 2 \mathrm{CCH} 2-1$ & $\mathrm{C}=[\mathrm{C}] \mathrm{cc} 1 \mathrm{ccc} 2 \mathrm{c} 3 \mathrm{c} 1 \mathrm{C}=\mathrm{Cc} 3 \mathrm{ccc} 2$ & $\operatorname{lnChl}=1 \mathrm{~S} / \mathrm{C} 15 \mathrm{H} 11 / \mathrm{c} 1-2-4-11-7-8-12-5-3-6-13-9-10-14(11) 15(12) 13 / \mathrm{h} 3,5-10 \mathrm{H}, 1,4 \mathrm{H} 2$ \\
\hline 3855 & A2R5CH2CCH-1 & $\mathrm{C \# CCc1ccc2c3c1 \textrm {C } = \mathrm { Cc } 3 \mathrm { ccc } 2}$ & $\operatorname{lnChl}=1 \mathrm{~S} / \mathrm{C} 15 \mathrm{H} 10 / \mathrm{c} 1-2-4-11-7-8-12-5-3-6-13-9-10-14(11) 15(12) 13 / \mathrm{h} 1,3,5-10 \mathrm{H}, 4 \mathrm{H} 2$ \\
\hline 3856 & A2R5C3H4-1 & $\mathrm{C}=\mathrm{CC}=\mathrm{C} 1 \mathrm{C}=\mathrm{C} c 2 \mathrm{c} 3[\mathrm{C}] 1 \mathrm{C}=\mathrm{C} \mathrm{c} 3 \mathrm{ccc} 2$ & InChl=1S/C15H11/c1-2-4-11-7-8-12-5-3-6-13-9-10-14(11)15(12)13/h2-10H,1H2 \\
\hline 3857 & A2R5CHCCH3-3 & $\mathrm{C}[\mathrm{C}]=\mathrm{Cc} 1 \mathrm{ccc} 2 \mathrm{c} 3 \mathrm{c} 1 \mathrm{cccc} 3 \mathrm{C}=\mathrm{C} 2$ & $\operatorname{lnChl=1S/C15H11/c1-2-4-11-7-8-13-10-9-12-5-3-6-14(11)15(12)13/h3-10H,1H3~}$ \\
\hline 3858 & A2R5CHCCH2-3 & $\mathrm{C}=\mathrm{C}=\mathrm{Cc} 1 \mathrm{ccc} 2 \mathrm{c} 3 \mathrm{c} 1 \mathrm{cccc} 3 \mathrm{C}=\mathrm{C} 2$ & $\operatorname{lnChl}=15 / C 15 H 10 / c 1-2-4-11-7-8-13-10-9-12-5-3-6-14(11) 15(12) 13 / h 3-10 \mathrm{H}, 1 \mathrm{H} 2$ \\
\hline 3859 & A2R5CCCH3-3 & 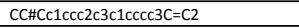 & $\operatorname{lnChl}=15 / C 15 H 10 / c 1-2-4-11-7-8-13-10-9-12-5-3-6-14(11) 15(12) 13 / \mathrm{h} 3,5-10 \mathrm{H}, 1 \mathrm{H} 3$ \\
\hline 3860 & A2R5IC3H4-3R & {$[\mathrm{CH} 2] \mathrm{C}(=\mathrm{C}) \mathrm{c} 1 \mathrm{ccc} 2 \mathrm{c} 3 \mathrm{c} 1 \mathrm{cccc} 3 \mathrm{C}=\mathrm{C} 2$} & $\operatorname{lnChl}=1 \mathrm{~S} / \mathrm{C} 15 \mathrm{H} 11 / \mathrm{c} 1-10(2) 13-9-8-12-7-6-11-4-3-5-14(13) 15(11) 12 / \mathrm{h} 3-9 \mathrm{H}, 1-2 \mathrm{H} 2$ \\
\hline 3861 & A2R5CH2CCH2-3 & $\mathrm{C}=[\mathrm{C}] \mathrm{cc} 1 \mathrm{ccc} 2 \mathrm{c} 3 \mathrm{c} 1 \mathrm{cccc} 3 \mathrm{C}=\mathrm{C} 2$ & $\operatorname{lnChl}=1 \mathrm{~S} / \mathrm{C} 15 \mathrm{H} 11 / \mathrm{c} 1-2-4-11-7-8-13-10-9-12-5-3-6-14(11) 15(12) 13 / \mathrm{h3}, 5-10 \mathrm{H}, 1,4 \mathrm{H} 2$ \\
\hline 3862 & A2R5CH2CCH-3 & 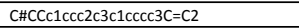 & InChl=1S/C15H10/c1-2-4-11-7-8-13-10-9-12-5-3-6-14(11)15(12)13/h1,3,5-10H,4H2 \\
\hline 3863 & A2R5C3H4-3 & $\mathrm{C}=\mathrm{CC}=\mathrm{C} 1 \mathrm{C}=\mathrm{C}[\mathrm{C}] 2 \mathrm{c} 3 \mathrm{c} 1 \mathrm{cccc} 3 \mathrm{C}=\mathrm{C} 2$ & InChl=1S/C15H11/c1-2-4-11-7-8-13-10-9-12-5-3-6-14(11)15(12)13/h2-10H,1H2 \\
\hline 3864 & A2R5CHCCH3-2 & $\mathrm{C}[\mathrm{C}]=\mathrm{C} c 1 \mathrm{cc} 2 \mathrm{C}=\mathrm{C} c 3 \mathrm{c} 2 \mathrm{c}(\mathrm{c} 1) \mathrm{ccc} 3$ & InChl=1S/C15H11/c1-2-4-11-9-13-6-3-5-12-7-8-14(10-11)15(12)13/h3-10H,1H3 \\
\hline 3865 & A2R5CCCH3-2 & 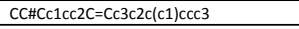 & $\operatorname{lnChl}=15 / C 15 \mathrm{H} 10 / c 1-2-4-11-9-13-6-3-5-12-7-8-14(10-11) 15(12) 13 / \mathrm{h} 3,5-10 \mathrm{H}, 1 \mathrm{H} 3$ \\
\hline 3866 & A2R5CHCCH2-2 & $\mathrm{C}=\mathrm{C}=\mathrm{Cc} 1 \mathrm{cc} 2 \mathrm{C}=\mathrm{Cc} 3 \mathrm{c} 2 \mathrm{c}(\mathrm{c} 1) \mathrm{ccc} 3$ & $\operatorname{lnChl}=15 / C 15 H 10 / c 1-2-4-11-9-13-6-3-5-12-7-8-14(10-11) 15(12) 13 / h 3-10 \mathrm{H}, 1 \mathrm{H} 2$ \\
\hline 3867 & A2R5IC3H4-2R & {$[\mathrm{CH} 2] \mathrm{C}(=\mathrm{C}) \mathrm{c} 1 \mathrm{cc} 2 \mathrm{C}=\mathrm{Cc} 3 \mathrm{c} 2 \mathrm{c}(\mathrm{c} 1) \mathrm{ccc} 3$} & $\operatorname{lnChl}=15 / C 15 H 11 / c 1-10(2) 14-8-12-5-3-4-11-6-7-13(9-14) 15(11) 12 / h 3-9 \mathrm{H}, 1-2 \mathrm{H} 2$ \\
\hline 3868 & $\mathrm{~A} 2 \mathrm{R} 5 \mathrm{CH} 2 \mathrm{CCH} 2-2$ & $\mathrm{C}=[\mathrm{C}] \mathrm{Cc} 1 \mathrm{cc} 2 \mathrm{C}=\mathrm{Cc} 3 \mathrm{c} 2 \mathrm{c}(\mathrm{c} 1) \mathrm{ccc} 3$ & $\operatorname{lnChl}=1 \mathrm{~S} / \mathrm{C} 15 \mathrm{H} 11 / \mathrm{c} 1-2-4-11-9-13-6-3-5-12-7-8-14(10-11) 15(12) 13 / \mathrm{h} 3,5-10 \mathrm{H}, 1,4 \mathrm{H} 2$ \\
\hline 3869 & $\mathrm{~A} 2 \mathrm{R} 5 \mathrm{CH} 2 \mathrm{CCH}-2$ & C\#CCc1cc2C=Cc3c2c(c1)ccc3 & 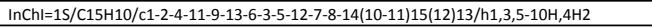 \\
\hline 3870 & A2R5C3H4-2 & $\mathrm{C}=\mathrm{CC}=\mathrm{C} 1 \mathrm{C}=\mathrm{C} 2 \mathrm{C}=\mathrm{Cc} 3 \mathrm{c} 2 \mathrm{c}([\mathrm{CH}] 1) \mathrm{ccc} 3$ & $\operatorname{lnChl}=15 / C 15 H 11 / c 1-2-4-11-9-13-6-3-5-12-7-8-14(10-11) 15(12) 13 / \mathrm{h} 2-10 \mathrm{H}, 1 \mathrm{H} 2$ \\
\hline 3871 & NAPHCHCCH3-V & $\mathrm{C}[\mathrm{C}]=\mathrm{Cc} 1 \mathrm{ccc} 2 \mathrm{c}(\mathrm{c} 1) \operatorname{cccc} 2$ & $\operatorname{lnChl}=1 \mathrm{~S} / \mathrm{C} 13 \mathrm{H} 11 / \mathrm{c} 1-2-5-11-8-9-12-6-3-4-7-13(12) 10-11 / \mathrm{h} 3-10 \mathrm{H}, 1 \mathrm{H} 3$ \\
\hline 3872 & NAPHCCCH3-V & CC\#Cc1ccc2c(c1)cccc2 & $\operatorname{lnChl}=15 / C 13 \mathrm{H} 10 / c 1-2-5-11-8-9-12-6-3-4-7-13(12) 10-11 / h 3-4,6-10 \mathrm{H}, 1 \mathrm{H} 3$ \\
\hline 3873 & ISOC13H11-V & {$[\mathrm{CH} 2] \mathrm{C}(=\mathrm{C}) \mathrm{c} 1 \mathrm{ccc} 2 \mathrm{c}(\mathrm{c} 1) \mathrm{cccc} 2$} & InChl=1S/C13H11/c1-10(2)12-8-7-11-5-3-4-6-13(11)9-12/h3-9H,1-2H2 \\
\hline 3874 & NAPHCH2CCH2-V & $\mathrm{C}=[\mathrm{c}] \mathrm{Cc} 1 \mathrm{ccc} 2 \mathrm{c}(\mathrm{c} 1) \operatorname{cccc} 2$ & $\operatorname{lnChl}=15 / \mathrm{C} 13 \mathrm{H} 11 / \mathrm{c} 1-2-5-11-8-9-12-6-3-4-7-13(12) 10-11 / \mathrm{h} 3-4,6-10 \mathrm{H}, 1,5 \mathrm{H} 2$ \\
\hline 3875 & IND-C2HA & 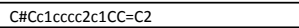 & InChl=1S/C11H8/c1-2-9-5-3-6-10-7-4-8-11(9)10/h1,3-7H,, $8 \mathrm{H} 2$ \\
\hline 3876 & $\mathrm{C} 2 \mathrm{HIC9H} 8$ & $\mathrm{CHCc1} 1 \mathrm{cccc}(\mathrm{c} 1) \mathrm{C}(=\mathrm{C})[\mathrm{CH} 2]$ & $\operatorname{lnChl}=15 / \mathrm{C} 11 \mathrm{H} 9 / \mathrm{c1}-4-10-6-5-7-11(8-10) 9(2) 3 / \mathrm{h} 1,5-8 \mathrm{H}, 2-3 \mathrm{H} 2$ \\
\hline 3877 & $\mathrm{C} 2 \mathrm{HC} 6 \mathrm{H} 4 \mathrm{C} 3 \mathrm{H} 4$ & $\mathrm{C}=\mathrm{CC}=\mathrm{C} 1[\mathrm{CH}] \mathrm{C}=\mathrm{CC}(=\mathrm{C} 1) \mathrm{CHC}$ & $\operatorname{lnChl}=1 \mathrm{~S} / \mathrm{C} 11 \mathrm{H} 9 / \mathrm{c1}-3-6-11-8-5-7-10(4-2) 9-11 / \mathrm{h} 2-3,5-9 \mathrm{H}, 1 \mathrm{H} 2$ \\
\hline 3878 & $\mathrm{CH} 3 \mathrm{C9H} 6$ & $\mathrm{C}[\mathrm{C}] 1 \mathrm{C}=\mathrm{CC}=\mathrm{C} 2 \mathrm{C} 1=\mathrm{CC}=\mathrm{C} 2$ & $\operatorname{lnChl}=15 / \mathrm{C} 10 \mathrm{H} 9 / \mathrm{c} 1-8-4-2-5-9-6-3-7-10(8) 9 / \mathrm{h} 2-7 \mathrm{H}, 1 \mathrm{H} 3$ \\
\hline 3879 & $\mathrm{C} 9 \mathrm{H} 7 \mathrm{CH} 3-2$ & $\mathrm{CC} 1=\mathrm{Cc} 2 \mathrm{c}(\mathrm{C} 1) \mathrm{cccc} 2$ & $\operatorname{lnChl}=15 / \mathrm{C} 10 \mathrm{H} 10 / \mathrm{c} 1-8-6-9-4-2-3-5-10(9) 7-8 / \mathrm{h} 2-6 \mathrm{H}, 7 \mathrm{H} 2,1 \mathrm{H} 3$ \\
\hline 3880 & C9H6CH3-2 & $\mathrm{CC} 1=\mathrm{Cc} 2 \mathrm{c}([\mathrm{CH}] 1) \mathrm{cccc} 2$ & InChl=15/C10H9/c1-8-6-9-4-2-3-5-10(9)7-8/h2-7H,1H3 \\
\hline 3881 & MECYC5A2R5 & $\mathrm{CC} 1 \mathrm{C}=\mathrm{Cc} 2 \mathrm{c} 1 \mathrm{c} 1 \mathrm{cccc} 3 \mathrm{c} 1 \mathrm{c}(\mathrm{c} 2) \mathrm{C}=\mathrm{C} 3$ & $\operatorname{lnChl}=15 / C 16 H 12 / c 1-10-5-6-12-9-13-8-7-11-3-2-4-14(15(10) 12) 16(11) 13 / \mathrm{h} 2-10 \mathrm{H}, 1 \mathrm{H} 3$ \\
\hline 3882 & CYC5A2R5-2ME & $\mathrm{CC} 1=\mathrm{Cc} 2 \mathrm{c}(\mathrm{C} 1) \mathrm{c} 1 \mathrm{cccc} 3 \mathrm{c} 1 \mathrm{c}(\mathrm{c} 2) \mathrm{C}=\mathrm{C} 3$ & 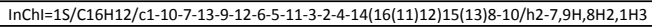 \\
\hline 3883 & RMECYC5A2R5 & $\mathrm{CC} 1=\mathrm{C} 2 \mathrm{C}(=\mathrm{C}[\mathrm{C}] 3 \mathrm{c} 4 \mathrm{c} 2 \operatorname{cccc} 4 \mathrm{C}=\mathrm{C} 3) \mathrm{C}=\mathrm{C} 1$ & $\begin{array}{ll}\operatorname{lnChl}=1 \mathrm{~S} / \mathrm{C} 16 \mathrm{H} 11 / \mathrm{c1} 1-10-5-6-12-9-13-8-7-11-3-2-4-14(15(10) 12) 16(11) 13 / \mathrm{h} 2-9 \mathrm{H}, 1 \mathrm{H} 3 \\
\end{array}$ \\
\hline 3884 & CYC5A2R5-2MER & $\mathrm{CC} 1=\mathrm{C}[\mathrm{C}] 2 \mathrm{C}(=\mathrm{C} 1) \operatorname{c} 1 \mathrm{cccc} 3 \mathrm{c} 1 \mathrm{C}(=\mathrm{C} 2) \mathrm{C}=\mathrm{C} 3$ & InChl=1S/C16H11/c1-10-7-13-9-12-6-5-11-3-2-4-14(16(11)12)15(13)8-10/h2-9H,1H3 \\
\hline 3885 & C7D125 & $\mathrm{CC}=\mathrm{CCC}=\mathrm{C}=\mathrm{C}$ & $\operatorname{lnChl}=1 \mathrm{~S} / \mathrm{C} 7 \mathrm{H} 10 / \mathrm{c} 1-3-5-7-6-4-2 / \mathrm{h} 4-6 \mathrm{H}, 1,7 \mathrm{H} 2,2 \mathrm{H} 3$ \\
\hline 3886 & C7T1D5 & $\mathrm{CC}=\mathrm{CCCC} \# \mathrm{C}$ & InChl=1S/C7H10/c1-3-5-7-6-4-2/h1,4,6H,5,7H2,2H3 \\
\hline 3887 & 13C7D145 & $\mathrm{C}=\mathrm{C}=\mathrm{CC}(\mathrm{C}=\mathrm{C}) \mathrm{C}$ & InChl=1S/C7H10/c1-4-6-7(3)5-2/h5-7H,1-2H2,3H3 \\
\hline 3888 & 13C7D1T5 & $\mathrm{CC}(\mathrm{C}=\mathrm{C}) \mathrm{CCHC}$ & InChl=1S/C7H10/c1-4-6-7(3)5-2/h1,5,7H,2,6H2,3H3 \\
\hline 3889 & CPTENE-4CH2 & $\mathrm{C}=\mathrm{C} 1 \mathrm{CC}=\mathrm{CC} 1$ & InChl=1S/C6H8/c1-6-4-2-3-5-6/h2-3H,1,4-5H2 \\
\hline 3890 & $\mathrm{C} 6 \mathrm{H} 5 \mathrm{CHCCH}$ & $\mathrm{CHCC}=\mathrm{C} 1[\mathrm{CH}] \mathrm{C}=\mathrm{CC}=\mathrm{C} 1$ & InChl=1S/C9H7/c1-2-6-9-7-4-3-5-8-9/h1,3-8H \\
\hline 3891 & $\mathrm{C} 6 \mathrm{H} 5 \mathrm{CCCH} 3$ & CC\#Cc1ccccc1 & $\operatorname{lnChl}=1 \mathrm{~S} / \mathrm{C} 9 \mathrm{H} 8 / \mathrm{c} 1-2-6-9-7-4-3-5-8-9 / \mathrm{h} 3-5,7-8 \mathrm{H}, 1 \mathrm{H3}$ \\
\hline 3892 & $\mathrm{C} 6 \mathrm{H} 5 \mathrm{CCCH} 2$ & $\mathrm{C}=\mathrm{C}=\mathrm{C}=\mathrm{C} 1[\mathrm{CH}] \mathrm{C}=\mathrm{CC}=\mathrm{C} 1$ & InChl=1S/C9H7/c1-2-6-9-7-4-3-5-8-9/h3-5,7-8H,1H2 \\
\hline 3893 & $\mathrm{C} 6 \mathrm{H} 5 \mathrm{CHCCH} 2$ & $\mathrm{C}=\mathrm{C}=\mathrm{Cc} 1 \mathrm{cccccc} 1$ & $\operatorname{lnChl}=15 / C 9 H 8 / c 1-2-6-9-7-4-3-5-8-9 / h 3-8 \mathrm{H}, 1 \mathrm{H} 2$ \\
\hline 3894 & $\mathrm{C} 6 \mathrm{H} 5-\mathrm{C} 5 \mathrm{H} 5$ & $\mathrm{C} 1=\mathrm{CC}(\mathrm{C}=\mathrm{C} 1) \mathrm{c} 1 \mathrm{ccccc} 1$ & InChl=1S/C11H10/c1-2-6-10(7-3-1)11-8-4-5-9-11/h1-9,11H \\
\hline 3895 & $\mathrm{C} 12 \mathrm{H} 9-\mathrm{C} 2 \mathrm{H}$ & C\#Cc1cccc(c1)c1ccccc1 & InChl=1S/C14H10/c1-2-12-7-6-10-14(11-12)13-8-4-3-5-9-13/h1,3-11H \\
\hline 3896 & $\mathrm{RC} 12 \mathrm{H} 8-\mathrm{C} 2 \mathrm{H}$ & $\mathrm{CHC} 1 \mathrm{cccc}(\mathrm{c} 1) \mathrm{C} 1=\mathrm{CC}=\mathrm{CC}=[\mathrm{C}] 1$ & $\operatorname{lnChl}=15 / C 14 H 9 / c 1-2-12-7-6-10-14(11-12) 13-8-4-3-5-9-13 / h 1,3-8,10-11 \mathrm{H}$ \\
\hline 3897 & PHNTHRC2H & C\#Cc1 $1 \mathrm{ccc} 2 \mathrm{c}(\mathrm{c} 1) \operatorname{ccc} 1 \mathrm{c} 2 \mathrm{cccc} 1$ & InChl=1S/C16H10/c1-2-12-7-10-16-14(11-12)9-8-13-5-3-4-6-15(13)16/h1,3-11H \\
\hline 3898 & $\mathrm{C} 12 \mathrm{H} 8-2 \mathrm{C} 2 \mathrm{H}$ & C\#Cc1cccc(c1)c1cccc(c1)C\#C & 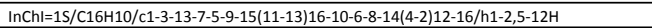 \\
\hline 3899 & CTCCCVCCVC & $\mathrm{C}=\mathrm{CC}=\mathrm{CCCHC}$ & InChl=1S/C7H8/c1-3-5-7-6-4-2/h1,4,6-7H,2,5H2 \\
\hline 3900 & A1CCVCVC & $\mathrm{C}=\mathrm{C}=\mathrm{CCc} 1 \mathrm{cccccc} 1$ & $\operatorname{lnChl}=15 / \mathrm{C} 10 \mathrm{H} 10 / \mathrm{c} 1-2-3-7-10-8-5-4-6-9-10 / \mathrm{h} 3-6,8-9 \mathrm{H}, 1,7 \mathrm{H} 2$ \\
\hline 3901 & A1CCCTC & C\#CCCC1ccccc1 & InChl=1S/C10H10/c1-2-3-7-10-8-5-4-6-9-10/h1,4-6,8-9H,3,7H2 \\
\hline 3902 & PHNTHRN & $\mathrm{c} 1 \mathrm{ccc} 2 \mathrm{c}(\mathrm{c} 1) \mathrm{c} 1 \mathrm{ccccc} 1 \mathrm{cc} 2$ & InChl=1S/C14H10/c1-3-7-13-11(5-1)9-10-12-6-2-4-8-14(12)13/h1-10H \\
\hline 3903 & CC6H5C4H6-1 & $\mathrm{C}=\mathrm{C}[\mathrm{CH}] \mathrm{Cc} 1 \mathrm{ccccc} 1$ & $\operatorname{lnChl}=15 / \mathrm{C} 10 \mathrm{H} 11 / \mathrm{c} 1-2-3-7-10-8-5-4-6-9-10 / \mathrm{h} 2-6,8-9 \mathrm{H}, 1,7 \mathrm{H} 2$ \\
\hline 3904 & $\mathrm{C} 6 \mathrm{H} 4 \mathrm{CH} 2 \mathrm{CH} 3$ & $\mathrm{CCC} 1=\mathrm{CC}=\mathrm{CC}=[\mathrm{C}] 1$ & InChl=1S/C8H9/c1-2-8-6-4-3-5-7-8/h3-6H,2H2,1H3 \\
\hline 3905 & H14PHNTHRN & $\mathrm{C} 1 \mathrm{C}=\mathrm{Cc} 2 \mathrm{c}(\mathrm{C} 1) \mathrm{c} 1 \mathrm{ccccc} 1 \mathrm{cc} 2$ & $\operatorname{lnChl}=1 \mathrm{~S} / \mathrm{C} 14 \mathrm{H} 12 / \mathrm{c1} 1-3-7-13-11(5-1) 9-10-12-6-2-4-8-14(12) 13 / \mathrm{h} 1-3,5-7,9-10 \mathrm{H}, 4,8 \mathrm{H} 2$ \\
\hline 3906 & $\mathrm{RA} 2 \mathrm{C} 2 \mathrm{H} 5$ & $\mathrm{CCC} 1=\mathrm{C}=\mathrm{C}[\mathrm{CH}] \mathrm{c} 2 \mathrm{c} 1 \mathrm{cccc} 2$ & InChl=1S/C12H11/c1-2-10-7-5-8-11-6-3-4-9-12(10)11/h3-6,8-9H,2H2,1H3 \\
\hline 3907 & $\mathrm{~A} 2 \mathrm{C} 2 \mathrm{H} 3$ & $\mathrm{C}=\mathrm{Cc} 1 \mathrm{cccc} 2 \operatorname{ccccc} 12$ & $\operatorname{lnChl}=1 \mathrm{~S} / \mathrm{C} 12 \mathrm{H} 10 / \mathrm{c} 1-2-10-7-5-8-11-6-3-4-9-12(10) 11 / \mathrm{h} 2-9 \mathrm{H}, 1 \mathrm{H} 2$ \\
\hline 3908 & $\mathrm{~A} 2 \mathrm{C} 2 \mathrm{H} 2$ & {$[\mathrm{CH}]=\mathrm{Cc} 1 \mathrm{cccc} 2 \mathrm{ccccc} 12$} & InChl=1S/C12H9/c1-2-10-7-5-8-11-6-3-4-9-12(10)11/h1-9H \\
\hline 3909 & CH2IND & $\mathrm{C}=\mathrm{C} 1 \mathrm{C}=\mathrm{Cc} 2 \operatorname{ccccc} 12$ & $\operatorname{lnChl}=15 / C 10 H 8 / c 1-8-6-7-9-4-2-3-5-10(8) 9 / h 2-7 \mathrm{H}, 1 \mathrm{H} 2$ \\
\hline
\end{tabular}




\begin{tabular}{|c|c|c|c|}
\hline 3910 & $\mathrm{C} 6 \mathrm{H} 5 \mathrm{CHCCH} 3$ & $\mathrm{C}[\mathrm{c}]=\mathrm{Cc} 1 \mathrm{ccccc} 1$ & InChl=1S/C9H9/c1-2-6-9-7-4-3-5-8-9/h3-8H,1H3 \\
\hline 3911 & $\mathrm{~A} 1 \mathrm{CH} 2 \mathrm{CCH} 2$ & $\mathrm{C}=[\mathrm{C}] \mathrm{cc} 1 \mathrm{cccccc} 1$ & InChl=15/C9H9/c1-2-6-9-7-4-3-5-8-9/h3-5,7-8H,1,6H2 \\
\hline 3912 & $\mathrm{CHCCHCHCHCH} 2$ & $\mathrm{C}=\mathrm{CC}=\mathrm{CCHC}$ & $\operatorname{lnChl}=15 / C 6 H 6 / c 1-3-5-6-4-2 / h 1,4-6 \mathrm{H}, 2 \mathrm{H} 2$ \\
\hline 3913 & $\mathrm{C} 4 \mathrm{H} 5-2 \mathrm{C} 2 \mathrm{H}$ & $\mathrm{C}=\mathrm{C}(\mathrm{CHC}) \mathrm{C}=\mathrm{C}$ & $\operatorname{lnChl}=1 \mathrm{~S} / \mathrm{C} 6 \mathrm{H} 6 / \mathrm{c1}-4-6(3) 5-2 / \mathrm{h} 1,5 \mathrm{H}, 2-3 \mathrm{H} 2$ \\
\hline 3914 & $\mathrm{CHCCH} 2 \mathrm{CH} 2 \mathrm{CHCH} 2$ & $\mathrm{C}=\mathrm{CCCCHC}$ & InChl=1S/C6H8/c1-3-5-6-4-2/h1,4H,2,5-6H2 \\
\hline 3915 & $\mathrm{CH} 2 \mathrm{CCHCH} 2 \mathrm{CHCH} 2$ & $\mathrm{C}=\mathrm{CCC}=\mathrm{C}=\mathrm{C}$ & $\operatorname{lnChl}=1 \mathrm{~S} / \mathrm{C} 6 \mathrm{H} 8 / \mathrm{c} 1-3-5-6-4-2 / \mathrm{h} 3,6 \mathrm{H}, 1-2,5 \mathrm{H} 2$ \\
\hline 3916 & A1CVCCTC & $\mathrm{C \# CC}=\mathrm{Cc} 1 \mathrm{ccccc} 1$ & InChl=1S/C10H8/c1-2-3-7-10-8-5-4-6-9-10/h1,3-9H \\
\hline 3917 & $\mathrm{C} 12 \mathrm{H} 9 \mathrm{O}$ & {$[0] \operatorname{c1cccc}(\mathrm{c} 1) \operatorname{c1ccccc1}$} & InChl=1S/C12H9O/c13-12-8-4-7-11(9-12)10-5-2-1-3-6-10/h1-9H \\
\hline 3918 & C14H11-AR & $\mathrm{C} 1=\mathrm{CC}=\mathrm{C}([\mathrm{C}]=\mathrm{C} 1) \mathrm{C}=\mathrm{C} c 1 \mathrm{ccccc} 1$ & InChl=1S/C14H11/c1-3-7-13(8-4-1)11-12-14-9-5-2-6-10-14/h1-9,11-12H \\
\hline 3919 & $\mathrm{C} 5 \mathrm{H} 5 \mathrm{C} 3 \mathrm{H} 3$ & $\mathrm{CACCC1C}=\mathrm{CC}=\mathrm{C} 1$ & $\operatorname{lnChl}=15 / \mathrm{C} 8 \mathrm{H} 8 / \mathrm{c} 1-2-5-8-6-3-4-7-8 / \mathrm{h} 1,3-4,6-8 \mathrm{H}, 5 \mathrm{H} 2$ \\
\hline 3920 & C5H4VCCVC & $\mathrm{C}=\mathrm{C}=\mathrm{CC} 1 \mathrm{C}=\mathrm{CC}=\mathrm{C} 1$ & $\operatorname{lnChl}=1 \mathrm{~S} / \mathrm{C} 8 \mathrm{H} 8 / \mathrm{c1}-2-5-8-6-3-4-7-8 / \mathrm{h} 3-8 \mathrm{H}, 1 \mathrm{H} 2$ \\
\hline 3921 & $\mathrm{C9H7C3H3}$ & $\mathrm{CHCCC} 1 \mathrm{C}=\mathrm{Cc} 2 \mathrm{c} 1 \mathrm{cccc} 2$ & $\operatorname{lnChl}=1 \mathrm{~S} / \mathrm{C} 12 \mathrm{H} 10 / \mathrm{c} 1-2-5-10-8-9-11-6-3-4-7-12(10) 11 / \mathrm{h} 1,3-4,6-10 \mathrm{H}, 5 \mathrm{H} 2$ \\
\hline 3922 & C9H6VCCVC & $\mathrm{C}=\mathrm{C}=\mathrm{CC} 1 \mathrm{C}=\mathrm{C} c 2 \mathrm{c} 1 \mathrm{cccc} 2$ & $\operatorname{lnChl}=15 / C 12 \mathrm{H} 10 / c 1-2-5-10-8-9-11-6-3-4-7-12(10) 11 / h 3-10 \mathrm{H}, 1 \mathrm{H} 2$ \\
\hline 3923 & C5PHNTHRN & $\mathrm{c} 1 \mathrm{cc} 2 \mathrm{cc} 3 \mathrm{c} 4 \mathrm{c} 2 \mathrm{c}(\mathrm{c} 1) \operatorname{ccc} 4 \mathrm{ccc} 3$ & $\operatorname{lnChl}=15 / C 15 \mathrm{H} 10 / c 1-3-10-7-8-11-4-2-6-13-9-12(5-1) 14(10) 15(11) 13 / h 1-8 \mathrm{H}, 9 \mathrm{H} 2$ \\
\hline 3924 & RPHNTHRC2H & $\mathrm{CHC} C 1 \mathrm{ccc} 2 \mathrm{c}(\mathrm{c} 1) \mathrm{c} 1=[\mathrm{C}] \mathrm{C}=\mathrm{CC}=\mathrm{c} 1 \mathrm{cc} 2$ & $\operatorname{lnChl}=1 \mathrm{~S} / \mathrm{C} 16 \mathrm{H} / \mathrm{c} 1-2-12-7-8-14-10-9-13-5-3-4-6-15(13) 16(14) 11-12 / \mathrm{h} 1,3-5,7-11 \mathrm{H}$ \\
\hline 3925 & RC5PHNTHRN & $\mathrm{c1} 1 \mathrm{cc} 2[\mathrm{CH}] \mathrm{c} 3 \mathrm{c} 4 \mathrm{c} 2 \mathrm{c}(\mathrm{c} 1) \mathrm{ccc} 4 \mathrm{ccc} 3$ & $\operatorname{lnChl}=15 / C 15 H 9 / c 1-3-10-7-8-11-4-2-6-13-9-12(5-1) 14(10) 15(11) 13 / h 1-9 H$ \\
\hline 3926 & MECSPHNTHRN & $\mathrm{CC} 1 \mathrm{c} 2 \mathrm{cccc} 3 \mathrm{c} 2 \mathrm{c} 2 \mathrm{c} 1 \mathrm{cccc} 2 \mathrm{cc} 3$ & $\operatorname{lnChl}=15 / C 16 \mathrm{H} 12 / \mathrm{c} 1-10-13-6-2-4-11-8-9-12-5-3-7-14(10) 16(12) 15(11) 13 / \mathrm{h} 2-10 \mathrm{H}, 1 \mathrm{H} 3$ \\
\hline 3927 & RMEC5PHNTHRN & $\mathrm{c}[\mathrm{c}] 1 \mathrm{c} 2 \mathrm{cccc} 3 \mathrm{c} 2 \mathrm{c} 2 \mathrm{c} 1 \mathrm{cccc} 2 \mathrm{cc} 3$ & $\operatorname{lnChl}=15 / C 16 \mathrm{H} 11 / c 1-10-13-6-2-4-11-8-9-12-5-3-7-14(10) 16(12) 15(11) 13 / \mathrm{h} 2-9 \mathrm{H}, 1 \mathrm{H} 3$ \\
\hline 3928 & A2R5-C2H & 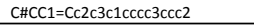 & InChl=1S/C14H8/c1-2-10-9-12-7-3-5-11-6-4-8-13(10)14(11)12/h1,3-9H \\
\hline 3929 & PHNTHRNC5OH & $0<1 c 2 c c c c 3 c 2 c 2 c 1 c c c c 2 c c 3$ & $\operatorname{lnChl}=15 / C 15 H 100 / c 16-15-11-5-1-3-9-7-8-10-4-2-6-12(15) 14(10) 13(9) 11 / \mathrm{h} 1-8,15-16 \mathrm{H}$ \\
\hline 3930 & A1CTCCVC & $\mathrm{C}=\mathrm{CCACC} 1 \mathrm{ccccc} 1$ & InChl=1S/C10H8/c1-2-3-7-10-8-5-4-6-9-10/h2,4-6,8-9H,1H2 \\
\hline 3931 & A2CVCCTC & $\mathrm{CHCC}=\mathrm{Cc} 1 \mathrm{cccc} 2 \mathrm{c} 1 \mathrm{cccc} 2$ & $\operatorname{lnChl}=1 \mathrm{~S} / \mathrm{C} 14 \mathrm{H} 10 / \mathrm{c} 1-2-3-7-12-9-6-10-13-8-4-5-11-14(12) 13 / \mathrm{h} 1,3-11 \mathrm{H}$ \\
\hline 3932 & A2CTCCVC & $\mathrm{C}=\mathrm{CC} \# \mathrm{Cc} 1 \mathrm{cccc} 2 \mathrm{c} 1 \mathrm{cccc} 2$ & $\operatorname{lnChl}=1 \mathrm{~S} / \mathrm{C} 14 \mathrm{H} 10 / \mathrm{c} 1-2-3-7-12-9-6-10-13-8-4-5-11-14(12) 13 / \mathrm{h} 2,4-6,8-11 \mathrm{H}, 1 \mathrm{H} 2$ \\
\hline 3933 & $\mathrm{C} 6 \mathrm{H} 5 \mathrm{CH} 2 \mathrm{C} 6 \mathrm{H} 5$ & $\operatorname{c1ccc(cc1)\operatorname {cc1}1\mathrm {ccccc}1}$ & $\operatorname{lnChl}=1 \mathrm{~S} / \mathrm{C} 13 \mathrm{H} 12 / \mathrm{cc}-3-7-12(8-4-1) 11-13-9-5-2-6-10-13 / \mathrm{h} 1-10 \mathrm{H}, 11 \mathrm{H} 2$ \\
\hline 3934 & $\mathrm{C} 6 \mathrm{H} 5 \mathrm{CHC} 6 \mathrm{H} 5$ & $\mathrm{c} 1 \mathrm{ccc}(\mathrm{cc} 1)[\mathrm{CH}] \mathrm{c} 1 \mathrm{ccccc} 1$ & InChl=1S/C13H11/c1-3-7-12(8-4-1)11-13-9-5-2-6-10-13/h1-11H \\
\hline 3935 & A2R5-2C2H & $\mathrm{CHC} 11 \mathrm{cc} 2 \mathrm{C}=\mathrm{Cc} 3 \mathrm{c} 2 \mathrm{c}(\mathrm{c} 1) \mathrm{ccc} 3$ & $\operatorname{lnChl}=1 \mathrm{~S} / \mathrm{C} 14 \mathrm{H} 8 / \mathrm{c} 1-2-10-8-12-5-3-4-11-6-7-13(9-10) 14(11) 12 / \mathrm{h} 1,3-9 \mathrm{H}$ \\
\hline 3936 & A2R5-1C2H & 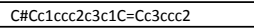 & InChl=1S/C14H8/c1-2-10-6-7-11-4-3-5-12-8-9-13(10)14(11)12/h1,3-9H \\
\hline 3937 & A2R5-C2H2 & {$[\mathrm{CH}]=\mathrm{CC} 1=\mathrm{Cc} 2 \mathrm{c} 3 \mathrm{c} 1 \mathrm{ccccc} 3 \mathrm{ccc} 2$} & InChl=1S/C14H9/c1-2-10-9-12-7-3-5-11-6-4-8-13(10)14(11)12/h1-9H \\
\hline 3938 & MECPENE-3CH2 & $\mathrm{C}=\mathrm{C} 1 \mathrm{CCC}(=\mathrm{C} 1) \mathrm{C}$ & InChl=1S/C7H10/c1-6-3-4-7(2)5-6/h5H,1,3-4H2,2H3 \\
\hline 3939 & MECPENE-4CH2 & $\mathrm{C}=\mathrm{C} 1 \mathrm{CC}=\mathrm{C}(\mathrm{C} 1) \mathrm{C}$ & InChl=1S/C7H10/c1-6-3-4-7(2)5-6/h4H,1,3,5H2,2H3 \\
\hline 3940 & $\mathrm{CH} 2 \mathrm{C} 6 \mathrm{H} 4 \mathrm{C} 2 \mathrm{H}$ & $\mathrm{CHCC} 1=\mathrm{CC}=\mathrm{C}[\mathrm{CH}] \mathrm{C} 1=\mathrm{C}$ & $\operatorname{lnChl}=1 \mathrm{~S} / \mathrm{C} 9 \mathrm{H} 7 / \mathrm{c1}-3-9-7-5-4-6-8(9) 2 / \mathrm{h} 1,4-7 \mathrm{H}, 2 \mathrm{H} 2$ \\
\hline 3941 & $\mathrm{~A} 2 \mathrm{CYC5}-\mathrm{C} 2 \mathrm{H}$ & $\mathrm{CHC} c 1 \mathrm{cccc} 2 \mathrm{c} 1 \mathrm{ccc} 1 \mathrm{c} 2 \mathrm{CC}=\mathrm{C} 1$ & InChl=15/C15H10/c1-2-11-5-3-8-15-13-7-4-6-12(13)9-10-14(11)15/h1,3-6,8-10H,7H2 \\
\hline 3942 & $\mathrm{C} 2 \mathrm{HC} 6 \mathrm{H} 4 \mathrm{CHCCH} 3$ & $\mathrm{C}[\mathrm{C}]=\mathrm{Cc} 1 \mathrm{cccc}(\mathrm{c} 1) \mathrm{C \# C}$ & InChl=1S/C11H9/c1-3-6-11-8-5-7-10(4-2)9-11/h2,5-9H,1H3 \\
\hline 3943 & $\mathrm{C} 2 \mathrm{HC} 6 \mathrm{H} 4 \mathrm{CCCH} 3$ & CC\#Cc1cccc(c1)C\#C & $\operatorname{lnChl}=1 \mathrm{~S} / \mathrm{C} 11 \mathrm{H} 8 / \mathrm{c} 1-3-6-11-8-5-7-10(4-2) 9-11 / \mathrm{h} 2,5,7-9 \mathrm{H}, 1 \mathrm{H} 3$ \\
\hline 3944 & $\mathrm{C} 2 \mathrm{HC} 6 \mathrm{H} 4 \mathrm{CHCCH} 2$ & $\mathrm{C}=\mathrm{C}=\mathrm{Cc} 1 \mathrm{cccc}(\mathrm{c} 1) \mathrm{CHC}$ & $\operatorname{lnChl}=1 \mathrm{~S} / \mathrm{C} 11 \mathrm{H} 8 / \mathrm{c} 1-3-6-11-8-5-7-10(4-2) 9-11 / \mathrm{h} 2,5-9 \mathrm{H}, 1 \mathrm{H} 2$ \\
\hline 3945 & $\mathrm{C} 2 \mathrm{HC} 6 \mathrm{H} 4 \mathrm{CH}_{2} \mathrm{CCH} 2$ & $\mathrm{C}=[\mathrm{C}] \mathrm{Cc} 1 \mathrm{cccc}(\mathrm{c} 1) \mathrm{C \# C}$ & $\operatorname{lnChl}=1 \mathrm{~S} / \mathrm{C} 11 \mathrm{H} 9 / \mathrm{c} 1-3-6-11-8-5-7-10(4-2) 9-11 / \mathrm{h} 2,5,7-9 \mathrm{H}, 1,6 \mathrm{H} 2$ \\
\hline 3946 & $\mathrm{C} 2 \mathrm{HC} 6 \mathrm{H} 4 \mathrm{CH} 2 \mathrm{CCH}$ & C\#CCc1cccc(c1)CHC & $\operatorname{lnChl}=1 \mathrm{~S} / \mathrm{C} 11 \mathrm{H} 8 / \mathrm{c1}-3-6-11-8-5-7-10(4-2) 9-11 / \mathrm{h} 1-2,5,7-9 \mathrm{H}, 6 \mathrm{H} 2$ \\
\hline 3947 & $\mathrm{CH} 3 \mathrm{C} 6 \mathrm{H} 4 \mathrm{CH} 2 \mathrm{CCH}$ & C\#CCc1ccccc1C & InChl=1S/C10H10/c1-3-6-10-8-5-4-7-9(10)2/h1,4-5,7-8H,6H2,2H3 \\
\hline 3948 & $\mathrm{CH} 3 \mathrm{C} 6 \mathrm{H} 4 \mathrm{CHCCH} 2$ & $\mathrm{C}=\mathrm{C}=\mathrm{Cc} 1 \operatorname{ccccc} 1 \mathrm{C}$ & $\operatorname{lnChl}=1 \mathrm{~S} / \mathrm{C} 10 \mathrm{H} 10 / \mathrm{c} 1-3-6-10-8-5-4-7-9(10) 2 / \mathrm{h} 4-8 \mathrm{H}, 1 \mathrm{H} 2,2 \mathrm{H} 3$ \\
\hline 3949 & $\mathrm{CH} 3 \mathrm{C9H} 7$ & $\mathrm{Cc} 1 \mathrm{cccc} 2 \mathrm{c} 1 \mathrm{CC}=\mathrm{C} 2$ & $\operatorname{lnChl}=15 / \mathrm{C} 10 \mathrm{H} 10 / \mathrm{c} 1-8-4-2-5-9-6-3-7-10(8) 9 / \mathrm{h} 2-6 \mathrm{H}, 7 \mathrm{H} 2,1 \mathrm{H} 3$ \\
\hline 3950 & $\mathrm{C} 9 \mathrm{H} 6 \mathrm{OH}$ & $\mathrm{c} 1 \mathrm{ccc} 2 \mathrm{c}(\mathrm{c} 1) \mathrm{C}=\mathrm{C}[\mathrm{c}] 2 \mathrm{O}$ & InChl=15/C9H70/c10-9-6-5-7-3-1-2-4-8(7)9/h1-6,10H \\
\hline 3951 & $\mathrm{C} 10 \mathrm{H} 9 \mathrm{OO}$ & {$[0] \mathrm{OC} 1 \mathrm{C}=\mathrm{CCc} 2 \mathrm{c} 1 \mathrm{cccc} 2$} & InChl=1S/C10H9O2/c11-12-10-7-3-5-8-4-1-2-6-9(8)10/h1-4,6-7,10H,5H2 \\
\hline 3952 & $\mathrm{C} 1 \mathrm{OH} 90 \mathrm{OH}$ & $00 \mathrm{C} 1 \mathrm{C}=\mathrm{CCc} 2 \mathrm{c} 1 \mathrm{cccc} 2$ & $\operatorname{lnChl}=1 \mathrm{~s} / \mathrm{C} 10 \mathrm{H} 1002 / \mathrm{c} 11-12-10-7-3-5-8-4-1-2-6-9(8) 10 / \mathrm{h} 1-4,6-7,10-11 \mathrm{H}, 5 \mathrm{H} 2$ \\
\hline 3953 & C3H5A1COJ & $\mathrm{C}=\mathrm{CCc} 1 \mathrm{ccccc} 1 \mathrm{C}[\mathrm{O}]$ & $\operatorname{lnChl}=15 / \mathrm{C} 10 \mathrm{H} 110 / \mathrm{c} 1-2-5-9-6-3-4-7-10(9) 8-11 / \mathrm{h} 2-4,6-7 \mathrm{H}, 1,5,8 \mathrm{H} 2$ \\
\hline 3954 & CH2A1C3H5 & $\mathrm{C}=\mathrm{C} 1[\mathrm{CH}] \mathrm{C}=\mathrm{CC}=\mathrm{C} 1 \mathrm{CC}=\mathrm{C}$ & $\operatorname{lnChl=1S/C10H11/c1-3-6-10-8-5-4-7-9(10)2/h3-5,7-8H,1-2,6H2~}$ \\
\hline 3955 & $\mathrm{CH} 3 \mathrm{~A} 1 \mathrm{C} 3 \mathrm{H} 4$ & $\mathrm{C}=\mathrm{CC}=\mathrm{C} 1[\mathrm{CH}] \mathrm{C}=\mathrm{CC}=\mathrm{C} 1 \mathrm{C}$ & $\operatorname{lnChl=1S/C10H11/c1-3-6-10-8-5-4-7-9(10)2/h3-8H,1H2,2H3~}$ \\
\hline 3956 & CH3A1C3H5 & $\mathrm{C}=\mathrm{CCc} 1 \mathrm{ccccc} 1 \mathrm{C}$ & InChl=15/C10H12/c1-3-6-10-8-5-4-7-9(10)2/h3-5,7-8H,1,6H2,2H3 \\
\hline 3957 & CHOA1C3H5O2-3 & $\mathrm{O}=\mathrm{Cc} 1 \mathrm{ccccc} 1 \mathrm{CC} 1 \mathrm{CO} 1$ & $\ln \mathrm{Chl}=15 / \mathrm{C} 10 \mathrm{H} 1002 / \mathrm{c} 11-6-9-4-2-1-3-8(9) 5-10-7-12-10 / \mathrm{h} 1-4,6,10 \mathrm{H}, 5,7 \mathrm{H} 2$ \\
\hline 3958 & CHOA1С3КЕТ3-1 & $\mathrm{O}=\mathrm{CCC}(\operatorname{c} 1 \operatorname{cccccc} 1 \mathrm{C}=0) 0 \mathrm{O}$ & InChl=15/C10H1004/c11-6-5-10(14-13)9-4-2-1-3-8(9)7-12/h1-4,6-7,10,13H,5H2 \\
\hline 3959 & CHOA1C3Q13 & $00 \operatorname{0cc}(\operatorname{c} 1 \operatorname{ccccc} 1 \mathrm{C}=0) 00$ & $\operatorname{lnChl}=15 / \mathrm{C} 10 \mathrm{H} 1205 / \mathrm{c} 11-7-8-3-1-2-4-9(8) 10(15-13) 5-6-14-12 / \mathrm{h} 1-4,7,10,12-13 \mathrm{H}, 5-6 \mathrm{H} 2$ \\
\hline 3960 & CHOA1C3Q3-102 & $00 \mathrm{OCcc}(\operatorname{c1ccccc} 1 \mathrm{C}=0) 0[0]$ & InChl=1S/C10H1105/c11-7-8-3-1-2-4-9(8)10(15-13)5-6-14-12/h1-4,7,10,12H,5-6H2 \\
\hline 3961 & CHOA1C3Q3-10J & $00 \operatorname{ccc}(\operatorname{c} 1 \operatorname{ccccc} 1 \mathrm{C}=0)[0]$ & InChl=15/C10H1104/c11-7-8-3-1-2-4-9(8)10(12)5-6-14-13/h1-4,7,10,13H,5-6H2 \\
\hline 3962 & CHOA1CCCJ & {$[\mathrm{CH} 2] \mathrm{CCc} 1 \mathrm{ccccc} 1 \mathrm{C}=\mathrm{O}$} & $\operatorname{lnChl}=15 / \mathrm{C} 10 \mathrm{H} 110 / \mathrm{c} 1-2-5-9-6-3-4-7-10(9) 8-11 / \mathrm{h} 3-4,6-8 \mathrm{H}, 1-2,5 \mathrm{H} 2$ \\
\hline 3963 & CHOA1CCCO2J & {$[0] \operatorname{Occcc} 1 \operatorname{ccccc} 1 \mathrm{C}=\mathrm{O}$} & InChl=15/C10H11O3/c11-8-10-5-2-1-4-9(10)6-3-7-13-12/h1-2,4-5,8H,3,6-7H2 \\
\hline 3964 & CHOA1CCVC & $\mathrm{C}=\mathrm{CCc} 1 \mathrm{ccccc} 1 \mathrm{C}=\mathrm{O}$ & InChl=1S/C10H100/c1-2-5-9-6-3-4-7-10(9)8-11/h2-4,6-8H,1,5H2 \\
\hline 3965 & CHOA1CJCCQ & $\mathrm{OOCC}[\mathrm{CH}] \mathrm{c} 1 \mathrm{ccccc} 1 \mathrm{C}=\mathrm{O}$ & InChl=1S/C10H1103/c11-8-10-5-2-1-4-9(10)6-3-7-13-12/h1-2,4-6,8,12H,3,7H2 \\
\hline 3966 & CJCA1CVC & $\mathrm{C}=\mathrm{Cc} 1 \mathrm{cccccc} 1 \mathrm{C}[\mathrm{CH} 2]$ & $\operatorname{lnChl}=1 \mathrm{~S} / \mathrm{C} 10 \mathrm{H} 11 / \mathrm{c} 1-3-9-7-5-6-8-10(9) 4-2 / \mathrm{h} 3,5-8 \mathrm{H}, 1-2,4 \mathrm{H} 2$ \\
\hline 3967 & CVCA1CCO2 & {$[0] \mathrm{OCCc} 1 \operatorname{ccccc} 1 \mathrm{C}=\mathrm{C}$} & $\operatorname{lnChl}=15 / C 10 H 1102 / c 1-2-9-5-3-4-6-10(9) 7-8-12-11 / \mathrm{h} 2-6 \mathrm{H}, 1,7-8 \mathrm{H} 2$ \\
\hline 3968 & CVCA1CH2CHO & $\mathrm{O}=\mathrm{CCc} 1 \operatorname{ccccc} 1 \mathrm{C}=\mathrm{C}$ & $\operatorname{lnChl}=1 \mathrm{~S} / \mathrm{C} 10 \mathrm{H} 10 \mathrm{O} / \mathrm{c1}-2-9-5-3-4-6-10(9) 7-8-11 / \mathrm{h} 2-6,8 \mathrm{H}, 1,7 \mathrm{H} 2$ \\
\hline 3969 & CVCA1CH2CO & $\mathrm{O}=[\mathrm{C}] \operatorname{cc} 1 \operatorname{ccc} c \mathrm{c} 1 \mathrm{C}=\mathrm{C}$ & InChl=15/C10H9O/c1-2-9-5-3-4-6-10(9)7-8-11/h2-6H,1,7H2 \\
\hline 3970 & CVCA1CJVC & $\mathrm{C}=\mathrm{CC} 1=\mathrm{CC}=\mathrm{C}[\mathrm{CH}] \mathrm{C} 1=\mathrm{C}=\mathrm{C}$ & $\operatorname{lnChl}=1 \mathrm{~S} / \mathrm{C} 10 \mathrm{H} 9 / \mathrm{c} 1-3-9-7-5-6-8-10(9) 4-2 / \mathrm{h} 3,5-8 \mathrm{H}, 1-2 \mathrm{H} 2$ \\
\hline 3971 & CVCA1CVC & $\mathrm{C}=\mathrm{C} c 1 \operatorname{ccccc} 1 \mathrm{C}=\mathrm{C}$ & InChl=1S/C10H10/c1-3-9-7-5-6-8-10(9)4-2/h3-8H,1-2H2 \\
\hline 3972 & CVCCA1CJO & $\mathrm{C}=\mathrm{CCc} 1 \mathrm{cccccc} 1[\mathrm{C}]=0$ & InChl=1S/C10H9O/c1-2-5-9-6-3-4-7-10(9)8-11/h2-4,6-7H,1,5H2 \\
\hline 3973 & TET-BOOH & $0001 \mathrm{cccc} 2 \mathrm{c} 1 \mathrm{cccc} 2$ & $\operatorname{lnChl}=15 / \mathrm{C} 10 \mathrm{H} 1202 / \mathrm{c} 11-12-10-7-3-5-8-4-1-2-6-9(8) 10 / \mathrm{h} 1-2,4,6,10-11 \mathrm{H}, 3,5,7 \mathrm{H} 2$ \\
\hline 3974 & TETBQ-B2J & 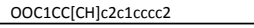 & InChl=15/C10H1102/c11-12-10-7-3-5-8-4-1-2-6-9(8)10/h1-2,4-6,10-11H,3,7H2 \\
\hline 3975 & TETBQ-S2J & $\mathrm{OOC1C}[\mathrm{CH}] \mathrm{Cc} 2 \mathrm{c} 1 \mathrm{cccc} 2$ & InChl=1S/C10H1102/c11-12-10-7-3-5-8-4-1-2-6-9(8)10/h1-4,6,10-11H,5,7H2 \\
\hline 3976 & TETBQ-S2O2 & $00 \mathrm{OC1} 1 \mathrm{cc}(\mathrm{O}[0]) \mathrm{Cc} 2 \mathrm{c} 1 \mathrm{cccc} 2$ & InChl=1S/C10H1104/c11-13-8-5-7-3-1-2-4-9(7)10(6-8)14-12/h1-4,8,10,12H,5-6H2 \\
\hline 3977 & TETKETB-S2 & $00 C 1 \mathrm{cC}(=0) c 2 c(\mathrm{C} 1) \mathrm{cccc} 2$ & InChl=15/C10H1003/c11-10-6-8(13-12)5-7-3-1-2-4-9(7)10/h1-4,8,12H,5-6H2 \\
\hline 3978 & TETKETS-B2 & $00 \mathrm{C} 1 \mathrm{CC}(=0) \mathrm{cc} 2 \mathrm{c} 1 \mathrm{cccc} 2$ & InChl=15/C10H1003/c11-8-5-7-3-1-2-4-9(7)10(6-8)13-12/h1-4,10,12H,5-6H2 \\
\hline 3979 & TETQBB & $00 \mathrm{OC1ccc}(\mathrm{c} 2 \mathrm{c} 1 \mathrm{cccc} 2) 00$ & $\operatorname{lnChl}=15 / \mathrm{C} 10 \mathrm{H} 12 \mathrm{O} 4 / \mathrm{c} 11-13-9-5-6-10(14-12) 8-4-2-1-3-7(8) 9 / \mathrm{h} 1-4,9-12 \mathrm{H}, 5-6 \mathrm{H} 2$ \\
\hline 3980 & TETQB-B2O2 & $00 \mathrm{c} 1 \mathrm{ccc}(\mathrm{c} 2 \mathrm{c} 1 \mathrm{cccc} 2) \mathrm{O}[0]$ & InChl=1S/C10H1104/c11-13-9-5-6-10(14-12)8-4-2-1-3-7(8)9/h1-4,9-11H,5-6H2 \\
\hline 3981 & TETQB-B2OJ & $00 \mathrm{OC1CCC}(\mathrm{c} 2 \mathrm{c} 1 \mathrm{cccc} 2)[0]$ & $\operatorname{lnChl=15/C10H1103/c11-9-5-6-10(13-12)8-4-2-1-3-7(8)9/h1-4,9-10,12H,5-6H2~}$ \\
\hline 3982 & TETQBS & $00 \mathrm{OC} 1 \mathrm{cc}(\mathrm{OO}) \mathrm{Cc} 2 \mathrm{c} 1 \mathrm{cccc} 2$ & InChl=1S/C10H12O4/c11-13-8-5-7-3-1-2-4-9(7)10(6-8)14-12/h1-4,8,10-12H,5-6H2 \\
\hline 3983 & TETRA & $\mathrm{C} 1 \mathrm{CCc} 2 \mathrm{c}(\mathrm{C} 1) \mathrm{cccc} 2$ & $\operatorname{lnChl}=15 / \mathrm{C} 10 \mathrm{H} 12 / \mathrm{c} 1-2-6-10-8-4-3-7-9(10) 5-1 / \mathrm{h} 1-2,5-6 \mathrm{H}, 3-4,7-8 \mathrm{H} 2$ \\
\hline 3984 & TETRARB & $\mathrm{C} 1 \mathrm{CCc} 2 \mathrm{c}([\mathrm{CH}] 1) \operatorname{cccc} 2$ & InChl=1S/C10H11/c1-2-6-10-8-4-3-7-9(10)5-1/h1-2,5-7H,3-4,8H2 \\
\hline 3985 & TETRARBO2 & {$[0] 0 \mathrm{C} 1 \mathrm{ccccc} 2 \mathrm{c} 1 \mathrm{cccc} 2$} & InChl=15/C10H1102/c11-12-10-7-3-5-8-4-1-2-6-9(8)10/h1-2,4,6,10H,3,5,7H2 \\
\hline 3986 & TETRARS & $\mathrm{C} 1[\mathrm{CH}] \mathrm{Cc} 2 \mathrm{c}(\mathrm{C} 1) \mathrm{cccc} 2$ & InChl=1S/C1OH11/c1-2-6-10-8-4-3-7-9(10)5-1/h1-3,5-6H,4,7-8H2 \\
\hline 3987 & TETRARSO2 & {$[0] \mathrm{OC} 1 \mathrm{cCc} 2 \mathrm{c}(\mathrm{C} 1) \mathrm{cccc} 2$} & InChl=1S/C10H1102/c11-12-10-6-5-8-3-1-2-4-9(8)7-10/h1-4,10H,5-7H2 \\
\hline 3988 & TETROXYB & {$[0] \mathrm{c} 1 \mathrm{cccc} 2 \mathrm{c} 1 \mathrm{cccc} 2$} & $\operatorname{lnChl=1S/C10H110/c11-10-7-3-5-8-4-1-2-6-9(8)10/h1-2,4,6,10H,3,5,7H2~}$ \\
\hline 3989 & TETSQ-B2J & $\mathrm{OOC1C}[\mathrm{CH}] \mathrm{c} 2 \mathrm{c}(\mathrm{C} 1) \mathrm{cccc} 2$ & $\operatorname{lnChl}=1 \mathrm{~S} / \mathrm{C} 10 \mathrm{H} 1102 / \mathrm{c} 11-12-10-6-5-8-3-1-2-4-9(8) 7-10 / \mathrm{h} 1-5,10-11 \mathrm{H}, 6-7 \mathrm{H} 2$ \\
\hline 3990 & TETSQ-B2O2 & {$[0] \mathrm{OC1} 1 \mathrm{cC}(00) \mathrm{cc} 2 \mathrm{c} 1 \mathrm{cccc} 2$} & InChl=1S/C10H1104/c11-13-8-5-7-3-1-2-4-9(7)10(6-8)14-12/h1-4,8,10-11H,5-6H2 \\
\hline 3991 & TETSQ-B2OJ & $0001 \mathrm{cC}([0]) \operatorname{c2c}(\mathrm{C} 1) \operatorname{cccc} 2$ & InChl=15/C10H1103/c11-10-6-8(13-12)5-7-3-1-2-4-9/7)10/h1-4,8,10,12H,5-6H2 \\
\hline 3992 & IC8D3-3R & $\mathrm{CC}(=[\mathrm{C}] \mathrm{C}(\mathrm{C})(\mathrm{C}) \mathrm{C}) \mathrm{C}$ & $\operatorname{lnChl}=15 / \mathrm{C} 8 \mathrm{H} 15 / \mathrm{c} 1-7(2) 6-8(3,4) 5 / \mathrm{h} 1-5 \mathrm{H} 3$ \\
\hline 3993 & IC8D4-5AR & $\mathrm{CC}(=[\mathrm{CH}]) \mathrm{CC}(\mathrm{C})(\mathrm{C}) \mathrm{C}$ & $\operatorname{lnChl}=1 \mathrm{~S} / \mathrm{C} 8 \mathrm{H} 15 / \mathrm{c1}-7(2) 6-8(3,4) 5 / \mathrm{h} 1 \mathrm{H}, 6 \mathrm{H} 2,2-5 \mathrm{H} 3$ \\
\hline 3994 & IC8OOH4-5OR & {$[\mathrm{O}] \mathrm{CC}(\mathrm{CC}(\mathrm{C})(\mathrm{C}) \mathrm{C})(\mathrm{OO}) \mathrm{C}$} & InChl=15/C8H1703/c1-7(2,3)5-8(4,6-9)11-10/h10H,5-6H2,1-4H3 \\
\hline 3995 & IC8OH5Q4-1R & $\mathrm{OOCC}(\mathrm{CC}([\mathrm{CH} 2])(\mathrm{C}) \mathrm{C})(\mathrm{O}) \mathrm{C}$ & $\operatorname{lnChl}=15 / \mathrm{C} 8 \mathrm{H} 1703 / \mathrm{c} 1-7(2,3) 5-8(4,9) 6-11-10 / \mathrm{h} 9-10 \mathrm{H}, 1,5-6 \mathrm{H} 2,2-4 \mathrm{H} 3$ \\
\hline 3996 & IC8OH5-CYO14 & $\mathrm{OCC} 1(\mathrm{C}) \mathrm{OCC}(\mathrm{C} 1)(\mathrm{C}) \mathrm{C}$ & InChl=15/C8H16O2/c1-7(2)4-8(3,5-9)10-6-7/h9H,4-6H2,1-3H3 \\
\hline 3997 & IC8OOH5-4OR & $\operatorname{Oocc}(\operatorname{CC}(\mathrm{C})(\mathrm{C}) \mathrm{C})([\mathrm{O}]) \mathrm{C}$ & InChl=1S/C8H1703/c1-7(2,3)5-8(4,9)6-11-10/h10H,5-6H2,1-4H3 \\
\hline 3998 & IC8OH4Q5-3R & $\mathrm{OOCC}([\mathrm{CH}] \mathrm{C}(\mathrm{C})(\mathrm{C}) \mathrm{C})(\mathrm{O}) \mathrm{C}$ & $\operatorname{lnChl}=1 \mathrm{~S} / \mathrm{C} 8 \mathrm{H} 1703 / \mathrm{c} 1-7(2,3) 5-8(4,9) 6-11-10 / \mathrm{h5}, 9-10 \mathrm{H}, 6 \mathrm{H} 2,1-4 \mathrm{H} 3$ \\
\hline 3999 & IC8OH4-CYO35 & $\mathrm{CC}(\mathrm{C} 1 \mathrm{OCC} 1(\mathrm{C}) \mathrm{O})(\mathrm{C}) \mathrm{C}$ & $\operatorname{lnChl}=1 \mathrm{~S} / \mathrm{C} 8 \mathrm{H} 16 \mathrm{O} 2 / \mathrm{c1}-7(2,3) 6-8(4,9) 5-10-6 / \mathrm{h} 6,9 \mathrm{H}, 5 \mathrm{H} 2,1-4 \mathrm{H} 3$ \\
\hline 4000 & IC8D4-3OH & $\mathrm{CC}(=\mathrm{C}) \mathrm{C}(\mathrm{C}(\mathrm{C})(\mathrm{C}) \mathrm{C}) \mathrm{O}$ & InChl=15/C8H16O/c1-6(2)7(9)8(3,4)5/h7,9H,1H2,2-5H3 \\
\hline
\end{tabular}




\begin{tabular}{|c|c|c|c|}
\hline 4001 & IC8D3-50H & $\mathrm{OCC}(=\mathrm{CC}(\mathrm{C})(\mathrm{C}) \mathrm{C}) \mathrm{C}$ & InChl=15/C8H16O/c1-7(6-9)5-8(2,3)4/h5,9H,6H2,1-4H3 \\
\hline 4002 & IC8D4-5AOH & $\mathrm{OC}=\mathrm{C}(\mathrm{CC}(\mathrm{C})(\mathrm{C}) \mathrm{C}) \mathrm{C}$ & InChl=1S/C8H160/c1-7(6-9)5-8(2,3)4/h6,9H,5H2,1-4H3 \\
\hline 4003 & ME133-CPTR1 & $\mathrm{C}[\mathrm{C}] 1 \mathrm{CCC}(\mathrm{C} 1)(\mathrm{C}) \mathrm{C}$ & InChl=15/C8H15/c1-7-4-5-8(2,3)6-7/h4-6H2,1-3H3 \\
\hline 4004 & IC8D3-5CHO & $\mathrm{O}=\mathrm{CC}(=\mathrm{CC}(\mathrm{C})(\mathrm{C}) \mathrm{C}) \mathrm{C}$ & InChl=1S/C8H14O/c1-7(6-9)5-8(2,3)4/h5-6H,1-4H3 \\
\hline 4005 & IC8D4-5CHO & $\mathrm{O}=\mathrm{CC}(=\mathrm{C}) \mathrm{CC}(\mathrm{C})(\mathrm{C}) \mathrm{C}$ & $\mathrm{InChl}=1 \mathrm{~S} / \mathrm{C} 8 \mathrm{H} 14 \mathrm{O} / \mathrm{c1} 1-7(6-9) 5-8(2,3) 4 / \mathrm{h} 6 \mathrm{H}, 1,5 \mathrm{H} 2,2-4 \mathrm{H} 3$ \\
\hline 4006 & IC8D4-302 & {$[\mathrm{O}] \mathrm{OC}(\mathrm{C}(\mathrm{C})(\mathrm{C}) \mathrm{C}) \mathrm{C}(=\mathrm{C}) \mathrm{C}$} & $\operatorname{lnChl}=1 \mathrm{~S} / \mathrm{C} 8 \mathrm{H} 15 \mathrm{O} 2 / \mathrm{c} 1-6(2) 7(10-9) 8(3,4) 5 / \mathrm{h7H}, 1 \mathrm{H} 2,2-5 \mathrm{H} 3$ \\
\hline 4007 & IC8D3-502 & {$[0] \mathrm{OCC}(=\mathrm{CC}(\mathrm{C})(\mathrm{C}) \mathrm{C}) \mathrm{C}$} & InChl=1S/C8H15O2/c1-7(6-10-9)5-8(2,3)4/h5H,6H2,1-4H3 \\
\hline 4008 & IC8D4-502R & {$[\mathrm{O}] \mathrm{OCC}(=\mathrm{C}) \mathrm{CC}(\mathrm{C})(\mathrm{C}) \mathrm{C}$} & InChl=1S/C8H1502/c1-7(6-10-9)5-8(2,3)4/h1,5-6H2,2-4H3 \\
\hline 4009 & IC8D3-302R & {$[0] O C(=C(C) C) C(C)(C) C$} & InChl=1S/C8H15O2/c1-6(2)7(10-9)8(3,4)5/h1-5H3 \\
\hline 4010 & IC8D4-5AO2R & {$[\mathrm{O}] \mathrm{OC}=\mathrm{C}(\mathrm{CC}(\mathrm{C})(\mathrm{C}) \mathrm{C}) \mathrm{C}$} & $\operatorname{lnChl}=1 \mathrm{~S} / \mathrm{C} 8 \mathrm{H} 15 \mathrm{O} 2 / \mathrm{c} 1-7(6-10-9) 5-8(2,3) 4 / \mathrm{h} 6 \mathrm{H}, 5 \mathrm{H} 2,1-4 \mathrm{H} 3$ \\
\hline 4011 & IC8CYOO14-5R & $\mathrm{CC} 1(\mathrm{C}) \mathrm{COOC}(\mathrm{C} 1)([\mathrm{CH} 2]) \mathrm{C}$ & InChl=1S/C8H1502/c1-7(2)5-8(3,4)10-9-6-7/h3,5-6H2,1-2,4H3 \\
\hline 4012 & 124C7D1305 & $\mathrm{CC}(=\mathrm{CC}(=\mathrm{C}) \mathrm{C}) \mathrm{COO}$ & InChl=1S/C7H12O2/c1-6(2)4-7(3)5-9-8/h4,8H,1,5H2,2-3H3 \\
\hline 4013 & IC8D4CYO1-3 & $\mathrm{CC}(=\mathrm{C}) \mathrm{C} 1 \mathrm{OCC} 1(\mathrm{C}) \mathrm{C}$ & InChl=1S/C8H14O/c1-6(2)7-8(3,4)5-9-7/h7H,1,5H2,2-4H3 \\
\hline 4014 & IC8D4Q1-302 & $\operatorname{Oocc}(\mathrm{C}(\mathrm{C}(=\mathrm{C}) \mathrm{C}) \mathrm{O}[\mathrm{O}])(\mathrm{C}) \mathrm{C}$ & $\operatorname{lnChl}=15 / \mathrm{C} 8 \mathrm{H} 1504 / \mathrm{c1} 1-6(2) 7(12-10) 8(3,4) 5-11-9 / \mathrm{h} 7,9 \mathrm{H}, 1,5 \mathrm{H} 2,2-4 \mathrm{H} 3$ \\
\hline 4015 & IC8D4Q3Y1 & $0 O C(C(C=0)(C) C) C(=C) C$ & $\operatorname{lnChl}=1 \mathrm{~S} / \mathrm{C} 8 \mathrm{H} 1403 / \mathrm{c} 1-6(2) 7(11-10) 8(3,4) 5-9 / \mathrm{h} 5,7,10 \mathrm{H}, 1 \mathrm{H} 2,2-4 \mathrm{H} 3$ \\
\hline 4016 & IC7D1Y5-4J & $\mathrm{CC}(=\mathrm{C}[\mathrm{O}]) \mathrm{CC}(=\mathrm{C}) \mathrm{C}$ & $\operatorname{lnChl}=1 \mathrm{~S} / \mathrm{C} 7 \mathrm{H} 110 / \mathrm{c} 1-6(2) 4-7(3) 5-8 / \mathrm{h} 5 \mathrm{H}, 1,4 \mathrm{H} 2,2-3 \mathrm{H} 3$ \\
\hline 4017 & DIB4-1OR & {$[\mathrm{O}] \mathrm{CC}(\mathrm{CC}(=\mathrm{C}) \mathrm{C})(\mathrm{C}) \mathrm{C}$} & $\operatorname{lnChl}=15 / \mathrm{C} 8 \mathrm{H} 150 / \mathrm{c} 1-7(2) 5-8(3,4) 6-9 / \mathrm{h} 1,5-6 \mathrm{H} 2,2-4 \mathrm{H} 3$ \\
\hline 4018 & $\mathrm{IC} 8 \mathrm{OH} 4 \mathrm{Q} 5-3 \mathrm{O} 2 \mathrm{R}$ & $00 \mathrm{cc}(\mathrm{C}(\mathrm{C}(\mathrm{C})(\mathrm{C}) \mathrm{C}) \mathrm{O}[\mathrm{O}])(\mathrm{O}) \mathrm{C}$ & InChl=1S/C8H1705/c1-7(2,3)6(13-11)8(4,9)5-12-10/h6,9-10H,5H2,1-4H3 \\
\hline 4019 & IC8OH4KET53 & $0 O C(C(C=0)(0) c) c(C)(C) C$ & InChl=15/C8H16O4/c1-7(2,3)6(12-11)8(4,10)5-9/h5-6,10-11H,1-4H3 \\
\hline 4020 & C3Y1-2OH-2J & {$[\mathrm{O}] \mathrm{C}=\mathrm{C}(\mathrm{O}) \mathrm{C}$} & InChl=1S/C3H502/c1-3(5)2-4/h2,5H,1H3 \\
\hline 4021 & IC8D4Q13 & $00 c C(C(C(=) C) C) 00)(C) C$ & $\operatorname{lnChl}=1 \mathrm{~S} / \mathrm{C} 8 \mathrm{H} 1604 / \mathrm{c1}-6(2) 7(12-10) 8(3,4) 5-11-9 / \mathrm{h} 7,9-10 \mathrm{H}, 1,5 \mathrm{H} 2,2-4 \mathrm{H} 3$ \\
\hline 4022 & IC8D3Q15 & $\mathrm{OOCC}(=\mathrm{C}) \mathrm{CC}(\mathrm{COO})(\mathrm{C}) \mathrm{C}$ & InChl=1S/C8H1604/c1-7(5-11-9)4-8(2,3)6-12-10/h9-10H,1,4-6H2,2-3H3 \\
\hline 4023 & IC8D4Q1-30J & $00 c c(C(C(=) C) C)[O])(C) C$ & $\operatorname{lnChl}=15 / \mathrm{C} 8 \mathrm{H} 1503 / \mathrm{c} 1-6(2) 7(9) 8(3,4) 5-11-10 / \mathrm{h} 7,10 \mathrm{H}, 1,5 \mathrm{H} 2,2-4 \mathrm{H} 3$ \\
\hline 4024 & IC8D3Q1-5OJ & $\mathrm{OOCC}(\mathrm{CC}(=\mathrm{C}) \mathrm{C}[\mathrm{O}])(\mathrm{C}) \mathrm{C}$ & InChl=1S/C8H15O3/c1-7(5-9)4-8(2,3)6-11-10/h10H,1,4-6H2,2-3H3 \\
\hline 4025 & IC8D3Q1Y5 & $000 \mathrm{CCC}(=\mathrm{C}) \mathrm{C}=\mathrm{O})(\mathrm{C}) \mathrm{C}$ & $\operatorname{lnChl}=15 / C 8 \mathrm{H} 1403 / \mathrm{c1}-7(5-9) 4-8(2,3) 6-11-10 / \mathrm{h} 5,10 \mathrm{H}, 1,4,6 \mathrm{H} 2,2-3 \mathrm{H} 3$ \\
\hline 4026 & IC8D3Q1Y5J & $00 c C(C C(=C)[C]=0)(C) C$ & InChl=1S/C8H1303/c1-7(5-9)4-8(2,3)6-11-10/h10H,1,4,6H2,2-3H3 \\
\hline 4027 & NEC7Q1D3-4R & $000 C(C[C]=C)(C) C$ & InChl=1S/C7H13O2/c1-4-5-7(2,3)6-9-8/h8H,1,5-6H2,2-3H3 \\
\hline 4028 & IC8D4Q3-1 & $\mathrm{OOC}(\mathrm{C}([\mathrm{CH} 2])(\mathrm{C}) \mathrm{C}) \mathrm{C}(=\mathrm{C}) \mathrm{C}$ & $\operatorname{lnChl}=1 \mathrm{~S} / \mathrm{C} 8 \mathrm{H} 15 \mathrm{O} 2 / \mathrm{c} 1-6(2) 7(10-9) 8(3,4) 5 / \mathrm{h7}, 9 \mathrm{H}, 1,3 \mathrm{H} 2,2,4-5 \mathrm{H} 3$ \\
\hline 4029 & 124C7D14Q3 & $\mathrm{OOC}(\mathrm{C}(=\mathrm{C}) \mathrm{C}) \mathrm{C}(=\mathrm{C}) \mathrm{C}$ & InChl=1S/C7H12O2/c1-5(2)7(9-8)6(3)4/h7-8H,1,3H2,2,4H3 \\
\hline 4030 & IC8D4Q3-5 & $\mathrm{OOC}(\mathrm{C}(\mathrm{C})(\mathrm{C}) \mathrm{C}) \mathrm{C}(=\mathrm{C})[\mathrm{CH} 2]$ & $\operatorname{lnChl}=1 \mathrm{~S} / \mathrm{C} 8 \mathrm{H} 15 \mathrm{O} 2 / \mathrm{c1}-6(2) 7(10-9) 8(3,4) 5 / \mathrm{h} 7,9 \mathrm{H}, 1-2 \mathrm{H} 2,3-5 \mathrm{H} 3$ \\
\hline 4031 & IC8D4Q3-102 & {$[\mathrm{O}] \mathrm{OCC}(\mathrm{C}(\mathrm{C}(=\mathrm{C}) \mathrm{C}) \mathrm{OO})(\mathrm{C}) \mathrm{C}$} & InChl=1S/C8H15O4/c1-6(2)7(12-10)8(3,4)5-11-9/h7,10H,1,5H2,2-4H3 \\
\hline 4032 & IC8D4Q1Y3 & $\operatorname{Oocc}(\mathrm{C}(=) \mathrm{O}) \mathrm{C}(=\mathrm{C}) \mathrm{C})(\mathrm{C}) \mathrm{C}$ & InChl=1S/C8H1403/c1-6(2)7(9)8(3,4)5-11-10/h10H,1,5H2,2-4H3 \\
\hline 4033 & C7H11O & {$[\mathrm{O}] \mathrm{C}(=\mathrm{C}(\mathrm{C}) \mathrm{C}) \mathrm{C}(=\mathrm{C}) \mathrm{C}$} & $\operatorname{lnChl}=1 \mathrm{~S} / \mathrm{C} 7 \mathrm{H} 11 \mathrm{O} / \mathrm{c} 1-5(2) 7(8) 6(3) 4 / \mathrm{h} 1 \mathrm{H} 2,2-4 \mathrm{H} 3$ \\
\hline 4034 & 124C7D1CYO3-4 & $\mathrm{CC}(=\mathrm{C}) \mathrm{C} 10 \mathrm{OC} 1(\mathrm{C}) \mathrm{C}$ & $\operatorname{lnChl}=1 \mathrm{~S} / \mathrm{C} 7 \mathrm{H} 12 \mathrm{O} / \mathrm{c1}-5(2) 6-7(3,4) 8-6 / \mathrm{h} 6 \mathrm{H}, 1 \mathrm{H} 2,2-4 \mathrm{H} 3$ \\
\hline 4035 & IC8D4Q3-502 & {$[0] O C C(=C) C(C(C)(C) C) O O$} & InChl=1S/C8H15O4/c1-6(5-11-9)7(12-10)8(2,3)4/h7,10H,1,5H2,2-4H3 \\
\hline 4036 & IC8D4Q5Y3 & $\mathrm{OOCC}(=\mathrm{C}) \mathrm{C}(=\mathrm{O}) \mathrm{C}(\mathrm{C})(\mathrm{C}) \mathrm{C}$ & InChl=1S/C8H1403/c1-6(5-11-10)7(9)8(2,3)4/h10H,1,5H2,2-4H3 \\
\hline 4037 & IC8D4Y3-5OJ & {$[\mathrm{O}] \mathrm{CC}(=\mathrm{C}) \mathrm{C}(=\mathrm{O}) \mathrm{C}(\mathrm{C})(\mathrm{C}) \mathrm{C}$} & InChl=15/C8H13O2/c1-6(5-9)7(10)8(2,3)4/h1,5H2,2-4H3 \\
\hline 4038 & IC8D4Y35 & $\mathrm{O}=\mathrm{CC}(=\mathrm{C}) \mathrm{C}(=\mathrm{O}) \mathrm{C}(\mathrm{C})(\mathrm{C}) \mathrm{C}$ & InChl=1S/C8H12O2/c1-6(5-9)7(10)8(2,3)4/h5H,1H2,2-4H3 \\
\hline 4039 & IC8D4Y3-5J & $\mathrm{O}=[\mathrm{C}] \mathrm{C}(=\mathrm{C}) \mathrm{C}(=\mathrm{O}) \mathrm{C}(\mathrm{C})(\mathrm{C}) \mathrm{C}$ & $\operatorname{lnChl}=1 \mathrm{~S} / \mathrm{C} 8 \mathrm{H} 11 \mathrm{O} 2 / \mathrm{c} 1-6(5-9) 7(10) 8(2,3) 4 / \mathrm{h} 1 \mathrm{H} 2,2-4 \mathrm{H} 3$ \\
\hline 4040 & TC4H9COCCH2 & $\mathrm{C}=\mathrm{C}=\mathrm{C}(\mathrm{C}(\mathrm{C})(\mathrm{C}) \mathrm{C})[\mathrm{O}]$ & InChl=1S/C7H11O/c1-5-6(8)7(2,3)4/h1H2,2-4H3 \\
\hline 4041 & IC8D4Q35 & $00 c C(=C) C(C(C)(C) C) 00$ & InChl=1S/C8H1604/c1-6(5-11-9)7(12-10)8(2,3)4/h7,9-10H,1,5H2,2-4H3 \\
\hline 4042 & IC8D4Q5-3R & $\mathrm{OOCC}(=\mathrm{C})[\mathrm{CH}] \mathrm{C}(\mathrm{C})(\mathrm{C}) \mathrm{C}$ & InChl=15/C8H15O2/c1-7(6-10-9)5-8(2,3)4/h5,9H,1,6H2,2-4H3 \\
\hline 4043 & IC8D3Q5-5O2R & $\mathrm{OOCC}(=\mathrm{CC}(\mathrm{C})(\mathrm{C}) \mathrm{C}) \mathrm{CO}[\mathrm{O}]$ & InChl=15/C8H15O4/c1-8(2,3)4-7(5-11-9)6-12-10/h4,9H,5-6H2,1-3H3 \\
\hline 4044 & IC8D3Q5Y5 & $\mathrm{OOCC}(=\mathrm{CC}(\mathrm{C})(\mathrm{C}) \mathrm{C}) \mathrm{C}=\mathrm{O}$ & InChl=1S/C8H14O3/c1-8(2,3)4-7(5-9)6-11-10/h4-5,10H,6H2,1-3H3 \\
\hline 4045 & IC8D3Q55 & $00 \mathrm{OCC}(=\mathrm{CC}(\mathrm{C})(\mathrm{C}) \mathrm{C}) \mathrm{COO}$ & InChl=1S/C8H1604/c1-8(2,3)4-7(5-11-9)6-12-10/h4,9-10H,5-6H2,1-3H3 \\
\hline 4046 & NEC6D3-4R & {$[\mathrm{CH}]=\mathrm{CC}(\mathrm{C})(\mathrm{C}) \mathrm{C}$} & $\operatorname{lnChl}=1 \mathrm{~S} / \mathrm{C} 6 \mathrm{H} 11 / \mathrm{c} 1-5-6(2,3) 4 / \mathrm{h} 1,5 \mathrm{H}, 2-4 \mathrm{H} 3$ \\
\hline 4047 & IC8D3Q5-50J J & $\mathrm{OOCC}(=\mathrm{CC}(\mathrm{C})(\mathrm{C}) \mathrm{C}) \mathrm{C}[\mathrm{O}]$ & InChl=1S/C8H15O3/c1-8(2,3)4-7(5-9)6-11-10/h4,10H,5-6H2,1-3H3 \\
\hline 4048 & C7H1102 & $\mathrm{CC}(=\mathrm{C}) \mathrm{C}(=0) \mathrm{C}([\mathrm{O}])(\mathrm{C}) \mathrm{C}$ & InChl=1S/C7H11O2/c1-5(2)6(8)7(3,4)9/h1H2,2-4H3 \\
\hline 4049 & $\mathrm{C} 7 \mathrm{H} 12 \mathrm{O} 3$ & $\operatorname{OOC}(\mathrm{C}(=\mathrm{O}) \mathrm{C}(=\mathrm{C}) \mathrm{C})(\mathrm{C}) \mathrm{C}$ & InChl=1S/C7H12O3/c1-5(2)6(8)7(3,4)10-9/h9H,1H2,2-4H3 \\
\hline 4050 & IC8CYC3O2-4R & $\mathrm{CC}(\mathrm{C}[\mathrm{C}] 1 \mathrm{COOC} 1)(\mathrm{C}) \mathrm{C}$ & $\operatorname{lnChl}=1 \mathrm{~S} / \mathrm{C} 8 \mathrm{H} 15 \mathrm{O} 2 / \mathrm{c} 1-8(2,3) 4-7-5-9-10-6-7 / \mathrm{h} 4-6 \mathrm{H} 2,1-3 \mathrm{H} 3$ \\
\hline 4051 & IC8CYC2O2-5R & $\mathrm{CC}(\mathrm{CC} 1([\mathrm{CH} 2]) \mathrm{COO} 1)(\mathrm{C}) \mathrm{C}$ & InChl=15/C8H1502/c1-7(2,3)5-8(4)6-9-10-8/h4-6H2,1-3H3 \\
\hline 4052 & 124C7D13Q1 & $\mathrm{OOCC}(=\mathrm{C}) \mathrm{C}=\mathrm{C}(\mathrm{C}) \mathrm{C}$ & InChl=15/C7H12O2/c1-6(2)4-7(3)5-9-8/h4,8H,3,5H2,1-2H3 \\
\hline 4053 & IC8D4Q5-302 & $00 C C(=C) C(C(C)(C) C) O[0]$ & InChl=1S/C8H15O4/c1-6(5-11-9)7(12-10)8(2,3)4/h7,9H,1,5H2,2-4H3 \\
\hline 4054 & IC8D4Q5-30J & $0 O C C(=C) C(C(C)(C) C)[0]$ & $\operatorname{lnChl}=15 / \mathrm{C} 8 \mathrm{H} 1503 / \mathrm{c} 1-6(5-11-10) 7(9) 8(2,3) 4 / \mathrm{h7}, 10 \mathrm{H}, 1,5 \mathrm{H} 2,2-4 \mathrm{H} 3$ \\
\hline 4055 & IC8D4Q3Y5 & $0 O C(C(C)(C) C) C(=C) C=0$ & InChl=1S/C8H14O3/c1-6(5-9)7(11-10)8(2,3)4/h5,7,10H,1H2,2-4H3 \\
\hline 4056 & 124C7D3OH1-2R & $\mathrm{OC}[\mathrm{C}](\mathrm{C}=\mathrm{C}(\mathrm{C}) \mathrm{C}) \mathrm{C}$ & $\operatorname{lnChl}=1 \mathrm{~S} / \mathrm{C} 7 \mathrm{H} 13 \mathrm{O} / \mathrm{c1} 1-6(2) 4-7(3) 5-8 / \mathrm{h} 4,8 \mathrm{H}, 5 \mathrm{H} 2,1-3 \mathrm{H} 3$ \\
\hline 4057 & 124C7D1OH4-3R & $\mathrm{CC}(=\mathrm{C})[\mathrm{CH}] \mathrm{C}(\mathrm{O})(\mathrm{C}) \mathrm{C}$ & InChl=1S/C7H13O/c1-6(2)5-7(3,4)8/h5,8H,1H2,2-4H3 \\
\hline 4058 & 124C7D3OH1-2RO2 & $\mathrm{OCC}(\mathrm{C}=\mathrm{C}(\mathrm{C}) \mathrm{C})(\mathrm{O}[\mathrm{O}]) \mathrm{C}$ & $\operatorname{lnChl}=1 \mathrm{~S} / \mathrm{C} 7 \mathrm{H} 1303 / \mathrm{c} 1-6(2) 4-7(3,5-8) 10-9 / \mathrm{h} 4,8 \mathrm{H}, 5 \mathrm{H} 2,1-3 \mathrm{H} 3$ \\
\hline 4059 & 124C7D1OH4-3RO2 & {$[\mathrm{O}] \mathrm{OC}(\mathrm{C}(\mathrm{O})(\mathrm{C}) \mathrm{C}) \mathrm{C}(=\mathrm{C}) \mathrm{C}$} & InChl=1S/C7H1303/c1-5(2)6(10-9)7(3,4)8/h6,8H,1H2,2-4H3 \\
\hline 4060 & 124C7D14-30J & {$[\mathrm{O}] \mathrm{C}(\mathrm{C}(=\mathrm{C}) \mathrm{C}) \mathrm{C}(=\mathrm{C}) \mathrm{C}$} & $\operatorname{lnChl}=1 \mathrm{~S} / \mathrm{C} 7 \mathrm{H} 11 \mathrm{O} / \mathrm{c1} 1-5(2) 7(8) 6(3) 4 / \mathrm{h} 7 \mathrm{H}, 1,3 \mathrm{H} 2,2,4 \mathrm{H} 3$ \\
\hline 4061 & IC8D3-5CO & $\mathrm{O}=[\mathrm{C}] \mathrm{C}(=\mathrm{CC}(\mathrm{C})(\mathrm{C}) \mathrm{C}) \mathrm{C}$ & InChl=1S/C8H130/c1-7(6-9)5-8(2,3)4/h5H,1-4H3 \\
\hline 4062 & IC8D4-5CO & $\mathrm{O}=[\mathrm{C}] \mathrm{C}(=\mathrm{C}) \mathrm{CC}(\mathrm{C})(\mathrm{C}) \mathrm{C}$ & $\operatorname{lnChl}=1 \mathrm{~S} / \mathrm{C} 8 \mathrm{H} 130 / \mathrm{c} 1-7(6-9) 5-8(2,3) 4 / \mathrm{h} 1,5 \mathrm{H} 2,2-4 \mathrm{H} 3$ \\
\hline 4063 & IC8D4Y5-1] & $\mathrm{O}=\mathrm{CC}(=\mathrm{C}) \mathrm{CC}([\mathrm{CH} 2])(\mathrm{C}) \mathrm{C}$ & InChl=15/C8H13O/c1-7(6-9)5-8(2,3)4/h6H,1-2,5H2,3-4H3 \\
\hline 4064 & IC8D3Y5-1J & $\mathrm{O}=\mathrm{CC}(=\mathrm{CC}([\mathrm{CH} 2])(\mathrm{C}) \mathrm{C}) \mathrm{C}$ & InChl=1S/C8H130/c1-7(6-9)5-8(2,3)4/h5-6H,2H2,1,3-4H3 \\
\hline 4065 & IC8D4Y5-3J & $\mathrm{O}=\mathrm{CC}(=\mathrm{C})[\mathrm{CH}] \mathrm{C}(\mathrm{C})(\mathrm{C}) \mathrm{C}$ & InChl=1S/C8H130/c1-7(6-9)5-8(2,3)4/h5-6H,1H2,2-4H3 \\
\hline 4066 & 124C7D3CYO1-2 & $\mathrm{CC}(=\mathrm{CC} 1(\mathrm{C}) \mathrm{CO} 1) \mathrm{C}$ & InChl=1S/C7H12O/c1-6(2)4-7(3)5-8-7/h4H,5H2,1-3H3 \\
\hline 4067 & 124C7D1-10J & {$[\mathrm{O}] \mathrm{CC}(=\mathrm{C}) \mathrm{CC}(\mathrm{C}) \mathrm{C}$} & $\operatorname{lnChl}=1 \mathrm{~S} / \mathrm{C} 7 \mathrm{H} 13 \mathrm{O} / \mathrm{c1}-6(2) 4-7(3) 5-8 / \mathrm{h} 6 \mathrm{H}, 3-5 \mathrm{H} 2,1-2 \mathrm{H3}$ \\
\hline 4068 & 124C7D1-Y1 & $\mathrm{O}=\mathrm{CC}(=\mathrm{C}) \mathrm{CC}(\mathrm{C}) \mathrm{C}$ & InChl=1S/C7H12O/c1-6(2)4-7(3)5-8/h5-6H,3-4H2,1-2H3 \\
\hline 4069 & I24C7D1-Y1J & $O=[C] C(=C) C C(C) C$ & InChl=1S/C7H110/c1-6(2)4-7(3)5-8/h6H,3-4H2,1-2H3 \\
\hline 4070 & IC6D4-4R & $\mathrm{C}=[\mathrm{C}] \mathrm{CC}(\mathrm{C}) \mathrm{C}$ & InChl=1S/C6H11/c1-4-5-6(2)3/h6H,1,5H2,2-3H3 \\
\hline 4071 & IC6D14Q3 & $\mathrm{OOC}(\mathrm{C}(=\mathrm{C}) \mathrm{C}) \mathrm{C}=\mathrm{C}$ & InChl=15/C6H10O2/c1-4-6(8-7)5(2)3/h4,6-7H,1-2H2,3H3 \\
\hline 4072 & IC6D1403J & $\mathrm{C}=\mathrm{CC}(\mathrm{C}(=\mathrm{C}) \mathrm{C})[\mathrm{O}]$ & InChl=1S/C6H9O/c1-4-6(7)5(2)3/h4,6H,1-2H2,3H3 \\
\hline 4073 & XC6D13-1Q & $\mathrm{CC}(=\mathrm{C}) \mathrm{C}(=\mathrm{C}) \mathrm{COO}$ & $\operatorname{lnChl}=15 / C 6 H 1002 / c 1-5(2) 6(3) 4-8-7 / \mathrm{h} 7 \mathrm{H}, 1,3-4 \mathrm{H} 2,2 \mathrm{H} 3$ \\
\hline 4074 & XC6D13-10J & $\mathrm{CC}(=\mathrm{C}) \mathrm{C}(=\mathrm{C}) \mathrm{C}[\mathrm{O}]$ & InChl=1S/C6H9O/c1-5(2)6(3)4-7/h1,3-4H2,2H3 \\
\hline 4075 & XC6D13-Y1 & $\mathrm{CC}(=\mathrm{C}) \mathrm{C}(=\mathrm{C}) \mathrm{C}=\mathrm{O}$ & $\operatorname{lnChl}=1 \mathrm{~S} / \mathrm{C} 6 \mathrm{H} 8 \mathrm{O} / \mathrm{c1}-5(2) 6(3) 4-7 / \mathrm{h} 4 \mathrm{H}, 1,3 \mathrm{H} 2,2 \mathrm{H} 3$ \\
\hline 4076 & XC6D13Y1J & $\mathrm{CC}(=\mathrm{C}) \mathrm{C}(=\mathrm{C})[\mathrm{C}]=\mathrm{O}$ & InChl=1S/C6H7O/c1-5(2)6(3)4-7/h1,3H2,2H3 \\
\hline 4077 & B13DE2M-3J & $\mathrm{C}=[\mathrm{C}] \mathrm{C}(=\mathrm{C}) \mathrm{C}$ & InChl=1S/C5H7/c1-4-5(2)3/h1-2H2,3H3 \\
\hline 4078 & DIBD4-DIMER & $\mathrm{C}=\mathrm{C}(\mathrm{CC}(\mathrm{C})(\mathrm{C}) \mathrm{C}) \mathrm{CCC}(=\mathrm{C}) \mathrm{CC}(\mathrm{C})(\mathrm{C}) \mathrm{C}$ & $\operatorname{lnChl}=1 \mathrm{~S} / \mathrm{C} 16 \mathrm{H} 30 / \mathrm{c} 1-13(11-15(3,4) 5) 9-10-14(2) 12-16(6,7) 8 / \mathrm{h} 1-2,9-12 \mathrm{H} 2,3-8 \mathrm{H} 3$ \\
\hline 4079 & RDIBD4-DIMER & $\mathrm{C}=\mathrm{C}(\mathrm{CC}(\mathrm{C})(\mathrm{C}) \mathrm{C})[\mathrm{CH}] \mathrm{CC}(=\mathrm{C}) \mathrm{CC}(\mathrm{C})(\mathrm{C}) \mathrm{C}$ & InChl=1S/C16H29/c1-13(11-15(3,4)5)9-10-14(2)12-16(6,7)8/h9H,1-2,10-12H2,3-8H3 \\
\hline 4080 & DIBD3-DIMER & $\mathrm{CC}(=\mathrm{CC}(\mathrm{C})(\mathrm{C}) \mathrm{C}) \mathrm{CCC}(=\mathrm{CC}(\mathrm{C})(\mathrm{C}) \mathrm{C}) \mathrm{C}$ & InChl=1S/C16H30/c1-13(11-15(3,4)5)9-10-14(2)12-16(6,7)8/h11-12H,9-10H2,1-8H3 \\
\hline 4081 & RDIBD3-DIMER & $\mathrm{CC}(=\mathrm{CCC}(=\mathrm{CC}(\mathrm{C})(\mathrm{C}) \mathrm{C}) \mathrm{C})[\mathrm{CH}] \mathrm{C}(\mathrm{C})(\mathrm{C}) \mathrm{C}$ & InChl=1S/C16H29/c1-13(11-15(3,4)5)9-10-14(2)12-16(6,7)8/h9,11-12H,10H2,1-8H3 \\
\hline 4082 & $\mathrm{IC6OH} 2 \mathrm{D} 3-1 \mathrm{R}$ & $\mathrm{CC}=\mathrm{CC}(\mathrm{O})([\mathrm{CH} 2]) \mathrm{C}$ & $\operatorname{lnChl}=1 \mathrm{~S} / \mathrm{C} 6 \mathrm{H} 110 / \mathrm{c} 1-4-5-6(2,3) 7 / \mathrm{h} 4-5,7 \mathrm{H}, 2 \mathrm{H} 2,1,3 \mathrm{H} 3$ \\
\hline 4083 & IC6D3OH2-102 & $\mathrm{CC}(\mathrm{C}=\mathrm{CC})(\mathrm{CO}[\mathrm{O}]) \mathrm{O}$ & InChl=1S/C6H1103/c1-3-4-6(2,7)5-9-8/h3-4,7H,5H2,1-2H3 \\
\hline 4084 & IC6D3OH1-2R & $\mathrm{C}[\mathrm{C}](\mathrm{C}=\mathrm{CC}) \mathrm{CO}$ & $\operatorname{lnChl}=1 \mathrm{~S} / \mathrm{C} 6 \mathrm{H} 110 / \mathrm{c1} 1-3-4-6(2) 5-7 / \mathrm{h} 3-4,7 \mathrm{H}, 5 \mathrm{H} 2,1-2 \mathrm{H} 3$ \\
\hline 4085 & IC6D3OH1-202 & $\mathrm{CC}=\mathrm{CC}(\mathrm{O}[\mathrm{O}])(\mathrm{CO}) \mathrm{C}$ & InChl=1S/C6H1103/c1-3-4-6(2,5-7)9-8/h3-4,7H,5H2,1-2H3 \\
\hline 4086 & DIB3DIMER-OHJ & $\mathrm{CC}(=\mathrm{CC}(\mathrm{C})(\mathrm{C}) \mathrm{C}) \mathrm{CC}[\mathrm{C}](\mathrm{C}(\mathrm{C}(\mathrm{C})(\mathrm{C}) \mathrm{C}) \mathrm{O}) \mathrm{C}$ & $\operatorname{lnChl}=1 \mathrm{~S} / \mathrm{C} 16 \mathrm{H} 310 / \mathrm{c1}-12(11-15(3,4) 5) 9-10-13(2) 14(17) 16(6,7) 8 / \mathrm{h} 11,14,17 \mathrm{H}, 9-10 \mathrm{H} 2,1-8 \mathrm{H} 3$ \\
\hline 4087 & DIB4DIMER-OHJ & $\mathrm{OC}[\mathrm{C}](\mathrm{CC}(\mathrm{C})(\mathrm{C}) \mathrm{C}) \mathrm{CCC}(=\mathrm{C}) \mathrm{CC}(\mathrm{C})(\mathrm{C}) \mathrm{C}$ & $\operatorname{lnChl}=15 / \mathrm{C} 16 \mathrm{H} 310 / \mathrm{c} 1-13(10-15(2,3) 4) 8-9-14(12-17) 11-16(5,6) 7 / \mathrm{h} 17 \mathrm{H}, 1,8-12 \mathrm{H} 2,2-7 \mathrm{H} 3$ \\
\hline 4088 & NEC6D3-402 & {$[\mathrm{O}] \mathrm{OC}=\mathrm{CC}(\mathrm{C})(\mathrm{C}) \mathrm{C}$} & $\operatorname{lnChl}=1 \mathrm{~S} / \mathrm{C} 6 \mathrm{H} 11 \mathrm{O} 2 / \mathrm{c1}-6(2,3) 4-5-8-7 / \mathrm{h} 4-5 \mathrm{H}, 1-3 \mathrm{H} 3$ \\
\hline
\end{tabular}

